Supporting Information for

\title{
Enantioselective Construction of Quinoxaline-Based Heterobiaryls and P,N-Ligands via Chirality Transfer Strategy
}

Zeng Gao, Fang Wang, Jinlong Qian, Huameng Yang, Chungu Xia*, Jinlong Zhang*, and Gaoxi Jiang*

Z. Gao, F. Wang, Dr. J. Qian, H. Yang, Prof. Dr. C. Xia, Dr. J. Zhang, Prof. Dr. G. Jiang State Key Laboratory for Oxo Synthesis and Selective Oxidation, Center for Excellence in Molecular Synthesis, Suzhou Research Institute of LICP, Lanzhou Institute of Chemical Physics (LICP),

Chinese Academy of Sciences, Lanzhou 730000, P. R. China

E-mail: cgxia@lzb.ac.cn, zhangj1@licp.cas.cn,gxjiang@licp.cas.cn

Z. Gao

University of Chinese Academy of Sciences, Beijing 100049, P. R.

\section{Table of Contents}

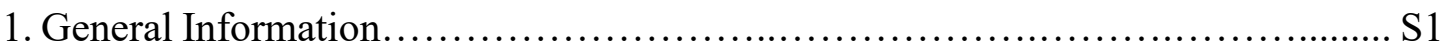

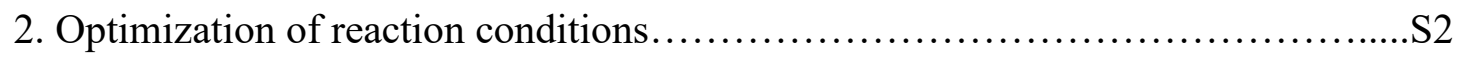

3. Optimization of oxidation conditions.......................................... 4

4. Evaluation of the DHPLC profiles.............................................. 7

5. Investigation of the steric effects on central-to-axial chirality transfer process......S8

6. Synthesis and characterization data of substrates ............................. 11

7. Synthesis and characterization data of products ............................... 16

8. Synthesis of quinoxalinap ligands ...................................... 31

9. Application of quinoxalinap ligands....................................... 36

10. Crystallographic data for compound (R)-5a ............................... 38

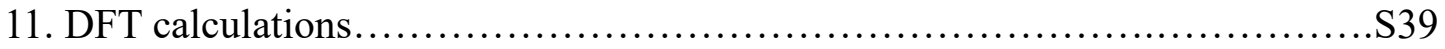

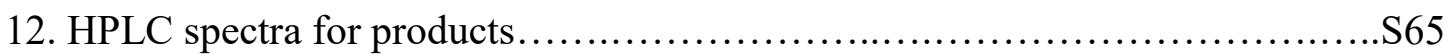

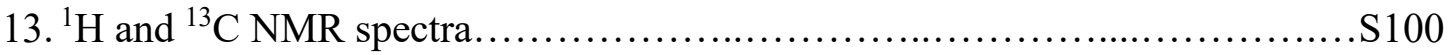




\section{General information}

Experimental: All reactions were set up under inert atmosphere utilizing glassware that was flame-dried and cooled under vacuum. All non-aqueous manipulations were using standard Schlenk techniques. Reactions were monitored using thin-layer chromatography (TLC) on silica gel plates. Visualization of the developed plates was performed under UV light $(254 \mathrm{~nm})$ or $\mathrm{KMnO}_{4}$ stain. Silica gel flash column chromatography was performed on SYNTHWARE 40-63 $\mu \mathrm{m}$ silica gel.

Instrumentation: All NMR spectra were run at $400 \mathrm{MHz}\left({ }^{1} \mathrm{H} \mathrm{NMR}\right), 100 \mathrm{MHz}\left({ }^{13} \mathrm{C} \mathrm{NMR}\right), 377 \mathrm{MHz}\left({ }^{19} \mathrm{~F}\right.$ $\mathrm{NMR})$ and $162 \mathrm{MHz}\left({ }^{31} \mathrm{P} \mathrm{NMR}\right)$ in $\mathrm{CDCl}_{3}$ or $\mathrm{d}_{6}$-DMSO solution. ${ }^{1} \mathrm{H}$ NMR spectra were internally referenced to TMS. ${ }^{13} \mathrm{C}$ NMR spectra were internally referenced to the residual solvent signal. Data for ${ }^{1} \mathrm{H}$ NMR are reported as follows: chemical shift $(\delta \mathrm{ppm})$, multiplicity $(\mathrm{s}=$ singlet, $\mathrm{d}=$ doublet, $\mathrm{t}=$ triplet, $\mathrm{q}=$ quartet, $\mathrm{m}=$ multiplet, $\mathrm{br}=$ broad), coupling constants $(J)$ were reported in $\mathrm{Hz}$. High resolution mass spectra (HRMS) were recorded on Bruker MicrOTOF-QII mass instrument (ESI).

Materials: Unless otherwise indicated, starting catalysts and materials were obtained from Sigma Aldrich, $\mathrm{TCl}$, Alfa Aesar, Adamas or Acros. Moreover, commercially available reagents were used without additional purification. 


\section{Optimization of reaction conditions}

Table S1[a]

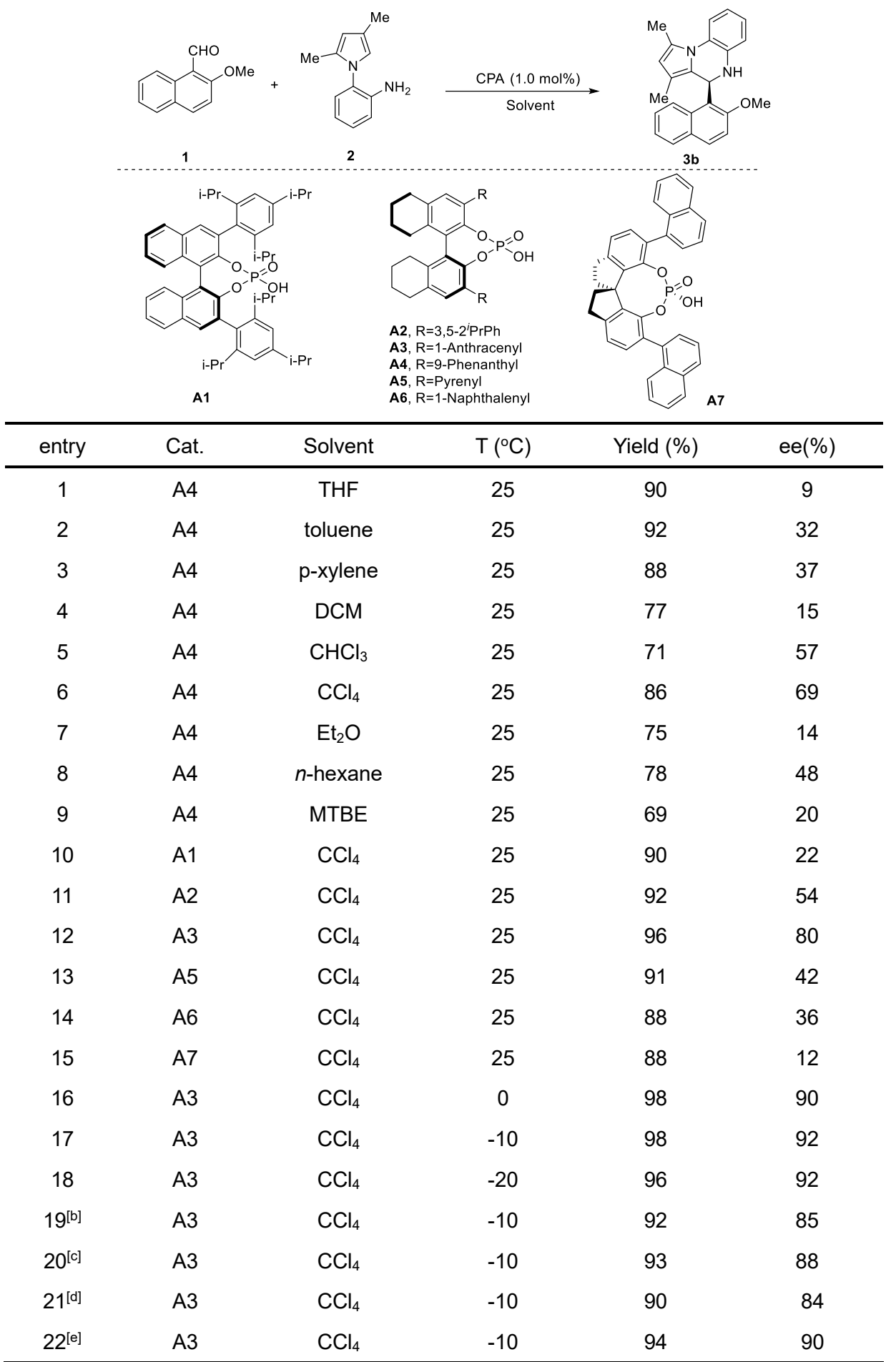

[a] Reaction conditions: 1 (0.20 mmol), $2(0.24 \mathrm{mmol}, 1.2$ equiv.) and chiral phosphoric acid (1.0 mol \%) were stirred in solvent $(1.0 \mathrm{~mL}, 0.1 \mathrm{M})$ for $8 \mathrm{~h}$, isolated yield was given, ee value was determined by chiral HPLC analysis. [b] $100 \mathrm{mg}$ of $3 \AA$ molecular sieves was added. [c] $100 \mathrm{mg}$ of $4 \AA$ molecular sieves was added. [d] $100 \mathrm{mg}$ of $5 \AA$ molecular sieves was added. [e] $100 \mathrm{mg}$ of $\mathrm{MgSO}_{4}$ was added. 


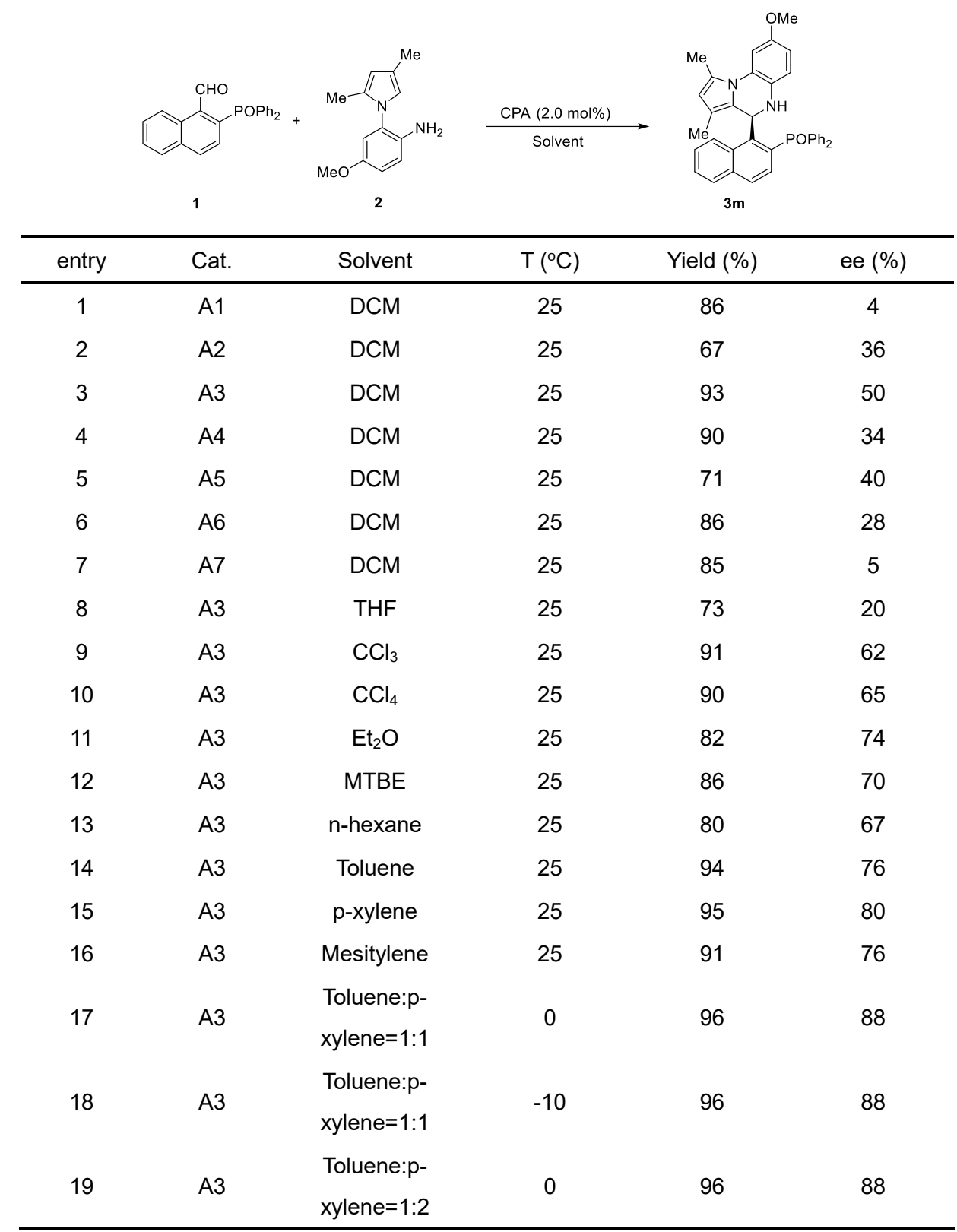

[a] Reaction conditions: 1 (0.10 mmol), $2(0.12 \mathrm{mmol}, 1.2$ equiv.) and chiral phosphoric acid (2.0 mol \%) were stirred in solvent $(1.0 \mathrm{~mL}, 0.1 \mathrm{M})$ for $12 \mathrm{~h}$, isolated yield was given, ee value was determined by chiral HPLC analysis. 


\section{Optimization of oxidation conditions}

\section{Table $S 3^{[a]}$}

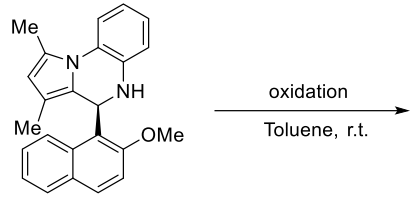

3b

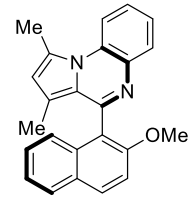

4b

\begin{tabular}{cccc}
\hline entry & Oxidant & Yield (\%) & ee (\%) \\
\hline 1 & $\mathrm{MnO}_{2}$ & 86 & 40 \\
2 & $\mathrm{DDQ}$ & - & - \\
3 & $\mathrm{tBuO}_{2} \mathrm{H}$ & 35 & 56 \\
4 & $\mathrm{KMnO}_{4}$ & 78 & 36 \\
5 & $\mathrm{BPO}$ & - & - \\
6 & $\mathrm{CPBA}$ & - & - \\
7 & $\mathrm{Phl}(\mathrm{OAc})_{2}$ & - & - \\
8 & $\mathrm{Phl}\left(\mathrm{CF}_{3} \mathrm{CO}_{2}\right)_{2}$ & - & - \\
\hline
\end{tabular}

[a] Reaction conditions: 3 b $(0.10 \mathrm{mmol})$ and oxidant $(1 \mathrm{mmol}, 10$ equiv. $)$ were stirred in toluene $(1.0 \mathrm{~mL}, 0.1 \mathrm{M})$ for $12 \mathrm{~h}$, isolated yield was given, ee value was determined by chiral HPLC analysis.

Table S4[a]

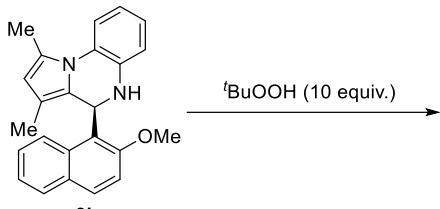

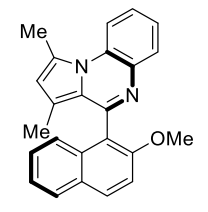

4b

\begin{tabular}{ccccc}
\hline entry & Solvent & $\mathrm{T}\left({ }^{\circ} \mathrm{C}\right)$ & Yield $(\%)$ & ee $(\%)$ \\
\hline 1 & $\mathrm{THF}$ & 25 & 49 & 20 \\
2 & $\mathrm{CHCl}_{3}$ & 25 & 54 & 62 \\
3 & $\mathrm{CCl}_{4}$ & 25 & 56 & 68 \\
4 & $\mathrm{Et}_{2} \mathrm{O}$ & 25 & 48 & 60 \\
5 & $\mathrm{CCl}_{4}$ & 0 & 45 & 72 \\
\hline
\end{tabular}

[a] Reaction conditions: $3 \mathbf{b}(0.10 \mathrm{mmol})$ and ${ }^{\mathrm{t}} \mathrm{BuOOH}(1 \mathrm{mmol}, 10$ equiv. $)$ were stirred in solvent $(1.0 \mathrm{~mL}, 0.1 \mathrm{M})$. After 3 days, the starting material was a little existed in reaction and the reaction almost stopped. The staring materials was recycled. isolated yield was given, ee value was determined by chiral HPLC analysis. 
Table S5 ${ }^{[a]}$

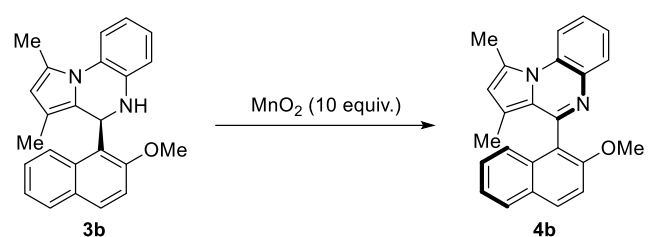

\begin{tabular}{ccccc}
\hline entry & Solvent & $\mathrm{T}\left({ }^{\circ} \mathrm{C}\right)$ & Yield (\%) & ee (\%) \\
\hline 1 & toluene & 0 & 86 & 40 \\
2 & $\mathrm{EA}$ & 0 & 80 & 14 \\
3 & $\mathrm{PhCl}$ & 0 & 82 & 20 \\
4 & $\mathrm{CHCl}_{3}$ & 0 & 80 & 28 \\
5 & $\mathrm{CCl}_{4}$ & 0 & 66 & 22 \\
6 & $\mathrm{THF}_{7}$ & 0 & 73 & 26 \\
8 & toluene & -5 & 85 & 40 \\
\hline
\end{tabular}

[a] Reaction conditions: $\mathbf{3 b}(0.10 \mathrm{mmol})$ and $\mathrm{MnO}_{2}(1 \mathrm{mmol}, 10$ equiv. $)$ were stirred in solvent

$(1.0 \mathrm{~mL}, 0.1 \mathrm{M})$ for $1 \mathrm{~h}$, isolated yield was given, ee value was determined by chiral HPLC analysis. 


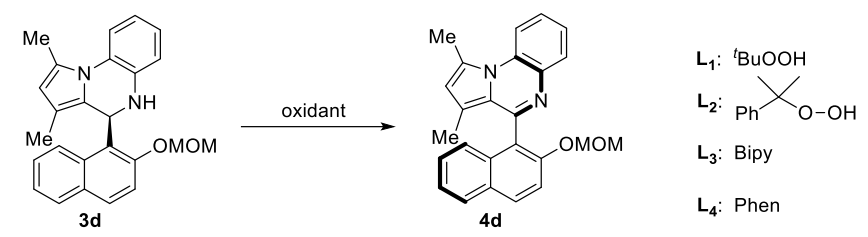

\begin{tabular}{|c|c|c|c|c|c|}
\hline Entry & Cat. & Solvent & $\mathrm{T}\left({ }^{\circ} \mathrm{C}\right)$ & Yield (\%) & ee $(\%)$ \\
\hline 1 & $\mathrm{CuAc}_{2}+\mathrm{L}_{1}$ & $\mathrm{CCl}_{4}$ & -10 & 86 & 32 \\
\hline 2 & $\mathrm{CuTf}_{2}+\mathrm{L}_{1}$ & $\mathrm{CCl}_{4}$ & -10 & 67 & 21 \\
\hline 3 & $\mathrm{Cul}+\mathrm{L}_{1}$ & $\mathrm{CCl}_{4}$ & -10 & 91 & 52 \\
\hline 4 & $\mathrm{CuAc}+\mathrm{L}_{1}$ & $\mathrm{CCl}_{4}$ & -10 & 85 & 48 \\
\hline 5 & $\mathrm{CuBr}+\mathrm{L}_{1}$ & $\mathrm{CCl}_{4}$ & -10 & 71 & 36 \\
\hline 6 & Cul $+L_{1}$ & $\mathrm{CCl}_{4}$ & -20 & 86 & 51 \\
\hline 7 & $\mathrm{Cul}+\mathrm{L}_{2}$ & $\mathrm{CCl}_{4}$ & -20 & 85 & 52 \\
\hline 8 & $\mathrm{Cul}+\mathrm{L}_{3}$ & $\mathrm{CCl}_{4}$ & -20 & 81 & 0 \\
\hline 9 & $\mathrm{Cul}+\mathrm{L}_{4}$ & $\mathrm{CCl}_{4}$ & -20 & 83 & 0 \\
\hline 8 & $\mathrm{Cul}+\mathrm{L}_{1}$ & toluene & -10 & 87 & 64 \\
\hline 9 & $\mathrm{Cul}+\mathrm{L}_{1}$ & THF & -10 & 81 & 30 \\
\hline 10 & Cul $+L_{1}$ & DCM & -10 & 83 & 41 \\
\hline 11 & $\mathrm{Cul}+\mathrm{L}_{1}$ & acetone & -10 & 82 & 32 \\
\hline 12 & Cul+ $\mathrm{L}_{1}$ & $\mathrm{Et}_{2} \mathrm{O}$ & -10 & 86 & 30 \\
\hline $13^{[b]}$ & $\mathrm{Cul}+\mathrm{L}_{1}$ & toluene & -10 & 62 & 44 \\
\hline $14^{[c]}$ & $\mathrm{Cul}+\mathrm{L}_{1}$ & toluene & -10 & 89 & 64 \\
\hline
\end{tabular}

[a] Reaction conditions: $3 \mathbf{d}(0.10 \mathrm{mmol})$, Cu salt $(5 \%)$ and oxidant $(0.5 \mathrm{mmol}, 5$ equiv.) were stirred in solvent $(1.0 \mathrm{~mL}, 0.1 \mathrm{M})$ for $8 \mathrm{~h}$, isolated yield was given, ee value was determined by chiral HPLC analysis. [b] $100 \mathrm{mg}$ of $4 \AA$ molecular sieves was added, reacted for $24 \mathrm{~h}$. [c] $100 \mathrm{mg}$ of $\mathrm{MgSO}_{4}$ was added, reacted for $4 \mathrm{~h}$. 


\section{Evaluation of the DHPLC profiles}

The rate constants are obtained directly from the parameters given, via the software DCXplorer by Trapp and coworkers and are shown in the following table. Experimental conditions: Chiralpak IA, $n$-hexane/i-propanol 95:5 (v/v), flow $1.0 \mathrm{~mL} \cdot \mathrm{min}^{-1}$.
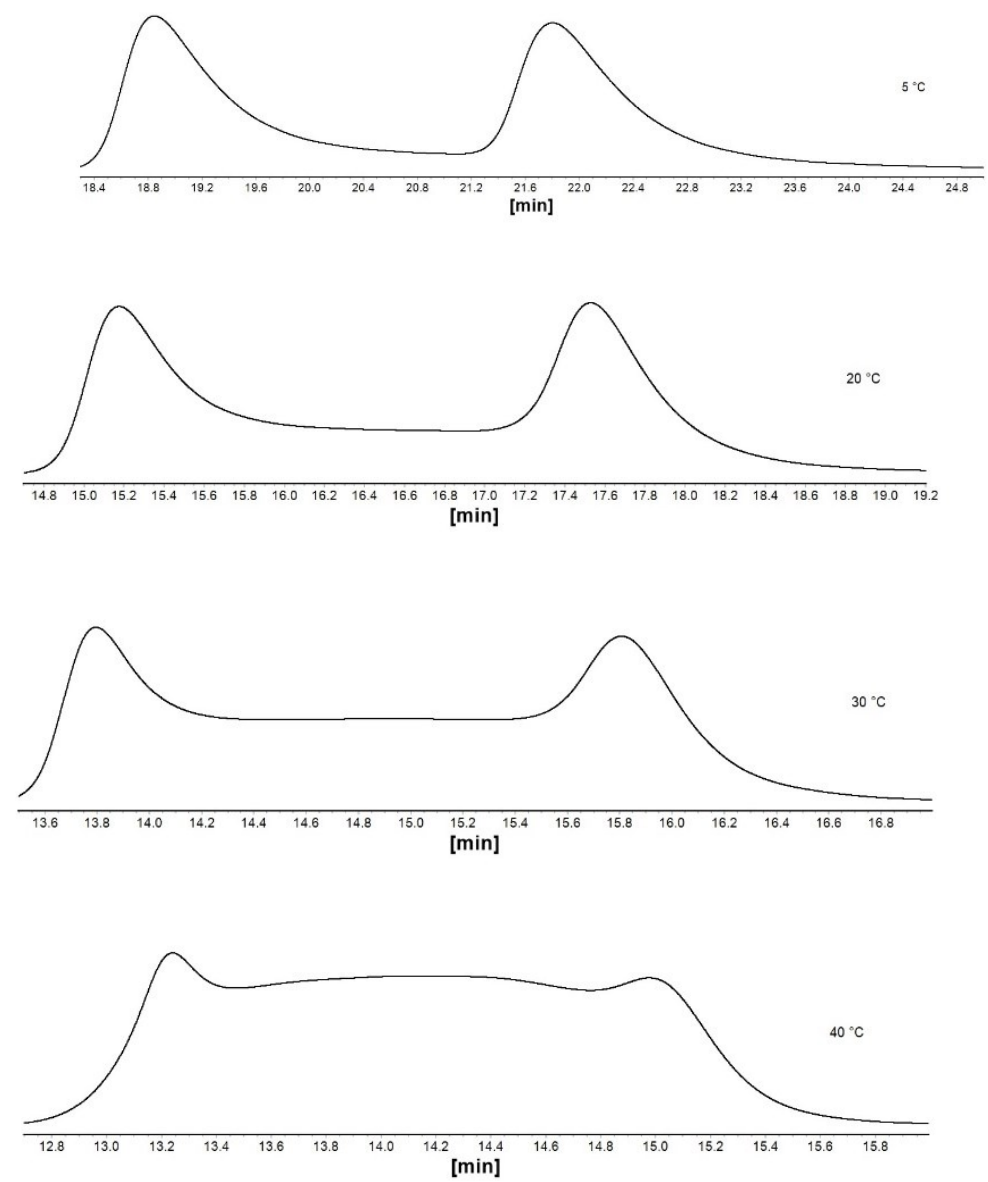

Table S7. Obtained data from DCXplorer.

\begin{tabular}{|c|c|c|c|c|c|c|c|c|c|c|c|c|c|}
\hline entry & $\begin{array}{c}\mathrm{T} \\
{\left[{ }^{\circ} \mathrm{C}\right]}\end{array}$ & $\begin{array}{c}\mathrm{t}_{\mathrm{R} 1} \\
{[\mathrm{~min}]}\end{array}$ & $\begin{array}{c}\mathrm{t}_{\mathrm{R} 2} \\
{[\mathrm{~min}]}\end{array}$ & $\begin{array}{l}\text { Wh1 } \\
{[\mathrm{s}]}\end{array}$ & $\begin{array}{l}\mathrm{Wh}_{\mathrm{h}} \\
{[\mathrm{s}]}\end{array}$ & $\begin{array}{l}\mathrm{h}_{1} \\
{[\%]}\end{array}$ & $\begin{array}{l}\mathrm{h}_{\mathrm{p}} \\
{[\%]}\end{array}$ & $\begin{array}{l}\mathrm{h}_{2} \\
{[\%]}\end{array}$ & $A_{1}$ & $\mathrm{~A}_{2}$ & $\mathrm{k}_{1}[1 / \mathrm{s}]$ & $\begin{array}{c}\Delta G^{\ddagger}{ }_{1} \\
{[\mathrm{~kJ} / \mathrm{mol}]}\end{array}$ & $\ln (k / T)$ \\
\hline 1 & 5.0 & 18.847 & 21.800 & 30.5 & 63.6 & 100.00 & 8.58 & 95.75 & 50.55 & 49.45 & $1.91 \mathrm{E}-04$ & 87.77 & -14.1919 \\
\hline 2 & 10.0 & 17.154 & 19.906 & 26.4 & 51.5 & 100.00 & 12.24 & 98.65 & 50.72 & 49.28 & $3.18 \mathrm{E}-04$ & 88.19 & -13.6985 \\
\hline 3 & 20.0 & 15.174 & 17.528 & 21.6 & 38.3 & 98.57 & 24.70 & 100.00 & 50 & 50 & 4.36E-04 & 90.62 & -13.4195 \\
\hline 4 & 30.0 & 13.794 & 15.807 & 16.8 & 30.4 & 100.00 & 46.74 & 96.08 & 49.94 & 50.06 & $1.37 \mathrm{E}-03$ & 90.91 & -12.3079 \\
\hline 5 & 40.0 & 13.241 & 14.976 & 19.0 & 30.8 & 100.00 & 86.57 & 85.50 & 49.98 & 50.02 & 2.02E-03 & 92.99 & -11.9538 \\
\hline
\end{tabular}




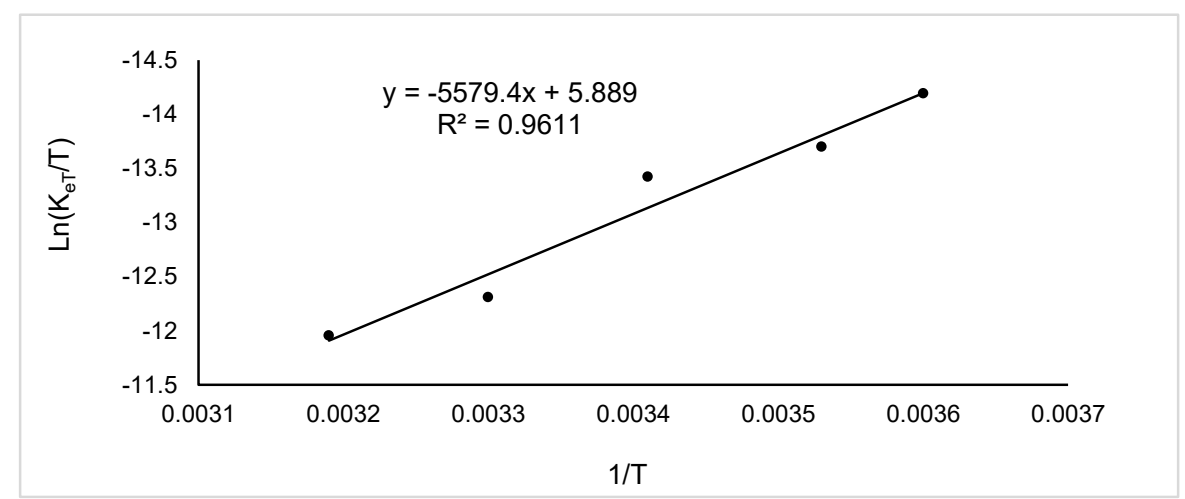

Figure S1. Eyring plot of $\ln \left(\mathrm{k} e_{e} / T\right)$ against $1 / T$ with linear regression $(\mathrm{y}=\mathrm{ax}+\mathrm{b})$.

From Figure $S 1$, the entropy and enthalpy of the enantiomerization process can be calculated through:

$$
\begin{aligned}
& \Delta H^{\ddagger_{e}}=-a R=-\left(-5579.4 \cdot 8.31 \cdot 10^{-3}\right)=46.36 \mathrm{~kJ} \cdot \mathrm{mol}^{-1} \\
& \Delta S^{\ddagger}=R\left[b-\left(k_{B} / \mathrm{h}\right)\right]=8.3110^{-3} \cdot(5.889-23.8)=-0.149 \mathrm{~kJ} \cdot \mathrm{mol}^{-1} \cdot \mathrm{K}^{-1}
\end{aligned}
$$

From the obtained values, the Gibbs free activation energy calculated through full thermodynamical analysis:

$$
\Delta G^{\ddagger}{ }_{e 298}=\Delta H^{\ddagger} e_{e} T \Delta S^{\ddagger}=46.36-(-0.149 \cdot 298)=90.76 \mathrm{~kJ} \cdot \mathrm{mol}^{-1}
$$

Racemization constant in $25^{\circ} \mathrm{C}$ can be calculated according to the following relationship:

$\mathrm{k}_{\text {racemization }}=2 \cdot \mathrm{k}_{1(298)}=2 \cdot \frac{k_{B} T}{h} \cdot e\left(\frac{-\Delta \mathrm{G}^{\ddagger}}{R T}\right)==1.5631 \cdot 10^{-3} \mathrm{~s}^{-1}$

Where: $\mathrm{R}=$ Gas constant $=8.31454 \mathrm{~J} \cdot \mathrm{K}^{-1} \cdot \mathrm{mol}^{-1}, \mathrm{~h}=$ Planck constant $=6.62608 \cdot 10^{-34} \mathrm{~J} \cdot \mathrm{s}, k_{\mathrm{B}}=$ Boltzmann constant $=1.38066 \cdot 10^{-23} \mathrm{~J} \cdot \mathrm{K}^{-1}, \mathrm{~T}=298.15 \mathrm{~K}\left(25^{\circ} \mathrm{C}\right)$

The half-life at $25^{\circ} \mathrm{C}$ is calculated as follows:

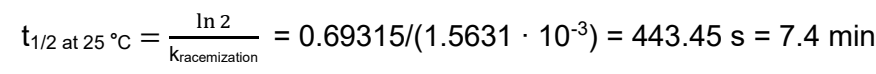

\section{Investigation of the steric effects on central-to-axial chirality transfer process}

(1) Investigation on the steric effects of nathphyl substituted substrate

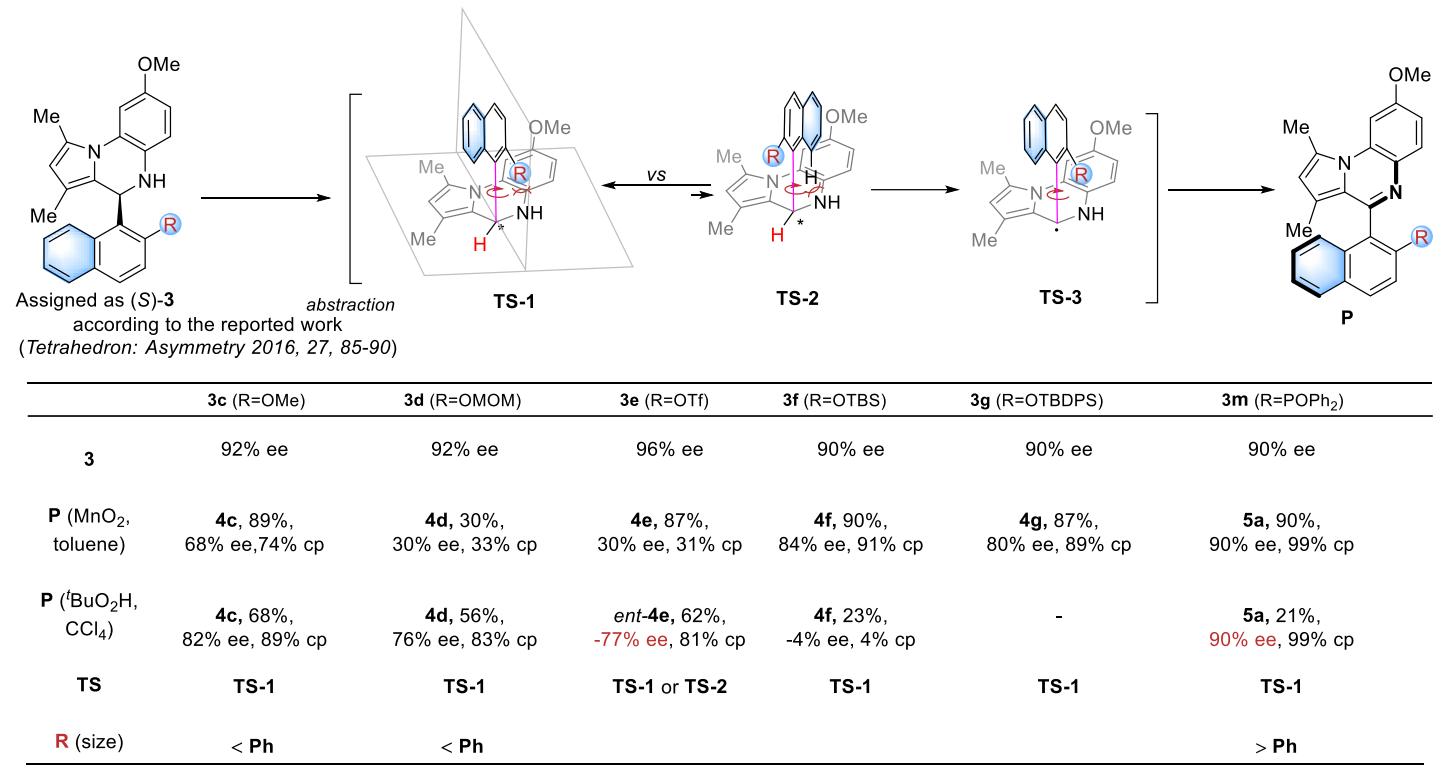

Scheme S1. Investigation on the steric effects of nathphyl substituted substrate 
The atropisomeric generation of new chiral axis by the $\mathrm{CH}-\mathrm{NH}$ bond oxidative central-to-axial chirality transfer was susceptive to the steric effects of oxidative precursor 4,5-dihydropyrroloquinoxalines, oxidants and reaction conditions. Although the free rotation of $\mathrm{C}_{\mathrm{Ar}} \mathrm{C}$ single bond at the chiral center, large difference in steric interaction between $\mathrm{OMe}$ and naphthyl moiety with oxidant might enable the efficient discrimination of two interconvert conformers $a p-(S)-3 \mathbf{c}$ and $s p-(S)-3 c$. Consequently, $(R)-\mathbf{4 c}$ was predominantly produced with satisfied results under both $\mathrm{MnO}_{2}\left(68 \%\right.$ ee, $74 \% \mathrm{cp}$ ) and ${ }^{t} \mathrm{BuO}_{2} \mathrm{H}$ oxidation ( $82 \%$ ee, $\left.89 \% \mathrm{cp}\right)$, maybe owing to the relatively smaller steric interaction of OMe residue with oxidant. ${ }^{[1]}$ By changing Me (3c) to MOM (3d), $\mathrm{Tf}$ (3e), TBS (3q) and TBDPS (3f), the chirality conversion percentage was decreased for $\mathbf{4 d}(30 \% \mathrm{ee}, 33 \% \mathrm{cp})$ and $\mathbf{4 e}$ $(30 \%$ ee, $31 \% \mathrm{cp})$, but increased for $\mathbf{4 f}(84 \% \mathrm{ee}, 91 \% \mathrm{cp})$ and $\mathbf{4 g}(80 \%, 89 \% \mathrm{cp})$ with the same $(R)$-configuration. This phenomenon might imply the easy $\mathrm{C}_{\mathrm{Ar}} \mathrm{C}$ bond rotation in $\mathbf{3 d - e}$ and higher rotation barrier in $\mathbf{3 f}-\mathbf{g}$ due to the increasing steric repulsion between phenol-protect group with the crowded dihydropyrrolo[1,2-a]quinoxaline face from MOM/Tf to bulkier TBS/TBDPS to forbid the $\mathrm{C}_{\mathrm{Ar}} \mathrm{C}$ single bond free rotation. However, the increased steric impulsion between bulkier Tf/TBS with larger ${ }^{t} \mathrm{BuO}_{2} \mathrm{H}$ was injurious to the conformer ap-(S)-3, and enantiomer $(S)-4 e(-77 \%$ ee, $81 \% \mathrm{cp})$ and $(S)-4 f(-4 \%$ ee, $4 \% \mathrm{cp})$ were obtained from another conformer. As expected, the bulkiest $\mathrm{POPh}_{2}$ should provide the highest rotation barrier because of the largest steric congestion between $\mathrm{POPh}_{2}$ with dihydropyrrolo[1,2-a]quinoxaline, and the excellent reaction outcomes was detected for $(R)-5 a$ with a quantitative conversion percentage of $>99 \%$ by using both $\mathrm{MnO}_{2}$ and ${ }^{t} \mathrm{BuO}_{2} \mathrm{H}$ from the stable conformer ap-(S)-3m.

\section{(2) Investigation on the steric effects of $\mathrm{CF}_{3}$ substituted substrate}

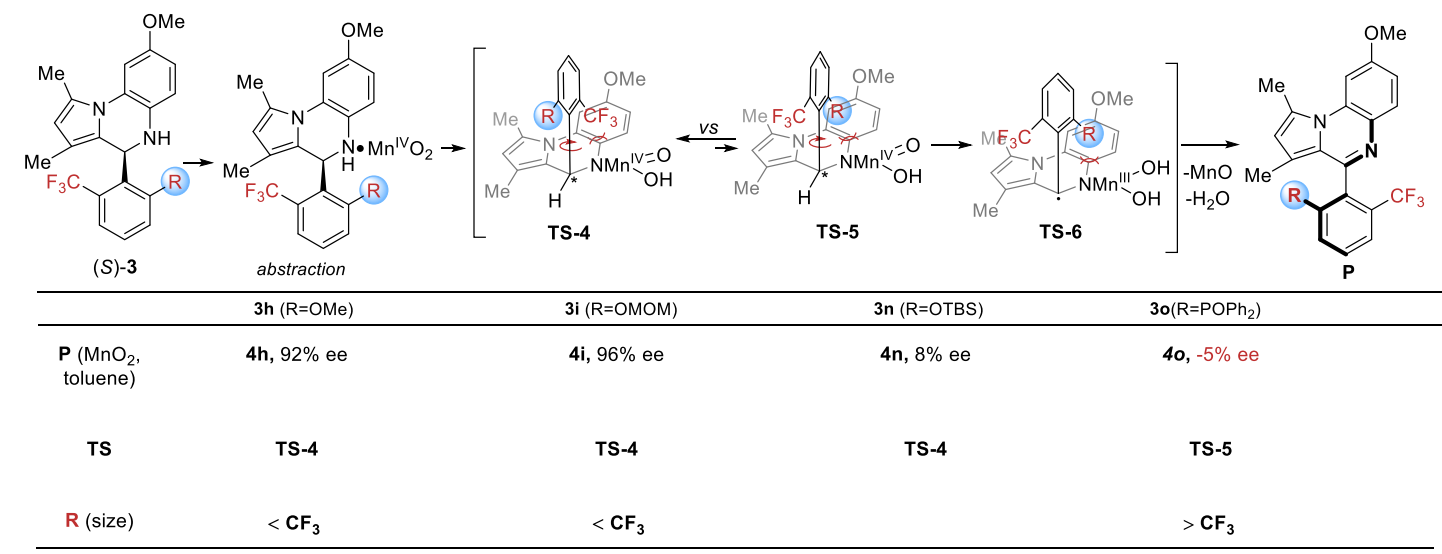

Scheme S2. Investigation on the steric effects of $\mathrm{CF}_{3}$ substituted substrate

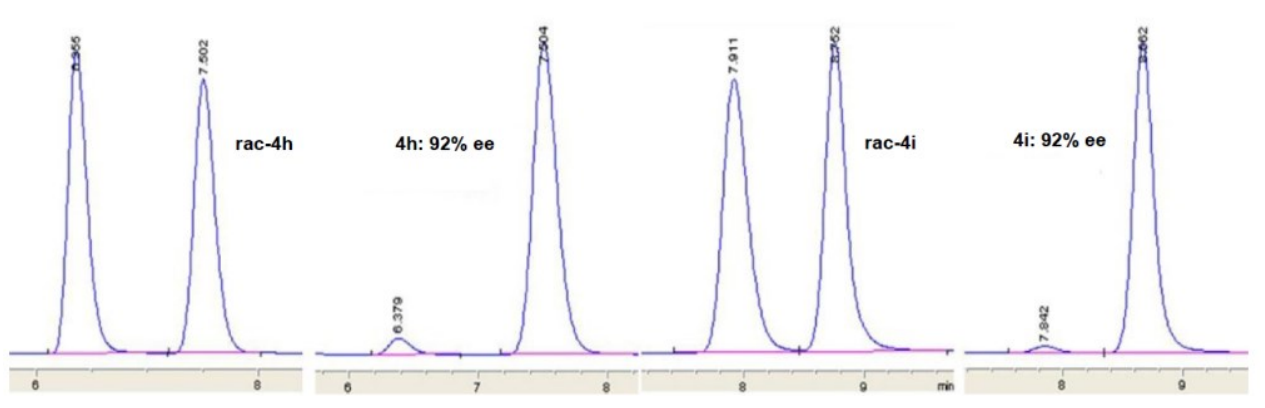



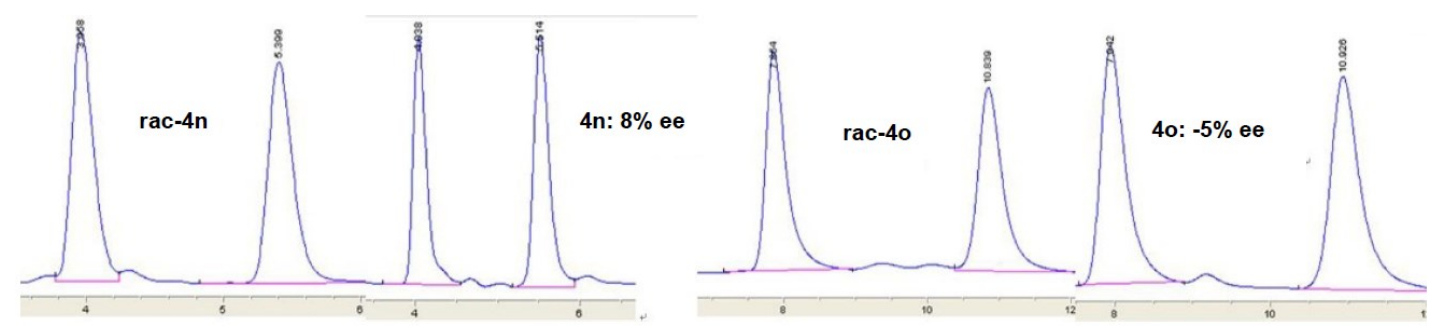

How steric hindrance of vicinal group effects the reaction results of the $\mathrm{CF}_{3}$ substituted oxidative precusor, we then explored groups OMe, OMOM, OTBS and $\mathrm{POPh}_{2}$ installed an another 2'-position in phenyl ring. The results showed in Scheme 2 demonstrated obviously the reaction principle that the conformer with bulkier $\mathrm{CF}_{3}$ (more like $\mathrm{POPh}_{2}$ in steric effect) positioned away from the crowded dihydropyrrolo[1,2-a]quinoxaline oxidized preferentially into the final axially chiral products $\mathbf{4 h}$ and $\mathbf{4 i}$ in $92 \%$ and $96 \%$ ee by $\mathrm{MnO}_{2}$ oxidation through mechanism TS-4 ${ }^{[2]}$ by virtue of the enough barriers of $\mathrm{CF}_{3}$ group to rotation of $\mathrm{C}-\mathrm{C}$ single bond. With the increasing steric bulk of OTBS, the enantiomeric excess was decreased to $8 \%$ and an ent-4o in $-5 \%$ ee was obtained from the bulkiest $\mathrm{POPh}_{2}$ substituted 3o, indicating the fact that the steric effect is consistent with the sequence of $\mathrm{OMe}<\mathrm{OTBS}<\mathrm{CF}_{3}<\mathrm{POPh}_{2}$.

\section{(3) Investigation on the steric effects of oxidants}
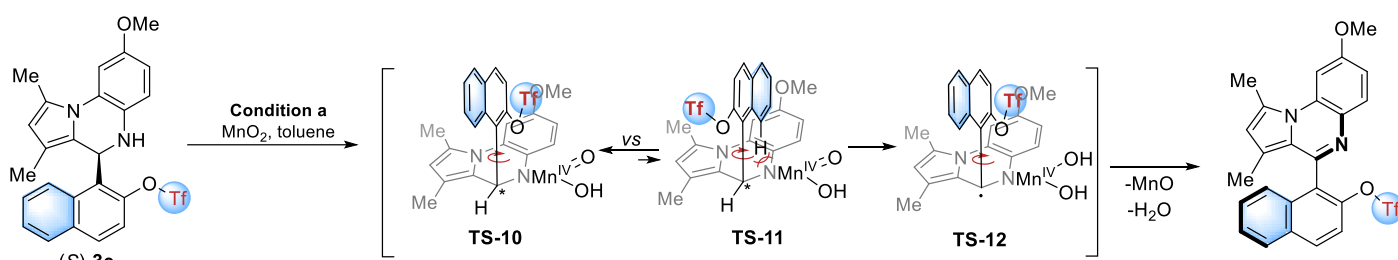

(S)-3e

TS-10 TS-11

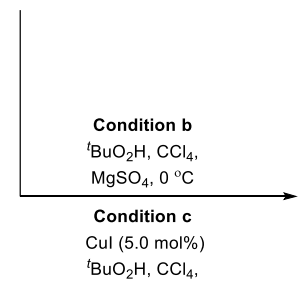

${ }^{t} \mathrm{BuO}_{2} \mathrm{H}, \mathrm{CCl}_{4}$,

$\mathrm{MgSO}_{4}, 0^{\circ} \mathrm{C}$
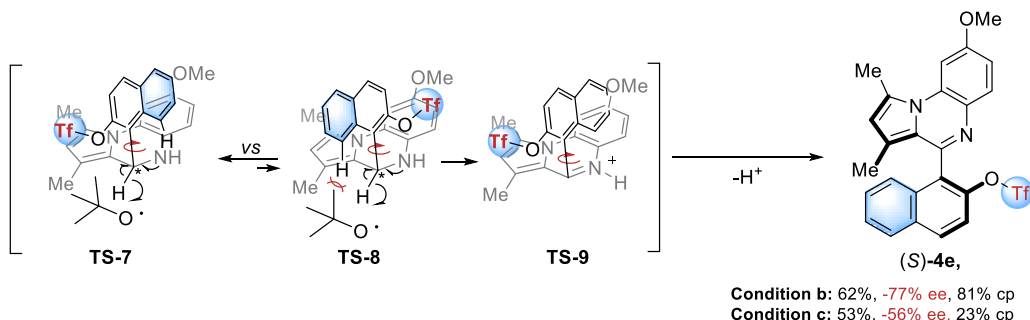

Scheme S3. Investigation on the steric effects of oxidants. Condition a: $3 p(0.10 \mathrm{mmol}), \mathrm{MnO}_{2}(1.0 \mathrm{mmol}, 10$ equiv.) were stirred in toluene $(1.0 \mathrm{~mL}, 0.1 \mathrm{M})$ at $-20^{\circ} \mathrm{C}$ for $2 \mathrm{~h}$. Condition b: $3 \mathrm{p}(0.10 \mathrm{mmol}),{ }^{t} \mathrm{BuO}_{2} \mathrm{H}(1.0 \mathrm{mmol}, 10$ equiv. $)$ and $100 \mathrm{mg} \mathrm{MgSO}_{4}$ were stirred in $\mathrm{CCl}_{4}(1.0 \mathrm{~mL}, 0.1 \mathrm{M})$ at $0^{\circ} \mathrm{C}$ for 3 days and the remaining staring materials was recycled. Condition c: $3 \mathrm{p}(0.10 \mathrm{mmol}), \mathrm{Cul}(5.0 \%)$, ${ }^{t} \mathrm{BuO}_{2} \mathrm{H}(0.5 \mathrm{mmol}, 5$ equiv. $)$ and $100 \mathrm{mg} \mathrm{MgSO}_{4}$ were stirred in $\mathrm{CCl}_{4}(1.0 \mathrm{~mL}, 0.1 \mathrm{M})$ at $0{ }^{\circ} \mathrm{C}$ for $8 \mathrm{~h}$.

Two atropisomers of compound $\mathbf{4 e}$ was obtained through oxidation of the common precusor $(S)$-3e under different oxidation conditions. $(R)-4 \mathrm{e}$ was readily obtained with $30 \%$ ee and $31 \% \mathrm{cp}$ under $\mathrm{MnO}_{2}$ oxidation a and (S)-4e obtained with $-77 \%$ ee and $81 \% \mathrm{cp}$ under ${ }^{t} \mathrm{BuO}_{2} \mathrm{H}$ oxidation $\mathbf{b}$, implying the steric repulsion between substituent in precusor and oxidant could be regulated through different bulk oxidant to deliver desired configurational axially chiral product. Additionally, in the presence of Cul as a catalyst to promote the oxidation process c, a decreased $-22 \%$ ee value and $23 \% \mathrm{cp}$ was obtained for $(S)-4 \mathbf{e}$, which might be resulted from the major transition state TS-8 promoted by larger ${ }^{t} \mathrm{BuO} \cdot$ and the minor transition state TS-7 with other smaller oxidative radicals instead of ${ }^{t} \mathrm{BuO} \cdot$ generated under $\mathrm{Cu}$ catalysis. ${ }^{[3]}$ 
[1] O. Quinonero, M. Jean, N. Vanthuyne, C. Roussel, D. Bonne, T. Constantieux, C. Bressy, X. Bugaut, J. Rodriguez. Angew. Chem. Int. Ed. 2016, 55, 1401-1405.

[2] a) I. M. Goldman. J. Org. Chem. 1969, 34, 3289-3295. b) M. K. Brown, M. M. Blewett, J. R. Colombe, E. J. Corey. J. Am. Chem. Soc. 2010, 132, 11165-11170.

[3] a) E. Boess, L. M. Wolf, S. Malakar, M. Salamone, M. Bietti, W. Thiel, M. Klussmann. ACS Catal. 2016, 6, 3253-3261. b) M. O, Ratnikov, M. P. Doyle. J. Am. Chem. Soc. 2013, 135, 1549-1557. c) H. Bartling, A. Eisenhofer, B. König, R. M. Gschwind. J. Am. Chem. Soc. 2016, 138, 36, 11860-11871. d) E. Boess, C. Schmitz, M. Klussmann. J. Am. Chem. Soc. 2012, 134, 5317-5325. e) M. Scott, A. Sud, E. Boess, M. Klussmann. J. Org. Chem. 2014, 79, 12033-12040.

\section{Synthesis and characterization data of substrates}

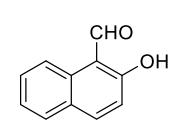

s1

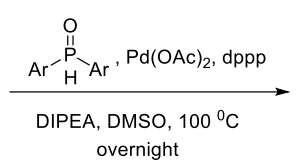

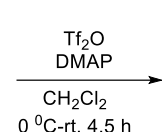

$0{ }^{\circ} \mathrm{C}-$ rt, $4.5 \mathrm{~h}$

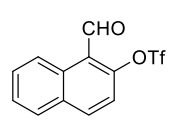

s2

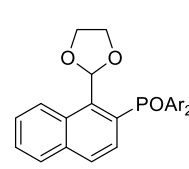

s4
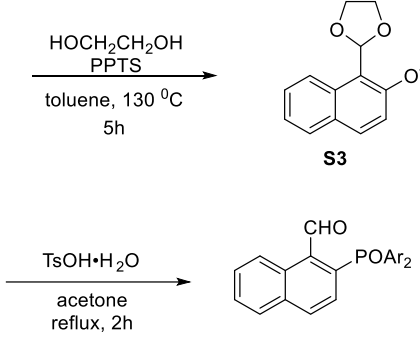

The compound is prepared as reported in literature. ${ }^{[1]}$ To a solution of 2-hydroxy-1-naphthaldehyde (S1, 20 mmol) and 4-( $\mathrm{N}, \mathrm{N}$-dimethylamino)pyridine $(40 \mathrm{mmol}, 2.0$ equiv. $)$ in $\mathrm{CH}_{2} \mathrm{Cl}_{2}(50 \mathrm{~mL})$, trifluoromethanesulfonic anhydride (24 mmol, 1.2 equiv.) was slowly added at $0{ }^{\circ} \mathrm{C}$ under argon atmosphere. The reaction mixture was stirred for $30 \mathrm{~min}$ at $0{ }^{\circ} \mathrm{C}$. The resulting mixture was warmed to room temperature, and stirred for additional $4 \mathrm{~h}$. The resulting mixture was quenched with distilled water $(50 \mathrm{~mL})$ at $0{ }^{\circ} \mathrm{C}$ followed by an extraction with $\mathrm{CH}_{2} \mathrm{Cl}_{2}$ $(3 \times 50 \mathrm{~mL})$. The combined organic layer was washed with $1 \mathrm{M}-\mathrm{HCl}(2 \times 40 \mathrm{~mL})$, brine $(2 \times 40 \mathrm{~mL})$ and dried over $\mathrm{MgSO}_{4}$ before filtration and concentration under reduced pressure. The resulting yellow oil was purified by silica gel column chromatography to provide $\mathbf{S 2}(81 \%$ yield) as a white solid.

The compound is prepared as reported in literature. ${ }^{[2]}$ To a solution of $\mathbf{S} 2$ and ethylene glycol (3.5 equiv.) in anhydrous toluene $(30 \mathrm{~mL})$ was added pyridinium p-toluenesulfonate $(5 \%)$. The mixture was refluxed for about $5 \mathrm{~h}$ in the oil bath with a Dean-Stark apparatus. The reaction was removed to room temperature and quenched by adding saturated $\mathrm{NaHCO}_{3}$, extracted with EtOAc $(3 \times 20 \mathrm{~mL})$. The combined organic layers were dried over anhydrous $\mathrm{Na}_{2} \mathrm{SO}_{4}$, filtered and concentrated in vacuo. The crude residue was purified by flash column chromatography to afford $\mathbf{S 3}$ (65\% yield).

A solution of $\mathrm{Pd}(\mathrm{OAc})_{2}(10 \%)$, dppp (10\%) in DMSO $(6 \mathrm{~mL})$ was vigorously stirred at room temperature for 30 min under Ar atmosphere. Then S3, DIPEA (4.0 equiv.) and diphenylphosphine oxide (2.2 equiv.) were added sequentially before heating at $100^{\circ} \mathrm{C}$ in the oil bath for $12 \mathrm{~h}$. The reaction was removed to room temperature and quenched by water, extracted with EtOAc $(3 \times 10 \mathrm{~mL})$. The combined organic layers were dried over anhydrous $\mathrm{Na}_{2} \mathrm{SO}_{4}$, filtered and concentrated in vacuo afford a residue $\mathbf{S} 4$ quantitatively which was used directly without further purification for the next step.

To a solution of $\mathbf{S} 4(5 \mathrm{mmol})$ in acetone $(8 \mathrm{~mL})$ was added $\mathrm{TsOH} \cdot \mathrm{H}_{2} \mathrm{O}$ (1 equiv.) in one portion, which was refluxed in the oil bath for $2 \mathrm{~h}$. The reaction was quenched by water and extracted with EtOAc $(3 \times 20 \mathrm{~mL})$. The combined organic layers were dried over anhydrous $\mathrm{Na}_{2} \mathrm{SO}_{4}$, filtered and concentrated in vacuo. The crude residue was purified by flash column chromatography on silica gel to afford 1 . 
2-(diphenylphosphoryl)-1-naphthaldehyde (1a):

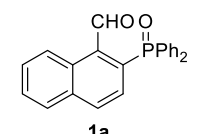

$1.4 \mathrm{~g}, 78 \%$ yield, white solid, $\mathrm{mp}: 180-182{ }^{\circ} \mathrm{C}$. Flash silica gel chromatography (petroleum ether/ethyl acetate $=3 / 1)$. ${ }^{1} \mathrm{H}$ NMR $(400 \mathrm{MHz}$, Chloroform- $d$ ) $1 \mathrm{H}$ NMR $(400 \mathrm{MHz}$, Chloroformd) $\delta 11.23(\mathrm{~d}, J=0.9 \mathrm{~Hz}, 1 \mathrm{H}), 8.94-8.82(\mathrm{~m}, 1 \mathrm{H}), 7.95(\mathrm{dd}, \mathrm{J}=8.5,2.4 \mathrm{~Hz}, 1 \mathrm{H}), 7.89(\mathrm{dd}, \mathrm{J}=$ 8.0, $1.7 \mathrm{~Hz}, 1 \mathrm{H}$ ), $7.73-7.62(\mathrm{~m}, 6 \mathrm{H}), 7.58$ (dddd, J = 8.8, 6.7, 3.3, $1.6 \mathrm{~Hz}, 2 \mathrm{H}$ ), 7.49 (ddd, J = 8.5, 6.7, $3.1 \mathrm{~Hz}$, 4H), $7.33-7.24(\mathrm{~m}, 1 \mathrm{H}) .{ }^{13} \mathrm{C}$ NMR (100 MHz, Chloroform-d) $\delta 194.3$ (d, J= 7.7 Hz), 138.4 (d, J = 6.2 Hz), 135.3 (d, $J=2.0 \mathrm{~Hz}), 134.6$ (d, $J=94.5 \mathrm{~Hz}), 132.8(\mathrm{~d}, J=76.8 \mathrm{~Hz}), 132.5(\mathrm{~d}, J=3.0 \mathrm{~Hz}), 132.3(\mathrm{~d}, J=15.6 \mathrm{~Hz}), 132.1$ (d, $J=10.0 \mathrm{~Hz}$ ), 130.6 (d, $J=10.2 \mathrm{~Hz}), 129.5,128.9$ (d, $J=12.4 \mathrm{~Hz}), 128.7,128.4,128.0$ (d, $J=11.8 \mathrm{~Hz}), 126.7$. ${ }^{31} \mathrm{P}$ NMR (162 MHz, Chloroform-d) $\delta$ 32.3. HRMS (ESI) m/z: $[\mathrm{M}+\mathrm{Na}]^{+}$Calcd for $\mathrm{C}_{23} \mathrm{H}_{17} \mathrm{O}_{2} \mathrm{PNa} 379.0864$; found 379.0856 .

2-(di-o-tolylphosphoryl)-1-naphthaldehyde (1b):

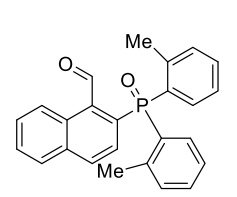

1b

$1.3 \mathrm{~g}, 65 \%$ yield, white solid, $\mathrm{mp}: 157-158^{\circ} \mathrm{C}$. Flash silica gel chromatography (petroleum ether/ethyl acetate $=3 / 1) .{ }^{1} \mathbf{H}$ NMR $(400 \mathrm{MHz}$, Chloroform- $d) \delta 11.23(\mathrm{~d}, J=0.9 \mathrm{~Hz}, 1 \mathrm{H})$, $8.91-8.81(\mathrm{~m}, 1 \mathrm{H}), 7.92$ (ddd, $J=16.4,8.4,2.3 \mathrm{~Hz}, 2 \mathrm{H}), 7.65$ (tt, $J=7.0,5.2 \mathrm{~Hz}, 2 \mathrm{H}$ ), 7.46 (tt, $J=7.5,1.7 \mathrm{~Hz}, 2 \mathrm{H}), 7.34$ (dd, $J=7.7,4.2 \mathrm{~Hz}, 2 \mathrm{H}), 7.22-7.05(\mathrm{~m}, 5 \mathrm{H}), 2.53$ (s, 6H). ${ }^{13} \mathrm{C}$ NMR $(100 \mathrm{MHz}$, Chloroform-d) $\delta 194.9(\mathrm{~d}, J=6.7 \mathrm{~Hz}), 143.6(\mathrm{~d}, J=7.8 \mathrm{~Hz}), 139.1$ (d, $J=5.9 \mathrm{~Hz}$ ), 135.2 (d, $J=2.2 \mathrm{~Hz}), 134.4$ (d, $J=93.6 \mathrm{~Hz}), 132.9$ (d, $J=13.4 \mathrm{~Hz}$ ), 132.5 (d, $J=2.7 \mathrm{~Hz}), 132.3$ (d, $J=10.7 \mathrm{~Hz}), 132.2$ (d, $J=12.1 \mathrm{~Hz}), 130.5$ (d, $J=103.0 \mathrm{~Hz}), 130.5$ (d, $J=10.2 \mathrm{~Hz}), 129.2,128.6,128.3$, $127.4(\mathrm{~d}, J=11.8 \mathrm{~Hz}), 126.6,125.8(\mathrm{~d}, J=13.1 \mathrm{~Hz}), 22.0(\mathrm{~d}, J=4.3 \mathrm{~Hz}) .{ }^{31} \mathrm{P}$ NMR $(162 \mathrm{MHz}$, Chloroform- $d) \delta$ 38.6. HRMS (ESI) m/z: [M+H] $]^{+}$Calcd for $\mathrm{C}_{25} \mathrm{H}_{22} \mathrm{O}_{2} \mathrm{P} 385.1357$; found 385.1363.

\section{2-(di-m-tolylphosphoryl)-1-naphthaldehyde (1c):}

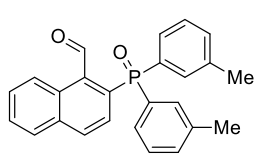

$1 \mathrm{c}$

$1.3 \mathrm{~g}, 70 \%$ yield, white solid, $\mathrm{mp}: 145-148^{\circ} \mathrm{C}$. Flash silica gel chromatography (petroleum ether/ethyl acetate = 3/1). ${ }^{1} \mathrm{H}$ NMR $(400 \mathrm{MHz}$, Chloroform- $d) \delta 11.25(\mathrm{~d}, J=0.9 \mathrm{~Hz}, 1 \mathrm{H})$, $8.99-8.76(\mathrm{~m}, 1 \mathrm{H}), 7.95(\mathrm{dd}, J=8.5,2.3 \mathrm{~Hz}, 1 \mathrm{H}), 7.87$ (dd, $J=8.0,1.7 \mathrm{~Hz}, 1 \mathrm{H}), 7.66-$ $7.57(\mathrm{~m}, 4 \mathrm{H}), 7.43-7.26(\mathrm{~m}, 7 \mathrm{H}), 2.35(\mathrm{~s}, 6 \mathrm{H}) .{ }^{13} \mathrm{C}$ NMR $(100 \mathrm{MHz}$, Chloroform-d) $\delta$ $194.2(\mathrm{~d}, J=7.8 \mathrm{~Hz}), 138.8(\mathrm{~d}, J=12.3 \mathrm{~Hz}), 138.0(\mathrm{~d}, J=6.1 \mathrm{~Hz}), 135.1$ (d, $J=2.2 \mathrm{~Hz}), 134.9(\mathrm{~d}, J=93.4 \mathrm{~Hz})$, $133.1(\mathrm{~d}, J=2.9 \mathrm{~Hz}), 132.6$ (d, $J=64.7 \mathrm{~Hz}), 132.2$ (d, $J=9.4 \mathrm{~Hz}), 131.9,130.4(\mathrm{~d}, J=10.2 \mathrm{~Hz}), 129.2,129.1$, 129.0, 128.6 (d, $J=13.1 \mathrm{~Hz}), 128.4(\mathrm{~d}, J=17.8 \mathrm{~Hz}), 127.9$ (d, $J=11.7 \mathrm{~Hz}), 126.5,21.4 .{ }^{31} \mathrm{P}$ NMR $(162 \mathrm{MHz}$, Chloroform-d) $\delta$ 32.6. HRMS (ESI) m/z: $[\mathrm{M}+\mathrm{H}]^{+}$Calcd for $\mathrm{C}_{25} \mathrm{H}_{22} \mathrm{O}_{2} \mathrm{P} 385.1357$; found 385.1354.

\section{2-(di-p-tolylphosphoryl)-1-naphthaldehyde (1d):}

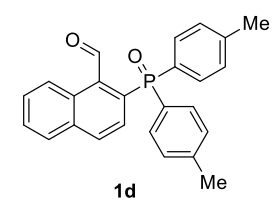

$1.3 \mathrm{~g}, 69 \%$ yield, white solid, $\mathrm{mp}: 123-125^{\circ} \mathrm{C}$. Flash silica gel chromatography (petroleum ether/ethyl acetate = 3/1). ${ }^{1} \mathrm{H}$ NMR $(400 \mathrm{MHz}$, Chloroform- $d) \delta 11.26(\mathrm{~d}, J=0.9 \mathrm{~Hz}, 1 \mathrm{H})$, 8.89 (dd, $J=8.1,1.6 \mathrm{~Hz}, 1 \mathrm{H}), 7.92(\mathrm{dd}, J=8.4,2.3 \mathrm{~Hz}, 1 \mathrm{H}), 7.85$ (dd, $J=8.1,1.7 \mathrm{~Hz}$, $1 \mathrm{H}), 7.61-7.48(\mathrm{~m}, 6 \mathrm{H}), 7.28(\mathrm{dd}, J=8.6,3.1 \mathrm{~Hz}, 5 \mathrm{H}), 2.38(\mathrm{~s}, 6 \mathrm{H}) .{ }^{13} \mathrm{C}$ NMR $(100 \mathrm{MHz}$, Chloroform-d) $\delta 194.3$ (d, $J=7.4 \mathrm{~Hz}$ ), 142.8 (d, $J=2.9 \mathrm{~Hz}$ ), 138.0 (d, $J=6.4 \mathrm{~Hz}$ ), 135.1 (d, $J=94.9 \mathrm{~Hz}$ ), 135.0 (d, $J=2.2 \mathrm{~Hz}$ ), 132.2 (d, $J=12.4 \mathrm{~Hz}$ ), 131.9 (d, $J=10.3 \mathrm{~Hz}$ ), 130.3 (d, J = 10.2 Hz), 129.5 (d, J = 12.7 Hz), $129.4(\mathrm{~d}, J=108.1 \mathrm{~Hz}), 129.2,128.4,128.2,127.9$ (d, $J=11.6 \mathrm{~Hz}), 126.4,21.5 .{ }^{31} \mathrm{P}$ NMR $(162 \mathrm{MHz}$, Chloroformd) $\delta$ 32.6. HRMS (ESI) m/z: $[\mathrm{M}+\mathrm{H}]^{+}$Calcd for $\mathrm{C}_{25} \mathrm{H}_{22} \mathrm{O}_{2} \mathrm{P} 385.1357$; found 385.1349. 
2-(bis(4-methoxyphenyl)phosphoryl)-1-naphthaldehyde (1e):

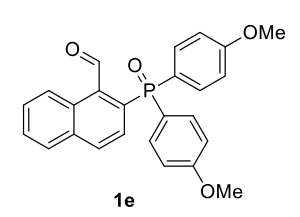

$1.6 \mathrm{~g}, 79 \%$ yield, white solid, $\mathrm{mp}: 175-177^{\circ} \mathrm{C}$. Flash silica gel chromatography (petroleum ether/ethyl acetate = 3/1). ${ }^{1} \mathrm{H}$ NMR $(400 \mathrm{MHz}$, Chloroform-d) $\delta 11.25$ (s, $1 \mathrm{H}), 8.90(\mathrm{~d}, J=8.3 \mathrm{~Hz}, 1 \mathrm{H}), 7.98-7.84(\mathrm{~m}, 2 \mathrm{H}), 7.61(\mathrm{td}, J=12.5,11.5,8.5 \mathrm{~Hz}, 6 \mathrm{H})$, $7.32-7.23(\mathrm{~m}, 1 \mathrm{H}), 7.03-6.91(\mathrm{~m}, 4 \mathrm{H}), 3.84(\mathrm{~s}, 6 \mathrm{H}) .{ }^{13} \mathrm{C}$ NMR $(100 \mathrm{MHz}$, Chloroformd) $\delta 194.7$ (d, $J=7.6 \mathrm{~Hz}), 162.7$ (d, $J=2.9 \mathrm{~Hz}), 137.9$ (d, $J=6.3 \mathrm{~Hz}), 135.6$ (d, $J=$ $95.3 \mathrm{~Hz}), 135.1(\mathrm{~d}, J=2.1 \mathrm{~Hz}), 133.9(\mathrm{~d}, J=11.4 \mathrm{~Hz}), 132.3(\mathrm{~d}, J=12.4 \mathrm{~Hz}), 130.4(\mathrm{~d}, J=10.1 \mathrm{~Hz}), 129.3$, 128.5, 128.3, 128.0 (d, $J=11.6 \mathrm{~Hz}), 126.6,124.0$ (d, $J=111.8 \mathrm{~Hz}), 114.4$ (d, $J=13.4 \mathrm{~Hz}), 55.5 .{ }^{31} \mathrm{P}$ NMR $(162$ $\mathrm{MHz}$, Chloroform-d) $\delta$ 32.0. HRMS (ESI) m/z: $[\mathrm{M}+\mathrm{H}]^{+}$Calcd for $\mathrm{C}_{25} \mathrm{H}_{22} \mathrm{O}_{4} \mathrm{P}$ 417.1256; found 417.1247.

2-(bis(4-fluorophenyl)phosphoryl)-1-naphthaldehyde (1f):

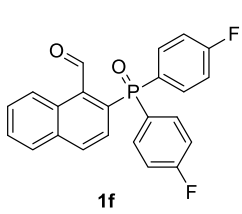

$1.4 \mathrm{~g}, 71 \%$ yield, white solid, $\mathrm{mp}: 130-132{ }^{\circ} \mathrm{C}$. Flash silica gel chromatography (petroleum ether/ethyl acetate $=3 / 1) .{ }^{1} \mathrm{H}$ NMR $(400 \mathrm{MHz}$, Chloroform- $d) \delta 11.20(\mathrm{~d}, J=0.9 \mathrm{~Hz}, 1 \mathrm{H})$, $8.86-8.78(\mathrm{~m}, 1 \mathrm{H}), 8.02-7.95(\mathrm{~m}, 1 \mathrm{H}), 7.92-7.87(\mathrm{~m}, 1 \mathrm{H}), 7.74-7.63(\mathrm{~m}, 6 \mathrm{H}), 7.29$ (d, $J=8.4 \mathrm{~Hz}, 1 \mathrm{H}), 7.20$ (ddt, $J=8.7,6.6,2.2 \mathrm{~Hz}, 4 \mathrm{H}) .{ }^{13} \mathrm{C}$ NMR $(100 \mathrm{MHz}$, Chloroform-d) $\delta 194.1$ (d, $J=7.3 \mathrm{~Hz}), 165.3$ (dd, $J=254.9,3.3 \mathrm{~Hz}), 138.4$ (d, $J=6.6 \mathrm{~Hz}), 135.2$ (d, $J=$ $2.3 \mathrm{~Hz}), 134.5$ (dd, $J=11.5,8.9 \mathrm{~Hz}), 133.5(\mathrm{~d}, J=97.1 \mathrm{~Hz}), 132.5(\mathrm{~d}, J=12.6 \mathrm{~Hz}), 130.5$ (d, $J=10.4 \mathrm{~Hz}), 129.6$, 128.9 (d, $J=3.7 \mathrm{~Hz}), 128.4$ (dd, $J=109.1,3.7 \mathrm{~Hz}), 128.4,127.6$ (d, $J=11.8 \mathrm{~Hz}$ ), 126.3, 116.4 (dd, $J=21.5$, 13.6 Hz). ${ }^{31} \mathrm{P}$ NMR (162 MHz, Chloroform-d) $\delta ~ 30.9 . ~{ }^{19} \mathrm{~F}$ NMR (377 MHz, Chloroform-d) $\delta-105.4$. HRMS (ESI) $\mathrm{m} / \mathrm{z}:[\mathrm{M}+\mathrm{Na}]^{+}$Calcd for $\mathrm{C}_{23} \mathrm{H}_{15} \mathrm{~F}_{2} \mathrm{O}_{2} \mathrm{PNa} 415.0675$, found 415.0663 .

\section{2-(di(naphthalen-2-yl)phosphoryl)-1-naphthaldehyde (1g):}

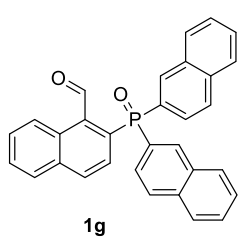

$1.8 \mathrm{~g}, 79 \%$ yield, white solid, $\mathrm{mp}: 220-222^{\circ} \mathrm{C}$. Flash silica gel chromatography (petroleum ether/ethyl acetate = 3/1). ${ }^{1} \mathrm{H}$ NMR $(400 \mathrm{MHz}$, Chloroform- $d) \delta 11.34(\mathrm{~d}, \mathrm{~J}=0.7 \mathrm{~Hz}, 1 \mathrm{H})$, $8.92(\mathrm{dd}, J=8.4,1.5 \mathrm{~Hz}, 1 \mathrm{H}), 8.35(\mathrm{~d}, J=14.2 \mathrm{~Hz}, 2 \mathrm{H}), 7.92(\mathrm{dd}, J=8.5,2.8 \mathrm{~Hz}, 3 \mathrm{H}), 7.90$ $-7.81(\mathrm{~m}, 5 \mathrm{H}), 7.70$ (ddd, $J=10.1,8.4,1.6 \mathrm{~Hz}, 2 \mathrm{H}), 7.65-7.48(\mathrm{~m}, 6 \mathrm{H}), 7.37$ (dd, $J=$ 12.3, 8.5 Hz, 1H). ${ }^{13} \mathrm{C}$ NMR (100 MHz, Chloroform- $d$ ) $\delta 194.2(\mathrm{~d}, J=7.7 \mathrm{~Hz}), 138.4(\mathrm{~d}, J=$ $6.1 \mathrm{~Hz}), 135.3(\mathrm{~d}, J=2.1 \mathrm{~Hz}), 134.9(\mathrm{~d}, J=2.3 \mathrm{~Hz}), 134.6(\mathrm{~d}, J=95.9 \mathrm{~Hz}), 134.1$ (d, $J=9.5 \mathrm{~Hz}), 132.6$ (d, $J=$ $14.1 \mathrm{~Hz}), 132.5$ (d, $J=12.1 \mathrm{~Hz}), 130.6$ (d, $J=10.2 \mathrm{~Hz}), 129.9$ (d, $J=106.1 \mathrm{~Hz}), 129.5,129.1,128.9,128.8$, 128.7, 128.4, 128.1 (d, $J=11.7 \mathrm{~Hz}), 128.0,127.3,126.7,126.6$ (d, $J=5.0 \mathrm{~Hz}) .{ }^{31} \mathrm{P}$ NMR (162 MHz, Chloroformd) $\delta$ 32.5. HRMS (ESI) m/z: $[\mathrm{M}+\mathrm{H}]^{+}$Calcd for $\mathrm{C}_{31} \mathrm{H}_{22} \mathrm{O}_{2} \mathrm{P}$ 457.1357; found 457.1352.

2-(bis(3,5-dimethylphenyl)phosphoryl)-1-naphthaldehyde (1h):

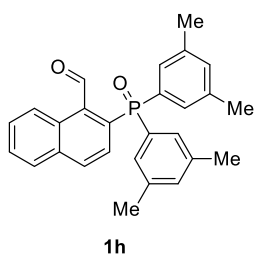

$1.5 \mathrm{~g}, 72 \%$ yield, white solid, $\mathrm{mp}: 198-200^{\circ} \mathrm{C}$. Flash silica gel chromatography (petroleum ether/ethyl acetate $=4 / 1) .{ }^{1} \mathrm{H}$ NMR $(400 \mathrm{MHz}$, Chloroform- $d) \delta 11.21(\mathrm{~d}, J=0.9 \mathrm{~Hz}, 1 \mathrm{H})$, $8.94(\mathrm{dd}, J=8.2,1.5 \mathrm{~Hz}, 1 \mathrm{H}), 7.96(\mathrm{dd}, J=8.4,2.3 \mathrm{~Hz}, 1 \mathrm{H}), 7.91-7.84(\mathrm{~m}, 1 \mathrm{H}), 7.68-$ $7.58(\mathrm{~m}, 2 \mathrm{H}), 7.33-7.26(\mathrm{~m}, 5 \mathrm{H}), 7.19(\mathrm{~s}, 2 \mathrm{H}), 2.32(\mathrm{~s}, 12 \mathrm{H}) .{ }^{13} \mathrm{C}$ NMR $(100 \mathrm{MHz}$, Chloroform- $d$ ) $\delta 194.4$ (d, $J=7.4 \mathrm{~Hz}), 138.6$ (d, $J=13.0 \mathrm{~Hz}), 138.0(\mathrm{~d}, J=6.4 \mathrm{~Hz}), 135.4$ (d, $J=93.9 \mathrm{~Hz}$ ), 135.1 (d, $J=2.2 \mathrm{~Hz}$ ), 134.2 (d, $J=2.9 \mathrm{~Hz}), 132.5$ (d, $J=104.0 \mathrm{~Hz}$ ), 132.3 (d, $J=12.3 \mathrm{~Hz}$ ), $130.5(\mathrm{~d}, J=9.9 \mathrm{~Hz}), 129.5(\mathrm{~d}, J=10.1 \mathrm{~Hz}), 129.3,128.5,128.3,128.2(\mathrm{~d}, J=11.6 \mathrm{~Hz}), 126.7,21.4 .{ }^{31} \mathrm{P}$ NMR (162 MHz, Chloroform-d) $\delta$ 32.8. HRMS (ESI) m/z: [M+H] $]^{+}$Calcd for $\mathrm{C}_{27} \mathrm{H}_{26} \mathrm{O}_{2} \mathrm{P}$ 413.1670; found 413.1675. 
2-(diphenylphosphoryl)-6-methoxybenzaldehyde (1i):<smiles>COc1ccccc1POc1ccccc1</smiles>

$1 \mathrm{i}$

$1.0 \mathrm{~g}, 62 \%$ yield, yellow solid, $\mathrm{mp}: 150-153^{\circ} \mathrm{C}$. Flash silica gel chromatography (petroleum ether/ethyl acetate $=1 / 1) .{ }^{1} \mathrm{H}$ NMR $(400 \mathrm{MHz}$, Chloroform- $d) \delta 10.46(\mathrm{~s}, 1 \mathrm{H}), 7.70-7.62(\mathrm{~m}$, $5 \mathrm{H}$ ), 7.56 (ddd, $J=8.4,7.6,3.0 \mathrm{~Hz}, 1 \mathrm{H}$ ), $7.53-7.47$ (m, 2H), 7.42 (dddd, $J=8.3,6.6,3.0$, $1.3 \mathrm{~Hz}, 5 \mathrm{H}), 7.35-7.27(\mathrm{~m}, 1 \mathrm{H}), 7.24(\mathrm{~d}, J=8.6 \mathrm{~Hz}, 1 \mathrm{H}), 3.93(\mathrm{~s}, 3 \mathrm{H}) .{ }^{13} \mathrm{C}$ NMR $(101 \mathrm{MHz}$, Chloroform- $d$ ) $\delta 189.6$ (d, $J=4.2 \mathrm{~Hz}$ ), $162.0(\mathrm{~d}, J=12.2 \mathrm{~Hz}$ ), 134.7 (d, $J=95.1 \mathrm{~Hz}), 134.0(\mathrm{~d}, J=13.8 \mathrm{~Hz}$ ), 133.7, 132.6, $131.7(\mathrm{~d}, J=10.1 \mathrm{~Hz}), 131.6(\mathrm{~d}, J=2.9 \mathrm{~Hz}), 128.4(\mathrm{~d}, J=12.6 \mathrm{~Hz}), 127.3(\mathrm{~d}, J=9.4 \mathrm{~Hz}), 116.1$ (d, $J=2.3 \mathrm{~Hz}$ ), 56.3. ${ }^{31} \mathrm{P}$ NMR (162 MHz, Chloroform-d) $\delta$ 31.7. HRMS (ESI) m/z: [M+Na] ${ }^{+}$Calcd for $\mathrm{C}_{20} \mathrm{H}_{17} \mathrm{O}_{3} \mathrm{PNa} 359.0813$; found 359.0808 .

\section{2-(diphenylphosphoryl)-6-(methoxymethoxy)benzaldehyde (1j):}

момо $\quad 1.1 \mathrm{~g}, 60 \%$ yield, white solid, $\mathrm{mp}: 141-143^{\circ} \mathrm{C}$. Flash silica gel chromatography (petroleum ether/ethyl acetate = 1/1). ${ }^{1} \mathrm{H}$ NMR $(400 \mathrm{MHz}$, Chloroform- $d) \delta 10.49(\mathrm{~s}, 1 \mathrm{H}), 7.70-7.63(\mathrm{~m}$, $4 \mathrm{H}$ ), 7.50 (dddd, $J=11.8,9.0,7.6,6.2 \mathrm{~Hz}, 4 \mathrm{H}$ ), 7.43 (tdd, $J=8.3,2.7,1.3 \mathrm{~Hz}, 4 \mathrm{H}$ ), $7.34-$ $7.25(\mathrm{~m}, 1 \mathrm{H}), 5.28(\mathrm{~s}, 2 \mathrm{H}), 3.49$ (s, 3H). ${ }^{13} \mathrm{C}$ NMR (101 MHz, Chloroform-d) $\delta 189.6$ (d, J = 4.0 Hz), 159.5 (d, J $=12.2 \mathrm{~Hz}$ ), 134.4 (d, $J=95.6 \mathrm{~Hz}), 133.7(\mathrm{~d}, J=14.6 \mathrm{~Hz}), 133.6,132.5,131.7$ (d, $J=10.1 \mathrm{~Hz}), 131.6(\mathrm{~d}, J=3.0$ $\mathrm{Hz}), 128.4(\mathrm{~d}, J=12.4 \mathrm{~Hz}), 128.3(\mathrm{~d}, J=9.7 \mathrm{~Hz}), 119.6(\mathrm{~d}, J=2.5 \mathrm{~Hz}), 95.1,56.6 .{ }^{31} \mathrm{P}$ NMR $(162 \mathrm{MHz}$, Chloroform-d) $\delta$ 31.6. HRMS (ESI) m/z: [M+H] $]^{+}$Calcd for $\mathrm{C}_{21} \mathrm{H}_{20} \mathrm{O}_{4} \mathrm{P} 367.1099$; found 367.1095.

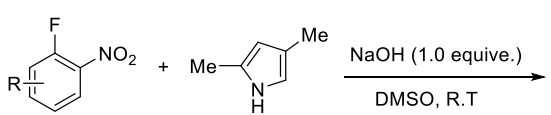

S5

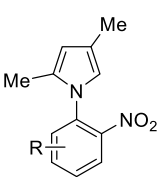

S6

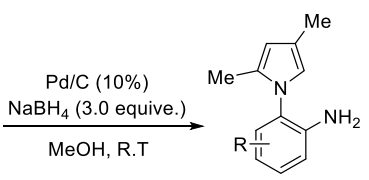

2

The compound is prepared as reported in literature ${ }^{[3,4]}$ A mixture of $\mathbf{S 5}(10 \mathrm{mmol})$, pyrrole $(10 \mathrm{mmol})$ and $\mathrm{NaOH}(10 \mathrm{mmol})$ in DMSO $(15 \mathrm{~mL})$ was stirred vigorously for $2 \mathrm{~h}$. Then, the reaction mixture was poured into water $(60 \mathrm{~mL})$ and extracted with ethyl acetate three times $(3 \times 30 \mathrm{~mL})$. The combined organic layers were dried with $\mathrm{Na}_{2} \mathrm{SO}_{4}$ and the solvent was removed in vacuo to afford a residue $\mathbf{S 6}$ quantitatively which was used directly without further purification for the next step. Then, to a stirred solution of $\mathbf{S 6}$ in methanol $(30 \mathrm{ml})$ was added $10 \%$ palladium on carbon. To this mixture was then added sodium borohydride $\left(30 \mathrm{mmol}, 3\right.$ equive) at $0{ }^{\circ} \mathrm{C}$ and the reaction was stirred at room temperature for $1 \mathrm{~h}$. The reaction mixture was filtered through Celite and removed in vacuo. Next, the mixture was poured into water $(60 \mathrm{~mL})$ and extracted with ethyl acetate three times $(3 \times 30$ $\mathrm{mL}$ ). The combined organic layers were dried with $\mathrm{Na}_{2} \mathrm{SO}_{4}$ and the solvent was removed in vacuo. The crude mass was purified by silica gel column chromatography to give 2 .

\section{2-(2,4-dimethyl-1H-pyrrol-1-yl)aniline (2a):}

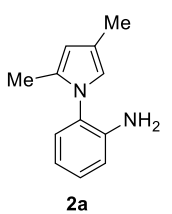

$1.3 \mathrm{~g}, 72 \%$ yield, brown solid, $\mathrm{mp}: 47-49^{\circ} \mathrm{C}$. Flash silica gel chromatography (petroleum ether/ethyl acetate $=20 / 1) .{ }^{1} \mathrm{H}$ NMR $(400 \mathrm{MHz}$, Chloroform- $d) \delta 7.16(\mathrm{t}, J=7.7 \mathrm{~Hz}, 1 \mathrm{H}), 7.08$ $(\mathrm{d}, J=7.7 \mathrm{~Hz}, 1 \mathrm{H}), 6.76(\mathrm{t}, J=7.6 \mathrm{~Hz}, 2 \mathrm{H}), 6.38(\mathrm{~s}, 1 \mathrm{H}), 5.88(\mathrm{~s}, 1 \mathrm{H}), 3.54(\mathrm{~s}, 2 \mathrm{H}), 2.11(\mathrm{~s}, 3 \mathrm{H})$, 2.00 (s, 3H). ${ }^{13} \mathrm{C}$ NMR (100 MHz, Chloroform-d) $\delta 143.8,129.9,129.1,128.8,126.3,119.0$, 118.6, 118.2, 115.7, 108.9, 12.0, 12.0. HRMS (ESI) m/z: $[\mathrm{M}+\mathrm{Na}]^{+}$Calcd for $\mathrm{C}_{12} \mathrm{H}_{14} \mathrm{~N}_{2} \mathrm{Na}$ 209.1055; found 209.1059 
2-(2,4-dimethyl-1H-pyrrol-1-yl)-4-methoxyaniline (2b):

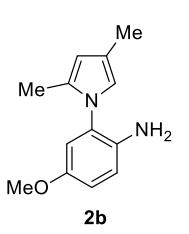

$1.7 \mathrm{~g}, 79 \%$ yield, brown liquid. Flash silica gel chromatography (petroleum ether/ethyl acetate $=20 / 1) .{ }^{1} \mathrm{H}$ NMR $(400 \mathrm{MHz}$, Chloroform- $d$ ) $\delta \delta 6.77$ (dd, $J=8.8,2.8 \mathrm{~Hz}, 1 \mathrm{H}), 6.67$ (dd, $J=5.8$, $2.9 \mathrm{~Hz}, 2 \mathrm{H}), 6.36(\mathrm{dd}, J=2.0,1.1 \mathrm{~Hz}, 1 \mathrm{H}), 5.90-5.83(\mathrm{~m}, 1 \mathrm{H}), 3.69(\mathrm{~s}, 3 \mathrm{H}), 3.28(\mathrm{~s}, 2 \mathrm{H}), 2.09$ $(\mathrm{d}, J=1.1 \mathrm{~Hz}, 3 \mathrm{H}), 2.00(\mathrm{~d}, J=1.0 \mathrm{~Hz}, 3 \mathrm{H}) .{ }^{13} \mathrm{C}$ NMR $(100 \mathrm{MHz}$, Chloroform-d) $\delta 152.1,137.3$, $137.3,129.7,126.8,118.9,118.4,116.7,115.4,113.6,108.9,108.9,55.7,11.9$. HRMS (ESI) $\mathrm{m} / \mathrm{z}:[\mathrm{M}+\mathrm{Na}]^{+}$Calcd for $\mathrm{C}_{13} \mathrm{H}_{16} \mathrm{~N}_{2} \mathrm{ONa} 239.1160$; found 239.1158 .

4-(benzyloxy)-2-(2,4-dimethyl-1H-pyrrol-1-yl)aniline (2c):

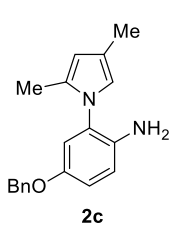

$2.2 \mathrm{~g}, 75 \%$ yield, brown liquid. Flash silica gel chromatography (petroleum ether/ethyl acetate = 20/1). ${ }^{1} \mathrm{H}$ NMR (400 MHz, Chloroform- $d$ ) $\delta 7.43-7.24(\mathrm{~m}, 5 \mathrm{H}), 6.85$ (dd, $\left.J=8.8,2.9 \mathrm{~Hz}, 1 \mathrm{H}\right)$, $6.74(\mathrm{~d}, J=2.9 \mathrm{~Hz}, 1 \mathrm{H}), 6.67(\mathrm{~d}, J=8.7 \mathrm{~Hz}, 1 \mathrm{H}), 6.36$ (s, 1H), 5.86 (s, 1H), 4.93 (s, 2H), 3.27 (s, $2 \mathrm{H}), 2.10$ (s, 3H), 1.97 (s, 3H). ${ }^{13} \mathrm{C}$ NMR (100 MHz, Chloroform-d) $\delta 151.2,137.6,137.1$, 129.7, 128.6, 128.0, 127.6, 126.7, 119.0, 118.5, 116.7, 116.6, 115.0, 109.0, 70.8, 12.0, 11.9. HRMS (ESI) m/z: [M+Na] ${ }^{+}$Calcd for $\mathrm{C}_{19} \mathrm{H}_{20} \mathrm{~N}_{2} \mathrm{ONa} 315.1473$, found 315.1479.

\section{4-bromo-2-(2,4-dimethyl-1H-pyrrol-1-yl)aniline (2d):}

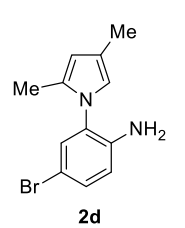

$2.1 \mathrm{~g}, 80 \%$ yield, brown solid, $\mathrm{mp}: 49-52{ }^{\circ} \mathrm{C}$. Flash silica gel chromatography (petroleum ether/ethyl acetate $=30 / 1) .{ }^{1} \mathrm{H}$ NMR $(400 \mathrm{MHz}$, Chloroform- $d) \delta 7.33-7.10(\mathrm{~m}, 2 \mathrm{H}), 6.63(\mathrm{~d}, J$ $=8.6 \mathrm{~Hz}, 1 \mathrm{H}), 6.33(\mathrm{~s}, 1 \mathrm{H}), 5.87(\mathrm{~s}, 1 \mathrm{H}), 3.58(\mathrm{~s}, 2 \mathrm{H}), 2.08(\mathrm{~s}, 3 \mathrm{H}), 1.99(\mathrm{~s}, 3 \mathrm{H}) \cdot{ }^{13} \mathrm{C} \mathrm{NMR}(100$ $\mathrm{MHz}$, Chloroform-d) $\delta 143.0,131.8,131.4,129.7,127.2,119.5,118.4,116.9,109.3,108.8,11.9$, 11.9. HRMS (ESI) m/z: [M+H] $]^{+}$Calcd for $\mathrm{C}_{12} \mathrm{H}_{14} \mathrm{BrN}_{2}$ 265.0340; found 265.0331 .

\section{2-(2,4-dimethyl-1H-pyrrol-1-yl)-4-fluoroaniline (2e):}

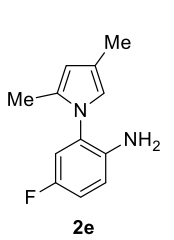

$1.5 \mathrm{~g}, 73 \%$ yield, brown liquid. Flash silica gel chromatography (petroleum ether/ethyl acetate = 30/1). ${ }^{1} \mathrm{H}$ NMR (400 MHz, Chloroform- $d$ ) $\delta 6.91$ (td, $\left.J=8.5,2.9 \mathrm{~Hz}, 1 \mathrm{H}\right), 6.84$ (dd, $J=8.8,2.9$ $\mathrm{Hz}, 1 \mathrm{H}), 6.69(\mathrm{dd}, J=8.8,5.2 \mathrm{~Hz}, 1 \mathrm{H}), 6.35(\mathrm{~s}, 1 \mathrm{H}), 5.88(\mathrm{~s}, 1 \mathrm{H}), 3.42(\mathrm{~s}, 2 \mathrm{H}), 2.09(\mathrm{~s}, 3 \mathrm{H}), 2.00$ (s, 3H). ${ }^{13} \mathrm{C}$ NMR $\left(100 \mathrm{MHz}\right.$, Chloroform-d) $\delta 155.3\left(\mathrm{~d}, \mathrm{C}-\mathrm{F},{ }^{1} \mathrm{~J}_{\mathrm{C}-\mathrm{F}}=237.6 \mathrm{~Hz}\right), 140.2\left(\mathrm{~d}, \mathrm{C}-\mathrm{F},{ }^{4} \mathrm{~J}_{\mathrm{C}-}\right.$ $F=2.7 \mathrm{~Hz}), 129.8,126.6\left(\mathrm{~d}, \mathrm{C}-\mathrm{F},{ }^{3} J_{C-F}=9.4 \mathrm{~Hz}\right), 119.5,118.4,116.2\left(\mathrm{~d}, \mathrm{C}-\mathrm{F},{ }^{3} J_{C-F}=8.2 \mathrm{~Hz}\right)$, $115.9\left(\mathrm{~d}, \mathrm{C}-\mathrm{F},{ }^{2} J_{\mathrm{C}-\mathrm{F}}=21.9 \mathrm{~Hz}\right), 115.5\left(\mathrm{~d}, \mathrm{C}-\mathrm{F},{ }^{2} \mathrm{~J}_{\mathrm{C}-\mathrm{F}}=23.1 \mathrm{~Hz}\right), 109.4,11.9,11.9 .{ }^{19} \mathrm{~F}$ NMR $(377 \mathrm{MHz}$, Chloroformd) $\delta$-126.3. HRMS (ESI) m/z: [M+Na] $]^{+}$Calcd for $\mathrm{C}_{12} \mathrm{H}_{13} \mathrm{FN}_{2} \mathrm{Na} 227.0960$; found 227.0955.

\section{4-(2,4-dimethyl-1H-pyrrol-1-yl)-N1,N1-dimethylbenzene-1,3-diamine (2f):}

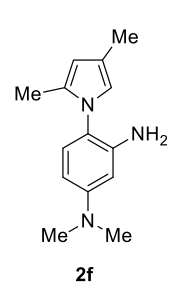

$1.4 \mathrm{~g}, 62 \%$ yield, brown solid, $\mathrm{mp}: 103-106{ }^{\circ} \mathrm{C}$. Flash silica gel chromatography (petroleum ether/ethyl acetate = 20/1). ${ }^{1} \mathrm{H}$ NMR $(400 \mathrm{MHz}$, Chloroform- $d) \delta 6.94(\mathrm{~d}, J=8.6 \mathrm{~Hz}, 1 \mathrm{H}), 6.35$ (dd, $J=2.0,1.1 \mathrm{~Hz}, 1 \mathrm{H}), 6.16(\mathrm{dd}, J=8.6,2.7 \mathrm{~Hz}, 1 \mathrm{H}), 6.09(\mathrm{~d}, J=2.7 \mathrm{~Hz}, 1 \mathrm{H}), 5.85(\mathrm{~s}, 1 \mathrm{H})$, 3.45 (s, 2H), 2.94 (s, 6H), $2.10(\mathrm{~s}, 3 \mathrm{H}), 2.00$ (s, 3H). ${ }^{13} \mathrm{C}$ NMR (100 MHz, Chloroform-d) $\delta 151.2$, 144.3, 130.4, 129.1, 119.2, 118.5, 116.6, 108.4, 102.9, 98.9, 40.7, 12.0, 12.0. HRMS (ESI) m/z: $[\mathrm{M}+\mathrm{Na}]^{+}$Calcd for $\mathrm{C}_{14} \mathrm{H}_{19} \mathrm{~N}_{3} \mathrm{Na} 252.1477$; found 252.1479 . 
2-(3-ethyl-2,4-dimethyl-1H-pyrrol-1-yl)-4-methoxyaniline (2g):

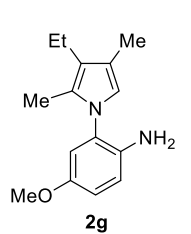

$1.6 \mathrm{~g}, 67 \%$ yield, brown liquid. Flash silica gel chromatography (petroleum ether/ethyl acetate = 30/1). ${ }^{1} \mathrm{H}$ NMR $(400 \mathrm{MHz}$, Chloroform- $d$ ) $\delta 6.78(\mathrm{dd}, \mathrm{J}=8.7,2.9 \mathrm{~Hz}, 1 \mathrm{H}), 6.72-6.67(\mathrm{~m}, 2 \mathrm{H})$, $6.36(\mathrm{~s}, 1 \mathrm{H}), 3.72(\mathrm{~s}, 3 \mathrm{H}), 3.26(\mathrm{~s}, 2 \mathrm{H}), 2.44(\mathrm{q}, \mathrm{J}=7.5 \mathrm{~Hz}, 2 \mathrm{H}), 2.07$ (s, 3H), $1.96(\mathrm{~s}, 3 \mathrm{H}), 1.11$ $(\mathrm{t}, J=7.5 \mathrm{~Hz}, 3 \mathrm{H}) .{ }^{13} \mathrm{C}$ NMR $(101 \mathrm{MHz}$, Chloroform- $d$ ) $\delta 152.4,137.5,127.4,125.5,121.9,118.0$, $117.5,116.8,115.4,114.0,55.9,18.1,15.8,10.3$, 9.8. HRMS (ESI) m/z: $[\mathrm{M}+\mathrm{Na}]^{+}$Calcd for $\mathrm{C}_{15} \mathrm{H}_{20} \mathrm{~N}_{2} \mathrm{ONa}$ 267.1473; found 267.1478.

2-(3,4-diethyl-1H-pyrrol-1-yl)-4-methoxyaniline (2h):

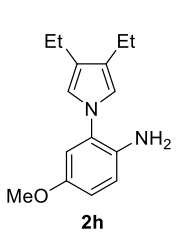

$1.6 \mathrm{~g}, 67 \%$ yield, brown liquid. Flash silica gel chromatography (petroleum ether/ethyl acetate $=30 / 1) .{ }^{1} \mathrm{H}$ NMR $(400 \mathrm{MHz}$, Chloroform- $d$ ) $\delta 6.73(\mathrm{dd}, J=2.0,1.0 \mathrm{~Hz}, 1 \mathrm{H}), 6.71(\mathrm{~d}, J=2.6 \mathrm{~Hz}$, 2H), 6.58 (s, 2H), $3.72(\mathrm{~s}, 3 \mathrm{H}), 3.49(\mathrm{~s}, 2 \mathrm{H}), 2.49$ (q, $J=7.5 \mathrm{~Hz}, 4 \mathrm{H}), 1.28-1.17(\mathrm{~m}, 6 \mathrm{H}) .{ }^{13} \mathrm{C}$ NMR (101 MHz, Chloroform-d) $\delta 152.7,135.3,128.9,126.0,118.3,117.3,114.2,112.3,55.9$, 18.6, 14.5. HRMS (ESI) m/z: [M+Na] $]^{+}$Calcd for $\mathrm{C}_{15} \mathrm{H}_{20} \mathrm{~N}_{2} \mathrm{ONa} 267.1473$; found 267.1468.

\section{Synthesis and characterization data of products}
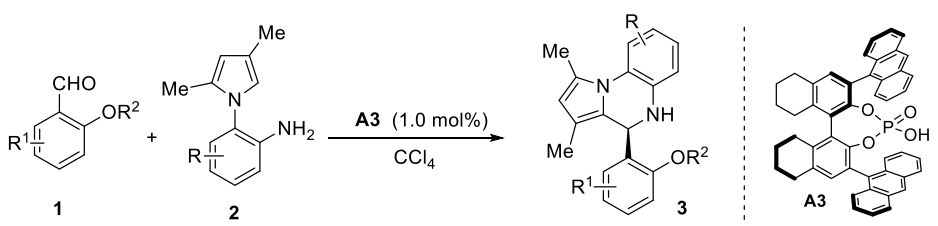

aldehyde 1 (0.2 mmol, 1.0 equiv.), 2-(2,4-dimethyl-1H-pyrrol-1-yl)aniline 2 (0.24 mmol, 1.2 equiv.) and chiral phosphoric acid $\mathbf{A} 3(1.0 \mathrm{~mol} \%)$ was stirred in $\mathrm{CCl}_{4}(1.0 \mathrm{~mL}, 0.1 \mathrm{M})$ at $-10{ }^{\circ} \mathrm{C}$ under $\mathrm{N}_{2}$. Upon reaction completion, reaction solvent was removed and the crude reaction mixture was purified by flash column chromatography

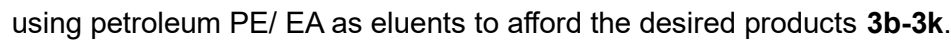

\section{Gram-scale reaction of $3 f$ :}

2-(tert-butyldimethylsilyl)-1-naphthaldehyde (3.0 mmol, 1.0 equiv.) 2-(2,4-dimethyl-1H-pyrrol-1-yl)aniline (3.6 mmol, 1.2 equiv.), and chiral phosphoric acid A3 $(0.5 \mathrm{~mol} \%)$ was stirred in the $30 \mathrm{ml}$ mixed solvent $(p-$ xylene/toluene $=2: 1(\mathrm{v} / \mathrm{v})$ ) at $0{ }^{\circ} \mathrm{C}$ for $24 \mathrm{~h}$ under $\mathrm{N}_{2}$. Upon reaction completion, reaction solvent was removed and the crude reaction mixture was purified by flash column chromatography using petroleum PE/ EA (30/1) as eluents to afford the desired products $3 f(1.34 \mathrm{~g}, 92 \%$ yield, $90 \%$ ee).

\section{(S)-4-(2-methoxynaphthalen-1-yl)-1,3-dimethyl-4,5-dihydropyrrolo[1,2-a]quinoxaline (3b):}

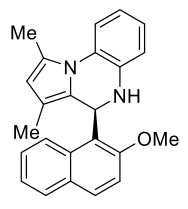

3b

$68.7 \mathrm{mg}, 97 \%$ yield, yellow solid, $\mathrm{mp}: 145-147^{\circ} \mathrm{C}, 92 \%$ ee. Flash silica gel chromatography (petroleum ether/ethyl acetate $=2 / 1$ ). Flash silica gel chromatography (petroleum ether/ethyl acetate $=30 / 1) .{ }^{1} \mathrm{H}$ NMR $(400 \mathrm{MHz}$, Chloroform- $d) \delta 8.54-8.40(\mathrm{~m}, 1 \mathrm{H}), 7.84-7.66(\mathrm{~m}, 2 \mathrm{H})$, $7.49(\mathrm{~d}, J=8.0 \mathrm{~Hz}, 1 \mathrm{H}), 7.33-7.14(\mathrm{~m}, 3 \mathrm{H}), 6.82(\mathrm{dt}, J=31.0,7.5 \mathrm{~Hz}, 2 \mathrm{H}), 6.64-6.48(\mathrm{~m}$, 2H), $5.69(\mathrm{~s}, 1 \mathrm{H}), 3.89(\mathrm{~s}, 1 \mathrm{H}), 3.78(\mathrm{~s}, 3 \mathrm{H}), 2.55(\mathrm{~s}, 3 \mathrm{H}), 1.03(\mathrm{~s}, 3 \mathrm{H}) .{ }^{13} \mathrm{C}$ NMR $(100 \mathrm{MHz}$, Chloroform-d) $\delta 155.5,138.9,133.3,130.6,129.7,128.2,127.4,125.9,125.4,125.3,123.9,123.6,118.9,117.2$, 115.4, 113.5, 113.3, 112.9, 56.9, 48.3, 16.3, 10.5. HRMS(ESI) m/z: [M+Na] ${ }^{+}$Calcd for $\mathrm{C}_{24} \mathrm{H}_{22} \mathrm{~N}_{2} \mathrm{ONa}$ 377.1630; found 377.1631. Optical rotation: $[\alpha]_{D}{ }^{25}=-63.2\left(\mathrm{c}=0.5, \mathrm{CHCl}_{3}\right)$. HPLC: Chiralpak IA, $n$-hexane/isopropanol $=$ 
$95 / 5$, Flow rate $=1.0 \mathrm{~mL} / \mathrm{min}, \mathrm{UV}=254 \mathrm{~nm}, t_{\mathrm{R}}($ major $)=11.31 \mathrm{~min}, t_{\mathrm{R}}($ minor $)=12.43 \mathrm{~min}$.

(S)-8-methoxy-4-(2-methoxynaphthalen-1-yl)-1,3-dimethyl-4,5-dihydropyrrolo[1,2-a]quinoxaline (3c):

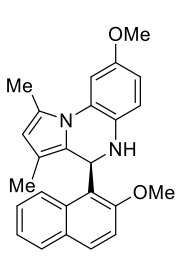

$3 c$

$75.3 \mathrm{mg}, 98 \%$ yield, yellow solid, $\mathrm{mp}: 180-181^{\circ} \mathrm{C}, 92 \%$ ee. Flash silica gel chromatography (petroleum ether/ethyl acetate $=30 / 1) .{ }^{1} \mathrm{H}$ NMR $(400 \mathrm{MHz}$, Chloroform- $d) \delta 8.47(\mathrm{dt}, \mathrm{J}=6.9$, $3.8 \mathrm{~Hz}, 1 \mathrm{H}), 7.83(\mathrm{~d}, J=9.0 \mathrm{~Hz}, 1 \mathrm{H}), 7.78-7.70(\mathrm{~m}, 1 \mathrm{H}), 7.32-7.22(\mathrm{~m}, 3 \mathrm{H}), 7.16(\mathrm{~d}, J=2.5$ $\mathrm{Hz}, 1 \mathrm{H}), 6.61(\mathrm{~d}, J=8.5 \mathrm{~Hz}, 1 \mathrm{H}), 6.51(\mathrm{dd}, J=8.5,2.6 \mathrm{~Hz}, 1 \mathrm{H}), 6.44(\mathrm{~s}, 1 \mathrm{H}), 5.71(\mathrm{~s}, 1 \mathrm{H}), 3.85$ (s, 3H), $3.79(\mathrm{~s}, 4 \mathrm{H}), 2.60(\mathrm{~s}, 3 \mathrm{H}), 1.02(\mathrm{~s}, 3 \mathrm{H}) .{ }^{13} \mathrm{C}$ NMR (100 MHz, Chloroform-d) $\delta$ 155.6, $153.1,133.4,132.9,130.5,129.8,128.4,128.1,126.3,125.8,125.7,125.3,123.6,120.5,115.9$, 113.6, 113.5, 113.0, 108.3, 104.9, 57.0, 55.9, 48.8, 16.2, 10.5. HRMS (ESI) m/z: $[M+H]^{+}$Calcd for $\mathrm{C}_{25} \mathrm{H}_{25} \mathrm{~N}_{2} \mathrm{O}_{2}$ 385.1916; found 385.1911. Optical rotation: $[\alpha]_{D}^{25}=-71.8\left(\mathrm{c}=0.5, \mathrm{CHCl}_{3}\right)$. HPLC: Chiralpak IC, $n-$ hexane/isopropanol $=80 / 20$, Flow rate $=1.0 \mathrm{~mL} / \mathrm{min}, \mathrm{UV}=254 \mathrm{~nm}, t_{\mathrm{R}}($ minor $)=5.53 \mathrm{~min}, t_{\mathrm{R}}$ (major) $=6.65 \mathrm{~min}$.

\section{(S)-8-methoxy-4-(2-(methoxymethoxy)naphthalen-1-yl)-1,3-dimethyl-4,5-dihydropyrrolo[1,2-} a]quinoxaline (3d):

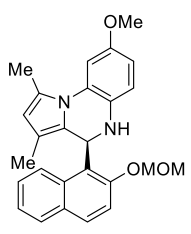

$81.1 \mathrm{mg}, 98 \%$ yield, yellow solid, $\mathrm{mp}: 165-167^{\circ} \mathrm{C}, 92 \%$ ee. Flash silica gel chromatography (petroleum ether/ethyl acetate $=40 / 1) .{ }^{1} \mathrm{H}$ NMR $(400 \mathrm{MHz}$, Chloroform- $d) \delta 8.51(\mathrm{~d}, J=8.0 \mathrm{~Hz}$, $1 \mathrm{H}), 7.76(\mathrm{~d}, J=9.1 \mathrm{~Hz}, 1 \mathrm{H}), 7.74-7.68(\mathrm{~m}, 1 \mathrm{H}), 7.35(\mathrm{~d}, J=9.0 \mathrm{~Hz}, 1 \mathrm{H}), 7.26(\mathrm{pd}, J=6.7$, $1.7 \mathrm{~Hz}, 2 \mathrm{H}), 7.15$ (d, J = 2.7 Hz, 1H), $6.53(\mathrm{~d}, J=8.5 \mathrm{~Hz}, 1 \mathrm{H}), 6.48-6.41(\mathrm{~m}, 2 \mathrm{H}), 5.69$ (s, $1 \mathrm{H}), 5.24-5.02(\mathrm{~m}, 2 \mathrm{H}), 3.74(\mathrm{~s}, 4 \mathrm{H}), 3.38(\mathrm{~s}, 3 \mathrm{H}), 2.58(\mathrm{~s}, 3 \mathrm{H}) .{ }^{13} \mathrm{C}$ NMR $(100 \mathrm{MHz}$, Chloroform- $d$ ) $\delta 153.4,153.1,133.3,132.9,130.6,130.5,128.3,128.1,125.8,125.7,125.3$, 124.1, 122.0, 116.4, 115.7, 113.7, 113.1, 108.3, 104.9, 95.8, 56.4, 55.9, 49.1, 16.1, 10.5. HRMS(ESI) m/z: $[\mathrm{M}+\mathrm{H}]^{+}$Calcd for $\mathrm{C}_{26} \mathrm{H}_{27} \mathrm{~N}_{2} \mathrm{O}_{3}$ 415.2022; found 415.2023. Optical rotation: $[\alpha]_{D}{ }^{25}=-71.5\left(\mathrm{c}=0.5, \mathrm{CHCl}_{3}\right)$. HPLC: Chiralpak OD-H, $n$-hexane/isopropanol $=70 / 30$, Flow rate $=1.0 \mathrm{~mL} / \mathrm{min}, \mathrm{UV}=254 \mathrm{~nm}, t_{\mathrm{R}}(\operatorname{minor})=7.43 \mathrm{~min}, t_{\mathrm{R}}$ (major) $=16.37 \mathrm{~min}$.

\section{(S)-1-(8-methoxy-1,3-dimethyl-4,5-dihydropyrrolo[1,2-a]quinoxalin-4-yl)naphthalen-2-yl} trifluoromethanesulfonate (3e):

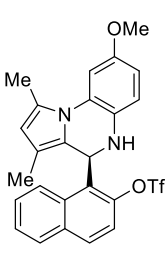

3e

$91.4 \mathrm{mg}, 91 \%$ yield, yellow solid, $\mathrm{mp}: 153-155^{\circ} \mathrm{C}, 96 \%$ ee. Flash silica gel chromatography (petroleum ether/ethyl acetate $=20 / 1) .{ }^{1} \mathrm{H}$ NMR $(400 \mathrm{MHz}$, Chloroform- $d) \delta 8.62(\mathrm{~d}, J=8.7 \mathrm{~Hz}$, $1 \mathrm{H}), 7.96-7.88(\mathrm{~m}, 1 \mathrm{H}), 7.89-7.79(\mathrm{~m}, 1 \mathrm{H}), 7.49$ (ddd, $J=8.2,6.8,1.2 \mathrm{~Hz}, 1 \mathrm{H}), 7.42-7.33$ $(\mathrm{m}, 2 \mathrm{H}), 7.18(\mathrm{~d}, J=2.6 \mathrm{~Hz}, 1 \mathrm{H}), 6.68(\mathrm{~d}, J=8.5 \mathrm{~Hz}, 1 \mathrm{H}), 6.56(\mathrm{dd}, J=8.5,2.6 \mathrm{~Hz}, 1 \mathrm{H}), 6.21$ (s, 1H), 5.69 (s, 1H), 4.01 (s, 1H), 3.80 (s, 3H), 2.61 (s, 3H), $0.89(\mathrm{~s}, 3 \mathrm{H}) .{ }^{13} \mathrm{C}$ NMR $(100 \mathrm{MHz}$, Chloroform-d) $\delta 153.7,145.6,133.4,133.0,131.8,131.7,128.4,128.2,128.1,127.1,126.8$, 126.1, 124.1, 122.0 (q, $J=322.2 \mathrm{~Hz}), 119.2,116.0,113.9,108.7,105.0,56.0,50.7,16.2,10.6 .{ }^{19} \mathrm{~F}$ NMR $(377$ $\mathrm{MHz}$, Chloroform-d) $\delta$-73.0. HRMS (ESI) m/z: $[\mathrm{M}+\mathrm{H}]^{+}$Calcd for $\mathrm{C}_{25} \mathrm{H}_{22} \mathrm{~F}_{3} \mathrm{~N}_{2} \mathrm{O}_{4} \mathrm{~S}$ 503.1252; found 503.1245. Optical rotation: $[\alpha]_{D}^{25}=-68.7\left(c=0.5, \mathrm{CHCl}_{3}\right)$. HPLC: Chiralpak OD-H, $n$-hexane/isopropanol $=60 / 40$, Flow rate $=1.0 \mathrm{~mL} / \mathrm{min}, \mathrm{UV}=254 \mathrm{~nm}, t_{\mathrm{R}}($ minor $)=5.29 \mathrm{~min}, t_{\mathrm{R}}($ major $)=5.96 \mathrm{~min}$. 
(S)-4-(2-((tert-butyldimethylsilyl)oxy)naphthalen-1-yl)-8-methoxy-1,3-dimethyl-4,5-dihydropyrrolo[1,2a]quinoxaline (3f):

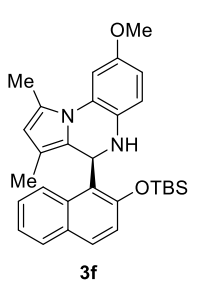

$91.9 \mathrm{mg}, 95 \%$ yield, yellow solid, $\mathrm{mp}: 110-112{ }^{\circ} \mathrm{C}, 90 \%$ ee. Flash silica gel chromatography (petroleum ether/ethyl acetate $=30 / 1) .{ }^{1} \mathrm{H}$ NMR $(400 \mathrm{MHz}$, Chloroform- $d) \delta 8.45-8.35(\mathrm{~m}$, $1 \mathrm{H}), 7.72(\mathrm{~d}, J=8.8 \mathrm{~Hz}, 2 \mathrm{H}), 7.26(\mathrm{td}, J=7.7,1.5 \mathrm{~Hz}, 2 \mathrm{H}), 7.17(\mathrm{~d}, J=2.6 \mathrm{~Hz}, 1 \mathrm{H}), 7.08(\mathrm{~d}, J$ $=8.9 \mathrm{~Hz}, 1 \mathrm{H}), 6.62(\mathrm{~d}, J=8.5 \mathrm{~Hz}, 1 \mathrm{H}), 6.53(\mathrm{dd}, J=8.6,2.6 \mathrm{~Hz}, 1 \mathrm{H}), 6.35(\mathrm{~s}, 1 \mathrm{H}), 5.70(\mathrm{~s}$, $1 \mathrm{H}), 3.81(\mathrm{~s}, 4 \mathrm{H}), 2.61(\mathrm{~s}, 3 \mathrm{H}), 1.01(\mathrm{~d}, J=1.0 \mathrm{~Hz}, 3 \mathrm{H}), 0.97(\mathrm{~s}, 9 \mathrm{H}), 0.25(\mathrm{~s}, 3 \mathrm{H}), 0.22(\mathrm{~s}, 3 \mathrm{H})$.

${ }^{13} \mathrm{C}$ NMR (100 MHz, Chloroform-d) $\delta 153.2,151.9,133.9,132.9,130.2,130.2,128.4,128.1$, 126.7, 125.9, 125.6, 125.5, 123.7, 122.2, 120.5, 115.6, 113.8, 113.3, 108.4, 105.0, 56.0, 49.7, 25.9, 18.5, 16.3, 10.7, -3.8. HRMS (ESI) m/z: $[\mathrm{M}+\mathrm{Na}]^{+}$Calcd for $\mathrm{C}_{30} \mathrm{H}_{36} \mathrm{~N}_{2} \mathrm{O}_{2} \mathrm{SiNa}$ 507.2444; found 507.2436. Optical rotation: $[\alpha]_{D}^{25}=-34.5\left(\mathrm{c}=0.5, \mathrm{CHCl}_{3}\right)$. HPLC: Chiralpak OD-H, $n$-hexane/isopropanol $=95 / 5$, Flow rate $=1.0 \mathrm{~mL} / \mathrm{min}$, $\mathrm{UV}=254 \mathrm{~nm}, t_{\mathrm{R}}($ minor $)=5.19 \mathrm{~min}, t_{\mathrm{R}}($ major $)=12.44 \mathrm{~min}$.

\section{(S)-4-(2-((tert-butyldiphenylsilyl)oxy)naphthalen-1-yl)-8-methoxy-1,3-dimethyl-4,5-dihydropyrrolo[1,2-} a]quinoxaline $(\mathbf{3 g})$ :

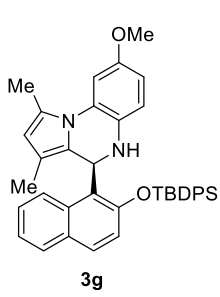

$116.5 \mathrm{mg}, 96 \%$ yield, yellow solid, $\mathrm{mp}: 101-104^{\circ} \mathrm{C}, 90 \%$ ee. Flash silica gel chromatography (petroleum ether/ethyl acetate $=30 / 1) .{ }^{1} \mathrm{H}$ NMR $(400 \mathrm{MHz}$, Chloroform- $d) \delta 8.40-8.32(\mathrm{~m}$, $1 \mathrm{H}), 7.77-7.67(\mathrm{~m}, 4 \mathrm{H}), 7.63-7.56(\mathrm{~m}, 1 \mathrm{H}), 7.44-7.37(\mathrm{~m}, 3 \mathrm{H}), 7.33(\mathrm{t}, J=7.6 \mathrm{~Hz}, 4 \mathrm{H})$, $7.20(\mathrm{dt}, J=9.4,3.2 \mathrm{~Hz}, 3 \mathrm{H}), 6.86(\mathrm{dt}, J=8.9,1.1 \mathrm{~Hz}, 1 \mathrm{H}), 6.68(\mathrm{~s}, 1 \mathrm{H}), 6.58-6.48(\mathrm{~m}$, $2 \mathrm{H}), 5.73(\mathrm{~s}, 1 \mathrm{H}), 3.78(\mathrm{~s}, 3 \mathrm{H}), 3.55(\mathrm{~s}, 1 \mathrm{H}), 2.62(\mathrm{~s}, 3 \mathrm{H}), 1.11(\mathrm{~s} 3 \mathrm{H}), 1.07(\mathrm{~s} 9 \mathrm{H}) .{ }^{13} \mathrm{C}$ NMR $(100 \mathrm{MHz}$, Chloroform-d) $\delta 153.1,151.7,135.6,135.6,133.7,132.8,132.7,132.6,130.2$, 130.0, 129.8, 128.2, 128.1, 128.1, 128.0, 127.9, 126.5, 125.8, 125.6, 125.4, 123.7, 121.9, 120.3, 115.6, 113.9, 113.4, 108.4, 104.8, 55.9, 49.6, 26.8, 19.7, 16.3, 10.8. HRMS (ESI) m/z: $[M+H]^{+}$Calcd for $\mathrm{C}_{40} \mathrm{H}_{41} \mathrm{~N}_{2} \mathrm{O}_{2} \mathrm{Si}$ 609.2937; found 609.2934. Optical rotation: $[\alpha]_{D}{ }^{25}=-40.2\left(\mathrm{c}=0.5, \mathrm{CHCl}_{3}\right)$. HPLC: Chiralpak OD-H, $n-$ hexane/isopropanol $=90 / 10$, Flow rate $=1.0 \mathrm{~mL} / \mathrm{min}, \mathrm{UV}=254 \mathrm{~nm}, t_{\mathrm{R}}(\operatorname{minor})=4.55 \mathrm{~min}, t_{\mathrm{R}}$ (major) $=5.88 \mathrm{~min}$.

(S)-8-methoxy-4-(2-methoxy-6-(trifluoromethyl)phenyl)-1,3-dimethyl-4,5-dihydropyrrolo[1,2a]quinoxaline $(3 \mathrm{~h})$ :

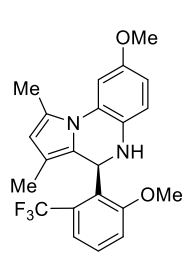

$77.2 \mathrm{mg}, 96 \%$ yield, yellow solid, $\mathrm{mp}: 122-124^{\circ} \mathrm{C}, 96 \%$ ee. Flash silica gel chromatography (petroleum ether/ethyl acetate $=50 / 1) .{ }^{1} \mathrm{H}$ NMR $(400 \mathrm{MHz}$, Chloroform- $d) \delta 7.29(\mathrm{q}, J=4.1,3.3$ $\mathrm{Hz}, 2 \mathrm{H}), 7.17(\mathrm{~d}, J=2.6 \mathrm{~Hz}, 1 \mathrm{H}), 6.97(\mathrm{dd}, J=6.4,3.1 \mathrm{~Hz}, 1 \mathrm{H}), 6.55(\mathrm{~d}, J=8.5 \mathrm{~Hz}, 1 \mathrm{H}), 6.45$ (dd, $J=8.5,2.6 \mathrm{~Hz}, 1 \mathrm{H}), 5.95(\mathrm{~s}, 1 \mathrm{H}), 5.79(\mathrm{~s}, 1 \mathrm{H}), 3.89(\mathrm{~s}, 1 \mathrm{H}), 3.78(\mathrm{~s}, 3 \mathrm{H}), 3.28(\mathrm{~s}, 3 \mathrm{H}), 2.61$ (s, 3H), 1.63 (s, 3H). ${ }^{13} \mathrm{C}$ NMR (100 MHz, Chloroform-d) $\delta 160.1,152.8,130.6,129.8,129.3$, 128.8, 128.7, 125.3, 124.2, 124.6 (q, $J=275.7 \mathrm{~Hz}$ ), 118.2 (q, $J=6.0 \mathrm{~Hz}$ ), 117.0, 115.7, 112.8, 112.6, 107.4, 104.0, 56.4, 55.9, 49.1, 16.4, 10.2. ${ }^{19} \mathrm{~F}$ NMR (377 MHz, Chloroform-d) $\delta ~-56.4$. HRMS (ESI) m/z: $[\mathrm{M}+\mathrm{Na}]^{+}$Calcd for $\mathrm{C}_{22} \mathrm{H}_{21} \mathrm{~F}_{3} \mathrm{~N}_{2} \mathrm{O}_{2} \mathrm{Na}$ 425.1453; found 425.1447. Optical rotation: $[\alpha]_{D}{ }^{25}=-112.2\left(\mathrm{c}=0.5, \mathrm{CHCl}_{3}\right)$. HPLC: Chiralpak IC, $n$-hexane/isopropanol $=80 / 20$, Flow rate $=1.0 \mathrm{~mL} / \mathrm{min}, \mathrm{UV}=254 \mathrm{~nm}, t_{\mathrm{R}}(\operatorname{minor})=4.34$ $\min , t_{R}$ (major) $=5.40 \mathrm{~min}$. 
(S)-8-methoxy-4-(2-(methoxymethoxy)-6-(trifluoromethyl)phenyl)-1,3-dimethyl-4,5-dihydropyrrolo[1,2a]quinoxaline (3i):

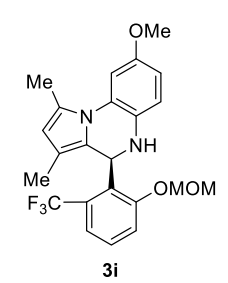

$82.9 \mathrm{mg}, 96 \%$ yield, yellow solid, $\mathrm{mp}: 137-139{ }^{\circ} \mathrm{C}, 98 \%$ ee. Flash silica gel chromatography (petroleum ether/ethyl acetate $=50 / 1) .{ }^{1} \mathrm{H}$ NMR $(400 \mathrm{MHz}$, Chloroform- $d) \delta 7.31(\mathrm{dd}, J=7.8$, $1.4 \mathrm{~Hz}, 1 \mathrm{H}), 7.24(\mathrm{t}, J=8.0 \mathrm{~Hz}, 1 \mathrm{H}), 7.21-7.14(\mathrm{~m}, 2 \mathrm{H}), 6.51(\mathrm{~d}, J=8.5 \mathrm{~Hz}, 1 \mathrm{H}), 6.41$ (dd, $J=8.5,2.6 \mathrm{~Hz}, 1 \mathrm{H}), 6.03(\mathrm{~s}, 1 \mathrm{H}), 5.75(\mathrm{~s}, 1 \mathrm{H}), 4.65(\mathrm{~d}, J=7.1 \mathrm{~Hz}, 1 \mathrm{H}), 4.50(\mathrm{~d}, J=7.1 \mathrm{~Hz}$, $1 \mathrm{H}), 3.92(\mathrm{~s}, J=8.2 \mathrm{~Hz}, 1 \mathrm{H}), 3.76(\mathrm{~s}, 3 \mathrm{H}), 2.90(\mathrm{~s}, 3 \mathrm{H}), 2.60(\mathrm{~s}, 3 \mathrm{H}), 1.65(\mathrm{~s}, 3 \mathrm{H}) .{ }^{13} \mathrm{C}$ NMR (100 MHz, Chloroform-d) $\delta 157.4,152.6,130.7,130.3,129.2$ (q, $J=29.3 \mathrm{~Hz}), 128.7,128.3$, 125.2, 124.7 (q, $J=275.7 \mathrm{~Hz}$ ),124.1, 118.8 (q, $J=6.1 \mathrm{~Hz}), 118.7,115.0,113.0,112.8,107.5,103.9,93.9,56.0$, 55.4, 48.8 (q, $J=3.1 \mathrm{~Hz}), 16.6,10.2 .{ }^{19} \mathrm{~F}$ NMR (377 MHz, Chloroform- $d$ ) $\delta-56.3$. HRMS (ESI) m/z: $[\mathrm{M}+\mathrm{H}]^{+}$Calcd for $\mathrm{C}_{23} \mathrm{H}_{24} \mathrm{~F}_{3} \mathrm{~N}_{2} \mathrm{O}_{3}$ 433.1739; found 433.1739. Optical rotation: $[\alpha]_{D}^{25}=-111.8$ (c=0.5, $\mathrm{CHCl}_{3}$ ). HPLC: Chiralpak IA, $n$-hexane/isopropanol $=98 / 2$, Flow rate $=1.0 \mathrm{~mL} / \mathrm{min}, \mathrm{UV}=254 \mathrm{~nm}, t_{\mathrm{R}}($ major $)=7.64 \mathrm{~min}, t_{\mathrm{R}}(\operatorname{minor})=8.47$ min.

(S)-6-(2-methoxynaphthalen-1-yl)-7-methyl-5,6-dihydroindolo[1,2-a]quinoxaline (3j):

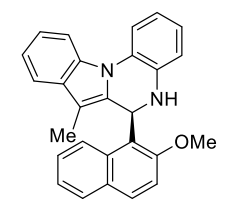

3j

$24.3 \mathrm{mg}, 31 \%$ yield, white solid, $\mathrm{mp}: 170-173^{\circ} \mathrm{C}, 96 \%$ ee. Flash silica gel chromatography (petroleum ether/ethyl acetate $=20 / 1) .{ }^{1} \mathrm{H}$ NMR $(400 \mathrm{MHz}$, Chloroform- $d) \delta 8.31(\mathrm{~d}, J=8.6$ $\mathrm{Hz}, 1 \mathrm{H}), 8.04(\mathrm{~d}, J=8.4 \mathrm{~Hz}, 1 \mathrm{H}), 7.99-7.93(\mathrm{~m}, 1 \mathrm{H}), 7.89(\mathrm{~d}, J=9.1 \mathrm{~Hz}, 1 \mathrm{H}), 7.77(\mathrm{~d}, J=$ $8.1 \mathrm{~Hz}, 1 \mathrm{H}), 7.40(\mathrm{~d}, J=7.8 \mathrm{~Hz}, 1 \mathrm{H}), 7.33-7.23(\mathrm{~m}, 3 \mathrm{H}), 7.19(\mathrm{t}, J=7.8 \mathrm{~Hz}, 1 \mathrm{H}), 7.12(\mathrm{t}, J$ $=7.4 \mathrm{~Hz}, 1 \mathrm{H}), 6.96(\mathrm{dd}, J=6.0,2.9 \mathrm{~Hz}, 2 \mathrm{H}), 6.78-6.70(\mathrm{~m}, 2 \mathrm{H}), 3.97(\mathrm{~s}, 1 \mathrm{H}), 3.88(\mathrm{~s}, 3 \mathrm{H})$, $1.34(\mathrm{~s}, 3 \mathrm{H}) .{ }^{13} \mathrm{C}$ NMR (101 MHz, Chloroform-d) $\delta 155.7,138.3,133.5,133.2,131.4,131.1,129.9,128.4,127.6$, 126.4, 123.9, 123.4, 122.4, 120.2, 119.7, 118.8, 116.5, 115.6, 113.4. HRMS (ESI) m/z: $[\mathrm{M}+\mathrm{H}]^{+}$Calcd for $\mathrm{C}_{27} \mathrm{H}_{23} \mathrm{~N}_{2} \mathrm{O}$ 391.1810; found 391.1811. Optical rotation: $[\alpha]_{D}{ }^{25}=-32.1\left(\mathrm{c}=0.5, \mathrm{CHCl}_{3}\right)$. HPLC: Chiralpak IC, $n$ hexane/isopropanol $=95 / 5$, Flow rate $=1.0 \mathrm{~mL} / \mathrm{min}, \mathrm{UV}=254 \mathrm{~nm}, t_{\mathrm{R}}($ major $)=6.17 \mathrm{~min}, t_{\mathrm{R}}($ minor $)=6.89 \mathrm{~min}$.

(R)-6-(2-methoxynaphthalen-1-yl)-6,11-dihydro-5H-indolo[3,2-c]quinolone (3k):

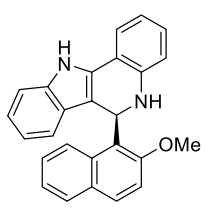

$3 \mathbf{k}$

$69.2 \mathrm{mg}, 92 \%$ yield, white solid, $\mathrm{mp}: 231-233^{\circ} \mathrm{C}, 90 \%$ ee. Flash silica gel chromatography (petroleum ether/ethyl acetate $=30 / 1) .{ }^{1} \mathrm{H}$ NMR $(400 \mathrm{MHz}$, Chloroform- $d) \delta 8.61(\mathrm{~d}, J=8.5$ $\mathrm{Hz}, 1 \mathrm{H}), 8.12(\mathrm{~s}, 1 \mathrm{H}), 7.83(\mathrm{~d}, J=9.1 \mathrm{~Hz}, 1 \mathrm{H}), 7.76-7.66(\mathrm{~m}, 1 \mathrm{H}), 7.54(\mathrm{~s}, 1 \mathrm{H}), 7.34$ (dd, $J$ $=8.2,5.7 \mathrm{~Hz}, 1 \mathrm{H}), 7.24-7.06(\mathrm{~m}, 4 \mathrm{H}), 7.02-6.90(\mathrm{~m}, 2 \mathrm{H}), 6.67$ (qd, J = 7.8, $1.0 \mathrm{~Hz}, 2 \mathrm{H}$ ), $6.60(\mathrm{~d}, J=8.2 \mathrm{~Hz}, 1 \mathrm{H}), 6.40(\mathrm{dd}, J=7.9,1.0 \mathrm{~Hz}, 1 \mathrm{H}), 4.01(\mathrm{~s}, 4 \mathrm{H}) .{ }^{13} \mathrm{C}$ NMR $(101 \mathrm{MHz}$, Chloroform- $d$ ) $\delta 144.8,137.3,133.4,130.8,130.5,130.2,128.8,128.5,126.3,126.2,123.5$, 123.3, 121.9, 120.3, 119.9, 118.7, 116.9, 113.8, 113.3, 113.0, 113.0, 110.8, 110.0, 57.2, 49.0. HRMS (ESI) m/z: $[\mathrm{M}+\mathrm{H}]^{+}$Calcd for $\mathrm{C}_{26} \mathrm{H}_{21} \mathrm{~N}_{2} \mathrm{O} 377.1654$; found 377.1657. Optical rotation: $[\alpha]_{\mathrm{D}}^{25}=-41.8\left(\mathrm{c}=0.5, \mathrm{CHCl}_{3}\right)$. HPLC: Chiralpak IA, $n$-hexane/isopropanol $=50 / 50$, Flow rate $=1.0 \mathrm{~mL} / \mathrm{min}, \mathrm{UV}=254 \mathrm{~nm}, t_{\mathrm{R}}$ (major) $=12.95 \mathrm{~min}, t_{\mathrm{R}}$ $($ minor $)=15.78 \mathrm{~min}$.

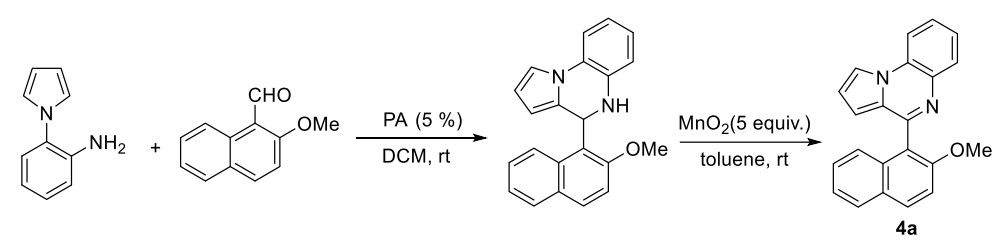

2-(1H-pyrrol-1-yl)aniline (0.24 mmol, 1.2 equiv.), 2-methoxy-1-naphthaldehyde (0.2 mmol, 1.0 equiv.) and phosphoric acid $(5.0 \mathrm{~mol} \%)$ was stirred in $\mathrm{DCM}$ at room temperature. Upon reaction completion, $\mathrm{MnO}_{2}$ was 
added directly and stirred for $2 \mathrm{~h}$. The reaction mixture was filtered through Celite and solvent was removed in vacuo. The crude mass was purified by silica gel column chromatography to give $\mathbf{4 a}$.

\section{4-(2-methoxynaphthalen-1-yl)pyrrolo[1,2-a]quinoxaline (4a):}

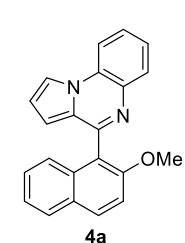

$4 a$

$47.2 \mathrm{mg}, 73 \%$ yield, white solid, $\mathrm{mp}: 162-163^{\circ} \mathrm{C}$. Flash silica gel chromatography (petroleum ether/ethyl acetate $=10 / 1) .{ }^{1} \mathrm{H}$ NMR $(400 \mathrm{MHz}$, Chloroform- $d) \delta 8.08(\mathrm{dd}, J=8.0,1.5 \mathrm{~Hz}, 1 \mathrm{H})$, $8.00-7.95(\mathrm{~m}, 2 \mathrm{H}), 7.92(\mathrm{dd}, J=8.2,1.4 \mathrm{~Hz}, 1 \mathrm{H}), 7.83(\mathrm{dd}, J=7.7,1.8 \mathrm{~Hz}, 1 \mathrm{H}), 7.55$ (ddd, $J=$ 8.3, 7.3, $1.5 \mathrm{~Hz}, 1 \mathrm{H}), 7.50-7.43(\mathrm{~m}, 2 \mathrm{H}), 7.40(\mathrm{~d}, J=9.1 \mathrm{~Hz}, 1 \mathrm{H}), 7.35-7.25(\mathrm{~m}, 2 \mathrm{H}), 6.76$ (dd, $J=4.0,2.7 \mathrm{~Hz}, 1 \mathrm{H}), 6.36$ (dd, $J=4.0,1.3 \mathrm{~Hz}, 1 \mathrm{H}), 3.82(\mathrm{~s}, 3 \mathrm{H}) .{ }^{13} \mathrm{C}$ NMR $(101 \mathrm{MHz}$,

Chloroform-d) $\delta$ 155.1, 152.9, 136.5, 133.3, 130.9, 130.6, 129.2, 128.0, 127.7, 127.5, 127.0, 125.2, 124.7, 123.9, 120.9, 114.2, 114.0, 113.9, 113.8, 108.2, 56.9. HRMS (ESI) m/z: $[\mathrm{M}+\mathrm{Na}]^{+} \mathrm{Calcd}$ for $\mathrm{C}_{22} \mathrm{H}_{16} \mathrm{~N}_{2} \mathrm{ONa} 347.1160$; found 347.1156 .

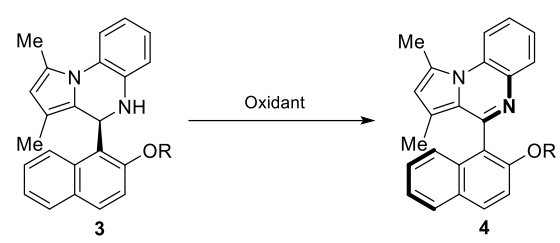

Procedure A: A tube was charged with $\mathrm{MnO}_{2}(1.0 \mathrm{mmol}, 10.0$ equiv.) in toluene $(1.0 \mathrm{~mL})$. The reaction mixture was cooled to $-20^{\circ} \mathrm{C}$ and stirred for 10 minutes, then the first step product $3(0.1 \mathrm{mmol})$ was added to the reaction mixture and stirred for $2 \mathrm{~h}$. The reaction mixture was filtered through Celite and solvent was removed in vacuo. The crude mass was purified by silica gel column chromatography to give 4 .

Procedure B: A mixture of $3(0.1 \mathrm{mmol})$, $t$-butylhydroperoxide $\left(0.5 \mathrm{mmol}, 5.0\right.$ equiv.) and $\mathrm{MgSO}_{4}$ in $\mathrm{CCl}_{4}(1$ $\mathrm{mL}$ ) was stirred at $0^{\circ} \mathrm{C}$ in tube. After 3 days, the starting material was a little existed in reaction and the reaction almost stopped. The resulting mixture was concentrated under reduced pressure and was purified by silica gel column chromatography to give $\mathbf{4}$ and recycled the starting material $\mathbf{3}$.

Procedure C: A mixture of $3(0.1 \mathrm{mmol}), \mathrm{KMnO}_{4}(0.2 \mathrm{mmol}, 2.0$ equiv. $)$ in toluene/actone $(2: 1)(1 \mathrm{~mL})$ was stirred at $0{ }^{\circ} \mathrm{C}$ in tube. Upon reaction completion, the resulting mixture was washed with water. The organic layers was removed and the crude reaction mixture was purified by flash column chromatography using petroleum PE/ EA as eluents to afford the desired products 4.

(R)-4-(2-methoxynaphthalen-1-yl)-1,3-dimethylpyrrolo[1,2-a]quinoxaline (4b):

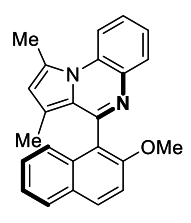

$4 b$

According to the general procedure A, $\mathbf{4 b}$ was obtained in $86 \%$ yield $(30.3 \mathrm{mg})$ with $40 \%$ ee from $\mathbf{3 b}$. According to the general procedure $B, \mathbf{4 b}$ was obtained in $35 \%$ yield $(12.5 \mathrm{mg})$ with $72 \%$ ee from 3 b. mp: $211-213{ }^{\circ} \mathrm{C}$. Flash silica gel chromatography (petroleum ether/ethyl acetate $=10 / 1) .{ }^{1} \mathrm{H}$ NMR $(400 \mathrm{MHz}$, Chloroform- $d) \delta 8.34-8.27(\mathrm{~m}, 1 \mathrm{H}), 8.04-7.99(\mathrm{~m}, 1 \mathrm{H})$, $7.95(\mathrm{dd}, J=9.1,0.8 \mathrm{~Hz}, 1 \mathrm{H}), 7.84-7.77(\mathrm{~m}, 1 \mathrm{H}), 7.47-7.35(\mathrm{~m}, 4 \mathrm{H}), 7.29$ (dtd, $J=8.1,6.8$, $5.1 \mathrm{~Hz}, 2 \mathrm{H}), 6.32(\mathrm{~s}, 1 \mathrm{H}), 3.84(\mathrm{~s}, 3 \mathrm{H}), 2.95(\mathrm{~s}, 3 \mathrm{H}), 1.42(\mathrm{~s}, 3 \mathrm{H}) .{ }^{13} \mathrm{C}$ NMR $(101 \mathrm{MHz}$, Chloroform-d) $\delta 154.7$, 152.5, 137.7, 133.6, 130.6, 130.3, 130.1, 129.1, 127.9, 127.8, 127.1, 126.3, 124.7, 124.6, 124.5, 123.9, 122.2, $118.5,118.2,115.3,113.6,56.8,17.9,12.1$. HRMS (ESI) m/z: $[\mathrm{M}+\mathrm{Na}]^{+}$Calcd for $\mathrm{C}_{24} \mathrm{H}_{20} \mathrm{~N}_{2} \mathrm{ONa} 375.1473$; found 375.1484. Optical rotation: $[\alpha]_{D}{ }^{25}=+80.2\left(\mathrm{c}=0.5, \mathrm{CHCl}_{3}\right)$. HPLC: Chiralpak IA, $n$-hexane/isopropanol $=$ $90 / 10$, Flow rate $=1.0 \mathrm{~mL} / \mathrm{min}, \mathrm{UV}=254 \mathrm{~nm}, t_{\mathrm{R}}($ minor $)=7.13 \mathrm{~min}, t_{\mathrm{R}}($ major $)=8.46 \mathrm{~min}$. 
(R)-8-methoxy-4-(2-methoxynaphthalen-1-yl)-1,3-dimethylpyrrolo[1,2-a]quinoxaline (4c):

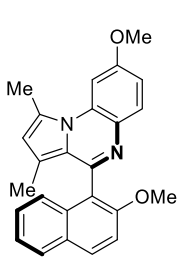

According to the general procedure A, 4c was obtained in $89 \%$ yield $(34.1 \mathrm{mg}$ ) with $68 \%$ ee from 3c. According to the general procedure B, $4 \mathrm{c}$ was obtained in $68 \%$ yield $(25.9 \mathrm{mg})$ with $82 \%$ ee from 3c. mp: $217-219{ }^{\circ} \mathrm{C}$. Flash silica gel chromatography (petroleum ether/ethyl acetate $=10 / 1) .{ }^{1} \mathrm{H}$ NMR $(400 \mathrm{MHz}$, Chloroform- $d) \delta 7.94(\mathrm{dd}, J=9.0,3.2 \mathrm{~Hz}, 2 \mathrm{H}), 7.85-7.79$ $(\mathrm{m}, 2 \mathrm{H}), 7.41-7.35(\mathrm{~m}, 2 \mathrm{H}), 7.32-7.26(\mathrm{~m}, 2 \mathrm{H}), 7.01(\mathrm{dd}, J=8.9,2.6 \mathrm{~Hz}, 1 \mathrm{H}), 6.29(\mathrm{~s}, 1 \mathrm{H})$, 3.94 (s, 3H), 3.84 (s, 3H), 2.96 (s, 3H), 1.40 (s, 3H). ${ }^{13} \mathrm{C}$ NMR (100 MHz, Chloroform-d) $\delta 158.1$, 154.8, 150.2, 133.7, 132.1, 131.0, 130.9, 130.5, 129.1, 127.9, 127.1, 127.0, 124.7, 124.3, 123.8, 122.3, 118.5, 117.5, 113.6, 110.7, 100.8, 56.8, 55.9, 17.7, 12.1. HRMS (ESI) m/z: $[\mathrm{M}+\mathrm{H}]^{+}$Calcd for $\mathrm{C}_{25} \mathrm{H}_{23} \mathrm{~N}_{2} \mathrm{O}_{2} 383.1760$; found 383.1762. Optical rotation: $[\alpha]_{D}{ }^{25}=+110.4\left(\mathrm{c}=0.5, \mathrm{CHCl}_{3}\right)$. HPLC: Chiralpak IA, $n$-hexane/isopropanol $=90 / 10$, Flow rate $=1.0 \mathrm{~mL} / \mathrm{min}, \mathrm{UV}=254 \mathrm{~nm}, t_{\mathrm{R}}($ minor $)=9.15 \mathrm{~min}, t_{\mathrm{R}}($ major $)=10.48 \mathrm{~min}$.

(R)-8-methoxy-4-(2-(methoxymethoxy)naphthalen-1-yl)-1,3-dimethylpyrrolo[1,2-a]quinoxaline (4d):

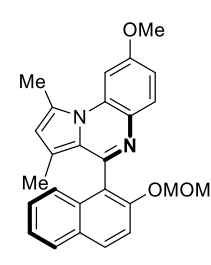

$4 d$

According to the general procedure A, $4 \mathbf{d}$ was obtained in $30 \%$ yield $(12.3 \mathrm{mg})$ with $30 \%$ ee from 3d. According to the general procedure B, $4 \mathbf{d}$ was obtained in $56 \%$ yield $(23.2 \mathrm{mg})$ with $76 \%$ ee from 3d. mp: $169-171^{\circ} \mathrm{C}$. Flash silica gel chromatography (petroleum ether/ethyl acetate $=10 / 1) .{ }^{1} \mathrm{H}$ NMR $(400 \mathrm{MHz}$, Chloroform- $d) \delta 7.97-7.85(\mathrm{~m}, 2 \mathrm{H}), 7.84-7.76(\mathrm{~m}$, 2H), 7.49 (dd, $J=9.0,1.2 \mathrm{~Hz}, 1 \mathrm{H}), 7.43(\mathrm{dd}, J=7.9,2.0 \mathrm{~Hz}, 1 \mathrm{H}), 7.34-7.23(\mathrm{~m}, 2 \mathrm{H}), 7.00$ (dt, $J=9.0,1.6 \mathrm{~Hz}, 1 \mathrm{H}), 6.27(\mathrm{~s}, 1 \mathrm{H}), 5.22(\mathrm{dd}, J=6.9,1.1 \mathrm{~Hz}, 1 \mathrm{H}), 5.08$ (dd, $J=6.9,1.1$ $\mathrm{Hz}, 1 \mathrm{H}), 3.89(\mathrm{~s}, 3 \mathrm{H}), 3.28(\mathrm{~s}, 3 \mathrm{H}), 2.93(\mathrm{~s}, 3 \mathrm{H}), 1.42$ (s, 3H). ${ }^{13} \mathrm{C}$ NMR $(101 \mathrm{MHz}$, Chloroform-d) $\delta 158.1,152.3$, 150.0, 133.5, 132.0, 130.9, 130.7, 130.2, 129.7, 127.8, 127.0, 126.9, 124.8, 124.2, 124.2, 123.8, 118.5, 117.4, 116.5, 110.6, 100.6, 95.0, 56.1, 55.7, 17.6, 11.9. HRMS (ESI) $\mathrm{m} / \mathrm{z}:[\mathrm{M}+\mathrm{H}]^{+}$Calcd for $\mathrm{C}_{26} \mathrm{H}_{25} \mathrm{~N}_{2} \mathrm{O}_{3} 413.1865$; found 413.1857. Optical rotation: $[\alpha]_{D}{ }^{25}=+24.1\left(\mathrm{c}=0.5, \mathrm{CHCl}_{3}\right)$. HPLC: Chiralpak IA, $n$-hexane/isopropanol $=$ $90 / 10$, Flow rate $=1.0 \mathrm{~mL} / \mathrm{min}, \mathrm{UV}=254 \mathrm{~nm}, t_{\mathrm{R}}(\operatorname{minor})=8.29 \mathrm{~min}, t_{\mathrm{R}}($ major $)=10.15 \mathrm{~min}$.

(R)-1-(8-methoxy-1,3-dimethylpyrrolo[1,2-a]quinoxalin-4-yl)naphthalen-2-yl trifluoromethanesulfonate (4e):

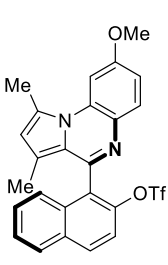

According to the general procedure A, (R)-4e was obtained in $87 \%$ yield $(43.6 \mathrm{mg})$ with $30 \%$ ee from $3 e$. According to the general procedure $B,(\mathbf{S})-4 e$ was obtained in $62 \%$ yield $(31.3 \mathrm{mg})$ with $-77 \%$ ee from 3e. $\mathrm{mp}: 146-147^{\circ} \mathrm{C}$. Flash silica gel chromatography (petroleum ether/ethyl acetate $=10 / 1) .{ }^{1} \mathrm{H}$ NMR $(400 \mathrm{MHz}$, Chloroform- $d) \delta 8.04(\mathrm{~d}, J=9.1 \mathrm{~Hz}, 1 \mathrm{H}), 7.97-7.90(\mathrm{~m}$, 2H), $7.86(\mathrm{~d}, J=2.6 \mathrm{~Hz}, 1 \mathrm{H}), 7.63(\mathrm{dq}, J=8.5,1.0 \mathrm{~Hz}, 1 \mathrm{H}), 7.58-7.51(\mathrm{~m}, 2 \mathrm{H}), 7.44$ (ddd, $J=$ 8.3, 6.8, 1.3 Hz, 1H), $7.04(\mathrm{dd}, J=8.9,2.6 \mathrm{~Hz}, 1 \mathrm{H}), 6.34(\mathrm{~s}, 1 \mathrm{H}), 3.96(\mathrm{~s}, 3 \mathrm{H}), 2.99(\mathrm{~s}, 3 \mathrm{H}), 1.38$

(s, 3H). ${ }^{13} \mathrm{C}$ NMR (100 MHz, Chloroform-d) $\delta 158.7,146.2,145.0,133.2,132.5,131.5,131.2,131.1,131.0$, 129.5, 128.2, 128.2, 128.0, 127.3, 126.3, 123.9, 118.5 (q, $J=322.2 \mathrm{~Hz}$ ), 119.6, 118.9, 117.6, 111.1, 100.8, 55.9, 17.8, 12.1. ${ }^{19} \mathrm{~F}$ NMR (377 MHz, Chloroform-d) $\delta$-74.3. HRMS (ESI) m/z: [M+Na] ${ }^{+}$Calcd for $\mathrm{C}_{25} \mathrm{H}_{19} \mathrm{~F}_{3} \mathrm{~N}_{2} \mathrm{O}_{4} \mathrm{SNa}$ 523.0915; found 523.0903. Optical rotation: $[\alpha]_{D}^{25}=+40.4$ (c $\left.=0.5, \mathrm{CHCl}_{3}\right)$. HPLC: Chiralpak OD-H, $n-$ hexane/isopropanol $=90 / 10$, Flow rate $=1.0 \mathrm{~mL} / \mathrm{min}, \mathrm{UV}=254 \mathrm{~nm}, t_{\mathrm{R}}(\operatorname{minor})=7.02 \mathrm{~min}, t_{\mathrm{R}}$ (major) $=7.54 \mathrm{~min}$.

\section{(R)-4-(2-((tert-butyldimethylsilyl)oxy)naphthalen-1-yl)-8-methoxy-1,3-dimethylpyrrolo[1,2-a]quinoxaline} (4f):

According to the general procedure A, (R)-4f was obtained in $90 \%$ yield $(43.4 \mathrm{mg}$ ) with $84 \%$ ee from $\mathbf{3 f}$. According to the general procedure B, (S)-4f was obtained in $23 \%$ yield $(11.3 \mathrm{mg}$ ) with $-4 \%$ ee from $\mathbf{3 f}$. $\mathrm{mp}$ : $165-$ 


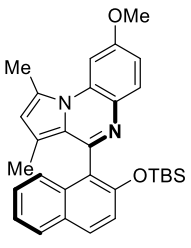

$4 f$

$167^{\circ} \mathrm{C}$. Flash silica gel chromatography (petroleum ether/ethyl acetate $\left.=10 / 1\right) .{ }^{1} \mathbf{H}$ NMR $(400$ $\mathrm{MHz}$, Chloroform- $d$ ) $\delta 8.08(\mathrm{~d}, J=8.9 \mathrm{~Hz}, 1 \mathrm{H}), 8.00-7.91(\mathrm{~m}, 3 \mathrm{H}), 7.69-7.61(\mathrm{~m}, 1 \mathrm{H}), 7.46$ $-7.42(\mathrm{~m}, 2 \mathrm{H}), 7.30$ (d, J = 8.9 Hz, 1H), 7.15 (dd, $J=8.9,2.6 \mathrm{~Hz}, 1 \mathrm{H}), 6.43(\mathrm{~s}, 1 \mathrm{H}), 4.06$ (s, $3 \mathrm{H}), 3.08(\mathrm{~s}, 3 \mathrm{H}), 1.66(\mathrm{~s}, 3 \mathrm{H}), 0.71(\mathrm{~s}, 9 \mathrm{H}), 0.28(\mathrm{~s}, 3 \mathrm{H}), 0.13(\mathrm{~s}, 3 \mathrm{H}) .{ }^{13} \mathrm{C}$ NMR $(100 \mathrm{MHz}$, Chloroform-d) $\delta 157.9,150.9,150.4,133.8,131.9,130.8,130.6,129.9,129.3,127.8,126.7$, 126.7, 124.8, 124.6, 124.4, 123.8, 120.6, 118.3, 117.6, 110.5, 100.5, 55.6, 25.1, 17.7, 17.6, 12.0, -4.1, -4.3. HRMS (ESI) m/z: $[\mathrm{M}+\mathrm{Na}]^{+}$Calcd for $\mathrm{C}_{30} \mathrm{H}_{34} \mathrm{~N}_{2} \mathrm{O}_{2} \mathrm{SiNa} 505.2287$; found 505.2296. Optical rotation: $[\alpha]_{D}{ }^{25}=+22.4\left(c=0.5, \mathrm{CHCl}_{3}\right)$. HPLC: Chiralpak IA, $n$-hexane/isopropanol $=95 / 5$, Flow rate $=1.0$ $\mathrm{mL} / \mathrm{min}, \mathrm{UV}=254 \mathrm{~nm}, t_{\mathrm{R}}$ (minor) $=4.64 \mathrm{~min}, t_{\mathrm{R}}$ (major) $=5.16 \mathrm{~min}$.

\section{(R)-4-(2-((tert-butyldiphenylsilyl)oxy)naphthalen-1-yl)-8-methoxy-1,3-dimethylpyrrolo[1,2-a]quinoxaline} $(4 \mathrm{~g})$ :

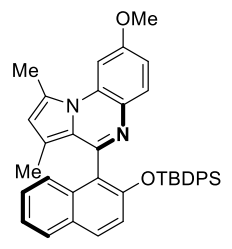

$4 \mathrm{~g}$

According to the general procedure A, $\mathbf{4 g}$ was obtained in $87 \%$ yield $(52.8 \mathrm{mg})$ with $80 \%$ ee. mp: $194-196{ }^{\circ} \mathrm{C}$. Flash silica gel chromatography (petroleum ether/ethyl acetate $=$ 10/1). ${ }^{1} \mathrm{H}$ NMR (400 MHz, Chloroform- $d$ ) $\delta 7.98$ (ddd, $\left.J=8.8,6.3,2.6 \mathrm{~Hz}, 1 \mathrm{H}\right), 7.87(\mathrm{p}$, $J=1.9 \mathrm{~Hz}, 1 \mathrm{H}), 7.68(\mathrm{t}, J=6.7 \mathrm{~Hz}, 3 \mathrm{H}), 7.64-7.59(\mathrm{~m}, 2 \mathrm{H}), 7.54$ (ddd, $J=9.7,7.1,2.2$ $\mathrm{Hz}, 2 \mathrm{H}$ ), $7.39-7.24(\mathrm{~m}, 8 \mathrm{H}), 7.01$ (ddt, $J=8.9,2.7,1.7 \mathrm{~Hz}, 1 \mathrm{H}), 6.83$ (ddd, $J=8.9,6.0$, $2.5 \mathrm{~Hz}, 1 \mathrm{H}), 6.33(\mathrm{~s}, 1 \mathrm{H}), 3.92(\mathrm{~s}, 3 \mathrm{H}), 2.97(\mathrm{~s}, 3 \mathrm{H}), 1.53(\mathrm{~s}, 3 \mathrm{H}), 0.65(\mathrm{~s}, 9 \mathrm{H}) .{ }^{13} \mathrm{C} \mathrm{NMR}$ (100 MHz, Chloroform-d) $\delta 158.0,150.8,150.6,135.6,135.4,133.7,133.3,132.6,132.2,130.9,130.7,129.9$, 129.5, 129.1, 127.8, 127.8, 127.7, 126.9, 126.9, 124.8, 124.6, 124.1, 123.8, 120.2, 118.5, 117.5, 110.5, 100.6, 55.7, 25.9, 19.3, 17.7, 12.2. HRMS (ESI) m/z: $[\mathrm{M}+\mathrm{H}]^{+}$Calcd for $\mathrm{C}_{40} \mathrm{H}_{39} \mathrm{~N}_{2} \mathrm{O}_{2} \mathrm{Si}$ 607.2781; found 607.2788. Optical rotation: $[\alpha]_{D}{ }^{25}=+35.4\left(\mathrm{C}=0.5, \mathrm{CHCl}_{3}\right)$. HPLC: Chiralpak IC, $n$-hexane/isopropanol $=80 / 20$, Flow rate $=1.0 \mathrm{~mL} / \mathrm{min}, \mathrm{UV}=254 \mathrm{~nm}, t_{\mathrm{R}}($ minor $)=4.51 \mathrm{~min}, t_{\mathrm{R}}($ major $)=5.13 \mathrm{~min}$.

(R)-8-methoxy-4-(2-methoxy-6-(trifluoromethyl)phenyl)-1,3-dimethylpyrrolo[1,2-a]quinoxaline (4h):

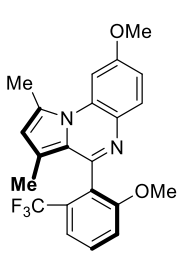

According to the general procedure A, $4 \mathrm{~h}$ was obtained in $85 \%$ yield $(34.2 \mathrm{mg})$ with $90 \%$ ee. $\mathrm{mp}: 182-184^{\circ} \mathrm{C}$. Flash silica gel chromatography (petroleum ether/ethyl acetate $=8 / 1$ ). ${ }^{1} \mathrm{H}$ NMR $(400 \mathrm{MHz}$, Chloroform- $d$ ) $\delta 7.88(\mathrm{~d}, J=8.9 \mathrm{~Hz}, 1 \mathrm{H}), 7.79(\mathrm{~d}, J=2.6 \mathrm{~Hz}, 1 \mathrm{H}), 7.52$ (td, $J=8.2$, $1.0 \mathrm{~Hz}, 1 \mathrm{H}), 7.39$ (d, J = 7.9 Hz, 1H), 7.17 (d, J = 8.3 Hz, 1H), 6.99 (dd, $J=8.9,2.6 \mathrm{~Hz}, 1 \mathrm{H}$ ), $6.32(\mathrm{~s}, 1 \mathrm{H}), 3.92(\mathrm{~s}, 3 \mathrm{H}), 3.71(\mathrm{~s}, 3 \mathrm{H}), 2.94(\mathrm{~s}, 3 \mathrm{H}), 1.64(\mathrm{~s}, 3 \mathrm{H}) .{ }^{13} \mathrm{C}$ NMR $(100 \mathrm{MHz}$, Chloroform- $d$ ) $\delta 158.5,158.3,147.9,131.6,131.0,130.8,130.5,130.2,127.2,126.9,123.9$ (q, $\left.\mathrm{C}-\mathrm{F},{ }^{1} J_{C-F}=275.7 \mathrm{~Hz}\right), 123.7,118.5\left(\mathrm{q}, \mathrm{C}-\mathrm{F},{ }^{2} J_{C-F}=5.1 \mathrm{~Hz}\right), 118.4,116.8,114.6,110.7,100.7,77.4,56.3,55.8$, 17.7, 12.1. ${ }^{19} \mathrm{~F}$ NMR (377 MHz, Chloroform- $d$ ) $\delta$-58.6. HRMS (ESI) $\mathrm{m} / \mathrm{z}: \quad[\mathrm{M}+\mathrm{H}]^{+}$Calcd for $\mathrm{C}_{22} \mathrm{H}_{20} \mathrm{~F}_{3} \mathrm{~N}_{2} \mathrm{O}_{2}$ 401.1477; found 401.1474. Optical rotation: $[\alpha]_{D}{ }^{25}=-30.2\left(\mathrm{c}=0.5, \mathrm{CHCl}_{3}\right.$ ). HPLC: Chiralpak IC, $n$-hexane/isopropanol $=80 / 20$, Flow rate $=1.0 \mathrm{~mL} / \mathrm{min}, \mathrm{UV}=254 \mathrm{~nm}, t_{\mathrm{R}}($ minor $)=6.38 \mathrm{~min}, t_{\mathrm{R}}($ major $)=7.50$ $\min$.

(S)-8-methoxy-4-(2-(methoxymethoxy)-6-(trifluoromethyl)phenyl)-1,3-dimethylpyrrolo[1,2-a]quinoxaline (4i):

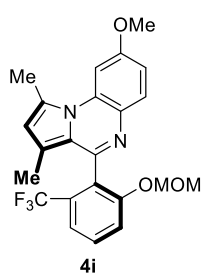

According to the general procedure A, $4 \mathbf{i}$ was obtained in $86 \%$ yield $(37.0 \mathrm{mg})$ with $96 \%$ ee. $\mathrm{mp}: 168-170{ }^{\circ} \mathrm{C}$. Flash silica gel chromatography (petroleum ether/ethyl acetate $=8 / 1$ ). ${ }^{1} \mathrm{H}$ NMR (400 MHz, Chloroform-d) $\delta 7.87$ (d, $J=8.9 \mathrm{~Hz}, 1 \mathrm{H}), 7.78(\mathrm{~d}, J=2.6 \mathrm{~Hz}, 1 \mathrm{H}), 7.52-$ $7.37(\mathrm{~m}, 3 \mathrm{H}), 6.97$ (dd, J = 8.9, $2.6 \mathrm{~Hz}, 1 \mathrm{H}), 6.30$ (s, 1H), 5.10 (d, J = 7.0 Hz, 1H), 4.98 (d, J $=7.0 \mathrm{~Hz}, 1 \mathrm{H}), 3.88(\mathrm{~s}, 3 \mathrm{H}), 3.22(\mathrm{~s}, 3 \mathrm{H}), 2.90(\mathrm{~s}, 3 \mathrm{H}), 1.67(\mathrm{~s}, 3 \mathrm{H}) \cdot{ }^{13} \mathrm{C}$ NMR $(100 \mathrm{MHz}$, 
Chloroform- $d$ ) $\delta 158.2,156.1,147.8,131.4,130.9,130.6,130.3,130.0,127.9,126.9,123.8$ (q, C-F, ${ }^{1} J_{C-F}=275.7$ $\mathrm{Hz}), 123.6,119.6\left(\mathrm{q}, \mathrm{C}-\mathrm{F},{ }^{2} J_{C-F}=5.1 \mathrm{~Hz}\right), 118.4,118.2,116.8,110.7,100.6,94.4,56.2,55.7,17.6,12.0 .{ }^{19} \mathrm{~F} \mathrm{NMR}$ (377 MHz, Chloroform-d) $\delta$-58.6. HRMS (ESI) m/z: [M+H] ${ }^{+}$Calcd for $\mathrm{C}_{23} \mathrm{H}_{22} \mathrm{~F}_{3} \mathrm{~N}_{2} \mathrm{O}_{3}$ 431.1583; found 431.1576. Optical rotation: $[\alpha]_{D}{ }^{25}=-42.1\left(c=0.5, \mathrm{CHCl}_{3}\right)$. HPLC: Chiralpak IC, $n$-hexane/isopropanol $=90 / 10$, Flow rate $=1.0 \mathrm{~mL} / \mathrm{min}, \mathrm{UV}=254 \mathrm{~nm}, t_{\mathrm{R}}($ minor $)=7.84 \mathrm{~min}, t_{\mathrm{R}}($ major $)=8.66 \mathrm{~min}$.

(R)-6-(2-methoxynaphthalen-1-yl)-11H-indolo[3,2-c]quinolone (4k):

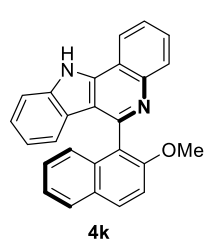

According to the general procedure B, $4 \mathbf{k}$ was obtained in $46 \%$ yield $(17.3 \mathrm{mg}$ ) with $68 \%$ ee from 3k. According to the general procedure C, $\mathbf{4 k}$ was obtained in $87 \%$ yield $(32.6 \mathrm{mg})$ with $64 \%$ ee from 3k. mp: $244-246{ }^{\circ} \mathrm{C}$. Flash silica gel chromatography (petroleum ether/ethyl acetate $=8 / 1) .{ }^{1} \mathrm{H}$ NMR $\left(400 \mathrm{MHz}\right.$, DMSO- $\left.d_{6}\right) \delta 8.67-8.60(\mathrm{~m}, 1 \mathrm{H}), 8.22(\mathrm{~d}, J=9.0 \mathrm{~Hz}, 1 \mathrm{H})$, $8.16-8.09(\mathrm{~m}, 1 \mathrm{H}), 8.05-7.98(\mathrm{~m}, 1 \mathrm{H}), 7.81-7.62(\mathrm{~m}, 4 \mathrm{H}), 7.33(\mathrm{tdd}, J=8.1,6.9,1.2 \mathrm{~Hz}$, 2H), 7.20 (ddd, $J=8.2,6.7,1.3 \mathrm{~Hz}, 1 \mathrm{H}$ ), 7.05 (dd, $J=8.5,1.0 \mathrm{~Hz}, 1 \mathrm{H}$ ), 6.88 (ddd, $J=8.0,7.1,1.0 \mathrm{~Hz}, 1 \mathrm{H}$ ), 6.52 (d, $J=7.9 \mathrm{~Hz}, 1 \mathrm{H}), 3.69$ (s, 3H). ${ }^{13} \mathrm{C}$ NMR $(101 \mathrm{MHz} \text {, DMSO-d })_{6} \delta 154.1,152.0,145.4,140.3,138.8,132.4$, 130.2, 129.4, 128.5, 128.1, 128.0, 126.6, 125.6, 125.0, 124.1, 123.6, 122.7, 122.0, 121.8, 120.2, 119.9, 116.6, 114.3, 114.0, 111.7, 56.2. HRMS (ESI) m/z: $[\mathrm{M}+\mathrm{Na}]^{+}$Calcd for $\mathrm{C}_{26} \mathrm{H}_{18} \mathrm{~N}_{2} \mathrm{ONa} 397.1317$; found 397.1304. Optical rotation: $[\alpha]_{D}{ }^{25}=+74.8\left(\mathrm{c}=0.5, \mathrm{CHCl}_{3}\right)$. HPLC: Chiralpak IA, $n$-hexane/isopropanol $=60 / 40$, Flow rate $=1.0$ $\mathrm{mL} / \mathrm{min}, \mathrm{UV}=254 \mathrm{~nm}, t_{\mathrm{R}}$ (major) $=4.51 \mathrm{~min}, t_{\mathrm{R}}(\operatorname{minor})=7.14 \mathrm{~min}$.

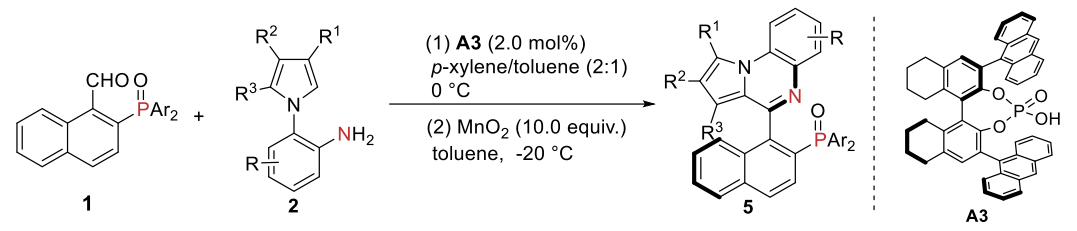

aldehyde $1(0.50 \mathrm{mmol}), 2(0.60 \mathrm{mmol}, 1.2$ equiv.) and chiral phosphoric acid $\mathbf{A} 3(2.0 \mathrm{~mol} \%)$ were stirred in a mixed solvent of $p$-xylene/toluene $(2: 1 \mathrm{v} / \mathrm{v}, 5.0 \mathrm{~mL}, 0.1 \mathrm{M})$ at $0{ }^{\circ} \mathrm{C}$ for $12 \mathrm{~h}$. Upon reaction completion, the reaction solvent was removed and the crude reaction mixture was purified by flash column chromatography using petroleum PE/EA as eluents to afford the first step product. The product was slightly oxidized in the air at room temperature, so the isolated product was immediately oxidized by $\mathrm{MnO}_{2}$.

A tube was charged with $\mathrm{MnO}_{2}$ (5.0 mmol, 10.0 equiv.) in toluene $(5.0 \mathrm{~mL})$. The reaction mixture was cooled to $-20^{\circ} \mathrm{C}$ and stirred for 10 minutes, then the first step products was added to the reaction mixture and stirred for $2 \mathrm{~h}$. The reaction mixture was filtered through Celite and solvent was removed in vacuo. The crude mass was purified by silica gel column chromatography to give $\mathbf{5}$.

(R)-(1-(8-methoxy-1,3-dimethylpyrrolo[1,2-a]quinoxalin-4-yl)naphthalen-2-yl)diphenylphosphine oxide (5a):

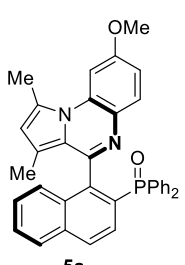

$5 a$

$231.5 \mathrm{mg}, 84 \%$ yield, yellow solid, $\mathrm{mp}: 90-92{ }^{\circ} \mathrm{C}, 90 \%$ ee. The enantiomeric excess can be increased up to $>99 \%$ through a recrystallization in acetonitrile. The crystals was easily afforded from solution with $64 \%$ yield. Flash silica gel chromatography (petroleum ether/ethyl acetate = 2/1). ${ }^{1} \mathrm{H}$ NMR (400 MHz, Chloroform- $d$ ) $\delta 8.26$ (ddd, $J=10.7,8.6,1.8 \mathrm{~Hz}, 1 \mathrm{H}$ ), 8.05 (ddt, $J=$ $11.4,6.6,1.7 \mathrm{~Hz}, 3 \mathrm{H}), 7.92-7.85(\mathrm{~m}, 1 \mathrm{H}), 7.62(\mathrm{~d}, J=8.9 \mathrm{~Hz}, 1 \mathrm{H}), 7.55-7.49(\mathrm{~m}, 3 \mathrm{H}), 7.49$ - $7.39(\mathrm{~m}, 3 \mathrm{H}), 7.38-7.28(\mathrm{~m}, 1 \mathrm{H}), 7.01$ (ddd, $J=12.6,8.1,1.3 \mathrm{~Hz}, 2 \mathrm{H}), 6.91$ (dt, $J=8.9,2.2$

$\mathrm{Hz}, 1 \mathrm{H}$ ), 6.85 (ddd, $J=7.4,4.5,1.5 \mathrm{~Hz}, 1 \mathrm{H}), 6.54$ (td, $J=7.7,2.9 \mathrm{~Hz}, 2 \mathrm{H}), 6.13(\mathrm{~s}, 1 \mathrm{H}), 3.90$ (s, 3H), 2.78 (s, 
3H), 1.30 (s, 3H). ${ }^{13} \mathrm{C}$ NMR (100 MHz, Chloroform- $d$ ) $\delta 158.3,150.0$ (d, $\left.J=5.0 \mathrm{~Hz}\right), 140.2$ (d, $\left.J=9.5 \mathrm{~Hz}\right), 134.6$ (d, $J=2.7 \mathrm{~Hz}), 132.8$ (d, $J=10.2 \mathrm{~Hz}), 132.7$ (d, $J=95.5 \mathrm{~Hz}), 132.1$ (d, $J=2.1 \mathrm{~Hz}), 131.5$ (d, $J=2.9 \mathrm{~Hz}), 131.1$, 130.9, 130.8, 130.7, 130.6, 130.4, 129.4, 128.9 (d, $J=11.4 \mathrm{~Hz}), 128.6$ (d, $J=8.9 \mathrm{~Hz}), 128.0,128.0,127.9$, 127.4, 126.9, $126.3(\mathrm{~d}, J=6.1 \mathrm{~Hz}), 126.2,124.7,118.4,117.7,110.3,100.3,55.7,17.4,12.4 .{ }^{31} \mathrm{P}$ NMR $(162$ $\mathrm{MHz}$, Chloroform- $d$ ) $\delta$ 30.9. HRMS (ESI) m/z: $[\mathrm{M}+\mathrm{H}]^{+}$Calcd for $\mathrm{C}_{36} \mathrm{H}_{30} \mathrm{~N}_{2} \mathrm{O}_{2} \mathrm{P}$ 553.204; found 553.2039. Optical rotation: $[\alpha]_{D}{ }^{25}=+304.3\left(c=0.5, \mathrm{CHCl}_{3}\right)$. HPLC: Chiralpak IA, $n$-hexane/isopropanol $=70 / 30$, Flow rate $=1.0$ $\mathrm{mL} / \mathrm{min}, \mathrm{UV}=254 \mathrm{~nm}, t_{\mathrm{R}}$ (minor) $=6.70 \mathrm{~min}, t_{\mathrm{R}}$ (major) $=10.41 \mathrm{~min}$.

(R)-(1-(8-methoxy-1,3-dimethylpyrrolo[1,2-a]quinoxalin-4-yl)naphthalen-2-yl)di-o-tolylphosphine oxide (5b):

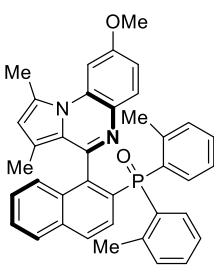

$5 b$

$261.4 \mathrm{mg}, 90 \%$ yield, yellow solid, $\mathrm{mp}: 137-139^{\circ} \mathrm{C}, 88 \%$ ee. Flash silica gel chromatography (petroleum ether/ethyl acetate $=2 / 1) .{ }^{1} \mathrm{H}$ NMR $(400 \mathrm{MHz}$, Chloroform- $d) \delta 8.34(\mathrm{t}, \mathrm{J}=9.6$ $\mathrm{Hz}, 1 \mathrm{H}), 8.10(\mathrm{dd}, J=8.7,1.8 \mathrm{~Hz}, 1 \mathrm{H}), 7.93(\mathrm{~d}, J=8.2 \mathrm{~Hz}, 1 \mathrm{H}), 7.59-7.46(\mathrm{~m}, 4 \mathrm{H}), 7.42-$ $7.27(\mathrm{~m}, 3 \mathrm{H}), 7.22-7.14(\mathrm{~m}, 2 \mathrm{H}), 6.82(\mathrm{dd}, J=8.8,2.5 \mathrm{~Hz}, 2 \mathrm{H}), 6.63$ (dd, $J=7.7,4.2 \mathrm{~Hz}$, $1 \mathrm{H}), 6.44(\mathrm{~s}, 1 \mathrm{H}), 6.14(\mathrm{~d}, J=23.4 \mathrm{~Hz}, 2 \mathrm{H}), 3.88(\mathrm{~s}, 3 \mathrm{H}), 2.78(\mathrm{~s}, 3 \mathrm{H}), 2.44(\mathrm{~s}, 3 \mathrm{H}), 2.30$ (s, $3 \mathrm{H}), 1.24(\mathrm{~s}, 3 \mathrm{H}) .{ }^{13} \mathrm{C}$ NMR (100 MHz, Chloroform- $\left.d\right) \delta 158.1,150.2(\mathrm{~d}, J=5.0 \mathrm{~Hz}), 143.3$ (d, $J=8.0 \mathrm{~Hz}$ ), 142.1 (d, $J=8.7 \mathrm{~Hz}), 140.5$ (d, $J=8.6 \mathrm{~Hz}$ ), 135.5 (d, $J=13.2 \mathrm{~Hz}), 134.7$ (d, $J=2.2 \mathrm{~Hz}), 132.5$ (d, $J=3.1 \mathrm{~Hz}), 132.4,131.9,131.5(\mathrm{~d}, J=2.4 \mathrm{~Hz}), 131.2(\mathrm{~d}, J=10.7 \mathrm{~Hz}), 131.0,130.9,130.7(\mathrm{~d}, J=28.2 \mathrm{~Hz})$, 130.4, 130.1 (d, $J=10.9 \mathrm{~Hz}), 129.7$ (d, $J=2.8 \mathrm{~Hz}), 129.2$ (d, $J=84.6 \mathrm{~Hz}), 129.1$ (d, $J=8.8 \mathrm{~Hz}), 128.9,128.0$, 127.9, 127.3, 126.9, 126.6, 124.7 (d, $J=13.1 \mathrm{~Hz}), 124.5,123.5$ (d, $J=13.5 \mathrm{~Hz}$ ), 118.1, 117.0, 110.2, 100.3, 55.7, $22.3(\mathrm{~d}, J=4.4 \mathrm{~Hz}), 22.1$ (d, $J=4.3 \mathrm{~Hz}), 17.6,12.8 .{ }^{31} \mathrm{P}$ NMR (162 MHz, Chloroform-d) $\delta 36.4$. HRMS (ESI) m/z: $[M+H]^{+}$Calcd for $\mathrm{C}_{38} \mathrm{H}_{34} \mathrm{~N}_{2} \mathrm{O}_{2} \mathrm{P}$ 581.2358; found 581.2357. Optical rotation: $[\alpha]_{D}{ }^{25}=+188.9(\mathrm{c}=0.5$, $\mathrm{CHCl}_{3}$ ). HPLC: Chiralpak IC, $n$-hexane/isopropanol $=80 / 20$, Flow rate $=1.0 \mathrm{~mL} / \mathrm{min}, \mathrm{UV}=254 \mathrm{~nm}, t_{\mathrm{R}}($ major$)=$ $20.98 \mathrm{~min}, t_{\mathrm{R}}$ (minor) $=23.71 \mathrm{~min}$.

(R)-(1-(8-methoxy-1,3-dimethylpyrrolo[1,2-a]quinoxalin-4-yl)naphthalen-2-yl)di-m-tolylphosphine oxide (5c):

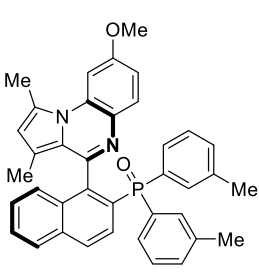

5c

$223.6 \mathrm{mg}, 77 \%$ yield, yellow solid, $\mathrm{mp}: 108-109{ }^{\circ} \mathrm{C}, 86 \%$ ee. Flash silica gel chromatography (petroleum ether/ethyl acetate $=2 / 1)$. ${ }^{1} \mathbf{H}$ NMR $(400 \mathrm{MHz}$, Chloroformd) $\delta 8.27$ (dd, $J=10.9,8.6 \mathrm{~Hz}, 1 \mathrm{H}$ ), 8.05 (dd, $J=8.7,1.8 \mathrm{~Hz}, 1 \mathrm{H}), 7.88(\mathrm{td}, J=12.8,7.7$ $\mathrm{Hz}, 3 \mathrm{H}), 7.66(\mathrm{~d}, J=8.8 \mathrm{~Hz}, 1 \mathrm{H}), 7.59-7.47(\mathrm{~m}, 3 \mathrm{H}), 7.30(\mathrm{dt}, J=10.3,7.1 \mathrm{~Hz}, 3 \mathrm{H})$, $7.03-6.90(\mathrm{~m}, 2 \mathrm{H}), 6.67$ (dt, $J=7.8,4.9 \mathrm{~Hz}, 2 \mathrm{H}), 6.38$ (td, $J=7.6,3.5 \mathrm{~Hz}, 1 \mathrm{H}), 6.14$ (s, 1H), 3.91 (s, 3H), 2.79 (s, 3H), 2.35 (s, 3H), $1.80(\mathrm{~s}, 3 \mathrm{H}), 1.32(\mathrm{~s}, 3 \mathrm{H}) .{ }^{13} \mathrm{C}$ NMR $(100$ MHz, Chloroform-d) $\delta 158.3,150.1$ (d, $J=5.4 \mathrm{~Hz}$ ), 140.1 (d, $J=9.3 \mathrm{~Hz}), 137.8$ (d, $J=12.0 \mathrm{~Hz}), 136.5$ (d, $J=$ 12.3 Hz), 134.6 (d, J = 2.2 Hz), 133.0, 133.0 (d, $J=10.0 \mathrm{~Hz}), 132.3$ (d, $J=2.9 \mathrm{~Hz}), 132.1$ (d, J = 2.7 Hz), 132.1 (d, $J=32.5 \mathrm{~Hz}), 131.4$ (d, $J=9.8 \mathrm{~Hz}), 131.1,130.8,130.5,130.3(\mathrm{~d}, J=101.0 \mathrm{~Hz}), 130.4(\mathrm{~d}, J=2.9 \mathrm{~Hz}), 130.2$ (d, $J=11.0 \mathrm{~Hz}$ ), 128.9 (d, $J=11.3 \mathrm{~Hz}), 128.7$ (d, $J=8.9 \mathrm{~Hz}), 128.3(\mathrm{~d}, J=11.1 \mathrm{~Hz}), 128.0$ (d, J = 7.2 Hz), 127.8 (d, $J=13.2 \mathrm{~Hz}$ ), 127.3, 126.8, 126.4, 126.1 (d, $J=13.5 \mathrm{~Hz}$ ), 124.7, 118.4, 117.7, 110.3, 100.4, 55.8, 21.5, 20.9, 17.4, 12.4. ${ }^{31} \mathrm{P}$ NMR (162 MHz, Chloroform-d) $\delta$ 31.2. HRMS (ESI) m/z: $[\mathrm{M}+\mathrm{H}]^{+}$Calcd for $\mathrm{C}_{38} \mathrm{H}_{34} \mathrm{~N}_{2} \mathrm{O}_{2} \mathrm{P} 581.2358$; found 581.2361. Optical rotation: $[\alpha]_{D}{ }^{25}=+323.3\left(\mathrm{c}=0.5, \mathrm{CHCl}_{3}\right)$. HPLC: Chiralpak IA, $n$-hexane/isopropanol $=80 / 20$, Flow rate $=1.0 \mathrm{~mL} / \mathrm{min}, \mathrm{UV}=254 \mathrm{~nm}, t_{\mathrm{R}}($ minor $)=7.98 \mathrm{~min}, t_{\mathrm{R}}($ major $)=11.05 \mathrm{~min}$. 

(5d):

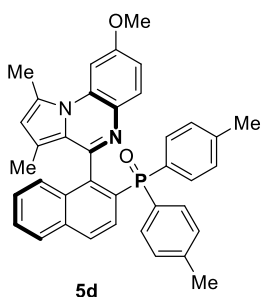

$5 d$

$200.5 \mathrm{mg}, 69 \%$ yield, yellow solid, $\mathrm{mp}: 92-95{ }^{\circ} \mathrm{C}, 88 \%$ ee. Flash silica gel chromatography (petroleum ether/ethyl acetate $=2 / 1) .{ }^{1} \mathrm{H}$ NMR $(400 \mathrm{MHz}$, Chloroformd) $\delta 8.24(\mathrm{dd}, J=10.9,8.7 \mathrm{~Hz}, 1 \mathrm{H}), 8.04(\mathrm{dd}, J=8.7,1.8 \mathrm{~Hz}, 1 \mathrm{H}), 7.95-7.84(\mathrm{~m}, 3 \mathrm{H})$, $7.65(\mathrm{~d}, J=8.8 \mathrm{~Hz}, 1 \mathrm{H}), 7.58(\mathrm{~d}, J=2.6 \mathrm{~Hz}, 1 \mathrm{H}), 7.55-7.45(\mathrm{~m}, 2 \mathrm{H}), 7.31$ (ddd, $J=8.3$, 6.7, $1.3 \mathrm{~Hz}, 1 \mathrm{H}$ ), $7.23(\mathrm{dd}, J=8.1,2.6 \mathrm{~Hz}, 2 \mathrm{H}), 6.97-6.86(\mathrm{~m}, 3 \mathrm{H}), 6.36$ (dd, $J=8.0$, $2.8 \mathrm{~Hz}, 2 \mathrm{H}), 6.16(\mathrm{~s}, 1 \mathrm{H}), 3.92(\mathrm{~s}, 3 \mathrm{H}), 2.84(\mathrm{~s}, 3 \mathrm{H}), 2.37(\mathrm{~s}, 3 \mathrm{H}), 2.05(\mathrm{~s}, 3 \mathrm{H}), 1.31(\mathrm{~s}$, 3H). ${ }^{13} \mathrm{C}$ NMR (100 MHz, Chloroform- $d$ ) $\delta$ 158.2, $150.2(\mathrm{~d}, J=5.2 \mathrm{~Hz}), 141.9$ (d, $\left.J=2.9 \mathrm{~Hz}\right), 140.2(\mathrm{~d}, J=2.8$ $\mathrm{Hz}), 140.1(\mathrm{~d}, J=9.6 \mathrm{~Hz}$ ), $134.7(\mathrm{~d}, J=2.2 \mathrm{~Hz}), 132.8(\mathrm{~d}, J=10.4 \mathrm{~Hz}), 132.2(\mathrm{~d}, J=11.5 \mathrm{~Hz}), 131.1,131.0$, 131.0, 130.9, 130.5, 130.1 (d, $J=41.3 \mathrm{~Hz}), 129.3(\mathrm{~d}, J=7.3 \mathrm{~Hz}), 128.9(\mathrm{~d}, J=15.1 \mathrm{~Hz}), 128.8,128.7,128.2$, $128.0(\mathrm{~d}, J=8.7 \mathrm{~Hz}), 127.3,127.0(\mathrm{~d}, J=13.0 \mathrm{~Hz}), 126.7,126.4,124.9,118.3,117.9,110.5,100.1,55.8,21.7$, 21.6, 17.5, 12.4. ${ }^{31} \mathrm{P}$ NMR (162 MHz, Chloroform-d) $\delta$ 30.7. HRMS (ESI) m/z: $[\mathrm{M}+\mathrm{H}]^{+}$Calcd for $\mathrm{C}_{38} \mathrm{H}_{34} \mathrm{~N}_{2} \mathrm{O}_{2} \mathrm{P}$ 581.2358; found 581.2364. Optical rotation: $[\alpha]_{D}{ }^{25}=+223.0\left(\mathrm{c}=0.5, \mathrm{CHCl}_{3}\right)$. HPLC: Chiralpak IA, $n-$ hexane/isopropanol $=80 / 20$, Flow rate $=1.0 \mathrm{~mL} / \mathrm{min}, \mathrm{UV}=254 \mathrm{~nm}, t_{R}($ minor $)=12.53 \mathrm{~min}, t_{R}($ major $)=25.00 \mathrm{~min}$.

\section{(R)-(1-(8-methoxy-1,3-dimethylpyrrolo[1,2-a]quinoxalin-4-yl)naphthalen-2-yl)bis(4-} methoxyphenyl)phosphine oxide (5e):

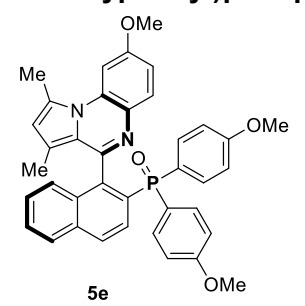

$220.5 \mathrm{mg}, 72 \%$ yield, yellow solid, mp: $114-116{ }^{\circ} \mathrm{C}, 80 \%$ ee. Flash silica gel chromatography (petroleum ether/ethyl acetate $=2 / 1) .{ }^{1} \mathrm{H} \mathrm{NMR}(400 \mathrm{MHz}$, Chloroformd) $\delta 8.30(\mathrm{dd}, J=10.9,8.7 \mathrm{~Hz}, 1 \mathrm{H}), 8.08-7.95(\mathrm{~m}, 3 \mathrm{H}), 7.88(\mathrm{dt}, J=8.2,1.0 \mathrm{~Hz}, 1 \mathrm{H})$, $7.67(\mathrm{~d}, J=8.8 \mathrm{~Hz}, 1 \mathrm{H}), 7.58(\mathrm{~d}, J=2.6 \mathrm{~Hz}, 1 \mathrm{H}), 7.54-7.48(\mathrm{~m}, 2 \mathrm{H}), 7.31$ (ddd, $J=$ 8.3, 6.7, $1.3 \mathrm{~Hz}, 1 \mathrm{H}), 6.99-6.84(\mathrm{~m}, 5 \mathrm{H}), 6.15(\mathrm{~s}, 1 \mathrm{H}), 6.04-5.94(\mathrm{~m}, 2 \mathrm{H}), 3.91(\mathrm{~s}$, $3 \mathrm{H}), 3.80(\mathrm{~s}, 3 \mathrm{H}), 3.54(\mathrm{~s}, 3 \mathrm{H}), 2.82(\mathrm{~s}, 3 \mathrm{H}), 1.29(\mathrm{~s}, 3 \mathrm{H}) .{ }^{13} \mathrm{C} \mathrm{NMR}(100 \mathrm{MHz}$, Chloroform- $d$ ) $\delta 162.1$ (d, $J=2.8 \mathrm{~Hz}$ ), $160.6(\mathrm{~d}, J=2.9 \mathrm{~Hz}), 158.2,150.3(\mathrm{~d}, J=5.4 \mathrm{~Hz}), 139.7$ (d, $J=9.4 \mathrm{~Hz})$, $134.6(\mathrm{~d}, J=12.3 \mathrm{~Hz}), 132.4(\mathrm{~d}, J=12.2 \mathrm{~Hz}), 132.2,131.2,131.2,130.9,130.4,130.2,128.9(\mathrm{~d}, J=11.1 \mathrm{~Hz})$, $128.7(\mathrm{~d}, J=8.9 \mathrm{~Hz}), 128.0,127.9,127.3,127.0,126.4,124.8,124.4(\mathrm{~d}, J=78.7 \mathrm{~Hz}), 123.3(\mathrm{~d}, J=80.0 \mathrm{~Hz})$, 118.3, 117.9, $113.5(\mathrm{~d}, J=13.3 \mathrm{~Hz}), 111.6(\mathrm{~d}, J=13.7 \mathrm{~Hz}), 110.4,100.2,55.7,55.2,54.7,17.2,12.4 .{ }^{31} \mathrm{P}$ NMR (162 MHz, Chloroform-d) $\delta$ 30.5. HRMS (ESI) m/z: $[M+H]^{+}$Calcd for $\mathrm{C}_{38} \mathrm{H}_{34} \mathrm{~N}_{2} \mathrm{O}_{4} \mathrm{P}$ 613.2256; found 613.2251. Optical rotation: $[\alpha]_{D}{ }^{25}=+300.3\left(\mathrm{c}=0.5, \mathrm{CHCl}_{3}\right)$. HPLC: Chiralpak IA, $n$-hexane/isopropanol $=70 / 30$, Flow rate $=1.0 \mathrm{~mL} / \mathrm{min}, \mathrm{UV}=254 \mathrm{~nm}, t_{\mathrm{R}}($ minor $)=10.13 \mathrm{~min}, t_{\mathrm{R}}($ major $)=15.38 \mathrm{~min}$.

(R)-bis(4-fluorophenyl)(1-(8-methoxy-1,3-dimethylpyrrolo[1,2-a]quinoxalin-4-yl)naphthalen-2yl)phosphine oxide (5f):

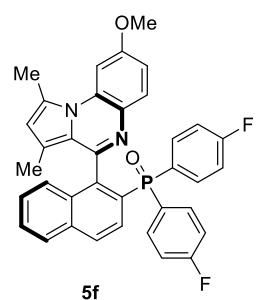

$205.9 \mathrm{mg}, 70 \%$ yield, yellow solid, $\mathrm{mp}: 121-123{ }^{\circ} \mathrm{C}, 86 \%$ ee. Flash silica gel chromatography (petroleum ether/ethyl acetate $=2 / 1) .{ }^{1} \mathrm{H}$ NMR $(400 \mathrm{MHz}$, Chloroform- $d)$ $\delta 8.29(\mathrm{dd}, J=11.0,8.7 \mathrm{~Hz}, 1 \mathrm{H}), 8.17-8.07(\mathrm{~m}, 3 \mathrm{H}), 7.92(\mathrm{dd}, J=8.3,1.1 \mathrm{~Hz}, 1 \mathrm{H}), 7.67$ $-7.60(\mathrm{~m}, 2 \mathrm{H}), 7.59-7.50(\mathrm{~m}, 2 \mathrm{H}), 7.36$ (ddd, $J=8.4,6.8,1.3 \mathrm{~Hz}, 1 \mathrm{H}), 7.15$ (ddt, $J=8.8$, 6.7, $2.1 \mathrm{~Hz}, 2 \mathrm{H}), 6.99-6.85(\mathrm{~m}, 3 \mathrm{H}), 6.22-6.13(\mathrm{~m}, 3 \mathrm{H}), 3.94(\mathrm{~s}, 3 \mathrm{H}), 2.85(\mathrm{~s}, 3 \mathrm{H}), 1.27$ (s, 3H). ${ }^{13} \mathrm{C}$ NMR (100 MHz, Chloroform-d) $\delta 165.2\left(\mathrm{~d}, \mathrm{C}-\mathrm{F},{ }^{1} \mathrm{~J}_{\mathrm{C}-\mathrm{F}}=253.3 \mathrm{~Hz}\right), 165.1$ (d, $C-F,{ }^{1} J_{C-F}=253.3 \mathrm{~Hz}$ ), $164.89(\mathrm{~d}, J=2.9 \mathrm{~Hz}), 158.6,150.0(\mathrm{~d}, J=5.1 \mathrm{~Hz}), 140.0(\mathrm{~d}, J=10.0 \mathrm{~Hz}), 135.6(\mathrm{dd}, \mathrm{C}-$ $\left.\mathrm{F},{ }^{3} J_{C-F}=12.2, C-P, 8.7 \mathrm{~Hz}\right), 134.9(\mathrm{~d}, J=2.2 \mathrm{~Hz}), 132.9\left(\mathrm{dd}, \mathrm{C}-\mathrm{F},{ }^{3} J_{C-F}=12.2, \mathrm{C}-\mathrm{P}, 8.9 \mathrm{~Hz}\right), 132.2(\mathrm{~d}, J=11.6$ $\mathrm{Hz}), 130.9(\mathrm{~d}, J=9.5 \mathrm{~Hz}$ ), 130.4, $129.6(\mathrm{~d}, J=104.0 \mathrm{~Hz}$ ), 129.3 (d, $J=11.2 \mathrm{~Hz}$ ), 129.0 (dd, C-P, $J=100.0, \mathrm{C}-$ $F,{ }^{4} J_{C-F}=3.0 \mathrm{~Hz}$ ), $127.4\left(\mathrm{dd}, \mathrm{C}-\mathrm{P}, J=71.7, \mathrm{C}-\mathrm{F},{ }^{3} J_{C-F}=3.0 \mathrm{~Hz}\right), 128.4,128.4,128.3,128.2,127.7,127.5,126.4$, 
124.8, 118.5, 118.1, 115.5 (dd, C-F, ${ }^{2} J_{C-F}=21.3, C-P, 13.6 \mathrm{~Hz}$ ), 113.5 (dd, C-F, $\left.{ }^{2} J_{C-F}=21.6, C-P, 13.9 \mathrm{~Hz}\right), 111.1$, 100.2. ${ }^{19} \mathrm{~F}$ NMR (377 MHz, Chloroform- $d$ ) $\delta-107.0,-108.2 .{ }^{31} \mathrm{P}$ NMR (162 MHz, Chloroform- $d$ ) $\delta$ 29.9. HRMS (ESI) $\mathrm{m} / \mathrm{z}$ : $[\mathrm{M}+\mathrm{H}]^{+}$Calcd for $\mathrm{C}_{36} \mathrm{H}_{28} \mathrm{~F}_{2} \mathrm{~N}_{2} \mathrm{O}_{2} \mathrm{P}$ 589.1856; found 589.1855. Optical rotation: $[\alpha]_{D}{ }^{25}=+231.0(\mathrm{c}=$ $0.5, \mathrm{CHCl}_{3}$ ). HPLC: Chiralpak IA, $n$-hexane/isopropanol $=80 / 20$, Flow rate $=1.0 \mathrm{~mL} / \mathrm{min}, \mathrm{UV}=254 \mathrm{~nm}, t_{\mathrm{R}}$ (minor) $=7.28 \mathrm{~min}, t_{\mathrm{R}}$ (major) $=18.68 \mathrm{~min}$.

(R)-(1-(8-methoxy-1,3-dimethylpyrrolo[1,2-a]quinoxalin-4-yl)naphthalen-2-yl)di(naphthalen-2yl)phosphine oxide $(5 \mathrm{~g})$ :

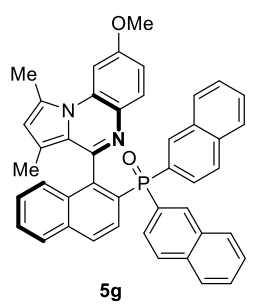

$247.9 \mathrm{mg}, 76 \%$ yield, yellow solid, $\mathrm{mp}: 141-143^{\circ} \mathrm{C}, 89 \%$ ee. The enantiomeric excess of $\mathbf{5 g}$ can be increased up to $>99 \%$ through a simple recrystallization in acetonitrile. The crystals were afforded with $67 \%$ yield. Flash silica gel chromatography (petroleum ether/ethyl acetate $=2 / 1) .{ }^{1} \mathrm{H}$ NMR $(400 \mathrm{MHz}$, Chloroform- $d) \delta 8.72(\mathrm{~d}, J=14.1 \mathrm{~Hz}, 1 \mathrm{H})$, 8.33 (dd, $J=11.0,8.7 \mathrm{~Hz}, 1 \mathrm{H}$ ), 8.22 (ddd, $J=10.1,8.3,1.5 \mathrm{~Hz}, 1 \mathrm{H}$ ), 8.07 (dd, $J=8.8,1.9$ $\mathrm{Hz}, 1 \mathrm{H}), 7.88$ (dt, $J=16.8,8.4 \mathrm{~Hz}, 4 \mathrm{H}), 7.61(\mathrm{~d}, J=8.5 \mathrm{~Hz}, 1 \mathrm{H}), 7.58-7.46(\mathrm{~m}, 5 \mathrm{H}), 7.41$ - $7.29(\mathrm{~m}, 3 \mathrm{H}), 7.24-7.16(\mathrm{~m}, 2 \mathrm{H}), 7.06-6.96(\mathrm{~m}, 2 \mathrm{H}), 6.80(\mathrm{~d}, J=8.7 \mathrm{~Hz}, 2 \mathrm{H}), 6.00(\mathrm{~s}, 1 \mathrm{H}), 3.75(\mathrm{~s}, 3 \mathrm{H})$, 2.34 (s, 3H), 1.39 (s, 3H). ${ }^{13} \mathrm{C}$ NMR (100 MHz, Chloroform-d) $\delta 158.1,149.7$ (d, $\left.J=5.2 \mathrm{~Hz}\right), 140.3(\mathrm{~d}, J=9.5$ $\mathrm{Hz}), 134.7$ (d, $J=2.8 \mathrm{~Hz}), 134.7(\mathrm{~d}, J=8.4 \mathrm{~Hz}), 133.4$ (d, $J=2.5 \mathrm{~Hz}), 132.5(\mathrm{~d}, J=10.7 \mathrm{~Hz}), 132.3(\mathrm{~d}, J=31.7$ $\mathrm{Hz}), 132.3$ (d, $J=7.2 \mathrm{~Hz}$ ), 131.0 (d, $J=13.8 \mathrm{~Hz}), 130.5,130.5$ (d, $J=8.8 \mathrm{~Hz}), 130.4,130.0,129.5,129.4(\mathrm{~d}, J$ $=6.5 \mathrm{~Hz}$ ), 129.2, 129.1 (d, $J=11.2 \mathrm{~Hz}), 128.6,128.6,128.5$ (d, $J=30.2 \mathrm{~Hz}), 128.1,128.1,128.0,127.7,127.7$ (d, $J=30.4 \mathrm{~Hz}$ ), 127.7, 127.4, 127.1, 126.9, 126.8, 126.5, 126.5, 125.8, 125.7, 125.5, 124.7, 118.2, 117.9, 110.9, 99.4, 55.4, 16.9, 12.5. ${ }^{31} \mathrm{P}$ NMR (162 MHz, Chloroform-d) $\delta ~ 31.1$. HRMS (ESI) m/z: $[\mathrm{M}+\mathrm{Na}]^{+}$Calcd for $\mathrm{C}_{44} \mathrm{H}_{33} \mathrm{~N}_{2} \mathrm{O}_{2} \mathrm{PNa}$ 675.2177; found 675.2172. Optical rotation: $[\alpha]_{D}{ }^{25}=+422.3$ (c $=0.5, \mathrm{CHCl}_{3}$ ). HPLC: Chiralpak $\mathrm{IA}, n$-hexane/isopropanol $=60 / 40$, Flow rate $=1.0 \mathrm{~mL} / \mathrm{min}, \mathrm{UV}=254 \mathrm{~nm}, t_{\mathrm{R}}($ minor $)=12.35 \mathrm{~min}, t_{\mathrm{R}}($ major $)=$ $15.75 \mathrm{~min}$.

(R)-bis(3,5-dimethylphenyl)(1-(8-methoxy-1,3-dimethylpyrrolo[1,2-a]quinoxalin-4-yl)naphthalen-2yl)phosphine oxide (5h):

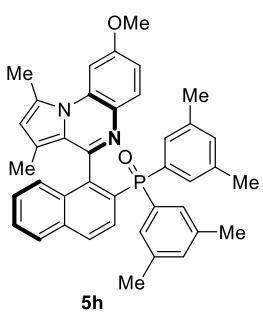

$270.7 \mathrm{mg}, 89 \%$ yield, yellow solid, mp: $126-128{ }^{\circ} \mathrm{C}, 91 \%$ ee. The enantiomeric excess can be increased up to $>99 \%$ through a simple recrystallization in acetonitrile. The crystals are racemic and the residue in the mother liquid was needed. Flash silica gel chromatography (petroleum ether/ethyl acetate $=2 / 1)$. ${ }^{1} \mathrm{H}$ NMR $(400 \mathrm{MHz}$, Chloroformd) $\delta 8.26(\mathrm{dd}, J=10.9,8.7 \mathrm{~Hz}, 1 \mathrm{H}), 8.05(\mathrm{dd}, J=8.7,1.8 \mathrm{~Hz}, 1 \mathrm{H}), 7.89(\mathrm{~d}, J=8.2 \mathrm{~Hz}$, $1 \mathrm{H}), 7.78-7.66(\mathrm{~m}, 3 \mathrm{H}), 7.59(\mathrm{~d}, J=2.6 \mathrm{~Hz}, 1 \mathrm{H}), 7.52(\mathrm{dd}, J=8.2,6.8 \mathrm{~Hz}, 1 \mathrm{H}), 7.49-$ $7.44(\mathrm{~m}, 1 \mathrm{H}), 7.31$ (ddd, $J=8.3,6.8,1.3 \mathrm{~Hz}, 1 \mathrm{H}), 7.09$ (s, 1H), 6.94 (dd, $J=8.8,2.5 \mathrm{~Hz}, 1 \mathrm{H}), 6.67$ (dd, $J=12.9$, $1.6 \mathrm{~Hz}, 2 \mathrm{H}), 6.48(\mathrm{~s}, 1 \mathrm{H}), 6.14(\mathrm{~s}, 1 \mathrm{H}), 3.92(\mathrm{~s}, 3 \mathrm{H}), 2.80(\mathrm{~s}, 3 \mathrm{H}), 2.32(\mathrm{~s}, 6 \mathrm{H}), 1.71(\mathrm{~s}, 6 \mathrm{H}), 1.33(\mathrm{~s}, 3 \mathrm{H}) .{ }^{13} \mathrm{C}$ NMR (100 MHz, Chloroform-d) $\delta 158.5,150.3$ (d, $J=5.1 \mathrm{~Hz}), 140.2(\mathrm{~d}, J=9.5 \mathrm{~Hz}), 137.6$ (d, $J=13.1 \mathrm{~Hz}), 136.3$ (d, $J=13.1 \mathrm{~Hz}$ ), 134.7 (d, $J=3.0 \mathrm{~Hz}), 133.3$ (d, $J=2.8 \mathrm{~Hz}), 132.6$ (d, $J=58.1 \mathrm{~Hz}), 132.2$ (d, J = 2.5 Hz), 131.6 (d, $J=2.9 \mathrm{~Hz}$ ), 131.5 (d, $J=72.7 \mathrm{~Hz}), 131.3,130.8,130.6$ (d, $J=7.0 \mathrm{~Hz}), 130.4,130.2,129.1,129.0,128.9$ (d, $J=2.9 \mathrm{~Hz}), 128.8,128.1,127.9,127.2,126.5$ (d, $J=5.6 \mathrm{~Hz}), 124.8,118.6,117.6,110.5,100.5,55.9,21.4,20.8$, 17.4, 12.4. ${ }^{31} \mathrm{P}$ NMR (162 MHz, Chloroform-d) $\delta$ 31.2. HRMS (ESI) m/z: $[\mathrm{M}+\mathrm{H}]^{+}$Calcd for $\mathrm{C}_{40} \mathrm{H}_{38} \mathrm{~N}_{2} \mathrm{O}_{2} \mathrm{P} 609.2671$; found 609.2668. Optical rotation: $[\alpha]_{D}{ }^{25}=+306.6\left(\mathrm{c}=0.5, \mathrm{CHCl}_{3}\right)$. HPLC: Chiralpak IA, $n$-hexane/isopropanol $=70 / 30$, Flow rate $=1.0 \mathrm{~mL} / \mathrm{min}, \mathrm{UV}=254 \mathrm{~nm}, t_{\mathrm{R}}($ minor $)=4.69 \mathrm{~min}, t_{\mathrm{R}}$ (major) $=5.95 \mathrm{~min}$. 
(R)-bis(3,5-dimethylphenyl)(1-(1,3-dimethylpyrrolo[1,2-a]quinoxalin-4-yl)naphthalen-2-yl)phosphine oxide (5i):

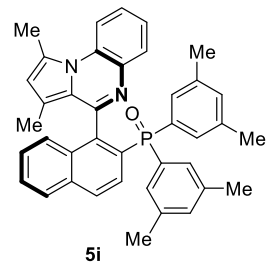

$228.7 \mathrm{mg}, 79 \%$ yield, yellow solid, $\mathrm{mp}: 118-120{ }^{\circ} \mathrm{C}, 82 \%$ ee. Flash silica gel chromatography (petroleum ether/ethyl acetate $=2 / 1) .{ }^{1} \mathrm{H}$ NMR $(400 \mathrm{MHz}$, Chloroformd) $\delta 8.28$ (dd, $J=10.8,8.7 \mathrm{~Hz}, 1 \mathrm{H}), 8.04(\mathrm{t}, J=7.9 \mathrm{~Hz}, 3 \mathrm{H}), 7.88(\mathrm{~d}, J=7.5 \mathrm{~Hz}, 1 \mathrm{H})$, $7.81-7.73(\mathrm{~m}, 3 \mathrm{H}), 7.53-7.45(\mathrm{~m}, 2 \mathrm{H}), 7.39$ (ddd, $J=8.5,7.1,1.5 \mathrm{~Hz}, 1 \mathrm{H}), 7.35-$ $7.27(\mathrm{~m}, 2 \mathrm{H}), 7.09(\mathrm{~s}, 1 \mathrm{H}), 6.67(\mathrm{~d}, J=12.9 \mathrm{~Hz}, 2 \mathrm{H}), 6.43(\mathrm{~s}, 1 \mathrm{H}), 6.17(\mathrm{~s}, 1 \mathrm{H}), 2.78(\mathrm{~s}$, $3 \mathrm{H}), 2.32(\mathrm{~s}, 6 \mathrm{H}), 1.65(\mathrm{~s}, 6 \mathrm{H}), 1.35(\mathrm{~s}, 3 \mathrm{H}) .{ }^{13} \mathrm{C}$ NMR (100 MHz, Chloroform-d) $\delta 152.3$ (d, $J=5.5 \mathrm{~Hz}$ ), 139.9 (d, $J=9.5 \mathrm{~Hz}), 137.5$ (d, $J=13.1 \mathrm{~Hz}), 136.7,136.3(\mathrm{~d}, J=13.1 \mathrm{~Hz}), 134.6,133.3(\mathrm{~d}, J=$ $3.1 \mathrm{~Hz}), 132.4$ (d, $J=82.1 \mathrm{~Hz}), 132.2(\mathrm{~d}, J=1.2 \mathrm{~Hz}), 131.6(\mathrm{~d}, J=3.1 \mathrm{~Hz}), 131.5(\mathrm{~d}, J=69.0 \mathrm{~Hz}), 130.5,130.4$ (d, $J=99.9 \mathrm{~Hz}), 130.4,130.0,129.7,129.0$ (d, $J=10.3 \mathrm{~Hz}), 128.8(\mathrm{~d}, J=18.2 \mathrm{~Hz}), 128.8,128.0,127.9,127.2$ (d, $J=7.2 \mathrm{~Hz}$ ), 126.6, 126.4, 125.1, 124.3, 118.5, 118.2, 115.0, 21.4, 20.7, 17.5, 12.4. ${ }^{31} \mathrm{P}$ NMR (162 MHz, Chloroform-d) $\delta$ 31.0. HRMS (ESI) m/z: $[\mathrm{M}+\mathrm{Na}]^{+}$Calcd for $\mathrm{C}_{39} \mathrm{H}_{35} \mathrm{~N}_{2} \mathrm{OPNa} 601.2385$, found 601.2379. Optical rotation: $[\alpha]_{\mathrm{D}}{ }^{25}=+289.3\left(\mathrm{c}=0.5, \mathrm{CHCl}_{3}\right.$ ). HPLC: Chiralpak IA, $n$-hexane/isopropanol $=80 / 20$, Flow rate $=1.0$ $\mathrm{mL} / \mathrm{min}, \mathrm{UV}=254 \mathrm{~nm}, t_{\mathrm{R}}($ minor $)=5.74 \mathrm{~min}, t_{\mathrm{R}}$ (major) $=9.09 \mathrm{~min}$.

(R)-(1-(8-(benzyloxy)-1,3-dimethylpyrrolo[1,2-a]quinoxalin-4-yl)naphthalen-2-yl)bis(3,5dimethylphenyl)phosphine oxide (5j):

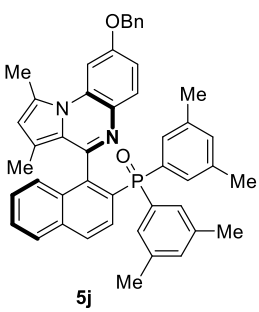

$283.4 \mathrm{mg}, 83 \%$ yield, yellow solid, $\mathrm{mp}: 113-115{ }^{\circ} \mathrm{C}, 94 \%$ ee. Flash silica gel chromatography (petroleum ether/ethyl acetate $=2 / 1) .{ }^{1} \mathrm{H}$ NMR $(400 \mathrm{MHz}$, Chloroformd) $\delta 8.25(\mathrm{dd}, J=10.8,8.6 \mathrm{~Hz}, 1 \mathrm{H}), 8.03(\mathrm{dd}, J=8.8,1.8 \mathrm{~Hz}, 1 \mathrm{H}), 7.87(\mathrm{~d}, J=8.2 \mathrm{~Hz}$, $1 \mathrm{H}), 7.75(\mathrm{~s}, 1 \mathrm{H}), 7.72(\mathrm{~s}, 1 \mathrm{H}), 7.67(\mathrm{~d}, J=8.9 \mathrm{~Hz}, 1 \mathrm{H}), 7.58(\mathrm{~d}, J=2.6 \mathrm{~Hz}, 1 \mathrm{H}), 7.52-$ $7.38(\mathrm{~m}, 6 \mathrm{H}), 7.37-7.32(\mathrm{~m}, 1 \mathrm{H}), 7.31-7.26(\mathrm{~m}, 1 \mathrm{H}), 7.08(\mathrm{~s}, 1 \mathrm{H}), 7.01(\mathrm{dd}, J=8.8$, $2.5 \mathrm{~Hz}, 1 \mathrm{H}), 6.65(\mathrm{~d}, J=12.9 \mathrm{~Hz}, 2 \mathrm{H}), 6.38(\mathrm{~s}, 1 \mathrm{H}), 6.11(\mathrm{~s}, 1 \mathrm{H}), 5.21(\mathrm{~d}, J=2.9 \mathrm{~Hz}, 2 \mathrm{H})$, 2.67 (s, 3H), 2.31 (s, 6H), $1.66(\mathrm{~s}, 6 \mathrm{H}), 1.32(\mathrm{~s}, 3 \mathrm{H}) .{ }^{13} \mathrm{C}$ NMR $(100 \mathrm{MHz}$, Chloroform-d) $\delta$ 157.4, $150.2(\mathrm{~d}, \mathrm{~J}=$ $5.6 \mathrm{~Hz}), 140.1$ (d, $J=9.5 \mathrm{~Hz}), 137.5$ (d, $J=13.1 \mathrm{~Hz}), 136.8,136.2(\mathrm{~d}, J=13.1 \mathrm{~Hz}), 134.6$ (d, J = 3.0 Hz), 133.3 (d, $J=3.0 \mathrm{~Hz}), 132.6$ (d, $J=66.9 \mathrm{~Hz}), 132.1$ (d, $J=4.4 \mathrm{~Hz}), 131.5(\mathrm{~d}, J=2.9 \mathrm{~Hz}), 131.3,130.6(\mathrm{~d}, J=97.0 \mathrm{~Hz}$ ), 130.6, 130.5 (d, $J=79.8 \mathrm{~Hz}$ ), 130.5, 130.4, 130.3, 129.0 (d, $J=10.6 \mathrm{~Hz}), 128.8$ (d, $J=10.1 \mathrm{~Hz}), 128.8,128.2$, 128.0, 127.8, 127.2, 127.2, 126.5, 126.5, 124.8, 118.5, 117.5, 111.9, 101.3, 70.6, 21.4, 20.7, 17.3, 12.4. ${ }^{31}$ P NMR (162 MHz, Chloroform-d) ס 31.1. HRMS (ESI) m/z: $[\mathrm{M}+\mathrm{H}]^{+}$Calcd for $\mathrm{C}_{46} \mathrm{H}_{42} \mathrm{~N}_{2} \mathrm{O}_{2} \mathrm{P}$ 685.2984; found 685.2978. Optical rotation: $[\alpha]_{D}^{25}=+314.7\left(\mathrm{C}=0.5, \mathrm{CHCl}_{3}\right)$. HPLC: Chiralpak IC, $n$-hexane/isopropanol $=80 / 20$, Flow rate $=1.0 \mathrm{~mL} / \mathrm{min}, \mathrm{UV}=254 \mathrm{~nm}, t_{\mathrm{R}}(\operatorname{minor})=23.02 \mathrm{~min}, t_{\mathrm{R}}($ major $)=27.46 \mathrm{~min}$

(R)-bis(3,5-dimethylphenyl)(1-(8-fluoro-1,3-dimethylpyrrolo[1,2-a]quinoxalin-4-yl)naphthalen-2yl)phosphine oxide (5k):

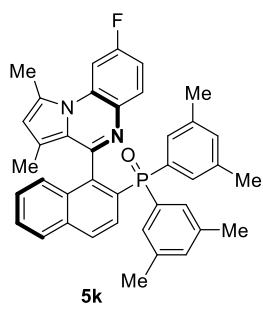

$226.8 \mathrm{mg}, 76 \%$ yield, yellow solid, $\mathrm{mp}: 137-139{ }^{\circ} \mathrm{C}, 84 \%$ ee. Flash silica gel chromatography (petroleum ether/ethyl acetate $=2 / 1)$. ${ }^{1} \mathrm{H}$ NMR $(400 \mathrm{MHz}$, Chloroformd) $\delta 8.25(\mathrm{dd}, J=10.9,8.7 \mathrm{~Hz}, 1 \mathrm{H}), 8.06(\mathrm{dd}, J=8.8,1.8 \mathrm{~Hz}, 1 \mathrm{H}), 7.90(\mathrm{~d}, J=8.2 \mathrm{~Hz}$, $1 \mathrm{H}), 7.78-7.67(\mathrm{~m}, 4 \mathrm{H}), 7.52(\mathrm{ddd}, J=8.1,6.8,1.2 \mathrm{~Hz}, 1 \mathrm{H}), 7.45(\mathrm{~d}, J=8.6 \mathrm{~Hz}, 1 \mathrm{H})$, 7.31 (ddd, $J=8.4,6.8,1.3 \mathrm{~Hz}, 1 \mathrm{H}), 7.11-7.05(\mathrm{~m}, 2 \mathrm{H}), 6.68(\mathrm{~d}, J=12.9 \mathrm{~Hz}, 2 \mathrm{H}), 6.49$ $(\mathrm{s}, 1 \mathrm{H}), 6.20(\mathrm{~s}, 1 \mathrm{H}), 2.77(\mathrm{~s}, 3 \mathrm{H}), 2.32(\mathrm{~s}, 6 \mathrm{H}), 1.72(\mathrm{~s}, 6 \mathrm{H}), 1.35(\mathrm{~s}, 3 \mathrm{H}) .{ }^{13} \mathrm{C}$ NMR $(100$ MHz, Chloroform- $d$ ) $\delta 160.8$ (d, C-F, $\left.{ }^{1} J_{C-F}=245.4 \mathrm{~Hz}\right), 151.7(\mathrm{~d}, J=2.1 \mathrm{~Hz}), 151.7(\mathrm{~d}, J=2.1 \mathrm{~Hz}), 139.7(\mathrm{~d}, J=$ $9.4 \mathrm{~Hz}), 137.6$ (d, $J=13.2 \mathrm{~Hz}), 136.3$ (d, $J=13.1 \mathrm{~Hz}), 134.6$ (d, $J=2.2 \mathrm{~Hz}), 133.4$ (d, $J=3.0 \mathrm{~Hz}), 133.3$ (d, $J=$ $2.2 \mathrm{~Hz}), 132.5$ (d, $J=51.9 \mathrm{~Hz}), 132.0$ (d, $J=11.4 \mathrm{~Hz}), 131.7$ (d, $J=2.9 \mathrm{~Hz}), 131.5$ (d, $J=51.7 \mathrm{~Hz}), 131.0$ (d, C- 
$\left.\mathrm{F},{ }^{3} J_{C-F}=9.0 \mathrm{~Hz}\right), 130.4(\mathrm{~d}, J=10.1 \mathrm{~Hz}), 130.3(\mathrm{~d}, J=11.1 \mathrm{~Hz}), 130.0,129.1(\mathrm{~d}, J=10.2 \mathrm{~Hz}), 129.0(\mathrm{~d}, J=11.6$ $\mathrm{Hz}), 128.8\left(\mathrm{~d}, \mathrm{C}-\mathrm{F},{ }^{3} \mathrm{~J}_{\mathrm{C}-\mathrm{F}}=8.9 \mathrm{~Hz}\right), 128.0(\mathrm{~d}, \mathrm{~J}=17.4 \mathrm{~Hz}), 127.3,127.2,126.3,124.5,118.9,118.5,111.8(\mathrm{~d}, \mathrm{C}-$ $\left.\mathrm{F}^{2} \mathrm{~J}_{\mathrm{C}-\mathrm{F}}=22.7 \mathrm{~Hz}\right), 101.9\left(\mathrm{~d}, \mathrm{C}-\mathrm{F},{ }^{2} J_{\mathrm{C}-\mathrm{F}}=28.4 \mathrm{~Hz}\right), 21.4,20.7,17.2,12.4 .{ }^{19} \mathrm{~F}$ NMR $(377 \mathrm{MHz}$, Chloroform-d $) \delta-$ 112.1. ${ }^{31} \mathrm{P}$ NMR (162 MHz, Chloroform-d) $\delta$ 30.9. HRMS (ESI) m/z: $[\mathrm{M}+\mathrm{H}]^{+}$Calcd for $\mathrm{C}_{39} \mathrm{H}_{35} \mathrm{FN}_{2} \mathrm{OP} 597.2471$; found 597.2464. Optical rotation: $[\alpha]_{D}{ }^{25}=+277.2\left(\mathrm{c}=0.5, \mathrm{CHCl}_{3}\right)$. HPLC: Chiralpak IA, $n$-hexane /isopropanol $=80 / 20$, Flow rate $=1.0 \mathrm{~mL} / \mathrm{min}, \mathrm{UV}=254 \mathrm{~nm}, t_{\mathrm{R}}($ minor $)=5.47 \mathrm{~min}, t_{\mathrm{R}}$ (major) $=8.12 \mathrm{~min}$.

\section{(R)-(1-(8-bromo-1,3-dimethylpyrrolo[1,2-a]quinoxalin-4-yl)naphthalen-2-yl)bis(3,5-} dimethylphenyl)phosphine oxide (5I):

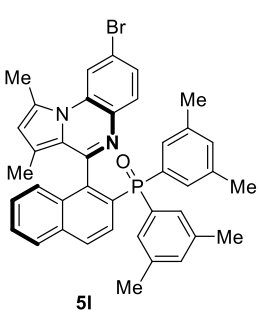

51 $226.6 \mathrm{mg}, 69 \%$ yield, yellow solid, $\mathrm{mp}: 125-127{ }^{\circ} \mathrm{C}, 84 \%$ ee. Flash silica gel chromatography (petroleum ether/ethyl acetate $=2 / 1)$. ${ }^{1} \mathrm{H}$ NMR $(400 \mathrm{MHz}$, Chloroformd) $\delta 8.19$ (dd, $J=10.9,8.6 \mathrm{~Hz}, 1 \mathrm{H}$ ), 7.95 (ddd, $J=8.4,7.2,1.6 \mathrm{~Hz}, 2 \mathrm{H}$ ), 7.79 (d, $J=8.2$ $\mathrm{Hz}, 1 \mathrm{H}), 7.70-7.68(\mathrm{~m}, 1 \mathrm{H}), 7.66(\mathrm{~d}, J=1.7 \mathrm{~Hz}, 1 \mathrm{H}), 7.44-7.34(\mathrm{~m}, 2 \mathrm{H}), 7.29$ (ddd, $J$ $=8.6,7.2,1.8 \mathrm{~Hz}, 1 \mathrm{H}), 7.26-7.17(\mathrm{~m}, 2 \mathrm{H}), 7.00(\mathrm{~s}, 1 \mathrm{H}), 6.58(\mathrm{~d}, J=13.0 \mathrm{~Hz}, 2 \mathrm{H}), 6.33$ (s, 1H), $6.08(\mathrm{~s}, 1 \mathrm{H}), 2.69(\mathrm{~s}, 3 \mathrm{H}), 2.23(\mathrm{~s}, 6 \mathrm{H}), 1.56(\mathrm{~s}, 6 \mathrm{H}), 1.26(\mathrm{~s}, 3 \mathrm{H}) .{ }^{13} \mathrm{C}$ NMR $(100$ MHz, Chloroform-d) $\delta 152.3$ (d, $J=5.2 \mathrm{~Hz}), 139.9$ (d, $J=9.4 \mathrm{~Hz}), 137.5(\mathrm{~d}, J=13.1 \mathrm{~Hz}), 136.7,136.2(\mathrm{~d}, J=$ $13.1 \mathrm{~Hz}$ ), 134.6 (d, $J=2.2 \mathrm{~Hz}), 133.3(\mathrm{~d}, J=2.9 \mathrm{~Hz}), 132.4(\mathrm{~d}, J=83.8 \mathrm{~Hz}), 132.1$ (d, $J=2.2 \mathrm{~Hz}), 131.6$ (d, $J=$ $2.9 \mathrm{~Hz}), 131.5$ (d, $J=69.7 \mathrm{~Hz}), 130.5,130.4(\mathrm{~d}, J=101.0 \mathrm{~Hz}), 130.4,130.0,129.6,129.1,128.9(\mathrm{~d}, J=17.2$ $\mathrm{Hz}), 128.8(\mathrm{~d}, J=20.2 \mathrm{~Hz}), 127.9$ (d, $J=17.1 \mathrm{~Hz}), 127.3,127.2,126.6,126.4,125.0,124.3,118.5,118.2,115.0$, 21.4, 20.7, 17.6, 12.4. ${ }^{31} \mathrm{P}$ NMR (162 MHz, Chloroform-d) $\delta 31.1$. HRMS (ESI) m/z: $[\mathrm{M}+\mathrm{H}]^{+}$Calcd for $\mathrm{C}_{39} \mathrm{H}_{35} \mathrm{BrN}_{2} \mathrm{OP}$ 657.1670; found 657.1672. Optical rotation: $[\alpha]_{\mathrm{D}}{ }^{25}=+346.1\left(\mathrm{c}=0.5, \mathrm{CHCl}_{3}\right)$. HPLC: Chiralpak $\mathrm{IA}, n$-hexane/isopropanol $=80 / 20$, Flow rate $=1.0 \mathrm{~mL} / \mathrm{min}, \mathrm{UV}=254 \mathrm{~nm}, t_{\mathrm{R}}($ minor $)=5.72 \mathrm{~min}, t_{\mathrm{R}}($ major $)=$ $8.69 \mathrm{~min}$.

(R)-(1-(7-(dimethylamino)-1,3-dimethylpyrrolo[1,2-a]quinoxalin-4-yl)naphthalen-2-yl)bis(3,5dimethylphenyl)phosphine oxide $(5 \mathrm{~m})$ :

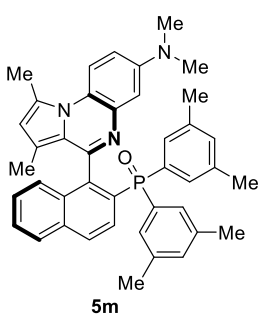

$192.5 \mathrm{mg}, 62 \%$ yield, yellow solid, mp: $117-119{ }^{\circ} \mathrm{C}, 76 \%$ ee. Flash silica gel chromatography (petroleum ether/ethyl acetate $=2 / 1)$. ${ }^{1} \mathbf{H}$ NMR $(400 \mathrm{MHz}$, Chloroformd) $\delta 8.25(\mathrm{dd}, J=10.8,8.6 \mathrm{~Hz}, 1 \mathrm{H}), 8.04(\mathrm{dd}, J=8.6,2.1 \mathrm{~Hz}, 1 \mathrm{H}), 7.90(\mathrm{t}, J=8.5 \mathrm{~Hz}$, 2H), $7.78(\mathrm{~d}, J=12.4 \mathrm{~Hz}, 2 \mathrm{H}$ ), $7.55-7.45(\mathrm{~m}, 2 \mathrm{H}), 7.30$ (ddd, $J=8.4,6.7,1.3 \mathrm{~Hz}, 1 \mathrm{H}$ ), $7.10(\mathrm{dd}, J=6.5,2.4 \mathrm{~Hz}, 2 \mathrm{H}), 6.89(\mathrm{dd}, J=9.2,3.1 \mathrm{~Hz}, 1 \mathrm{H}), 6.70(\mathrm{~d}, J=12.8 \mathrm{~Hz}, 2 \mathrm{H}$ ), $6.44(\mathrm{~s}, 1 \mathrm{H}), 6.10(\mathrm{~s}, 1 \mathrm{H}), 2.99(\mathrm{~s}, 6 \mathrm{H}), 2.74(\mathrm{~s}, 3 \mathrm{H}), 2.33(\mathrm{~s}, 6 \mathrm{H}), 1.71(\mathrm{~s}, 6 \mathrm{H}), 1.35(\mathrm{~s}$, 3H). ${ }^{13} \mathrm{C}$ NMR (100 MHz, Chloroform-d) $\delta 152.3(\mathrm{~d}, J=5.2 \mathrm{~Hz}), 148.0,140.4(\mathrm{~d}, J=9.5$ $\mathrm{Hz}), 138.0,137.5$ (d, $J=13.2 \mathrm{~Hz}), 136.3$ (d, $J=13.1 \mathrm{~Hz}), 134.7$ (d, $J=2.0 \mathrm{~Hz}), 133.3$ (d, $J=2.6 \mathrm{~Hz}), 132.7$ (d, $J=82.7 \mathrm{~Hz}$ ), 132.2, 131.7 (d, $J=2.6 \mathrm{~Hz}), 131.6(\mathrm{~d}, J=93.9 \mathrm{~Hz}), 130.5$ (d, $J=100.0 \mathrm{~Hz}), 130.5(\mathrm{~d}, J=10.2 \mathrm{~Hz})$, $129.1(\mathrm{~d}, J=10.3 \mathrm{~Hz}), 128.8$ (d, $J=20.2 \mathrm{~Hz}), 128.8,128.1,127.9,127.3,126.6,126.4,124.8,122.0,117.8$,

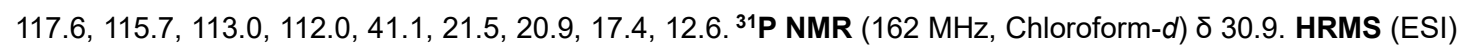
$\mathrm{m} / \mathrm{z}:[\mathrm{M}+\mathrm{H}]^{+}$Calcd for $\mathrm{C}_{41} \mathrm{H}_{41} \mathrm{~N}_{3} \mathrm{OP} 622.2987$; found 622.2982. Optical rotation: $[\alpha]_{D}{ }^{25}=+204.3\left(\mathrm{c}=0.5, \mathrm{CHCl}_{3}\right)$. HPLC: Chiralpak IA, $n$-hexane/isopropanol $=80 / 20$, Flow rate $=1.0 \mathrm{~mL} / \mathrm{min}, \mathrm{UV}=254 \mathrm{~nm}, t_{\mathrm{R}}(\mathrm{minor})=6.62 \mathrm{~min}$, $t_{\mathrm{R}}$ (major) $=8.81 \mathrm{~min}$.

(R)-(1-(2-ethyl-8-methoxy-1,3-dimethylpyrrolo[1,2-a]quinoxalin-4-yl)naphthalen-2-yl)diphenylphosphine oxide (5n):

$234.3 \mathrm{mg}, 81 \%$ yield, yellow solid, $\mathrm{mp}: 166-168{ }^{\circ} \mathrm{C}, 74 \%$ ee. Flash silica gel chromatography (petroleum 


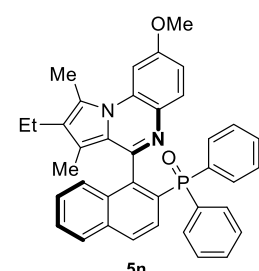

ether/ethyl acetate $=2 / 1) .{ }^{1} \mathrm{H}$ NMR $(400 \mathrm{MHz}$, Chloroform- $d) \delta 8.30(\mathrm{dd}, J=10.9,8.7 \mathrm{~Hz}$, $1 \mathrm{H}$ ), $8.09-8.02(\mathrm{~m}, 3 \mathrm{H}), 7.89$ (dt, $J=8.3,1.1 \mathrm{~Hz}, 1 \mathrm{H}), 7.63$ (d, J=8.9 Hz, 1H), 7.57 (d, $J=2.6 \mathrm{~Hz}, 1 \mathrm{H}$ ), $7.53-7.39(\mathrm{~m}, 5 \mathrm{H}), 7.31$ (ddd, $J=8.3,6.7,1.3 \mathrm{~Hz}, 1 \mathrm{H}), 6.97$ (ddd, $J=$ 12.6, 8.1, 1.4 Hz, 2H), 6.90 (dd, $J=8.9,2.6 \mathrm{~Hz}, 1 \mathrm{H}$ ), 6.81 (tq, $J=7.5,1.5 \mathrm{~Hz}, 1 \mathrm{H}$ ), 6.49 (td, $J=7.7,3.0 \mathrm{~Hz}, 2 \mathrm{H}$ ), 3.90 (s, 3H), $2.74(\mathrm{~s}, 3 \mathrm{H}), 2.46$ (qd, $J=7.2,3.0 \mathrm{~Hz}, 2 \mathrm{H}$ ), 1.27 (s, 3H), 1.07 (t, $J=7.5 \mathrm{~Hz}, 3 \mathrm{H}) .{ }^{13} \mathrm{C}$ NMR (101 MHz, Chloroform-d) $\delta 158.2,149.5$ (d, J $=5.6 \mathrm{~Hz}), 140.7(\mathrm{~d}, J=9.6 \mathrm{~Hz}), 134.7(\mathrm{~d}, J=2.2 \mathrm{~Hz}), 132.9$ (d, $J=105.1 \mathrm{~Hz}), 132.9$ (d, $J=10.3 \mathrm{~Hz}), 132.3$ (d, $J=11.2 \mathrm{~Hz}), 131.5(\mathrm{~d}, J=2.9 \mathrm{~Hz}), 131.2,131.1,130.8,130.7,130.6,130.3,130.0$ (d, $J=101.9 \mathrm{~Hz}), 129.5$, $129.3(\mathrm{~d}, J=3.0 \mathrm{~Hz}), 128.9(\mathrm{~d}, J=11.5 \mathrm{~Hz}), 128.7(\mathrm{~d}, J=8.9 \mathrm{~Hz}), 128.0,127.9,127.3,126.6,126.2(\mathrm{~d}, J=12.4$ $\mathrm{Hz}$ ), 124.3, 123.9, 116.1, 110.0, 100.6, 55.8, 17.5, 15.4, 14.7, 9.9. ${ }^{31} \mathrm{P}$ NMR (162 MHz, Chloroform-d) $\delta 31.0$. HRMS (ESI) m/z: [M+Na] $]^{+}$Calcd for $\mathrm{C}_{38} \mathrm{H}_{33} \mathrm{~N}_{2} \mathrm{O}_{2} \mathrm{PNa}$ 603.2177; found 603.2183. Optical rotation: $[\alpha]_{D}{ }^{25}=$ $+212.4\left(\mathrm{c}=0.5, \mathrm{CHCl}_{3}\right)$. HPLC: Chiralpak IC, $n$-hexane/isopropanol $=70 / 30$, Flow rate $=1.0 \mathrm{~mL} / \mathrm{min}, \mathrm{UV}=254$ $\mathrm{nm}, t_{\mathrm{R}}(\operatorname{minor})=16.93 \mathrm{~min}, t_{\mathrm{R}}($ major $)=20.25 \mathrm{~min}$.

(R)-(1-(2,3-diethyl-8-methoxypyrrolo[1,2-a]quinoxalin-4-yl)naphthalen-2-yl)diphenylphosphine

oxide (5o):

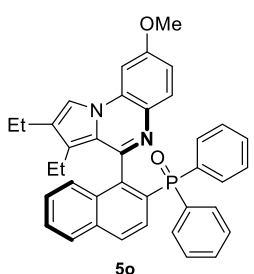

$240.5 \mathrm{mg}, 83 \%$ yield, yellow solid, $\mathrm{mp}: 156-158{ }^{\circ} \mathrm{C}, 76 \%$ ee. Flash silica gel chromatography (petroleum ether/ethyl acetate $=2 / 1) .{ }^{1} \mathrm{H}$ NMR $(400 \mathrm{MHz}$, Chloroform- $d)$ $\delta 8.25$ (dd, $J=10.9,8.7 \mathrm{~Hz}, 1 \mathrm{H}$ ), 8.07 (dd, $J=8.8,1.9 \mathrm{~Hz}, 1 \mathrm{H}$ ), 8.01 (ddt, $J=12.2,6.9$, $1.5 \mathrm{~Hz}, 2 \mathrm{H}), 7.92(\mathrm{~d}, J=8.2 \mathrm{~Hz}, 1 \mathrm{H}), 7.59(\mathrm{~d}, J=8.8 \mathrm{~Hz}, 1 \mathrm{H}), 7.56-7.46(\mathrm{~m}, 3 \mathrm{H}), 7.45-$ 7.39 (m, 2H), 7.36 (s, 1H), 7.31 (ddd, $J=8.4,6.8,1.3 \mathrm{~Hz}, 1 \mathrm{H}), 7.04-6.94(\mathrm{~m}, 3 \mathrm{H}), 6.90$ (dd, $J=8.8,2.6 \mathrm{~Hz}, 1 \mathrm{H}), 6.82$ (tt, $J=6.0,1.4 \mathrm{~Hz}, 1 \mathrm{H}$ ), 6.52 (td, $J=7.7,2.9 \mathrm{~Hz}, 2 \mathrm{H}$ ), 3.95 (s, 3H), 2.53 (q, J = 7.5 Hz, 2H), $1.93(\mathrm{dq}, J=15.0,7.5 \mathrm{~Hz}, 1 \mathrm{H}), 1.57(\mathrm{dq}, J=15.0,7.5 \mathrm{~Hz}, 1 \mathrm{H}), 1.30(\mathrm{t}, J=7.5$ $\mathrm{Hz}, 3 \mathrm{H}), 0.22(\mathrm{t}, J=7.5 \mathrm{~Hz}, 3 \mathrm{H}) .{ }^{13} \mathrm{C}$ NMR $(101 \mathrm{MHz}$, Chloroform- $d) \delta 159.6,149.6(\mathrm{~d}, J=5.1 \mathrm{~Hz}), 140.5(\mathrm{~d}, J$ $=9.7 \mathrm{~Hz}), 134.9(\mathrm{~d}, J=2.1 \mathrm{~Hz}), 133.0(\mathrm{~d}, J=105.0 \mathrm{~Hz}), 132.9(\mathrm{~d}, J=10.2 \mathrm{~Hz}), 132.2,132.1,131.6(\mathrm{~d}, J=2.3$ $\mathrm{Hz}), 131.2,131.1,131.0,131.0,130.0$ (d, $J=2.9 \mathrm{~Hz}), 129.3,129.0$ (d, $J=11.1 \mathrm{~Hz}), 128.8$ (d, $J=8.8 \mathrm{~Hz}), 128.3$, 128.2, 128.2, 128.0, 127.3, 127.1, 126.2 (d, J = 12.4 Hz), 124.1, 123.3, 111.8, 111.1, 96.9, 56.0, 18.5, 18.2, 14.8, 14.6. ${ }^{31} \mathrm{P}$ NMR (162 MHz, Chloroform-d) $\delta$ 30.5. HRMS (ESI) m/z: $[\mathrm{M}+\mathrm{H}]^{+}$Calcd for $\mathrm{C}_{38} \mathrm{H}_{34} \mathrm{~N}_{2} \mathrm{O}_{2} \mathrm{P} 581.2358$; found 581.2356. Optical rotation: $[\alpha]_{\mathrm{D}}^{25}=+228.5\left(\mathrm{c}=0.5, \mathrm{CHCl}_{3}\right)$. HPLC: Chiralpak IC, $n$-hexane/isopropanol $=70 / 30$, Flow rate $=1.0 \mathrm{~mL} / \mathrm{min}, \mathrm{UV}=254 \mathrm{~nm}, t_{\mathrm{R}}($ minor $)=15.26 \mathrm{~min}, t_{\mathrm{R}}($ major $)=17.01 \mathrm{~min}$.

\section{(R)-(1-(8-methoxypyrrolo[1,2-a]quinoxalin-4-yl)naphthalen-2-yl)diphenylphosphine oxide (5p):}

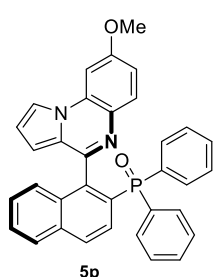

$136.5 \mathrm{mg}, 52 \%$ yield, white solid, $\mathrm{mp}: 254-256^{\circ} \mathrm{C}, 24 \%$ ee. Flash silica gel chromatography (petroleum ether/ethyl acetate $=2 / 1) .{ }^{1} \mathrm{H}$ NMR $(400 \mathrm{MHz}$, Chloroform- $d) \delta 8.21(\mathrm{dd}, J=11.0$, $8.7 \mathrm{~Hz}, 1 \mathrm{H}), 8.08(\mathrm{dd}, J=8.4,1.4 \mathrm{~Hz}, 1 \mathrm{H}), 8.01-7.89(\mathrm{~m}, 3 \mathrm{H}), 7.65(\mathrm{~d}, J=8.9 \mathrm{~Hz}, 1 \mathrm{H}), 7.58$ $-7.49(\mathrm{~m}, 3 \mathrm{H}), 7.47-7.43(\mathrm{~m}, 1 \mathrm{H}), 7.42-7.36(\mathrm{~m}, 2 \mathrm{H}), 7.32(\mathrm{ddd}, J=8.4,6.8,1.3 \mathrm{~Hz}, 1 \mathrm{H})$, $7.06-6.94(\mathrm{~m}, 4 \mathrm{H}), 6.85$ (tq, $J=7.4,1.4 \mathrm{~Hz}, 1 \mathrm{H}), 6.63(\mathrm{dd}, J=4.0,2.7 \mathrm{~Hz}, 1 \mathrm{H}), 6.60-6.52$ $(\mathrm{m}, 2 \mathrm{H}), 6.13(\mathrm{dd}, J=4.0,1.3 \mathrm{~Hz}, 1 \mathrm{H}), 3.95(\mathrm{~s}, 3 \mathrm{H}) .{ }^{13} \mathrm{C}$ NMR $(101 \mathrm{MHz}$, Chloroform-d) $\delta$ 159.7, 150.2 (d, $J=5.2 \mathrm{~Hz}), 139.3$ (d, $J=9.0 \mathrm{~Hz}$ ), 135.0 (d, $J=2.3 \mathrm{~Hz}), 132.8$ (d, $J=10.2 \mathrm{~Hz}), 132.8$ (d, $J=$ $105.1 \mathrm{~Hz}), 131.9$ (d, $J=11.2 \mathrm{~Hz}), 131.5,131.5,130.9,130.8,130.1,129.9$ (d, $J=2.9 \mathrm{~Hz}), 129.7,129.3$ (d, $J=$ $11.4 \mathrm{~Hz}$ ), 129.0 (d, $J=9.6 \mathrm{~Hz}), 128.3,128.2$ (d, $J=7.3 \mathrm{~Hz}), 128.0$ (d, $J=12.3 \mathrm{~Hz}), 128.0,127.1,127.0,126.4$, 126.2, 114.0, 113.3, 112.6, 108.6, 97.5, 56.0. ${ }^{31} \mathrm{P}$ NMR (162 MHz, Chloroform-d) $\delta 30.6$. HRMS (ESI) m/z: [M+H] $]^{+}$ Calcd for $\mathrm{C}_{34} \mathrm{H}_{26} \mathrm{~N}_{2} \mathrm{O}_{2} \mathrm{P}$ 525.1732; found 525.1728. Optical rotation: $[\alpha]_{D}{ }^{25}=+93.4$ (c $=0.5, \mathrm{CHCl}_{3}$ ). HPLC: Chiralpak IA, $n$-hexane /isopropanol $=80 / 20$, Flow rate $=1.0 \mathrm{~mL} / \mathrm{min}, \mathrm{UV}=254 \mathrm{~nm}, t_{\mathrm{R}}(\operatorname{minor})=16.59 \mathrm{~min}, t_{\mathrm{R}}$ 
$($ major $)=18.25 \mathrm{~min}$.

\section{(R)-(3-methoxy-2-(8-methoxy-1,3-dimethylpyrrolo[1,2-a]quinoxalin-4-yl)phenyl)diphenylphosphine} oxide (5q):

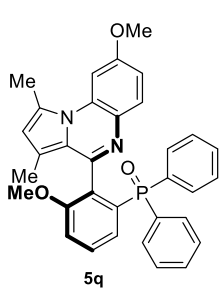

$236.1 \mathrm{mg}, 89 \%$ yield, white solid, $\mathrm{mp}: 120-122^{\circ} \mathrm{C}, 67 \%$ ee. Flash silica gel chromatography (petroleum ether/ethyl acetate $=1 / 1) .{ }^{1} \mathrm{H}$ NMR $(400 \mathrm{MHz}$, Chloroform- $d) \delta 8.07$ (ddt, $J=$ $12.4,6.7,1.5 \mathrm{~Hz}, 2 \mathrm{H}), 7.89$ (ddd, $J=12.0,7.9,1.0 \mathrm{~Hz}, 1 \mathrm{H}), 7.58-7.50(\mathrm{~m}, 2 \mathrm{H}), 7.49-7.38$ $(\mathrm{m}, 4 \mathrm{H}), 7.13(\mathrm{~d}, J=8.3 \mathrm{~Hz}, 1 \mathrm{H}), 6.92$ (ddd, $J=12.7,8.1,1.4 \mathrm{~Hz}, 2 \mathrm{H}), 6.88-6.78(\mathrm{~m}, 2 \mathrm{H})$, $6.50(\mathrm{td}, J=7.7,3.0 \mathrm{~Hz}, 2 \mathrm{H}), 6.11(\mathrm{~s}, 1 \mathrm{H}), 3.84(\mathrm{~s}, 3 \mathrm{H}), 3.65(\mathrm{~s}, 3 \mathrm{H}), 2.72(\mathrm{~s}, 3 \mathrm{H}), 1.66(\mathrm{~s}$, $3 \mathrm{H}) .{ }^{13} \mathrm{C}$ NMR $(101 \mathrm{MHz}$, Chloroform- $d) \delta 157.9,157.5$ (d, $\left.J=14.2 \mathrm{~Hz}\right), 148.8(\mathrm{~d}, J=4.6$ Hz), 134.4 (d, $J=100.8 \mathrm{~Hz}$ ), 132.9 (d, $J=10.3 \mathrm{~Hz}), 132.7$ (d, $J=76.4 \mathrm{~Hz}), 131.7$ (d, $J=76.7 \mathrm{~Hz}), 131.4$ (d, $J=$ $2.8 \mathrm{~Hz}), 130.8,130.7,130.4$ (d, $J=10.7 \mathrm{~Hz}), 130.1,130.0$ (d, $J=13.2 \mathrm{~Hz}), 129.3$ (d, $J=2.9 \mathrm{~Hz}), 127.7$ (d, $J=$ $12.7 \mathrm{~Hz}), 126.2,126.1,126.1,125.9(\mathrm{~d}, J=8.0 \mathrm{~Hz}), 123.9,118.0,116.9,114.0(\mathrm{~d}, J=2.6 \mathrm{~Hz}), 110.0,100.1$, 55.7, 55.6, 17.3, 12.2. ${ }^{31} \mathrm{P}$ NMR (162 MHz, Chloroform-d) $\delta 30.4$. HRMS (ESI) $\mathrm{m} / \mathrm{z}:[\mathrm{M}+\mathrm{H}]^{+}$Calcd for $\mathrm{C}_{33} \mathrm{H}_{30} \mathrm{~N}_{2} \mathrm{O}_{3} \mathrm{P}$ 533.1994; found 533.1989. Optical rotation: $[\alpha]_{D}{ }^{25}=+167.1$ (c $=0.5, \mathrm{CHCl}_{3}$ ). HPLC: Chiralpak $\mathrm{IA}, n$-hexane/isopropanol $=70 / 30$, Flow rate $=1.0 \mathrm{~mL} / \mathrm{min}, \mathrm{UV}=254 \mathrm{~nm}, t_{\mathrm{R}}(\operatorname{minor})=8.42 \mathrm{~min}, t_{\mathrm{R}}($ major $)=$ $10.29 \mathrm{~min}$.

\section{(R)-(2-(8-methoxy-1,3-dimethylpyrrolo[1,2-a]quinoxalin-4-yl)-3-(methoxymethoxy)phenyl)} diphenylphosphine oxide $(5 r)$ :

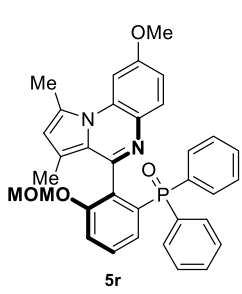

$244.1 \mathrm{mg}, 87 \%$ yield, white solid, $\mathrm{mp}: 100-102^{\circ} \mathrm{C}, 54 \%$ ee. Flash silica gel chromatography (petroleum ether/ethyl acetate $=1 / 1) .{ }^{1} \mathrm{H}$ NMR $(400 \mathrm{MHz}$, Chloroform- $d) \delta 8.03(\mathrm{ddt}, \mathrm{J}=$ 12.4, 6.8, $1.5 \mathrm{~Hz}, 2 \mathrm{H}$ ), 7.92 (ddd, $J=12.2,7.9,1.0 \mathrm{~Hz}, 1 \mathrm{H}), 7.58-7.50(\mathrm{~m}, 2 \mathrm{H}), 7.50-$ $7.38(\mathrm{~m}, 5 \mathrm{H}), 6.96$ (ddd, $J=12.6,8.1,1.4 \mathrm{~Hz}, 2 \mathrm{H}), 6.90-6.79(\mathrm{~m}, 2 \mathrm{H}), 6.53(\mathrm{td}, J=7.7$, $3.0 \mathrm{~Hz}, 2 \mathrm{H}), 6.12(\mathrm{~s}, 1 \mathrm{H}), 5.15(\mathrm{~d}, J=7.0 \mathrm{~Hz}, 1 \mathrm{H}), 4.88(\mathrm{~d}, J=7.1 \mathrm{~Hz}, 1 \mathrm{H}), 3.86(\mathrm{~s}, 3 \mathrm{H})$, 3.19 (s, 3H), 2.74 (s, 3H), 1.70 (s, 3H). ${ }^{13} \mathrm{C}$ NMR (101 MHz, Chloroform-d) $\delta 158.0,155.4$ (d, $J=13.8 \mathrm{~Hz}), 148.7$ (d, $J=4.4 \mathrm{~Hz}$ ), 134.4 (d, $J=100.7 \mathrm{~Hz}$ ), 132.8 (d, $J=79.3 \mathrm{~Hz}), 132.8$ (d, $J=10.3 \mathrm{~Hz}$ ), $131.7(\mathrm{~d}, J=92.3 \mathrm{~Hz}), 131.4(\mathrm{~d}, J=3.6 \mathrm{~Hz}), 130.8,130.8,130.7(\mathrm{~d}, J=10.7 \mathrm{~Hz}), 130.1,129.9(\mathrm{~d}, J=13.2 \mathrm{~Hz})$, $129.5(\mathrm{~d}, J=2.8 \mathrm{~Hz}), 127.8(\mathrm{~d}, J=12.4 \mathrm{~Hz}), 127.2(\mathrm{~d}, J=8.0 \mathrm{~Hz}), 126.3,126.2,124.0,118.1,117.7,116.9$,

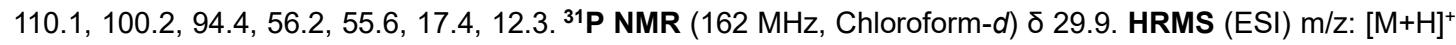
Calcd for $\mathrm{C}_{34} \mathrm{H}_{32} \mathrm{~N}_{2} \mathrm{O}_{4} \mathrm{P}$ 563.2100; found 563.2097. Optical rotation: $[\alpha]_{\mathrm{D}}{ }^{25}=+143.2\left(\mathrm{c}=0.5, \mathrm{CHCl}_{3}\right)$. HPLC: Chiralpak IA, $n$-hexane/isopropanol $=50 / 50$, Flow rate $=1.0 \mathrm{~mL} / \mathrm{min}, \mathrm{UV}=254 \mathrm{~nm}, t_{\mathrm{R}}(\operatorname{minor})=5.08 \mathrm{~min}, t_{\mathrm{R}}$ (major) $=6.36 \mathrm{~min}$.

[1] K. Urakawa, M. Sumimoto, M. Arisawa, M. Matsuda, H. Ishikawa. Angew. Chem. Int. Ed. 2016, 55, 7432.

[2] X-L. He, H-R. Zhao, X. Song, B. Jiang, W. Du, Y-C. Chen. ACS Catal. 2019, 9, 4374.

[3] Z. An, Y. Jiang, X. Guan, R. Yan. Chem. Commun., 2018, 54, 10738.

[4] R. J. Faggyas, N. L. Sloan, N. Buijs, A.Sutherland. Eur. J. Org. Chem. 2019, 5344. 


\section{Synthesis of quinoxalinap}

\section{(1) $\mathrm{MnO}_{2}$ preparative method}

$$
\mathrm{KMnO}_{2}+\mathrm{MnSO}_{4}+\mathrm{KOH} \frac{\mathrm{H}_{2} \mathrm{O}}{100^{\circ} \mathrm{C}} \mathrm{MnO}_{2}+\mathrm{K}_{2} \mathrm{SO}_{4}+\mathrm{H}_{2} \mathrm{O}
$$

$0.1 \mathrm{~mol} / \mathrm{L} \mathrm{KMnO}_{4}$ aqueous solution $(250 \mathrm{ml}, 1$ equiv. $)$ was heated in an oil bath to $100^{\circ} \mathrm{C}$ in $1000 \mathrm{ml}$ roundbotton flask. $40 \% \mathrm{NaOH}$ aqueous solution (2 equiv.) and $1 \mathrm{~mol} / \mathrm{L} \mathrm{MnSO}_{4}$ aqueous solution (1.5 equiv.) were added slowly during $1 \mathrm{~h}$. After $2 \mathrm{~h}$, the reaction mixture was filtered and washed with hot water until filtrate colourless. The residue was dried at $120^{\circ} \mathrm{C}$ to afford brown powdery $\mathrm{MnO}_{2}$.

\section{(2) Synthesis of $L_{N a-1}, L_{N a-2}$ and $L_{N a-3}$}
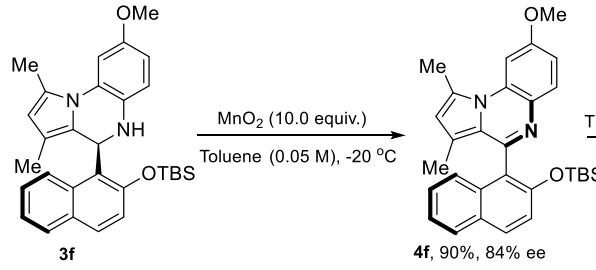

4f, $90 \%, 84 \%$ ee

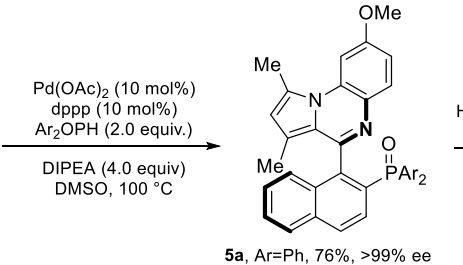

5a, $\mathrm{Ar}=\mathrm{Ph}, 76 \%,>99 \%$ ee
5f, $\mathrm{Ar}=4-\mathrm{FPh}, 73 \%,>99 \%$ e

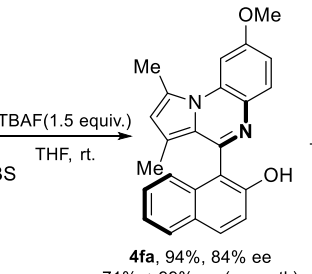

$4 \mathrm{fa}, 94 \%, 84 \%$ ee
$71 \%,>99 \%$ ee (recrystl.)

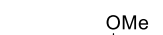

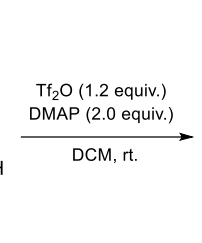

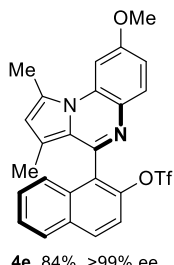

$4 e, 84 \%,>99 \%$ ee

Procedure A: A $50 \mathrm{~mL}$ round-bottom flask was charged with $\mathrm{MnO}_{2}(20 \mathrm{mmol}, 10.0$ equiv.) in toluene (20 $\mathrm{mL})$. The reaction mixture was cooled to $-20^{\circ} \mathrm{C}$ and stirred for 10 minutes, then $3 \mathbf{f}(1.2 \mathrm{~g}, 2.5 \mathrm{mmol})$ was added to the reaction mixture and stirred for $2 \mathrm{~h}$. The reaction mixture was filtered through Celite and removed in vacuo. The crude mass was purified by silica gel column chromatography to give $\mathbf{4 f}$ ( $90 \%$ yield, $1.08 \mathrm{~g}$ ).

According to the literature ${ }^{[1]}$, a mixture of $\mathbf{4 f}(1.08 \mathrm{~g}, 2.25 \mathrm{mmol})$ and tetrabutylammonium fluoride (TBAF, 3.8 mmol, 1.5 equiv. $)$ in THF $(20 \mathrm{~mL})$ was stirred at room temperature for $30 \mathrm{~min}$. The resulting mixture was quenched with water $(20 \mathrm{~mL})$ followed by an extraction with $\mathrm{CH}_{2} \mathrm{Cl}_{2}(3 \times 20 \mathrm{~mL})$ and dried over $\mathrm{MgSO}_{4}$ before filtration and concentration under reduced pressure. The crude mass was purified by silica gel column chromatography to give $\mathbf{4 f a}$ ( $94 \%$ yield, $0.78 \mathrm{~g}$ ). The enantiomeric excess of $\mathbf{4 f a}$ can be increased up to $>99 \%$ through a simple recrystallization in acetonitrile. The crystals are racemic and the residue in the mother liquid was needed.

Procedure B: According to the literature ${ }^{[2]}$, a solution of $4 \mathrm{fa}(0.55 \mathrm{~g}, 1.5 \mathrm{mmol})$ and $4-(\mathrm{N}, \mathrm{N}-$ dimethylamino)pyridine (3.0 mmol, 2.0 equiv.) in $\mathrm{CH}_{2} \mathrm{Cl}_{2}(10 \mathrm{~mL})$, trifluoromethanesulfonic anhydride (1.8 mmol, 1.2 equiv.) was slowly added at $0{ }^{\circ} \mathrm{C}$. The resulting mixture was warmed to room temperature, and stirred for additional $30 \mathrm{~min}$. The resulting mixture was quenched with distilled water $(20 \mathrm{~mL})$ at $0{ }^{\circ} \mathrm{C}$ followed by an extraction with $\mathrm{CH}_{2} \mathrm{Cl}_{2}(3 \times 20 \mathrm{~mL})$. The combined organic layer was washed with $1 \mathrm{M}-\mathrm{HCl}(2 \times 20 \mathrm{~mL})$, brine $(2 \times 20$ $\mathrm{mL}$ ) and dried over $\mathrm{MgSO}_{4}$ before filtration and concentration under reduced pressure. The resulting crude was purified by silica gel column chromatography to provide $4 \mathrm{e}(84 \%$ yield, $0.63 \mathrm{~g})$.

Procedure C: According to the literature ${ }^{[3]}$, a solution of $\mathrm{Pd}(\mathrm{OAc})_{2}(10 \%)$, dppp (10\%) in DMSO (5 mL) was vigorously stirred at room temperature for $30 \mathrm{~min}$ under $\mathrm{N}_{2}$ atmosphere. Then $4 \mathrm{e}(0.5 \mathrm{mmol}, 250 \mathrm{mg})$, DIPEA (2.0 mmol, 4.0 equiv.) and diphenylphosphine oxide $(1.1 \mathrm{mmol}, 2.2$ equiv.) were added sequentially before 
heating at $100{ }^{\circ} \mathrm{C}$ in an oil bath for $4 \mathrm{~h}$. The reaction was removed to room temperature and quenched by water, extracted with EtOAc $(3 \times 10 \mathrm{~mL})$. The combined organic layers were dried over anhydrous $\mathrm{Na}_{2} \mathrm{SO}_{4}$, filtered and concentrated in vacuo. The crude mass was purified by silica gel column chromatography to afford $\mathbf{5 a}$ or $\mathbf{5 f}$.

Procedure D: According to the literature ${ }^{[4]}$, to a solution of $\mathbf{5 a}$ or $\mathbf{5 f}(0.3 \mathrm{mmol})$ and $\mathrm{HSiCl}_{3}(0.9 \mathrm{mmol})$ in toluene $(3 \mathrm{ml}), \mathrm{Et}_{3} \mathrm{~N}(2.7 \mathrm{mmol})$ was slowly added under $\mathrm{N}_{2}$. The resulting mixture was stirred for $12 \mathrm{~h}$ at $100{ }^{\circ} \mathrm{C}$ by the oil bath. After cooling, the resulting solution was carefully quenched with $10 \%$ aq. $\mathrm{NaOH}$ and stirred until excess $\mathrm{HSiCl} 3$ was consumed. The resulting mixture was diluted with water and extracted with ethyl acetate (20 $\mathrm{mL} \times 3$ ). The combined organic layer was dried over $\mathrm{Na}_{2} \mathrm{SO}_{4}$ and volatiles were removed in vacuo. The crude mass was purified by silica gel column chromatography to afford $\mathbf{L}_{\mathrm{Na}-1}$ or $\mathbf{L}_{\mathrm{Na}-2}$.

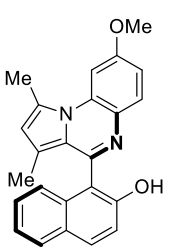

$4 \mathrm{fa},>99 \%$ ee

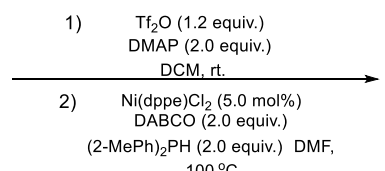
$100^{\circ} \mathrm{C}$

According to the literature ${ }^{[5]}$, a solution of $\mathrm{Ni}(\mathrm{dppe}) \mathrm{Cl}_{2}(5.0 \mathrm{~mol} \%)$ in $2 \mathrm{ml} \mathrm{DMF}$ at $23^{\circ} \mathrm{C}$ was treated with dio-tolylphosphane. The resulting solution was stirred at $100{ }^{\circ} \mathrm{C}$ for $30 \mathrm{~min}$. Then a solution of $4 \mathrm{e}(150 \mathrm{mg}, 0.3$ $\mathrm{mmol})$ and DABCO $(0.4 \mathrm{mmol})$ in $1 \mathrm{ml}$ DMF was added. The resulting solution was stirred at $100{ }^{\circ} \mathrm{C}$ by an oil bath for $12 \mathrm{~h}$. The mixture was concentrated under reduced pressure. The residue was purified by silica gel column chromatography to afford $\mathbf{L}_{\mathrm{Na}-3}$.

(3) Synthesis of $L_{\mathrm{Na}-1}, L_{\mathrm{Na}-4}$ and $L_{\mathrm{Na}-5}$
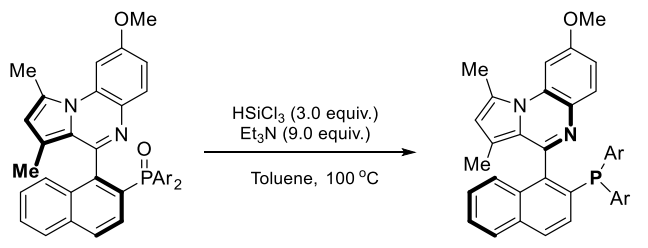

$\mathrm{L}_{\mathrm{Na}-1}, \mathrm{Ar}=\mathrm{Ph}, 95 \%,>99 \%$ ee

$L_{\mathrm{Na}-4}, \mathrm{Ar}=$ Naphthyl, $94 \%,>99 \%$ ee $\mathrm{L}_{\mathrm{Na}-5}, \mathrm{Ar}=3,5-\mathrm{Me}_{2} \mathrm{Ph}, 92 \%,>99 \%$ ee

According to the literature ${ }^{[4]}$, to a solution of the recrystallized $\mathbf{5 a}$ or $\mathbf{5 g}$ or $\mathbf{5 h}(0.3 \mathrm{mmol})$ and $\mathrm{HSiCl}_{3}(0.9$ $\mathrm{mmol})$ in toluene $(3 \mathrm{ml}), \mathrm{Et}_{3} \mathrm{~N}(2.7 \mathrm{mmol})$ was slowly added under $\mathrm{N}_{2}$. The resulting mixture was stirred for $12 \mathrm{~h}$ at $100{ }^{\circ} \mathrm{C}$ in an oil bath. After cooling, the resulting solution was carefully quenched with $10 \%$ aq. $\mathrm{NaOH}$ and stirred until excess $\mathrm{HSiCl}_{3}$ was consumed. The resulting mixture was diluted with water and extracted with ethyl acetate $(20 \mathrm{~mL} \times 3)$. The combined organic layer was dried over $\mathrm{Na}_{2} \mathrm{SO}_{4}$ and volatiles were removed in vacuo. The crude mass was purified by silica gel column chromatography to afford $\mathbf{L}_{\mathrm{Na}-1}$ or $\mathbf{L}_{\mathrm{Na}-4}$ or $\mathbf{L}_{\mathrm{Na}-5}$.

\section{(4) Synthesis of (S)- $L_{N a-1}$}
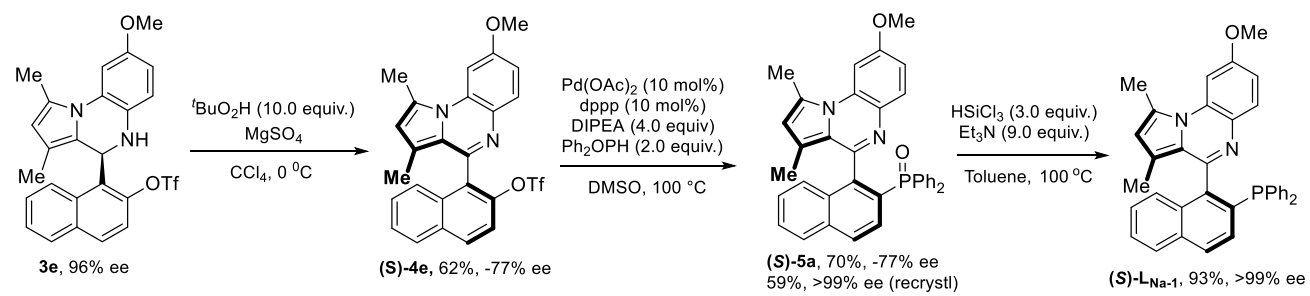

A mixture of $3 \mathbf{e}(1.0 \mathrm{~g}, 2 \mathrm{mmol}), t$-butylhydroperoxide (20 mmol, 1.5 equiv.) and $\mathrm{MgSO}_{4}$ in $\mathrm{CCl}_{4}(20 \mathrm{~mL})$ was stirred at $0{ }^{\circ} \mathrm{C}$ in four tubes. After 3 days, the starting material was a little existed in reaction and the reaction 
almost stopped. The resulting mixture was concentrated under reduced pressure and was purified by silica gel column chromatography to give (S)-4e $(62 \%$ yield, $0.62 \mathrm{~g})$ and recycled the starting material $3 \mathbf{e}(27 \%$ yield, 0.27 g).

(S)-5a was synthesized by procedure C

(S)-LNa-1 was synthesized by procedure $D$.

\section{(5) Confirm configuration of $3 e$}

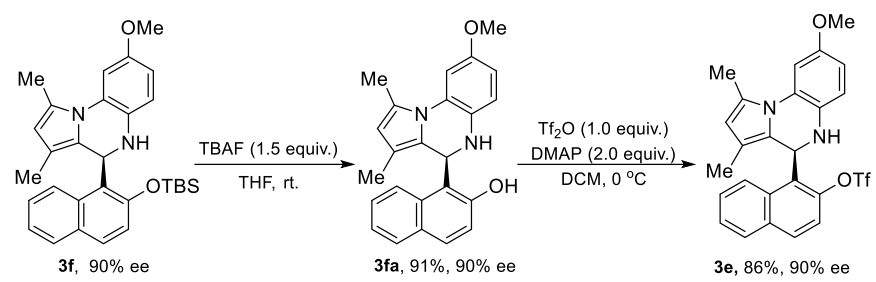

(6) Synthesis of $L_{F-1}$ and $L_{F-2}$
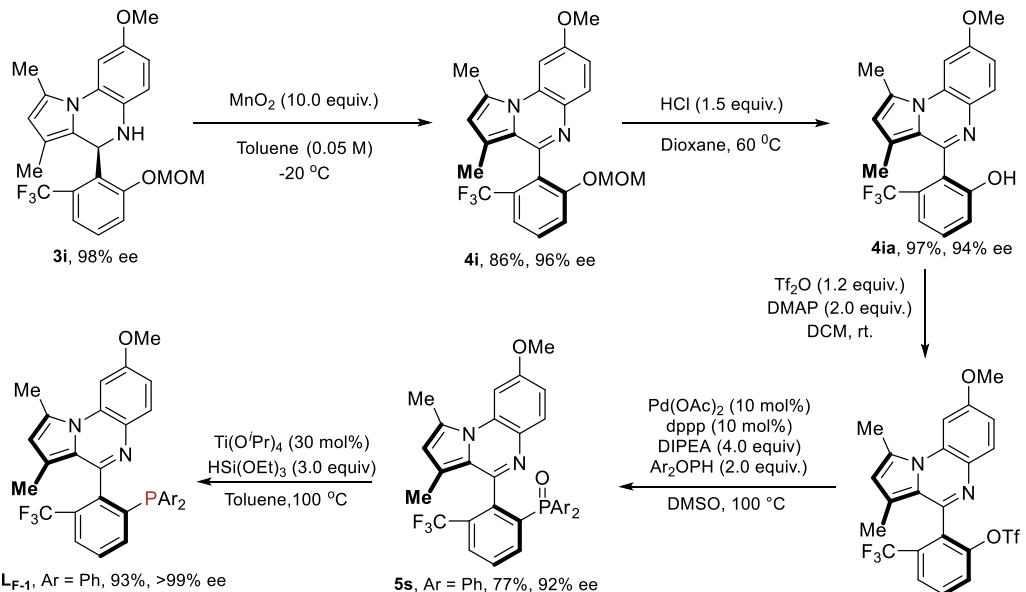

$\mathrm{L}_{\mathrm{F}-2}, \mathrm{Ar}=3,5-\mathrm{Me}_{2} \mathrm{Ph}, 95 \%,>99 \%$ ee

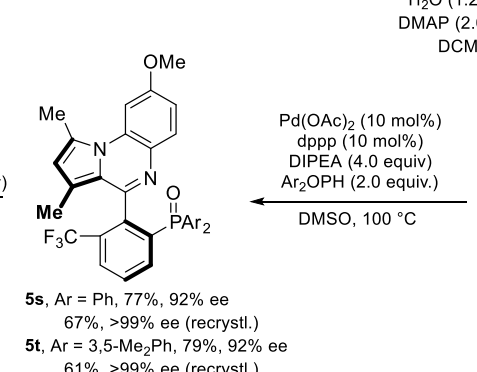

equiv.) CM, it.

General procedure A for the $4 \mathrm{i}: 3 \mathbf{i}(1.0 \mathrm{~g}, 2.5 \mathrm{mmol})$ and $\mathrm{MnO}_{2}(25 \mathrm{mmol})$ were stirred in toluene $(30 \mathrm{~mL})$ at $-20{ }^{\circ} \mathrm{C}$ for $2 \mathrm{~h}$. After completion, the solution was concentrated and the residue was purified by flash chromatography to afford the pure product $4 \mathbf{i}(86 \%$ yield, $0.93 \mathrm{~g})$.

According to the literature ${ }^{[6]}$, a mixture of $4 \mathbf{i}(0.93 \mathrm{~g}, 2.2 \mathrm{mmol})$ and concentrated hydrochloric acid $(3.3 \mathrm{mmol}$, 1.5 equiv.) in dioxane $(10 \mathrm{~mL})$ was stirred at $60^{\circ} \mathrm{C}$ for $4 \mathrm{~h}$. The resulting mixture was concentrated under reduced pressure to afford $4 \mathrm{ia}(97 \%$ yield, $0.82 \mathrm{~g}$ ) quantitatively which was used directly without further purification for the next step.

The 4ib was synthesized by procedure $B$.

The $\mathbf{5 s}$ and $\mathbf{5 t}$ were synthesized by procedure $\mathbf{C}$. The enantiomeric excess of $\mathbf{5 s}$ and $\mathbf{5 t}$ can be increased up to $>99 \%$ through a simple recrystallization in acetonitrile. The crystals are racemic and the residue in the mother liquid was needed.

According to the literature ${ }^{[7]}$, in a $10 \mathrm{ml}$ flask was placed $\mathbf{5 s}$ or $\mathbf{5 t}(0.3 \mathrm{mmol})$ under $\mathrm{N}_{2}$. Anhydrous toluene $(3 \mathrm{ml})$ was added, and then the mixture was magnetically stirred at room temperature. After the addition of $\mathrm{Ti}\left(\mathrm{O}^{\prime} \mathrm{Pr}\right)_{4}(30 \%, 0.09 \mathrm{mmol})$ and $(\mathrm{EtO})_{3} \mathrm{SiH}(0.9 \mathrm{mmol}, 3.0$ equiv. $)$, the reaction flask was stirred for $1 \mathrm{~h}$ at $100{ }^{\circ} \mathrm{C}$ in an oil bath. After cooling to room temperature, the sovent was concentrated in vacuo. The residue was purified by silica gel column chromatography to afford LF-1 or LF-2. 
(R)-4-(2-(diphenylphosphanyl)naphthalen-1-yl)-8-methoxy-1,3-dimethylpyrrolo[1,2-a]quinoxaline $\left(\mathrm{L}_{\mathrm{Na}-1}\right)$ :

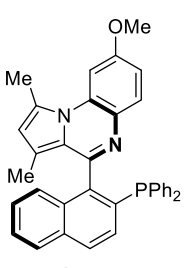

$\mathrm{L}_{\mathrm{Na}-1}$

$153.1 \mathrm{mg}, 95 \%$ yield, yellow solid, $>99 \%$ ee. (The product was quickly oxidized in isopropanol and ee value was determined by oxidation product.) Flash silica gel chromatography (petroleum ether/ethyl acetate $=10 / 1) .{ }^{1} \mathrm{H}$ NMR $(400 \mathrm{MHz}$, Chloroform- $d) \delta 7.86-7.79(\mathrm{~m}, 3 \mathrm{H}), 7.57$ (dd, $J=15.6,8.5 \mathrm{~Hz}, 2 \mathrm{H}), 7.48-7.38(\mathrm{~m}, 2 \mathrm{H}), 7.32-7.21(\mathrm{~m}, 11 \mathrm{H}), 6.92(\mathrm{dd}, J=8.9,2.6 \mathrm{~Hz}, 1 \mathrm{H})$, $6.30(\mathrm{~s}, 1 \mathrm{H}), 3.90(\mathrm{~s}, 3 \mathrm{H}), 2.97(\mathrm{~s}, 3 \mathrm{H}), 1.33(\mathrm{~s}, 3 \mathrm{H}) .{ }^{13} \mathrm{C}$ NMR $(100 \mathrm{MHz}$, Chloroform-d) $\delta 158.1$, $152.5(\mathrm{~d}, J=6.8 \mathrm{~Hz}), 143.9(\mathrm{~d}, J=32.0 \mathrm{~Hz}), 138.0(\mathrm{~d}, J=12.3 \mathrm{~Hz}), 137.4(\mathrm{~d}, J=10.9 \mathrm{~Hz})$, $134.5(\mathrm{~d}, J=13.1 \mathrm{~Hz}), 133.9$ (d, $J=9.4 \mathrm{~Hz}), 133.7,133.6,132.4(\mathrm{~d}, J=7.3 \mathrm{~Hz}), 131.5,131.0,130.8,129.9$, 128.8, 128.4, 128.4, 128.3, 128.2, 128.1, 127.9, 127.5, 127.0 (d, $J=7.3 \mathrm{~Hz}), 126.3$ (d, J = 2.5 Hz), 124.6 (d, J $=1.8 \mathrm{~Hz}), 118.6,117.5,110.6,100.7,55.8,17.8,12.5 .{ }^{31} \mathrm{P}$ NMR $(162 \mathrm{MHz}$, Chloroform-d) $\delta-13.0$. HRMS (ESI) $\mathrm{m} / \mathrm{z}:[\mathrm{M}+\mathrm{H}]^{+}$Calcd for $\mathrm{C}_{36} \mathrm{H}_{30} \mathrm{~N}_{2} \mathrm{OP} 537.2096$; found 537.2090. Optical rotation: $[\alpha]_{D}^{25}=+227.8\left(\mathrm{c}=0.5, \mathrm{CHCl}_{3}\right)$.

\section{(R)-4-(2-(bis(4-fluorophenyl)phosphanyl)naphthalen-1-yl)-8-methoxy-1,3-dimethylpyrrolo[1,2-} a]quinoxaline $\left(\mathrm{L}_{\mathrm{Na}-2}\right)$ :

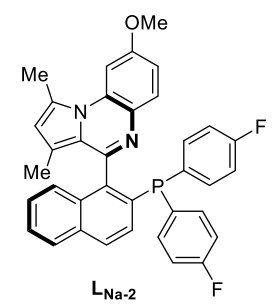

$161.6 \mathrm{mg}, 94 \%$ yield, yellow solid, >99\% ee. (The product was quickly oxidized in isopropanol and ee value was determined by oxidation product.) Flash silica gel chromatography (petroleum ether/ethyl acetate $=10 / 1) .{ }^{1} \mathrm{H}$ NMR $(400 \mathrm{MHz}$, Chloroformd) $\delta 7.89-7.81(\mathrm{~m}, 3 \mathrm{H}), 7.60-7.52(\mathrm{~m}, 2 \mathrm{H}), 7.47$ (ddd, $J=8.2,6.8,1.2 \mathrm{~Hz}, 1 \mathrm{H}), 7.36-$ $7.30(\mathrm{~m}, 2 \mathrm{H}), 7.22$ (tdd, $J=7.0,5.6,2.2 \mathrm{~Hz}, 4 \mathrm{H}), 7.00-6.88(\mathrm{~m}, 5 \mathrm{H}), 6.32(\mathrm{~s}, 1 \mathrm{H}), 3.94$ (s, 3H), $2.98(\mathrm{~s}, 3 \mathrm{H}), 1.32(\mathrm{~s}, 3 \mathrm{H}) .{ }^{13} \mathrm{C}$ NMR $\left(100 \mathrm{MHz}\right.$, Chloroform-d) $\delta 163.3$ (d, C-F, ${ }^{1} \mathrm{~J}_{\mathrm{C}}$ $F=248.7 \mathrm{~Hz}), 163.2\left(\mathrm{~d}, \mathrm{C}-\mathrm{F},{ }^{1} \mathrm{~J}_{\mathrm{C}-\mathrm{F}}=248.1 \mathrm{~Hz}\right), 158.3,152.1$ (d, $\left.J=6.5 \mathrm{~Hz}\right), 143.6(\mathrm{~d}, J=30.8 \mathrm{~Hz}), 135.8$ (dd, $\left.\mathrm{C}-\mathrm{F},{ }^{3} \mathrm{JC}_{\mathrm{C}-\mathrm{F}}=8.0, \mathrm{C}-\mathrm{P}, 5.1 \mathrm{~Hz}\right), 135.6\left(\mathrm{dd}, \mathrm{C}-\mathrm{F},{ }^{3} \mathrm{~J}_{\mathrm{C}-\mathrm{F}}=8.0, \mathrm{C}-\mathrm{P}, 4.4 \mathrm{~Hz}\right), 134.2(\mathrm{~d}, J=13.6 \mathrm{~Hz}), 133.7,133.1$ (d, J $=11.9 \mathrm{~Hz}$ ), 132.7 (d, J = 3.5 Hz), 132.6, 132.5, 131.4, 131.0, 130.7, 129.2, 129.0 (d, J = 1.0 Hz), 128.0, 127.6, $127.2(\mathrm{~d}, J=1.0 \mathrm{~Hz}), 126.2(\mathrm{~d}, J=2.5 \mathrm{~Hz}), 124.5(\mathrm{~d}, J=1.0 \mathrm{~Hz}), 118.7,117.5,115.6\left(\mathrm{dd}, \mathrm{C}-\mathrm{F},{ }^{2} J_{C-F}=24.2, \mathrm{C}-\right.$ $\mathrm{P}, 7.5 \mathrm{~Hz}), 115.4$ (dd, C-F, $\left.{ }^{2} \mathrm{~J}_{-} \mathrm{F}=24.3, \mathrm{C}-\mathrm{P}, 7.5 \mathrm{~Hz}\right), 110.8,100.8,55.9,17.8,12.5 .{ }^{19} \mathrm{~F} \mathrm{NMR}(377 \mathrm{MHz}$, Chloroform- $d$ ) $\delta-112.7,-113.1 .{ }^{31} \mathrm{P}$ NMR $\left(162 \mathrm{MHz}\right.$, Chloroform- $d$ ) $\delta-14.2$. HRMS (ESI) m/z: $[\mathrm{M}+\mathrm{H}]^{+}$Calcd for $\mathrm{C}_{36} \mathrm{H}_{28} \mathrm{~F}_{2} \mathrm{~N}_{2} \mathrm{OP} 573.1907$; found 573.1902. Optical rotation: $[\alpha]_{D}{ }^{25}=+155.8\left(\mathrm{c}=0.5, \mathrm{CHCl}_{3}\right.$ ).

(R)-4-(2-(di-o-tolylphosphanyl)naphthalen-1-yl)-8-methoxy-1,3-dimethylpyrrolo[1,2-a]quinoxaline ( $\left.\mathrm{L}_{\mathrm{Na}-3}\right)$ :

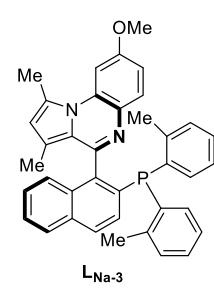

$116.7 \mathrm{mg}, 69 \%$ yield, yellow solid, >99\% ee. (The product was quickly oxidized in isopropanol and ee value was determined by oxidation product.) Flash silica gel chromatography (petroleum ether/ethyl acetate $=10 / 1) .{ }^{1} \mathrm{H}$ NMR $(400 \mathrm{MHz}$, Chloroform- $d) \delta 7.85-7.78(\mathrm{~m}$, $3 \mathrm{H}), 7.55(\mathrm{dd}, J=8.5,1.1 \mathrm{~Hz}, 1 \mathrm{H}), 7.44(\mathrm{ddd}, J=8.2,6.8,1.2 \mathrm{~Hz}, 1 \mathrm{H}), 7.32-7.24(\mathrm{~m}, 3 \mathrm{H})$, $7.22-7.15(\mathrm{~m}, 2 \mathrm{H}), 7.13-6.99(\mathrm{~m}, 5 \mathrm{H}), 6.94$ (ddd, $J=7.8,3.8,1.4 \mathrm{~Hz}, 1 \mathrm{H}), 6.88(\mathrm{dd}, J=$ 8.9, $2.6 \mathrm{~Hz}, 1 \mathrm{H}), 6.27(\mathrm{~s}, 1 \mathrm{H}), 3.91(\mathrm{~s}, 3 \mathrm{H}), 2.96(\mathrm{~s}, 3 \mathrm{H}), 2.12(\mathrm{~d}, J=1.4 \mathrm{~Hz}, 3 \mathrm{H}), 2.03$ (s, 3H), $1.30(\mathrm{~s}, 3 \mathrm{H}) .{ }^{13} \mathrm{C}$ NMR (100 MHz, Chloroform-d) $\delta 158.1,152.2(\mathrm{~d}, J=6.6 \mathrm{~Hz}), 143.3(\mathrm{~d}, J=31.9 \mathrm{~Hz}), 142.5$ (d, $J=14.5 \mathrm{~Hz}$ ), 142.3 (d, $J=16.0 \mathrm{~Hz}), 136.2(\mathrm{~d}, J=10.2 \mathrm{~Hz}), 135.5$ (d, $J=12.6 \mathrm{~Hz}), 134.8,134.2(\mathrm{~d}, J=12.6$ $\mathrm{Hz}), 133.7,133.6,132.7$ (d, $J=6.7 \mathrm{~Hz}), 131.5,130.8,130.7,130.1$ (d, $J=4.3 \mathrm{~Hz}), 130.0,129.6(\mathrm{~d}, J=5.1 \mathrm{~Hz})$, 128.5, 128.4, 128.3, 127.9, 127.4, 126.8, 126.7, 126.2 (d, $J=2.6 \mathrm{~Hz}$ ), 126.1, 125.9, 124.4, 118.6, 117.3, 110.5, 100.5, 55.8, 21.6 (d, $J=22.9 \mathrm{~Hz}$ ), 21.3 (d, $J=21.9 \mathrm{~Hz}$ ), 17.8, 12.5. ${ }^{31} \mathrm{P}$ NMR (162 MHz, Chloroform- $d$ ) $\delta$-29.6. HRMS (ESI) m/z: [M+Na] $]^{+}$Calcd for $\mathrm{C}_{38} \mathrm{H}_{33} \mathrm{~N}_{2} \mathrm{OPNa}$ 587.2228; found 587.2223. Optical rotation: $[\alpha]_{D}{ }^{25}=+97.3$ $\left(c=0.5, \mathrm{CHCl}_{3}\right)$. 
(R)-4-(2-(di(naphthalen-2-yl)phosphanyl)naphthalen-1-yl)-8-methoxy-1,3-dimethylpyrrolo[1,2-

a]quinoxaline $\left(\mathrm{L}_{\mathrm{Na}-4}\right)$ :

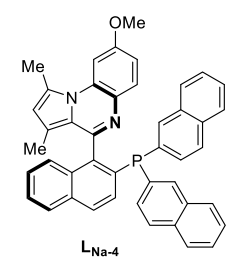

$179.7 \mathrm{mg}, 94 \%$ yield, yellow solid, >99\% ee. (The product was quickly oxidized in isopropanol and ee value was determined by oxidation product.) Flash silica gel chromatography (petroleum ether/ethyl acetate $=10 / 1$ ). Flash silica gel chromatography (petroleum ether/ethyl acetate $=10 / 1) .{ }^{1} \mathrm{H}$ NMR $(400 \mathrm{MHz}$, Chloroform- $d) \delta 7.83(\mathrm{~d}, J=7.7 \mathrm{~Hz}, 2 \mathrm{H}), 7.75$ $(\mathrm{t}, J=9.1 \mathrm{~Hz}, 4 \mathrm{H}), 7.71-7.65(\mathrm{~m}, 3 \mathrm{H}), 7.64-7.51(\mathrm{~m}, 5 \mathrm{H}), 7.49-7.37(\mathrm{~m}, 7 \mathrm{H}), 7.32$ (ddd, $J=8.4,6.8,1.3 \mathrm{~Hz}, 1 \mathrm{H}), 6.85(\mathrm{dt}, J=9.0,2.0 \mathrm{~Hz}, 1 \mathrm{H}), 6.26(\mathrm{~s}, 1 \mathrm{H}), 3.88(\mathrm{~s}, 3 \mathrm{H}), 2.91(\mathrm{~s}, 3 \mathrm{H}), 1.33(\mathrm{~s}, 3 \mathrm{H}) .{ }^{13} \mathrm{C}$ NMR $(100 \mathrm{MHz}$, Chloroform- $d$ ) $\delta 158.1,152.3(\mathrm{~d}, J=6.6 \mathrm{~Hz}), 143.6(\mathrm{~d}, J=29.1 \mathrm{~Hz}), 135.1$ (d, $J=12.1 \mathrm{~Hz})$, 134.7, $134.7(\mathrm{~d}, J=11.4 \mathrm{~Hz}), 134.4(\mathrm{~d}, J=4.8 \mathrm{~Hz}), 134.4(\mathrm{~d}, J=13.8 \mathrm{~Hz}), 134.2,133.7,133.3,133.3(\mathrm{~d}, J=$ $3.9 \mathrm{~Hz}), 133.3,133.2$ (d, $J=4.4 \mathrm{~Hz}), 132.6$ (d, $J=7.0 \mathrm{~Hz}$ ), 131.4, 130.9, 130.8, 130.3 (d, J = 8.3 Hz), 130.2 (d, $J=6.9 \mathrm{~Hz}$ ), 129.9, 128.8, 128.3, 128.1, 128.0, 127.8, 127.7, 127.7, 127.6 (d, J = 6.2 Hz), 127.5, 127.0 (d, $J=$ $2.9 \mathrm{~Hz}), 126.6,126.4,126.3(\mathrm{~d}, J=2.1 \mathrm{~Hz}), 126.2,125.9,124.7$ (d, $J=1.0 \mathrm{~Hz}), 118.6,117.6,110.7,100.6,55.8$, 17.7, 12.5. ${ }^{31}$ P NMR (162 MHz, Chloroform-d) $\delta-10.0$. HRMS (ESI) m/z: $[\mathrm{M}+\mathrm{H}]^{+}$Calcd for $\mathrm{C}_{44} \mathrm{H}_{34} \mathrm{~N}_{2} \mathrm{OP} 637.2408$; found 637.2403. Optical rotation: $[\alpha]_{\mathrm{D}}^{25}=+225.0\left(\mathrm{c}=0.5, \mathrm{CHCl}_{3}\right)$.

(R)-4-(2-(bis(3,5-dimethylphenyl)phosphanyl)naphthalen-1-yl)-8-methoxy-1,3-dimethylpyrrolo[1,2a]quinoxaline $\left(\mathrm{L}_{\mathrm{Na}-5}\right)$ :

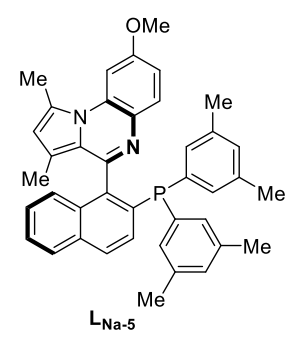

$163.7 \mathrm{mg}, 92 \%$ yield, yellow solid, >99\% ee. (The product was quickly oxidized in isopropanol and ee value was determined by oxidation product.) Flash silica gel chromatography (petroleum ether/ethyl acetate $=10 / 1) .{ }^{1} \mathrm{H}$ NMR $(400 \mathrm{MHz}$, Chloroformd) $\delta 7.83$ (ddd, $J=8.2,4.5,1.0 \mathrm{~Hz}, 3 \mathrm{H}$ ), 7.70 (d, $J=8.9 \mathrm{~Hz}, 1 \mathrm{H}$ ), 7.53 (ddd, $J=8.4,5.5$, $2.2 \mathrm{~Hz}, 2 \mathrm{H}$ ), 7.44 (ddd, $J=8.1,6.8,1.2 \mathrm{~Hz}, 1 \mathrm{H}$ ), 7.30 (ddd, $J=8.3,6.8,1.3 \mathrm{~Hz}, 1 \mathrm{H}$ ), $6.97(\mathrm{dd}, J=8.9,2.5 \mathrm{~Hz}, 1 \mathrm{H}), 6.95-6.91(\mathrm{~m}, 2 \mathrm{H}), 6.90-6.84(\mathrm{~m}, 4 \mathrm{H}), 6.28(\mathrm{~s}, 1 \mathrm{H})$, $3.94(\mathrm{~s}, 3 \mathrm{H}), 2.98(\mathrm{~s}, 3 \mathrm{H}), 2.18(\mathrm{~s}, 12 \mathrm{H}), 1.25(\mathrm{~s}, 3 \mathrm{H}) .{ }^{13} \mathrm{C}$ NMR $(100 \mathrm{MHz}$, Chloroformd) $\delta 158.2,152.7(\mathrm{~d}, J=6.7 \mathrm{~Hz}), 143.5(\mathrm{~d}, J=31.4 \mathrm{~Hz}), 137.6$ (d, $J=11.6 \mathrm{~Hz}), 137.5,137.5,137.4,137.4,137.1$ (d, $J=10.6 \mathrm{~Hz}), 135.5(\mathrm{~d}, J=14.6 \mathrm{~Hz}), 133.6,132.5(\mathrm{~d}, J=7.7 \mathrm{~Hz}), 131.7$ (d, $J=8.7 \mathrm{~Hz}), 131.6,131.5(\mathrm{~d}, J=$ $8.0 \mathrm{~Hz}$ ), 131.0, 130.9, 130.2 (d, $J=3.0 \mathrm{~Hz}), 130.1,128.6,127.9,127.3,126.8$ (d, $J=3.3 \mathrm{~Hz}), 126.3$ (d, $J=2.3$ $\mathrm{Hz}), 124.8(\mathrm{~d}, J=1.8 \mathrm{~Hz}), 118.6,117.5,110.7,100.7,55.9,21.5,21.4,17.8,12.3 .{ }^{31} \mathrm{P}$ NMR (162 MHz, Chloroform-d) $\delta-12.3$. HRMS (ESI) m/z: $[\mathrm{M}+\mathrm{H}]^{+}$Calcd for $\mathrm{C}_{40} \mathrm{H}_{38} \mathrm{~N}_{2} \mathrm{OP}$ 593.2722; found 593.2716. Optical rotation: $[\alpha]_{D}^{25}=+143.3\left(\mathrm{c}=0.5, \mathrm{CHCl}_{3}\right)$.

\section{(S)-4-(2-(diphenylphosphanyl)-6-(trifluoromethyl)phenyl)-8-methoxy-1,3-dimethylpyrrolo[1,2-} a]quinoxaline $\left(L_{F-1}\right)$ :

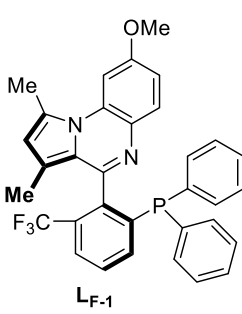

$154.7 \mathrm{mg}, 93 \%$ yield, yellow solid, $>99 \%$ ee. (The product was quickly oxidized in isopropanol and ee value was determined by oxidation product.) Flash silica gel chromatography (petroleum ether/ethyl acetate $=10 / 1) .{ }^{1} \mathrm{H}$ NMR $(400 \mathrm{MHz}$, Chloroform- $d$ ) $\delta 7.69$ (q, $J=3.3,2.5 \mathrm{~Hz}, 2 \mathrm{H}$ ), $7.45-7.38(\mathrm{~m}, 3 \mathrm{H}), 7.23-7.11(\mathrm{~m}, 7 \mathrm{H}), 7.08$ (ddd, $J=9.7$, 4.7, $1.9 \mathrm{~Hz}, 3 \mathrm{H}), 6.81$ (dd, $J=8.9,2.4 \mathrm{~Hz}, 1 \mathrm{H}), 6.24(\mathrm{~s}, 1 \mathrm{H}), 3.81(\mathrm{~s}, 3 \mathrm{H}), 2.87(\mathrm{~s}, 3 \mathrm{H})$, $1.50(\mathrm{~s}, 3 \mathrm{H}) .{ }^{13} \mathrm{C}$ NMR $(100 \mathrm{MHz}$, Chloroform- $d) \delta 158.3,150.2(\mathrm{~d}, J=6.5 \mathrm{~Hz}), 143.3(\mathrm{~d}, J$ $=32.7 \mathrm{~Hz}), 141.2(\mathrm{~d}, J=16.3 \mathrm{~Hz}), 137.6,137.0(\mathrm{~d}, J=12.4 \mathrm{~Hz}), 136.2(\mathrm{~d}, J=10.6 \mathrm{~Hz}), 134.1(\mathrm{~d}, J=16.6 \mathrm{~Hz})$, 134.0, 133.9, 133.8, 133.7, 131.0 (d, $J=10.9 \mathrm{~Hz}), 130.7,128.8,128.5,128.5,128.3$ (d, $J=7.2 \mathrm{~Hz}), 127.4$, $127.3\left(\mathrm{q}, \mathrm{C}-\mathrm{F},{ }^{2} \mathrm{~J}_{\mathrm{C}-\mathrm{F}}=5.0 \mathrm{~Hz}\right.$ ), 124.1 (q, C-F, $\left.{ }^{1} \mathrm{~J}_{\mathrm{C}-\mathrm{F}}=275.2 \mathrm{~Hz}\right), 124.0,118.7,117.1,110.7,100.6,55.8,17.8,12.5$. ${ }^{19} \mathrm{~F}$ NMR (377 MHz, Chloroform- $d$ ) $\delta-58.3 .{ }^{31} \mathrm{P}$ NMR $\left(162 \mathrm{MHz}\right.$, Chloroform-d) $\delta-14.1$. HRMS (ESI) m/z: [M+H] ${ }^{+}$ 


\section{(S)-4-(2-(bis(3,5-dimethylphenyl)phosphanyl)-6-(trifluoromethyl)phenyl)-8-methoxy-1,3-} dimethylpyrrolo[1,2-a]quinoxaline (LF-2):

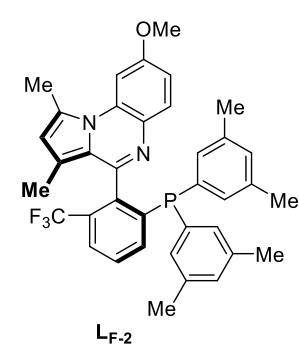

$174.2 \mathrm{mg}, 95 \%$ yield, yellow solid, >99\% ee. (The product was quickly oxidized in isopropanol and ee value was determined by oxidation product.) Flash silica gel chromatography (petroleum ether/ethyl acetate $=10 / 1)$. ${ }^{1} \mathbf{H}$ NMR $(400 \mathrm{MHz}$, Chloroform- $d$ ) $\delta \delta 7.79(\mathrm{~d}, J=2.6 \mathrm{~Hz}, 1 \mathrm{H}), 7.75(\mathrm{~d}, J=7.6 \mathrm{~Hz}, 1 \mathrm{H}), 7.65-7.58(\mathrm{~m}, 2 \mathrm{H})$, $7.54-7.46(\mathrm{~m}, 1 \mathrm{H}), 6.94$ (ddd, $J=8.9,2.6,1.1 \mathrm{~Hz}, 1 \mathrm{H}), 6.87(\mathrm{~d}, J=9.6 \mathrm{~Hz}, 2 \mathrm{H}), 6.80$ - $6.74(\mathrm{~m}, 4 \mathrm{H}), 6.28(\mathrm{~s}, 1 \mathrm{H}), 3.92(\mathrm{~s}, 3 \mathrm{H}), 2.96(\mathrm{~s}, 3 \mathrm{H}), 2.18(\mathrm{~s}, 12 \mathrm{H}) .{ }^{13} \mathrm{C}$ NMR $(100$ $\mathrm{MHz}$, Chloroform- $d$ ) $\delta 158.3,150.5(\mathrm{~d}, J=6.3 \mathrm{~Hz}), 143.0(\mathrm{~d}, J=33.2 \mathrm{~Hz}), 142.1$ (d, $J=$ 17.6 Hz), 137.7, 137.6, 137.5 (d, $J=7.4 \mathrm{~Hz}), 136.6$ (d, $J=11.8 \mathrm{~Hz}), 136.0$ (d, $J=10.2 \mathrm{~Hz}), 131.9,131.8,131.6$, 131.6, 131.2, 131.0, 130.8, 130.5 (d, J = 9.0 Hz), 128.7, 127.2, 127.1 (q, C-F, $\left.{ }^{2} J_{C-F}=5.0,4.3 \mathrm{~Hz}\right), 124.2,124.1$ $\left(\mathrm{d}, \mathrm{C}-\mathrm{F},{ }^{1} \mathrm{~J}_{\mathrm{C}-\mathrm{F}}=271.7 \mathrm{~Hz}\right), 118.6,117.3,110.7,100.6,55.8,21.4,21.4,17.8,12.3 .{ }^{19} \mathrm{~F} \mathrm{NMR}(377 \mathrm{MHz}$, Chloroform- $d$ ) $\delta-58.3$. ${ }^{31} \mathrm{P}$ NMR (162 MHz, Chloroform- $d$ ) $\delta-13.8$. HRMS (ESI) m/z: $[\mathrm{M}+\mathrm{H}]^{+}$Calcd for $\mathrm{C}_{37} \mathrm{H}_{35} \mathrm{~F}_{3} \mathrm{~N}_{2} \mathrm{OP} 611.2439$; found 611.2434. Optical rotation: $[\alpha]_{\mathrm{D}}{ }^{25}=-98.2\left(\mathrm{c}=0.5, \mathrm{CHCl}_{3}\right)$.

[1] M. Nagamochi, Y. Fang, Mark Lautens. Org. Lett. 2007, 9, 2955.

[2] K. Urakawa, M. Sumimoto, M. Arisawa, M. Matsuda, H. Ishikawa. Angew. Chem. Int. Ed. 2016, 55, 7432.

[3] X-L. He, H-R. Zhao, X. Song, B. Jiang, W. Du, Y-C. Chen. ACS Catal. 2019, 9, 4374

[4] Y. Unoh, K. Hirano, and M. Miura. J. Am. Chem. Soc. 2017, 139, 6106.

[5] T. F. Knöpfel, P. Aschwanden, T. Ichikawa, T. Watanabe, and E. M. Carreira. Angew. Chem. Int. Ed. 2004, 43, 5971.

[6] T. Oguma and T. Katsuki. J. Am. Chem. Soc. 2012, 134, 20017.

[7] J. Yin, S. L. Buchwald. J. Am. Chem. Soc. 2000, 122, 12051.

\section{9. application of quinoxalinap ligands}
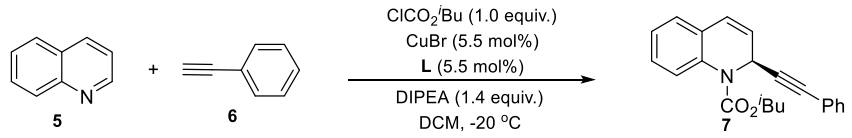

The compound is prepared as reported in literature. ${ }^{[1]} \mathrm{CuBr}(5 \mathrm{~mol} \%)$ was added to a flask in the glove box and the flask was fitted with a septum before being taken from the glove box and placed directly under dry nitrogen. $\mathrm{P}, \mathrm{N}$-ligand $(5.5 \mathrm{~mol} \%)$ in dichloromethane $(1.0 \mathrm{~mL})$ was added to the flask and the mixture was stirred for 30 minutes at room temperature. Then mixture was cooled to $0^{\circ} \mathrm{C}$ and alkyne $6(0.125 \mathrm{mmol}, 1.0$ equiv. $)$ was added. During this stirring time, in a separate flask $\mathrm{ClCO}_{2}{ }^{i} \mathrm{Bu}(0.125 \mathrm{mmol}, 1.0$ equiv) was added to quinoline $(0.125 \mathrm{mmol}, 1.0$ equiv. $)$ in $\mathrm{DCM}(1.0 \mathrm{~mL})$ and stirred for 5 minutes at $\mathrm{rt}$. The quinolinium salt thus formed was transferred to the flask containing the copper catalyst and alkyne, followed by addition of DIPEA ( $0.175 \mathrm{mmol}, 1.4$ equiv) via syringe. The reaction mixture was allowed to warm to room temperature with stirring for 24 hours and monitored by TLC. The reaction mixture was directly subjected to flash column chromatography on silica gel to afford the desired product 7 . 
(S)-isobutyl-2-(phenylethynyl)quinoline-1(2H)-carboxylate (7):

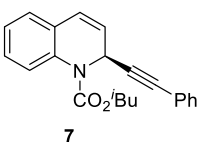

$14.1 \mathrm{mg}, 34 \%$ yield, white solid, $-94 \%$ ee. Flash silica gel chromatography (petroleum ether/ethyl acetate $=10 / 1) .{ }^{1} \mathrm{H}$ NMR $(400 \mathrm{MHz}$, Chloroform- $d) \delta 7.61(\mathrm{~d}, J=9.2 \mathrm{~Hz}, 1 \mathrm{H}), 7.21$ - $7.17(\mathrm{~m}, 2 \mathrm{H}), 7.14-7.09(\mathrm{~m}, 3 \mathrm{H}), 7.05-6.97(\mathrm{~m}, 2 \mathrm{H}), 6.46(\mathrm{~d}, J=8.7 \mathrm{~Hz}, 1 \mathrm{H}), 6.05-5.97$ (m, 2H), $4.03-3.89(\mathrm{~m}, 2 \mathrm{H}), 1.93$ (hept, $J=6.7 \mathrm{~Hz}, 1 \mathrm{H}), 0.89$ (d, $J=6.8 \mathrm{~Hz}, 6 \mathrm{H}) .{ }^{13} \mathrm{C} \mathrm{NMR}(100 \mathrm{MHz}$, Chloroform-d) $\delta 154.0,134.5,131.9,128.4,128.2,127.9,126.7,126.6,126.0,124.5,122.6,85.8,83.5,72.8$, 44.8, 28.0, 19.3. HPLC: Chiralpak OD-H, $n$-hexane/isopropanol $=90 / 10$, Flow rate $=1.0 \mathrm{~mL} / \mathrm{min}, \mathrm{UV}=254 \mathrm{~nm}$, $t_{\mathrm{R}}($ minor $)=5.41 \mathrm{~min}, t_{\mathrm{R}}($ major $)=6.39 \mathrm{~min}$.
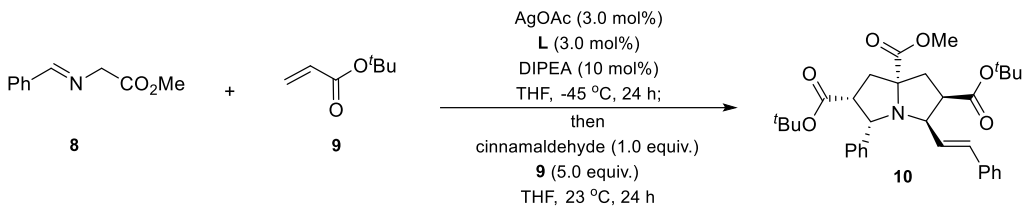

The compound is prepared as reported in literature. ${ }^{[2]}$ Silver $(\mathrm{I})$ acetate (1.0 equiv) and $\mathrm{P}, \mathrm{N}$-ligand (1.0 equiv) were added to a vial. Tetrahydrofuran $(2.0 \mathrm{~mL})$ was then introduced, and this solution was stirred at room temperature for $1 \mathrm{~h}$. The dipolarophile 9 (t-Bu Acrylate, $0.15 \mathrm{mmol}, 1.5$ equiv), DIPEA (0.1 equiv) and the $\alpha$ iminoester $8\left(0.1 \mathrm{mmol}, 1.0\right.$ equiv) were added at $-45^{\circ} \mathrm{C}$ in the glovebox. After stirring for 24 hours, the reaction was allowed to warm to room temperature and taken out of the glovebox. Dipolarophile 9 ( $0.5 \mathrm{mmol}, 5.0$ equiv) and cinnamaldehyde (1.0 equiv) were added. After stirring for 24 hours, the reaction was quenched with a tetrahydrofuran solution of acetic acid $(0.11 \mathrm{mmol}, 1.1$ equiv) and concentrated directly. The residue was purified by silica gel column chromatography to afford the desired product 10.

2,6-di-tert-butyl 7a-methyl (2R,3S,5R,6R,7aR)-3-phenyl-5-((E)-styryl)tetrahydro-1H-pyrrolizine2,6,7a(5H)-tricarboxylate (10):

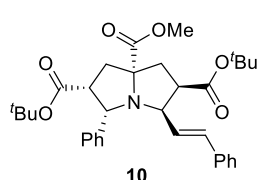

$51.8 \mathrm{mg}, 95 \%$ yield, yellow solid, 90\% ee. Flash silica gel chromatography (petroleum ether/ethyl acetate = 40/1). ${ }^{1} \mathrm{H}$ NMR $(400 \mathrm{MHz}$, Chloroform- $d) \delta 7.44-7.34(\mathrm{~m}, 2 \mathrm{H}), 7.28$ - $7.18(\mathrm{~m}, 7 \mathrm{H}), 7.18-7.12(\mathrm{~m}, 1 \mathrm{H}), 6.30(\mathrm{~d}, J=15.6 \mathrm{~Hz}, 1 \mathrm{H}), 6.03$ (dd, $J=15.6,10.3$ $\mathrm{Hz}, 1 \mathrm{H}), 4.79$ (d, $J=8.2 \mathrm{~Hz}, 1 \mathrm{H}), 4.20$ (dd, $J=10.3,7.6 \mathrm{~Hz}, 1 \mathrm{H}), 3.79$ (s, 3H), 3.61 (ddd, $J=12.0,7.6,6.6 \mathrm{~Hz}, 1 \mathrm{H}), 3.39(\mathrm{td}, J=7.9,3.7 \mathrm{~Hz}, 1 \mathrm{H}), 3.00(\mathrm{dd}, J=13.2,3.7 \mathrm{~Hz}, 1 \mathrm{H}), 2.44-2.23(\mathrm{~m}, 2 \mathrm{H})$, $2.13(\mathrm{dd}, J=13.3,7.8 \mathrm{~Hz}, 1 \mathrm{H}), 1.27$ (s, 9H), 0.96 (s, 9H). ${ }^{13} \mathrm{C}$ NMR (100 MHz, Chloroform-d) $\delta 177.1,171.1$, 171.0, 141.1, 136.5, 135.3, 128.5, 128.0, 127.9, 127.8, 126.8, 126.6, 126.4, 80.7, 80.2, 76.7, 65.3, 65.1, 53.0, 52.3, 51.4, 39.2, 39.1, 28.1, 27.5. HPLC: Chiralpak OD-H, $n$-hexane/isopropanol = 95/5, Flow rate = $1.0 \mathrm{~mL} / \mathrm{min}$, $\mathrm{UV}=254 \mathrm{~nm}, t_{\mathrm{R}}$ (minor) $=5.15 \mathrm{~min}, t_{\mathrm{R}}($ major $)=5.79 \mathrm{~min}$.

[1] M. Pappoppula, F. S. P. Cardoso , B. O. Garrett, and A. Aponick. Angew. Chem. Int. Ed. $2015,54,15202$.

[2] Andrew D. Lim, Julian A. Codelli and Sarah E. Reisman. Chem. Sci., 2013, 4, 650. 


\section{Crystallographic data for compound (R)-5a}

Single crystals of (R)-5a were obtained by recrystallization from acetonitrile at $25^{\circ} \mathrm{C}$. (very slow evaporation in air). The ellipsoids drawn at $50 \%$ probability level.

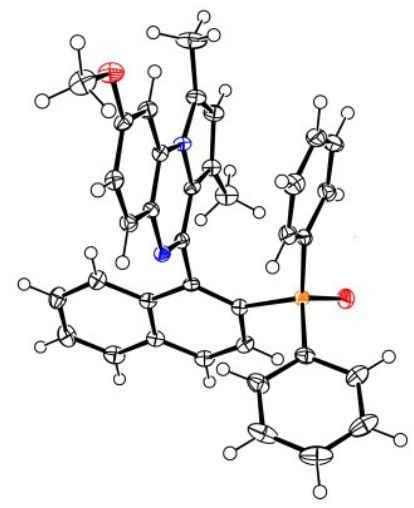

(R)-5a (CCDC: 2017670)

Table S8. Crystal Data and Structure Refinement for (R)-5a

Bond precision: $\mathrm{C}-\mathrm{C}=0.0044 \mathrm{~A} \quad$ Wavelength $=1.54178$

Cell: $\quad a=9.4101(2) \quad b=14.3778(4) \quad c=24.3877(6)$

alpha $=90 \quad$ beta $=90 \quad$ gamma $=90$

Temperature: $120 \mathrm{~K}$

Calculated Reported

$\begin{array}{lll}\text { Volume } & 3299.57(14) & 3299.57(14) \\ \text { Space group } & \text { P 212121 } & \text { P 21 21 21 } \\ \text { Hall group } & \text { P 2ac 2ab } & \text { P 2ac 2ab }\end{array}$

Moiety formula $\mathrm{C} 36 \mathrm{H} 29 \mathrm{~N} 2 \mathrm{O} 2 \mathrm{P}, 2(\mathrm{C} 2 \mathrm{H} 3 \mathrm{~N}) \quad \mathrm{C} 40 \mathrm{H} 35 \mathrm{~N} 4 \mathrm{O} 2 \mathrm{P}$

Sum formula $\mathrm{C} 40 \mathrm{H} 35 \mathrm{~N} 4 \mathrm{O} 2 \mathrm{P} \quad \mathrm{C} 40 \mathrm{H} 35 \mathrm{~N} 4 \mathrm{O} 2 \mathrm{P}$

$\begin{array}{lll}\mathrm{Mr} & 634.69 & 634.69\end{array}$

Dx,g cm-3 $\quad 1.278 \quad 1.278$

$\begin{array}{lll}Z & 4 & 4\end{array}$

$\begin{array}{lll}\mathrm{Mu}(\mathrm{mm}-1) & 1.067 & 1.067\end{array}$

$\begin{array}{lll}\text { F000 } & 1336.0 & 1336.0\end{array}$

F000' $\quad 1340.75$

h,k,Imax $\quad 11,17,30 \quad 11,17,30$

Nref 6744[3795] 6692

$\begin{array}{lll}\text { Tmin,Tmax } & 0.858,0.899 & 0.858,0.899\end{array}$

Tmin' $\quad 0.527$

Correction method= \# Reported T Limits: Tmin=0.858 Tmax=0.899

AbsCorr $=$ MULTI-SCAN

Data completeness $=1.76 / 0.99$

Theta $(\max )=74.357$

$R($ reflections $)=0.0507(6216)$

$w R 2$ (reflections) $=0.1438(6692)$

$S=1.100$

Npar $=429$ 


\section{DFT calculations}

\section{Computational Methods:}

The DFT calculations were performed employing Gaussian 16 program ${ }^{[1]}$. The spin-unrestricted B3LYP functional ${ }^{[2-4]}$ combined with def2SVP basis set was selected to optimize the complexes. The dispersion effec $t$ was evaluated using the D3 version of Grimme's dispersion correction with Becke-Johnson damping ${ }^{[5]}$. All geometries were fully optimized without any symmetry constraints. Vibrational frequency analysis was perform ed at the same level of theory to confirm the nature (minima or first-order saddle points) of the stationary point s. The intrinsic reaction coordinate (IRC) ${ }^{[6]}$ was conducted in both directions (forward and reverse) from the tr ansition states to the corresponding local minima, to identify the minimum-energy paths. The energies discuss ed in the text are based on the free energies in $\mathrm{kcal} \mathrm{mol}^{-1}$.

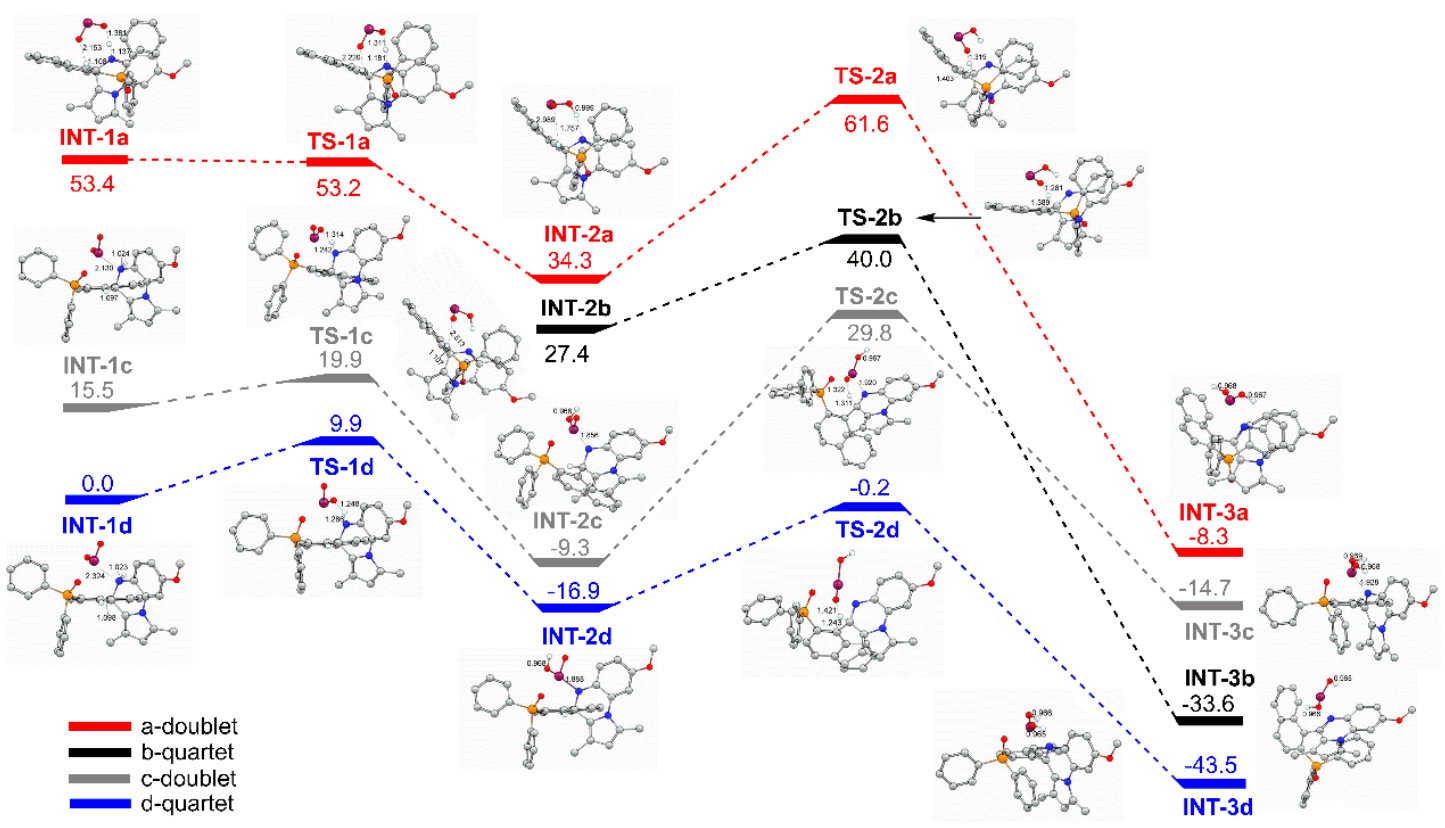

Table S9. Energies of the key intermediates and transition states for the central-to-axial chirality transfer of (S)-

$3 m .^{a}$

\begin{tabular}{|l|l|l|l|l|l|l|l|}
\hline & $\boldsymbol{E}$ & $\Delta \boldsymbol{E}$ & $\boldsymbol{E}+\mathrm{ZPE}$ & $\Delta \boldsymbol{E}_{\text {zpe }}$ & $\boldsymbol{G}$ & $\Delta \boldsymbol{G}$ & Imag.Freq. \\
\hline INT-1c & -3291.976979 & 14.5 & -3291.385155 & 14.8 & -3291.457367 & 15.5 & \\
\hline INT-1d & -3292.000045 & 0.0 & -3291.408724 & 0.0 & -3291.481996 & 0.0 & \\
\hline TS-1c & -3291.964167 & 22.5 & -3291.377559 & 19.6 & -3291.450234 & 19.9 & -1305.4 \\
\hline TS-1d & -3291.977019 & 14.4 & -3291.392017 & 10.5 & -3291.466264 & 9.9 & -1494.7 \\
\hline INT-2c & -3292.013014 & -8.1 & -3291.423862 & -9.5 & -3291.496786 & -9.3 & \\
\hline INT-2d & -3292.023201 & -14.5 & -3291.434859 & -16.4 & -3291.509005 & -16.9 & \\
\hline TS-2c & -3291.944611 & 34.8 & -3291.362035 & 29.3 & -3291.434578 & 29.8 & -1536.3 \\
\hline TS-2d & -3291.991061 & 5.6 & -3291.40878 & 0.0 & -3291.482273 & -0.2 & -953.7 \\
\hline INT-3c & -3292.01931 & -12.1 & -3291.432924 & -15.2 & -3291.505373 & -14.7 & \\
\hline INT-3d & -3292.064918 & -40.7 & -3291.479237 & -44.2 & -3291.551309 & -43.5 & \\
\hline INT-1a & -3291.908269 & 57.6 & -3291.321881 & 54.5 & -3291.3969 & 53.4 & \\
\hline TS-1a & -3291.908254 & 57.6 & -3291.323324 & 53.6 & -3291.397227 & 53.2 & -125.6 \\
\hline INT-2a & -3291.941549 & 36.7 & -3291.354131 & 34.3 & -3291.427374 & 34.3 & \\
\hline INT-2b & -3291.949468 & 31.7 & -3291.362435 & 29.0 & -3291.438361 & 27.4 & \\
\hline
\end{tabular}




\begin{tabular}{|l|l|l|l|l|l|l|l|}
\hline TS-2a & -3291.891906 & 67.9 & -3291.311131 & 61.2 & -3291.383844 & 61.6 & -1871.5 \\
\hline TS-2b & -3291.924586 & 47.4 & -3291.343776 & 40.8 & -3291.418282 & 40.0 & -2649.7 \\
\hline INT-3a & -3292.011718 & -7.3 & -3291.424777 & -10.1 & -3291.495254 & -8.3 & \\
\hline INT-3b & -3292.050422 & -31.6 & -3291.464239 & -34.8 & -3291.535514 & -33.6 & \\
\hline
\end{tabular}

${ }^{a}$ Absolute energies are in a.u. and the relative ones are in $\mathrm{kcal} \mathrm{mol}^{-1}$.

Table S10. Energies of stationary points and Cartesian coordinates of the optimized structures:

\begin{tabular}{|c|c|c|c|c|c|c|c|}
\hline \multicolumn{4}{|c|}{ INT-1a } & \multicolumn{4}{|c|}{ TS-1a } \\
\hline $\mathrm{P}$ & 0.31849800 & 1.82907300 & -0.00187900 & $\mathrm{P}$ & 0.28727000 & 1.83388100 & -0.00298800 \\
\hline 0 & -0.68494400 & 1.62041000 & 1.10548600 & 0 & -0.71874900 & 1.61611800 & 1.09995000 \\
\hline 0 & -5.94534100 & -0.54018200 & 0.75949700 & 0 & -5.94667100 & -0.62167600 & 0.73676700 \\
\hline $\mathrm{N}$ & -0.60954300 & -1.02390900 & -0.35263900 & $\mathrm{~N}$ & -0.59854600 & -1.03293300 & -0.35555000 \\
\hline $\mathrm{N}$ & -1.42727900 & -0.81306100 & 2.31031300 & $\mathrm{~N}$ & -1.43277400 & -0.82952100 & 2.30898400 \\
\hline C & 1.79348200 & 0.72308500 & 0.01332700 & C & 1.77650900 & 0.74707900 & 0.02030700 \\
\hline C & 3.05413300 & 1.32761900 & -0.24969100 & C & 3.02975900 & 1.36676900 & -0.24311700 \\
\hline $\mathrm{H}$ & 3.10814800 & 2.39972000 & -0.43239200 & $\mathrm{H}$ & 3.07024000 & 2.43879400 & -0.42974800 \\
\hline C & 4.21115000 & 0.58705700 & -0.25701900 & C & 4.19615800 & 0.64110600 & -0.24699500 \\
\hline $\mathrm{H}$ & 5.17321800 & 1.07107300 & -0.44255700 & $\mathrm{H}$ & 5.15208600 & 1.13654000 & -0.43412300 \\
\hline C & 4.17689500 & -0.80914200 & -0.02708900 & C & 4.17949200 & -0.75442800 & -0.01088800 \\
\hline C & 5.37283700 & -1.57861000 & -0.03304700 & C & 5.38506000 & -1.50876400 & -0.01336400 \\
\hline $\mathrm{H}$ & 6.32054600 & -1.06512000 & -0.21353200 & $\mathrm{H}$ & 6.32614400 & -0.98414400 & -0.19654000 \\
\hline C & 5.33814800 & -2.93739700 & 0.17999000 & C & 5.36789000 & -2.86683400 & 0.20644000 \\
\hline $\mathrm{H}$ & 6.26156000 & -3.52144400 & 0.17353400 & $\mathrm{H}$ & 6.29866400 & -3.43909200 & 0.20244900 \\
\hline C & 4.09491200 & -3.58111700 & 0.38990700 & C & 4.13316200 & -3.52505200 & 0.42128400 \\
\hline $\mathrm{H}$ & 4.06049700 & -4.66454000 & 0.52468600 & $\mathrm{H}$ & 4.11233500 & -4.60791200 & 0.56308100 \\
\hline C & 2.91758700 & -2.86450900 & 0.40566400 & C & 2.94711700 & -2.82292200 & 0.43400000 \\
\hline $\mathrm{H}$ & 1.98439400 & -3.41589400 & 0.50647700 & $\mathrm{H}$ & 2.02205100 & -3.38602200 & 0.54217200 \\
\hline C & 2.91274200 & -1.44934700 & 0.21594200 & C & 2.92347700 & -1.40928500 & 0.23505400 \\
\hline C & 1.72058600 & -0.65043700 & 0.26436500 & C & 1.72063800 & -0.62604800 & 0.27685700 \\
\hline C & 0.39391600 & -1.31497700 & 0.64078200 & C & 0.40063200 & -1.30663300 & 0.64787900 \\
\hline
\end{tabular}




\begin{tabular}{|c|c|c|c|c|c|c|c|}
\hline C & -0.05841600 & -0.99920100 & 2.03227600 & C & -0.06084300 & -0.99388500 & 2.03789500 \\
\hline C & 0.65344000 & -0.86732400 & 3.20009000 & C & 0.64376900 & -0.84660400 & 3.20836100 \\
\hline C & -0.30657900 & -0.56291400 & 4.21931100 & C & -0.32563300 & -0.55505300 & 4.22275100 \\
\hline $\mathrm{H}$ & -0.08066100 & -0.37744900 & 5.26837900 & $\mathrm{H}$ & -0.10740100 & -0.36282900 & 5.27224100 \\
\hline C & -1.56719300 & -0.51858900 & 3.67104900 & C & -1.58399700 & -0.53332800 & 3.66874500 \\
\hline C & 2.13512300 & -0.97437100 & 3.41304300 & C & 2.12611500 & -0.92806200 & 3.42836000 \\
\hline $\mathrm{H}$ & 2.68231700 & -0.16786100 & 2.89895400 & $\mathrm{H}$ & 2.66250100 & -0.11590500 & 2.91174800 \\
\hline $\mathrm{H}$ & 2.54545700 & -1.92481700 & 3.04062900 & $\mathrm{H}$ & 2.55397600 & -1.87394300 & 3.06395500 \\
\hline $\mathrm{H}$ & 2.36910400 & -0.90579400 & 4.48562600 & $\mathrm{H}$ & 2.35417200 & -0.84903600 & 4.50152400 \\
\hline C & -2.83519200 & -0.17276100 & 4.37897300 & C & -2.86098300 & -0.20695800 & 4.36974300 \\
\hline $\mathrm{H}$ & -2.58783100 & 0.07091000 & 5.42146100 & $\mathrm{H}$ & -2.62272800 & 0.04294000 & 5.41290700 \\
\hline $\mathrm{H}$ & -3.56315600 & -1.00066900 & 4.40169300 & $\mathrm{H}$ & -3.57542900 & -1.04664000 & 4.39074800 \\
\hline $\mathrm{H}$ & -3.33292800 & 0.70785100 & 3.94216500 & $\mathrm{H}$ & -3.37125000 & 0.66434100 & 3.92868300 \\
\hline C & -2.37688800 & -0.79537900 & 1.29869600 & C & -2.37712300 & -0.82741800 & 1.29195900 \\
\hline C & -3.74837100 & -0.68878400 & 1.50222300 & C & -3.75072000 & -0.74124700 & 1.48900800 \\
\hline $\mathrm{H}$ & -4.18205800 & -0.63155800 & 2.49199600 & $\mathrm{H}$ & -4.18999900 & -0.69224900 & 2.47680200 \\
\hline C & -4.64618800 & -0.65208900 & 0.42161600 & C & -4.64414200 & -0.71387700 & 0.40420700 \\
\hline C & -4.18264800 & -0.73613900 & -0.91223200 & C & -4.17306700 & -0.78590100 & -0.92726100 \\
\hline $\mathrm{H}$ & -4.87107600 & -0.71213900 & -1.75510100 & $\mathrm{H}$ & -4.85751600 & -0.76724300 & -1.77354800 \\
\hline C & -2.82990200 & -0.86206600 & -1.13781500 & C & -2.81732100 & -0.89242000 & -1.14618200 \\
\hline $\mathrm{H}$ & -2.42235600 & -0.95549200 & -2.14513600 & $\mathrm{H}$ & -2.40506800 & -0.97438200 & -2.15245400 \\
\hline C & -1.90128500 & -0.89999400 & -0.06072800 & C & -1.89103400 & -0.92378400 & -0.06568200 \\
\hline C & -6.93159900 & -0.50376700 & -0.25250700 & C & -6.92800600 & -0.59396700 & -0.27988700 \\
\hline $\mathrm{H}$ & -6.79422300 & 0.36336300 & -0.92122300 & $\mathrm{H}$ & -6.79924500 & 0.27802700 & -0.94407500 \\
\hline $\mathrm{H}$ & -7.89749900 & -0.41225400 & 0.26015100 & $\mathrm{H}$ & -7.89779900 & -0.51806400 & 0.22803200 \\
\hline $\mathrm{H}$ & -6.92844000 & -1.42820200 & -0.85510800 & $\mathrm{H}$ & -6.90930600 & -1.51535200 & -0.88698000 \\
\hline C & -0.41095300 & 1.68857500 & -1.66509500 & C & -0.43208100 & 1.68305200 & -1.66976600 \\
\hline C & -1.70524100 & 2.21060500 & -1.82473800 & C & -1.73374800 & 2.18395400 & -1.83514000 \\
\hline $\mathrm{H}$ & -2.20787900 & 2.66523900 & -0.96811900 & $\mathrm{H}$ & -2.24839800 & 2.62755900 & -0.97988800 \\
\hline
\end{tabular}




\begin{tabular}{|c|c|c|c|c|c|c|c|}
\hline C & -2.34515600 & 2.11751300 & -3.05981500 & C & -2.36547000 & 2.08363100 & -3.07400100 \\
\hline $\mathrm{H}$ & -3.35289200 & 2.52203400 & -3.18272300 & $\mathrm{H}$ & -3.37917700 & 2.47154700 & -3.20120900 \\
\hline C & -1.70506600 & 1.48699000 & -4.13407800 & C & -1.70919200 & 1.46710100 & -4.14642300 \\
\hline $\mathrm{H}$ & -2.21383100 & 1.40002700 & -5.09738900 & $\mathrm{H}$ & -2.21094400 & 1.37487500 & -5.11292900 \\
\hline $\mathrm{C}$ & -0.42515400 & 0.95329700 & -3.97133600 & C & -0.42122400 & 0.95446800 & -3.97799300 \\
\hline $\mathrm{H}$ & 0.06194200 & 0.42484100 & -4.79259900 & $\mathrm{H}$ & 0.07956400 & 0.43821200 & -4.79890900 \\
\hline C & 0.22659300 & 1.05827300 & -2.73964100 & $\mathrm{C}$ & 0.22179300 & 1.06637000 & -2.74241400 \\
\hline $\mathrm{H}$ & 1.20754700 & 0.60059500 & -2.60791300 & $\mathrm{H}$ & 1.20959200 & 0.62508000 & -2.60601000 \\
\hline $\mathrm{C}$ & 0.97703500 & 3.53186800 & 0.07194700 & $\mathrm{C}$ & 0.92502700 & 3.54496700 & 0.07101400 \\
\hline C & 1.02643400 & 4.13713900 & 1.33605300 & C & 0.96309100 & 4.15163400 & 1.33486600 \\
\hline $\mathrm{H}$ & 0.67147800 & 3.57640800 & 2.20363100 & $\mathrm{H}$ & 0.61235000 & 3.58711400 & 2.20170400 \\
\hline C & 1.50401700 & 5.44277300 & 1.46605200 & $\mathrm{C}$ & 1.42400700 & 5.46317400 & 1.46555200 \\
\hline $\mathrm{H}$ & 1.54289200 & 5.91268800 & 2.45183500 & $\mathrm{H}$ & 1.45403200 & 5.93404800 & 2.45119200 \\
\hline C & 1.92361100 & 6.15127600 & 0.33558800 & $\mathrm{C}$ & 1.83811600 & 6.17628500 & 0.33595700 \\
\hline $\mathrm{H}$ & 2.29407600 & 7.17421200 & 0.43834200 & $\mathrm{H}$ & 2.19547400 & 7.20382700 & 0.43921200 \\
\hline $\mathrm{C}$ & 1.85921900 & 5.55598000 & -0.92821900 & $\mathrm{C}$ & 1.78486800 & 5.57951400 & -0.92765600 \\
\hline $\mathrm{H}$ & 2.17456000 & 6.11366800 & -1.81334000 & $\mathrm{H}$ & 2.09578500 & 6.14062200 & -1.81219100 \\
\hline C & 1.38371600 & 4.24925500 & -1.06198900 & $\mathrm{C}$ & 1.32610100 & 4.26685900 & -1.06204800 \\
\hline $\mathrm{H}$ & 1.32321100 & 3.78735100 & -2.05011600 & $\mathrm{H}$ & 1.27425900 & 3.80381200 & -2.05012900 \\
\hline $\mathrm{H}$ & 0.51609700 & -2.41235200 & 0.54488400 & $\mathrm{H}$ & 0.54211100 & -2.40073400 & 0.55907000 \\
\hline $\mathrm{H}$ & -0.32421600 & -1.32072000 & -1.41259000 & $\mathrm{H}$ & -0.28467400 & -1.35245400 & -1.44797100 \\
\hline $\mathrm{Mn}$ & 0.31737800 & -3.55863900 & -2.21429900 & $\mathrm{Mn}$ & 0.42180000 & -3.56397500 & -2.21896000 \\
\hline O & 0.34416900 & -4.14078300 & -0.72700000 & $\mathrm{O}$ & 0.43742500 & -4.18841300 & -0.75222100 \\
\hline $\mathrm{O}$ & 0.01228600 & -1.97686700 & -2.57971000 & $\mathrm{O}$ & 0.07544600 & -1.97277500 & -2.54481500 \\
\hline \multicolumn{4}{|c|}{ INT-2a } & \multicolumn{4}{|c|}{ TS-2a } \\
\hline $\mathrm{P}$ & -0.35952700 & 1.82397500 & 0.05018700 & $\mathrm{P}$ & -0.39157600 & 1.82449200 & 0.05787500 \\
\hline $\mathrm{O}$ & -1.36541200 & 1.31036800 & 1.04689800 & O & -1.39181000 & 1.29469500 & 1.05134800 \\
\hline $\mathrm{O}$ & -5.70535100 & -2.29468100 & 0.10462900 & $\mathrm{O}$ & -5.64465300 & -2.37203200 & 0.01050200 \\
\hline $\mathrm{N}$ & -0.35426600 & -1.17850900 & -0.44216000 & $\mathrm{~N}$ & -0.29906200 & -1.18876100 & -0.41210100 \\
\hline
\end{tabular}




\begin{tabular}{|c|c|c|c|c|c|c|c|}
\hline $\mathrm{N}$ & -1.49711100 & -1.30873500 & 2.15644400 & $\mathrm{~N}$ & -1.49912300 & -1.31785500 & 2.15590100 \\
\hline C & 1.36174300 & 1.17251600 & 0.19214000 & C & 1.34210500 & 1.20844800 & 0.20263100 \\
\hline C & 2.41791900 & 2.09430000 & -0.05445000 & C & 2.37576900 & 2.14219400 & -0.09051800 \\
\hline $\mathrm{H}$ & 2.18181400 & 3.13972600 & -0.24778300 & $\mathrm{H}$ & 2.11682100 & 3.17990600 & -0.29605400 \\
\hline C & 3.73043900 & 1.69096300 & -0.06851200 & C & 3.69307900 & 1.75879200 & -0.14059400 \\
\hline $\mathrm{H}$ & 4.52711400 & 2.40815600 & -0.28021600 & $\mathrm{H}$ & 4.47105700 & 2.48338600 & -0.39268600 \\
\hline C & 4.06806300 & 0.33912700 & 0.18361200 & C & 4.05974800 & 0.41713900 & 0.12563800 \\
\hline C & 5.41994400 & -0.10038300 & 0.12841900 & C & 5.41516300 & -0.00440400 & 0.03101100 \\
\hline $\mathrm{H}$ & 6.19459600 & 0.63444200 & -0.10462400 & $\mathrm{H}$ & 6.16980800 & 0.735774400 & -0.24639500 \\
\hline C & 5.74929200 & -1.41899800 & 0.34962900 & C & 5.77206500 & -1.31265400 & 0.27040200 \\
\hline $\mathrm{H}$ & 6.78932900 & -1.74768500 & 0.29182200 & $\mathrm{H}$ & 6.81404300 & -1.62802900 & 0.18190100 \\
\hline C & 4.72973100 & -2.34912800 & 0.66017800 & C & 4.77898900 & -2.24878600 & 0.64323800 \\
\hline $\mathrm{H}$ & 4.97748700 & -3.39685600 & 0.84099700 & $\mathrm{H}$ & 5.04976600 & -3.28695700 & 0.84495000 \\
\hline C & 3.40923800 & -1.94775500 & 0.74098000 & C & 3.45602900 & -1.86446800 & 0.76242700 \\
\hline $\mathrm{H}$ & 2.67125000 & -2.69477200 & 1.02182400 & $\mathrm{H}$ & 2.73999000 & -2.61149500 & 1.09575300 \\
\hline C & 3.02161500 & -0.59647100 & 0.48513500 & C & 3.03884300 & -0.52662700 & 0.48340500 \\
\hline C & 1.65333000 & -0.16314000 & 0.47014800 & C & 1.66246900 & -0.11592400 & 0.50057100 \\
\hline C & 0.53711300 & -1.18902400 & 0.69510500 & C & 0.56838200 & -1.16375800 & 0.74401000 \\
\hline C & -0.12352400 & -1.06187700 & 2.03482800 & C & -0.12430400 & -1.04318000 & 2.07166200 \\
\hline C & 0.38579000 & -0.74861000 & 3.27401700 & C & 0.34296800 & -0.73698600 & 3.32890100 \\
\hline C & -0.72379900 & -0.77943400 & 4.17919100 & C & -0.79133600 & -0.79437500 & 4.20396600 \\
\hline $\mathrm{H}$ & -0.68130800 & -0.56347100 & 5.24584900 & $\mathrm{H}$ & -0.78333600 & -0.58616800 & 5.27297500 \\
\hline C & -1.86901200 & -1.10803700 & 3.49149400 & C & -1.90935200 & -1.13537100 & 3.48266500 \\
\hline C & 1.79756100 & -0.39857000 & 3.64549100 & C & 1.73229000 & -0.39871700 & 3.77890500 \\
\hline $\mathrm{H}$ & 2.11818800 & 0.55404700 & 3.19290600 & $\mathrm{H}$ & 1.73929600 & 0.55073500 & 4.33988300 \\
\hline $\mathrm{H}$ & 2.52213000 & -1.16272800 & 3.32499300 & $\mathrm{H}$ & 2.43611900 & -0.29377300 & 2.94658800 \\
\hline $\mathrm{H}$ & 1.88811500 & -0.29481700 & 4.73694500 & $\mathrm{H}$ & 2.13181700 & -1.17351900 & 4.45573100 \\
\hline C & -3.25181300 & -1.18622400 & 4.04864100 & C & -3.30446100 & -1.23901700 & 4.00446200 \\
\hline $\mathrm{H}$ & -3.20845700 & -0.90653600 & 5.11054500 & $\mathrm{H}$ & -3.29096100 & -0.96833500 & 5.06945700 \\
\hline
\end{tabular}




\begin{tabular}{|c|c|c|c|c|c|c|c|}
\hline $\mathrm{H}$ & -3.68901400 & -2.19726200 & 3.99482600 & $\mathrm{H}$ & -3.72445000 & -2.25589000 & 3.93108700 \\
\hline $\mathrm{H}$ & -3.94517700 & -0.49007700 & 3.54986900 & $\mathrm{H}$ & -3.99545900 & -0.54868600 & 3.49475100 \\
\hline C & -2.28928900 & -1.52982600 & 1.03308100 & $\mathrm{C}$ & -2.26233100 & -1.55472600 & 1.01678100 \\
\hline C & -3.64035600 & -1.84779300 & 1.08283900 & $\mathrm{C}$ & -3.61057600 & -1.88968700 & 1.03501500 \\
\hline $\mathrm{H}$ & -4.16547600 & -1.99193700 & 2.01852400 & $\mathrm{H}$ & -4.15864400 & -2.03225800 & 1.95732200 \\
\hline C & -4.40324000 & -1.99082100 & -0.09034500 & $\mathrm{C}$ & -4.34172700 & -2.05369800 & -0.15526400 \\
\hline C & -3.81245300 & -1.81673100 & -1.35982000 & $\mathrm{C}$ & -3.72207000 & -1.88469600 & -1.41159000 \\
\hline $\mathrm{H}$ & -4.39482500 & -1.90752100 & -2.27535300 & $\mathrm{H}$ & -4.28014800 & -1.99126000 & -2.34042700 \\
\hline C & -2.46402900 & -1.53262400 & -1.42913600 & $\mathrm{C}$ & -2.37642700 & -1.58334600 & -1.44955200 \\
\hline $\mathrm{H}$ & -1.96695600 & -1.39793500 & -2.39026100 & $\mathrm{H}$ & -1.85683600 & -1.45015000 & -2.39891000 \\
\hline C & -1.64875200 & -1.40762700 & -0.26417100 & $\mathrm{C}$ & -1.59326500 & -1.43574800 & -0.26515600 \\
\hline C & -6.55294500 & -2.45923600 & -1.01150600 & $\mathrm{C}$ & -6.46176100 & -2.55861500 & -1.12463000 \\
\hline $\mathrm{H}$ & -6.62240200 & -1.53413700 & -1.61035500 & $\mathrm{H}$ & -6.52772400 & -1.64062000 & -1.73473800 \\
\hline $\mathrm{H}$ & -7.54672500 & -2.70375300 & -0.61460900 & $\mathrm{H}$ & -7.46211800 & -2.81171900 & -0.75023100 \\
\hline $\mathrm{H}$ & -6.21275000 & -3.28252600 & -1.66376400 & $\mathrm{H}$ & -6.09512200 & -3.38404500 & -1.75964900 \\
\hline C & -0.84628300 & 1.56528500 & -1.68678100 & $\mathrm{C}$ & -0.86494900 & 1.54625300 & -1.68045400 \\
\hline C & -2.20598000 & 1.73632100 & -1.98702500 & $\mathrm{C}$ & -2.22460000 & 1.70190400 & -1.98947700 \\
\hline $\mathrm{H}$ & -2.90063100 & 2.00593200 & -1.18835600 & $\mathrm{H}$ & -2.92582800 & 1.97427100 & -1.19748100 \\
\hline C & -2.66366800 & 1.52979700 & -3.28899600 & C & -2.67421500 & 1.47711300 & -3.29120600 \\
\hline $\mathrm{H}$ & -3.72365700 & 1.66053800 & -3.52013200 & $\mathrm{H}$ & -3.73415100 & 1.59620900 & -3.52874400 \\
\hline C & -1.76986000 & 1.13517700 & -4.29046000 & $\mathrm{C}$ & -1.77226500 & 1.07900300 & -4.28388900 \\
\hline $\mathrm{H}$ & -2.13070800 & 0.96140900 & -5.30733500 & $\mathrm{H}$ & -2.12658100 & 0.89107900 & -5.30054700 \\
\hline C & -0.41762400 & 0.94855900 & -3.98766400 & C & -0.42017600 & 0.90664000 & -3.97223600 \\
\hline $\mathrm{H}$ & 0.27901600 & 0.61085400 & -4.75790200 & $\mathrm{H}$ & 0.28324800 & 0.56602800 & -4.73500700 \\
\hline C & 0.04746200 & 1.16779400 & -2.68876600 & C & 0.03673300 & 1.14411400 & -2.67356300 \\
\hline $\mathrm{H}$ & 1.09350200 & 0.97198100 & -2.45090000 & $\mathrm{H}$ & 1.08331600 & 0.95973000 & -2.42931900 \\
\hline C & -0.20159100 & 3.63866200 & 0.21804400 & C & -0.27131200 & 3.64274400 & 0.21816700 \\
\hline C & -0.41335600 & 4.17320900 & 1.49744700 & C & -0.48919900 & 4.17735100 & 1.49655800 \\
\hline $\mathrm{H}$ & -0.66236100 & 3.49598500 & 2.31756800 & $\mathrm{H}$ & -0.71952000 & 3.49806800 & 2.32041100 \\
\hline
\end{tabular}




\begin{tabular}{|c|c|c|c|c|c|c|c|}
\hline C & -0.32733500 & 5.55183100 & 1.70112800 & C & -0.43304900 & 5.55825100 & 1.69481400 \\
\hline $\mathrm{H}$ & -0.49167000 & 5.96595800 & 2.69893200 & $\mathrm{H}$ & -0.60201100 & 5.97234700 & 2.69185900 \\
\hline C & -0.04134800 & 6.40247200 & 0.62835900 & C & -0.17115400 & 6.41108200 & 0.61761500 \\
\hline $\mathrm{H}$ & 0.02179300 & 7.48164400 & 0.78844200 & $\mathrm{H}$ & -0.13144800 & 7.49198300 & 0.77349300 \\
\hline C & 0.15206000 & 5.87418300 & -0.65179500 & C & 0.02802400 & 5.88246900 & -0.66146300 \\
\hline $\mathrm{H}$ & 0.36143200 & 6.53918200 & -1.49317800 & $\mathrm{H}$ & 0.21846000 & 6.54880300 & -1.50628100 \\
\hline C & 0.06928200 & 4.49488600 & -0.85877000 & C & -0.02475800 & 4.50085700 & -0.86299200 \\
\hline $\mathrm{H}$ & 0.20879000 & 4.08304400 & -1.86097500 & $\mathrm{H}$ & 0.11896800 & 4.08851900 & -1.86439700 \\
\hline $\mathrm{H}$ & 1.00277800 & -2.18926100 & 0.64083200 & $\mathrm{H}$ & 1.05878700 & -2.15256600 & 0.71217700 \\
\hline $\mathrm{H}$ & 0.71041400 & -1.40024200 & -1.82206600 & $\mathrm{H}$ & 0.77947100 & -1.40475500 & -1.77904500 \\
\hline $\mathrm{Mn}$ & 2.60421400 & -2.91759400 & -1.82261900 & $\mathrm{Mn}$ & 2.67475000 & -2.90797900 & -1.75011900 \\
\hline $\mathrm{O}$ & 2.35960200 & -4.26356500 & -1.02986000 & $\mathrm{O}$ & 2.41738200 & -4.23791400 & -0.93461400 \\
\hline 0 & 1.55311300 & -1.62175700 & -2.30974500 & 0 & 1.62400000 & -1.62170300 & -2.26620400 \\
\hline \multicolumn{4}{|c|}{ INT-3a } & \multicolumn{4}{|c|}{ INT-2b } \\
\hline$P$ & 1.99142200 & 1.58755200 & 0.33232300 & $P$ & -0.15836500 & 1.83968800 & 0.11544800 \\
\hline $\mathrm{O}$ & 1.79482200 & 2.55087700 & 1.47817000 & $\mathrm{O}$ & -1.16883900 & 1.38006300 & 1.13318900 \\
\hline 0 & -5.71146100 & 1.81432000 & -1.06132300 & 0 & -5.87328500 & -1.84748200 & 0.13956100 \\
\hline $\mathrm{N}$ & -1.09753100 & -1.03216800 & 0.13500800 & $\mathrm{~N}$ & -0.47087500 & -1.08584700 & -0.50356700 \\
\hline $\mathrm{N}$ & -1.91979100 & 1.08911300 & 1.77932600 & $\mathrm{~N}$ & -1.55004900 & -1.29289500 & 2.11559500 \\
\hline C & 2.21706100 & -0.19370500 & 0.73699200 & C & 1.49268600 & 1.01002500 & 0.15477200 \\
\hline C & 3.49898100 & -0.75646500 & 0.55647700 & C & 2.62864700 & 1.82569300 & -0.10841600 \\
\hline $\mathrm{H}$ & 4.35288000 & -0.10106300 & 0.38411100 & $\mathrm{H}$ & 2.49304600 & 2.89316200 & -0.27710600 \\
\hline C & 3.67498600 & -2.12950900 & 0.47787000 & C & 3.89376900 & 1.29462700 & -0.16866300 \\
\hline $\mathrm{H}$ & 4.65901600 & -2.53835300 & 0.23455100 & $\mathrm{H}$ & 4.75169500 & 1.93246800 & -0.39416600 \\
\hline C & 2.57637100 & -3.00283600 & 0.59775700 & C & 4.10212600 & -0.08669000 & 0.06197800 \\
\hline C & 2.64191100 & -4.38816000 & 0.19582700 & C & 5.40356300 & -0.65565200 & -0.01750600 \\
\hline $\mathrm{H}$ & 3.60910200 & -4.79735700 & -0.10527800 & $\mathrm{H}$ & 6.24205400 & 0.00170600 & -0.26073600 \\
\hline C & 1.50468900 & -5.14166100 & 0.11393300 & C & 5.60919500 & -2.00021300 & 0.19923900 \\
\hline $\mathrm{H}$ & 1.55828500 & -6.16867500 & -0.25699700 & $\mathrm{H}$ & 6.61276000 & -2.42554500 & 0.12892300 \\
\hline
\end{tabular}




\begin{tabular}{|c|c|c|c|c|c|c|c|}
\hline C & 0.22820700 & -4.63136800 & 0.54455000 & $\mathrm{C}$ & 4.51052700 & -2.82900100 & 0.52710600 \\
\hline $\mathrm{H}$ & -0.58754800 & -5.33389400 & 0.72250500 & $\mathrm{H}$ & 4.66366800 & -3.89370000 & 0.71601500 \\
\hline C & 0.16912400 & -3.33813400 & 1.14092900 & $\mathrm{C}$ & 3.23292000 & -2.30527700 & 0.61939300 \\
\hline $\mathrm{H}$ & -0.60463200 & -3.08645300 & 1.87035900 & $\mathrm{H}$ & 2.42565600 & -2.97595800 & 0.90326800 \\
\hline C & 1.32707600 & -2.45658200 & 1.00282200 & $\mathrm{C}$ & 2.97421400 & -0.91940100 & 0.37405500 \\
\hline C & 1.13911600 & -1.05658300 & 1.02528500 & C & 1.65432000 & -0.35478000 & 0.39308300 \\
\hline C & -0.26783400 & -0.55330300 & 1.05417800 & $\mathrm{C}$ & 0.44285000 & -1.26878400 & 0.60085200 \\
\hline C & -0.66715500 & 0.44727100 & 1.96928900 & $\mathrm{C}$ & -0.16442000 & -1.15359400 & 1.96682400 \\
\hline C & -0.10406500 & 0.93151400 & 3.16171700 & C & 0.39901900 & -0.94431500 & 3.20502500 \\
\hline C & -1.01932900 & 1.87314300 & 3.66535500 & C & -0.68711600 & -0.93031400 & 4.13862400 \\
\hline $\mathrm{H}$ & -0.90163000 & 2.44418800 & 4.58463600 & $\mathrm{H}$ & -0.60115700 & -0.77041000 & 5.21254700 \\
\hline C & -2.12050500 & 1.97161200 & 2.81506400 & $\mathrm{C}$ & -1.87185600 & -1.12998600 & 3.46855800 \\
\hline C & 1.19300200 & 0.55348100 & 3.80034900 & C & 1.84226500 & -0.72495400 & 3.55593600 \\
\hline $\mathrm{H}$ & 2.00226300 & 1.15173200 & 3.35411600 & $\mathrm{H}$ & 2.49204300 & -1.54665700 & 3.21716000 \\
\hline $\mathrm{H}$ & 1.43442800 & -0.50889300 & 3.66023400 & $\mathrm{H}$ & 1.95816000 & -0.64278600 & 4.64683800 \\
\hline $\mathrm{H}$ & 1.16007200 & 0.76454700 & 4.87951200 & $\mathrm{H}$ & 2.23940800 & 0.20109100 & 3.10922500 \\
\hline C & -3.31610200 & 2.84122400 & 3.01529300 & C & -3.24308900 & -1.12121200 & 4.05870300 \\
\hline $\mathrm{H}$ & -3.22709400 & 3.33811700 & 3.99070700 & $\mathrm{H}$ & -3.15283900 & -0.89078200 & 5.12948800 \\
\hline $\mathrm{H}$ & -4.25714400 & 2.26874200 & 3.01133100 & $\mathrm{H}$ & -3.76165000 & -2.09106200 & 3.97598400 \\
\hline $\mathrm{H}$ & -3.39901200 & 3.63016700 & 2.24992700 & $\mathrm{H}$ & -3.88972100 & -0.35218400 & 3.60638700 \\
\hline C & -2.71435900 & 0.74636000 & 0.68003500 & $\mathrm{C}$ & -2.38629100 & -1.38979700 & 1.00540800 \\
\hline C & -3.86191200 & 1.45157900 & 0.30788400 & C & -3.75507900 & -1.61351200 & 1.07865200 \\
\hline $\mathrm{H}$ & -4.18998000 & 2.33855800 & 0.83546500 & $\mathrm{H}$ & -4.26334200 & -1.78281300 & 2.01960200 \\
\hline C & -4.62638400 & 1.04956600 & -0.79190700 & C & -4.55774100 & -1.62362300 & -0.07671500 \\
\hline C & -4.24627900 & -0.07923900 & -1.53773900 & C & -3.99061700 & -1.40640200 & -1.35002900 \\
\hline $\mathrm{H}$ & -4.82121700 & -0.41144900 & -2.40047800 & $\mathrm{H}$ & -4.60261300 & -1.39516200 & -2.25057500 \\
\hline C & -3.09071800 & -0.76221500 & -1.18539000 & C & -2.62699000 & -1.21560200 & -1.44420600 \\
\hline $\mathrm{H}$ & -2.76844900 & -1.61465200 & -1.78373200 & $\mathrm{H}$ & -2.14633800 & -1.05669100 & -2.40993600 \\
\hline C & -2.29898900 & -0.38121800 & -0.08777700 & $\mathrm{C}$ & -1.77381500 & -1.23016000 & -0.30055300 \\
\hline
\end{tabular}




\begin{tabular}{|c|c|c|c|c|c|c|c|}
\hline C & -6.52682500 & 1.48169500 & -2.16305400 & C & -6.75921300 & -1.87826000 & -0.95792700 \\
\hline $\mathrm{H}$ & -5.96544500 & 1.52048000 & -3.11357500 & $\mathrm{H}$ & -6.77831900 & -0.91291800 & -1.49384100 \\
\hline $\mathrm{H}$ & -7.33142100 & 2.22784800 & -2.19292000 & $\mathrm{H}$ & -7.75731300 & -2.07896300 & -0.54735900 \\
\hline $\mathrm{H}$ & -6.97251300 & 0.47710100 & -2.05390100 & $\mathrm{H}$ & -6.49593600 & -2.67793500 & -1.67229100 \\
\hline C & 0.63019900 & 1.61399900 & -0.88015200 & C & -0.73688000 & 1.73285600 & -1.60807800 \\
\hline C & -0.23880000 & 2.71280600 & -0.83665400 & $\mathrm{C}$ & -2.08354300 & 2.05305500 & -1.83606600 \\
\hline $\mathrm{H}$ & -0.09585900 & 3.46510100 & -0.05746900 & $\mathrm{H}$ & -2.71551700 & 2.34121700 & -0.99307200 \\
\hline C & -1.27503900 & 2.81858100 & -1.76815500 & $\mathrm{C}$ & -2.61086100 & 1.96852200 & -3.12530500 \\
\hline $\mathrm{H}$ & -1.95982200 & 3.66887500 & -1.72754200 & $\mathrm{H}$ & -3.66103000 & 2.21450000 & -3.30057100 \\
\hline C & -1.44135800 & 1.83131400 & -2.74303700 & $\mathrm{C}$ & -1.80143200 & 1.54764500 & -4.18606300 \\
\hline $\mathrm{H}$ & -2.25826700 & 1.90952100 & -3.46411700 & $\mathrm{H}$ & -2.21835400 & 1.46849800 & -5.19322100 \\
\hline C & -0.58513600 & 0.72603000 & -2.77454900 & $\mathrm{C}$ & -0.46377100 & 1.21220600 & -3.95550300 \\
\hline $\mathrm{H}$ & -0.73466400 & -0.07498100 & -3.50153200 & $\mathrm{H}$ & 0.16394200 & 0.85036800 & -4.77248900 \\
\hline C & 0.44759500 & 0.60941700 & -1.84407300 & $\mathrm{C}$ & 0.07228100 & 1.30918000 & -2.66927300 \\
\hline $\mathrm{H}$ & 1.04913600 & -0.30290400 & -1.84349100 & $\mathrm{H}$ & 1.10045300 & 0.99332100 & -2.49010100 \\
\hline C & 3.48242900 & 2.04572000 & -0.62527800 & $\mathrm{C}$ & 0.20355700 & 3.61511800 & 0.37075900 \\
\hline C & 4.29738400 & 3.04377300 & -0.07541400 & C & 0.11672000 & 4.09248500 & 1.68691700 \\
\hline $\mathrm{H}$ & 4.00936200 & 3.48244800 & 0.88233300 & $\mathrm{H}$ & -0.16308100 & 3.39823000 & 2.48253800 \\
\hline C & 5.44365400 & 3.46434400 & -0.75632000 & C & 0.36302900 & 5.43961300 & 1.95881300 \\
\hline $\mathrm{H}$ & 6.07793800 & 4.24255400 & -0.32476300 & $\mathrm{H}$ & 0.29564400 & 5.80896100 & 2.98506300 \\
\hline C & 5.77415000 & 2.89428200 & -1.98813000 & $\mathrm{C}$ & 0.68520900 & 6.31731700 & 0.91851600 \\
\hline $\mathrm{H}$ & 6.66895300 & 3.22458400 & -2.52153300 & $\mathrm{H}$ & 0.87369700 & 7.37232000 & 1.13225200 \\
\hline C & 4.95606300 & 1.90328700 & -2.54341200 & $\mathrm{C}$ & 0.75425600 & 5.84834800 & -0.39694200 \\
\hline $\mathrm{H}$ & 5.21017700 & 1.46118500 & -3.50976900 & $\mathrm{H}$ & 0.99148500 & 6.53616600 & -1.21219900 \\
\hline C & 3.81208100 & 1.48012600 & -1.86628800 & C & 0.51099600 & 4.50021900 & -0.67230700 \\
\hline $\mathrm{H}$ & 3.17702200 & 0.70842100 & -2.30503400 & $\mathrm{H}$ & 0.55289800 & 4.13597600 & -1.70142800 \\
\hline $\mathrm{H}$ & -3.11669700 & -3.05791700 & -0.14476200 & $\mathrm{H}$ & 0.78246300 & -2.31365000 & 0.46589500 \\
\hline $\mathrm{H}$ & 1.21228200 & -3.38001700 & -1.99977800 & $\mathrm{H}$ & 0.53171800 & -1.31900300 & -1.98565300 \\
\hline $\mathrm{Mn}$ & -0.74582700 & -3.04797500 & -0.62250300 & $\mathrm{Mn}$ & 2.21989900 & -2.96719400 & -1.90218500 \\
\hline
\end{tabular}




\begin{tabular}{|c|c|c|c|c|c|c|c|}
\hline $\mathrm{O}$ & -2.40267400 & -3.69027000 & -0.30441000 & 0 & 1.45877000 & -4.15088200 & -1.10914500 \\
\hline $\mathrm{O}$ & 0.47260500 & -2.76004400 & -1.93010900 & $\mathrm{O}$ & 1.30639100 & -1.59399000 & -2.54556800 \\
\hline \multicolumn{4}{|c|}{ TS-2b } & \multicolumn{4}{|c|}{ INT-3b } \\
\hline $\mathrm{P}$ & 0.60802400 & 1.79267800 & 0.47639200 & $\mathrm{P}$ & 1.96328000 & 1.62692000 & 0.35331300 \\
\hline $\mathrm{O}$ & -0.37114000 & 1.46468000 & 1.57164200 & $\mathrm{O}$ & 1.80198500 & 2.57571200 & 1.51702000 \\
\hline $\mathrm{O}$ & -6.23806900 & -0.21722200 & 0.06880100 & $\mathrm{O}$ & -5.76457800 & 1.69430300 & -1.03929800 \\
\hline $\mathrm{N}$ & -0.75806400 & -0.47489600 & -0.60309200 & $\mathrm{~N}$ & -1.08794000 & -1.06460500 & 0.11429000 \\
\hline $\mathrm{N}$ & -1.91705500 & -1.37798400 & 1.80646800 & $\mathrm{~N}$ & -1.92789100 & 1.05129100 & 1.75903600 \\
\hline C & 1.82897100 & 0.46473000 & 0.06759800 & C & 2.21208800 & -0.15680300 & 0.73317900 \\
\hline C & 3.15500700 & 0.83894000 & -0.27993400 & C & 3.51190200 & -0.68135000 & 0.57772700 \\
\hline $\mathrm{H}$ & 3.41824500 & 1.89564600 & -0.32567300 & $\mathrm{H}$ & 4.34965000 & -0.00186100 & 0.41960400 \\
\hline C & 4.10848800 & -0.10484300 & -0.57979800 & C & 3.73057100 & -2.04946300 & 0.51233700 \\
\hline $\mathrm{H}$ & 5.11208300 & 0.19732500 & -0.88783100 & $\mathrm{H}$ & 4.73197500 & -2.43069200 & 0.29678400 \\
\hline C & 3.80463400 & -1.48613900 & -0.47686300 & C & 2.65652000 & -2.95444100 & 0.61229900 \\
\hline C & 4.77308700 & -2.48003900 & -0.79077700 & C & 2.78029500 & -4.34129000 & 0.23019700 \\
\hline $\mathrm{H}$ & 5.76439700 & -2.15254500 & -1.11387000 & $\mathrm{H}$ & 3.76761800 & -4.71793400 & -0.04678400 \\
\hline C & 4.47725200 & -3.82330800 & -0.70133700 & C & 1.67205200 & -5.13913000 & 0.13085000 \\
\hline $\mathrm{H}$ & 5.22876100 & -4.57347200 & -0.95681100 & $\mathrm{H}$ & 1.77217000 & -6.16619400 & -0.22984000 \\
\hline C & 3.19869600 & -4.22859500 & -0.24847000 & C & 0.37510400 & -4.67344300 & 0.52869900 \\
\hline $\mathrm{H}$ & 2.97266400 & -5.29057100 & -0.12891900 & $\mathrm{H}$ & -0.42681500 & -5.39657900 & 0.68390300 \\
\hline C & 2.23605600 & -3.28824600 & 0.08063900 & C & 0.24822600 & -3.36471400 & 1.09388200 \\
\hline $\mathrm{H}$ & 1.28035500 & -3.62253200 & 0.47601700 & $\mathrm{H}$ & -0.54951500 & -3.13759400 & 1.80464600 \\
\hline C & 2.48757900 & -1.88405500 & -0.05779400 & C & 1.37775100 & -2.44531900 & 0.97827000 \\
\hline C & 1.47178900 & -0.88357000 & 0.14953800 & C & 1.15228800 & -1.05083600 & 1.00032200 \\
\hline C & 0.00454500 & -1.24256800 & 0.27525900 & C & -0.26382500 & -0.57852800 & 1.03094000 \\
\hline C & -0.53108700 & -1.51368100 & 1.60939000 & C & -0.67400500 & 0.41425600 & 1.95253600 \\
\hline C & 0.04915900 & -1.90312600 & 2.80976200 & C & -0.11166300 & 0.90629200 & 3.14161300 \\
\hline C & -1.01621300 & -1.99924700 & 3.74919200 & C & -1.02656400 & 1.85338000 & 3.63684400 \\
\hline $\mathrm{H}$ & -0.91418600 & -2.25965400 & 4.80198300 & $\mathrm{H}$ & -0.90779700 & 2.43334900 & 4.55036900 \\
\hline
\end{tabular}




\begin{tabular}{|c|c|c|c|c|c|c|c|}
\hline C & -2.21007100 & -1.66903800 & 3.13662100 & C & -2.12758900 & 1.94448900 & 2.78611200 \\
\hline C & 1.49938100 & -2.10495500 & 3.13639200 & C & 1.18299200 & 0.53131800 & 3.78758200 \\
\hline $\mathrm{H}$ & 1.91296200 & -3.04390300 & 2.73118000 & $\mathrm{H}$ & 1.43366600 & -0.52760000 & 3.63879300 \\
\hline $\mathrm{H}$ & 1.63506900 & -2.13851900 & 4.22778500 & $\mathrm{H}$ & 1.13813200 & 0.73086400 & 4.86861600 \\
\hline $\mathrm{H}$ & 2.12476300 & -1.28580300 & 2.75058100 & $\mathrm{H}$ & 1.99177500 & 1.14108800 & 3.35634500 \\
\hline C & -3.54778700 & -1.57953700 & 3.79356000 & C & -3.32151300 & 2.81837600 & 2.97865500 \\
\hline $\mathrm{H}$ & -3.41879200 & -1.79057700 & 4.86445400 & $\mathrm{H}$ & -3.22272400 & 3.33665800 & 3.94193400 \\
\hline $\mathrm{H}$ & -4.27578700 & -2.30911900 & 3.40111600 & $\mathrm{H}$ & -4.26285300 & 2.24661000 & 2.99699900 \\
\hline $\mathrm{H}$ & -3.99631500 & -0.57713500 & 3.70311700 & $\mathrm{H}$ & -3.41167500 & 3.58989100 & 2.19667200 \\
\hline C & -2.73989100 & -0.86980500 & 0.78859600 & C & -2.72931200 & 0.68728400 & 0.67164100 \\
\hline C & -4.12817400 & -0.79999500 & 0.87988400 & C & -3.89128900 & 1.37283700 & 0.30783600 \\
\hline $\mathrm{H}$ & -4.67160000 & -1.18031900 & 1.73578800 & $\mathrm{H}$ & -4.22241900 & 2.26283900 & 0.82807200 \\
\hline C & -4.89573300 & -0.22943600 & -0.14749400 & C & -4.66732400 & 0.94530700 & -0.77363600 \\
\hline C & -4.27322600 & 0.27897400 & -1.29985300 & C & -4.28892300 & -0.19463600 & -1.50342600 \\
\hline $\mathrm{H}$ & -4.84937800 & 0.73146600 & -2.10578300 & $\mathrm{H}$ & -4.88020300 & -0.55436200 & -2.34385000 \\
\hline C & -2.89339500 & 0.18573900 & -1.41212800 & C & -3.11981900 & -0.85836100 & -1.16069000 \\
\hline $\mathrm{H}$ & -2.38397500 & 0.55452500 & -2.30280600 & $\mathrm{H}$ & -2.81447300 & -1.73568500 & -1.72964200 \\
\hline C & -2.09084700 & -0.39315000 & -0.40207600 & C & -2.30835000 & -0.44320600 & -0.08989800 \\
\hline C & -7.07964500 & 0.33103000 & -0.91624700 & C & -6.59377500 & 1.33346000 & -2.12149200 \\
\hline $\mathrm{H}$ & -6.86306800 & 1.40022600 & -1.09422600 & $\mathrm{H}$ & -6.04721800 & 1.35731700 & -3.08110000 \\
\hline $\mathrm{H}$ & -8.10762800 & 0.23425300 & -0.54183300 & $\mathrm{H}$ & -7.40473000 & 2.07264700 & -2.15392900 \\
\hline $\mathrm{H}$ & -6.99578400 & -0.20929600 & -1.87672100 & $\mathrm{H}$ & -7.02983500 & 0.32784700 & -1.98579200 \\
\hline C & -0.13607900 & 2.34958300 & -1.08835400 & C & 0.57070800 & 1.68039300 & -0.82170500 \\
\hline C & -1.31926600 & 3.09413200 & -0.98947000 & C & -0.29078400 & 2.78271400 & -0.73016500 \\
\hline $\mathrm{H}$ & -1.75124800 & 3.28075300 & -0.00392600 & $\mathrm{H}$ & -0.12449900 & 3.51400200 & 0.06418100 \\
\hline C & -1.94972200 & 3.55747900 & -2.14599200 & C & -1.34808100 & 2.91705100 & -1.63323600 \\
\hline $\mathrm{H}$ & -2.87620200 & 4.13123500 & -2.06639900 & $\mathrm{H}$ & -2.02702100 & 3.76948300 & -1.55524400 \\
\hline C & -1.40887600 & 3.26876600 & -3.40240500 & C & -1.54352500 & 1.95511200 & -2.62816000 \\
\hline $\mathrm{H}$ & -1.90816800 & 3.62293100 & -4.30768500 & $\mathrm{H}$ & -2.37728500 & 2.05481600 & -3.32697300 \\
\hline
\end{tabular}




\begin{tabular}{|c|c|c|c|c|c|c|c|}
\hline C & -0.23566100 & 2.51250200 & -3.50160400 & C & -0.69310400 & 0.84824700 & -2.70863700 \\
\hline $\mathrm{H}$ & 0.17595700 & 2.26289800 & -4.48220800 & $\mathrm{H}$ & -0.86434400 & 0.07000100 & -3.45536800 \\
\hline $\mathrm{C}$ & 0.40343600 & 2.05535300 & -2.34754000 & C & 0.36215500 & 0.70248700 & -1.80714300 \\
\hline $\mathrm{H}$ & 1.29172800 & 1.42720600 & -2.43272300 & $\mathrm{H}$ & 0.95876400 & -0.21291400 & -1.84334400 \\
\hline C & 1.66187900 & 3.20046500 & 0.99525300 & C & 3.42667800 & 2.09705600 & -0.64120900 \\
\hline $\mathrm{C}$ & 1.89938400 & 3.33507400 & 2.37122400 & $\mathrm{C}$ & 4.24003900 & 3.11093000 & -0.11907600 \\
\hline $\mathrm{H}$ & 1.44192100 & 2.61768500 & 3.05625400 & $\mathrm{H}$ & 3.96854500 & 3.55290900 & 0.84200200 \\
\hline C & 2.68718500 & 4.38544100 & 2.84616100 & C & 5.36450200 & 3.54092900 & -0.83004500 \\
\hline $\mathrm{H}$ & 2.86991600 & 4.48694900 & 3.91881700 & $\mathrm{H}$ & 5.99780600 & 4.33152200 & -0.42005500 \\
\hline $\mathrm{C}$ & 3.23273200 & 5.31265400 & 1.95232200 & C & 5.67470500 & 2.96391600 & -2.06381900 \\
\hline $\mathrm{H}$ & 3.84572300 & 6.13680100 & 2.32561300 & $\mathrm{H}$ & 6.55244500 & 3.30135700 & -2.62061100 \\
\hline $\mathrm{C}$ & 2.98267600 & 5.19312900 & 0.58192500 & C & 4.85835700 & 1.95605800 & -2.59084100 \\
\hline $\mathrm{H}$ & 3.39466500 & 5.92590600 & -0.11627800 & $\mathrm{H}$ & 5.09701600 & 1.50782300 & -3.55832600 \\
\hline $\mathrm{C}$ & 2.19605900 & 4.14172800 & 0.10355500 & $\mathrm{C}$ & 3.73627900 & 1.52361100 & -1.88376900 \\
\hline $\mathrm{H}$ & 1.98829200 & 4.05920900 & -0.96582300 & $\mathrm{H}$ & 3.10306800 & 0.73800300 & -2.30003100 \\
\hline $\mathrm{H}$ & -0.20907700 & -2.43680600 & -0.40057800 & $\mathrm{H}$ & -2.95418000 & -3.25024100 & 0.18753000 \\
\hline $\mathrm{H}$ & -0.04209900 & -0.70610300 & -2.22110300 & $\mathrm{H}$ & 1.48214000 & -3.10167800 & -1.89385000 \\
\hline $\mathrm{Mn}$ & 0.93992300 & -2.76104800 & -2.23968600 & $\mathrm{Mr}$ & -0.66511000 & -3.11539500 & -0.71050600 \\
\hline $\mathrm{O}$ & -0.31665300 & -3.34910600 & -1.29349600 & $\mathrm{O}$ & -2.32135700 & -3.75572300 & -0.33775800 \\
\hline $\mathrm{O}$ & 0.51327400 & -1.20045100 & -2.90585500 & $\mathrm{O}$ & 0.61764700 & -2.67120600 & -1.92614800 \\
\hline \multicolumn{4}{|c|}{ INT-1c } & \multicolumn{4}{|c|}{ TS-1c } \\
\hline $\mathrm{P}$ & -2.21363100 & -0.74454800 & -0.40427300 & $\mathrm{P}$ & 2.46757700 & -0.33141200 & 0.43661500 \\
\hline $\mathrm{O}$ & -1.13293600 & -1.80751100 & -0.12332900 & $\mathrm{O}$ & 1.61680200 & -1.03174000 & 1.52124200 \\
\hline $\mathrm{O}$ & 6.34994400 & -2.43230300 & -0.30066600 & $\mathrm{O}$ & -6.64400400 & -1.51067100 & 0.38338100 \\
\hline $\mathrm{N}$ & 1.24864500 & -0.42626100 & 0.91244600 & $\mathrm{~N}$ & -1.09389500 & -0.83079700 & 0.35981600 \\
\hline $\mathrm{N}$ & 3.02052300 & 0.97182100 & -0.80949300 & $\mathrm{~N}$ & -2.83138200 & 1.37491000 & 0.85502600 \\
\hline $\mathrm{C}$ & -1.79793300 & 0.88780800 & 0.28590700 & $\mathrm{C}$ & 1.57826200 & 0.24062900 & -1.05162700 \\
\hline C & -2.86305100 & 1.69795500 & 0.75976700 & C & 2.33688400 & 0.27901500 & -2.25627600 \\
\hline $\mathrm{H}$ & -3.89391200 & 1.36912500 & 0.62284700 & $\mathrm{H}$ & 3.37237500 & -0.06329700 & -2.25714500 \\
\hline
\end{tabular}




\begin{tabular}{|c|c|c|c|c|c|c|c|}
\hline C & -2.60001000 & 2.87568900 & 1.41411800 & $\mathrm{C}$ & 1.76920000 & 0.73456400 & -3.41952200 \\
\hline $\mathrm{H}$ & -3.41876700 & 3.50424600 & 1.77201500 & $\mathrm{H}$ & 2.35487000 & 0.77229100 & -4.34104400 \\
\hline C & -1.26396200 & 3.26895100 & 1.68253000 & C & 0.40562600 & 1.12706700 & -3.45456600 \\
\hline C & -0.98965900 & 4.44738300 & 2.42946600 & C & -0.19769700 & 1.55024800 & -4.66967900 \\
\hline $\mathrm{H}$ & -1.83193600 & 5.05358500 & 2.77177900 & $\mathrm{H}$ & 0.41980800 & 1.59526700 & -5.57017000 \\
\hline C & 0.30320900 & 4.81092300 & 2.72988600 & C & -1.53337800 & 1.88145500 & -4.71747900 \\
\hline $\mathrm{H}$ & 0.50269100 & 5.71494000 & 3.30966400 & $\mathrm{H}$ & -1.98897900 & 2.20066100 & -5.65773200 \\
\hline C & 1.37794000 & 3.99898800 & 2.29450500 & $\mathrm{C}$ & -2.32043700 & 1.79499300 & -3.54495300 \\
\hline $\mathrm{H}$ & 2.40389700 & 4.27647200 & 2.54624100 & $\mathrm{H}$ & -3.38398800 & 2.04001400 & -3.58558000 \\
\hline $\mathrm{C}$ & 1.14596700 & 2.86048000 & 1.55387500 & C & -1.76198900 & 1.40150000 & -2.34774600 \\
\hline $\mathrm{H}$ & 1.99989000 & 2.27014900 & 1.23179400 & $\mathrm{H}$ & -2.39637700 & 1.34516700 & -1.47063800 \\
\hline C & -0.17861000 & 2.45574200 & 1.20846300 & $\mathrm{C}$ & -0.37974000 & 1.06578800 & -2.25151300 \\
\hline C & -0.47197700 & 1.27906200 & 0.44185300 & C & 0.24574700 & 0.65441500 & -1.02748100 \\
\hline C & 0.65253800 & 0.43954000 & -0.15992200 & C & -0.51744900 & 0.52382100 & 0.29375400 \\
\hline C & 1.66425000 & 1.28252500 & -0.88284900 & C & -1.47829700 & 1.60902100 & 0.64741800 \\
\hline C & 1.50499700 & 2.41590500 & -1.65294000 & C & -1.20012700 & 2.93677300 & 0.89166900 \\
\hline C & 2.82307400 & 2.83538200 & -2.01913700 & C & -2.44075100 & 3.53941000 & 1.26147600 \\
\hline $\mathrm{H}$ & 3.07250000 & 3.72236400 & -2.59968400 & $\mathrm{H}$ & -2.59352000 & 4.58101000 & 1.54020700 \\
\hline C & 3.74461200 & 1.95272200 & -1.49768200 & C & -3.43302700 & 2.57786800 & 1.24363300 \\
\hline C & 0.22978900 & 3.12034100 & -2.00871700 & C & 0.14402400 & 3.58954400 & 0.77473800 \\
\hline $\mathrm{H}$ & -0.60724600 & 2.41971400 & -2.13949600 & $\mathrm{H}$ & 0.94112900 & 2.96725500 & 1.21282900 \\
\hline $\mathrm{H}$ & 0.35028900 & 3.67815900 & -2.94997800 & $\mathrm{H}$ & 0.15896000 & 4.56027800 & 1.29213000 \\
\hline $\mathrm{H}$ & -0.07090900 & 3.84260100 & -1.23075200 & $\mathrm{H}$ & 0.42547700 & 3.76967300 & -0.27760200 \\
\hline C & 5.23367100 & 2.04705200 & -1.58506600 & C & -4.86199800 & 2.76760500 & 1.63710800 \\
\hline $\mathrm{H}$ & 5.49678500 & 3.06174500 & -1.91580800 & $\mathrm{H}$ & -4.97289300 & 3.78056500 & 2.04884600 \\
\hline $\mathrm{H}$ & 5.67498600 & 1.34521000 & -2.31281900 & $\mathrm{H}$ & -5.18558000 & 2.05422900 & 2.41148200 \\
\hline $\mathrm{H}$ & 5.72344600 & 1.87017200 & -0.61515600 & $\mathrm{H}$ & -5.56332000 & 2.68146800 & 0.79046300 \\
\hline $\mathrm{C}$ & 3.45362100 & -0.24735700 & -0.24582600 & C & -3.38767400 & 0.09076400 & 0.63616000 \\
\hline C & 4.70514100 & -0.79617000 & -0.53180400 & C & -4.76658000 & -0.13766400 & 0.60577100 \\
\hline
\end{tabular}




\begin{tabular}{|c|c|c|c|c|c|c|c|}
\hline $\mathrm{H}$ & 5.39529900 & -0.32181600 & -1.21896500 & $\mathrm{H}$ & -5.48223800 & 0.66165000 & 0.75052600 \\
\hline C & 5.11031900 & -2.00746900 & 0.04617200 & $\mathrm{C}$ & -5.28575500 & -1.41651600 & 0.37517500 \\
\hline C & 4.24264100 & -2.69576500 & 0.90052800 & $\mathrm{C}$ & -4.41333000 & -2.48693200 & 0.14708600 \\
\hline $\mathrm{H}$ & 4.51842500 & -3.64568800 & 1.35539700 & $\mathrm{H}$ & -4.78036700 & -3.49489900 & -0.04024400 \\
\hline C & 2.97522400 & -2.16779700 & 1.15263400 & $\mathrm{C}$ & -3.03979600 & -2.25387800 & 0.14706700 \\
\hline $\mathrm{H}$ & 2.26866400 & -2.72379400 & 1.77530700 & $\mathrm{H}$ & -2.34891300 & -3.07472200 & -0.04913300 \\
\hline C & 2.56636500 & -0.95661300 & 0.59850100 & $\mathrm{C}$ & -2.49916500 & -0.98608200 & 0.39403600 \\
\hline C & 6.81855000 & -3.65556400 & 0.22164100 & $\mathrm{C}$ & -7.23170800 & -2.76865700 & 0.15568000 \\
\hline $\mathrm{H}$ & 6.87629100 & -3.63416900 & 1.32445000 & $\mathrm{H}$ & -6.97282900 & -3.17027900 & -0.84116400 \\
\hline $\mathrm{H}$ & 7.82694300 & -3.80432300 & -0.18639600 & $\mathrm{H}$ & -8.31906800 & -2.62306900 & 0.20889500 \\
\hline $\mathrm{H}$ & 6.17814600 & -4.50232700 & -0.08293200 & $\mathrm{H}$ & -6.93160300 & -3.50782500 & 0.92060900 \\
\hline C & -2.35932900 & -0.56465500 & -2.19937900 & C & 3.20743800 & 1.12980100 & 1.20576300 \\
\hline C & -1.87435600 & -1.59511300 & -3.01766700 & C & 3.19881700 & 1.22221800 & 2.60533400 \\
\hline $\mathrm{H}$ & -1.41866100 & -2.47279300 & -2.55502100 & $\mathrm{H}$ & 2.76118700 & 0.41182800 & 3.19103600 \\
\hline C & -1.96383700 & -1.47560400 & -4.40626300 & C & 3.72499100 & 2.35696500 & 3.22626700 \\
\hline $\mathrm{H}$ & -1.58289900 & -2.27543000 & -5.04531900 & $\mathrm{H}$ & 3.71130500 & 2.43397500 & 4.31572400 \\
\hline C & -2.53277200 & -0.33316400 & -4.97687900 & C & 4.25921100 & 3.39398200 & 2.45612900 \\
\hline $\mathrm{H}$ & -2.59746000 & -0.24099400 & -6.06362000 & $\mathrm{H}$ & 4.66505000 & 4.28281300 & 2.94486700 \\
\hline C & -3.01200100 & 0.69701400 & -4.16070000 & C & 4.26744600 & 3.30133000 & 1.06022100 \\
\hline $\mathrm{H}$ & -3.44763400 & 1.59339900 & -4.60771300 & $\mathrm{H}$ & 4.67575700 & 4.11652700 & 0.45883100 \\
\hline C & -2.92468600 & 0.58518200 & -2.77216700 & $\mathrm{C}$ & 3.73912500 & 2.17315300 & 0.43192900 \\
\hline $\mathrm{H}$ & -3.28535800 & 1.39552900 & -2.13453800 & $\mathrm{H}$ & 3.72149100 & 2.11421500 & -0.65822600 \\
\hline C & -3.82560800 & -1.20145400 & 0.27223900 & C & 3.77080500 & -1.43643500 & -0.15063100 \\
\hline C & -3.91291700 & -1.40608700 & 1.66296900 & $\mathrm{C}$ & 3.36727800 & -2.66106300 & -0.71654500 \\
\hline $\mathrm{H}$ & -3.02212100 & -1.24365000 & 2.28564500 & $\mathrm{H}$ & 2.29864000 & -2.89735400 & -0.80159300 \\
\hline C & -5.13127300 & -1.80679400 & 2.21211000 & $\mathrm{C}$ & 4.34212400 & -3.55886000 & -1.15241700 \\
\hline $\mathrm{H}$ & -5.20601600 & -1.97005400 & 3.28967600 & $\mathrm{H}$ & 4.03710800 & -4.51201100 & -1.59027000 \\
\hline C & -6.24957900 & -2.00140000 & 1.39358600 & C & 5.70072800 & -3.24540500 & -1.02985100 \\
\hline $\mathrm{H}$ & -7.19944200 & -2.31545800 & 1.83352600 & $\mathrm{H}$ & 6.45691900 & -3.95469100 & -1.37515600 \\
\hline
\end{tabular}




\begin{tabular}{|c|c|c|c|c|c|c|c|}
\hline C & -6.15644300 & -1.79874000 & 0.01369400 & C & 6.09501600 & -2.02999200 & -0.46383100 \\
\hline $\mathrm{H}$ & -7.02864600 & -1.95475900 & -0.62532000 & $\mathrm{H}$ & 7.15563800 & -1.78862000 & -0.36335700 \\
\hline C & -4.94285200 & -1.40076000 & -0.55219400 & C & 5.13049600 & -1.12196600 & -0.01935700 \\
\hline $\mathrm{H}$ & -4.86720400 & -1.24924600 & -1.63072000 & $\mathrm{H}$ & 5.43621200 & -0.17456400 & 0.42906800 \\
\hline $\mathrm{H}$ & 0.22552100 & -0.26462700 & -0.88410000 & $\mathrm{H}$ & 0.24333000 & 0.59553400 & 1.08129100 \\
\hline $\mathrm{H}$ & 1.29786800 & 0.12539900 & 1.77396000 & $\mathrm{H}$ & -0.57593200 & -1.64290000 & -0.42435200 \\
\hline $\mathrm{Mr}$ & -0.17704200 & -1.84340200 & 1.61801500 & $\mathrm{Mn}$ & 0.09694200 & -2.22440300 & 1.25463800 \\
\hline 0 & 0.33678400 & -3.24752300 & 2.14191400 & 0 & -0.48041000 & -3.04145600 & 2.44482500 \\
\hline O & -1.00297100 & -0.79743300 & 2.55302800 & $\mathrm{O}$ & 0.19438100 & -2.70595700 & -0.38071600 \\
\hline INT & & & & TS & $-2 c$ & & \\
\hline $\mathrm{P}$ & -2.50147300 & -0.20389800 & -0.34399800 & P & -2.50334000 & -0.63303900 & 0.21388800 \\
\hline $\mathrm{O}$ & -1.85016500 & -0.60349100 & -1.68558800 & $\mathrm{O}$ & -1.75600400 & -1.79558800 & 0.88802600 \\
\hline O & 6.34327300 & -1.59731900 & -0.20446700 & O & 6.71026600 & -1.55800500 & 0.76169600 \\
\hline $\mathrm{N}$ & 0.92387300 & -0.50784200 & -1.01779800 & $\mathrm{~N}$ & 1.22187000 & -0.69950900 & 0.57799700 \\
\hline $\mathrm{N}$ & 2.75728000 & 1.55856300 & -0.70733900 & $\mathrm{~N}$ & 2.94912100 & 0.66040700 & -1.23073000 \\
\hline C & -1.38937200 & -0.22222500 & 1.09216700 & C & -1.70828400 & 0.98481300 & 0.53509600 \\
\hline C & -1.91372200 & -0.76708000 & 2.29579200 & C & -2.57041300 & 2.00078800 & 1.04646800 \\
\hline $\mathrm{H}$ & -2.91848000 & -1.18950100 & 2.30666000 & $\mathrm{H}$ & -3.64146900 & 1.81285200 & 1.10658400 \\
\hline C & -1.15720700 & -0.77594400 & 3.44041000 & C & -2.08141800 & 3.21478300 & 1.45548300 \\
\hline $\mathrm{H}$ & -1.55365800 & -1.20664600 & 4.36279200 & $\mathrm{H}$ & -2.76058800 & 3.99458100 & 1.80803900 \\
\hline C & 0.14882600 & -0.22568400 & 3.44235700 & C & -0.68403900 & 3.44767000 & 1.48108700 \\
\hline C & 0.92507300 & -0.23525500 & 4.63360500 & C & -0.15461300 & 4.66100500 & 2.00160400 \\
\hline $\mathrm{H}$ & 0.49165700 & -0.68411800 & 5.53080900 & $\mathrm{H}$ & -0.85317100 & 5.43073700 & 2.33906400 \\
\hline C & 2.18950200 & 0.30676300 & 4.66090400 & C & 1.20432300 & 4.85342300 & 2.09924500 \\
\hline $\mathrm{H}$ & 2.77759100 & 0.29229500 & 5.58157900 & $\mathrm{H}$ & 1.60297300 & 5.78585000 & 2.50573400 \\
\hline C & 2.72050300 & 0.89407400 & 3.48942700 & C & 2.08691400 & 3.82329600 & 1.69435900 \\
\hline $\mathrm{H}$ & 3.71696400 & 1.34073700 & 3.50827600 & $\mathrm{H}$ & 3.16492000 & 3.95482900 & 1.81087500 \\
\hline C & 1.99524200 & 0.91504000 & 2.31785000 & C & 1.60172600 & 2.64940400 & 1.16104800 \\
\hline $\mathrm{H}$ & 2.43049000 & 1.39791500 & 1.45416700 & $\mathrm{H}$ & 2.30930100 & 1.87403000 & 0.88834300 \\
\hline
\end{tabular}




\begin{tabular}{|c|c|c|c|c|c|c|c|}
\hline C & 0.68984200 & 0.34483600 & 2.23311400 & C & 0.20178700 & 2.42444800 & 1.00409300 \\
\hline C & -0.09693000 & 0.32073200 & 1.02811200 & C & -0.33115100 & 1.21492700 & 0.42314500 \\
\hline C & 0.42878900 & 0.71193400 & -0.37414600 & C & 0.65373800 & 0.28210200 & -0.29251600 \\
\hline C & 1.40688600 & 1.83100200 & -0.51391700 & C & 1.58485200 & 0.97898800 & -1.19872200 \\
\hline C & 1.21236100 & 3.19631300 & -0.55146200 & C & 1.32787100 & 1.93041200 & -2.17115600 \\
\hline C & 2.49970900 & 3.77538300 & -0.78149900 & C & 2.57199800 & 2.19645300 & -2.80729000 \\
\hline $\mathrm{H}$ & 2.71753700 & 4.83620100 & -0.89727500 & $\mathrm{H}$ & 2.73435800 & 2.88659600 & -3.63412000 \\
\hline C & 3.43782600 & 2.76485500 & -0.88142700 & C & 3.56088700 & 1.41916600 & -2.23004900 \\
\hline C & -0.08793700 & 3.91790400 & -0.37048400 & C & 0.00113000 & 2.54630600 & -2.49114100 \\
\hline $\mathrm{H}$ & -0.94623500 & 3.28012300 & -0.62665500 & $\mathrm{H}$ & -0.32850400 & 3.24848300 & -1.70606700 \\
\hline $\mathrm{H}$ & -0.13863500 & 4.81527100 & -1.00678600 & $\mathrm{H}$ & -0.78224700 & 1.78019100 & -2.59015500 \\
\hline $\mathrm{H}$ & -0.23525500 & 4.24990000 & 0.67228200 & $\mathrm{H}$ & 0.04860000 & 3.10268200 & -3.43904000 \\
\hline C & 4.88758800 & 2.90095300 & -1.21642700 & C & 4.99735100 & 1.36863500 & -2.63666100 \\
\hline $\mathrm{H}$ & 5.07190400 & 3.92717000 & -1.56444000 & $\mathrm{H}$ & 5.12365300 & 2.00271100 & -3.52532900 \\
\hline $\mathrm{H}$ & 5.19447800 & 2.21031800 & -2.01743700 & $\mathrm{H}$ & 5.32891000 & 0.35249700 & -2.90432700 \\
\hline $\mathrm{H}$ & 5.55409900 & 2.72431400 & -0.35538100 & $\mathrm{H}$ & 5.68319200 & 1.75201800 & -1.86283000 \\
\hline C & 3.23391600 & 0.22929700 & -0.66345800 & C & 3.49444700 & -0.23614600 & -0.29391500 \\
\hline C & 4.57256300 & -0.08353200 & -0.42992100 & C & 4.86285500 & -0.48664700 & -0.17884900 \\
\hline $\mathrm{H}$ & 5.31335500 & 0.68486500 & -0.23629800 & $\mathrm{H}$ & 5.59175100 & 0.00746500 & -0.80829800 \\
\hline C & 5.01199900 & -1.41367700 & -0.43226800 & C & 5.36126200 & -1.40125100 & 0.75859900 \\
\hline C & 4.09076700 & -2.44255300 & -0.65807800 & C & 4.47840800 & -2.08040900 & 1.61270300 \\
\hline $\mathrm{H}$ & 4.39835300 & -3.48720300 & -0.68121600 & $\mathrm{H}$ & 4.83677500 & -2.79541600 & 2.35201900 \\
\hline C & 2.74314600 & -2.12922400 & -0.84079800 & C & 3.11744200 & -1.81829000 & 1.51897400 \\
\hline $\mathrm{H}$ & 2.02929400 & -2.93648200 & -1.00922300 & $\mathrm{H}$ & 2.40747200 & -2.33055400 & 2.17118100 \\
\hline C & 2.26971300 & -0.80698700 & -0.83117400 & C & 2.58326400 & -0.90389600 & 0.58513700 \\
\hline C & 6.84552500 & -2.91031700 & -0.18695900 & C & 7.28687200 & -2.46364300 & 1.67317900 \\
\hline $\mathrm{H}$ & 6.37621700 & -3.52125600 & 0.60624400 & $\mathrm{H}$ & 7.08023700 & -2.17957200 & 2.72085900 \\
\hline $\mathrm{H}$ & 7.92287100 & -2.83436900 & 0.01355700 & $\mathrm{H}$ & 8.37124500 & -2.43309900 & 1.50298700 \\
\hline $\mathrm{H}$ & 6.69776500 & -3.42183800 & -1.15587200 & $\mathrm{H}$ & 6.92736400 & -3.49561100 & 1.50954400 \\
\hline
\end{tabular}




\begin{tabular}{|c|c|c|c|c|c|c|c|}
\hline C & -3.13938600 & 1.48179400 & -0.50328400 & C & -2.83322200 & -0.80356200 & -1.55569200 \\
\hline C & -3.34208100 & 1.99929600 & -1.79110000 & C & -2.92072500 & -2.08365400 & -2.11899900 \\
\hline $\mathrm{H}$ & -3.11126800 & 1.37886000 & -2.65927100 & $\mathrm{H}$ & -2.70049600 & -2.95758900 & -1.50583600 \\
\hline C & -3.80591400 & 3.30725400 & -1.94352000 & C & -3.25492100 & -2.22125300 & -3.46464200 \\
\hline $\mathrm{H}$ & -3.95396000 & 3.71696500 & -2.94510800 & $\mathrm{H}$ & -3.31323800 & -3.21727800 & -3.90919000 \\
\hline C & -4.06944400 & 4.09364000 & -0.81781700 & C & -3.49671700 & -1.08812500 & -4.25055300 \\
\hline $\mathrm{H}$ & -4.42624300 & 5.11884700 & -0.94103300 & $\mathrm{H}$ & -3.74958500 & -1.20197200 & -5.30740600 \\
\hline C & -3.86673000 & 3.57657100 & 0.46598400 & $\mathrm{C}$ & -3.40666800 & 0.18759500 & -3.68940200 \\
\hline $\mathrm{H}$ & -4.06128500 & 4.19654700 & 1.34387400 & $\mathrm{H}$ & -3.58628700 & 1.07341100 & -4.30283900 \\
\hline C & -3.39702400 & 2.27224600 & 0.62696500 & $\mathrm{C}$ & -3.07491400 & 0.33215600 & -2.33890100 \\
\hline $\mathrm{H}$ & -3.21024800 & 1.87592700 & 1.62753200 & $\mathrm{H}$ & -2.99505100 & 1.32859600 & -1.89887300 \\
\hline C & -3.87683500 & -1.32232100 & 0.01089300 & C & -4.14184000 & -0.57345700 & 1.00481200 \\
\hline C & -3.62228100 & -2.70167600 & -0.10152100 & $\mathrm{C}$ & -4.22155700 & -0.98570000 & 2.34420400 \\
\hline $\mathrm{H}$ & -2.62137900 & -3.05223700 & -0.37892500 & $\mathrm{H}$ & -3.31931700 & -1.33581200 & 2.84910400 \\
\hline $\mathrm{C}$ & -4.65478800 & -3.60567600 & 0.15067000 & $\mathrm{C}$ & -5.44961100 & -0.96285400 & 3.00665500 \\
\hline $\mathrm{H}$ & -4.46445200 & -4.67791500 & 0.06457400 & $\mathrm{H}$ & -5.50987400 & -1.28606300 & 4.04845500 \\
\hline C & -5.92752200 & -3.14560300 & 0.50730600 & $\mathrm{C}$ & -6.60053200 & -0.53539100 & 2.33687600 \\
\hline $\mathrm{H}$ & -6.73105300 & -3.86040100 & 0.70089200 & $\mathrm{H}$ & -7.56135500 & -0.52073500 & 2.85699500 \\
\hline C & -6.17650000 & -1.77437700 & 0.61170000 & C & -6.52512400 & -0.13743900 & 0.99892300 \\
\hline $\mathrm{H}$ & -7.17198200 & -1.41562400 & 0.88291400 & $\mathrm{H}$ & -7.42565600 & 0.18376600 & 0.47045000 \\
\hline C & -5.15185400 & -0.85799900 & 0.36162400 & $\mathrm{C}$ & -5.29899100 & -0.15890200 & 0.33009500 \\
\hline $\mathrm{H}$ & -5.34684600 & 0.21370400 & 0.43458400 & $\mathrm{H}$ & -5.24536000 & 0.13943200 & -0.71844900 \\
\hline $\mathrm{H}$ & -0.44336300 & 1.05368700 & -0.94275900 & $\mathrm{H}$ & 0.07084800 & -0.50500200 & -1.05708400 \\
\hline $\mathrm{H}$ & 0.09916900 & -3.06864700 & -0.12076300 & $\mathrm{H}$ & 1.37338600 & -4.22147800 & 1.46488500 \\
\hline $\mathrm{Mn}$ & -0.20926500 & -1.62583000 & -1.97263600 & $\mathrm{Mn}$ & 0.13417700 & -2.37685100 & 0.36618500 \\
\hline $\mathrm{O}$ & 0.21103200 & -1.77145200 & -3.45516100 & $\mathrm{O}$ & -0.10840100 & -1.90028100 & -1.26058200 \\
\hline $\mathrm{O}$ & -0.48879700 & -3.04358800 & -0.88955600 & $\mathrm{O}$ & 0.50184300 & -3.80793600 & 1.46725200 \\
\hline \multicolumn{4}{|c|}{ INT-3c } & \multicolumn{4}{|c|}{ INT-1d } \\
\hline$P$ & 2.08115400 & -1.03552600 & -0.33605700 & $\mathrm{P}$ & 2.21102000 & -0.77011600 & 0.44531600 \\
\hline
\end{tabular}




\begin{tabular}{|c|c|c|c|c|c|c|c|}
\hline 0 & 1.65285000 & -1.42739800 & -1.76828700 & 0 & 1.19731000 & -1.88672500 & 0.11230500 \\
\hline $\mathrm{O}$ & -6.46189300 & -0.91045300 & -0.34851400 & O & -6.37996500 & -2.36044600 & 0.34447900 \\
\hline $\mathrm{N}$ & -1.01095900 & 0.15386700 & -1.03444500 & $\mathrm{~N}$ & -1.30058800 & -0.39713800 & -0.97760600 \\
\hline $\mathrm{N}$ & -2.21410000 & 0.31301700 & 1.50488300 & $\mathrm{~N}$ & -2.98346800 & 0.98651800 & 0.83531100 \\
\hline C & 2.15097700 & 0.76088300 & -0.20834000 & C & 1.81196300 & 0.82208000 & -0.34735100 \\
\hline C & 3.40303800 & 1.42559500 & -0.28535600 & C & 2.89005300 & 1.59665400 & -0.85163500 \\
\hline $\mathrm{H}$ & 4.32333100 & 0.84009900 & -0.29934900 & $\mathrm{H}$ & 3.91564000 & 1.26231700 & -0.69404500 \\
\hline C & 3.46185200 & 2.79926100 & -0.32398100 & C & 2.64702400 & 2.74694400 & -1.55993400 \\
\hline $\mathrm{H}$ & 4.42735800 & 3.30951400 & -0.35798800 & $\mathrm{H}$ & 3.47709100 & 3.34392500 & -1.94473600 \\
\hline C & 2.27183100 & 3.57473100 & -0.33911500 & C & 1.31872000 & 3.15479500 & -1.84201900 \\
\hline C & 2.30801600 & 4.99456500 & -0.39667100 & C & 1.06483700 & 4.31035100 & -2.63082300 \\
\hline $\mathrm{H}$ & 3.28060300 & 5.49283700 & -0.41285800 & $\mathrm{H}$ & 1.91755200 & 4.88312900 & -3.00336500 \\
\hline C & 1.14313900 & 5.73064300 & -0.43624500 & C & -0.22222200 & 4.69443800 & -2.93008100 \\
\hline $\mathrm{H}$ & 1.18487200 & 6.82148900 & -0.48234800 & $\mathrm{H}$ & -0.40651600 & 5.58084900 & -3.54119200 \\
\hline C & -0.11202500 & 5.07444400 & -0.43040000 & C & -1.31162400 & 3.92914700 & -2.44870100 \\
\hline $\mathrm{H}$ & -1.03104500 & 5.66274600 & -0.48199800 & $\mathrm{H}$ & -2.33354900 & 4.22700700 & -2.69340000 \\
\hline C & -0.17978300 & 3.69860100 & -0.36628500 & C & -1.09906500 & 2.81204900 & -1.67067300 \\
\hline $\mathrm{H}$ & -1.14619300 & 3.19451900 & -0.36282500 & $\mathrm{H}$ & -1.96273800 & 2.26007400 & -1.30888800 \\
\hline C & 1.00208600 & 2.90902100 & -0.30084400 & C & 0.21991200 & 2.38333800 & -1.33138500 \\
\hline C & 0.94847500 & 1.47712900 & -0.20892300 & C & 0.49194000 & 1.22654000 & -0.52681600 \\
\hline C & -0.37727200 & 0.83399100 & -0.01063800 & C & -0.65575600 & 0.42798000 & 0.08535400 \\
\hline C & -0.97920600 & 0.95579600 & 1.26683700 & C & -1.62543000 & 1.30095400 & 0.83422300 \\
\hline C & -0.52708800 & 1.47291800 & 2.49878400 & C & -1.43063700 & 2.44574700 & 1.58069600 \\
\hline C & -1.49348800 & 1.09468300 & 3.46430100 & C & -2.72857000 & 2.86427900 & 2.01169500 \\
\hline $\mathrm{H}$ & -1.43006900 & 1.29615300 & 4.53314700 & $\mathrm{H}$ & -2.95018500 & 3.75493400 & 2.59787100 \\
\hline C & -2.51341600 & 0.37644500 & 2.86327000 & C & -3.67288500 & 1.97215000 & 1.54915500 \\
\hline C & 0.71936500 & 2.24758100 & 2.80398500 & C & -0.14365600 & 3.16090500 & 1.86664100 \\
\hline $\mathrm{H}$ & 0.67120900 & 3.28246800 & 2.42558900 & $\mathrm{H}$ & 0.69855500 & 2.46542700 & 1.98536700 \\
\hline $\mathrm{H}$ & 1.62134100 & 1.80066900 & 2.36086400 & $\mathrm{H}$ & -0.22908600 & 3.74394800 & 2.79633700 \\
\hline
\end{tabular}




\begin{tabular}{|c|c|c|c|c|c|c|c|}
\hline $\mathrm{H}$ & 0.86934900 & 2.30112300 & 3.89313600 & $\mathrm{H}$ & 0.12565800 & 3.86225200 & 1.05864600 \\
\hline C & -3.66901500 & -0.28152600 & 3.54170500 & C & -5.15526800 & 2.06312100 & 1.71537000 \\
\hline $\mathrm{H}$ & -3.47176400 & -0.30157500 & 4.62315900 & $\mathrm{H}$ & -5.40160700 & 3.07304700 & 2.07249300 \\
\hline $\mathrm{H}$ & -3.81066300 & -1.32069400 & 3.20602200 & $\mathrm{H}$ & -5.55665000 & 1.35053700 & 2.45557600 \\
\hline $\mathrm{H}$ & -4.62687600 & 0.24771200 & 3.39850600 & $\mathrm{H}$ & -5.69519300 & 1.89736300 & 0.77031500 \\
\hline C & -3.00751000 & -0.06745400 & 0.41238100 & C & -3.45589200 & -0.21311000 & 0.25784300 \\
\hline C & -4.36962700 & -0.35029700 & 0.52942000 & C & -4.70169400 & -0.75800500 & 0.57626500 \\
\hline $\mathrm{H}$ & -4.87560400 & -0.33495200 & 1.48693900 & $\mathrm{H}$ & -5.35635800 & -0.30509000 & 1.31120400 \\
\hline C & -5.15451200 & -0.63145500 & -0.59731600 & C & -5.14872000 & -1.93674000 & -0.03753500 \\
\hline C & -4.57791700 & -0.57736400 & -1.86954600 & C & -4.33136100 & -2.59635700 & -0.96222400 \\
\hline $\mathrm{H}$ & -5.16505300 & -0.73710800 & -2.77272800 & $\mathrm{H}$ & -4.64437900 & -3.51959900 & -1.44739100 \\
\hline C & -3.21659100 & -0.28462800 & -1.98509500 & C & -3.06567700 & -2.07862600 & -1.24270300 \\
\hline $\mathrm{H}$ & -2.77731100 & -0.20830300 & -2.97859200 & $\mathrm{H}$ & -2.37998900 & -2.61169400 & -1.91031100 \\
\hline C & -2.38586400 & -0.08216500 & -0.87132600 & C & -2.61583600 & -0.90051500 & -0.64981300 \\
\hline C & -7.31559700 & -1.16382100 & -1.43845200 & C & -6.88822100 & -3.55260200 & -0.20995300 \\
\hline $\mathrm{H}$ & -7.38481300 & -0.29454100 & -2.11757400 & $\mathrm{H}$ & -6.99454600 & -3.48155100 & -1.30721700 \\
\hline $\mathrm{H}$ & -8.30999400 & -1.36809200 & -1.01952700 & $\mathrm{H}$ & -7.87976600 & -3.70765600 & 0.23561800 \\
\hline $\mathrm{H}$ & -6.98572400 & -2.04157100 & -2.02352400 & $\mathrm{H}$ & -6.24728200 & -4.42045700 & 0.02711900 \\
\hline C & 0.91943600 & -1.70750800 & 0.87499100 & C & 2.19951300 & -0.50036700 & 2.23423400 \\
\hline C & -0.06514700 & -2.59786900 & 0.41474500 & C & 1.60326600 & -1.46713300 & 3.05585600 \\
\hline $\mathrm{H}$ & -0.11604400 & -2.85551100 & -0.64451600 & $\mathrm{H}$ & 1.16304100 & -2.35581600 & 2.59918700 \\
\hline C & -1.00349800 & -3.11157200 & 1.31072500 & C & 1.56548800 & -1.27054100 & 4.43825600 \\
\hline $\mathrm{H}$ & -1.78279100 & -3.78520900 & 0.94758200 & $\mathrm{H}$ & 1.09588400 & -2.01935400 & 5.08000800 \\
\hline C & -0.95497100 & -2.75612700 & 2.66199400 & C & 2.12102600 & -0.11652600 & 4.99796100 \\
\hline $\mathrm{H}$ & -1.69523300 & -3.15520100 & 3.35928700 & $\mathrm{H}$ & 2.08616200 & 0.03596100 & 6.07922900 \\
\hline C & 0.03303500 & -1.88051500 & 3.12165700 & C & 2.71484100 & 0.84886600 & 4.17743500 \\
\hline $\mathrm{H}$ & 0.05748600 & -1.58296200 & 4.17105700 & $\mathrm{H}$ & 3.14083200 & 1.75422200 & 4.61544800 \\
\hline C & 0.96636000 & -1.35147300 & 2.23229000 & C & 2.75269700 & 0.66132100 & 2.79531000 \\
\hline $\mathrm{H}$ & 1.70388300 & -0.63248500 & 2.59203600 & $\mathrm{H}$ & 3.20148000 & 1.42179000 & 2.15193200 \\
\hline
\end{tabular}




\begin{tabular}{|c|c|c|c|c|c|c|c|}
\hline $\mathrm{C}$ & 3.73171500 & -1.71099800 & -0.03120100 & $\mathrm{C}$ & 3.88002600 & -1.21985600 & -0.07936800 \\
\hline C & 4.35977000 & -2.40169800 & -1.07689000 & $\mathrm{C}$ & 4.06924400 & -1.51661700 & -1.44320900 \\
\hline $\mathrm{H}$ & 3.83975000 & -2.50287900 & -2.03128800 & $\mathrm{H}$ & 3.22211200 & -1.42578600 & -2.13776200 \\
\hline $\mathrm{C}$ & 5.63211000 & -2.94528300 & -0.88224400 & $\mathrm{C}$ & 5.33428600 & -1.90961300 & -1.88056900 \\
\hline $\mathrm{H}$ & 6.12137600 & -3.48434900 & -1.69671200 & $\mathrm{H}$ & 5.48843900 & -2.14224000 & -2.93670700 \\
\hline $\mathrm{C}$ & 6.27693400 & -2.79831300 & 0.34850500 & $\mathrm{C}$ & 6.39884500 & -2.00780200 & -0.97722700 \\
\hline $\mathrm{H}$ & 7.27236600 & -3.22330200 & 0.49784000 & $\mathrm{H}$ & 7.38600800 & -2.31719700 & -1.32949600 \\
\hline $\mathrm{C}$ & 5.65050300 & -2.10724200 & 1.39190500 & $\mathrm{C}$ & 6.20432500 & -1.71472000 & 0.37534700 \\
\hline $\mathrm{H}$ & 6.15487900 & -1.99199000 & 2.35395700 & $\mathrm{H}$ & 7.03439200 & -1.79650400 & 1.08076000 \\
\hline $\mathrm{C}$ & 4.37895000 & -1.56503000 & 1.20575700 & C & 4.94287200 & -1.32177900 & 0.82961300 \\
\hline $\mathrm{H}$ & 3.89601700 & -1.02637000 & 2.02316900 & $\mathrm{H}$ & 4.78828200 & -1.10015700 & 1.88728600 \\
\hline $\mathrm{H}$ & -1.98277200 & -2.10865200 & -2.58024500 & $\mathrm{H}$ & -0.24460500 & -0.29679600 & 0.80029300 \\
\hline $\mathrm{H}$ & 0.13581400 & 1.48432100 & -3.46839900 & $\mathrm{H}$ & -1.33158100 & 0.13871500 & -1.84812800 \\
\hline $\mathrm{Mn}$ & -0.02872500 & -0.64563400 & -2.48782800 & $\mathrm{Mn}$ & 0.29108200 & -1.93279100 & -1.69286600 \\
\hline $\mathrm{O}$ & -1.11650600 & -1.97477600 & -2.99148300 & $\mathrm{O}$ & -0.47515000 & -3.24266700 & -2.24120600 \\
\hline $\mathrm{O}$ & 0.72281700 & 0.72562000 & -3.32979300 & $\mathrm{O}$ & 1.22640300 & -0.99194200 & -2.64301400 \\
\hline \multicolumn{4}{|c|}{ TS-1d } & \multicolumn{4}{|c|}{ INT-2d } \\
\hline $\mathrm{P}$ & 2.27368600 & -0.89823800 & 0.08974400 & $\mathrm{P}$ & 2.52705000 & -0.15130800 & 0.47865400 \\
\hline $\mathrm{O}$ & 1.53258600 & -1.71915500 & -0.98773100 & $\mathrm{O}$ & 1.74507700 & -0.66960700 & 1.70255300 \\
\hline $\mathrm{O}$ & -6.33350900 & -2.18856500 & -0.20079800 & $\mathrm{O}$ & -6.64311600 & -1.08915400 & 0.62236600 \\
\hline $\mathrm{N}$ & -1.22562900 & -0.05893700 & -1.02987300 & $\mathrm{~N}$ & -1.09418100 & -0.49721800 & 1.04716300 \\
\hline $\mathrm{N}$ & -2.80281400 & 0.77803400 & 1.14247400 & $\mathrm{~N}$ & -2.73645300 & 1.69095900 & 0.45658700 \\
\hline C & 1.95654600 & 0.87368600 & -0.12223300 & $\mathrm{C}$ & 1.53693300 & -0.17881500 & -1.04650000 \\
\hline C & 3.05543300 & 1.74214800 & -0.37402500 & C & 2.20641500 & -0.57605900 & -2.23676000 \\
\hline $\mathrm{H}$ & 4.07494800 & 1.36590600 & -0.28846800 & $\mathrm{H}$ & 3.27065200 & -0.81170400 & -2.20590800 \\
\hline C & 2.83298700 & 3.04984100 & -0.72819900 & $\mathrm{C}$ & 1.51893200 & -0.67692500 & -3.41862300 \\
\hline $\mathrm{H}$ & 3.67465800 & 3.72423500 & -0.90273000 & $\mathrm{H}$ & 2.03318600 & -0.97522600 & -4.33524200 \\
\hline C & 1.51231900 & 3.53684200 & -0.91204900 & C & 0.12342000 & -0.42735700 & -3.46116700 \\
\hline $\mathrm{C}$ & 1.27861100 & 4.87424800 & -1.33434600 & $\mathrm{C}$ & -0.59676100 & -0.58050700 & -4.67707500 \\
\hline
\end{tabular}




\begin{tabular}{|c|c|c|c|c|c|c|c|}
\hline $\mathrm{H}$ & 2.13829600 & 5.52853500 & -1.49820900 & $\mathrm{H}$ & -0.04587700 & -0.87810500 & -5.57280600 \\
\hline C & -0.00245500 & 5.33147200 & -1.54495100 & $\mathrm{C}$ & -1.95649200 & -0.37161500 & -4.72666900 \\
\hline $\mathrm{H}$ & -0.17280800 & 6.35936700 & -1.87358000 & $\mathrm{H}$ & -2.50106200 & -0.49682700 & -5.66553200 \\
\hline C & -1.10406900 & 4.46220800 & -1.35111300 & C & -2.64785300 & 0.00030000 & -3.55057700 \\
\hline $\mathrm{H}$ & -2.11731200 & 4.82173000 & -1.54293200 & $\mathrm{H}$ & -3.72859600 & 0.15564000 & -3.57882500 \\
\hline C & -0.91161700 & 3.16514400 & -0.93048300 & C & -1.97430400 & 0.16860600 & -2.35991900 \\
\hline $\mathrm{H}$ & -1.77006300 & 2.51178800 & -0.80356500 & $\mathrm{H}$ & -2.54581000 & 0.44557400 & -1.48575200 \\
\hline C & 0.39848000 & 2.66200600 & -0.68091400 & $\mathrm{C}$ & -0.56762200 & -0.03300300 & -2.26151900 \\
\hline C & 0.63970100 & 1.32715600 & -0.22143200 & $\mathrm{C}$ & 0.17118100 & 0.12696200 & -1.03884100 \\
\hline C & -0.52589700 & 0.41605400 & 0.17061900 & C & -0.47324400 & 0.59813200 & 0.28719700 \\
\hline C & -1.44465700 & 1.08829700 & 1.15983500 & C & -1.37767400 & 1.78810000 & 0.18879500 \\
\hline C & -1.21548900 & 2.02682000 & 2.15057000 & C & -1.06132200 & 3.10584200 & -0.07114600 \\
\hline C & -2.49013300 & 2.31568300 & 2.72752500 & $\mathrm{C}$ & -2.28031300 & 3.83938100 & 0.05485200 \\
\hline $\mathrm{H}$ & -2.68443300 & 3.03482700 & 3.52205800 & $\mathrm{H}$ & -2.39693300 & 4.91615100 & -0.06005200 \\
\hline C & -3.45555200 & 1.55472800 & 2.10111100 & C & -3.30057600 & 2.96719200 & 0.38362000 \\
\hline C & 0.06544800 & 2.70364900 & 2.53906000 & $\mathrm{C}$ & 0.29950800 & 3.64953100 & -0.38348300 \\
\hline $\mathrm{H}$ & 0.95139200 & 2.11266000 & 2.27770900 & $\mathrm{H}$ & 0.95712000 & 3.65153300 & 0.50185700 \\
\hline $\mathrm{H}$ & 0.09031300 & 2.88699700 & 3.62502800 & $\mathrm{H}$ & 0.23477500 & 4.68637600 & -0.74539600 \\
\hline $\mathrm{H}$ & 0.17468700 & 3.68099500 & 2.03810300 & $\mathrm{H}$ & 0.81054500 & 3.05663500 & -1.15831500 \\
\hline C & -4.92846900 & 1.59743900 & 2.35026400 & C & -4.72042400 & 3.31765900 & 0.69034500 \\
\hline $\mathrm{H}$ & -5.14646100 & 2.47179700 & 2.97983100 & $\mathrm{H}$ & -4.79121000 & 4.40855000 & 0.80588400 \\
\hline $\mathrm{H}$ & -5.30780600 & 0.70960200 & 2.88359900 & $\mathrm{H}$ & -5.07306000 & 2.85514400 & 1.62545800 \\
\hline $\mathrm{H}$ & -5.50926700 & 1.69641600 & 1.42014800 & $\mathrm{H}$ & -5.42562100 & 3.02964000 & -0.10776100 \\
\hline C & -3.32562700 & -0.19604600 & 0.25752400 & $\mathrm{C}$ & -3.33824900 & 0.42898400 & 0.66094000 \\
\hline $\mathrm{C}$ & -4.58136700 & -0.77880300 & 0.44219800 & $\mathrm{C}$ & -4.71447100 & 0.23375600 & 0.54365400 \\
\hline $\mathrm{H}$ & -5.19746500 & -0.55195500 & 1.30423800 & $\mathrm{H}$ & -5.38308800 & 1.04344100 & 0.27574300 \\
\hline C & -5.09294000 & -1.69827700 & -0.48158500 & $\mathrm{C}$ & -5.28870200 & -1.02483700 & 0.75930500 \\
\hline $\mathrm{C}$ & -4.32342800 & -2.06018400 & -1.59176400 & C & -4.46363900 & -2.10536100 & 1.08430700 \\
\hline $\mathrm{H}$ & -4.68219300 & -2.78003200 & -2.32622300 & $\mathrm{H}$ & -4.87048400 & -3.09891300 & 1.26832500 \\
\hline
\end{tabular}




\begin{tabular}{|c|c|c|c|c|c|c|c|}
\hline C & -3.04583600 & -1.51898100 & -1.74487600 & $\mathrm{C}$ & -3.08305700 & -1.91981300 & 1.15918900 \\
\hline $\mathrm{H}$ & -2.42176600 & -1.85822000 & -2.57417400 & $\mathrm{H}$ & -2.44601400 & -2.76405000 & 1.41963700 \\
\hline C & -2.51615800 & -0.58929300 & -0.84060300 & $\mathrm{C}$ & -2.47457800 & -0.67153900 & 0.94474200 \\
\hline C & -6.89718800 & -3.12858900 & -1.08187700 & $\mathrm{C}$ & -7.27824900 & -2.32696200 & 0.82639700 \\
\hline $\mathrm{H}$ & -7.02618500 & -2.71533100 & -2.09910000 & $\mathrm{H}$ & -6.92693700 & -3.09386800 & 0.11161200 \\
\hline $\mathrm{H}$ & -7.88431400 & -3.38949100 & -0.67661000 & $\mathrm{H}$ & -8.35278300 & -2.16080500 & 0.67001200 \\
\hline $\mathrm{H}$ & -6.28532000 & -4.04662600 & -1.15290300 & $\mathrm{H}$ & -7.12068100 & -2.70728100 & 1.85237700 \\
\hline C & 1.79350300 & -1.44427500 & 1.74360000 & $\mathrm{C}$ & 3.06691500 & 1.54839700 & 0.78463700 \\
\hline C & 1.25433700 & -2.73140600 & 1.88897600 & $\mathrm{C}$ & 2.98349100 & 2.05481900 & 2.08874500 \\
\hline $\mathrm{H}$ & 1.12091800 & -3.36109500 & 1.00683500 & $\mathrm{H}$ & 2.58994100 & 1.41802200 & 2.88347700 \\
\hline C & 0.86772200 & -3.17527200 & 3.15411000 & $\mathrm{C}$ & 3.38444700 & 3.36773900 & 2.34680600 \\
\hline $\mathrm{H}$ & 0.43782600 & -4.17258600 & 3.27029300 & $\mathrm{H}$ & 3.31154600 & 3.76822900 & 3.36037900 \\
\hline C & 1.01875000 & -2.34247000 & 4.26787000 & $\mathrm{C}$ & 3.87180800 & 4.16839600 & 1.30969300 \\
\hline $\mathrm{H}$ & 0.70673300 & -2.69142200 & 5.25488600 & $\mathrm{H}$ & 4.18119900 & 5.19605600 & 1.51391200 \\
\hline C & 1.55569700 & -1.05988300 & 4.12069900 & C & 3.95521600 & 3.66152600 & 0.00816300 \\
\hline $\mathrm{H}$ & 1.65980500 & -0.40492200 & 4.98839200 & $\mathrm{H}$ & 4.32450500 & 4.29259100 & -0.80317800 \\
\hline C & 1.94335600 & -0.60679600 & 2.85878600 & C & 3.54804900 & 2.35426000 & -0.25832600 \\
\hline $\mathrm{H}$ & 2.33801400 & 0.40440700 & 2.73825300 & $\mathrm{H}$ & 3.58584400 & 1.96667800 & -1.27914800 \\
\hline C & 4.05250100 & -1.17539800 & -0.09610700 & C & 3.99994800 & -1.16604400 & 0.19437100 \\
\hline C & 4.52623800 & -1.53862000 & -1.36581000 & $\mathrm{C}$ & 3.80804100 & -2.55751200 & 0.10860200 \\
\hline $\mathrm{H}$ & 3.81852100 & -1.67579400 & -2.18545500 & $\mathrm{H}$ & 2.79413200 & -2.96595000 & 0.18618700 \\
\hline C & 5.89426800 & -1.73393700 & -1.56360100 & $\mathrm{C}$ & 4.91275800 & -3.38799400 & -0.08023600 \\
\hline $\mathrm{H}$ & 6.26360400 & -2.01901700 & -2.55114700 & $\mathrm{H}$ & 4.76941100 & -4.46892600 & -0.14822100 \\
\hline C & 6.78685700 & -1.57001400 & -0.50003400 & $\mathrm{C}$ & 6.19860700 & -2.84460500 & -0.18004900 \\
\hline $\mathrm{H}$ & 7.85681300 & -1.72484500 & -0.65761200 & $\mathrm{H}$ & 7.05902700 & -3.50199100 & -0.32718800 \\
\hline C & 6.31325600 & -1.21708000 & 0.76782500 & C & 6.38606600 & -1.46322600 & -0.08682800 \\
\hline $\mathrm{H}$ & 7.01014800 & -1.10010000 & 1.60070500 & $\mathrm{H}$ & 7.39017600 & -1.03875600 & -0.15669700 \\
\hline $\mathrm{C}$ & 4.94643700 & -1.02246200 & 0.97393000 & C & 5.28800000 & -0.62013600 & 0.10361800 \\
\hline $\mathrm{H}$ & 4.57745500 & -0.76152300 & 1.96782400 & $\mathrm{H}$ & 5.43807300 & 0.45751700 & 0.18622400 \\
\hline
\end{tabular}




\begin{tabular}{|c|c|c|c|c|c|c|c|}
\hline $\mathrm{H}$ & -0.09915400 & -0.46170200 & 0.69041000 & $\mathrm{H}$ & 0.36242400 & 0.96067300 & 0.89811000 \\
\hline $\mathrm{H}$ & -0.83247900 & 0.48185800 & -2.12892700 & $\mathrm{H}$ & 0.26628300 & -3.80692300 & 0.37478700 \\
\hline $\mathrm{Mr}$ & 0.15965700 & -1.19047000 & -2.35365100 & $\mathrm{Mn}$ & 0.08288900 & -1.84469400 & 1.65037500 \\
\hline 0 & -0.27498300 & -2.52303500 & -3.11627900 & $\mathrm{O}$ & -0.64510000 & -2.83165400 & 2.67417800 \\
\hline $\mathrm{O}$ & -0.00460400 & 0.34167600 & -3.05154100 & $\mathrm{O}$ & 0.67970900 & -2.93358300 & 0.32453300 \\
\hline \multicolumn{4}{|c|}{ TS-2d } & \multicolumn{4}{|c|}{ INT-3d } \\
\hline $\mathrm{P}$ & -2.50334000 & -0.63303900 & 0.21388800 & $\mathrm{P}$ & 1.99403200 & -1.15183900 & -0.31326100 \\
\hline $\mathrm{O}$ & -1.75600400 & -1.79558800 & 0.88802600 & 0 & 1.57529300 & -1.64084300 & -1.71366400 \\
\hline O & 6.71026600 & -1.55800500 & 0.76169600 & $\mathrm{O}$ & -6.42340400 & -0.92479600 & -0.31309500 \\
\hline $\mathrm{N}$ & 1.22187000 & -0.69950900 & 0.57799700 & $\mathrm{~N}$ & -1.01559500 & 0.24867400 & -1.07042200 \\
\hline $\mathrm{N}$ & 2.94912100 & 0.66040700 & -1.23073000 & $\mathrm{~N}$ & -2.22731700 & 0.58394000 & 1.45166500 \\
\hline C & -1.70828400 & 0.98481300 & 0.53509600 & C & 2.16309300 & 0.64897700 & -0.29276700 \\
\hline C & -2.57041300 & 2.00078800 & 1.04646800 & C & 3.45755300 & 1.21712300 & -0.42549000 \\
\hline $\mathrm{H}$ & -3.64146900 & 1.81285200 & 1.10658400 & $\mathrm{H}$ & 4.33087900 & 0.56412900 & -0.44425900 \\
\hline C & -2.08141800 & 3.21478300 & 1.45548300 & C & 3.62346400 & 2.58013000 & -0.51006000 \\
\hline $\mathrm{H}$ & -2.76058800 & 3.99458100 & 1.80803900 & $\mathrm{H}$ & 4.62485700 & 3.01008000 & -0.58704300 \\
\hline C & -0.68403900 & 3.44767000 & 1.48108700 & C & 2.49830500 & 3.44713600 & -0.51861400 \\
\hline C & -0.15461300 & 4.66100500 & 2.00160400 & C & 2.64289200 & 4.85688900 & -0.62378800 \\
\hline $\mathrm{H}$ & -0.85317100 & 5.43073700 & 2.33906400 & $\mathrm{H}$ & 3.65069800 & 5.27655300 & -0.67501900 \\
\hline C & 1.20432300 & 4.85342300 & 2.09924500 & C & 1.53786300 & 5.68077800 & -0.66718400 \\
\hline $\mathrm{H}$ & 1.60297300 & 5.78585000 & 2.50573400 & $\mathrm{H}$ & 1.66321000 & 6.76289000 & -0.75106800 \\
\hline C & 2.08691400 & 3.82329600 & 1.69435900 & C & 0.23692100 & 5.12473300 & -0.61980500 \\
\hline $\mathrm{H}$ & 3.16492000 & 3.95482900 & 1.81087500 & $\mathrm{H}$ & -0.63522600 & 5.77987400 & -0.67817900 \\
\hline C & 1.60172600 & 2.64940400 & 1.16104800 & C & 0.06451400 & 3.76046400 & -0.50786700 \\
\hline $\mathrm{H}$ & 2.30930100 & 1.87403000 & 0.88834300 & $\mathrm{H}$ & -0.93829800 & 3.33484400 & -0.47263800 \\
\hline C & 0.20178700 & 2.42444800 & 1.00409300 & C & 1.18240200 & 2.88323200 & -0.43121500 \\
\hline C & -0.33115100 & 1.21492700 & 0.42314500 & C & 1.01913300 & 1.46144600 & -0.29074800 \\
\hline C & 0.65373800 & 0.28210200 & -0.29251600 & C & -0.35135900 & 0.93298100 & -0.07662800 \\
\hline C & 1.58485200 & 0.97898800 & -1.19872200 & C & -0.95482800 & 1.14066300 & 1.19150700 \\
\hline
\end{tabular}




\begin{tabular}{|c|c|c|c|c|c|c|c|}
\hline C & 1.32787100 & 1.93041200 & -2.17115600 & $\mathrm{C}$ & -0.48146100 & 1.69816200 & 2.39528200 \\
\hline C & 2.57199800 & 2.19645300 & -2.80729000 & $\mathrm{C}$ & -1.48055200 & 1.44460600 & 3.36798300 \\
\hline $\mathrm{H}$ & 2.73435800 & 2.88659600 & -3.63412000 & $\mathrm{H}$ & -1.41922100 & 1.71469600 & 4.42159900 \\
\hline C & 3.56088700 & 1.41916600 & -2.23004900 & $\mathrm{C}$ & -2.53638700 & 0.75377400 & 2.79928100 \\
\hline C & 0.00113000 & 2.54630600 & -2.49114100 & C & 0.81250000 & 2.40108700 & 2.67412100 \\
\hline $\mathrm{H}$ & -0.32850400 & 3.24848300 & -1.70606700 & $\mathrm{H}$ & 0.83783700 & 3.41833500 & 2.24870800 \\
\hline $\mathrm{H}$ & -0.78224700 & 1.78019100 & -2.59015500 & $\mathrm{H}$ & 1.68285200 & 1.87235800 & 2.25876700 \\
\hline $\mathrm{H}$ & 0.04860000 & 3.10268200 & -3.43904000 & $\mathrm{H}$ & 0.96046400 & 2.49382800 & 3.76086700 \\
\hline C & 4.99735100 & 1.36863500 & -2.63666100 & C & -3.74121600 & 0.23014800 & 3.50819500 \\
\hline $\mathrm{H}$ & 5.12365300 & 2.00271100 & -3.52532900 & $\mathrm{H}$ & -3.58149200 & 0.34525800 & 4.58969400 \\
\hline $\mathrm{H}$ & 5.32891000 & 0.35249700 & -2.90432700 & $\mathrm{H}$ & -3.91451100 & -0.83887700 & 3.30737300 \\
\hline $\mathrm{H}$ & 5.68319200 & 1.75201800 & -1.86283000 & $\mathrm{H}$ & -4.66988900 & 0.77125000 & 3.25893900 \\
\hline C & 3.49444700 & -0.23614600 & -0.29391500 & C & -3.00544100 & 0.09889100 & 0.39016900 \\
\hline C & 4.86285500 & -0.48664700 & -0.17884900 & C & -4.35879700 & -0.21714300 & 0.52102700 \\
\hline $\mathrm{H}$ & 5.59175100 & 0.00746500 & -0.80829800 & $\mathrm{H}$ & -4.87523300 & -0.13575600 & 1.46894100 \\
\hline C & 5.36126200 & -1.40125100 & 0.75859900 & C & -5.12308100 & -0.63069100 & -0.57929100 \\
\hline C & 4.47840800 & -2.08040900 & 1.61270300 & C & -4.53515600 & -0.68857400 & -1.84655100 \\
\hline $\mathrm{H}$ & 4.83677500 & -2.79541600 & 2.35201900 & $\mathrm{H}$ & -5.10690100 & -0.95915300 & -2.73296100 \\
\hline C & 3.11744200 & -1.81829000 & 1.51897400 & C & -3.18383200 & -0.36307100 & -1.97843500 \\
\hline $\mathrm{H}$ & 2.40747200 & -2.33055400 & 2.17118100 & $\mathrm{H}$ & -2.73675300 & -0.37234600 & -2.97316200 \\
\hline C & 2.58326400 & -0.90389600 & 0.58513700 & C & -2.37468400 & -0.01742600 & -0.88400500 \\
\hline C & 7.28687200 & -2.46364300 & 1.67317900 & C & -7.25676600 & -1.31885100 & -1.37683800 \\
\hline $\mathrm{H}$ & 7.08023700 & -2.17957200 & 2.72085900 & $\mathrm{H}$ & -7.34692900 & -0.52782200 & -2.14346800 \\
\hline $\mathrm{H}$ & 8.37124500 & -2.43309900 & 1.50298700 & $\mathrm{H}$ & -8.24834000 & -1.51144400 & -0.94600300 \\
\hline $\mathrm{H}$ & 6.92736400 & -3.49561100 & 1.50954400 & $\mathrm{H}$ & -6.89222600 & -2.24144100 & -1.86420500 \\
\hline C & -2.83322200 & -0.80356200 & -1.55569200 & C & 0.77356400 & -1.66976100 & 0.91928900 \\
\hline C & -2.92072500 & -2.08365400 & -2.11899900 & C & -0.26620200 & -2.51038200 & 0.48872000 \\
\hline $\mathrm{H}$ & -2.70049600 & -2.95758900 & -1.50583600 & $\mathrm{H}$ & -0.32425000 & -2.81192800 & -0.55985500 \\
\hline C & -3.25492100 & -2.22125300 & -3.46464200 & C & -1.24817800 & -2.90802000 & 1.39829300 \\
\hline
\end{tabular}




\begin{tabular}{|c|c|c|c|c|c|c|c|}
\hline $\mathrm{H}$ & -3.31323800 & -3.21727800 & -3.90919000 & $\mathrm{H}$ & -2.07107700 & -3.54126600 & 1.05926400 \\
\hline C & -3.49671700 & -1.08812500 & -4.25055300 & $\mathrm{C}$ & -1.18839400 & -2.48767600 & 2.72984700 \\
\hline $\mathrm{H}$ & -3.74958500 & -1.20197200 & -5.30740600 & $\mathrm{H}$ & -1.96232400 & -2.79702900 & 3.43632900 \\
\hline C & -3.40666800 & 0.18759500 & -3.68940200 & C & -0.14491800 & -1.66182500 & 3.15903200 \\
\hline $\mathrm{H}$ & -3.58628700 & 1.07341100 & -4.30283900 & $\mathrm{H}$ & -0.10966800 & -1.31391700 & 4.19256600 \\
\hline C & -3.07491400 & 0.33215600 & -2.33890100 & $\mathrm{C}$ & 0.83309500 & -1.24815900 & 2.25669500 \\
\hline $\mathrm{H}$ & -2.99505100 & 1.32859600 & -1.89887300 & $\mathrm{H}$ & 1.61974700 & -0.57000400 & 2.59073200 \\
\hline C & -4.14184000 & -0.57345700 & 1.00481200 & C & 3.59606500 & -1.88605400 & 0.10178000 \\
\hline $\mathrm{C}$ & -4.22155700 & -0.98570000 & 2.34420400 & $\mathrm{C}$ & 4.15875400 & -2.78178400 & -0.81759900 \\
\hline $\mathrm{H}$ & -3.31931700 & -1.33581200 & 2.84910400 & $\mathrm{H}$ & 3.62706300 & -2.98569300 & -1.74899300 \\
\hline C & -5.44961100 & -0.96285400 & 3.00665500 & $\mathrm{C}$ & 5.38160200 & -3.39357200 & -0.52980300 \\
\hline $\mathrm{H}$ & -5.50987400 & -1.28606300 & 4.04845500 & $\mathrm{H}$ & 5.82027800 & -4.09169400 & -1.24644000 \\
\hline C & -6.60053200 & -0.53539100 & 2.33687600 & $\mathrm{C}$ & 6.04163300 & -3.11219700 & 0.66901500 \\
\hline $\mathrm{H}$ & -7.56135500 & -0.52073500 & 2.85699500 & $\mathrm{H}$ & 6.99824500 & -3.59100200 & 0.89144200 \\
\hline C & -6.52512400 & -0.13743900 & 0.99892300 & C & 5.48066500 & -2.21633300 & 1.58639000 \\
\hline $\mathrm{H}$ & -7.42565600 & 0.18376600 & 0.47045000 & $\mathrm{H}$ & 5.99802100 & -1.99432900 & 2.52251900 \\
\hline C & -5.29899100 & -0.15890200 & 0.33009500 & C & 4.25913400 & -1.60406000 & 1.30646200 \\
\hline $\mathrm{H}$ & -5.24536000 & 0.13943200 & -0.71844900 & $\mathrm{H}$ & 3.83021100 & -0.90033600 & 2.02243400 \\
\hline $\mathrm{H}$ & 0.07084800 & -0.50500200 & -1.05708400 & $\mathrm{H}$ & -1.91989400 & -2.32882700 & -2.49546100 \\
\hline $\mathrm{H}$ & 1.37338600 & -4.22147800 & 1.46488500 & $\mathrm{H}$ & 0.59992300 & 1.61302200 & -2.96035500 \\
\hline $\mathrm{Mn}$ & 0.13417700 & -2.37685100 & 0.36618500 & $\mathrm{Mn}$ & -0.05751800 & -0.68964700 & -2.52642300 \\
\hline $\mathrm{O}$ & -0.10840100 & -1.90028100 & -1.26058200 & $\mathrm{O}$ & -0.99842300 & -2.22999100 & -2.76536400 \\
\hline $\mathrm{O}$ & 0.50184300 & -3.80793600 & 1.46725200 & $\mathrm{O}$ & 0.78958400 & 0.71717900 & -3.26771000 \\
\hline
\end{tabular}

[1] M. J. Frisch, G. W. Trucks, H. B. Schlegel, G. E. Scuseria, M. A. Robb, J. R. Cheeseman, G. Scalmani, V. Barone, G. A. Petersson, H. Nakatsuji, X. Li, M. Caricato, A. V. Marenich, J. Bloino, B. G. Janesko, R. Gomperts, B. Mennucci, H. P. Hratchian, J. V. Ortiz, A. F. Izmaylov, J. L. Sonnenberg, D. Williams-Young, F. Ding, F. Lipparini, F. Egidi, J. Goings, B. Peng, A. Petrone, T. Henderson, D. Ranasinghe, V. G. Zakrzewski, J. Gao, N. Rega, G. Zheng, W. Liang, M. Hada, M. Ehara, K. Toyota, R. Fukuda, J. Hasegawa, M. Ishida, T. Nakajima, Y. Honda, O. Kitao, H. Nakai, T. Vreven, K. Throssell, J. A. Montgomery, Jr., J. E. Peralta, F. Ogliaro, M. J. Bearpark, J. J. Heyd, E. N. Brothers, K. N. Kudin, V. N. Staroverov, T. A. Keith, R. Kobayashi, J. Normand, K. Raghavachari, A. P. Rendell, J. C. Burant, S. S. Iyengar, J. Tomasi, 
M. Cossi, J. M. Millam, M. Klene, C. Adamo, R. Cammi, J. W. Ochterski, R. L. Martin, K. Morokuma, O. Farkas, J. B. Foresman, and D. J. Fox, 2016, Gaussian 16, Revision A.03, Gaussian, Inc., Wallingford CT.

[2] A.D.Becke. J. Chem. Phys.1992, 96, 2155.

[3] A.D.Becke. J. Chem. Phys.1993, 98, 5648.

[4] C. Lee, W. Yang, R.G. Parr. Phys. Rev. B.1988, 37,785

[5] S. Grimme, S. Ehrlich and L. Goerigk. J. Comp. Chem. 2011,32,1456.

[6] K. Fukui. J. Phys. Chem.1970, 74, 4161 


\section{HPLC}

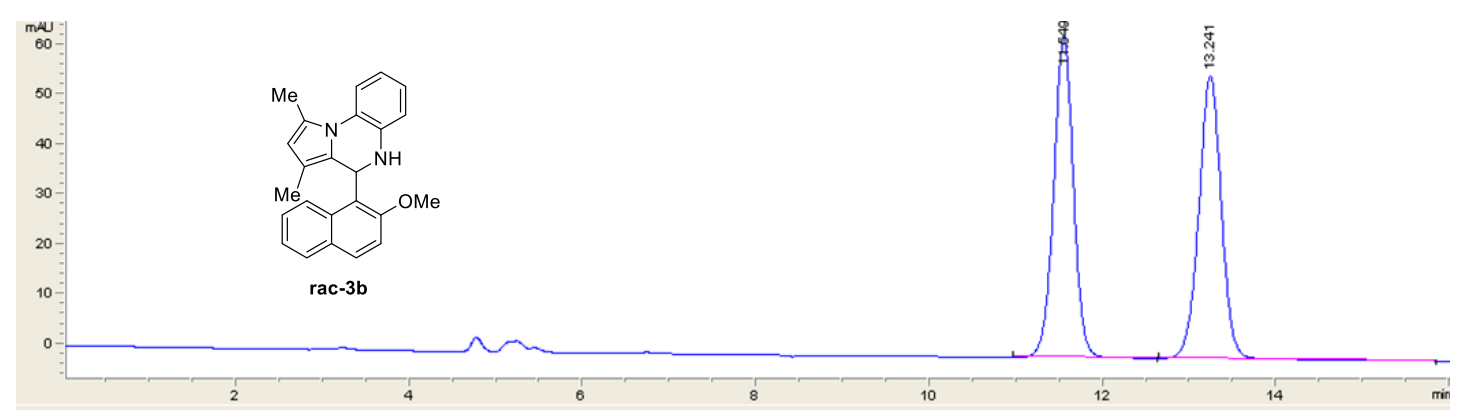

n-hexane/isopropanol 95:5, IA, $1.0 \mathrm{~mL} / \mathrm{min}$

\begin{tabular}{|c|c|c|c|c|c|}
\hline Peak \# & RetTime [min] & Width [min] & Area [mAU*s] & Height [mAU] & Area \% \\
\hline 1 & 11.549 & 0.2429 & 1020.9 & 64.4 & 49.959 \\
2 & 13.241 & 0.2766 & 1022.6 & 56.7 & 50.041 \\
\hline
\end{tabular}

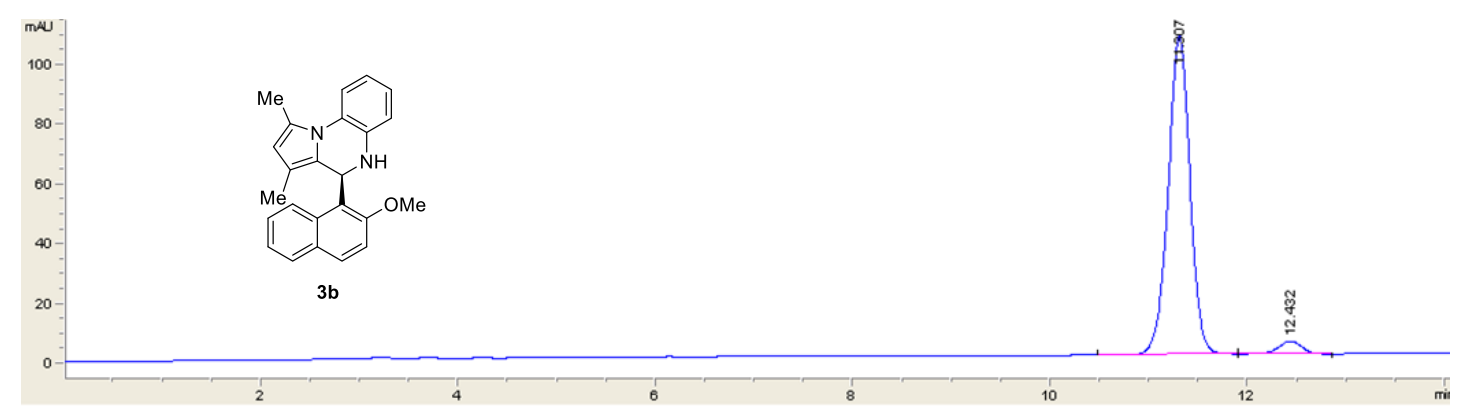

n-hexane/isopropanol 95:5, IA, $1.0 \mathrm{~mL} / \mathrm{min}$

\begin{tabular}{|c|c|c|c|c|c|}
\hline Peak \# & RetTime [min] & Width [min] & Area [mAU*s] & Height [mAU] & Area \% \\
\hline 1 & 11.307 & 0.2356 & 1644.5 & 106.8 & 95.898 \\
2 & 12.432 & 0.2532 & 70.3 & 4.3 & 4.102 \\
\hline
\end{tabular}

\begin{tabular}{|c|c|c|c|c|c|}
\hline Peak \# & RetTime [min] & Width [min] & Area [mAUs] & Height [mAU] & Area \% \\
\hline 1 & 5.536 & 0.1227 & 998.1 & 123.1 & 49.661 \\
\hline 2 & 6.592 & 0.2051 & 1011.9 & 78.6 & 50.339 \\
\hline
\end{tabular}




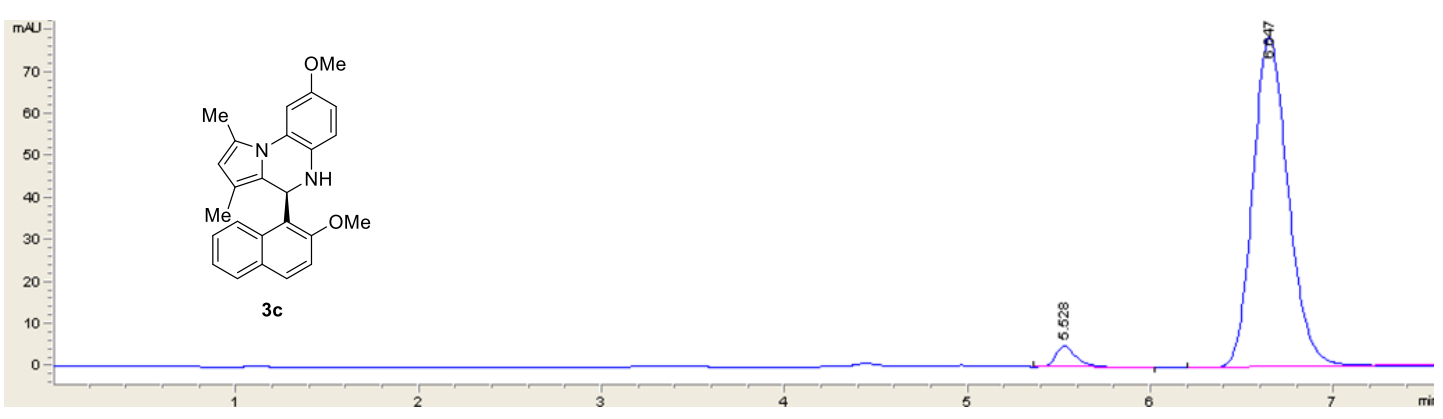

n-hexane/isopropanol 80:20, IC, $1.0 \mathrm{~mL} / \mathrm{min}$

\begin{tabular}{|c|c|c|c|c|c|}
\hline Peak \# & RetTime [min] & Width [min] & Area [mAU*s] & Height [mAU] & Area \% \\
\hline 1 & 5.528 & 0.1167 & 40.7 & 5.0 & 3.686 \\
2 & 6.647 & 0.2097 & 1063.9 & 78.7 & 96.314 \\
\hline
\end{tabular}

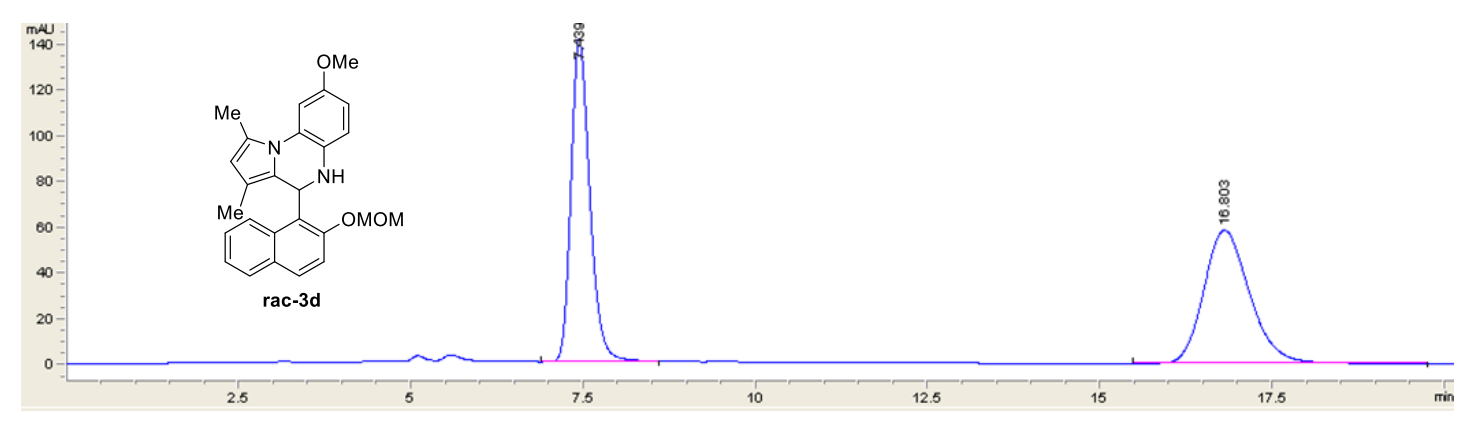

n-hexane/isopropanol 70:30, OD-H, $1.0 \mathrm{~mL} / \mathrm{min}$

\begin{tabular}{|c|c|c|c|c|c|}
\hline Peak \# & RetTime [min] & Width [min] & Area [mAU*s] & Height [mAU] & Area \% \\
\hline 1 & 7.439 & 0.2942 & 2688.9 & 141.2 & 49.864 \\
2 & 16.803 & 0.715 & 2703.5 & 58.1 & 50.136 \\
\hline
\end{tabular}

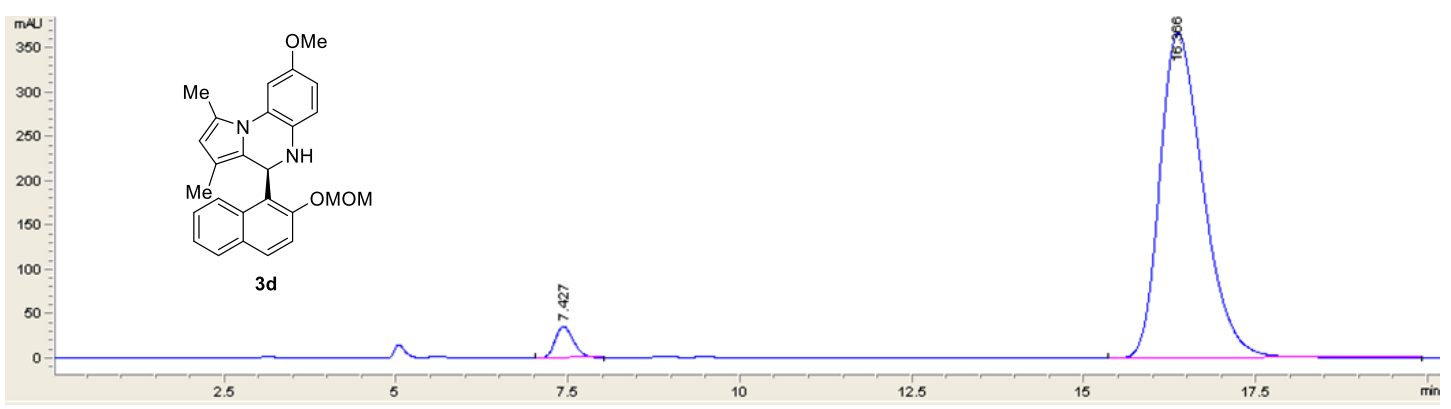

n-hexane/isopropanol 70:30, OD-H, $1.0 \mathrm{~mL} / \mathrm{min}$

\begin{tabular}{|c|c|c|c|c|c|}
\hline Peak \# & RetTime [min] & Width [min] & Area [mAU*s] & Height [mAU] & Area \% \\
\hline 1 & 7.427 & 0.2877 & 660.2 & 35.4 & 3.939 \\
2 & 16.366 & 0.6734 & 16100.4 & 367.3 & 96.061 \\
\hline
\end{tabular}




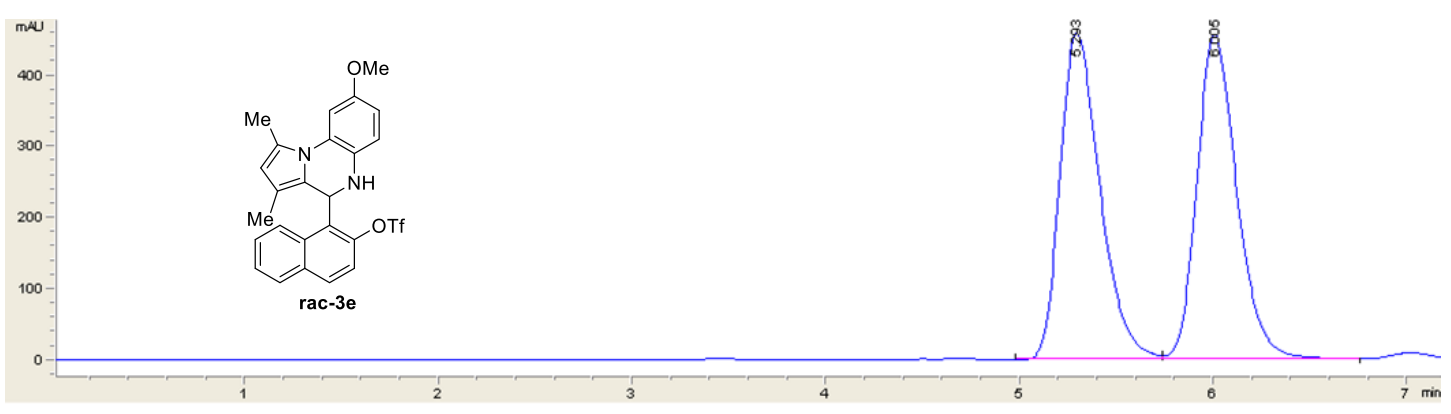

n-hexane/isopropanol 60:40, OD-H, $1.0 \mathrm{~mL} / \mathrm{min}$

\begin{tabular}{|c|c|c|c|c|c|}
\hline Peak \# & RetTime [min] & Width [min] & Area [mAU*s] & Height [mAU] & Area \% \\
\hline 1 & 5.293 & 0.2215 & 6593.7 & 459.2 & 50.110 \\
2 & 6.005 & 0.2220 & 6564.7 & 455.8 & 49.890 \\
\hline
\end{tabular}

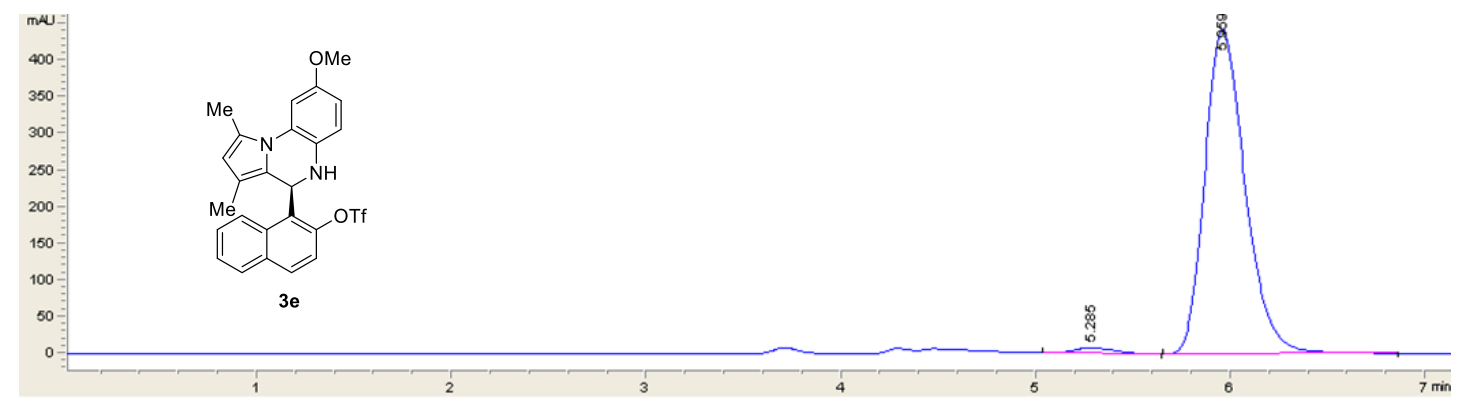

n-hexane/isopropanol 60:40, OD-H, $1.0 \mathrm{~mL} / \mathrm{min}$

\begin{tabular}{|c|c|c|c|c|c|}
\hline Peak \# & RetTime [min] & Width [min] & Area [mAU*s] & Height [mAU] & Area \% \\
\hline 1 & 5.285 & 0.2257 & 108.9 & 7.5 & 1.672 \\
2 & 5.959 & 0.2223 & 6400.0 & 443.6 & 98.328 \\
\hline
\end{tabular}

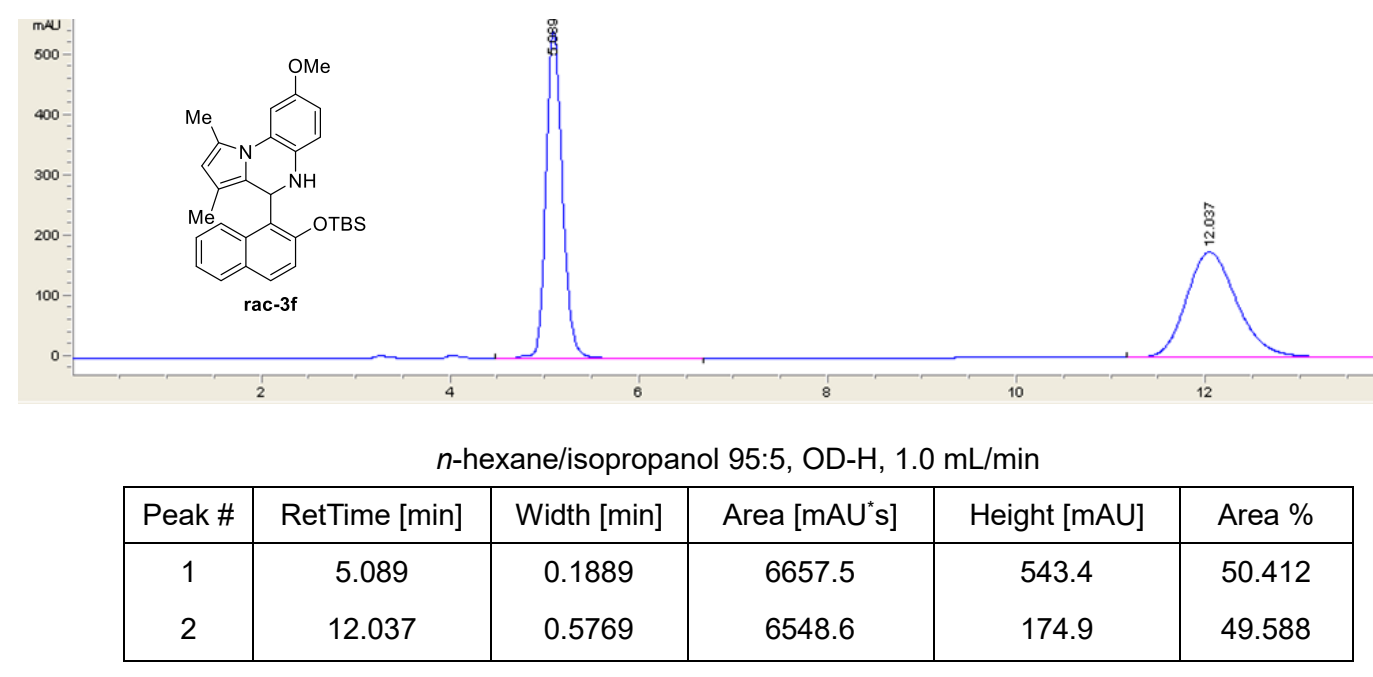




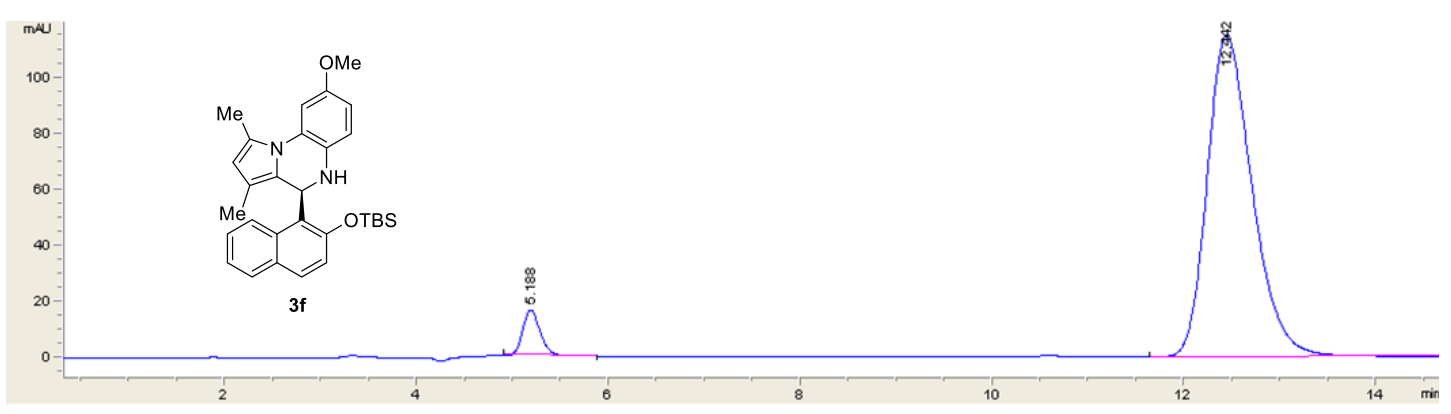

n-hexane/isopropanol 95:5, OD-H, $1.0 \mathrm{~mL} / \mathrm{min}$

\begin{tabular}{|c|c|c|c|c|c|}
\hline Peak \# & RetTime [min] & Width [min] & Area [mAU*s] & Height [mAU] & Area \% \\
\hline 1 & 5.188 & 0.1937 & 202.8 & 16.2 & 5.126 \\
2 & 12.442 & 0.4953 & 3753.1 & 114.8 & 94.874 \\
\hline
\end{tabular}

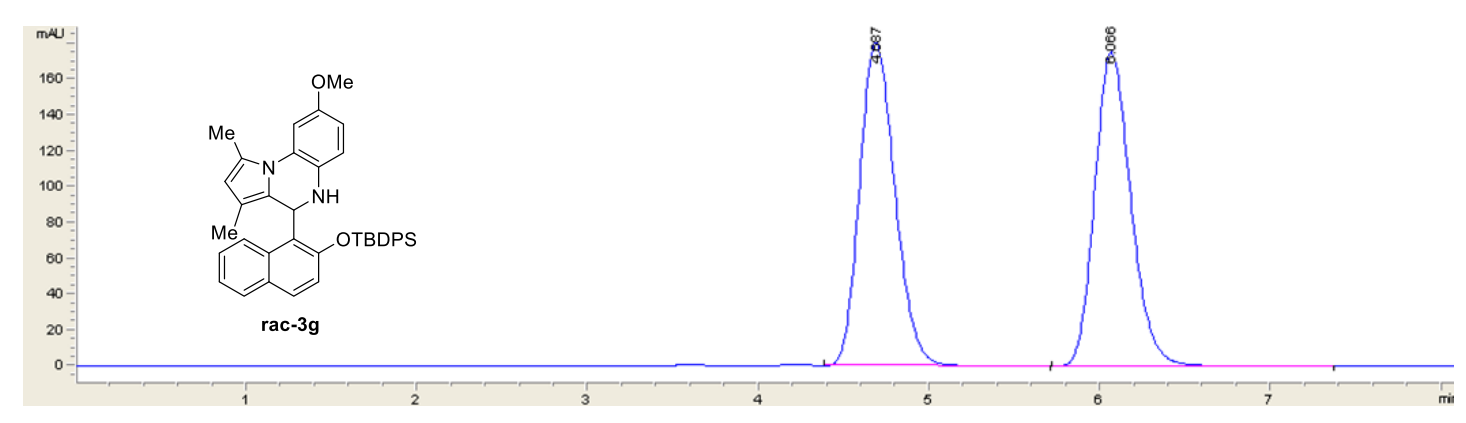

n-hexane/isopropanol 90:10, OD-H, $1.0 \mathrm{~mL} / \mathrm{min}$

\begin{tabular}{|c|c|c|c|c|c|}
\hline Peak \# & RetTime [min] & Width [min] & Area [mAU*s] & Height [mAU] & Area \% \\
\hline 1 & 4.687 & 0.2258 & 2596.8 & 180.5 & 50.171 \\
2 & 6.066 & 0.2276 & 2579.1 & 175.4 & 49.829 \\
\hline
\end{tabular}

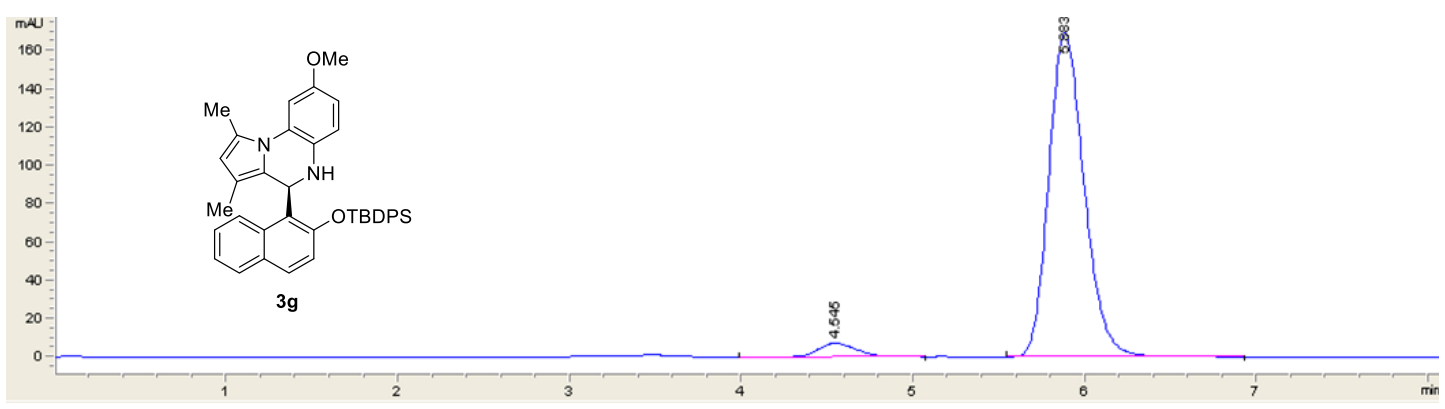

n-hexane/isopropanol 90:10, OD-H, $1.0 \mathrm{~mL} / \mathrm{min}$

\begin{tabular}{|c|c|c|c|c|c|}
\hline Peak \# & RetTime [min] & Width [min] & Area [mAU*s] & Height [mAU] & Area \% \\
\hline 1 & 4.545 & 0.2580 & 120.6 & 7.2 & 4.728 \\
2 & 5.883 & 0.2232 & 2430.8 & 169.6 & 95.272 \\
\hline
\end{tabular}




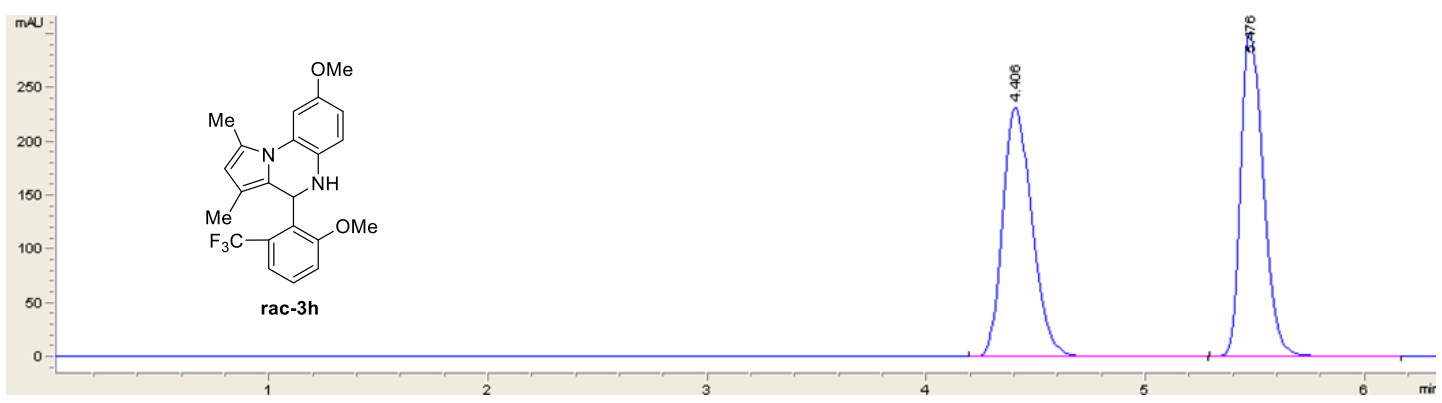

n-hexane/isopropanol 80:20, IC, $1.0 \mathrm{~mL} / \mathrm{min}$

\begin{tabular}{|c|c|c|c|c|c|}
\hline Peak \# & RetTime [min] & Width [min] & Area [mAU*s] & Height [mAU] & Area \% \\
\hline 1 & 4.406 & 0.1446 & 2157.3 & 231.7 & 49.940 \\
2 & 5.476 & 0.1114 & 2162.5 & 302.4 & 50.060 \\
\hline
\end{tabular}

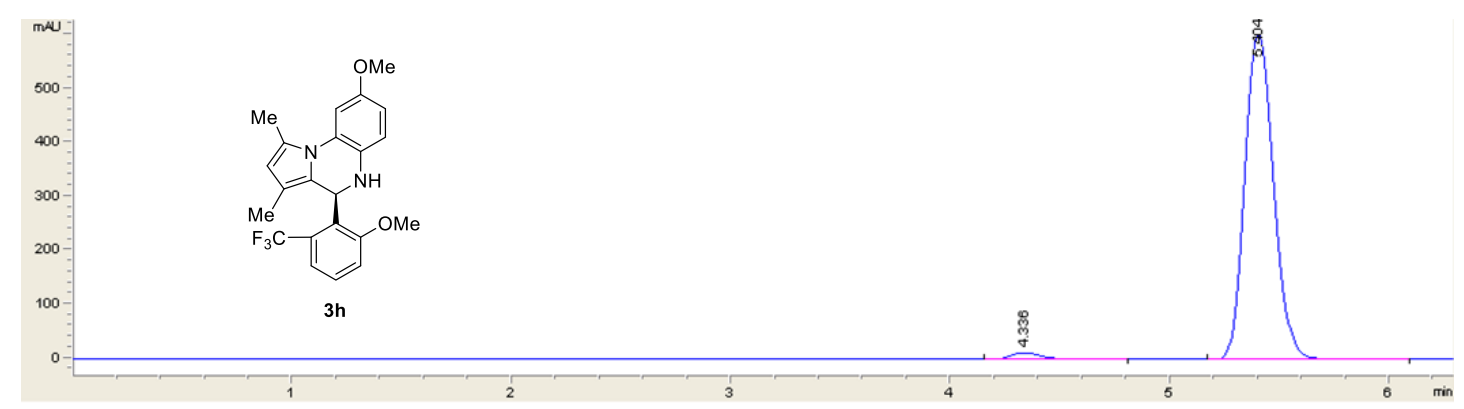

n-hexane/isopropanol 80:20, IC, $1.0 \mathrm{~mL} / \mathrm{min}$

\begin{tabular}{|c|c|c|c|c|c|}
\hline Peak \# & RetTime [min] & Width [min] & Area [mAU*s] & Height [mAU] & Area \% \\
\hline 1 & 4.336 & 0.1447 & 114.3 & 12.3 & 2.046 \\
2 & 5.404 & 0.1444 & 5474.5 & 600.1 & 97.954 \\
\hline
\end{tabular}

\begin{tabular}{|c|c|c|c|c|c|}
\hline \multicolumn{2}{|c|}{ RetTime [min] } & Width [min] & Area [mAUs] & Height [mAU] & Area \% \\
\hline 1 & 7.618 & 0.1882 & 1399.2 & 114.8 & 50.018 \\
\hline 2 & 8.423 & 0.2043 & 1398.2 & 105.7 & 49.982 \\
\hline
\end{tabular}




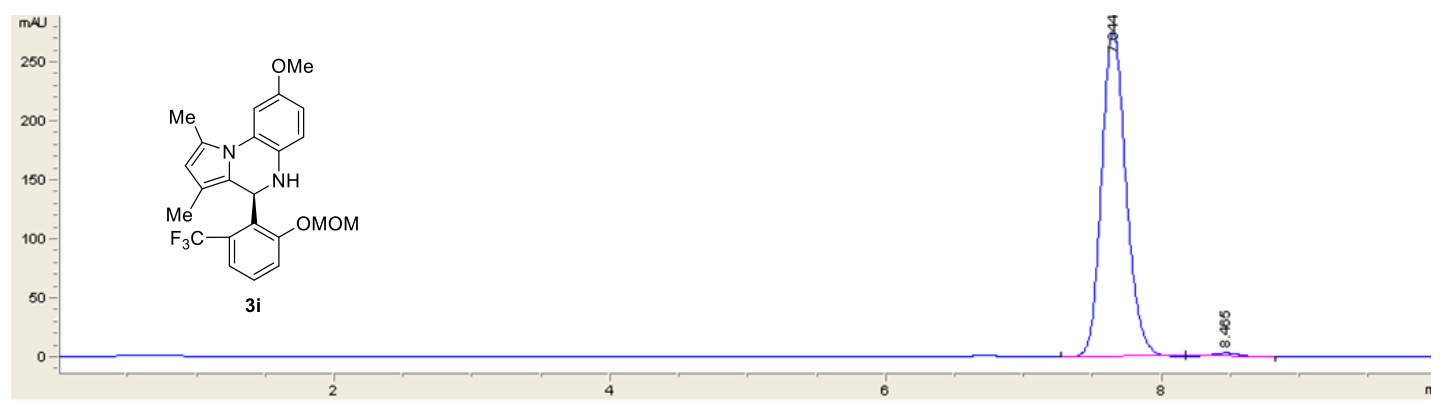

n-hexane/isopropanol 98:2, IA, $1.0 \mathrm{~mL} / \mathrm{min}$

\begin{tabular}{|c|c|c|c|c|c|}
\hline Peak \# & RetTime [min] & Width [min] & Area [mAUs] & Height [mAU] & Area \% \\
\hline 1 & 7.644 & 0.1900 & 3413.2 & 276.6 & 98.694 \\
2 & 8.465 & 0.2224 & 45.2 & 3.1 & 1.306 \\
\hline
\end{tabular}

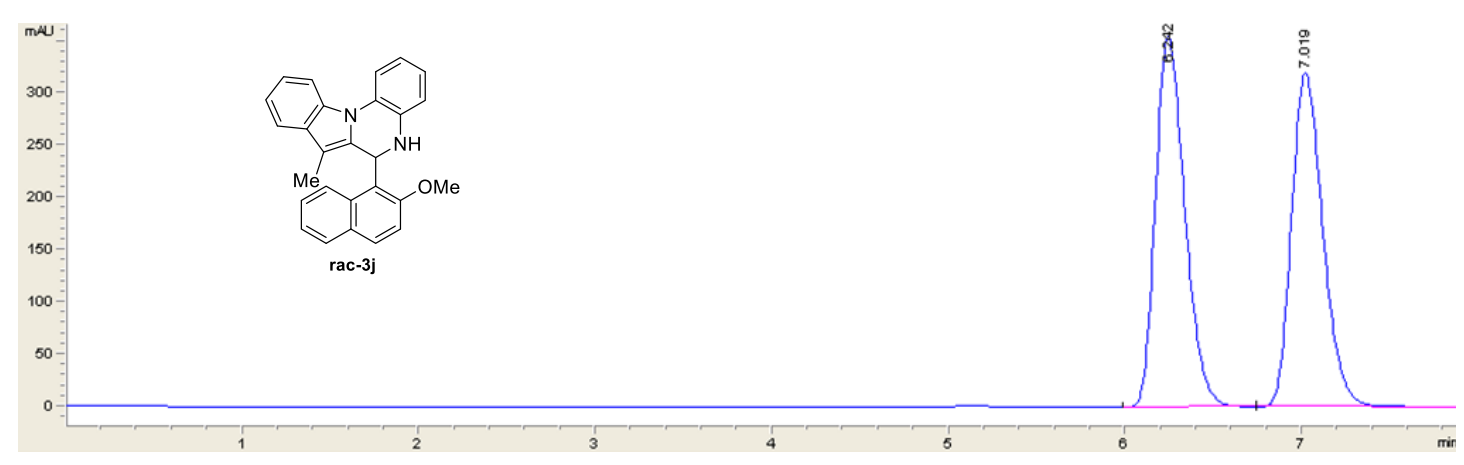

n-hexane/isopropanol 95:5, IC, $1.0 \mathrm{~mL} / \mathrm{min}$

\begin{tabular}{|c|c|c|c|c|c|}
\hline Peak \# & RetTime [min] & Width [min] & Area [mAU*s] & Height [mAU] & Area \% \\
\hline 1 & 6.242 & 0.1783 & 4094.0 & 355.7 & 50.933 \\
2 & 7.019 & 0.1899 & 3944.0 & 319.7 & 49.067 \\
\hline
\end{tabular}

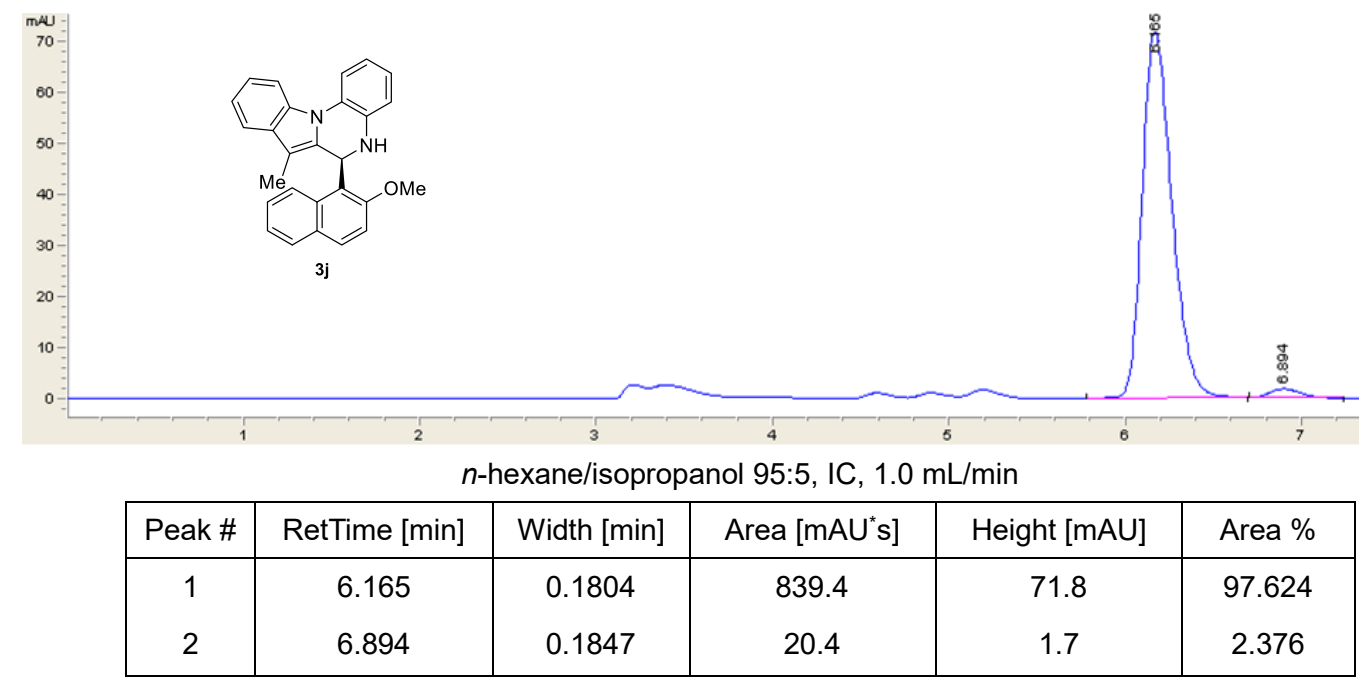




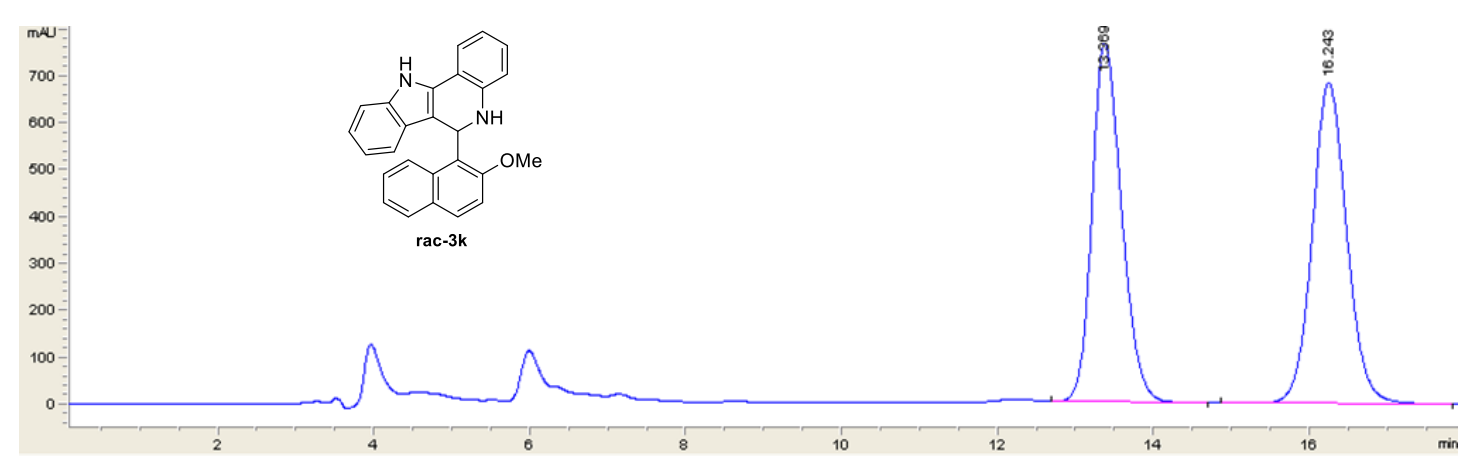

n-hexane/isopropanol 50:50, IA, $1.0 \mathrm{~mL} / \mathrm{min}$

\begin{tabular}{|c|c|c|c|c|c|}
\hline Peak \# & RetTime [min] & Width [min] & Area [mAU*s] & Height [mAU] & Area \% \\
\hline 1 & 13.369 & 0.4080 & 20198.1 & 765.1 & 48.957 \\
2 & 16.243 & 0.4797 & 21059.2 & 684.6 & 51.043 \\
\hline
\end{tabular}

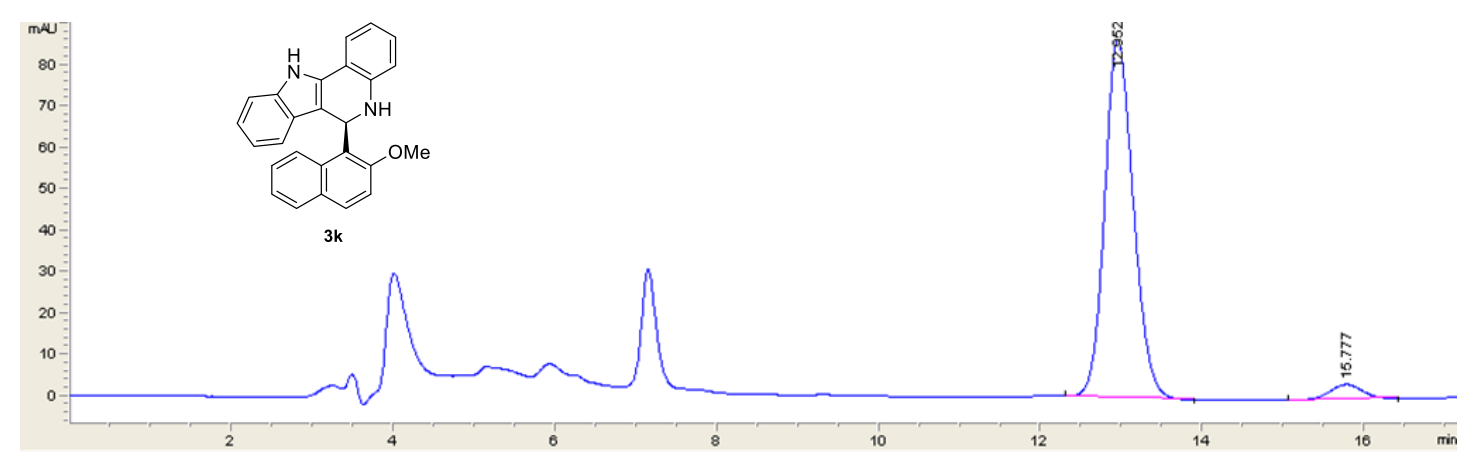

n-hexane/isopropanol 50:50, IA, $1.0 \mathrm{~mL} / \mathrm{min}$

\begin{tabular}{|c|c|c|c|c|c|}
\hline Peak \# & RetTime [min] & Width [min] & Area [mAU*s] & Height [mAU] & Area \% \\
\hline 1 & 12.952 & 0.3791 & 2131.3 & 86.6 & 95.546 \\
2 & 15.777 & 0.4387 & 99.3 & 3.5 & 4.454 \\
\hline
\end{tabular}

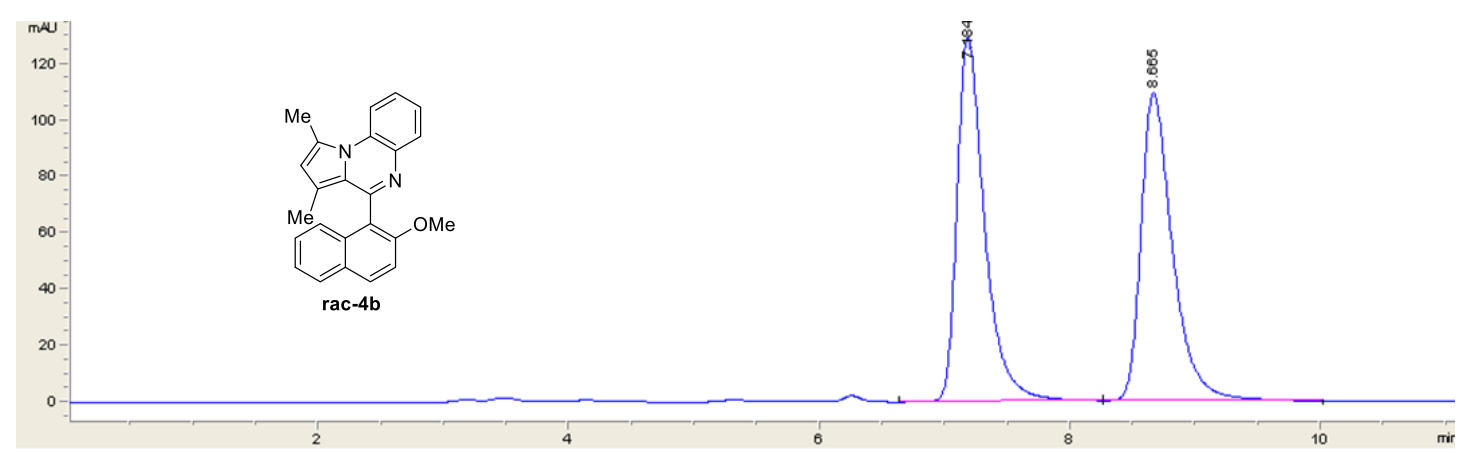

n-hexane/isopropanol 90:10, IA, $1.0 \mathrm{~mL} / \mathrm{min}$

\begin{tabular}{|c|c|c|c|c|c|}
\hline Peak \# & RetTime [min] & Width [min] & Area [mAU's] & Height [mAU] & Area \% \\
\hline 1 & 7.184 & 0.2311 & 1986.7 & 129.4 & 50.825 \\
2 & 8.665 & 0.2654 & 1922.2 & 109.2 & 49.175 \\
\hline
\end{tabular}




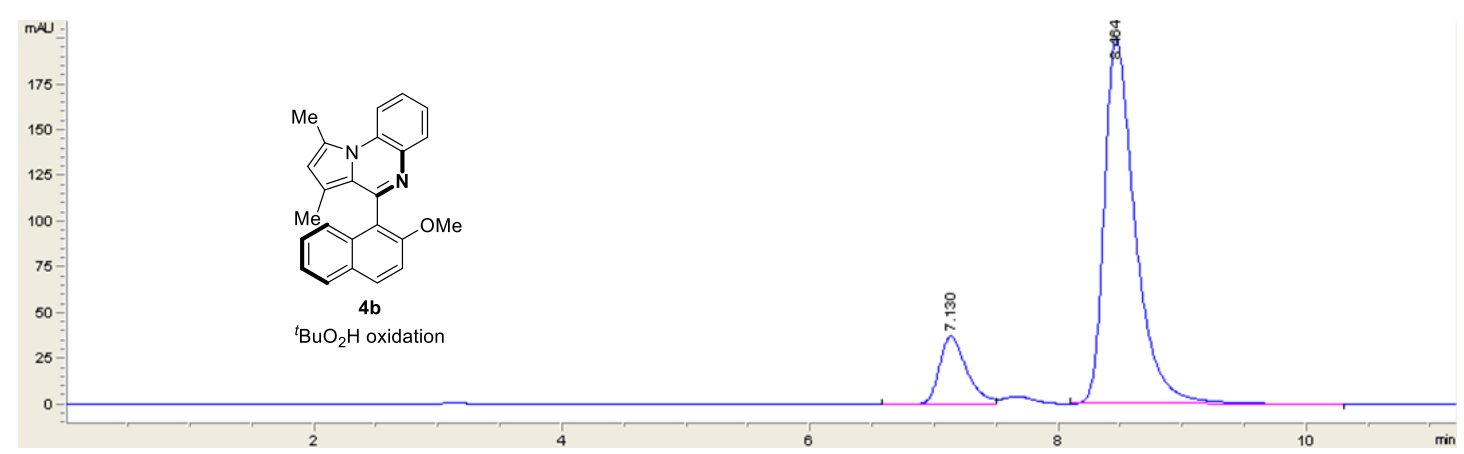

n-hexane/isopropanol 90:10, IA, $1.0 \mathrm{~mL} / \mathrm{min}$

\begin{tabular}{|c|c|c|c|c|c|}
\hline Peak \# & RetTime [min] & Width [min] & Area [mAU*s] & Height [mAU] & Area \% \\
\hline 1 & 7.130 & 0.2328 & 571.6 & 37.3 & 14.162 \\
2 & 8.464 & 0.2624 & 3464.1 & 199.7 & 85.838 \\
\hline
\end{tabular}

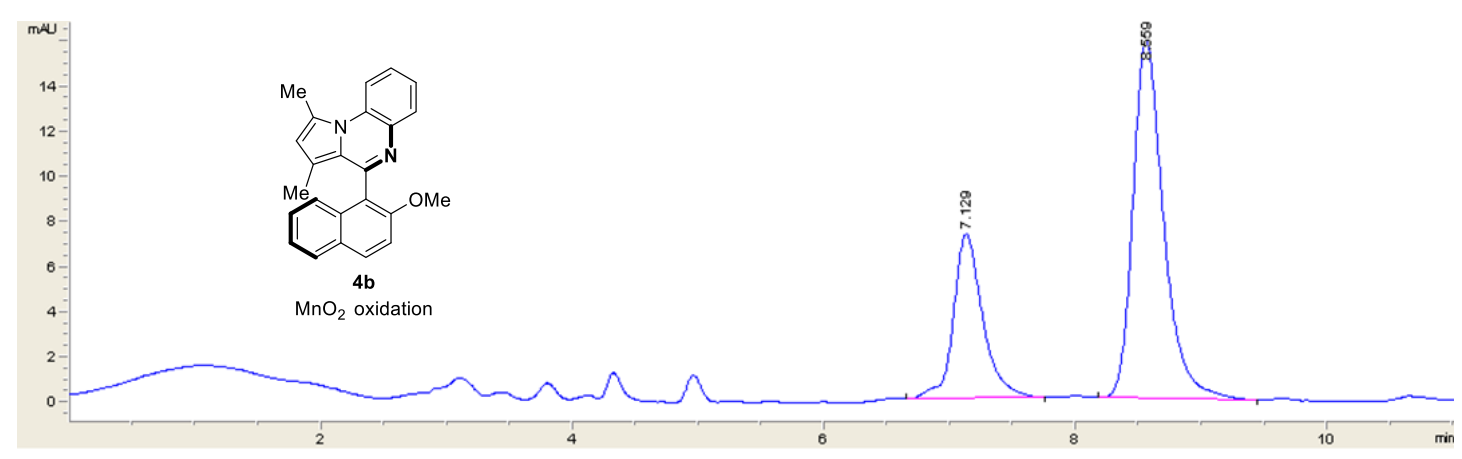

n-hexane/isopropanol 90:10, IA, $1.0 \mathrm{~mL} / \mathrm{min}$

\begin{tabular}{|c|c|c|c|c|c|}
\hline Peak \# & RetTime [min] & Width [min] & Area [mAU*s] & Height [mAU] & Area \% \\
\hline 1 & 7.129 & 0.2428 & 117.6 & 7.3 & 30.420 \\
2 & 8.559 & 0.2576 & 269.1 & 15.9 & 69.580 \\
\hline
\end{tabular}

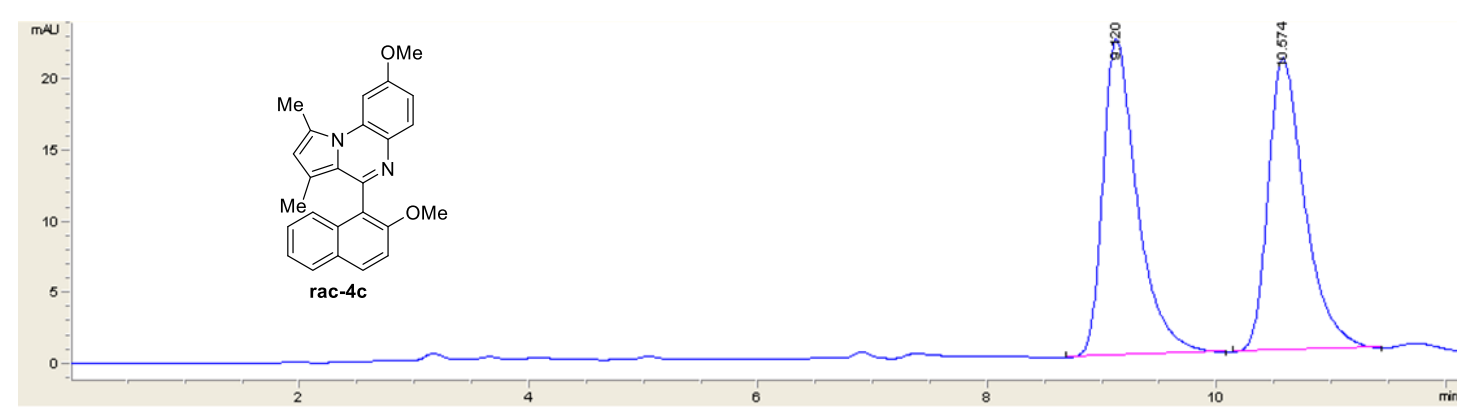

n-hexane/isopropanol 90:10, IA, $1.0 \mathrm{~mL} / \mathrm{min}$

\begin{tabular}{|c|c|c|c|c|c|}
\hline Peak \# & RetTime [min] & Width [min] & Area [mAU*s] & Height [mAU] & Area \% \\
\hline 1 & 9.120 & 0.3110 & 465.1 & 22.3 & 50.343 \\
2 & 10.574 & 0.3336 & 458.7 & 20.6 & 49.657 \\
\hline
\end{tabular}




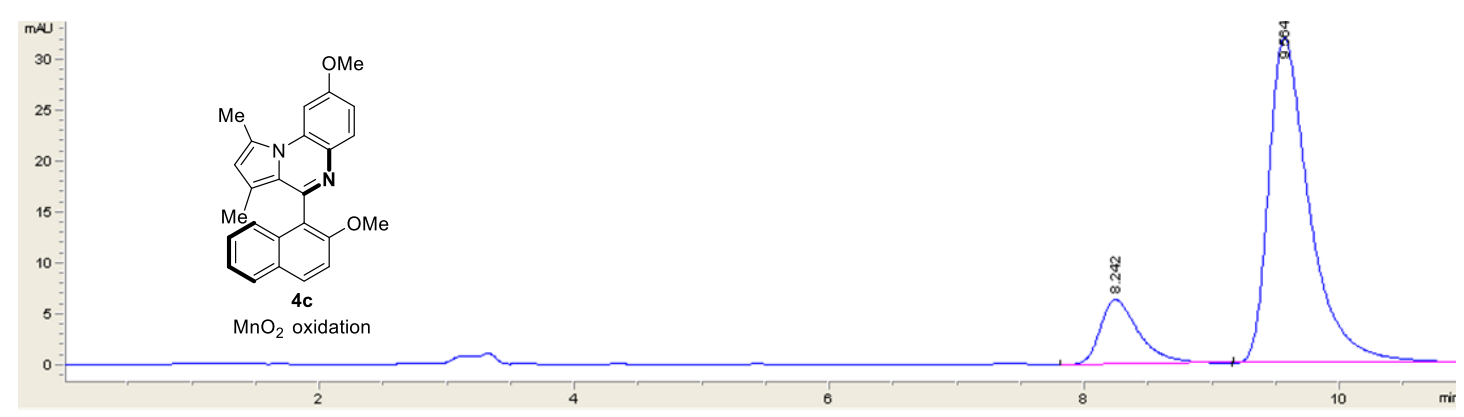

n-hexane/isopropanol 90:10, IA, $1.0 \mathrm{~mL} / \mathrm{min}$

\begin{tabular}{|c|c|c|c|c|c|}
\hline Peak \# & RetTime [min] & Width [min] & Area [mAU*s] & Height [mAU] & Area \% \\
\hline 1 & 8.242 & 0.3161 & 134.4 & 6.4 & 15.871 \\
2 & 9.564 & 0.3317 & 712.4 & 32.0 & 84.129 \\
\hline
\end{tabular}

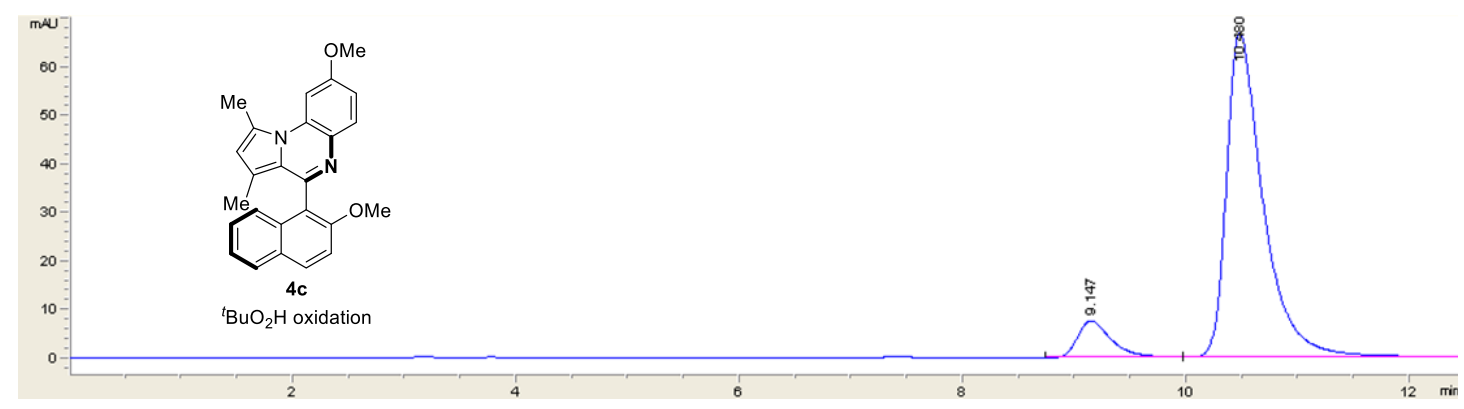

n-hexane/isopropanol 90:10, IA, $1.0 \mathrm{~mL} / \mathrm{min}$

\begin{tabular}{|c|c|c|c|c|c|}
\hline Peak \# & RetTime [min] & Width [min] & Area [mAU*s] & Height [mAU] & Area \% \\
\hline 1 & 9.147 & 0.3150 & 158.2 & 7.6 & 9.361 \\
2 & 10.480 & 0.3390 & 1531.6 & 66.9 & 90.639 \\
\hline
\end{tabular}

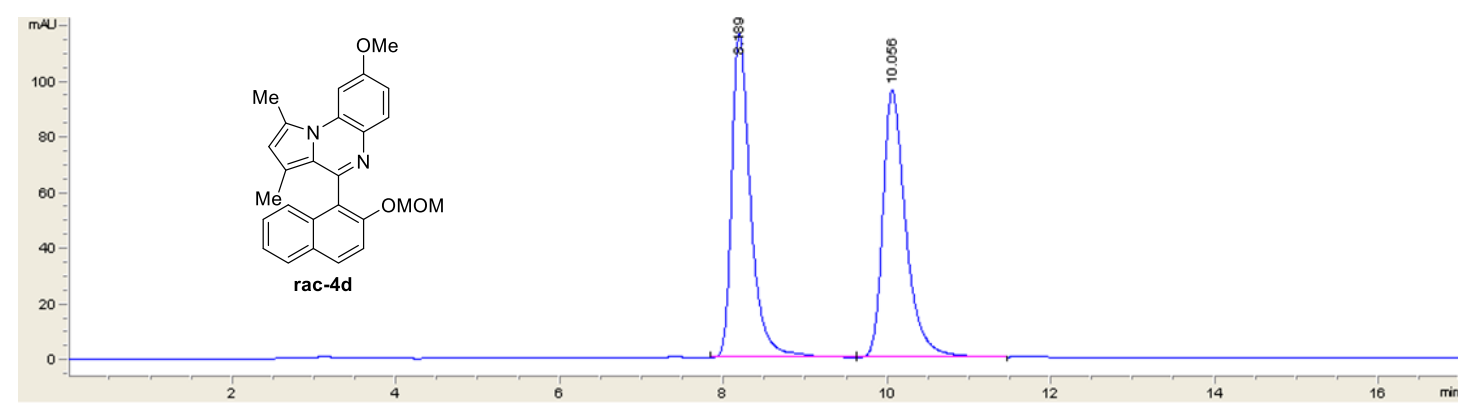

n-hexane/isopropanol 90:10, IA, $1.0 \mathrm{~mL} / \mathrm{min}$

\begin{tabular}{|c|c|c|c|c|c|}
\hline Peak \# & RetTime [min] & Width [min] & Area [mAUs] & Height [mAU] & Area \% \\
\hline 1 & 8.189 & 0.2411 & 1866.6 & 116.4 & 49.928 \\
2 & 10.056 & 0.2933 & 1872.0 & 96.1 & 50.072 \\
\hline
\end{tabular}




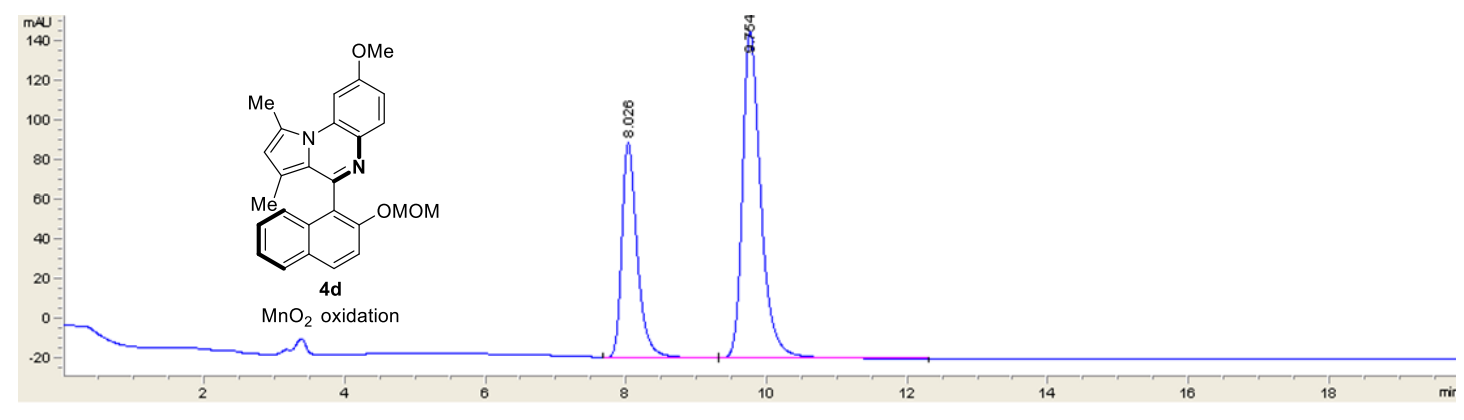

n-hexane/isopropanol 90:10, IA, $1.0 \mathrm{~mL} / \mathrm{min}$

\begin{tabular}{|c|c|c|c|c|c|}
\hline Peak \# & RetTime [min] & Width [min] & Area [mAUs] & Height [mAU] & Area \% \\
\hline 1 & 8.026 & 0.2294 & 1649.0 & 108.4 & 35.367 \\
2 & 9.754 & 0.2753 & 3013.6 & 164.9 & 64.633 \\
\hline
\end{tabular}

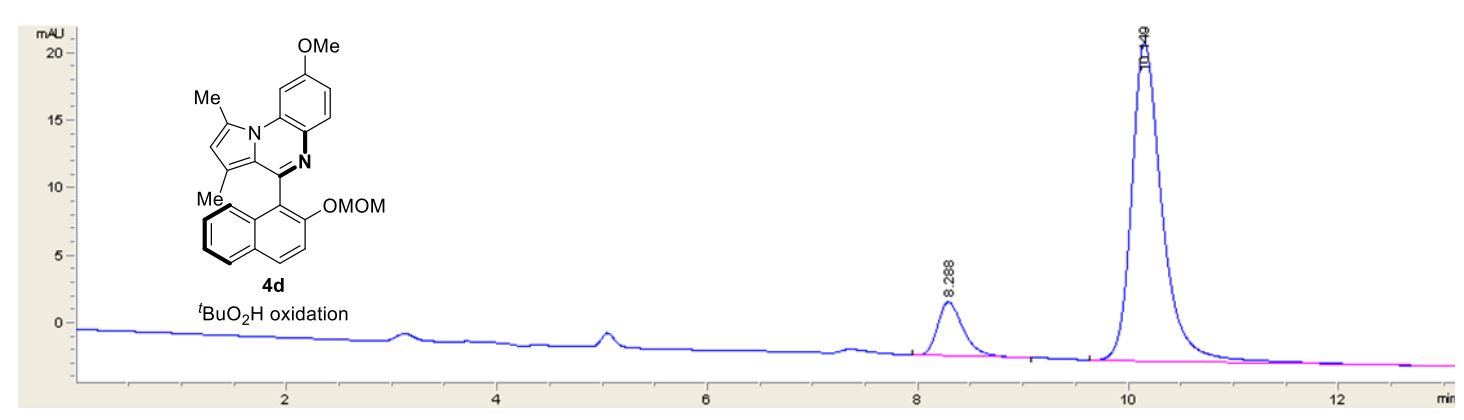

n-hexane/isopropanol 90:10, IA, $1.0 \mathrm{~mL} / \mathrm{min}$

\begin{tabular}{|c|c|c|c|c|c|}
\hline Peak \# & RetTime [min] & Width [min] & Area [mAU*s] & Height [mAU] & Area \% \\
\hline 1 & 8.288 & 0.2532 & 67.1 & 4.0 & 12.178 \\
2 & 10.149 & 0.3068 & 483.6 & 23.6 & 87.822 \\
\hline
\end{tabular}

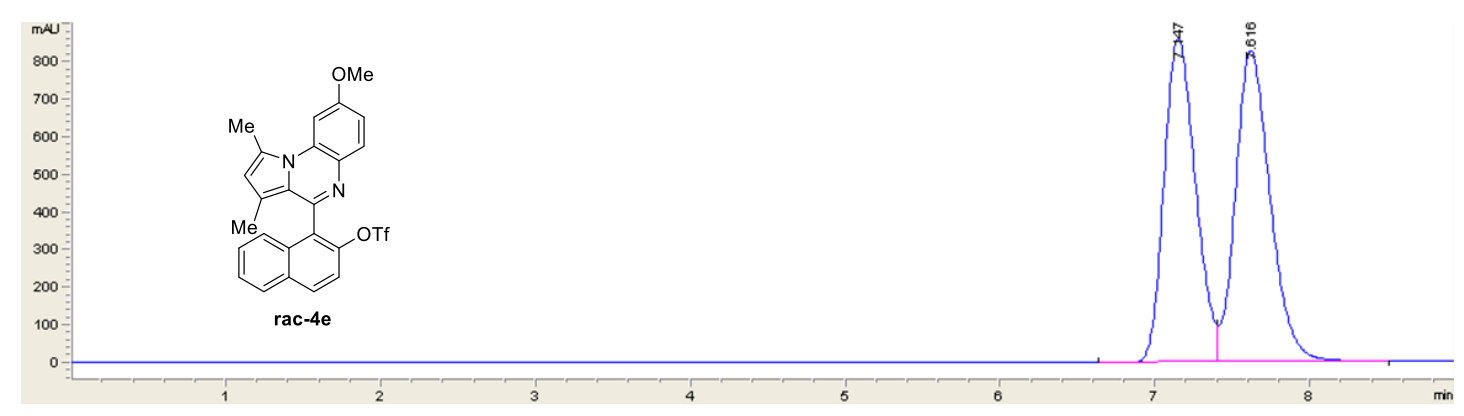

n-hexane/isopropanol 90:10, OD-H, $1.0 \mathrm{~mL} / \mathrm{min}$

\begin{tabular}{|c|c|c|c|c|c|}
\hline Peak \# & RetTime [min] & Width [min] & Area [mAU*s] & Height [mAU] & Area \% \\
\hline 1 & 7.147 & 0.2188 & 12192.1 & 863.2 & 49.573 \\
2 & 7.616 & 0.2397 & 12908.3 & 828.9 & 50.427 \\
\hline
\end{tabular}




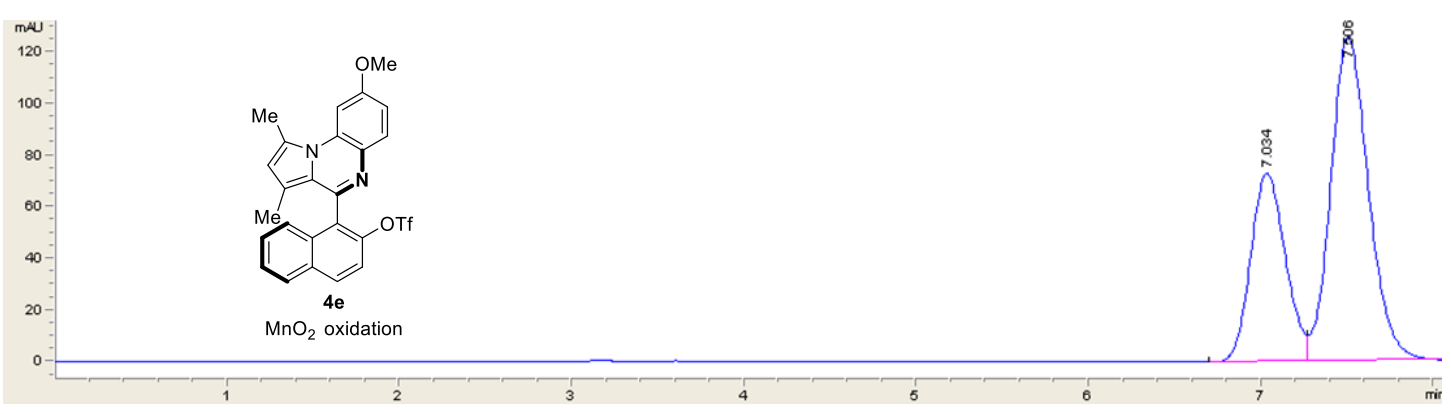

n-hexane/isopropanol 90:10, OD-H, $1.0 \mathrm{~mL} / \mathrm{min}$

\begin{tabular}{|c|c|c|c|c|c|}
\hline Peak \# & RetTime [min] & Width [min] & Area [mAU*s] & Height [mAU] & Area \% \\
\hline 1 & 7.034 & 0.2173 & 1020.9 & 73.0 & 34.783 \\
2 & 7.506 & 0.2335 & 1914.1 & 125.8 & 65.217 \\
\hline
\end{tabular}

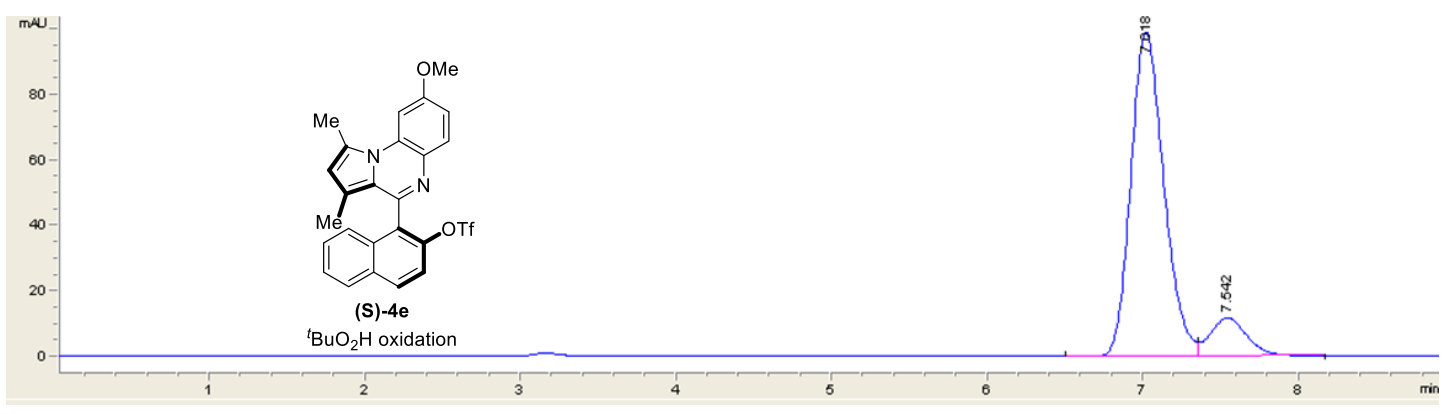

n-hexane/isopropanol 90:10, OD-H, $1.0 \mathrm{~mL} / \mathrm{min}$

\begin{tabular}{|c|c|c|c|c|c|}
\hline Peak \# & RetTime [min] & Width [min] & Area [mAU*s] & Height [mAU] & Area \% \\
\hline 1 & 7.018 & 0.2280 & 1459.4 & 99.0 & 88.503 \\
2 & 7.542 & 0.2471 & 189.6 & 11.7 & 11.497 \\
\hline
\end{tabular}

\begin{tabular}{|c|c|c|c|c|c|}
\hline Peak \# & RetTime [min] & Width [min] & Area [mAU*s] & Height [mAU] & Area \% \\
\hline 1 & 4.731 & 0.1531 & 2145.8 & 217.5 & 49.766 \\
\hline 2 & 5.268 & 0.1611 & 2166.0 & 208.6 & 50.243 \\
\hline
\end{tabular}




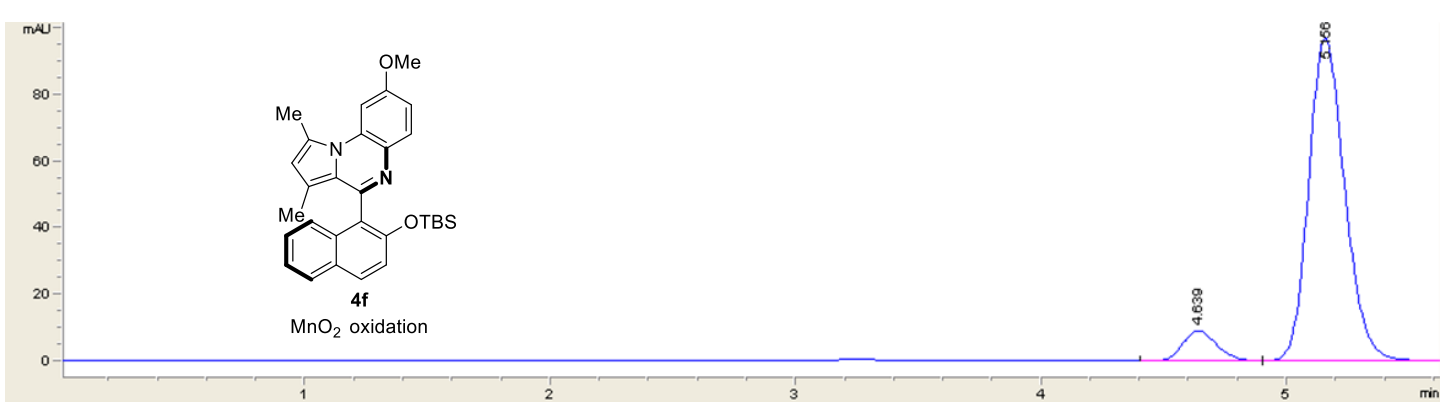

n-hexane/isopropanol 95:5, IA, $1.0 \mathrm{~mL} / \mathrm{min}$

\begin{tabular}{|c|c|c|c|c|c|}
\hline Peak \# & RetTime [min] & Width [min] & Area [mAUs] & Height [mAU] & Area \% \\
\hline 1 & 4.639 & 0.1513 & 87.1 & 9.0 & 8.085 \\
2 & 5.156 & 0.1590 & 990.5 & 97.1 & 91.915 \\
\hline
\end{tabular}

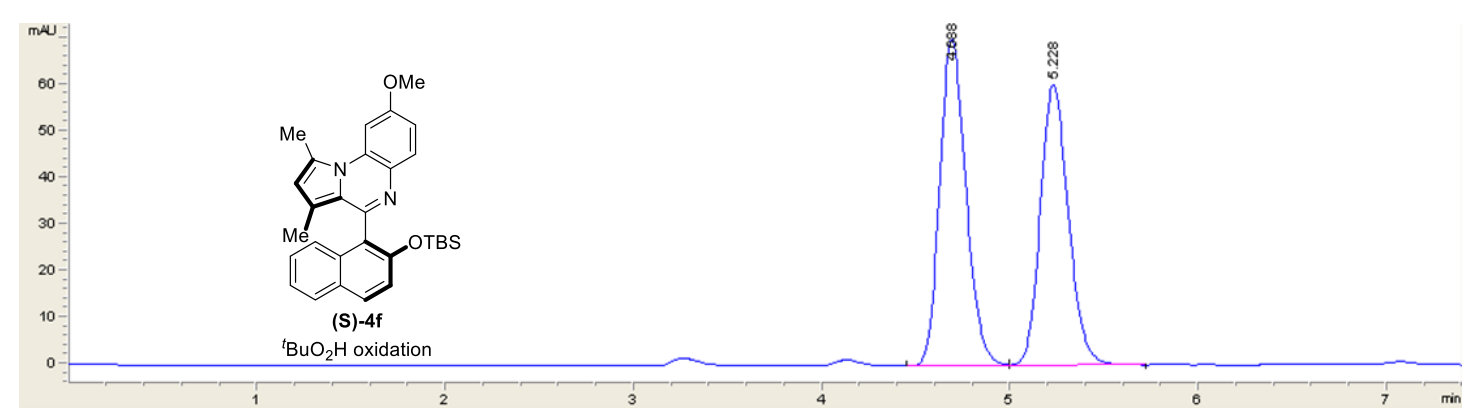

n-hexane/isopropanol 95:5, IA, $1.0 \mathrm{~mL} / \mathrm{min}$

\begin{tabular}{|c|c|c|c|c|c|}
\hline Peak \# & RetTime [min] & Width [min] & Area [mAU*s] & Height [mAU] & Area \% \\
\hline 1 & 4.688 & 0.1515 & 682.0 & 70.1 & 52.232 \\
2 & 5.228 & 0.1607 & 623.7 & 60.3 & 47.768 \\
\hline
\end{tabular}

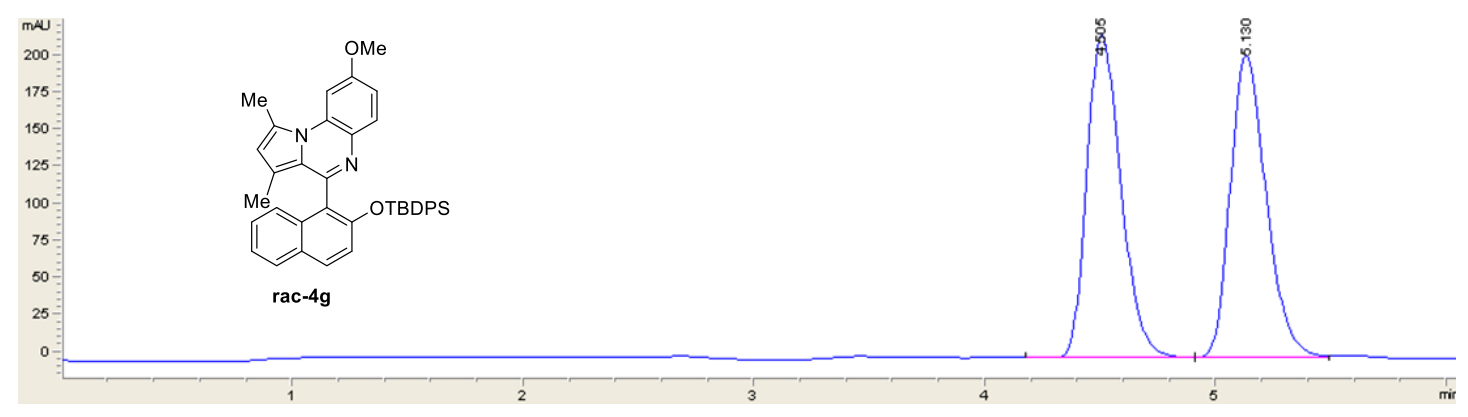

n-hexane/isopropanol 80:20, IC, $1.0 \mathrm{~mL} / \mathrm{min}$

\begin{tabular}{|c|c|c|c|c|c|}
\hline Peak \# & RetTime [min] & Width [min] & Area [mAU*s] & Height [mAU] & Area \% \\
\hline 1 & 4.505 & 0.1598 & 217.9 & 217.9 & 50.325 \\
2 & 5.130 & 0.1688 & 203.1 & 203.1 & 49.675 \\
\hline
\end{tabular}




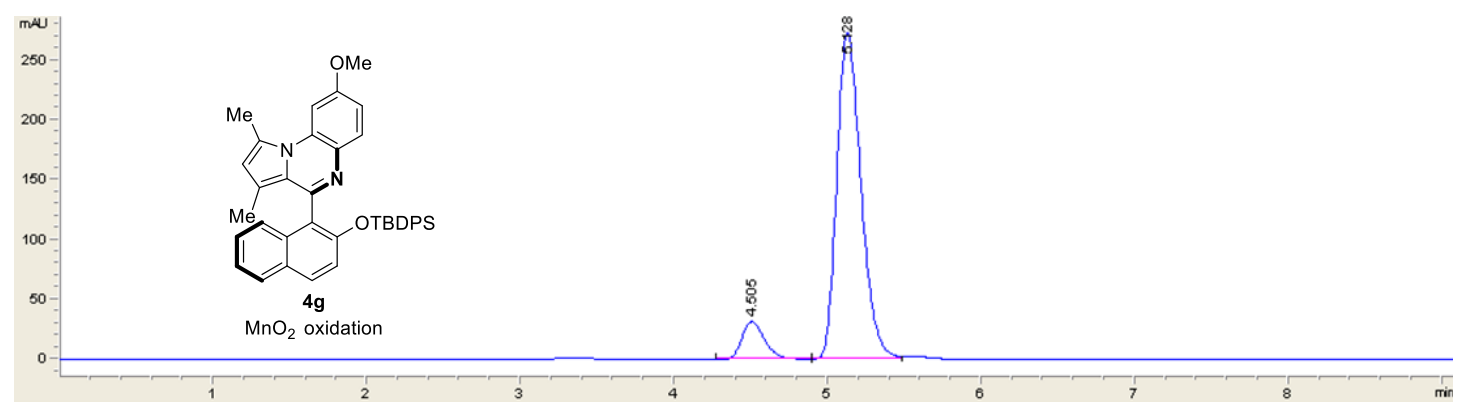

n-hexane/isopropanol 80:20, IC, $1.0 \mathrm{~mL} / \mathrm{min}$

\begin{tabular}{|c|c|c|c|c|c|}
\hline Peak \# & RetTime [min] & Width [min] & Area [mAU*s] & Height [mAU] & Area \% \\
\hline 1 & 4.505 & 0.1592 & 321.6 & 31.5 & 9.726 \\
2 & 5.128 & 0.1694 & 2984.5 & 273.3 & 90.274 \\
\hline
\end{tabular}

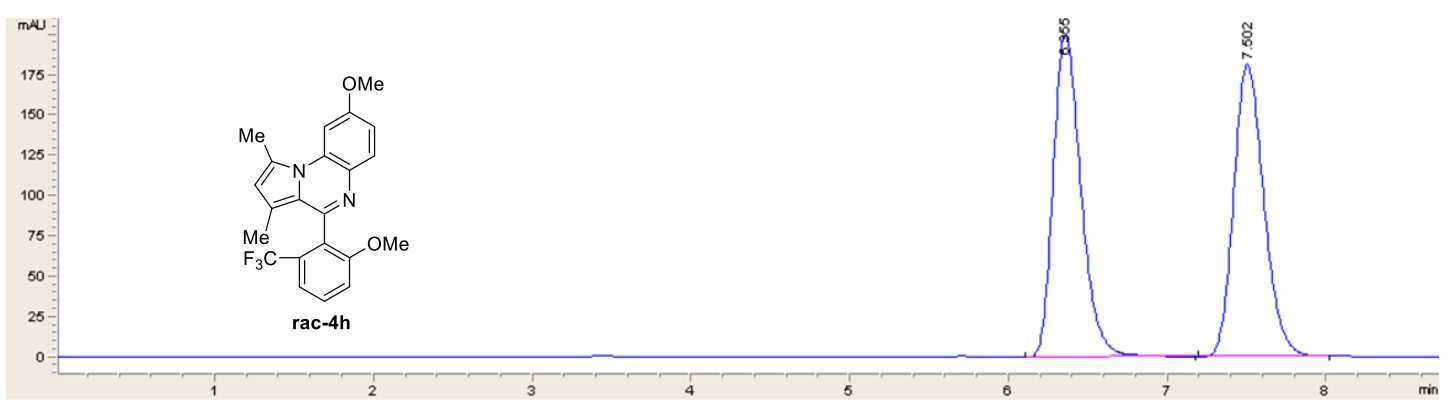

n-hexane/isopropanol 80:20, IC, $1.0 \mathrm{~mL} / \mathrm{min}$

\begin{tabular}{|c|c|c|c|c|c|}
\hline Peak \# & RetTime [min] & Width [min] & Area [mAU*s] & Height [mAU] & Area \% \\
\hline 1 & 6.355 & 0.1882 & 2445.1 & 200.6 & 50.525 \\
2 & 7.502 & 0.2041 & 2394.3 & 181.3 & 49.475 \\
\hline
\end{tabular}

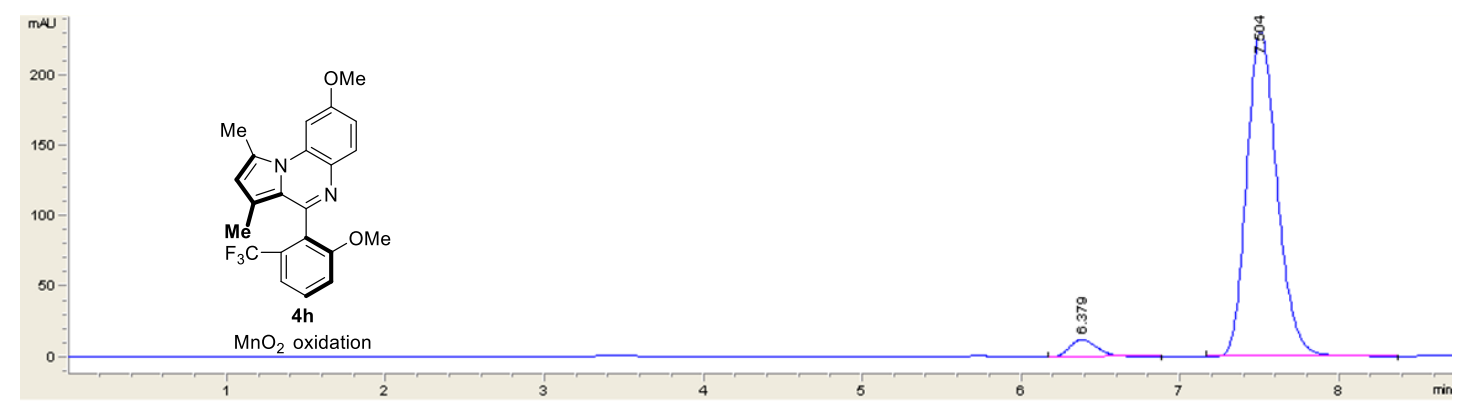

n-hexane/isopropanol 80:20, IC, $1.0 \mathrm{~mL} / \mathrm{min}$

\begin{tabular}{|c|c|c|c|c|c|}
\hline Peak \# & RetTime [min] & Width [min] & Area [mAU*s] & Height [mAU] & Area \% \\
\hline 1 & 6.379 & 0.1872 & 155.9 & 12.7 & 4.812 \\
2 & 7.504 & 0.2053 & 3083.4 & 231.7 & 95.188 \\
\hline
\end{tabular}




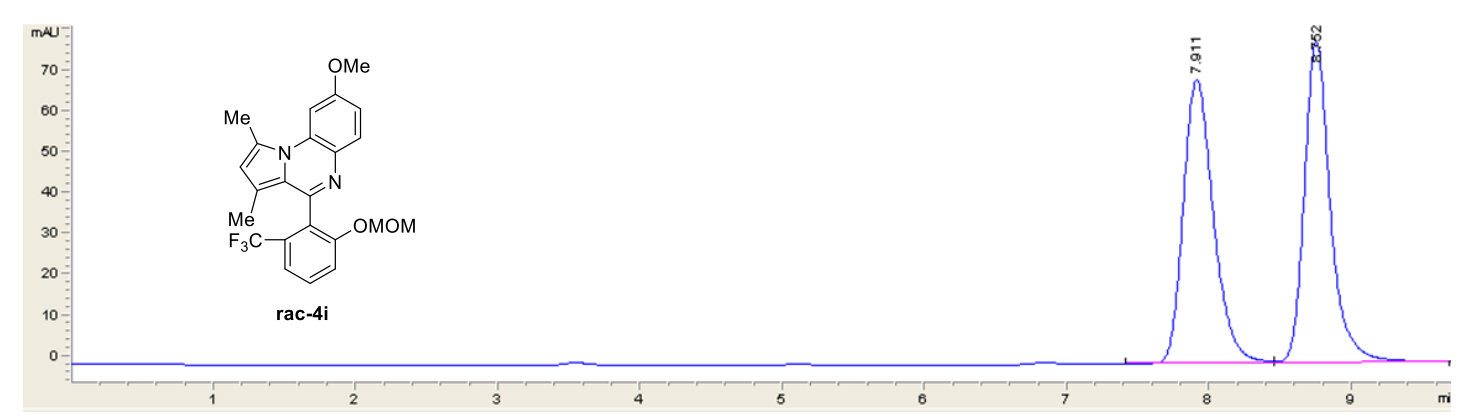

n-hexane/isopropanol 90:10, IC, $1.0 \mathrm{~mL} / \mathrm{min}$

\begin{tabular}{|c|c|c|c|c|c|}
\hline Peak \# & RetTime [min] & Width [min] & Area [mAU*s] & Height [mAU] & Area \% \\
\hline 1 & 7.911 & 0.2259 & 1023.1 & 69.5 & 50.626 \\
2 & 8.752 & 0.1920 & 997.8 & 78.7 & 49.374 \\
\hline
\end{tabular}

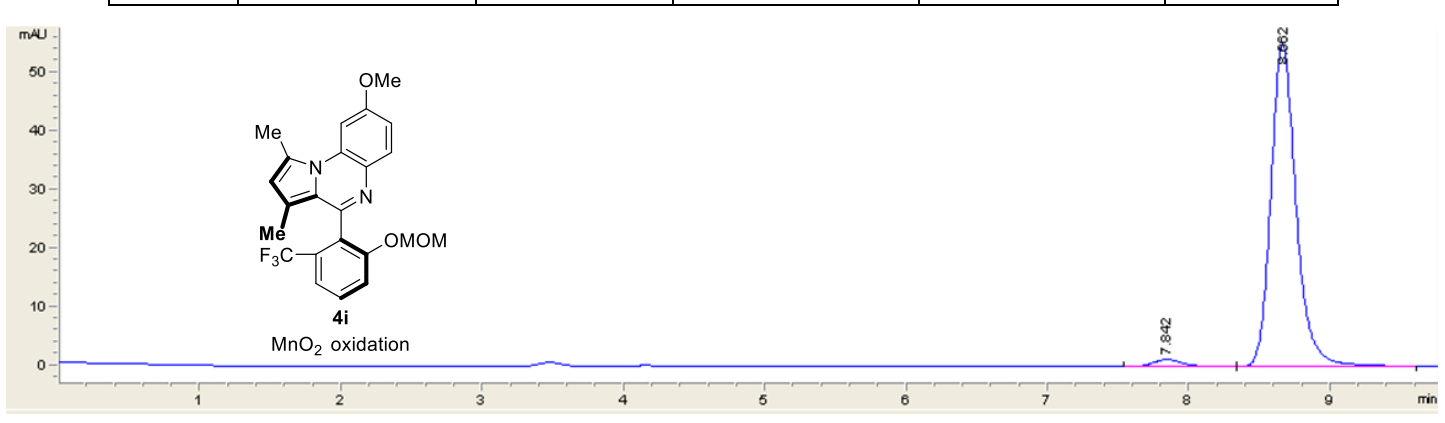

n-hexane/isopropanol 90:10, IC, $1.0 \mathrm{~mL} / \mathrm{min}$

\begin{tabular}{|c|c|c|c|c|c|}
\hline Peak \# & RetTime [min] & Width [min] & Area [mAU*s] & Height [mAU] & Area \% \\
\hline 1 & 7.842 & 0.2192 & 16.7 & 1.2 & 2.392 \\
2 & 8.662 & 0.1880 & 681.9 & 55.3 & 97.608 \\
\hline
\end{tabular}

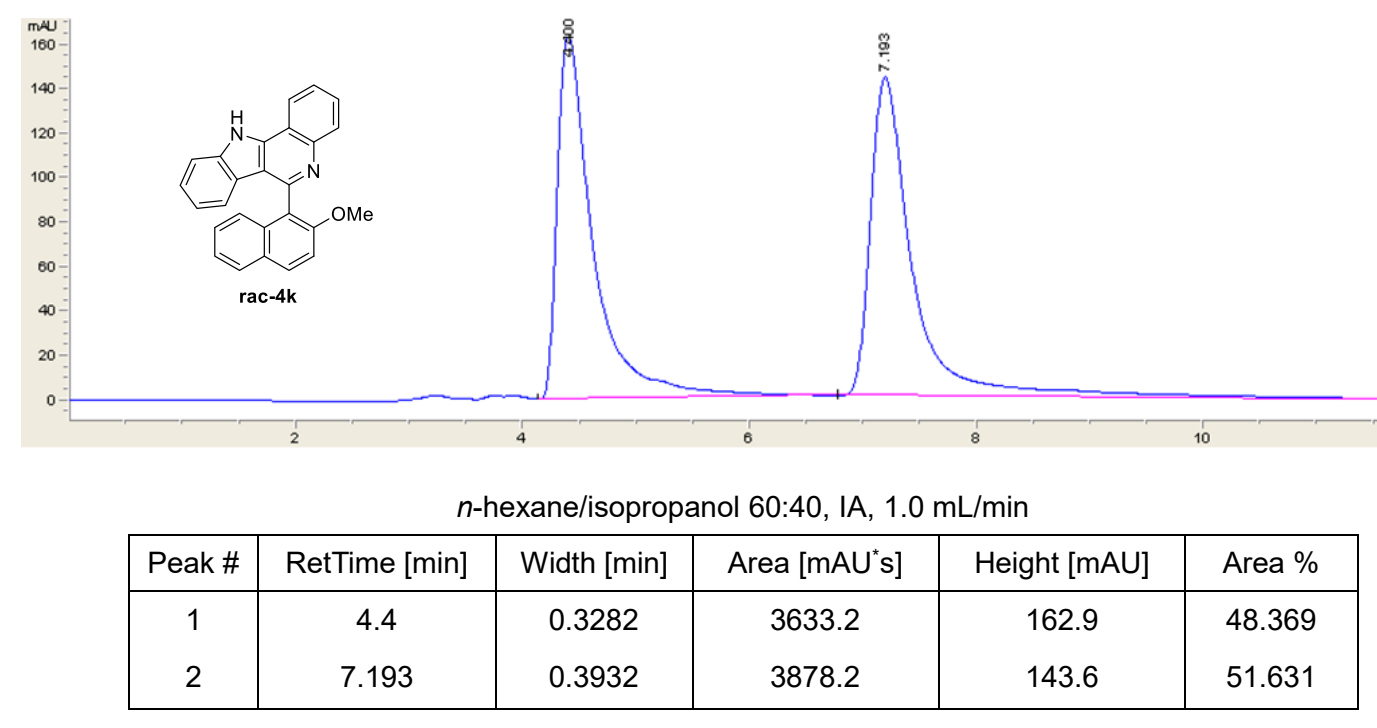



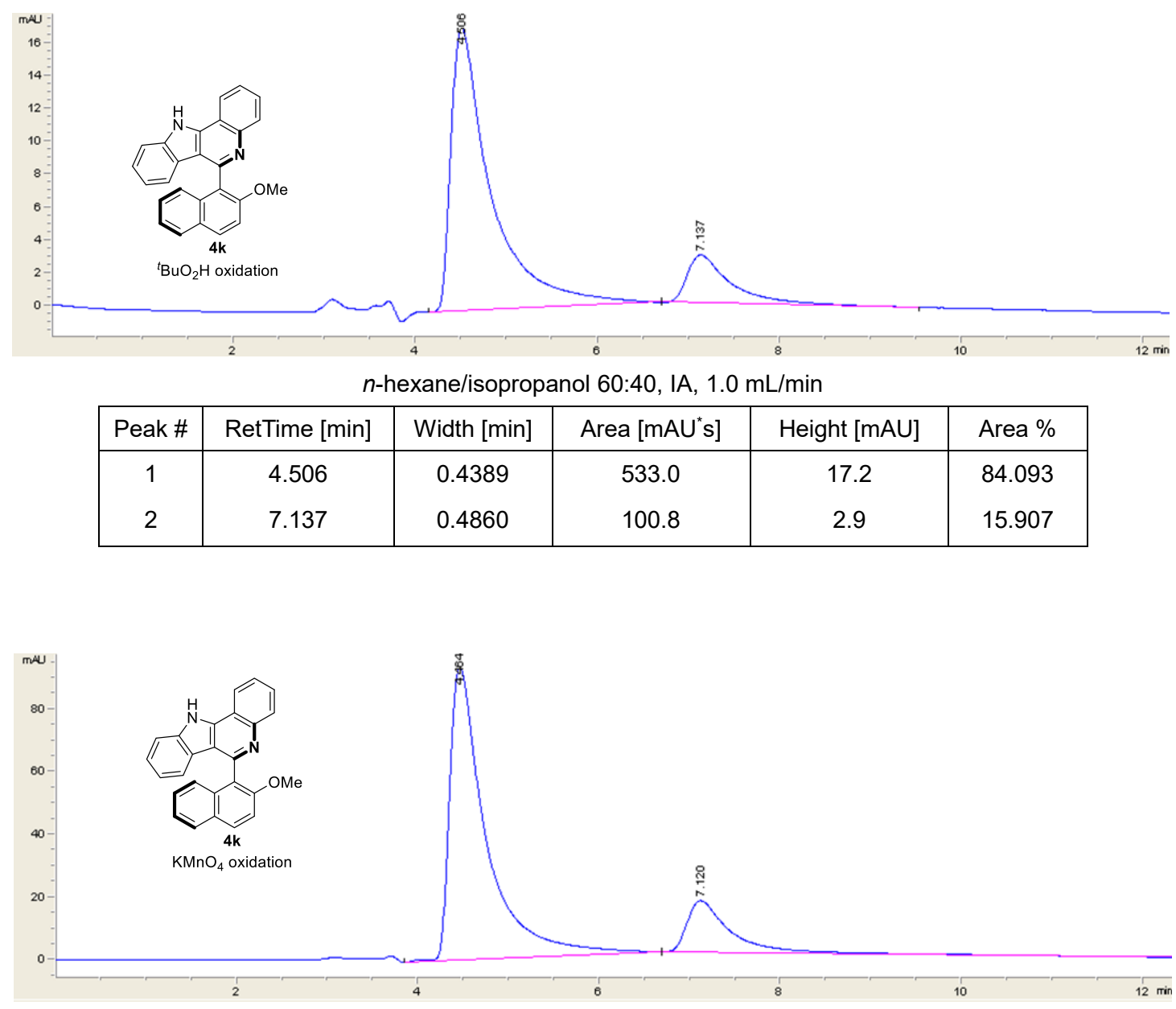

n-hexane/isopropanol 60:40, IA, $1.0 \mathrm{~mL} / \mathrm{min}$

\begin{tabular}{|c|c|c|c|c|c|}
\hline Peak \# & RetTime [min] & Width [min] & Area [mAU*s] & Height [mAU] & Area \% \\
\hline 1 & 4.4644 & 0.4075 & 2648.0 & 93.2 & 82.265 \\
2 & 7.120 & 0.4987 & 570.9 & 16.5 & 17.735 \\
\hline
\end{tabular}

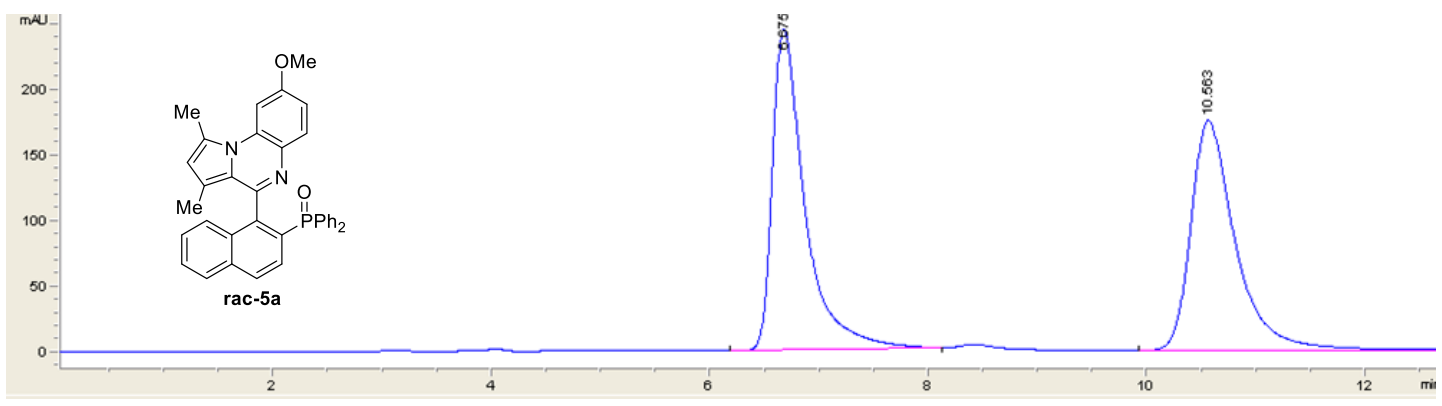

n-hexane/isopropanol 70:30, IA, $1.0 \mathrm{~mL} / \mathrm{min}$

\begin{tabular}{|c|c|c|c|c|c|}
\hline Peak \# & RetTime [min] & Width [min] & Area [mAU*s] & Height [mAU] & Area \% \\
\hline 1 & 6.675 & 0.3084 & 5080.0 & 244.5 & 50.271 \\
2 & 10.563 & 0.4268 & 5025.1 & 175.1 & 49.729 \\
\hline
\end{tabular}




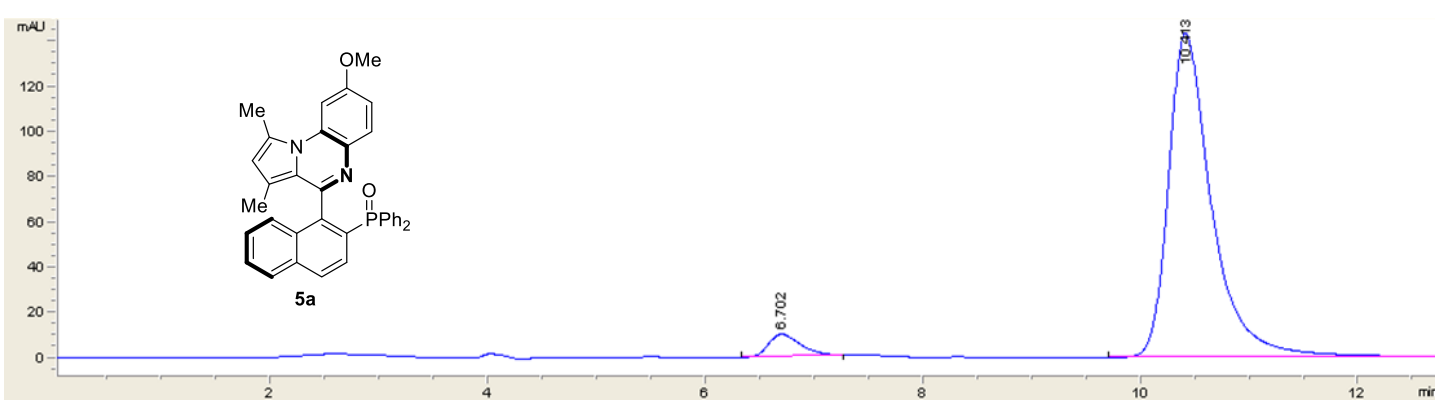

n-hexane/isopropanol 70:30, IA, $1.0 \mathrm{~mL} / \mathrm{min}$

\begin{tabular}{|c|c|c|c|c|c|}
\hline Peak \# & RetTime [min] & Width [min] & Area [mAU*s] & Height [mAU] & Area \% \\
\hline 1 & 6.702 & 0.3081 & 201.2 & 9.9 & 4.796 \\
2 & 10.413 & 0.4147 & 3993.2 & 143.5 & 95.204 \\
\hline
\end{tabular}

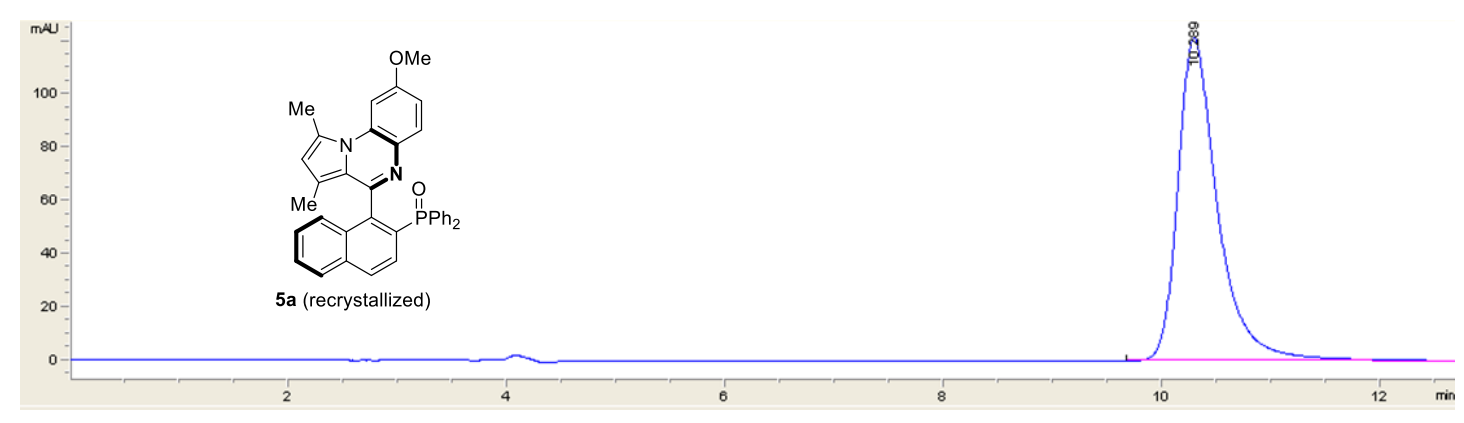

n-hexane/isopropanol 70:30, IA, $1.0 \mathrm{~mL} / \mathrm{min}$

\begin{tabular}{|c|c|c|c|c|c|}
\hline Peak \# & RetTime [min] & Width [min] & Area [mAU*s] & Height [mAU] & Area \% \\
\hline 1 & 10.289 & 0.3838 & 3126.7 & 121.7 & 100.000 \\
\hline
\end{tabular}

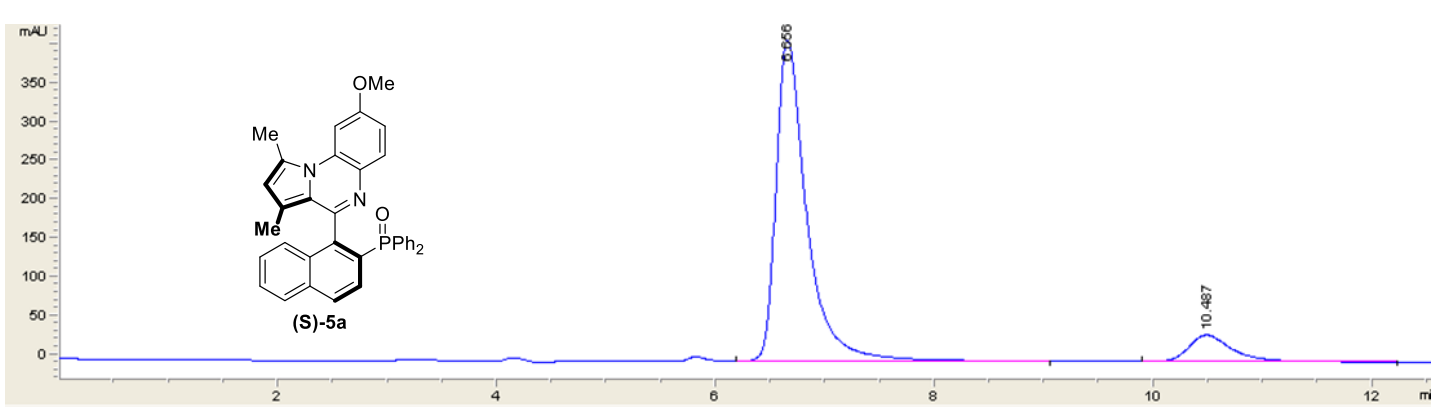

n-hexane/isopropanol 70:30, IA, $1.0 \mathrm{~mL} / \mathrm{min}$

\begin{tabular}{|c|c|c|c|c|c|}
\hline Peak \# & RetTime [min] & Width [min] & Area [mAU*s] & Height [mAU] & Area \% \\
\hline 1 & 6.656 & 0.2906 & 8112.2 & 414.1 & 89.375 \\
2 & 10.487 & 0.4162 & 964.4 & 34.5 & 10.625 \\
\hline
\end{tabular}




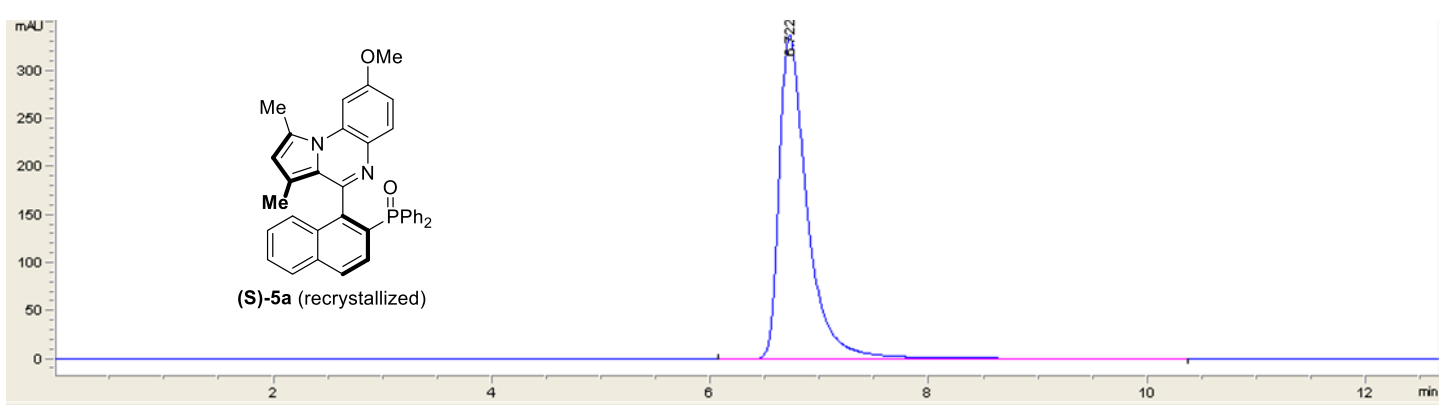

n-hexane/isopropanol 70:30, IA, $1.0 \mathrm{~mL} / \mathrm{min}$

\begin{tabular}{|c|c|c|c|c|c|}
\hline Peak \# & RetTime [min] & Width [min] & Area [mAUs] & Height [mAU] & Area \% \\
\hline 1 & 6.722 & 0.2685 & 6090.4 & 337.5 & 100.00 \\
\hline
\end{tabular}

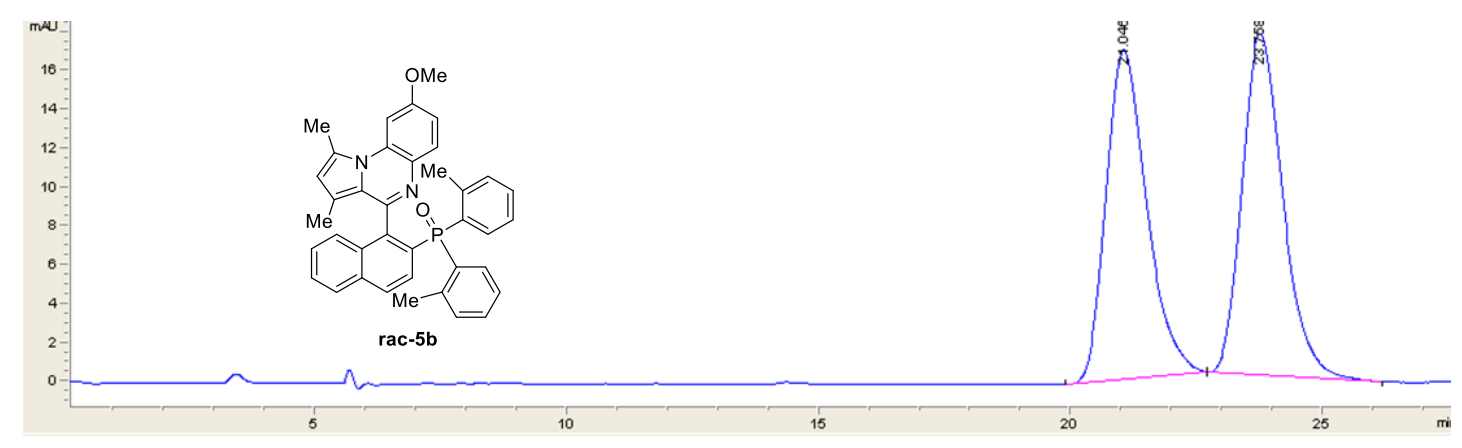

n-hexane/isopropanol 80:20, IC, $1.0 \mathrm{~mL} / \mathrm{min}$

\begin{tabular}{|c|c|c|c|c|c|}
\hline Peak \# & RetTime [min] & Width [min] & Area [mAUs] & Height [mAU] & Area \% \\
\hline 1 & 21.046 & 0.8568 & 954.8 & 17.0 & 49.996 \\
2 & 23.758 & 0.8491 & 986.0 & 17.6 & 50.004 \\
\hline
\end{tabular}

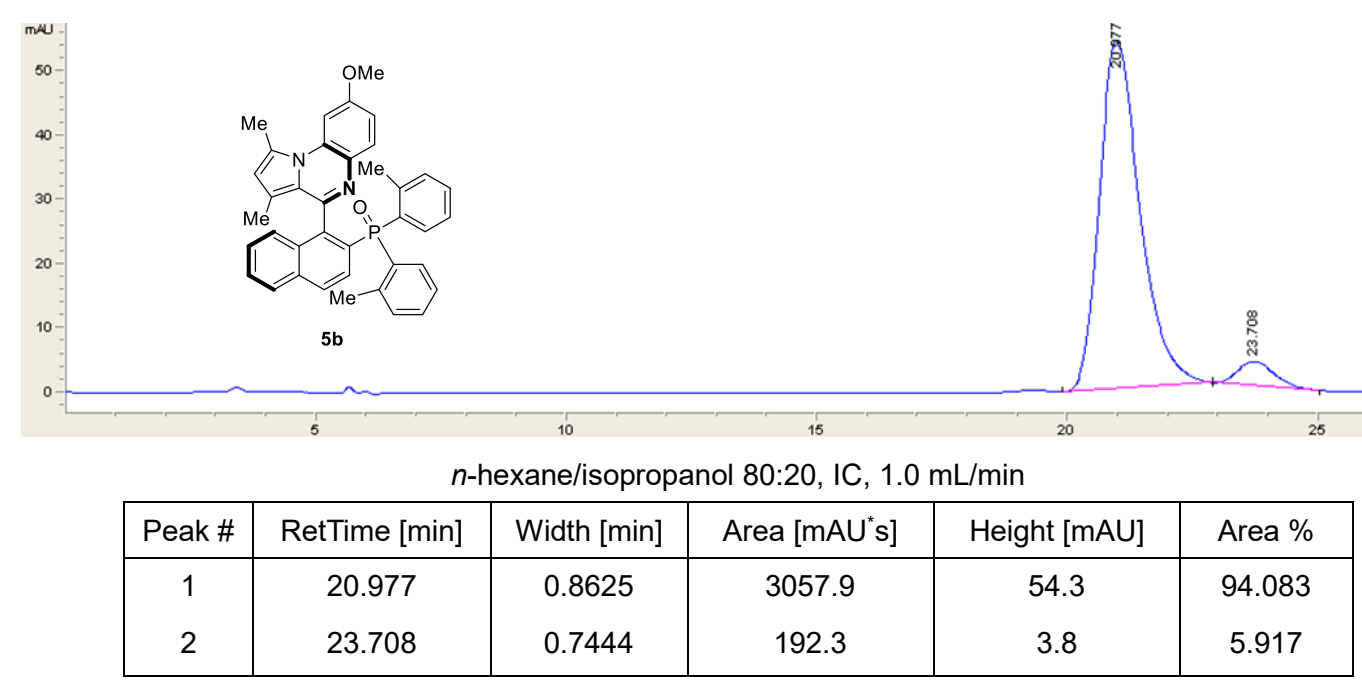




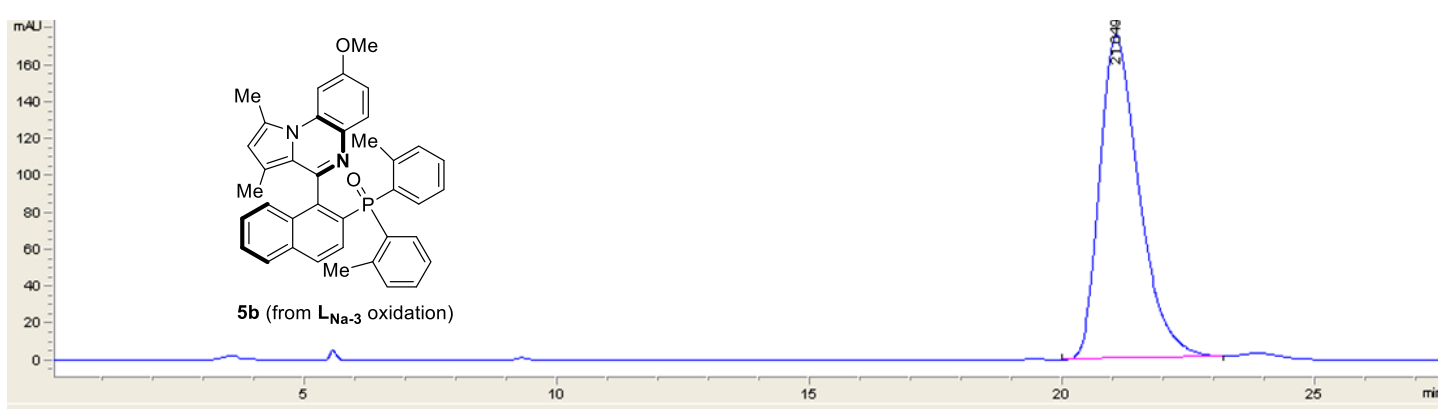

n-hexane/isopropanol 80:20, IC, $1.0 \mathrm{~mL} / \mathrm{min}$

\begin{tabular}{|c|c|c|c|c|c|}
\hline Peak \# & RetTime [min] & Width [min] & Area [mAU*s] & Height [mAU] & Area \% \\
\hline 1 & 21.049 & 0.8330 & 9556.4 & 175.6 & 100.000 \\
\hline
\end{tabular}

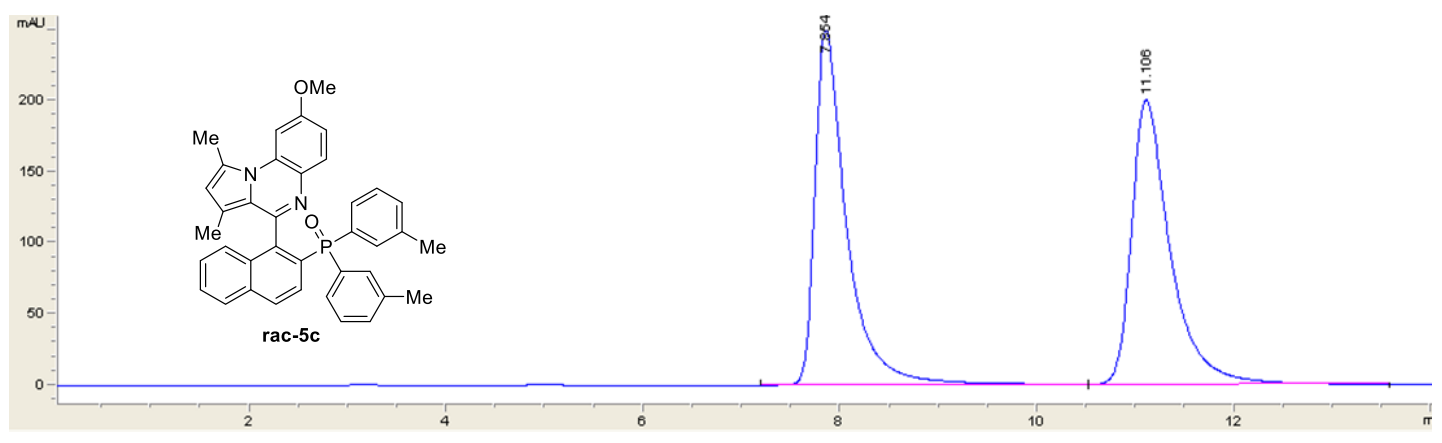

n-hexane/isopropanol 80:20, IA, $1.0 \mathrm{~mL} / \mathrm{min}$

\begin{tabular}{|c|c|c|c|c|c|}
\hline Peak \# & RetTime [min] & Width [min] & Area [mAU's] & Height [mAU] & Area \% \\
\hline 1 & 7.854 & 0.3311 & 5628.3 & 249.6 & 50.531 \\
2 & 11.106 & 0.4069 & 5510.0 & 200.3 & 49.469 \\
\hline
\end{tabular}

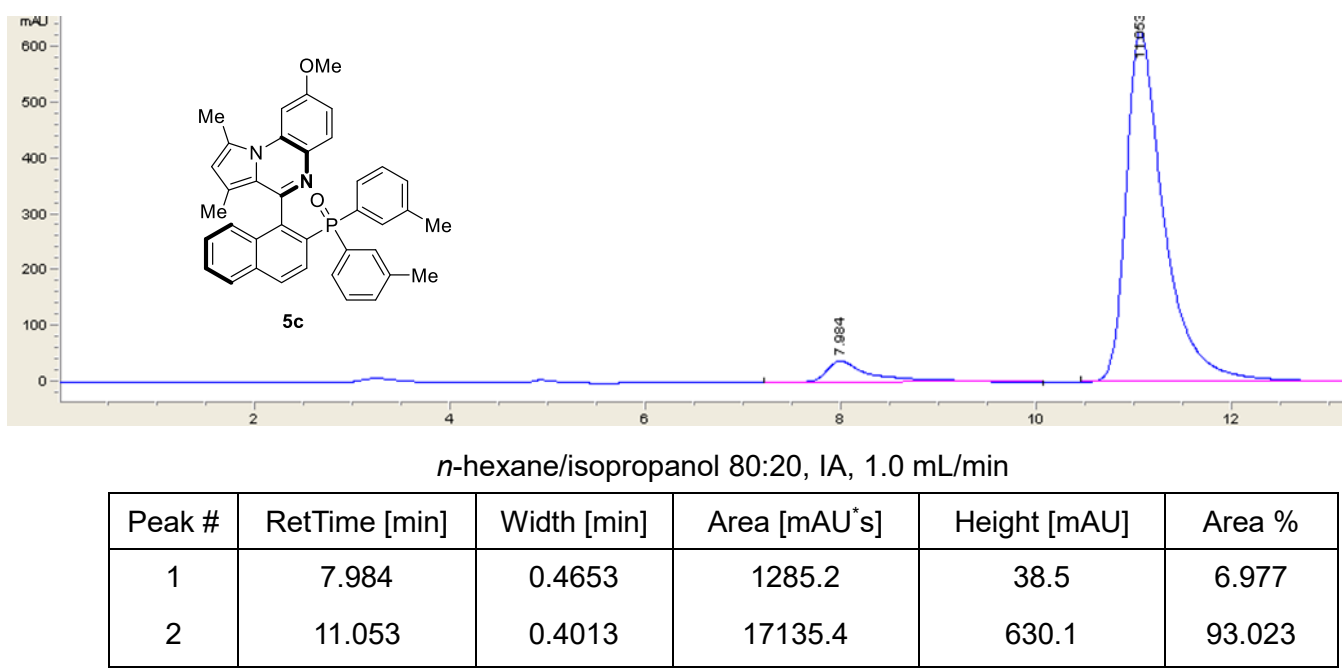




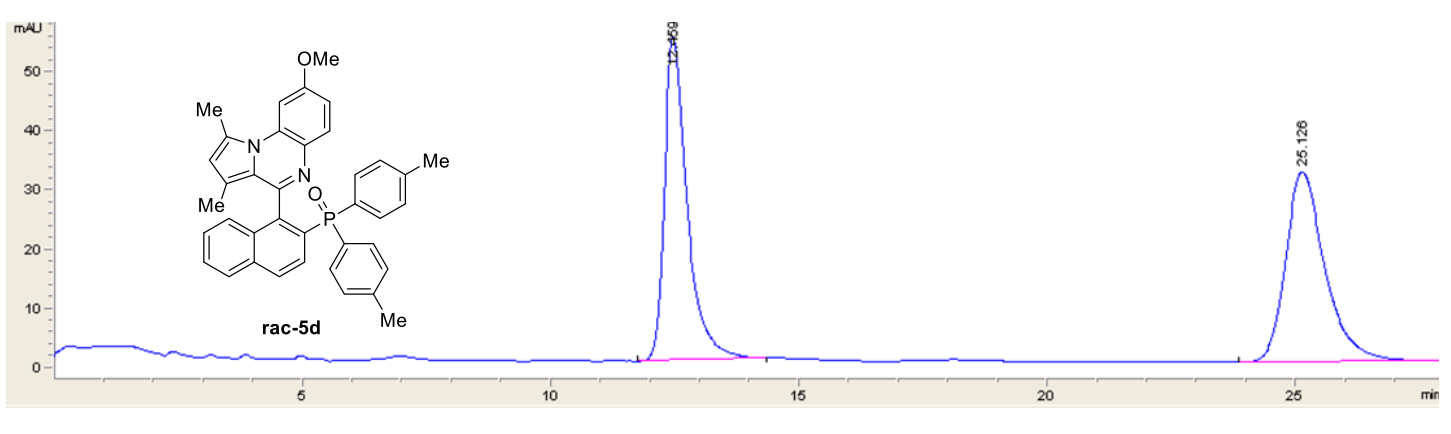

n-hexane/isopropanol 80:20, IA, $1.0 \mathrm{~mL} / \mathrm{min}$

\begin{tabular}{|c|c|c|c|c|c|}
\hline Peak \# & RetTime [min] & Width [min] & Area [mAU*s] & Height [mAU] & Area \% \\
\hline 1 & 12.459 & 0.4731 & 1739.6 & 54.7 & 49.522 \\
2 & 25.126 & 0.8325 & 1773.2 & 32.1 & 50.478 \\
\hline
\end{tabular}

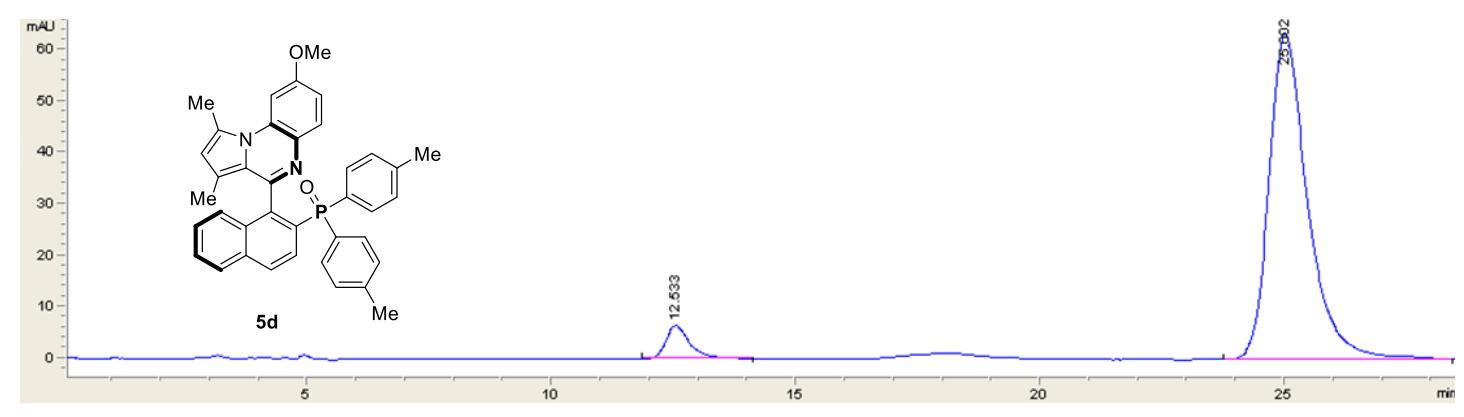

n-hexane/isopropanol 80:20, IA, $1.0 \mathrm{~mL} / \mathrm{min}$

\begin{tabular}{|c|c|c|c|c|c|}
\hline Peak \# & RetTime [min] & Width [min] & Area [mAU*s] & Height [mAU] & Area \% \\
\hline 1 & 12.533 & 0.5064 & 215.7 & 6.4 & 5.823 \\
2 & 25.002 & 0.8251 & 3488.5 & 63.7 & 94.177 \\
\hline
\end{tabular}

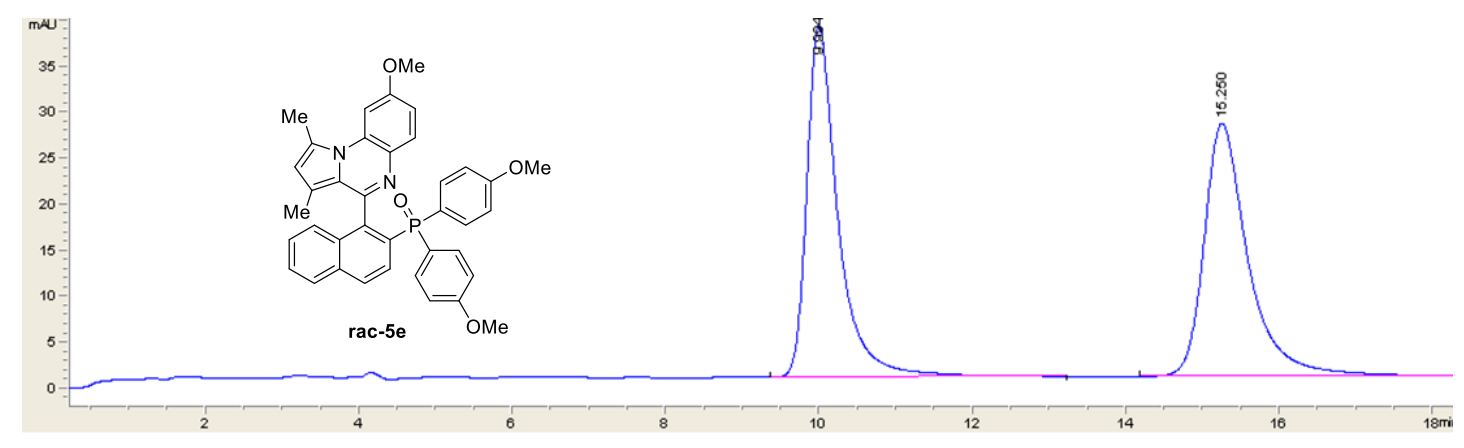

n-hexane/isopropanol 70:30, IA, $1.0 \mathrm{~mL} / \mathrm{min}$

\begin{tabular}{|c|c|c|c|c|c|}
\hline Peak \# & RetTime [min] & Width [min] & Area [mAUs] & Height [mAU] & Area \% \\
\hline 1 & 9.994 & 0.4197 & 1087.2 & 38.2 & 49.391 \\
2 & 15.250 & 0.5963 & 1114.0 & 27.5 & 50.609 \\
\hline
\end{tabular}




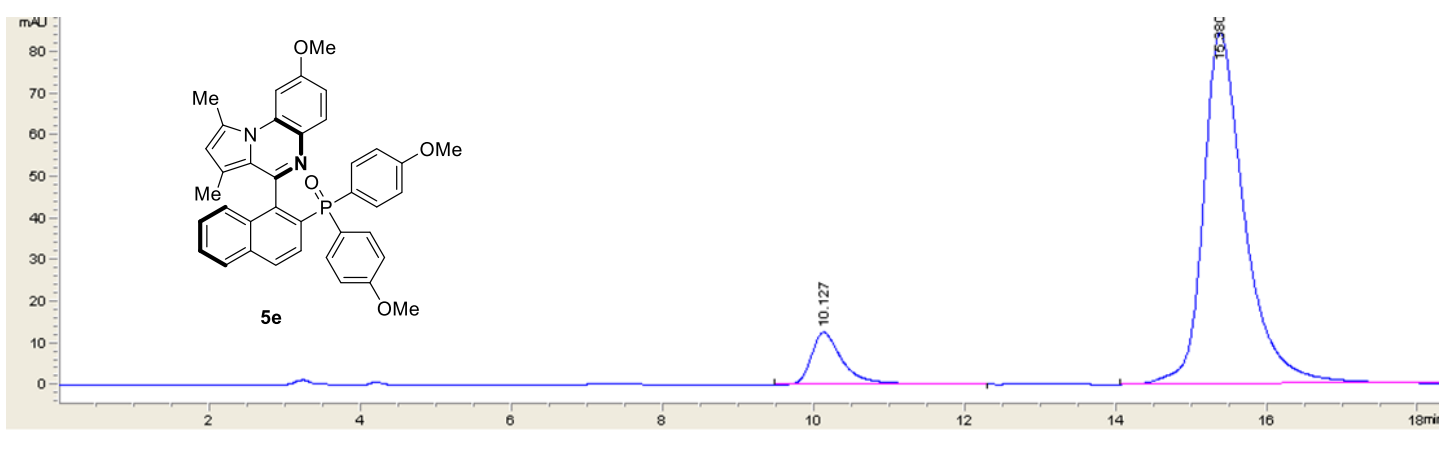

n-hexane/isopropanol 70:30, IA, $1.0 \mathrm{~mL} / \mathrm{min}$

\begin{tabular}{|c|c|c|c|c|c|}
\hline Peak \# & RetTime [min] & Width [min] & Area [mAU*s] & Height [mAU] & Area \% \\
\hline 1 & 10.127 & 0.4279 & 366.6 & 12.6 & 10.189 \\
2 & 15.380 & 0.5683 & 3231.7 & 84.5 & 89.811 \\
\hline
\end{tabular}

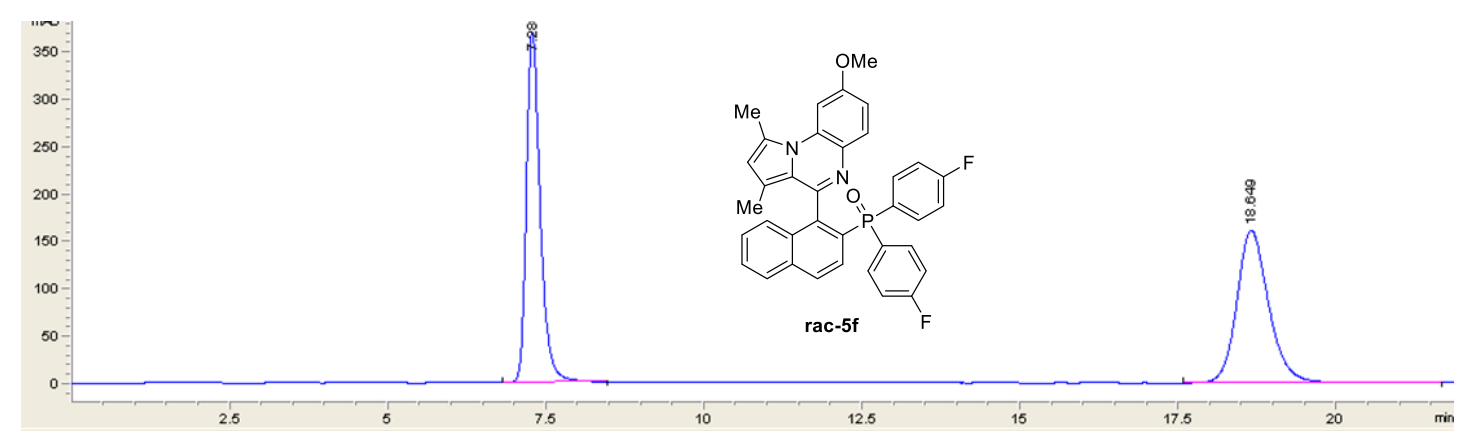

n-hexane/isopropanol 80:20, IA, $1.0 \mathrm{~mL} / \mathrm{min}$

\begin{tabular}{|c|c|c|c|c|c|}
\hline Peak \# & RetTime [min] & Width [min] & Area [mAU*s] & Height [mAU] & Area \% \\
\hline 1 & 7.280 & 0.2292 & 5632.1 & 370.8 & 49.951 \\
2 & 18.649 & 0.5331 & 5643.8 & 161.0 & 50.049 \\
\hline
\end{tabular}

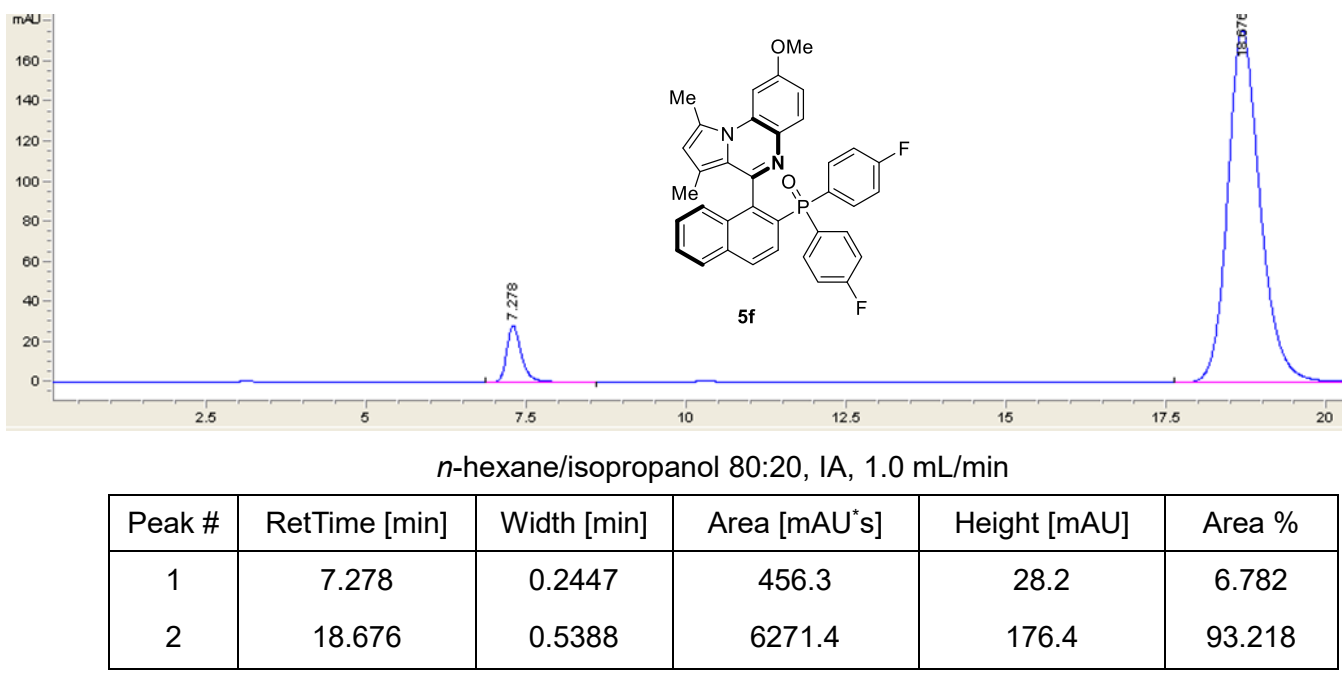




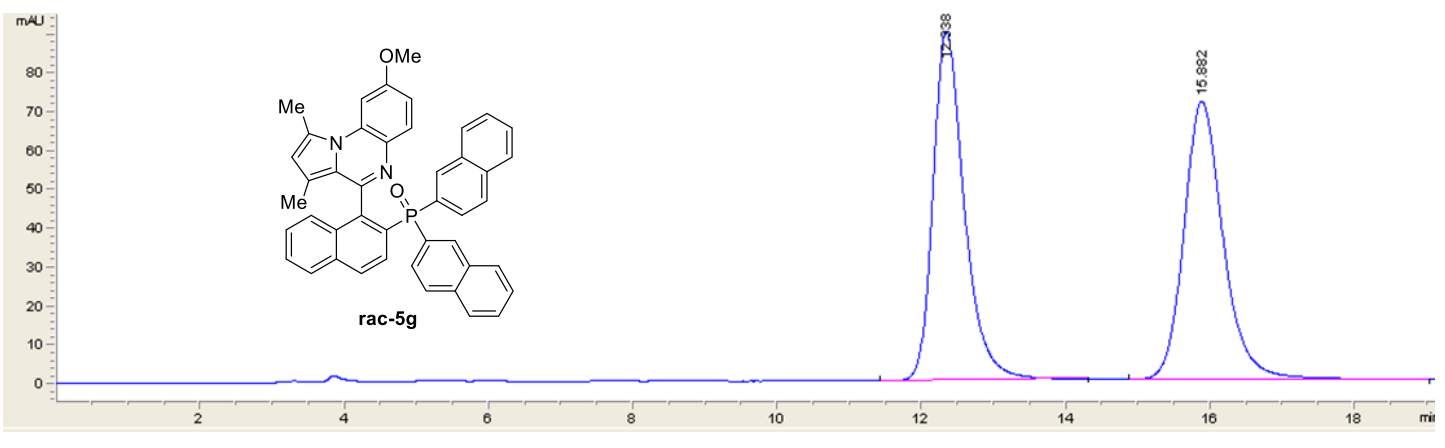

n-hexane/isopropanol 60:40, IA, $1.0 \mathrm{~mL} / \mathrm{min}$

\begin{tabular}{|c|c|c|c|c|c|}
\hline Peak \# & RetTime [min] & Width [min] & Area [mAU*s] & Height [mAU] & Area \% \\
\hline 1 & 12.338 & 0.4559 & 2710.4 & 89.9 & 49.992 \\
2 & 15.882 & 0.5759 & 2711.2 & 71.6 & 50.008 \\
\hline
\end{tabular}
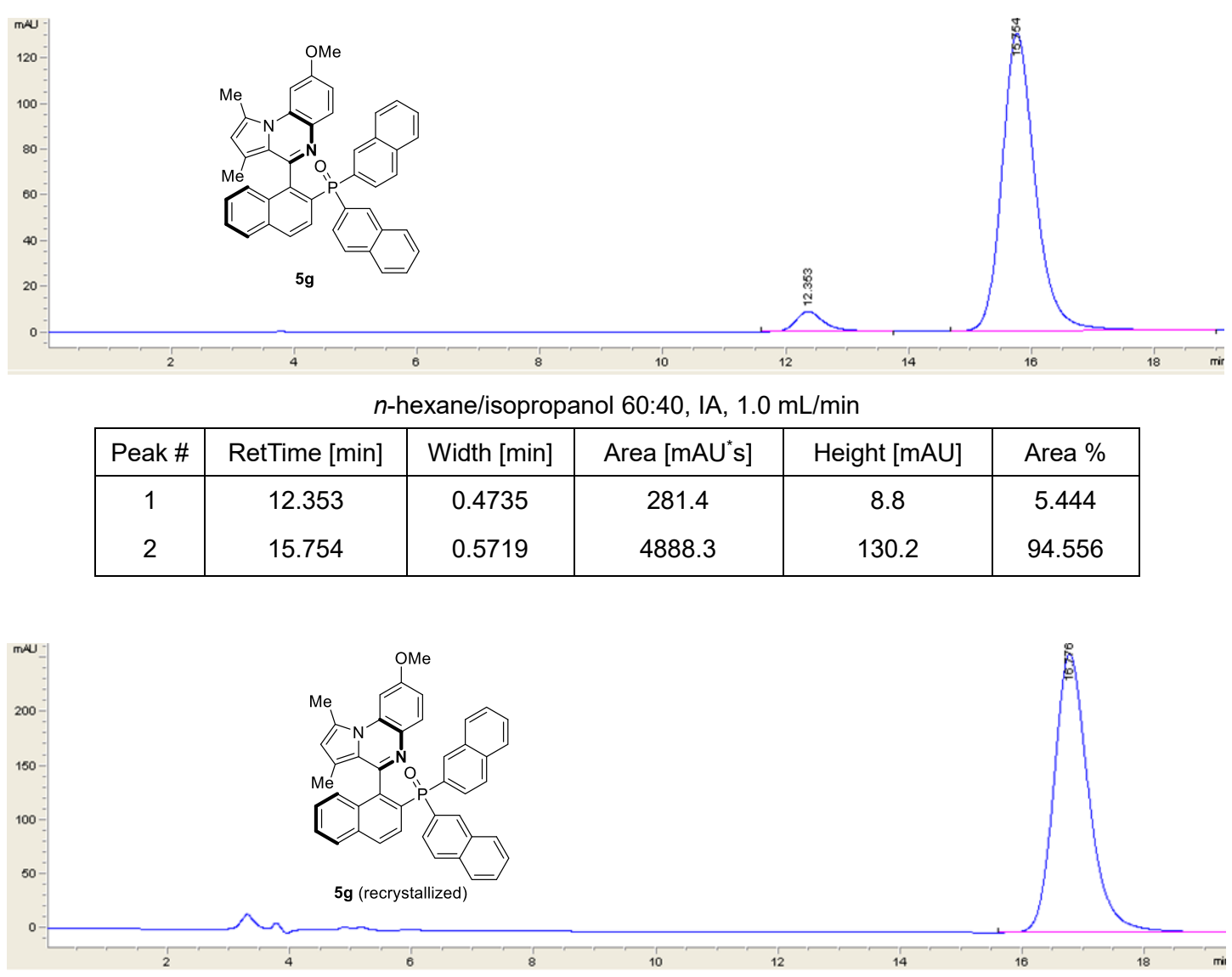

n-hexane/isopropanol 60:40, IA, $1.0 \mathrm{~mL} / \mathrm{min}$

\begin{tabular}{|c|c|c|c|c|c|}
\hline Peak \# & RetTime [min] & Width [min] & Area [mAU*s] & Height [mAU] & Area \% \\
\hline 1 & 16.776 & 0.6059 & 10283.7 & 258.6 & 100.000 \\
\hline
\end{tabular}




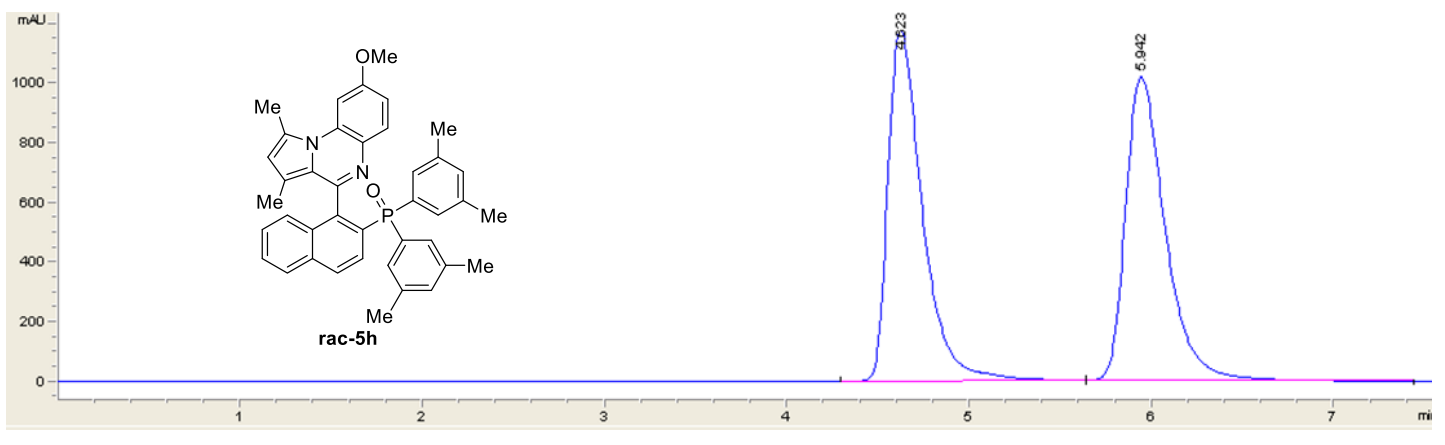

n-hexane/isopropanol 70:30, IA, $1.0 \mathrm{~mL} / \mathrm{min}$

\begin{tabular}{|c|c|c|c|c|c|}
\hline Peak \# & RetTime [min] & Width [min] & Area [mAU*s] & Height [mAU] & Area \% \\
\hline 1 & 4.623 & 0.203 & 15676.9 & 1180.1 & 50.128 \\
2 & 5.942 & 0.234 & 15596.9 & 1022.1 & 49.872 \\
\hline
\end{tabular}
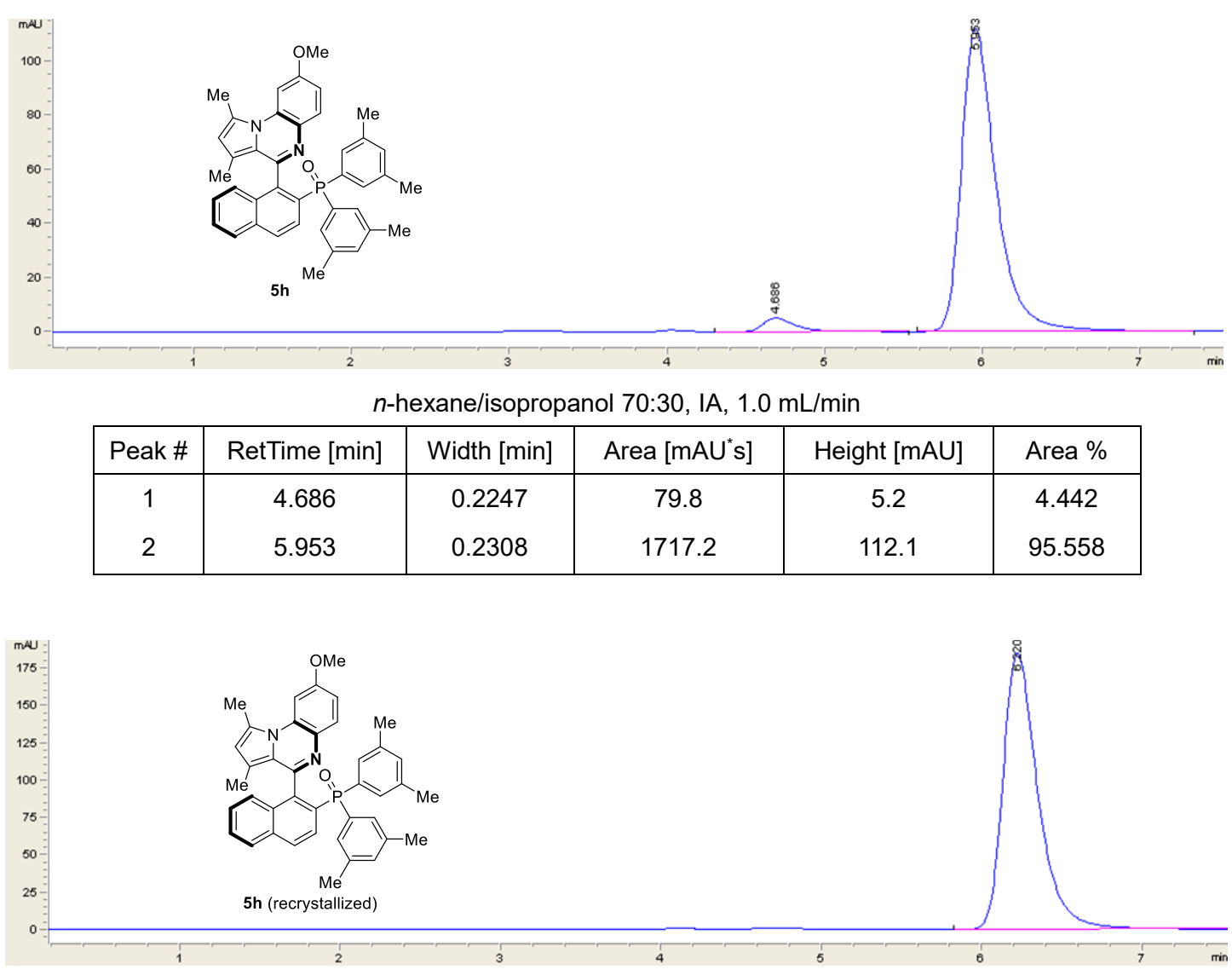

n-hexane/isopropanol 70:30, IA, $1.0 \mathrm{~mL} / \mathrm{min}$

\begin{tabular}{|c|c|c|c|c|c|}
\hline Peak \# & RetTime [min] & Width [min] & Area [mAU*s] & Height [mAU] & Area \% \\
\hline 1 & 6.220 & 0.2316 & 2817.5 & 185.1 & 100.000 \\
\hline
\end{tabular}




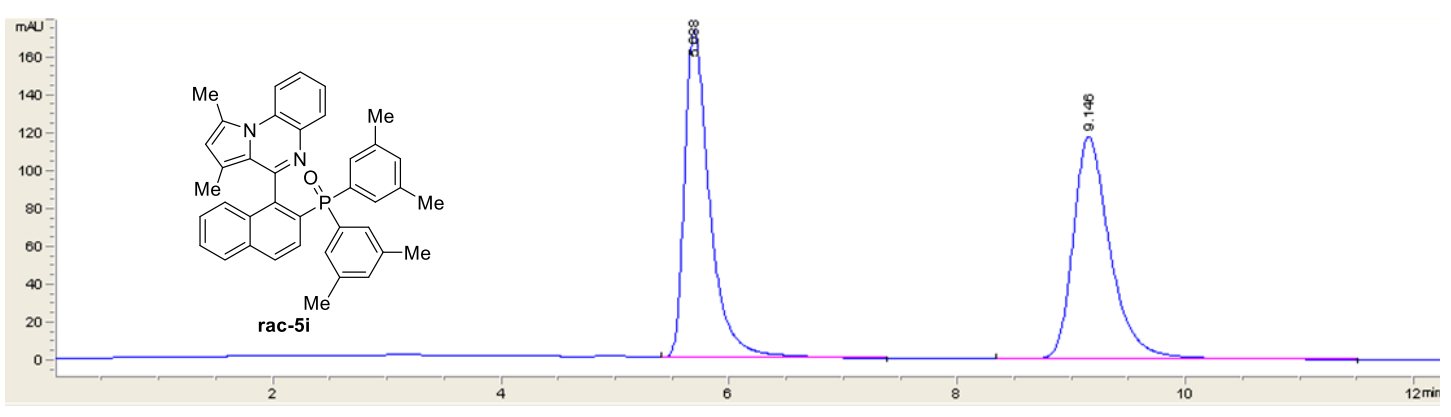

n-hexane/isopropanol 80:20, IA, $1.0 \mathrm{~mL} / \mathrm{min}$

\begin{tabular}{|c|c|c|c|c|c|}
\hline Peak \# & RetTime [min] & Width [min] & Area [mAU*s] & Height [mAU] & Area \% \\
\hline 1 & 5.688 & 0.2310 & 2651.6 & 172.8 & 49.978 \\
2 & 9.146 & 0.3397 & 2653.9 & 117.4 & 50.022 \\
\hline
\end{tabular}

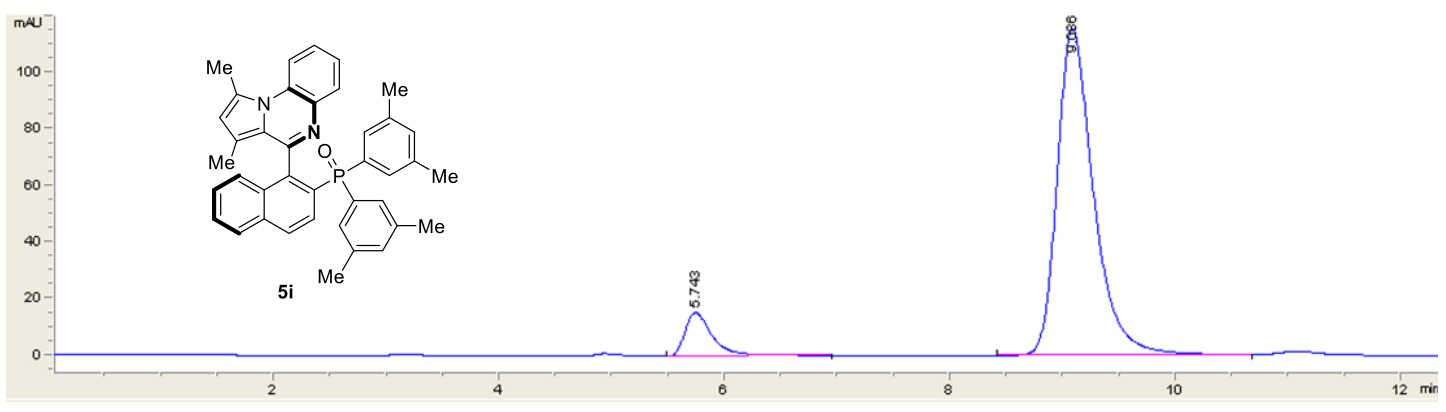

n-hexane/isopropanol 80:20, IA, $1.0 \mathrm{~mL} / \mathrm{min}$

\begin{tabular}{|c|c|c|c|c|c|}
\hline Peak \# & RetTime [min] & Width [min] & Area [mAU s] & Height [mAU] & Area \% \\
\hline 1 & 5.743 & 0.2543 & 261.2 & 15.4 & 9.154 \\
2 & 9.086 & 0.3400 & 2592.2 & 115.4 & 90.846 \\
\hline
\end{tabular}

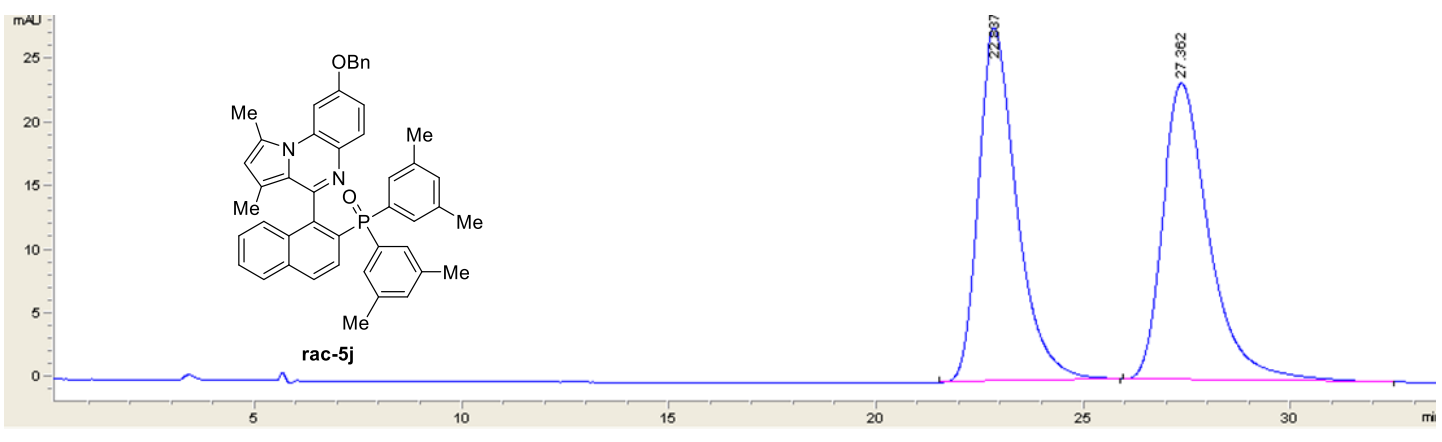

n-hexane/isopropanol 80:20, IC, $1.0 \mathrm{~mL} / \mathrm{min}$

\begin{tabular}{|c|c|c|c|c|c|}
\hline Peak \# & RetTime [min] & Width [min] & Area [mAU*s] & Height [mAU] & Area \% \\
\hline 1 & 22.837 & 0.9851 & 1809.1 & 27.8 & 49.860 \\
2 & 27.362 & 1.1833 & 1819.2 & 23.3 & 50.140 \\
\hline
\end{tabular}




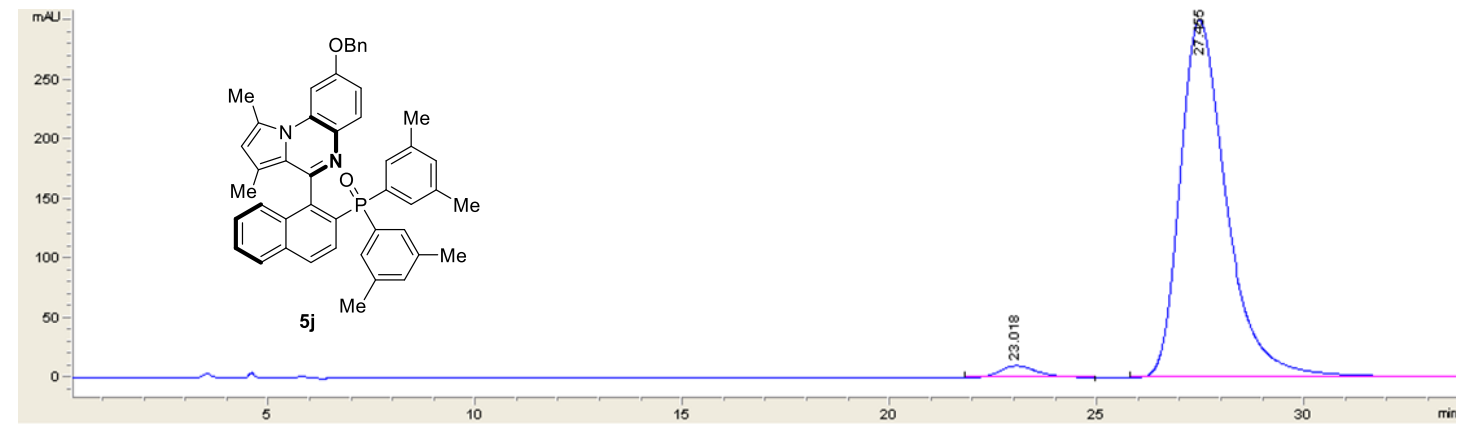

n-hexane/isopropanol 80:20, IC, $1.0 \mathrm{~mL} / \mathrm{min}$

\begin{tabular}{|c|c|c|c|c|c|}
\hline Peak \# & RetTime [min] & Width [min] & Area [mAU*s] & Height [mAU] & Area \% \\
\hline 1 & 23.018 & 0.8962 & 643.4 & 10.0 & 2.685 \\
2 & 27.455 & 1.1811 & 23320.4 & 299.9 & 97.315 \\
\hline
\end{tabular}
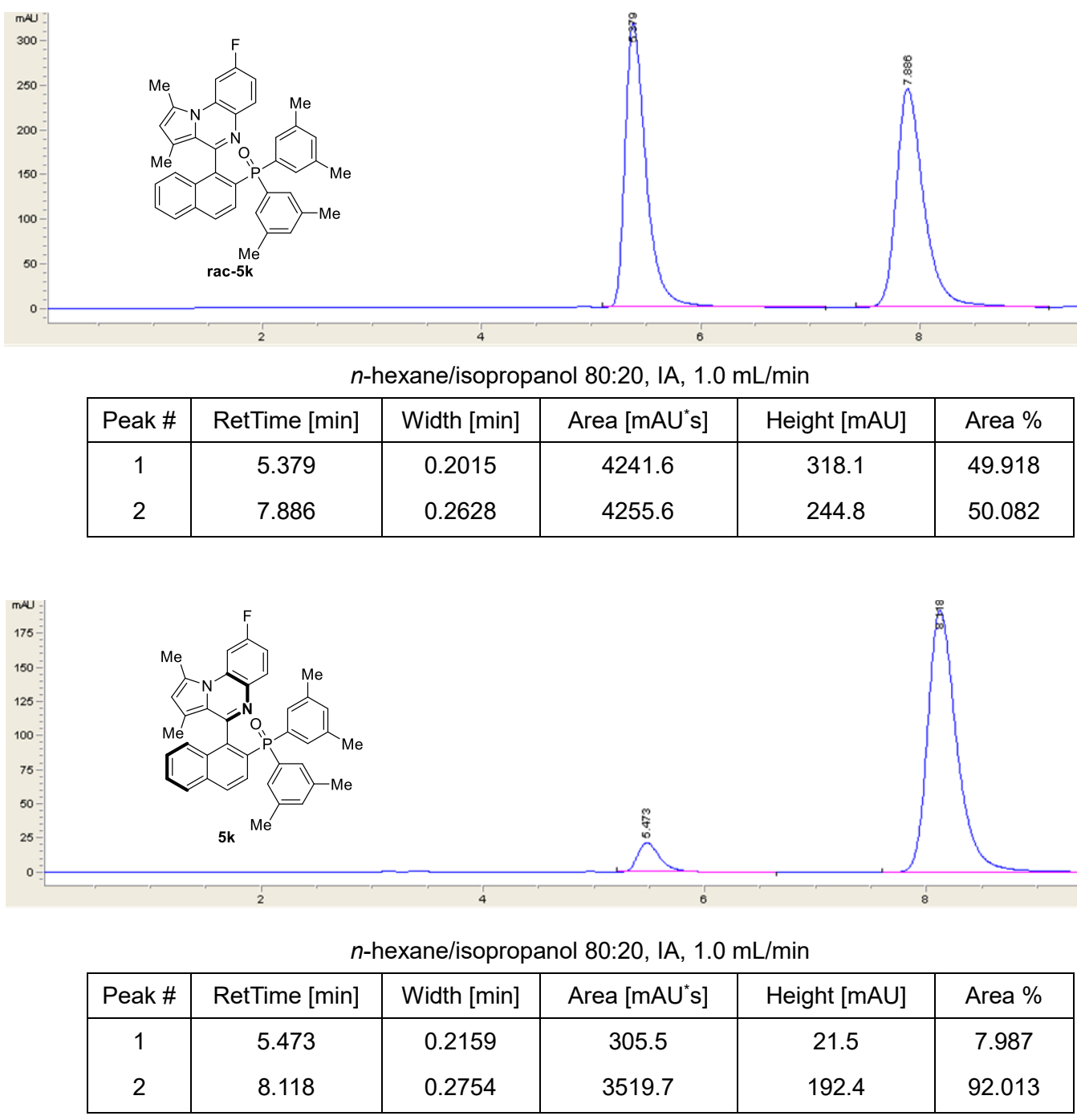


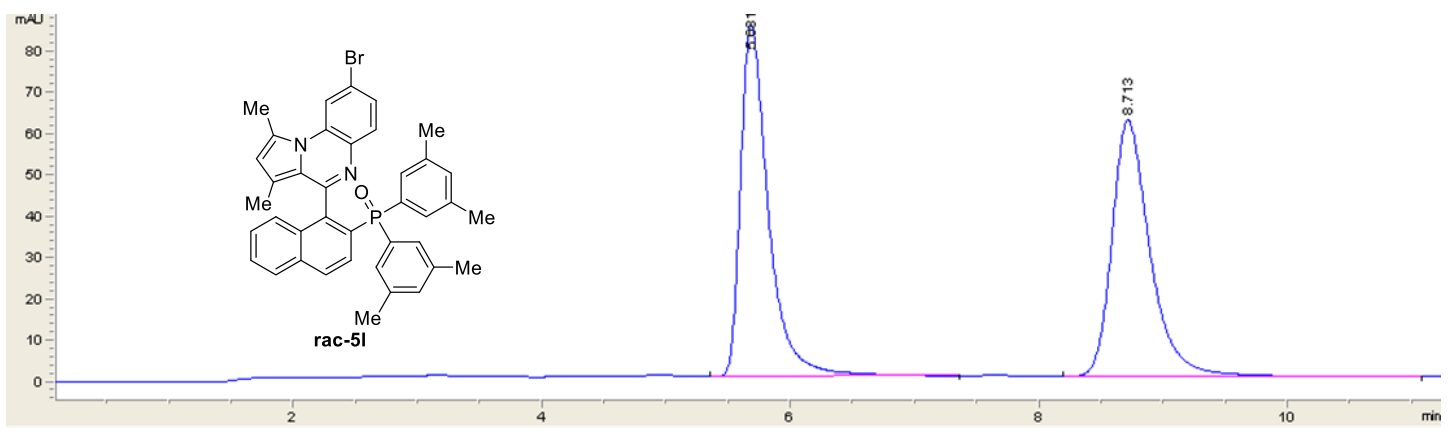

n-hexane/isopropanol 80:20, IA, $1.0 \mathrm{~mL} / \mathrm{min}$

\begin{tabular}{|c|c|c|c|c|c|}
\hline Peak \# & RetTime [min] & Width [min] & Area [mAU*s] & Height [mAU] & Area \% \\
\hline 1 & 5.681 & 0.2348 & 1337.0 & 85.3 & 50.142 \\
2 & 8.713 & 0.3219 & 1329.4 & 62.1 & 49.858 \\
\hline
\end{tabular}

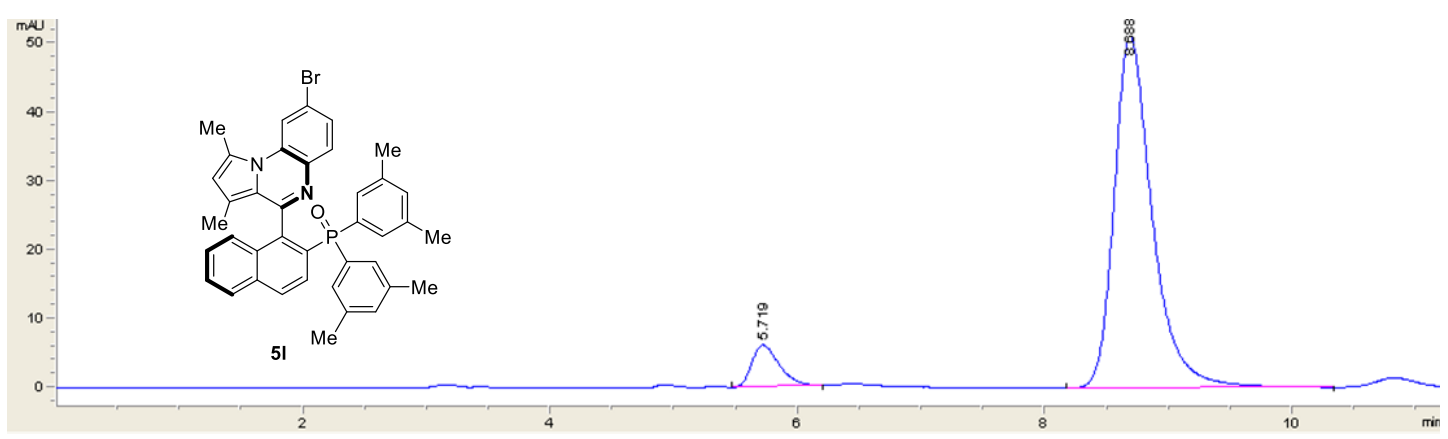

n-hexane/isopropanol 80:20, IA, $1.0 \mathrm{~mL} / \mathrm{min}$

\begin{tabular}{|c|c|c|c|c|c|}
\hline Peak \# & RetTime [min] & Width [min] & Area [mAU s] & Height [mAU] & Area \% \\
\hline 1 & 5.719 & 0.2363 & 93.9 & 6.1 & 7.857 \\
2 & 8.688 & 0.3248 & 1100.7 & 51.2 & 92.143 \\
\hline
\end{tabular}

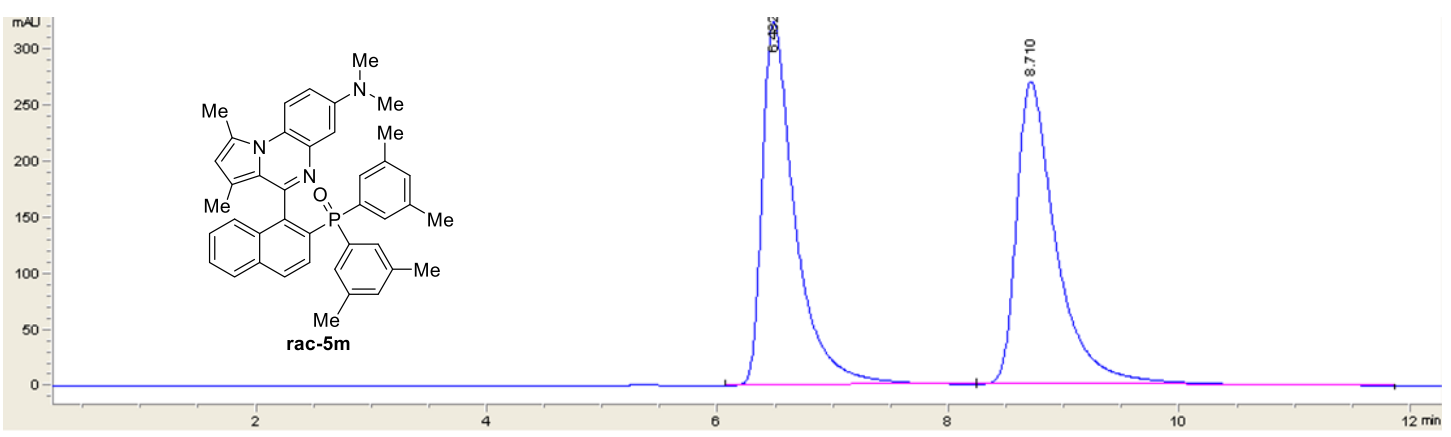

n-hexane/isopropanol 80:20, IA, $1.0 \mathrm{~mL} / \mathrm{min}$

\begin{tabular}{|c|c|c|c|c|c|}
\hline Peak \# & RetTime [min] & Width [min] & Area [mAU*s] & Height [mAU] & Area \% \\
\hline 1 & 6.482 & 0.2946 & 6466.8 & 324.4 & 49.895 \\
2 & 8.710 & 0.3569 & 6493.9 & 269.7 & 50.105 \\
\hline
\end{tabular}




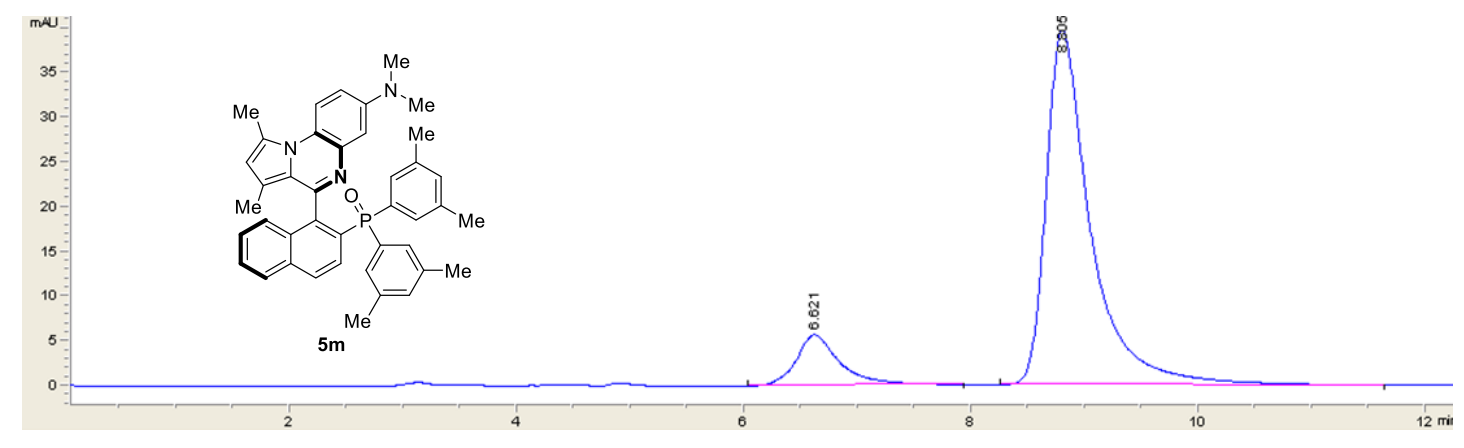

n-hexane/isopropanol 80:20, IA, $1.0 \mathrm{~mL} / \mathrm{min}$

\begin{tabular}{|c|c|c|c|c|c|}
\hline Peak \# & RetTime [min] & Width [min] & Area [mAU*s] & Height [mAU] & Area \% \\
\hline 1 & 6.621 & 0.3875 & 153.1 & 5.7 & 12.342 \\
2 & 8.805 & 0.4039 & 1087.6 & 39.7 & 87.658 \\
\hline
\end{tabular}

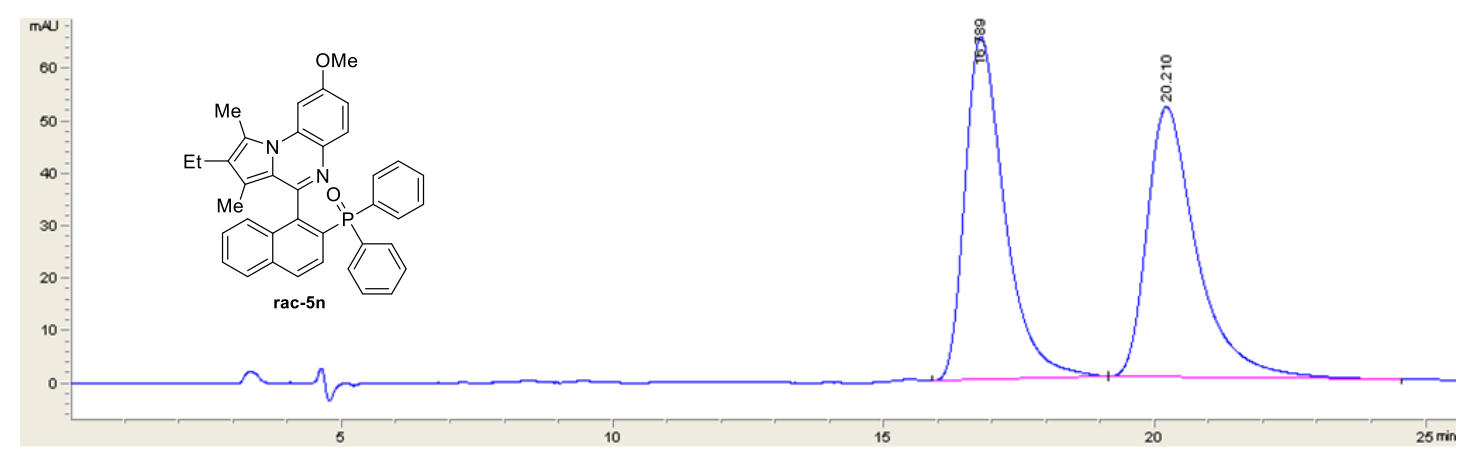

n-hexane/isopropanol 70:30, IC, $1.0 \mathrm{~mL} / \mathrm{min}$

\begin{tabular}{|c|c|c|c|c|c|}
\hline Peak \# & RetTime [min] & Width [min] & Area [mAU*s] & Height [mAU] & Area \% \\
\hline 1 & 16.789 & 0.7661 & 3320.0 & 65.4 & 50.206 \\
2 & 20.210 & 0.9576 & 3292.7 & 51.5 & 49.794 \\
\hline
\end{tabular}

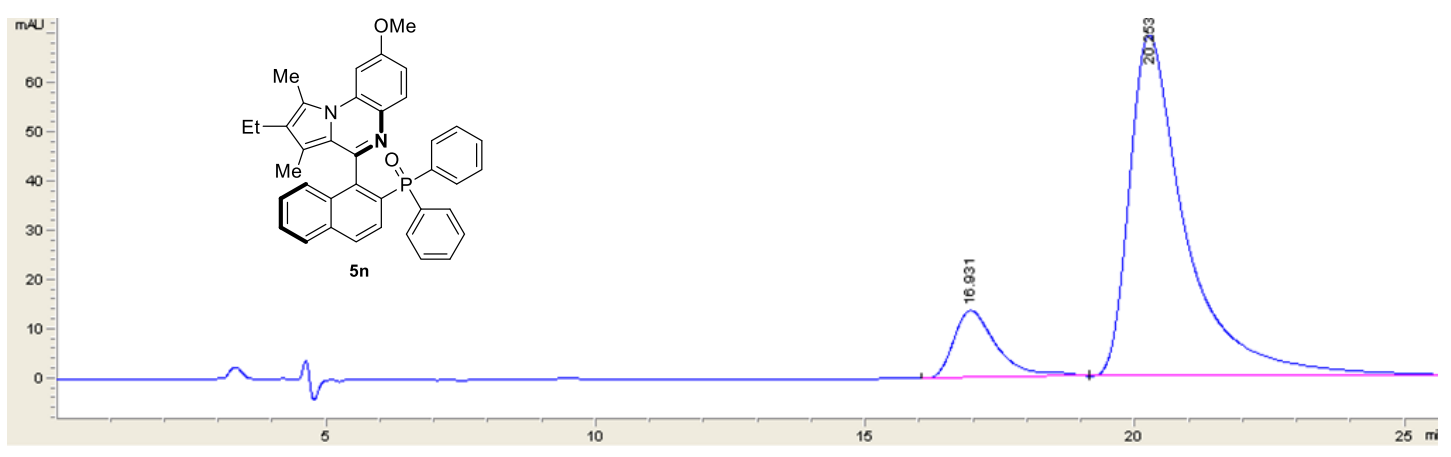

n-hexane/isopropanol 70:30, IC, $1.0 \mathrm{~mL} / \mathrm{min}$

\begin{tabular}{|c|c|c|c|c|c|}
\hline Peak \# & RetTime [min] & Width [min] & Area [mAU*s] & Height [mAU] & Area \% \\
\hline 1 & 16.931 & 0.8269 & 758.8 & 13.7 & 13.025 \\
2 & 20.253 & 1.0682 & 5067.2 & 69.2 & 86.975 \\
\hline
\end{tabular}




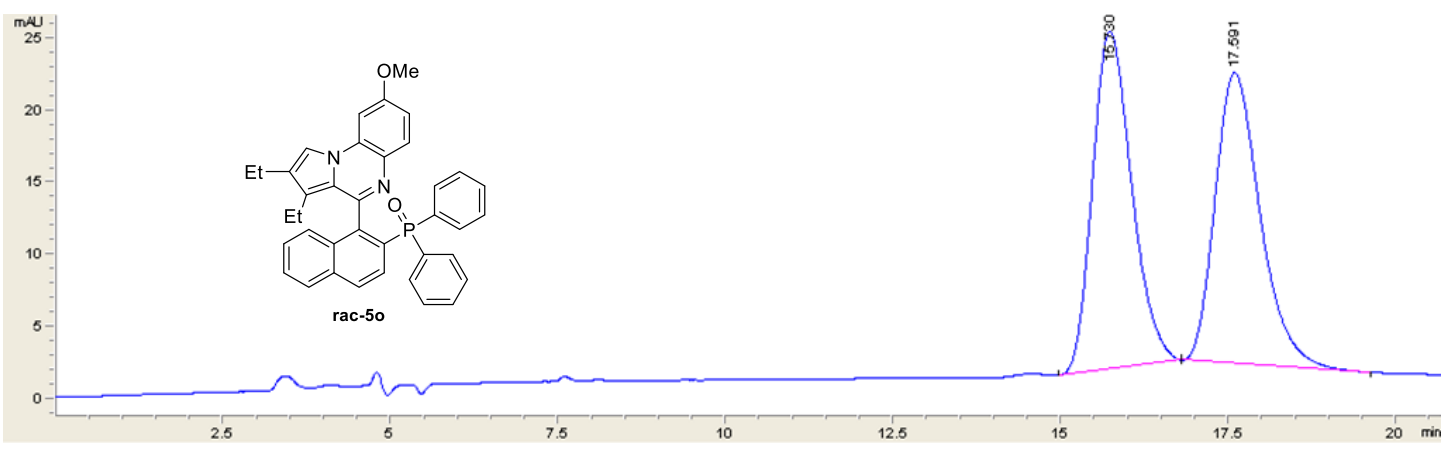

n-hexane/isopropanol 70:30, IC, $1.0 \mathrm{~mL} / \mathrm{min}$

\begin{tabular}{|c|c|c|c|c|c|}
\hline Peak \# & RetTime [min] & Width [min] & Area [mAU*s] & Height [mAU] & Area \% \\
\hline 1 & 15.730 & 0.6296 & 950.8 & 23.4 & 50.194 \\
2 & 17.591 & 0.7140 & 943.4 & 20.2 & 49.806 \\
\hline
\end{tabular}

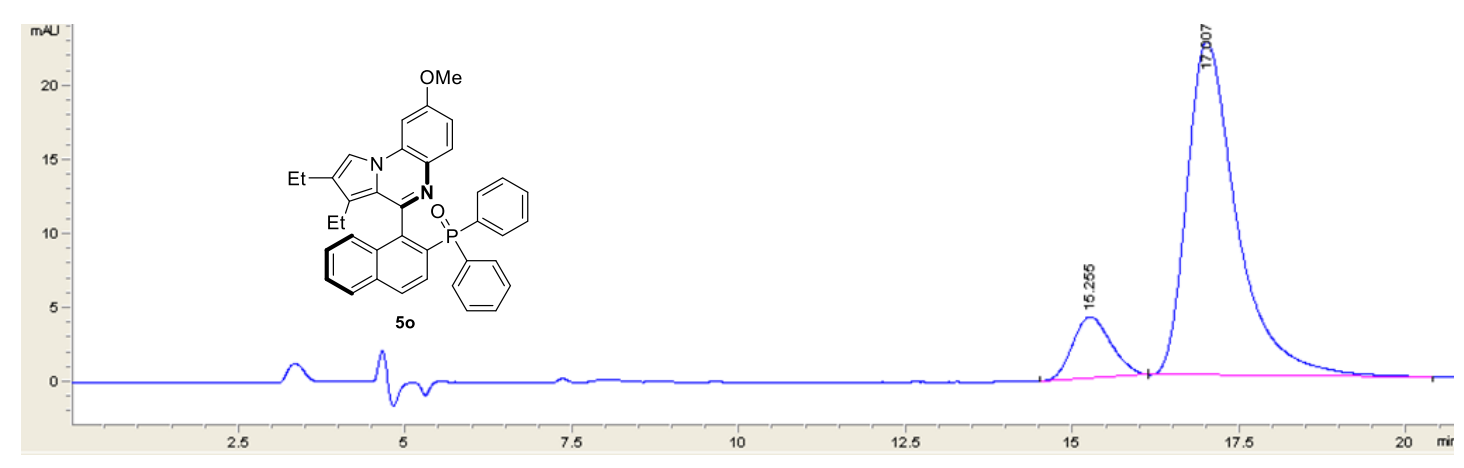

n-hexane/isopropanol 70:30, IC, $1.0 \mathrm{~mL} / \mathrm{min}$

\begin{tabular}{|c|c|c|c|c|c|}
\hline Peak \# & RetTime [min] & Width [min] & Area [mAU*s] & Height [mAU] & Area \% \\
\hline 1 & 15.255 & 0.6024 & 165.9 & 4.2 & 12.396 \\
2 & 17.007 & 0.7829 & 1172.8 & 22.5 & 87.604 \\
\hline
\end{tabular}

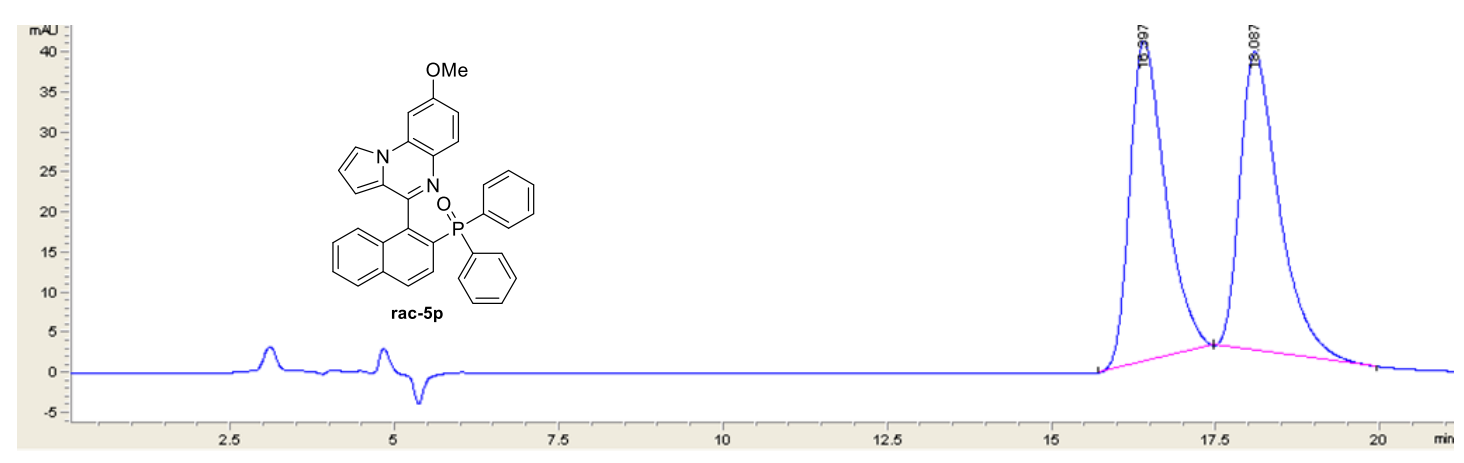

n-hexane/isopropanol 80:20, IA, $1.0 \mathrm{~mL} / \mathrm{min}$

\begin{tabular}{|c|c|c|c|c|c|}
\hline Peak \# & RetTime [min] & Width [min] & Area [mAUs] & Height [mAU] & Area \% \\
\hline 1 & 16.397 & 0.5688 & 1514.2 & 40.1 & 49.504 \\
2 & 18.087 & 0.6164 & 1544.5 & 37.4 & 50.496 \\
\hline
\end{tabular}




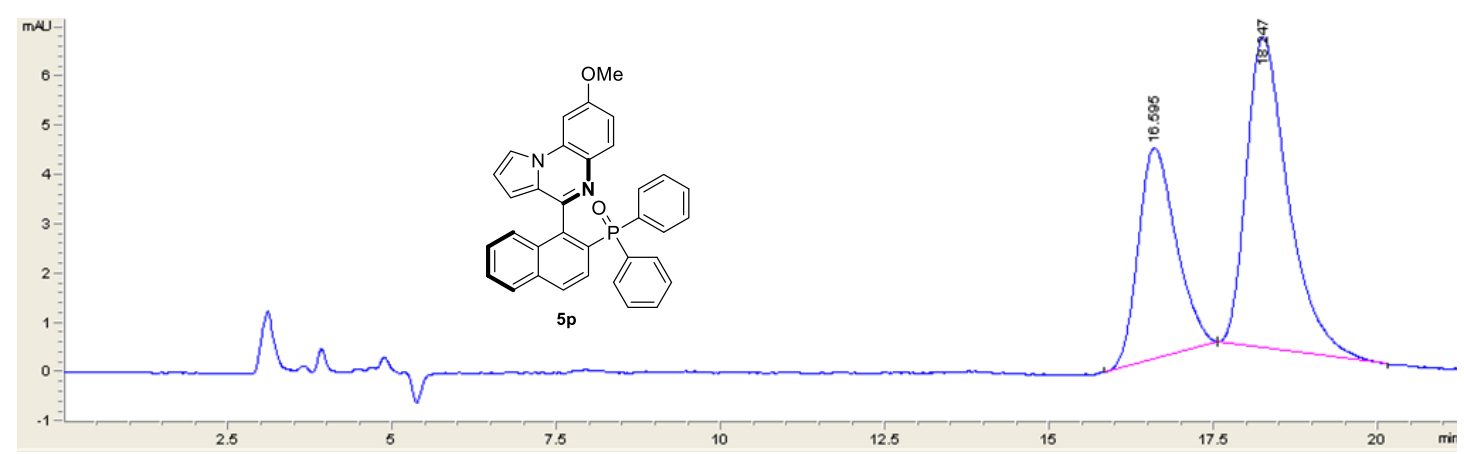

n-hexane/isopropanol 80:20, IA, $1.0 \mathrm{~mL} / \mathrm{min}$

\begin{tabular}{|c|c|c|c|c|c|}
\hline Peak \# & RetTime [min] & Width [min] & Area [mAU*s] & Height [mAU] & Area \% \\
\hline 1 & 16.595 & 0.5761 & 171.7 & 4.3 & 37.903 \\
2 & 18.247 & 0.6532 & 281.3 & 6.3 & 62.097 \\
\hline
\end{tabular}

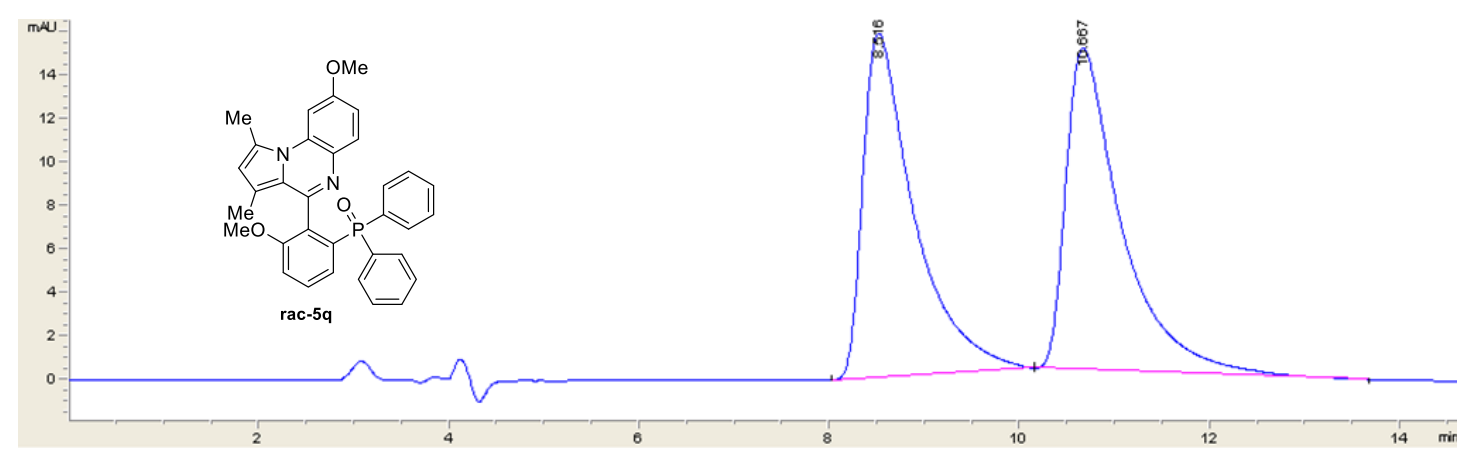

n-hexane/isopropanol 70:30, IA, $1.0 \mathrm{~mL} / \mathrm{min}$

\begin{tabular}{|c|c|c|c|c|c|}
\hline Peak \# & RetTime [min] & Width [min] & Area [mAUs] & Height [mAU] & Area \% \\
\hline 1 & 8.516 & 0.5409 & 595.9 & 15.8 & 49.678 \\
2 & 10.667 & 0.5839 & 603.7 & 14.8 & 50.322 \\
\hline
\end{tabular}

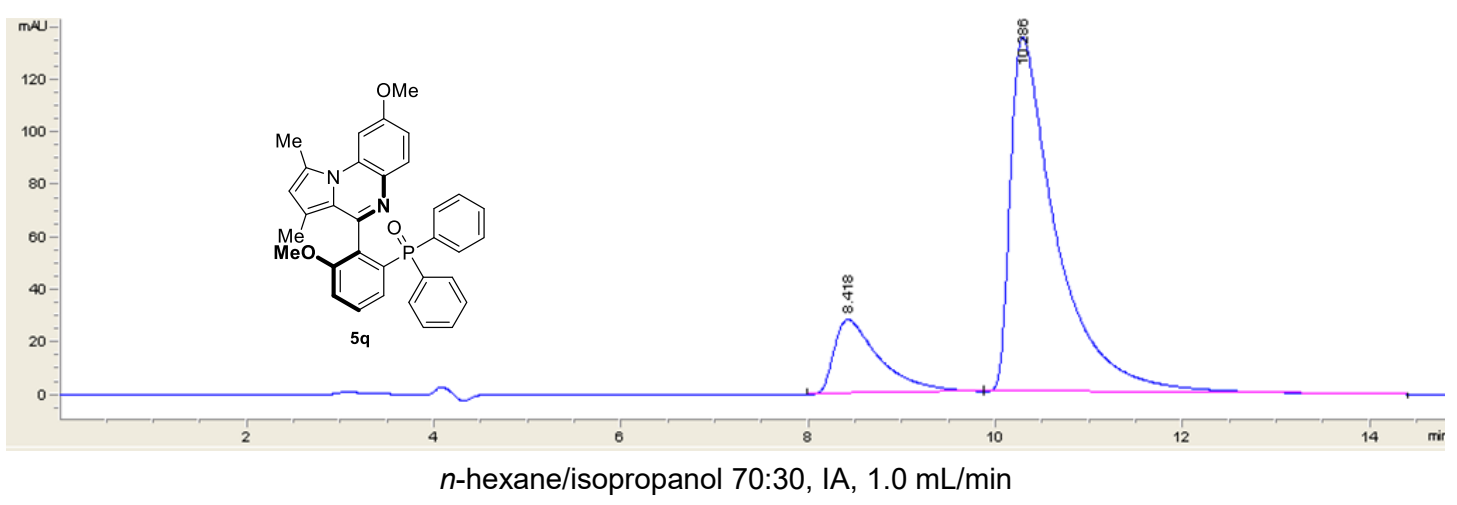

\begin{tabular}{|c|c|c|c|c|c|}
\hline Peak \# & RetTime [min] & Width [min] & Area [mAU*s] & Height [mAU] & Area \% \\
\hline 1 & 8.418 & 0.4987 & 962.6 & 28.3 & 17.161 \\
2 & 10.286 & 0.4928 & 4646.4 & 135.2 & 82.839 \\
\hline
\end{tabular}




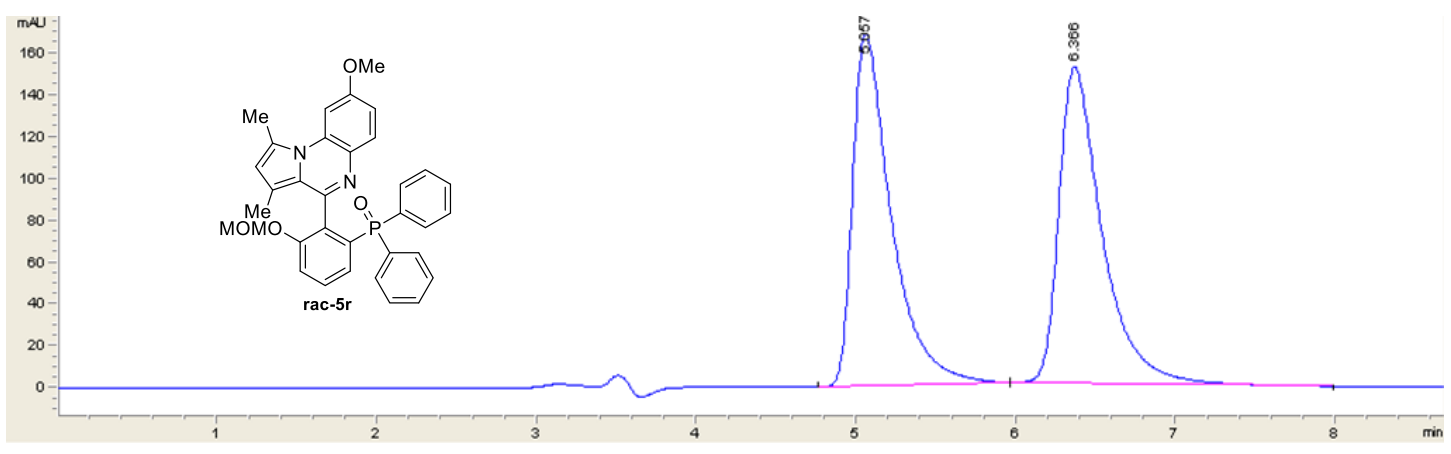

n-hexane/isopropanol 50:50, IA, $1.0 \mathrm{~mL} / \mathrm{min}$

\begin{tabular}{|c|c|c|c|c|c|}
\hline Peak \# & RetTime [min] & Width [min] & Area [mAU*s] & Height [mAU] & Area \% \\
\hline 1 & 5.057 & 0.2484 & 2834.8 & 168.4 & 49.864 \\
2 & 6.366 & 0.2798 & 2850.3 & 151.3 & 50.136 \\
\hline
\end{tabular}

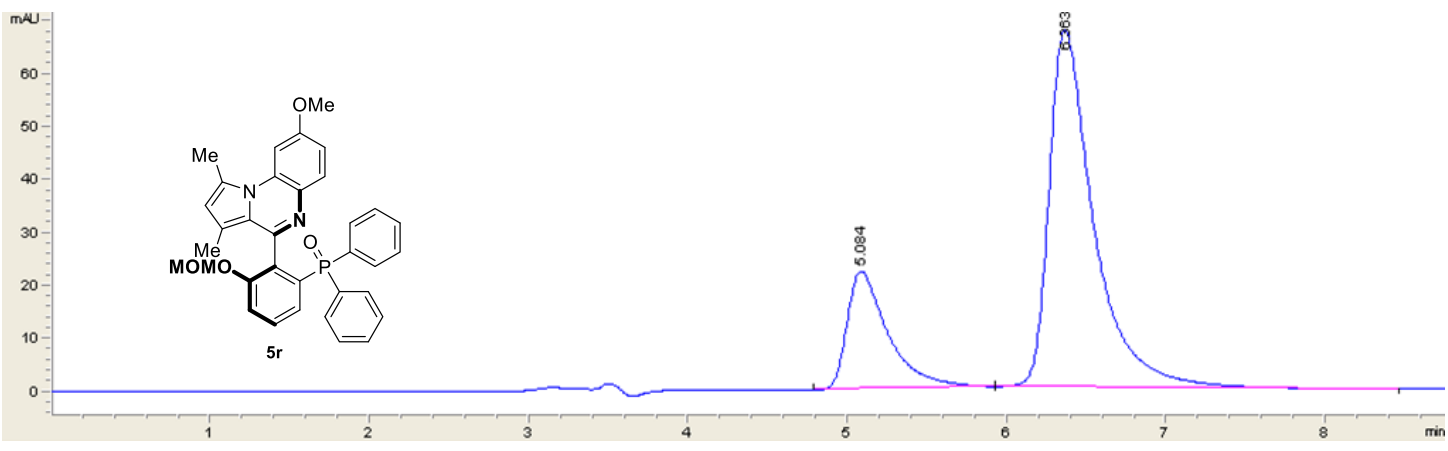

n-hexane/isopropanol 50:50, IA, $1.0 \mathrm{~mL} / \mathrm{min}$

\begin{tabular}{|c|c|c|c|c|c|}
\hline Peak \# & RetTime [min] & Width [min] & Area [mAU*s] & Height [mAU] & Area \% \\
\hline 1 & 5.084 & 0.2638 & 400.3 & 22.3 & 23.194 \\
2 & 6.363 & 0.2888 & 1325.7 & 67.6 & 76.806 \\
\hline
\end{tabular}

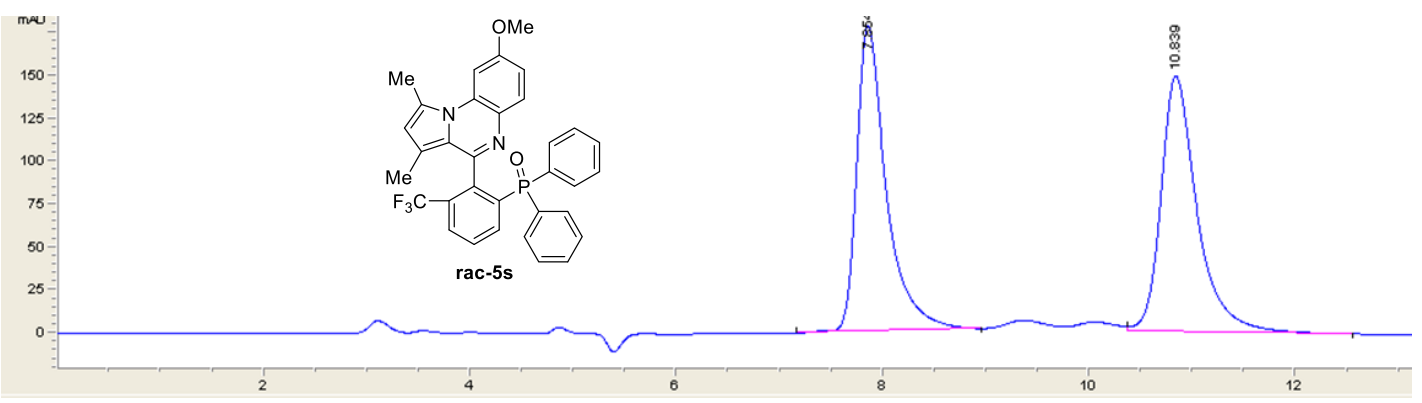

n-hexane/isopropanol 80:20, IA, $1.0 \mathrm{~mL} / \mathrm{min}$

\begin{tabular}{|c|c|c|c|c|c|}
\hline Peak \# & RetTime [min] & Width [min] & Area [mAU*s] & Height [mAU] & Area \% \\
\hline 1 & 7.854 & 0.2980 & 3574.5 & 178.2 & 49.711 \\
2 & 10.839 & 0.3596 & 3616.0 & 148.8 & 50.289 \\
\hline
\end{tabular}




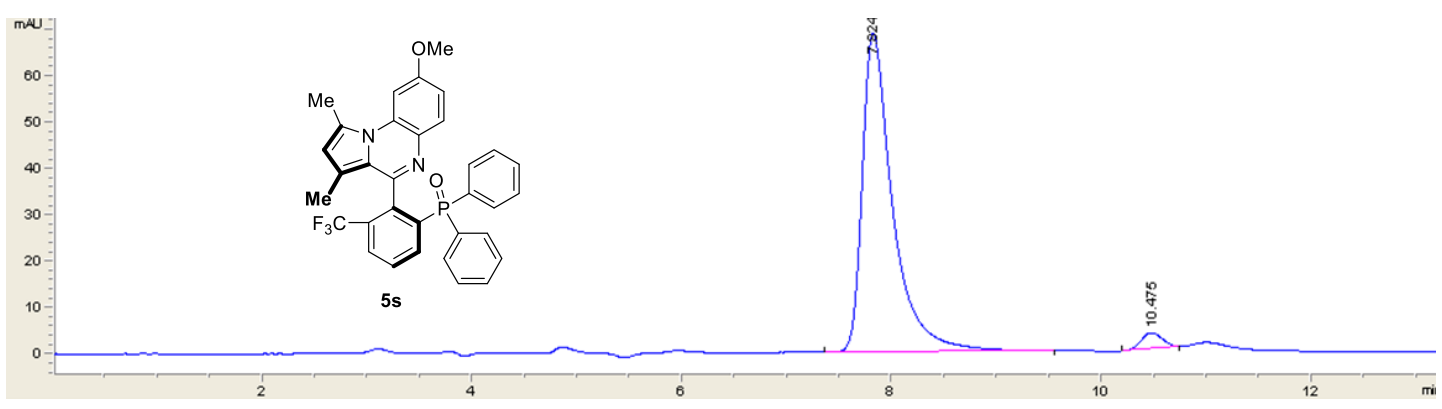

n-hexane/isopropanol 80:20, IA, $1.0 \mathrm{~mL} / \mathrm{min}$

\begin{tabular}{|c|c|c|c|c|c|}
\hline Peak \# & RetTime [min] & Width [min] & Area [mAUs] & Height [mAU] & Area \% \\
\hline 1 & 7.824 & 0.2964 & 1383.6 & 68.9 & 96.608 \\
2 & 10.475 & 0.2157 & 48.6 & 3.5 & 3.392 \\
\hline
\end{tabular}

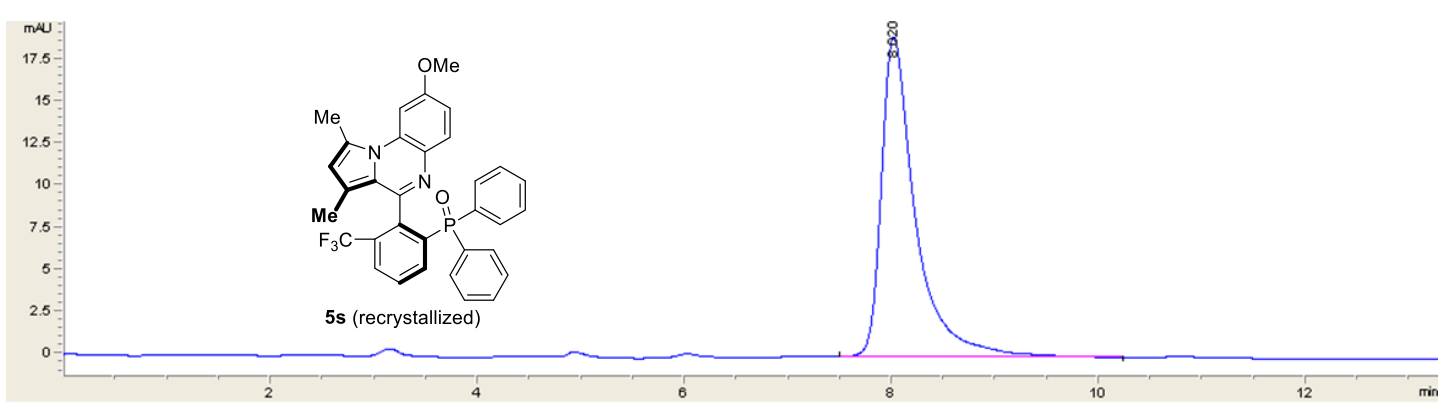

n-hexane/isopropanol 80:20, IA, $1.0 \mathrm{~mL} / \mathrm{min}$

\begin{tabular}{|c|c|c|c|c|c|}
\hline Peak \# & RetTime [min] & Width [min] & Area [mAU*s] & Height [mAU] & Area \% \\
\hline 1 & 8.02 & 0.3341 & 436.6 & 19.0 & 100.00 \\
\hline
\end{tabular}

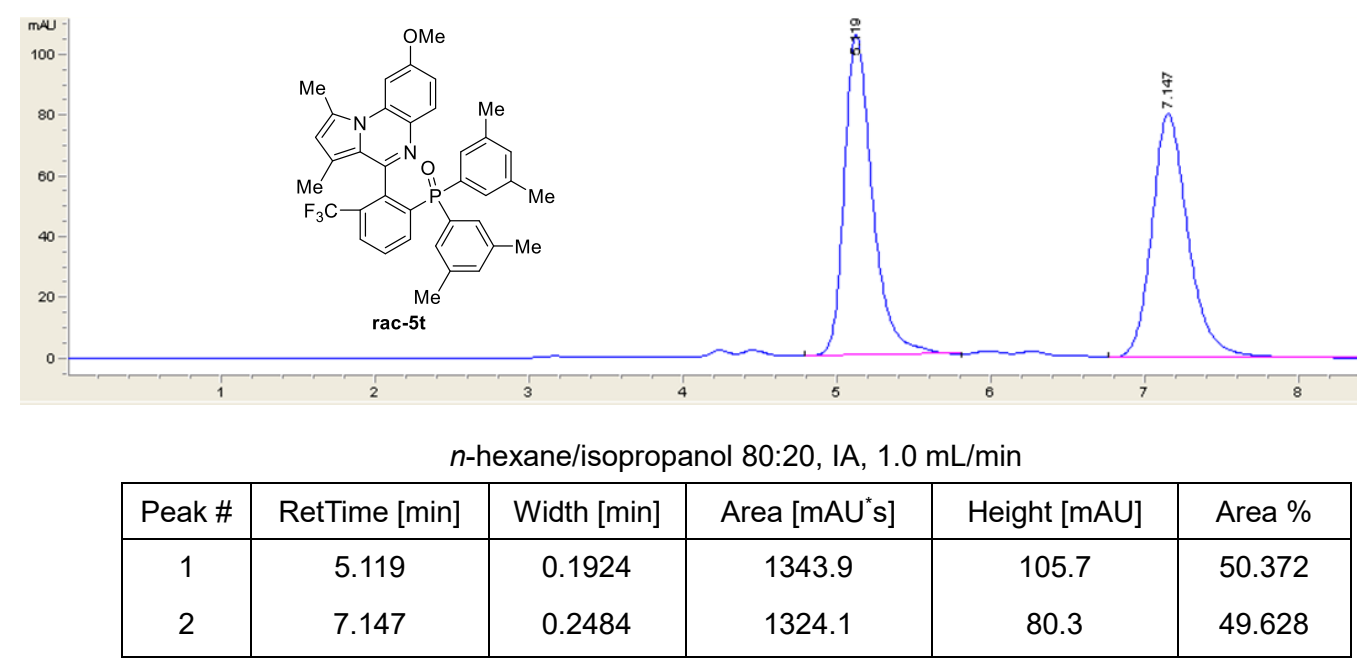




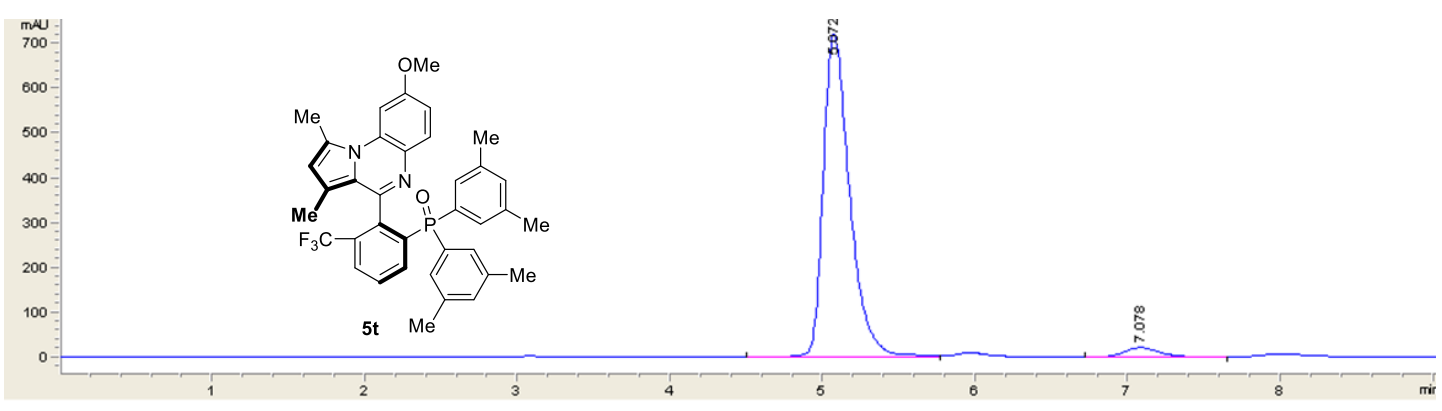

n-hexane/isopropanol 80:20, IA, $1.0 \mathrm{~mL} / \mathrm{min}$

\begin{tabular}{|c|c|c|c|c|c|}
\hline Peak \# & RetTime [min] & Width [min] & Area [mAUs] & Height [mAU] & Area \% \\
\hline 1 & 5.072 & 0.1852 & 8745.6 & 722.7 & 96.272 \\
2 & 7.078 & 0.2457 & 338.7 & 21.1 & 3.728 \\
\hline
\end{tabular}

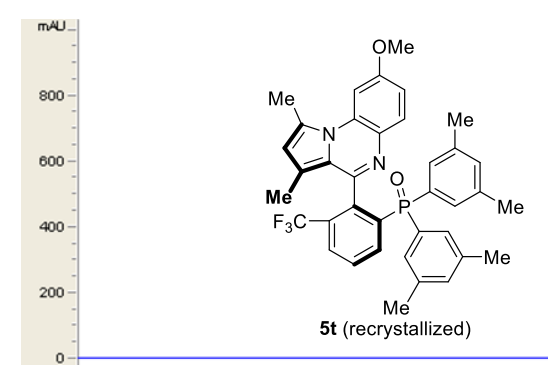

n-hexane/isopropanol 80:20, IA, $1.0 \mathrm{~mL} / \mathrm{min}$

\begin{tabular}{|c|c|c|c|c|c|}
\hline Peak \# & RetTime [min] & Width [min] & Area [mAU*s] & Height [mAU] & Area \% \\
\hline 1 & 5.179 & 0.1858 & 11947.9 & 982.9 & 100.000 \\
\hline
\end{tabular}

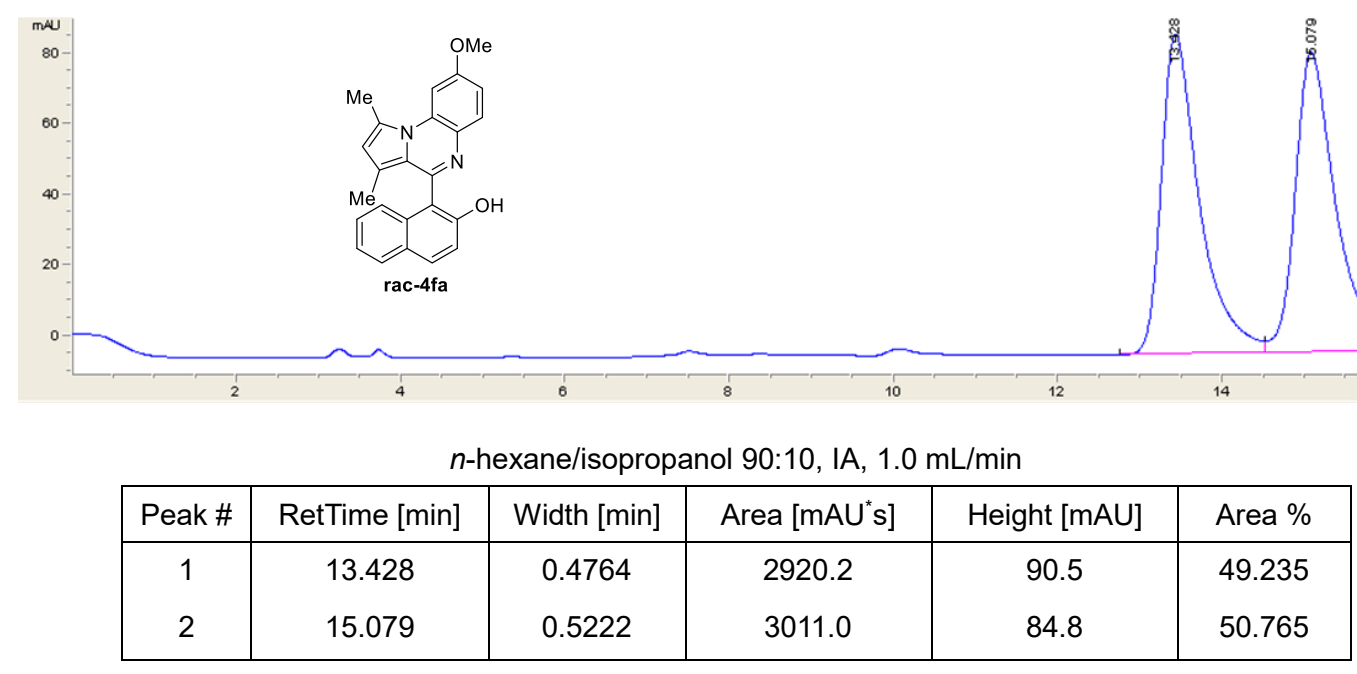




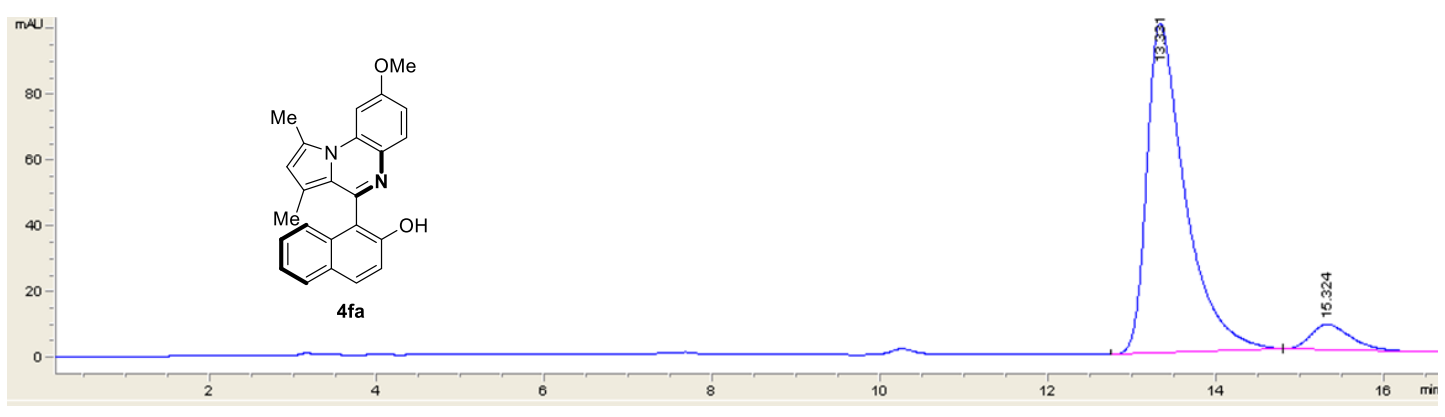

n-hexane/isopropanol 90:10, IA, $1.0 \mathrm{~mL} / \mathrm{min}$

\begin{tabular}{|c|c|c|c|c|c|}
\hline Peak \# & RetTime [min] & Width [min] & Area [mAU*s] & Height [mAU] & Area \% \\
\hline 1 & 13.331 & 0.4646 & 3183.8 & 100.2 & 92.190 \\
2 & 15.324 & 0.5252 & 269.7 & 7.7 & 7.810 \\
\hline
\end{tabular}

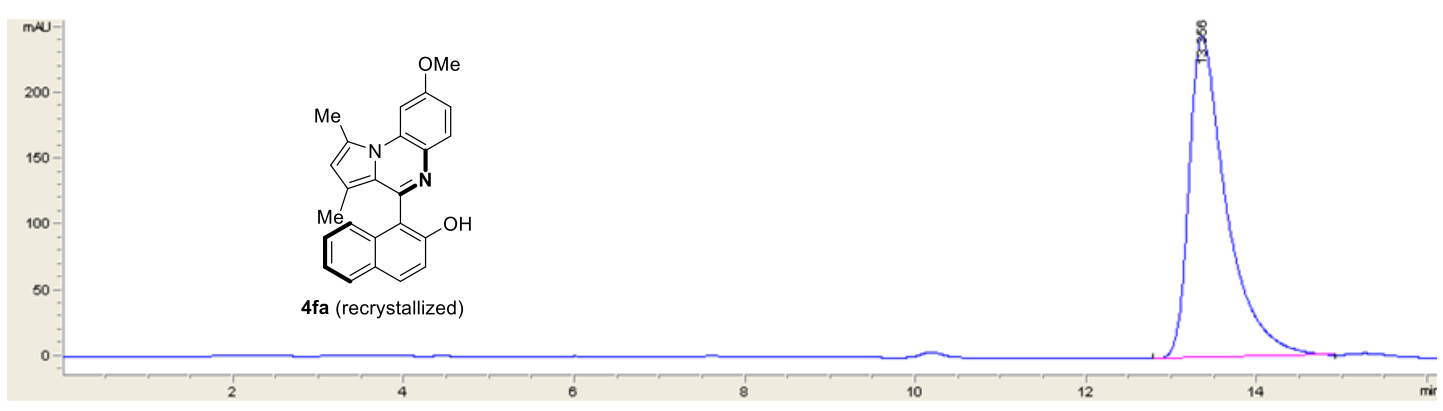

n-hexane/isopropanol 90:10, IA, $1.0 \mathrm{~mL} / \mathrm{min}$

\begin{tabular}{|c|c|c|c|c|c|}
\hline Peak \# & RetTime [min] & Width [min] & Area $\left[\mathrm{mAU}^{*}\right.$ s] & Height [mAU] & Area \% \\
\hline 1 & 13.356 & 0.4463 & 7468.8 & 244.5 & 100.000 \\
\hline
\end{tabular}

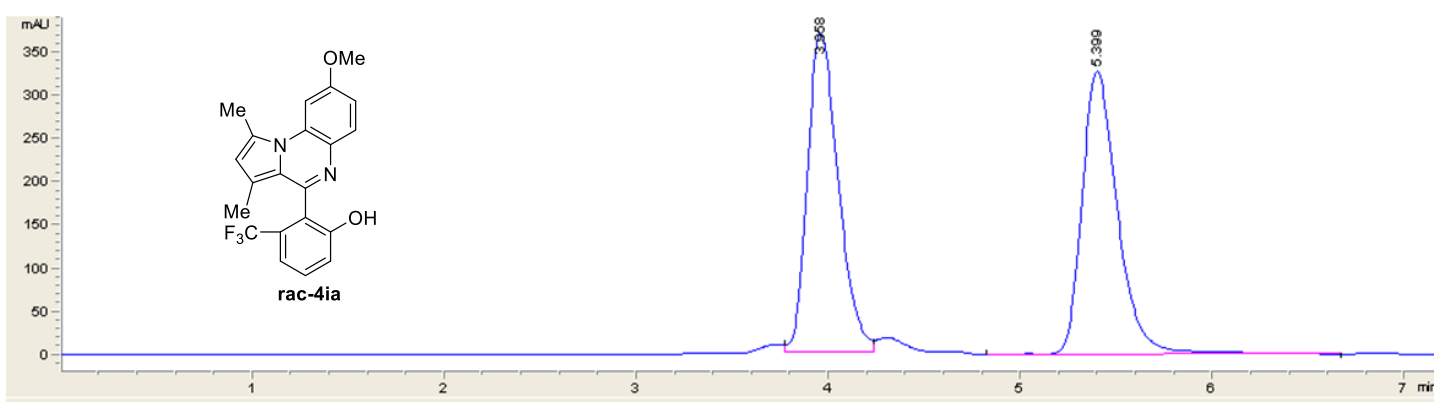

n-hexane/isopropanol 70:30, IC, $1.0 \mathrm{~mL} / \mathrm{min}$

\begin{tabular}{|c|c|c|c|c|c|}
\hline Peak \# & RetTime [min] & Width [min] & Area [mAU ${ }^{*}$ ] & Height [mAU] & Area \% \\
\hline 1 & 3.958 & 0.1730 & 4152.3 & 369.8 & 49.363 \\
2 & 5.399 & 0.1977 & 4259.4 & 327.6 & 50.637 \\
\hline
\end{tabular}




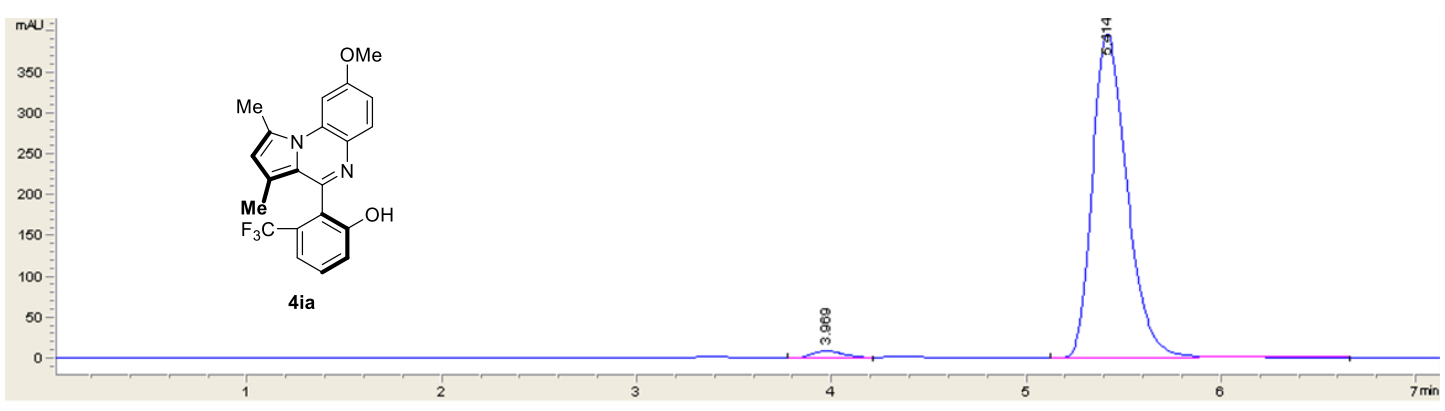

n-hexane/isopropanol 70:30, IC, $1.0 \mathrm{~mL} / \mathrm{min}$

\begin{tabular}{|c|c|c|c|c|c|}
\hline Peak \# & RetTime [min] & Width [min] & Area [mAU*s] & Height [mAU] & Area \% \\
\hline 1 & 3.969 & 0.1804 & 105.2 & 9.0 & 2.083 \\
2 & 5.414 & 0.1915 & 4944.3 & 396.6 & 97.917 \\
\hline
\end{tabular}

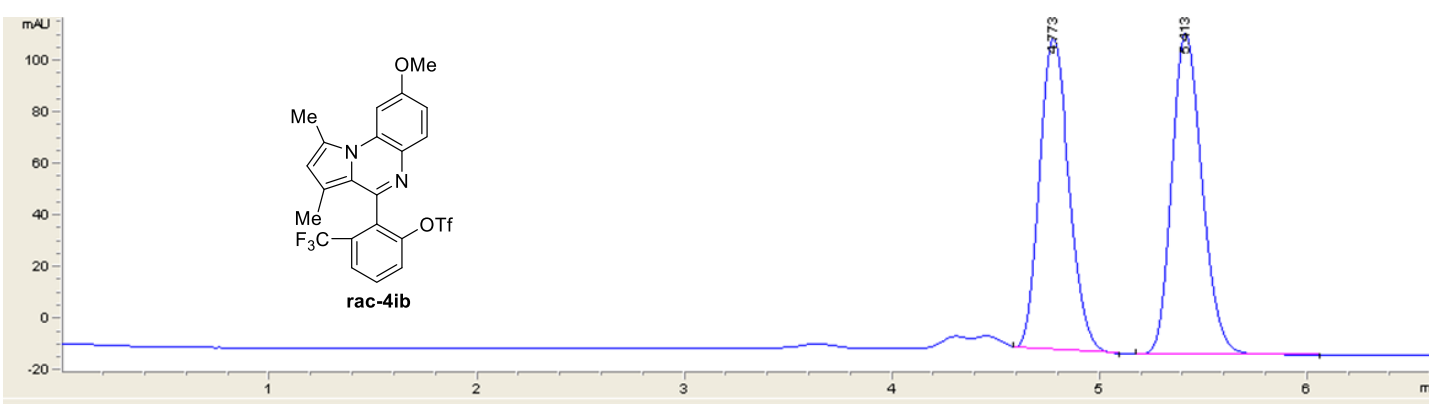

$n$-hexane/isopropanol 95:5, IC, $1.0 \mathrm{~mL} / \mathrm{min}$

\begin{tabular}{|c|c|c|c|c|c|}
\hline Peak \# & RetTime [min] & Width [min] & Area [mAUs] & Height [mAU] & Area \% \\
\hline 1 & 4.773 & 0.1571 & 1215.4 & 121.1 & 49.303 \\
2 & 5.413 & 0.1550 & 1249.7 & 124.6 & 50.697 \\
\hline
\end{tabular}
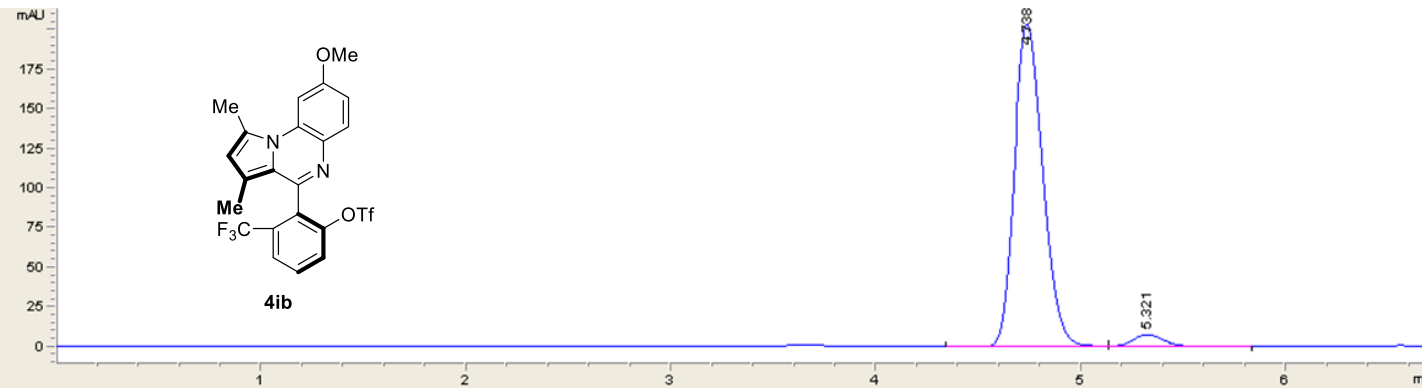

n-hexane/isopropanol 95:5, IC, $1.0 \mathrm{~mL} / \mathrm{min}$

\begin{tabular}{|c|c|c|c|c|c|}
\hline Peak \# & RetTime [min] & Width [min] & Area [mAU*s] & Height [mAU] & Area \% \\
\hline 1 & 4.738 & 0.1534 & 1981.6 & 203.9 & 96.202 \\
2 & 5.321 & 0.1588 & 78.2 & 7.6 & 3.798 \\
\hline
\end{tabular}




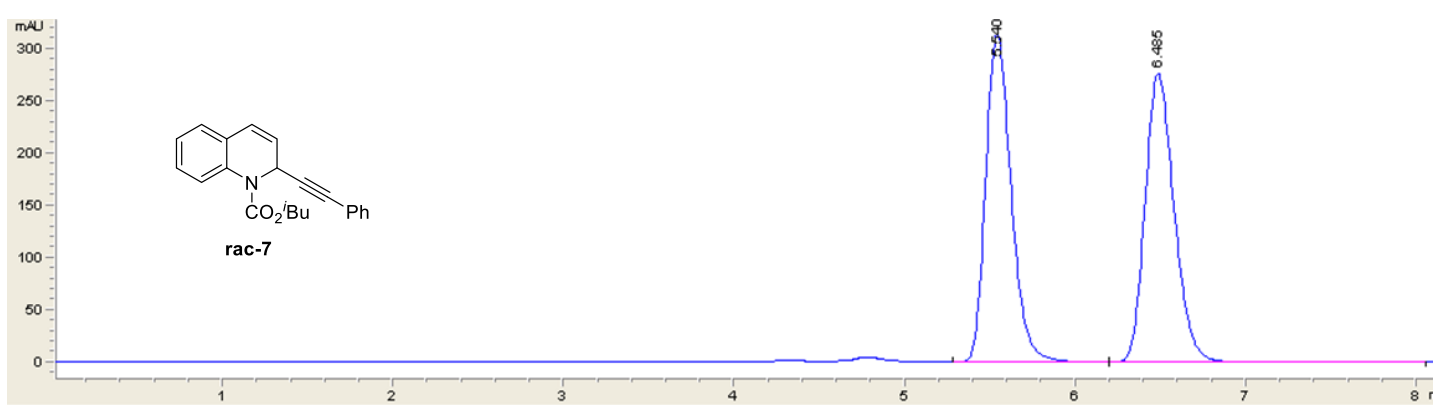

n-hexane/isopropanol 90:10, OD-H, $1.0 \mathrm{~mL} / \mathrm{min}$

\begin{tabular}{|c|c|c|c|c|c|}
\hline Peak \# & RetTime [min] & Width [min] & Area [mAU*s] & Height [mAU] & Area \% \\
\hline 1 & 5.540 & 0.1609 & 3261.5 & 314.6 & 50.063 \\
2 & 6.485 & 0.1834 & 3253.3 & 276.3 & 49.937 \\
\hline
\end{tabular}

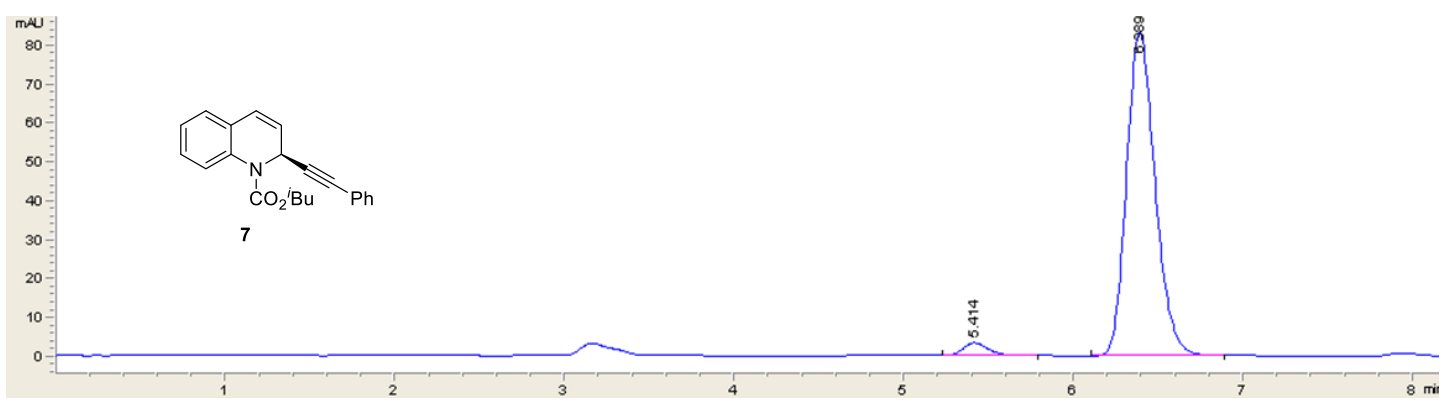

n-hexane/isopropanol 90:10, OD-H, $1.0 \mathrm{~mL} / \mathrm{min}$

\begin{tabular}{|c|c|c|c|c|c|}
\hline Peak \# & RetTime [min] & Width [min] & Area [mAU*s] & Height [mAU] & Area \% \\
\hline 1 & 5.414 & 0.1544 & 32.1 & 3.2 & 3.203 \\
2 & 6.389 & 0.1801 & 970.4 & 83.2 & 96.797 \\
\hline
\end{tabular}

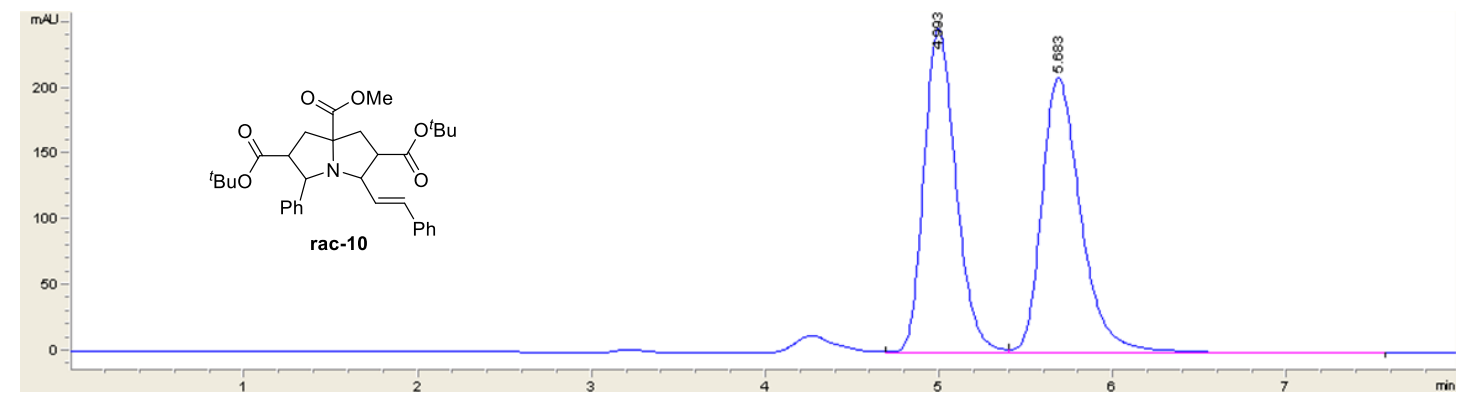

n-hexane/isopropanol 95:5, OD-H, $1.0 \mathrm{~mL} / \mathrm{min}$

\begin{tabular}{|c|c|c|c|c|c|}
\hline Peak \# & RetTime [min] & Width [min] & Area [mAU*s] & Height [mAU] & Area \% \\
\hline 1 & 4.993 & 0.1977 & 3174.5 & 247.4 & 49.062 \\
2 & 5.683 & 0.2415 & 3295.9 & 209.5 & 50.938 \\
\hline
\end{tabular}




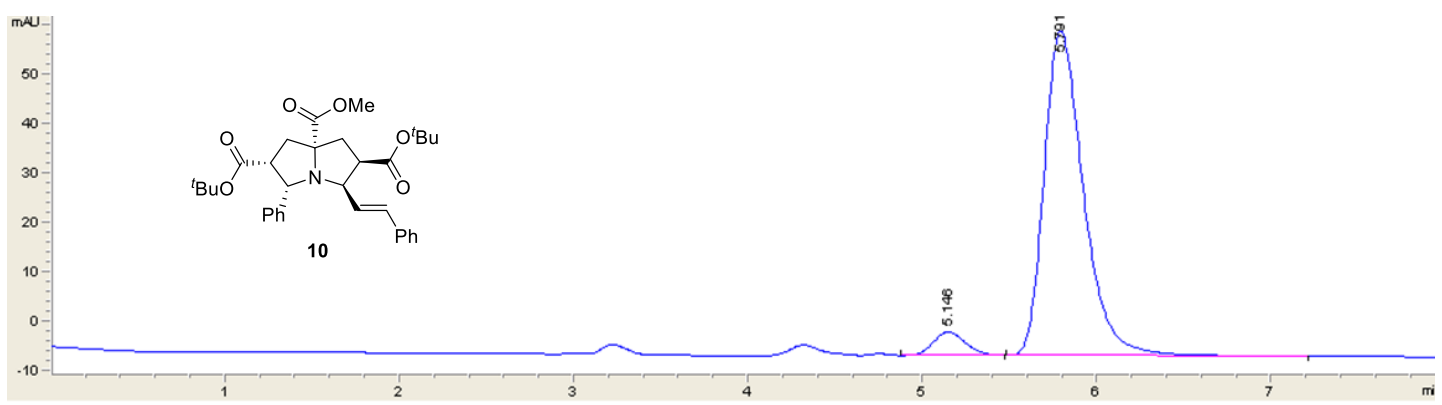

n-hexane/isopropanol 95:5, OD-H, $1.0 \mathrm{~mL} / \mathrm{min}$

\begin{tabular}{|c|c|c|c|c|c|}
\hline Peak \# & RetTime [min] & Width [min] & Area [mAU*s] & Height [mAU] & Area \% \\
\hline 1 & 5.146 & 0.2001 & 60.8 & 4.7 & 5.046 \\
2 & 5.791 & 0.2418 & 1036.2 & 65.8 & 94.954 \\
\hline
\end{tabular}




\section{3. ${ }^{1} \mathrm{H}$ and ${ }^{13} \mathrm{C}$ NMR spectra}

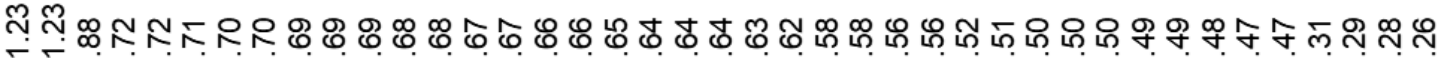

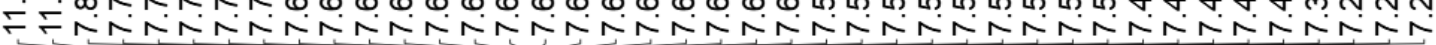

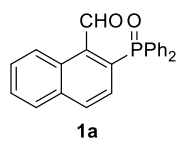

${ }^{1} \mathrm{H}$ NMR $\left(\mathrm{CDCl}_{3}, 400 \mathrm{MHz}\right)$

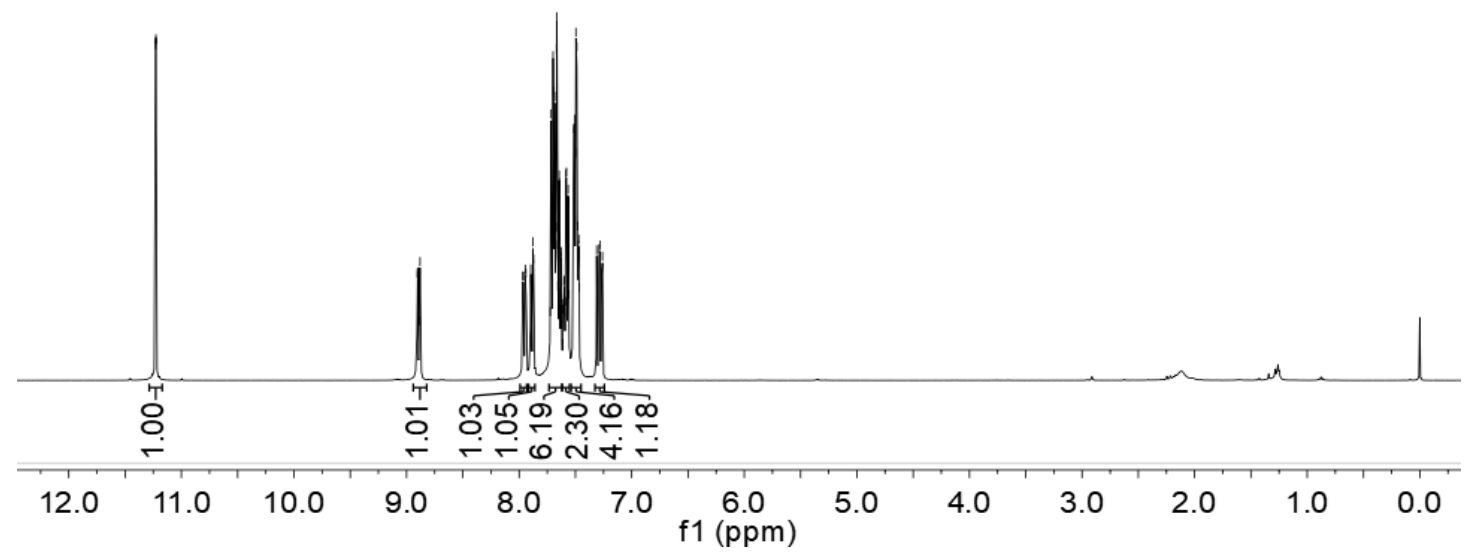

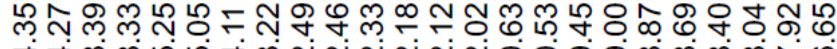
హ.

乐电市

กर゚

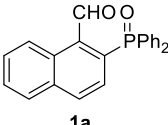

${ }^{13} \mathrm{C}$ NMR $\left(\mathrm{CDCl}_{3}, 100 \mathrm{MHz}\right)$

$210200190180170160150140130120110100 \quad 90 \quad 80 \quad 70 \quad 60 \quad 50 \quad 40 \quad 30 \quad 20 \quad 10 \quad 0$ f1 (ppm) 


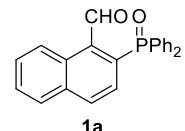

${ }^{31} \mathrm{P}$ NMR $\left(\mathrm{CDCl}_{3}, 162 \mathrm{MHz}\right)$

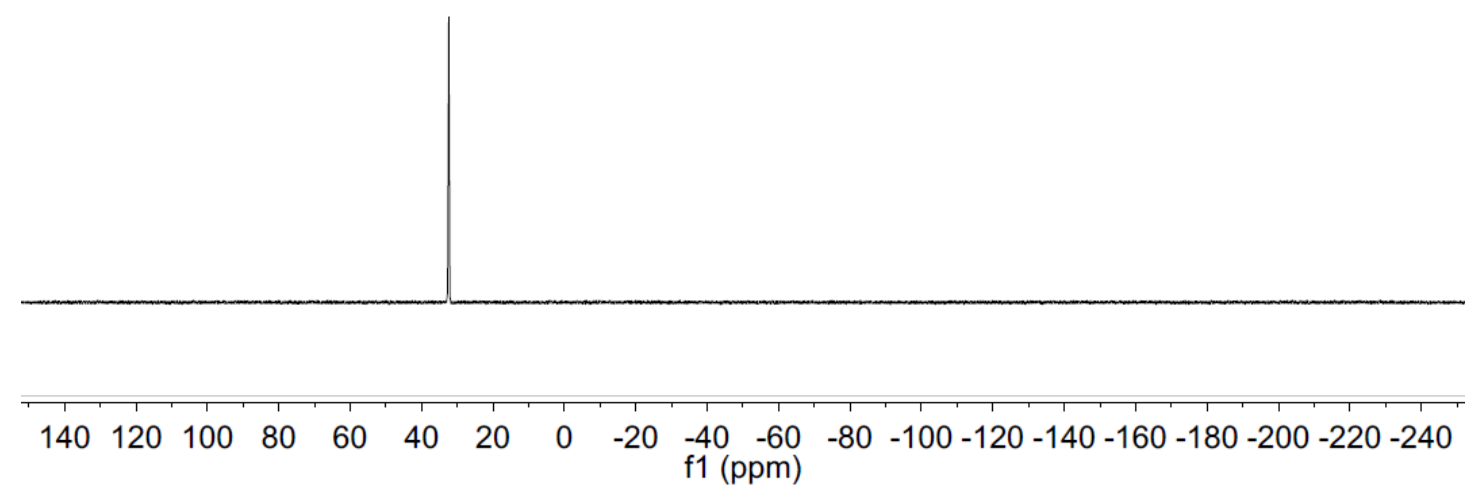

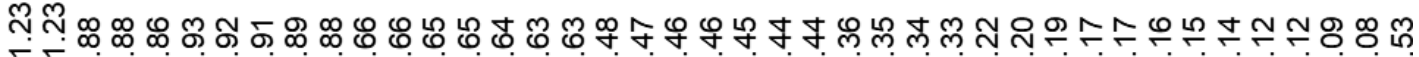
FЕ

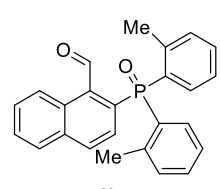

1b

${ }^{1} \mathrm{H}$ NMR $\left(\mathrm{CDCl}_{3}, 400 \mathrm{MHz}\right)$

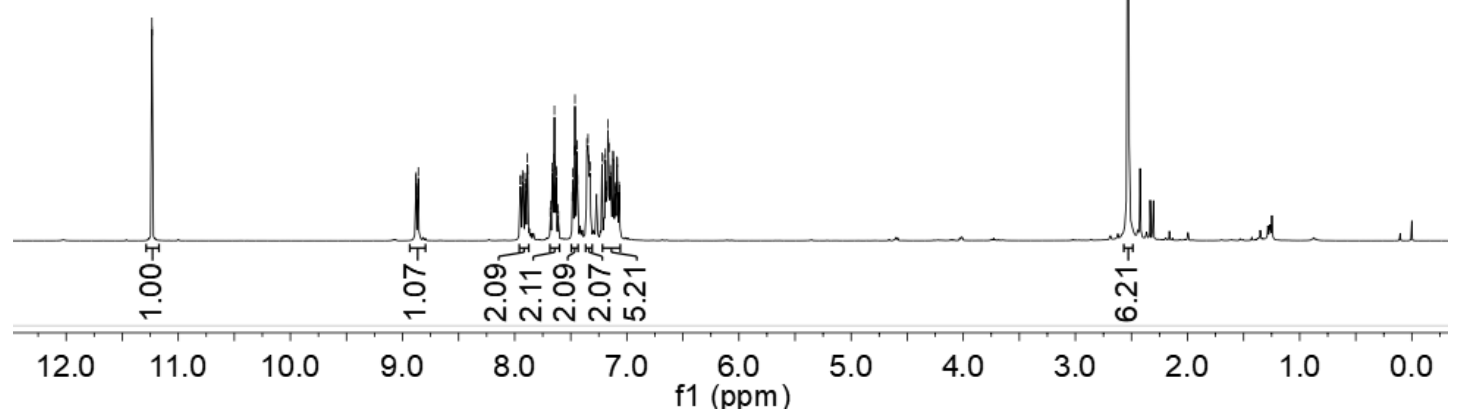




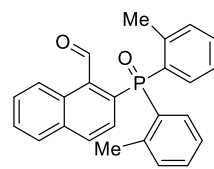

$1 \mathrm{~b}$

${ }^{13} \mathrm{C} \mathrm{NMR}\left(\mathrm{CDCl}_{3}, 100 \mathrm{MHz}\right)$

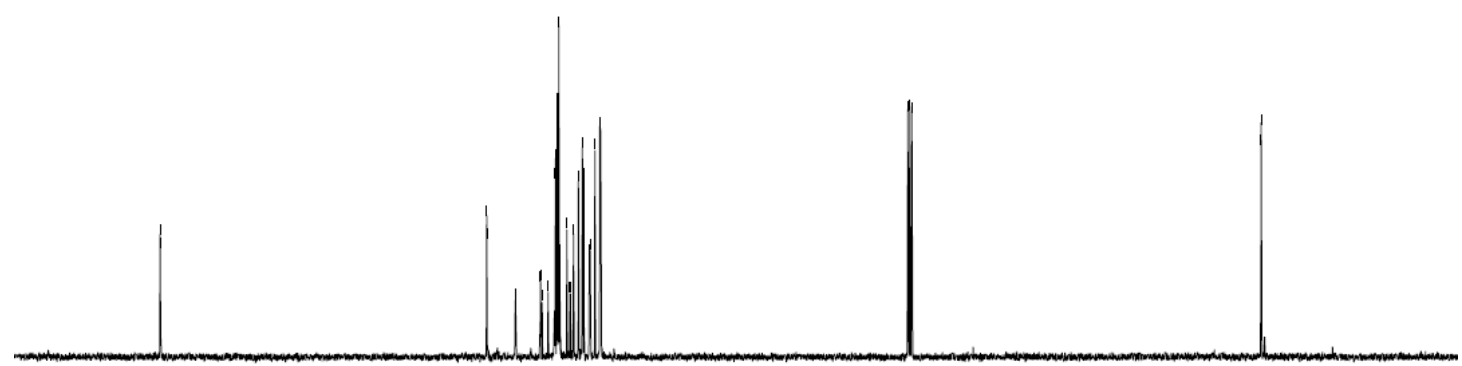

$210200190180170160150140130120110100 \quad 90 \quad 80 \quad 70 \quad 60 \quad 50 \quad 40 \quad 30 \quad 20 \quad 10 \quad 0 \quad$ f1 (ppm)

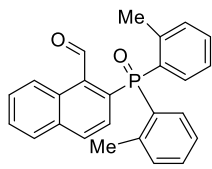

1b

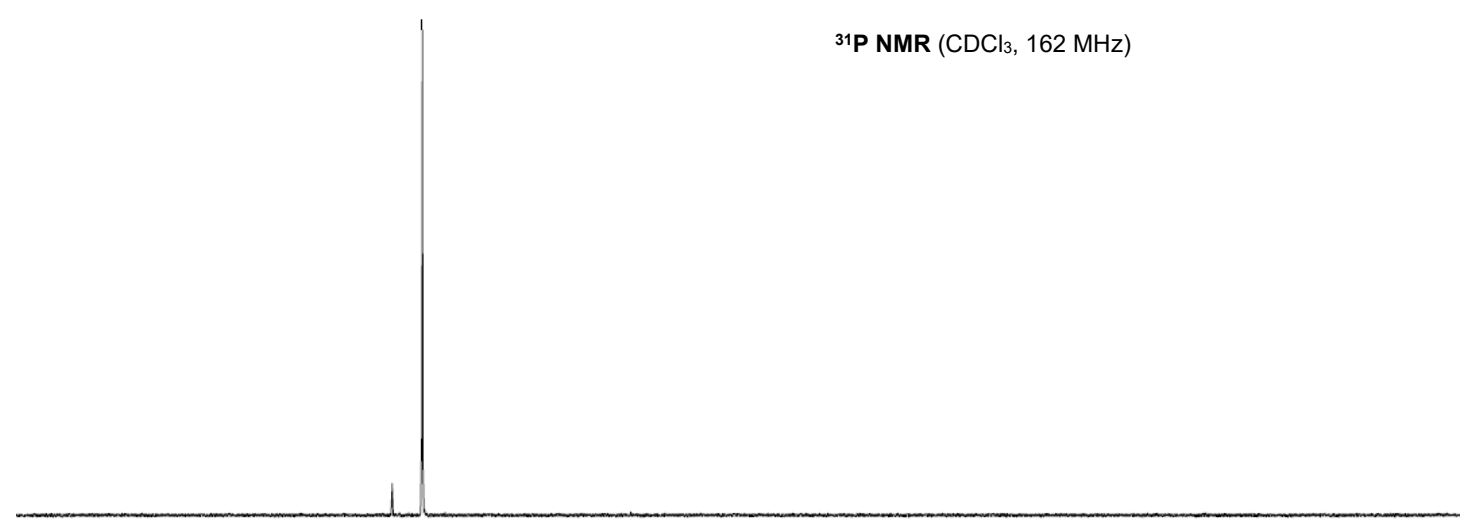

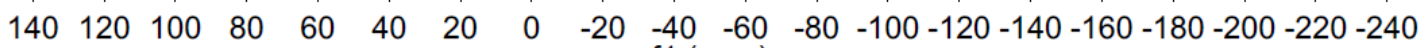
f1 (ppm) 


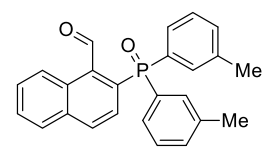

1c

${ }^{1} \mathrm{H}$ NMR $\left(\mathrm{CDCl}_{3}, 400 \mathrm{MHz}\right)$

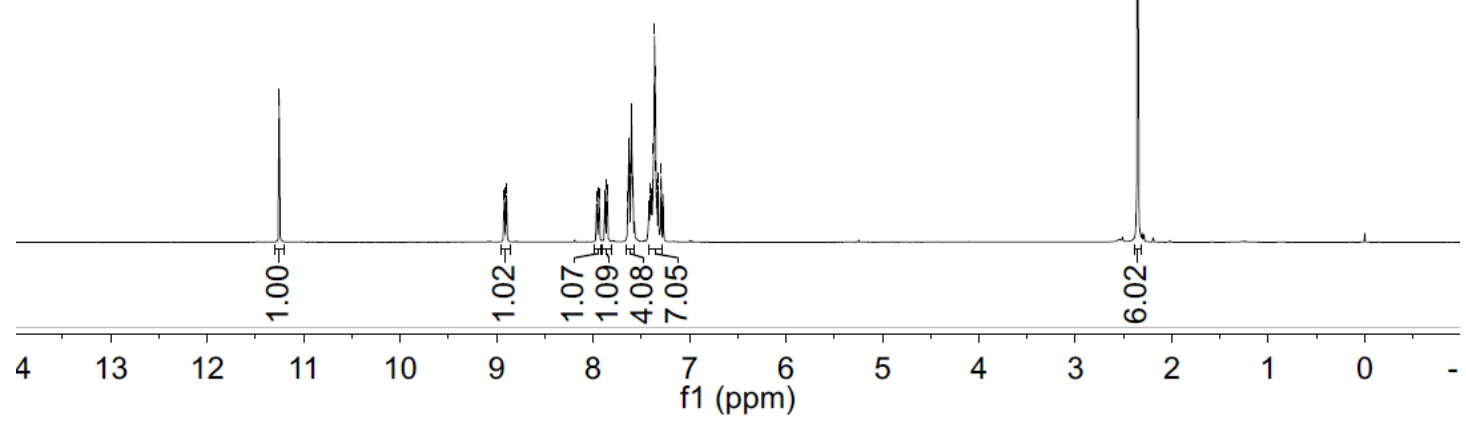

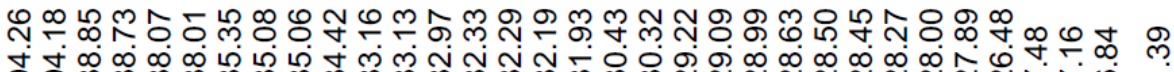

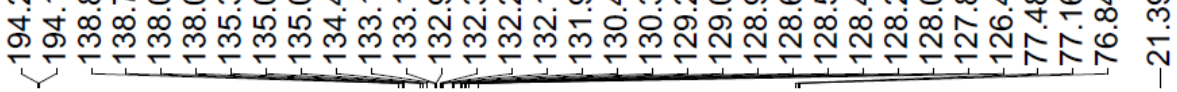

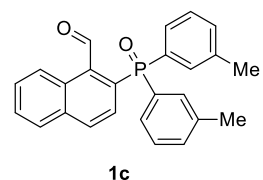

$1 c$

${ }^{13} \mathrm{C} \mathrm{NMR}\left(\mathrm{CDCl}_{3}, 100 \mathrm{MHz}\right)$

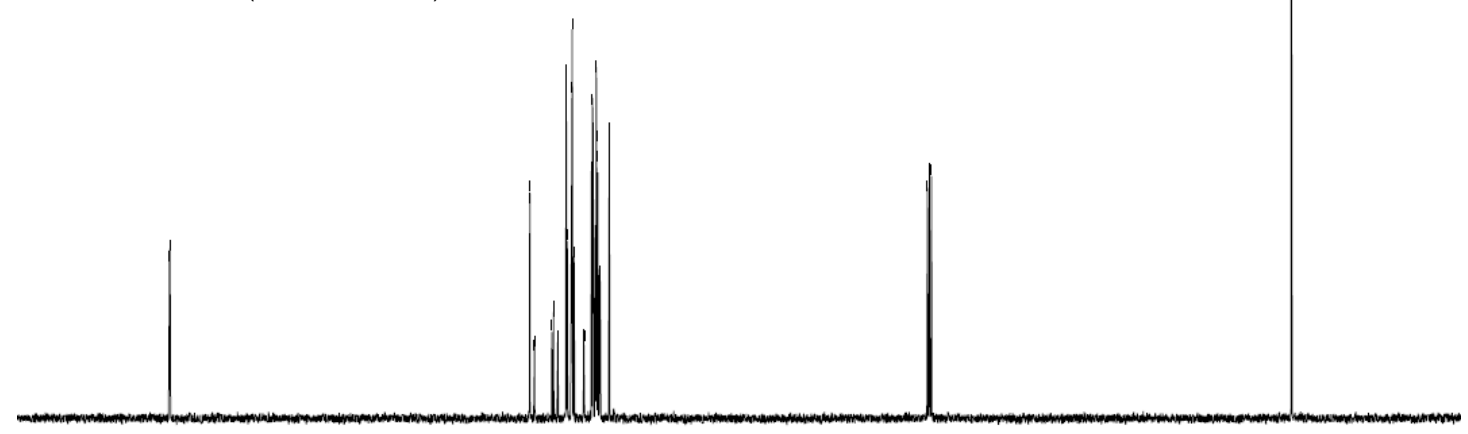

$210200190180170160150140130120110100 \quad 90 \quad 80 \quad 70 \quad 60 \quad 50 \quad 40 \quad 30 \quad 20 \quad 10 \quad 0$ f1 (ppm) 


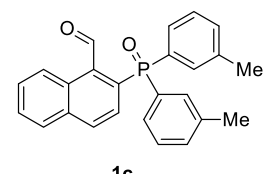

$1 c$

${ }^{31} \mathrm{P}$ NMR $\left(\mathrm{CDCl}_{3}, 162 \mathrm{MHz}\right)$

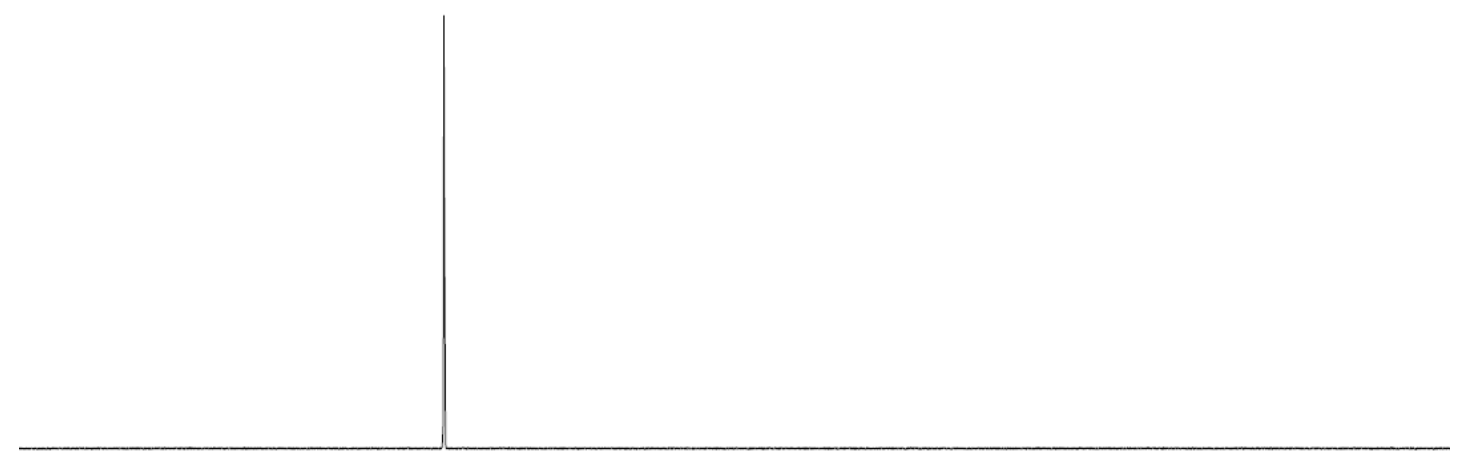

$\begin{array}{llllllllllllll}140 & 120 & 100 & 80 & 60 & 40 & 20 & 0 & -20 & \begin{array}{l}-40 \\ \mathrm{f} 1(\mathrm{ppm})\end{array} & -60 & -100-120-140-160-180-200-220-240\end{array}$

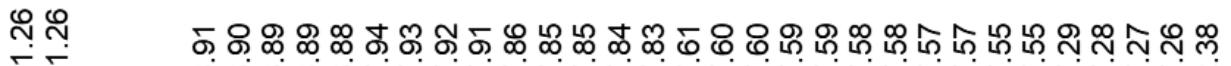

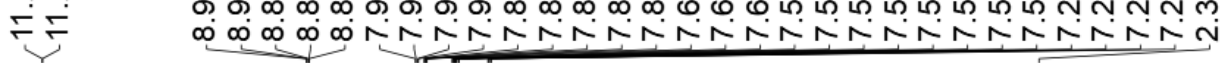

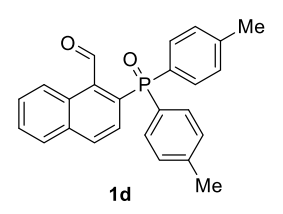

${ }^{1} \mathrm{H}$ NMR $\left(\mathrm{CDCl}_{3}, 400 \mathrm{MHz}\right)$

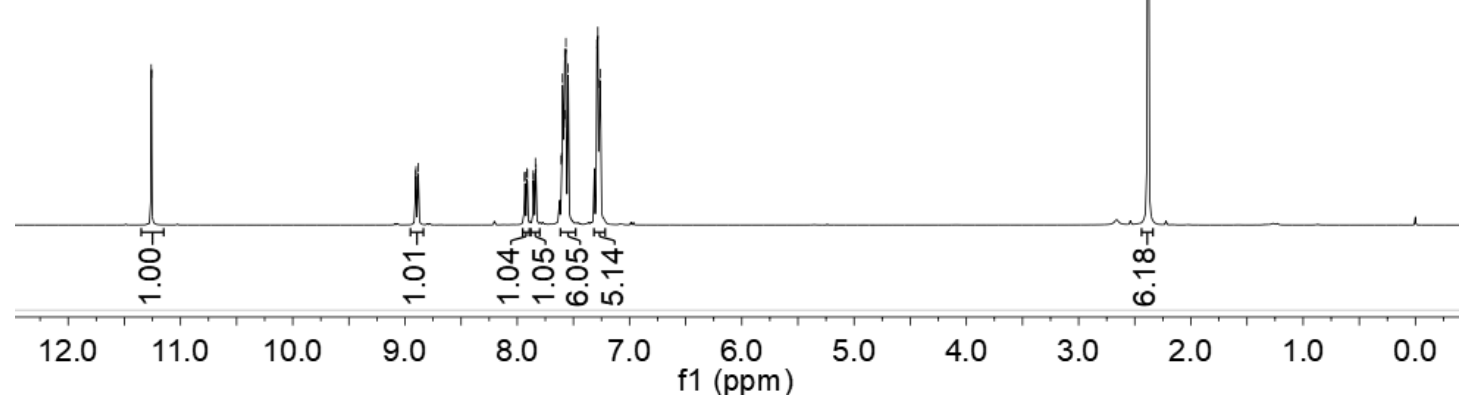




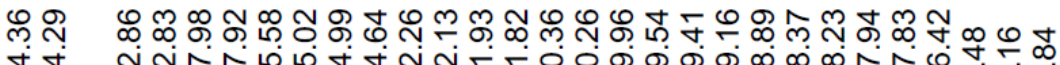

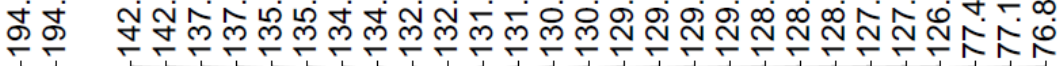

菓

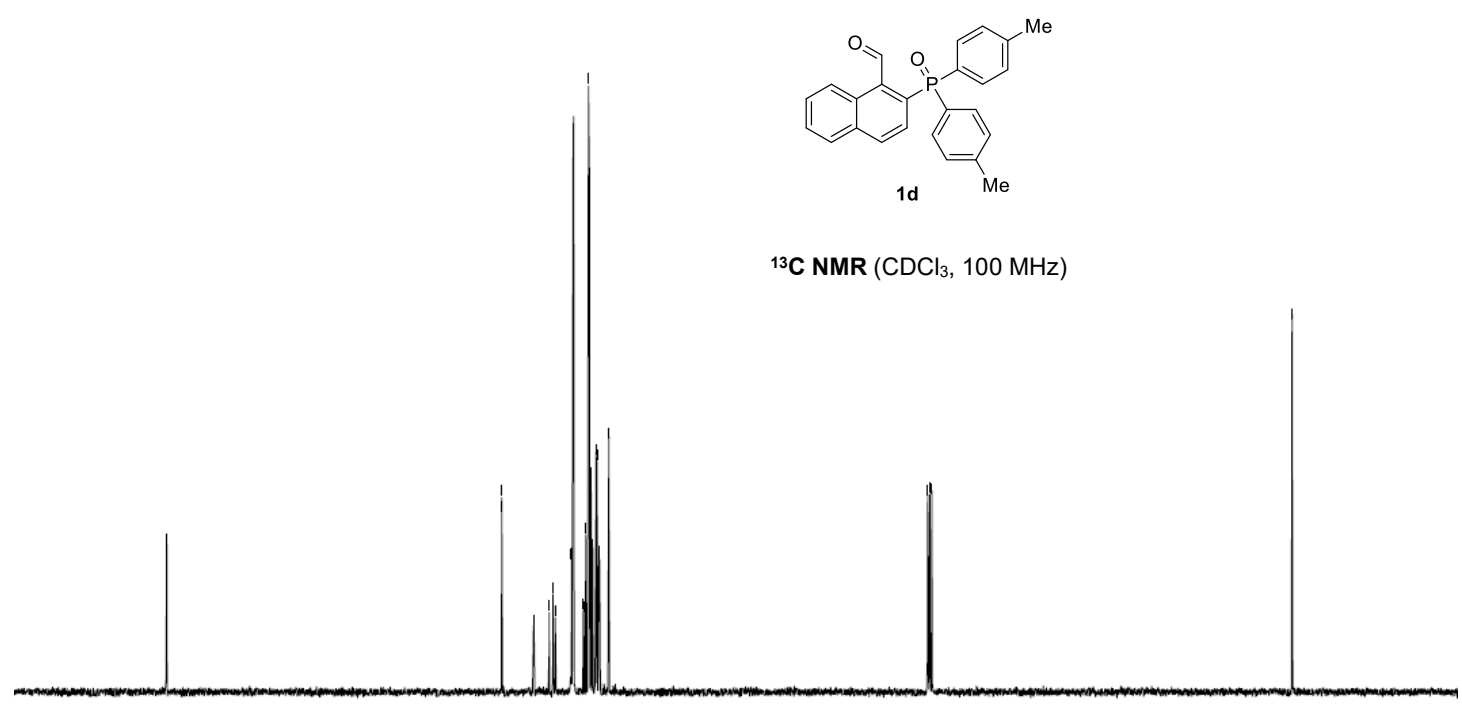

$210200190180170160150140130120110100 \quad 90 \quad 80 \quad 70 \quad 60 \quad 50 \quad 40 \quad 30 \quad 20 \quad 10 \quad 0$ f1 (ppm)

เุ

กั่

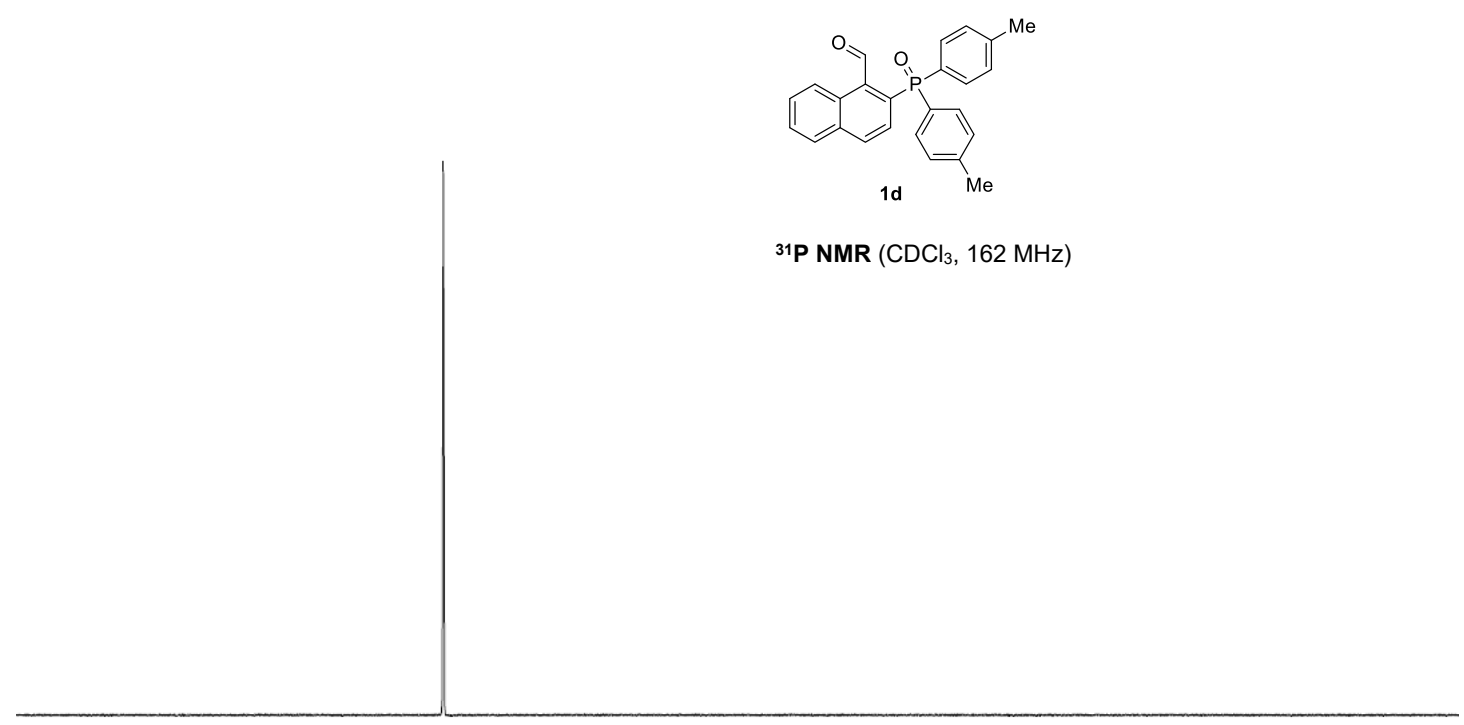

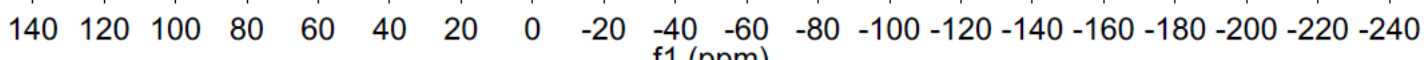
f1 (ppm) 


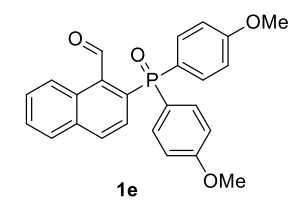

${ }^{1} \mathrm{H}$ NMR $\left(\mathrm{CDCl}_{3}, 400 \mathrm{MHz}\right)$

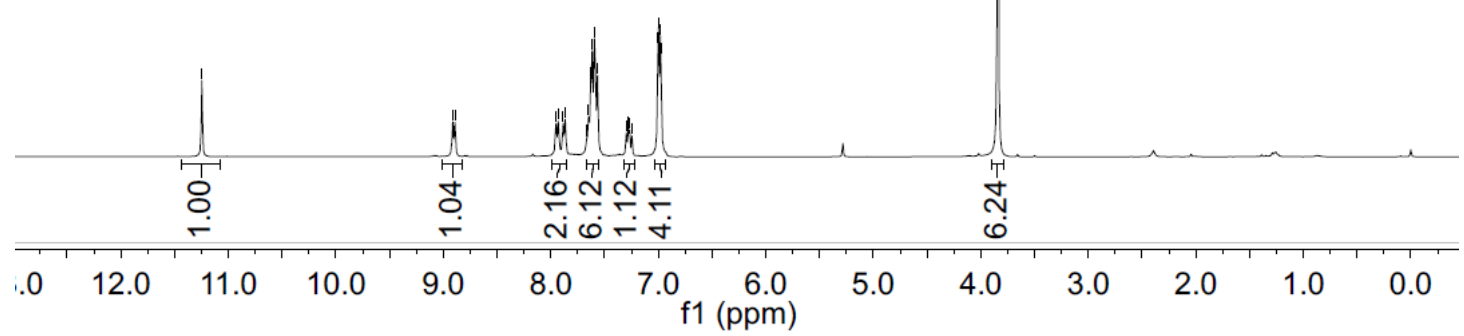

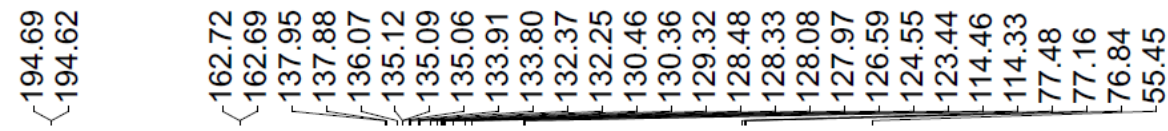

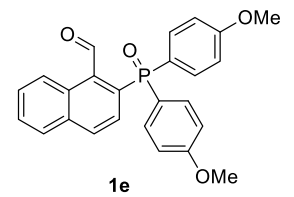

${ }^{13} \mathrm{C} \mathrm{NMR}\left(\mathrm{CDCl}_{3}, 100 \mathrm{MHz}\right)$

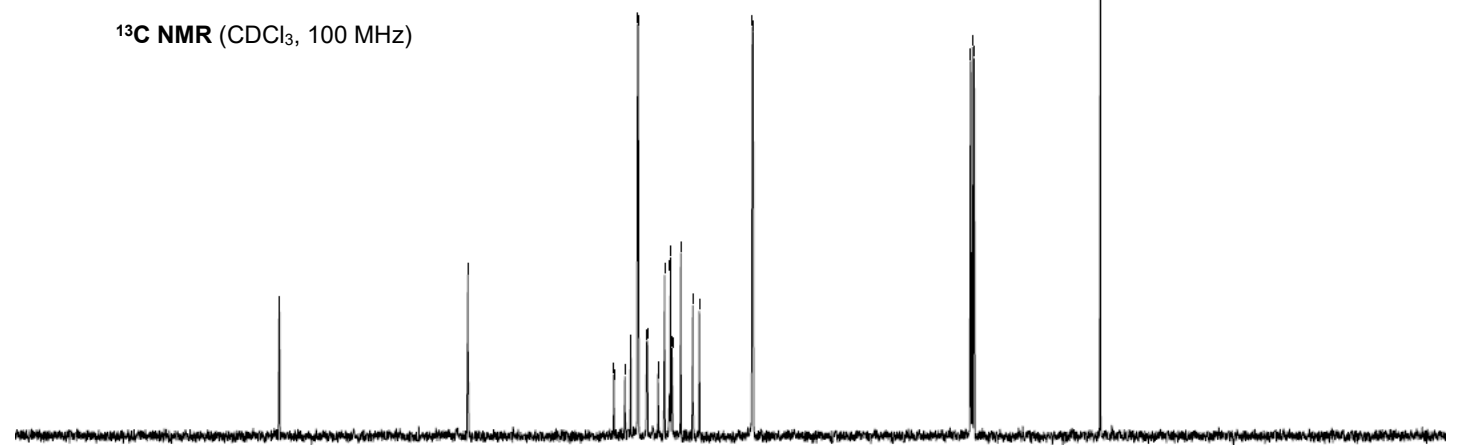

\begin{tabular}{lllllllllllllll}
\hline 0 & 220 & 200 & 180 & 160 & 140 & $\begin{array}{c}120 \\
\mathrm{f} 1(\mathrm{ppm})\end{array}$ & 100 & 80 & 60 & 40 & 20 & 0
\end{tabular}




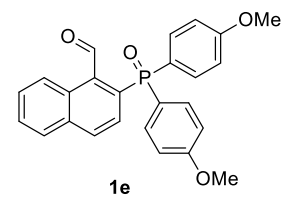

${ }^{31} \mathrm{P} \mathrm{NMR}\left(\mathrm{CDCl}_{3}, 162 \mathrm{MHz}\right)$

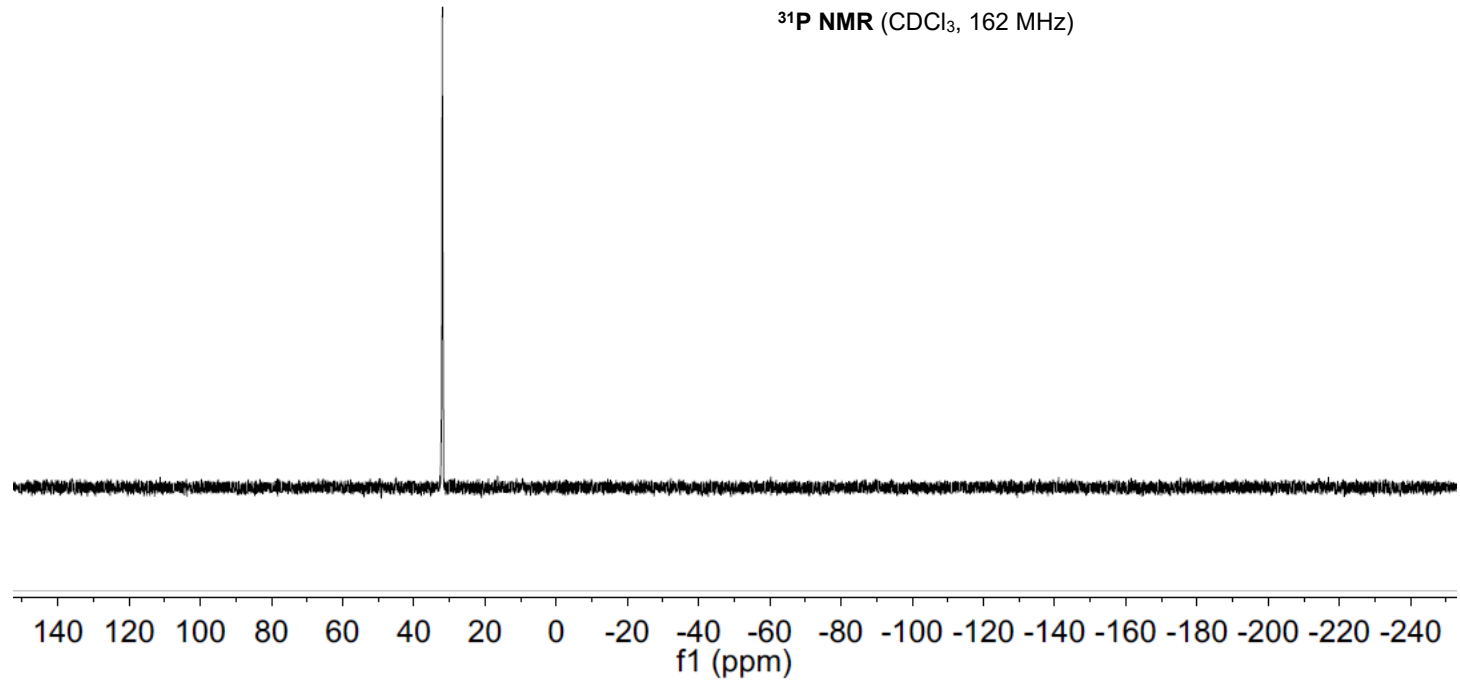

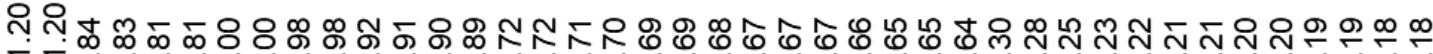

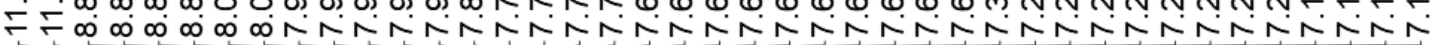

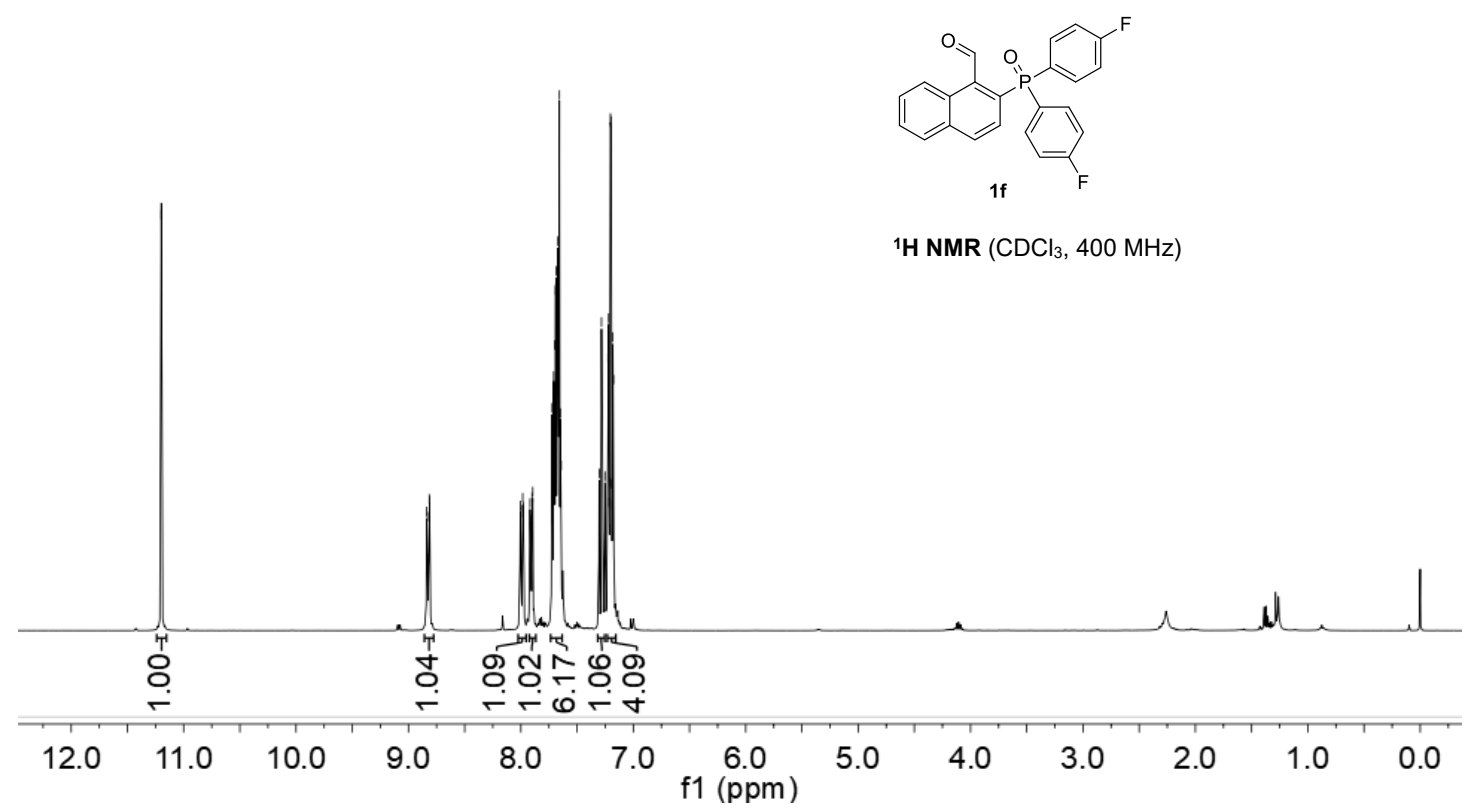




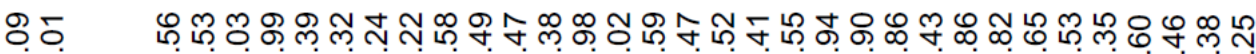

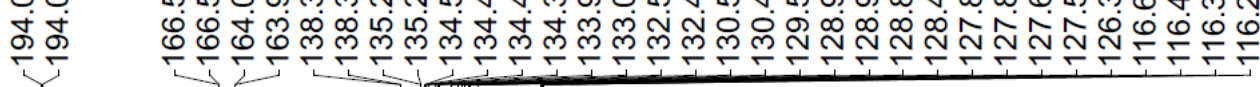

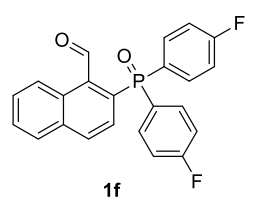

${ }^{13} \mathrm{C} \mathrm{NMR}\left(\mathrm{CDCl}_{3}, 100 \mathrm{MHz}\right)$

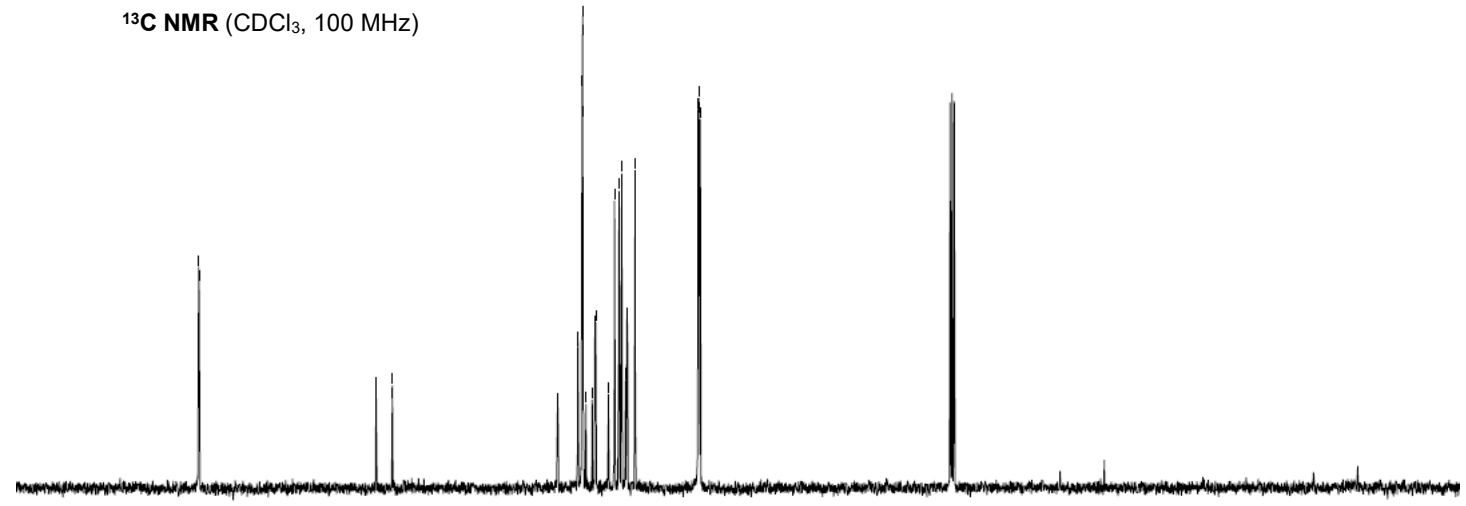

?20210200190180170160150140130120110100 90 80 70 $60 \begin{array}{lllllll}50 & 40 & 30 & 20 & 10 & 0\end{array}$ f1 (ppm)

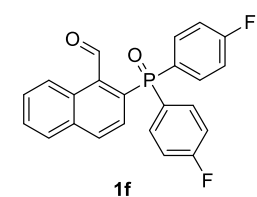

${ }^{19} \mathrm{~F}$ NMR $\left(\mathrm{CDCl}_{3}, 377 \mathrm{MHz}\right)$

ก้

\begin{tabular}{|c|c|c|c|c|c|c|c|c|c|c|}
\hline 1 & -20 & -40 & -60 & -80 & $\begin{array}{c}-100 \\
\mathrm{f} 1 \text { (ppm) }\end{array}$ & -120 & -140 & -160 & -180 & -2 \\
\hline
\end{tabular}




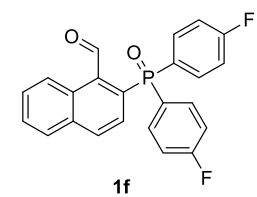

${ }^{31} \mathrm{P}$ NMR $\left(\mathrm{CDCl}_{3}, 162 \mathrm{MHz}\right)$

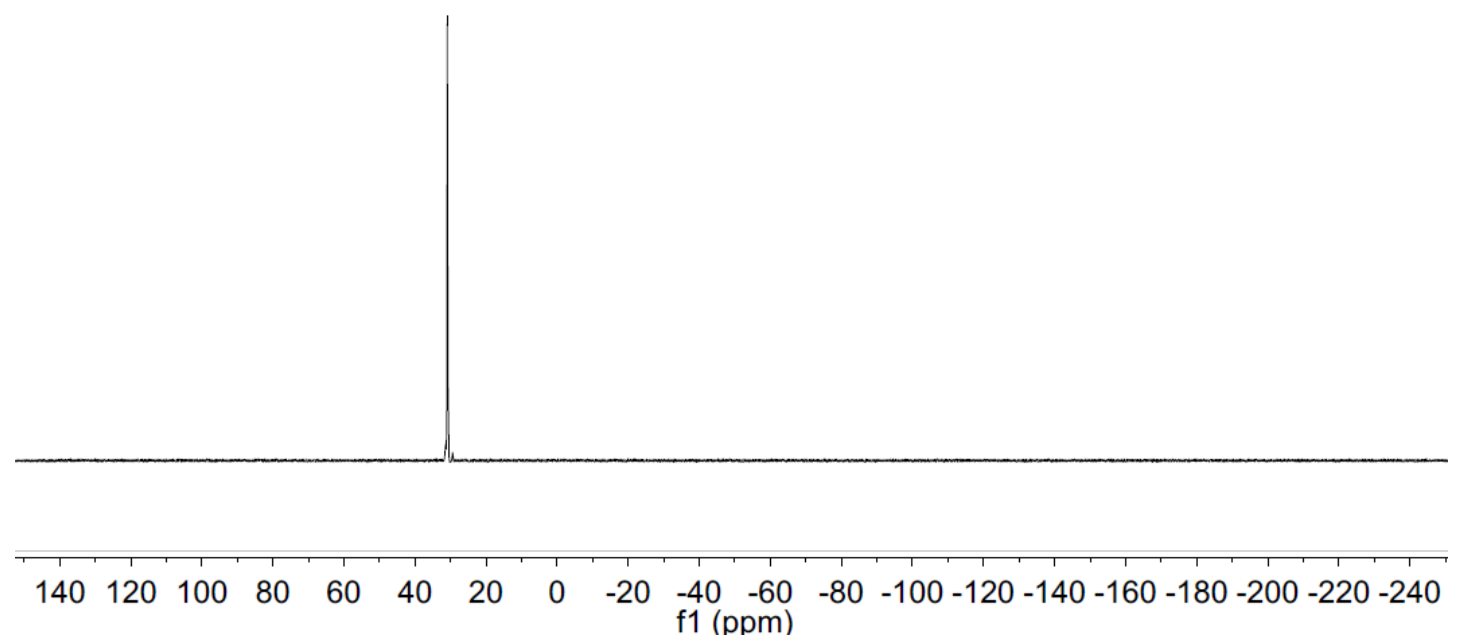

芦芦

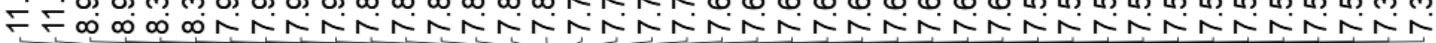

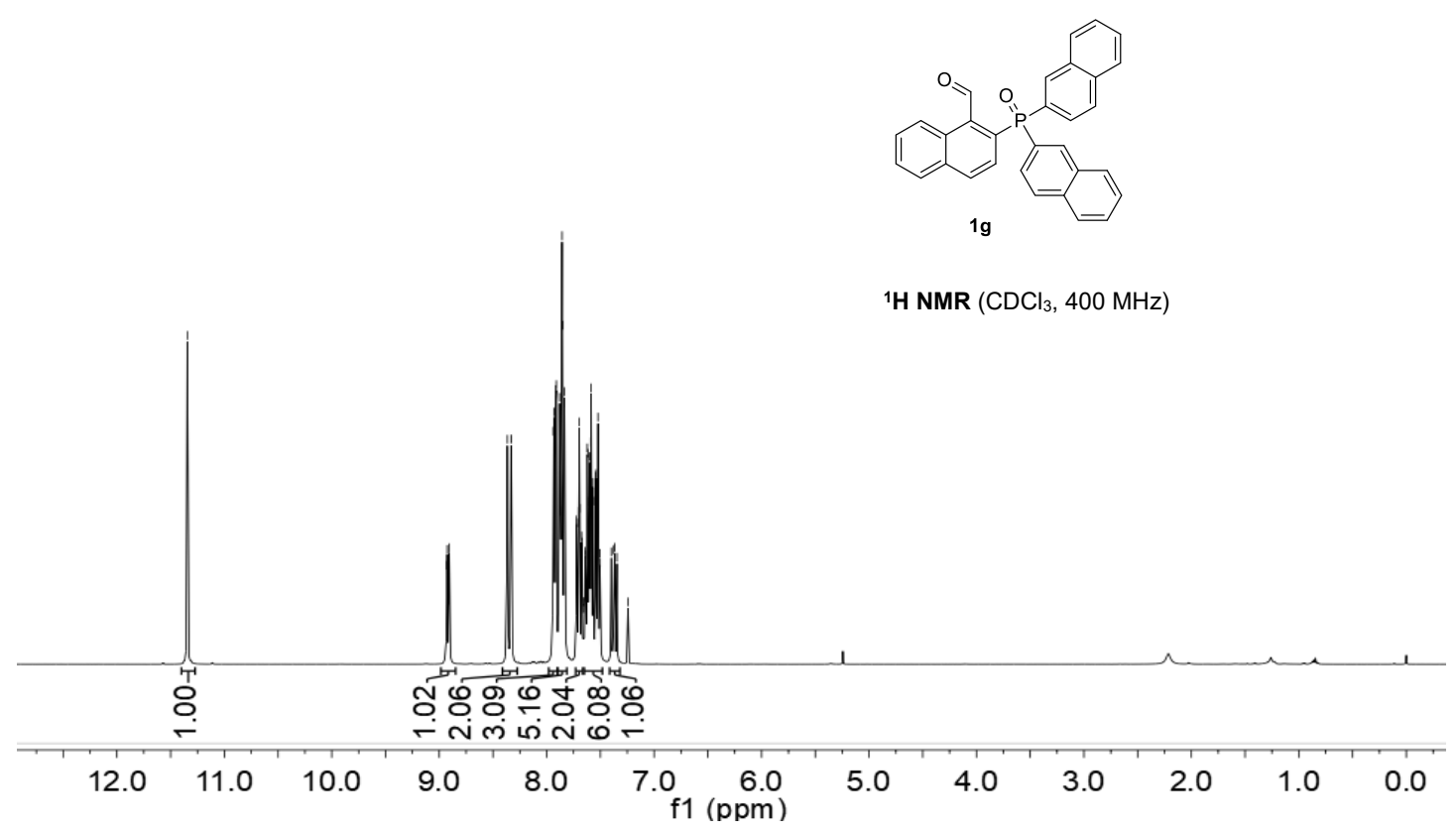




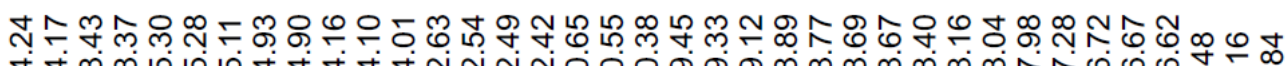

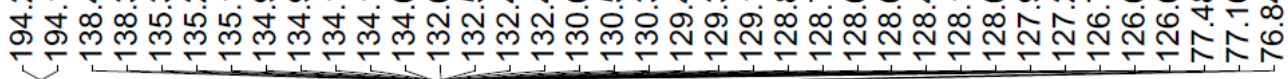

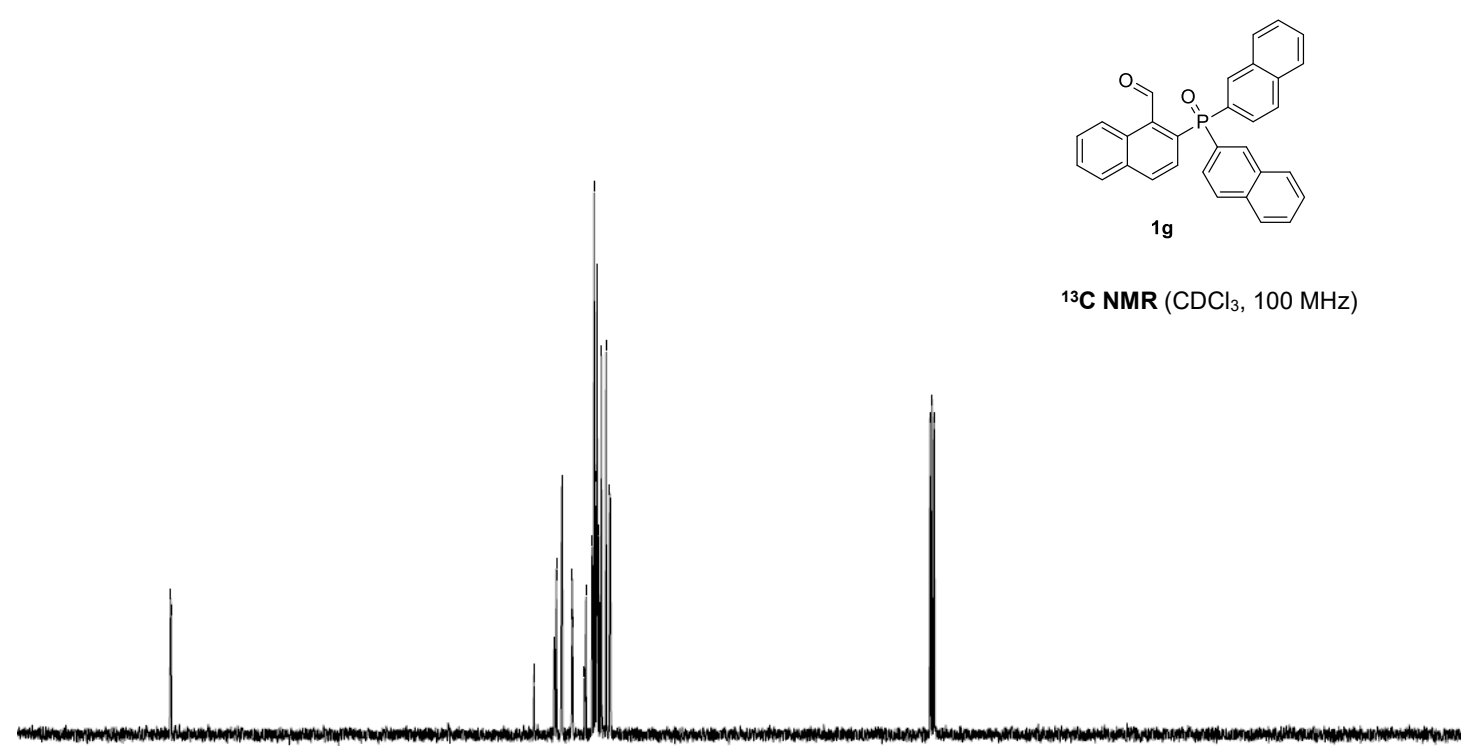

$210200190180170160150140130120110100 \quad 90 \quad 80 \quad 70 \quad 60 \quad 50 \quad 40 \quad 30 \quad 20 \quad 10 \quad 0$ f1 (ppm)

ヘัฐ

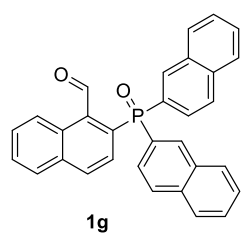

31P NMR ( $\left.\mathrm{CDCl}_{3}, 162 \mathrm{MHz}\right)$

$140120100 \quad 80 \quad 60 \quad 40 \quad 20 \quad 0 \quad-20 \quad-40 \quad-60 \quad-80$ - $1000-120-140-160-180-200-220-240$ f1 (ppm) 


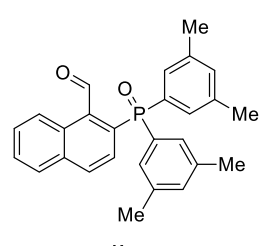

$1 \mathrm{~h}$

${ }^{1} \mathrm{H}$ NMR $\left(\mathrm{CDCl}_{3}, 400 \mathrm{MHz}\right)$

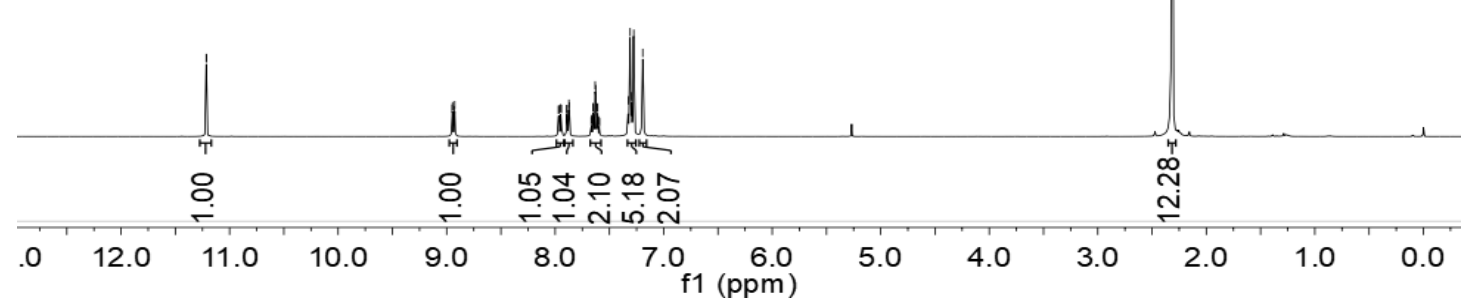

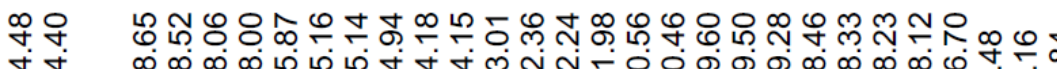

它安 $\quad m^{\infty}$

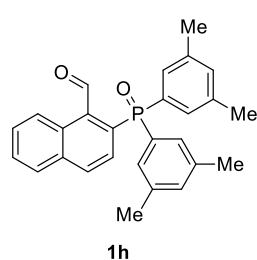

$1 \mathrm{~h}$

${ }^{13} \mathrm{C} \mathrm{NMR}\left(\mathrm{CDCl}_{3}, 100 \mathrm{MHz}\right)$

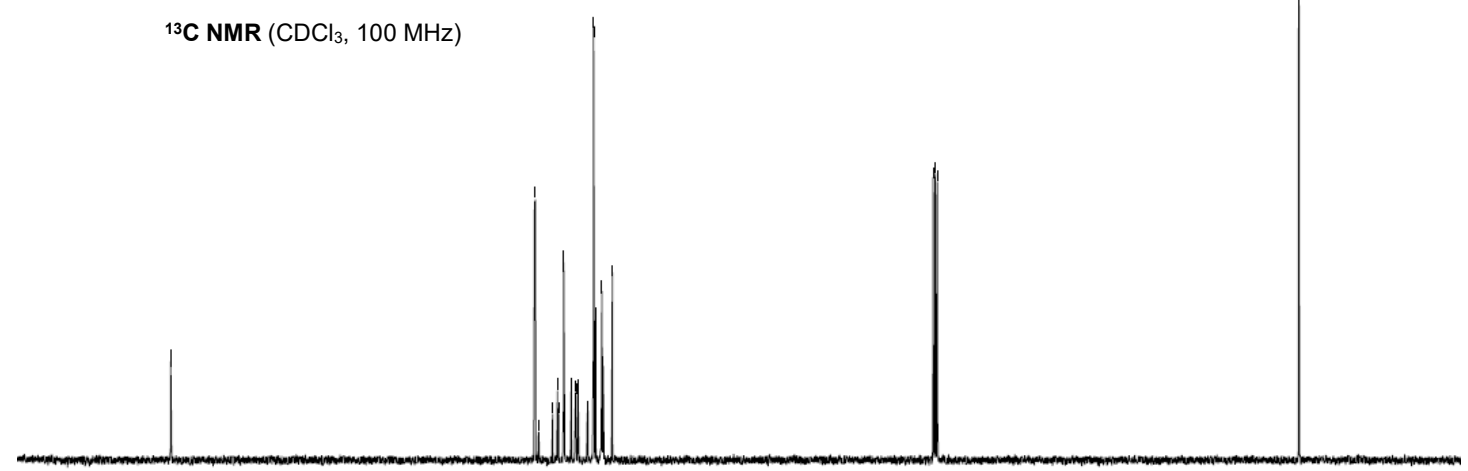

$210200190180170160150140130120110100 \quad 90 \quad 80 \quad 70 \quad 60 \begin{array}{lllllll}50 & 40 & 30 & 20 & 10 & 0\end{array}$ f1 $(\mathrm{ppm})$ 


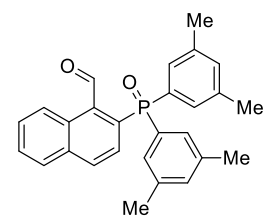

${ }^{31} \mathrm{P}$ NMR $\left(\mathrm{CDCl}_{3}, 162 \mathrm{MHz}\right)$

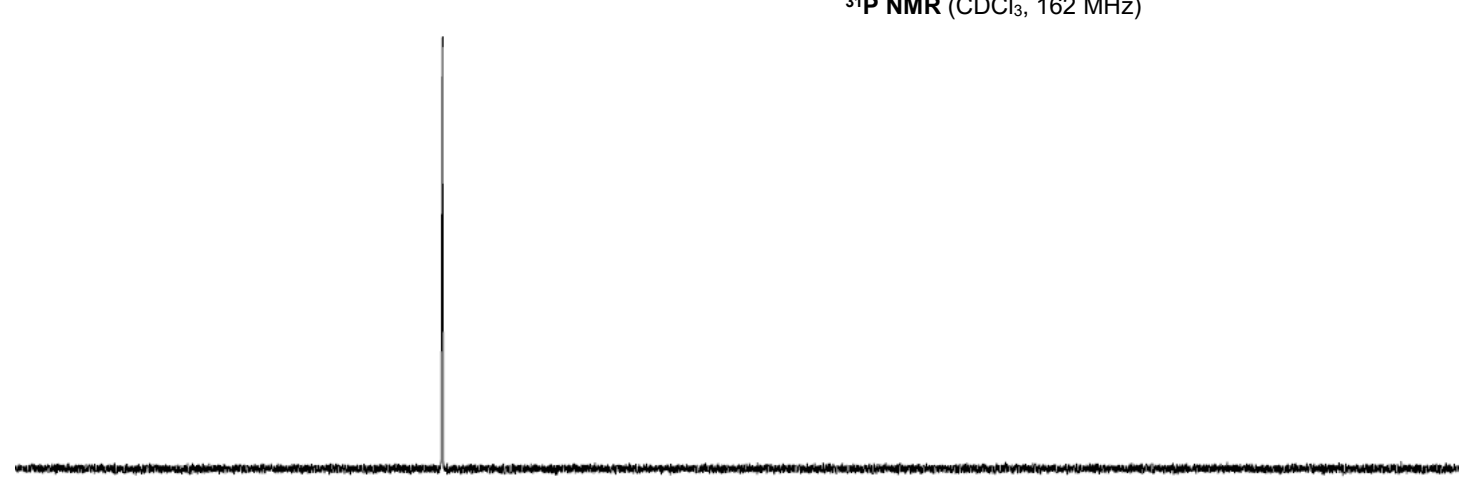

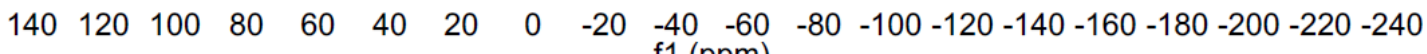
f1 (ppm)

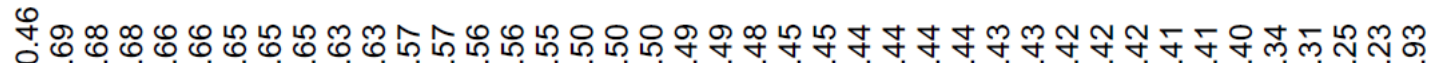
oricisin

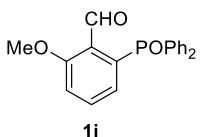

${ }^{1} \mathrm{H}$ NMR $\left(\mathrm{CDCl}_{3}, 400 \mathrm{MHz}\right)$

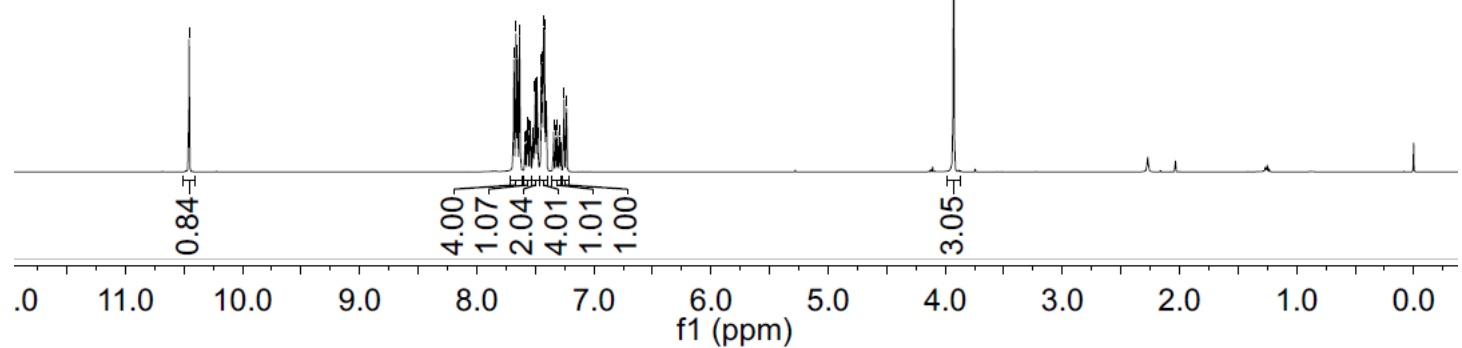




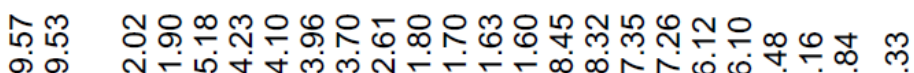

市
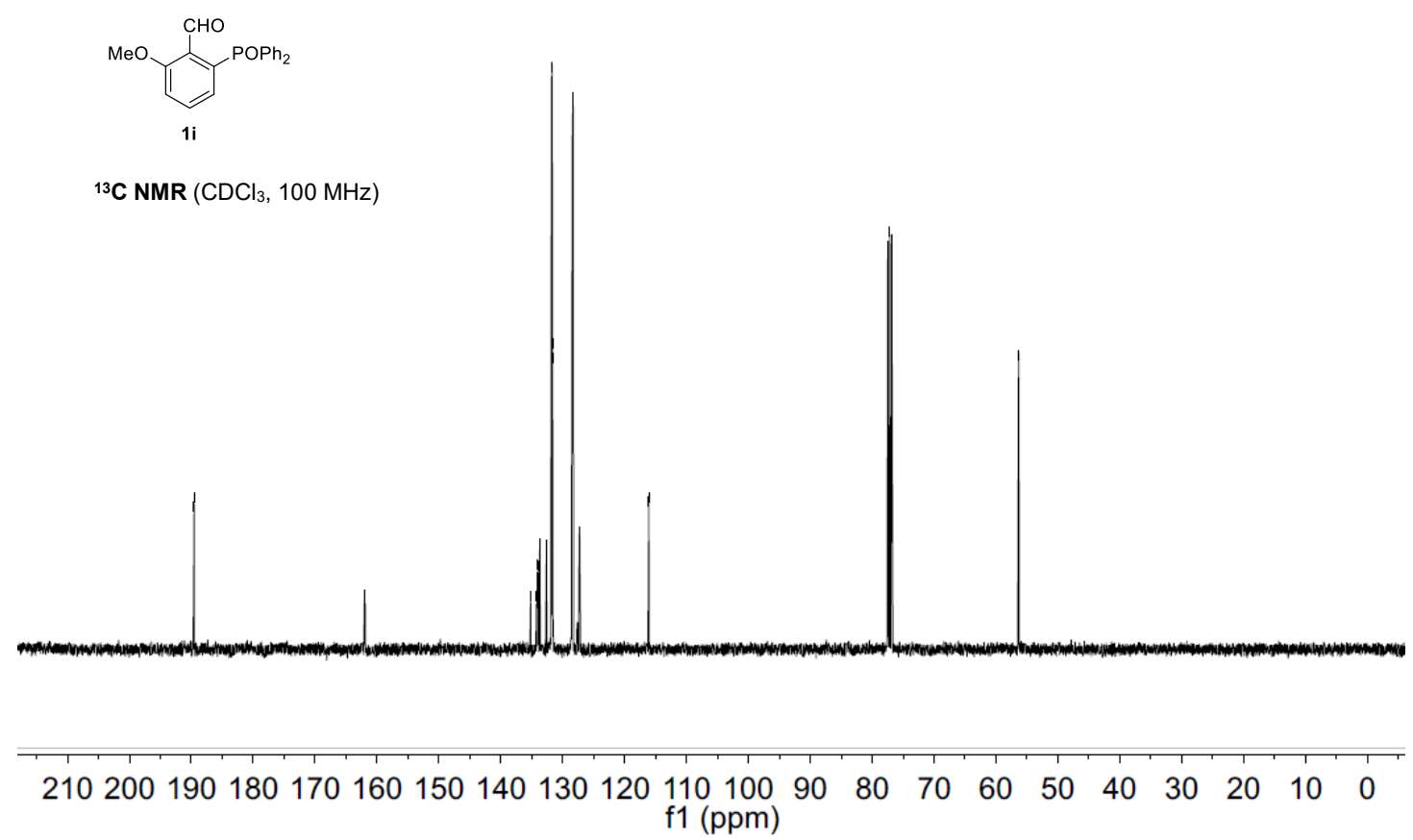

䇉

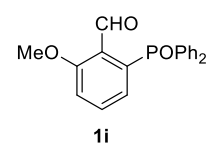

31P NMR ( $\left.\mathrm{CDCl}_{3}, 162 \mathrm{MHz}\right)$

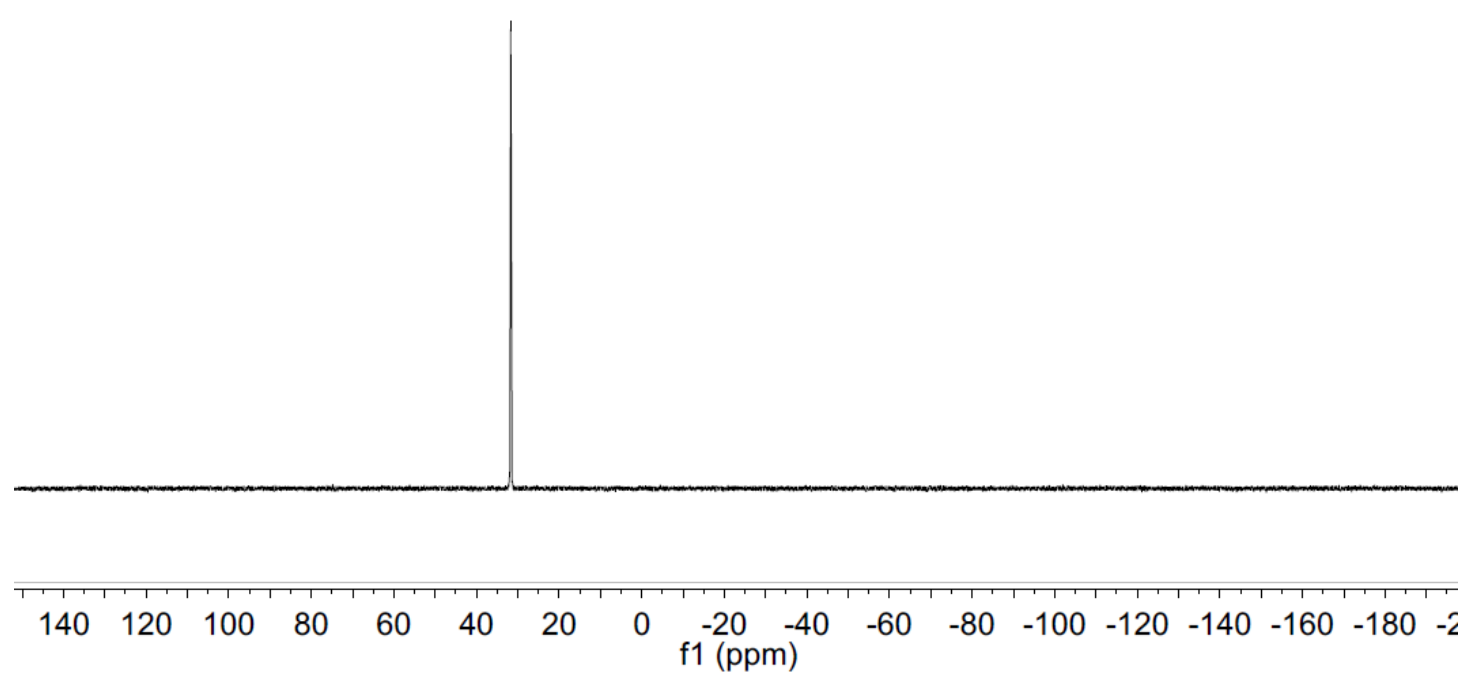




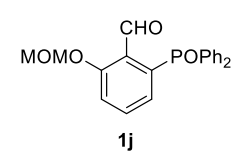

${ }^{1} \mathrm{H}$ NMR $\left(\mathrm{CDCl}_{3}, 400 \mathrm{MHz}\right)$

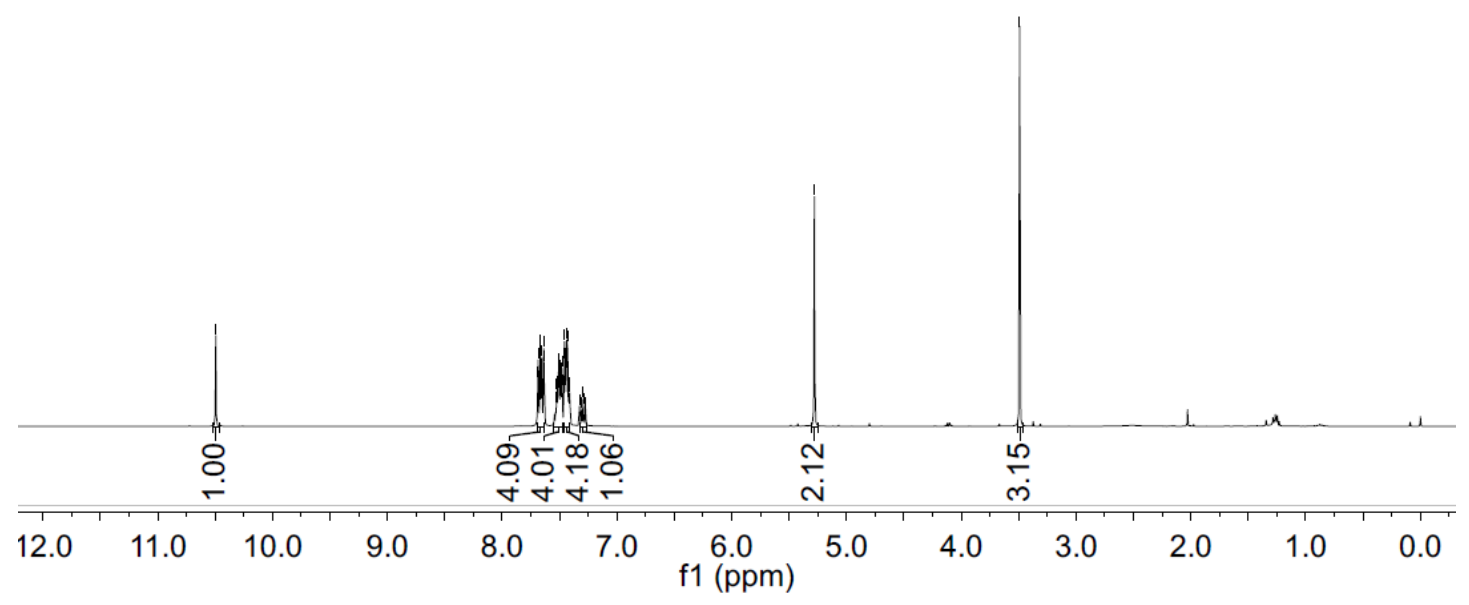

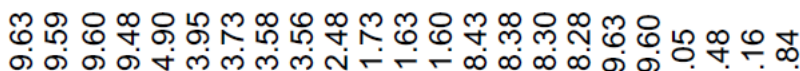
कळ

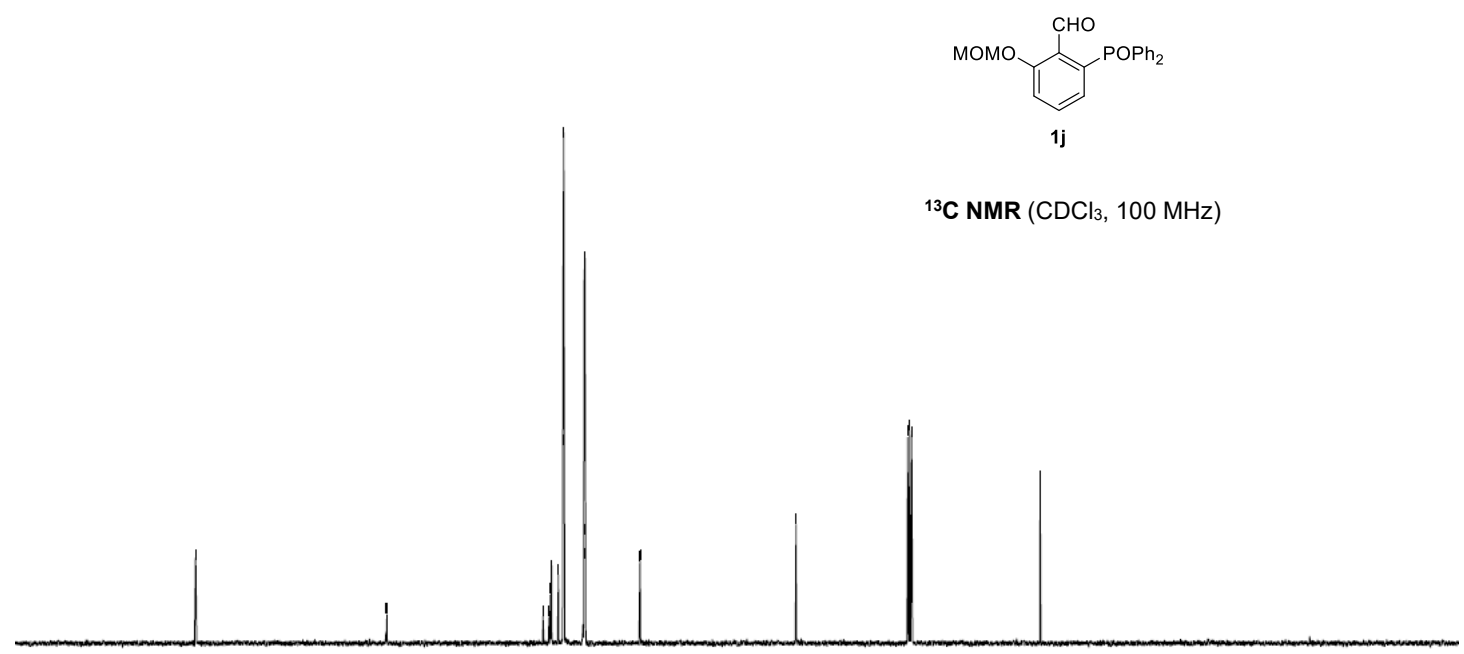

$210200190180170160150140130120110100 \quad 90 \quad 80 \quad 70 \quad 60 \begin{array}{llllllll}50 & 40 & 30 & 20 & 10 & 0 & -\end{array}$ f1 (ppm) 


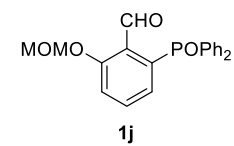

31P NMR ( $\left.\mathrm{CDCl}_{3}, 162 \mathrm{MHz}\right)$

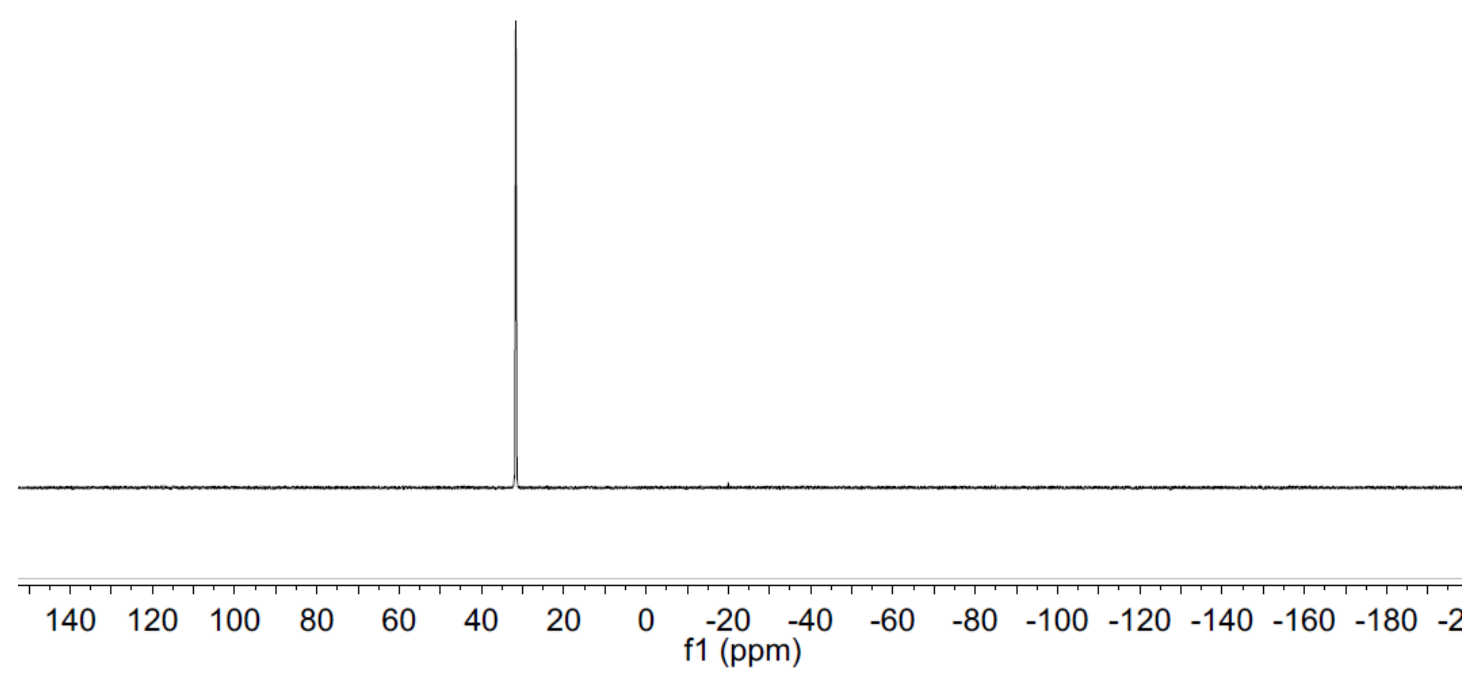

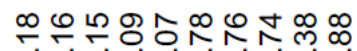

소숭

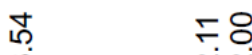

m रัก

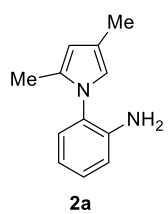

${ }^{1} \mathrm{H}$ NMR $\left(\mathrm{CDCl}_{3}, 400 \mathrm{MHz}\right)$

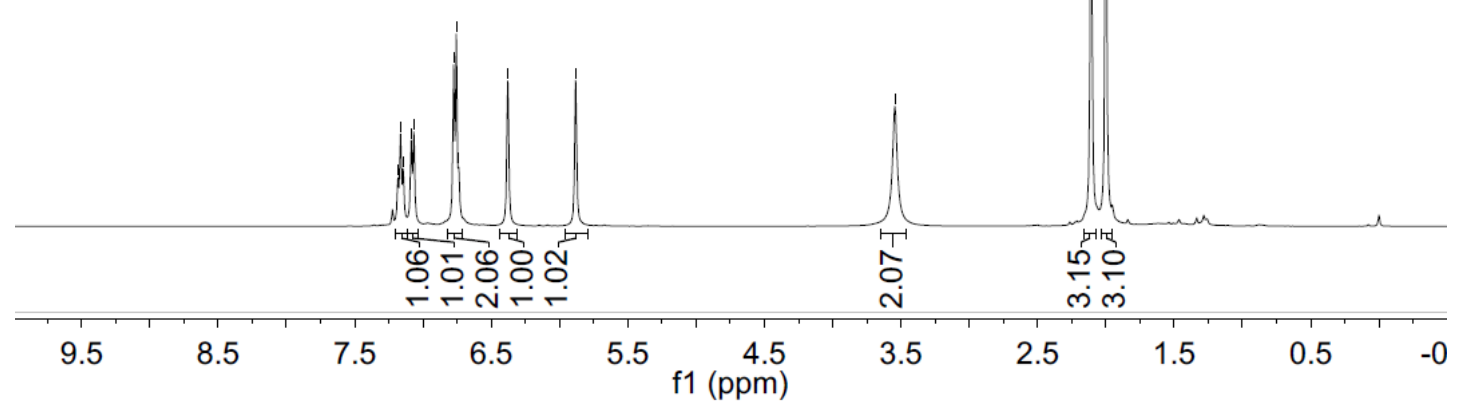




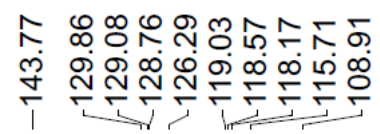

守ํํㅇ

손

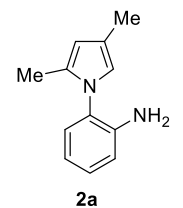

${ }^{13} \mathrm{C}$ NMR $\left(\mathrm{CDCl}_{3}, 100 \mathrm{MHz}\right)$

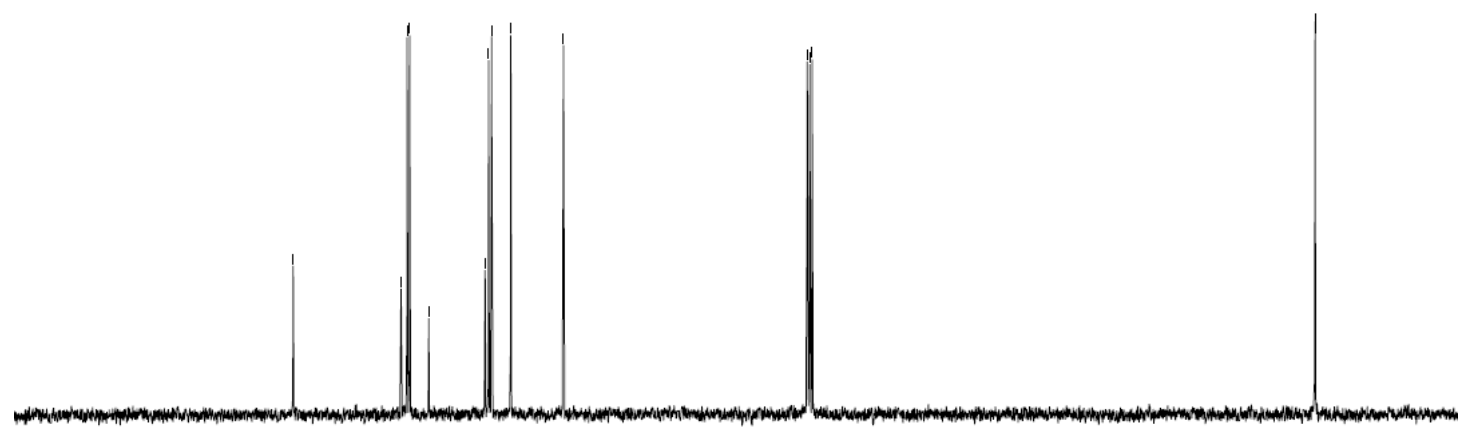

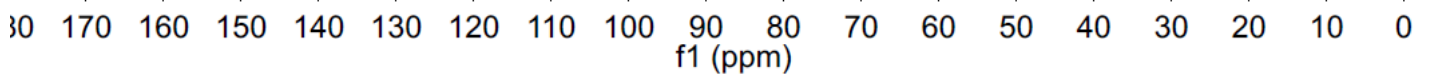
œ ب

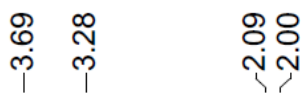

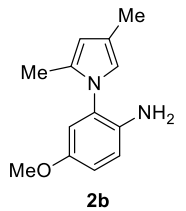

${ }^{1} \mathrm{H}$ NMR $\left(\mathrm{CDCl}_{3}, 400 \mathrm{MHz}\right)$

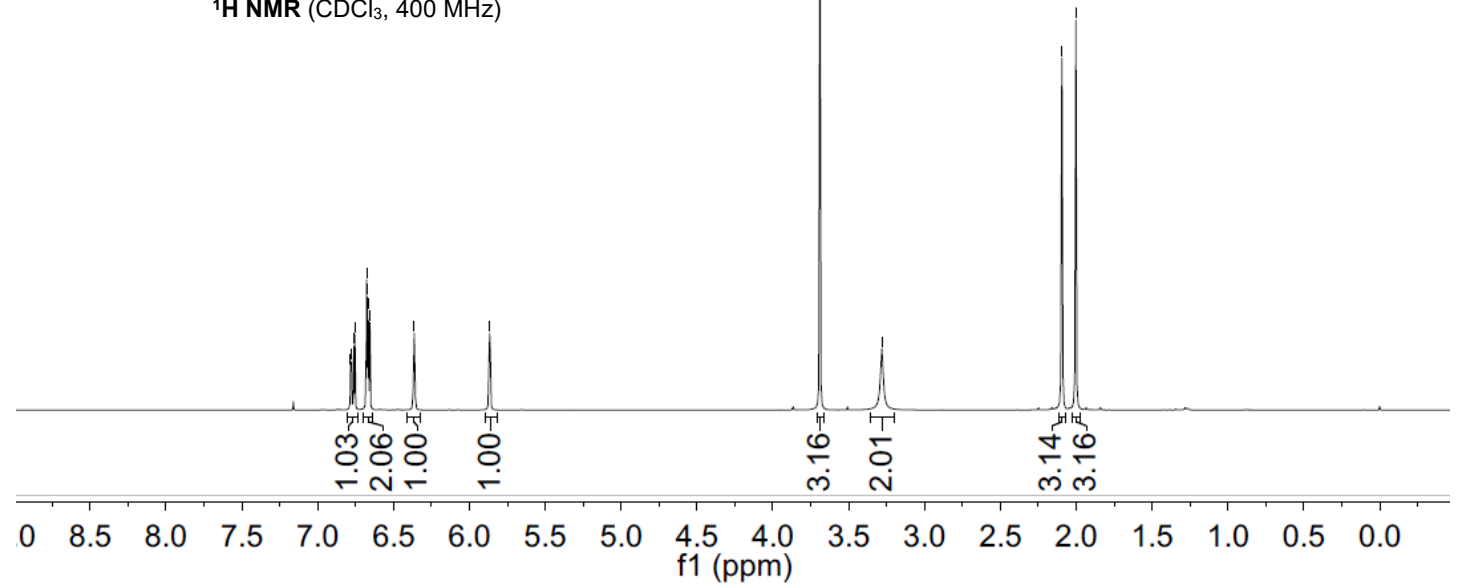




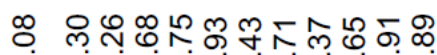

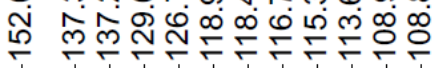

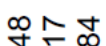

$\stackrel{\infty}{\bullet}$

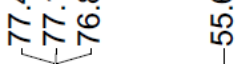

$\infty$

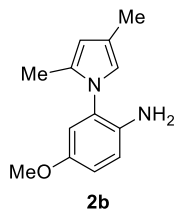

${ }^{13} \mathrm{C}$ NMR $\left(\mathrm{CDCl}_{3}, 100 \mathrm{MHz}\right)$

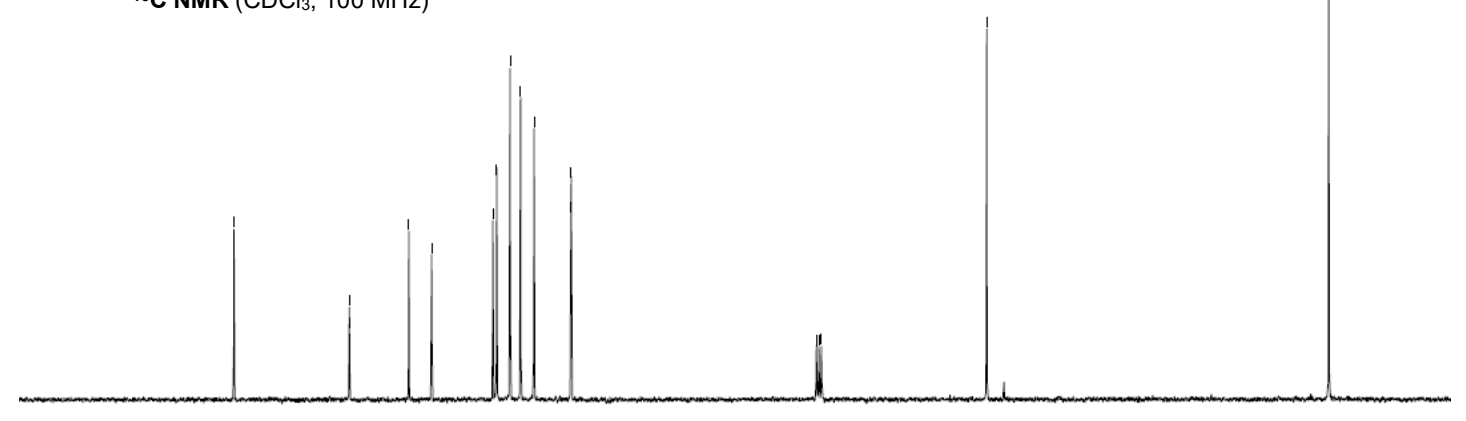

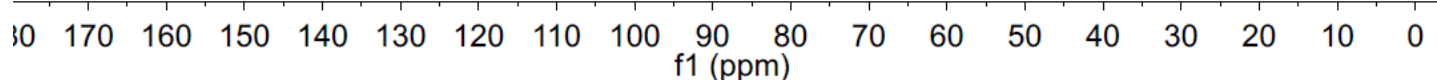

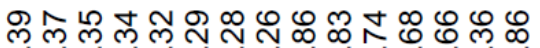

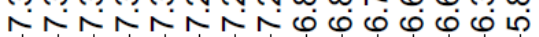

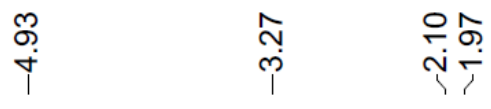

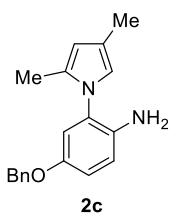

${ }^{1} \mathrm{H}$ NMR $\left(\mathrm{CDCl}_{3}, 400 \mathrm{MHz}\right)$

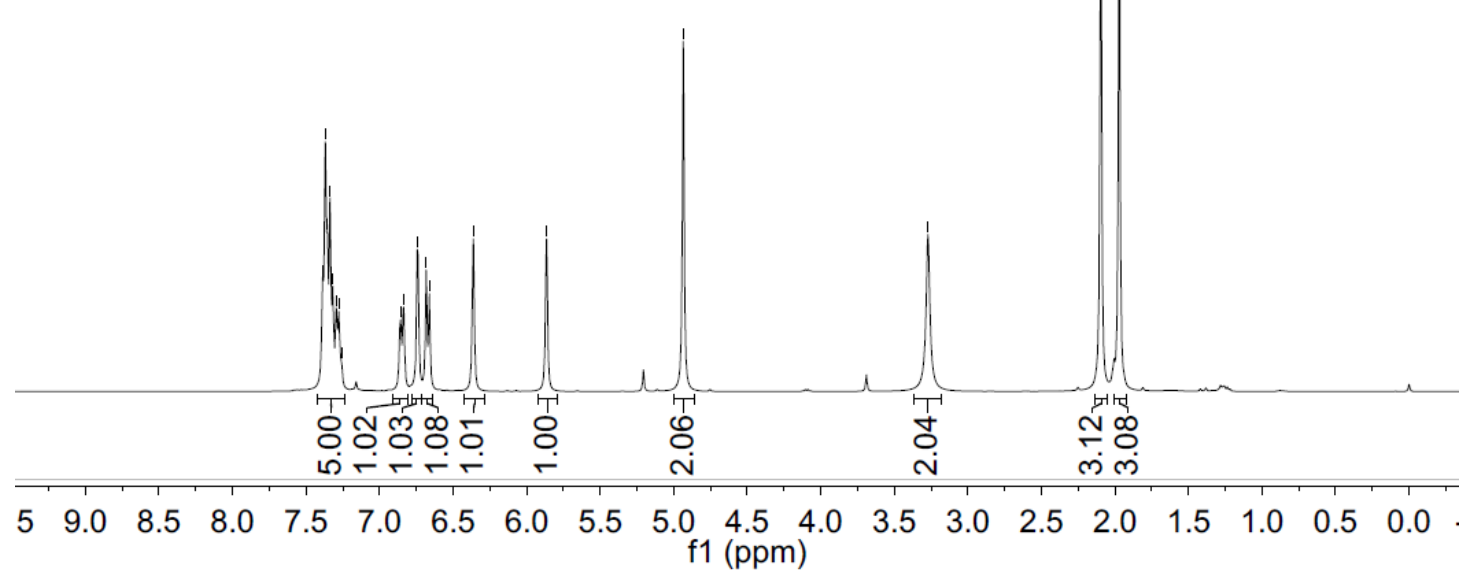



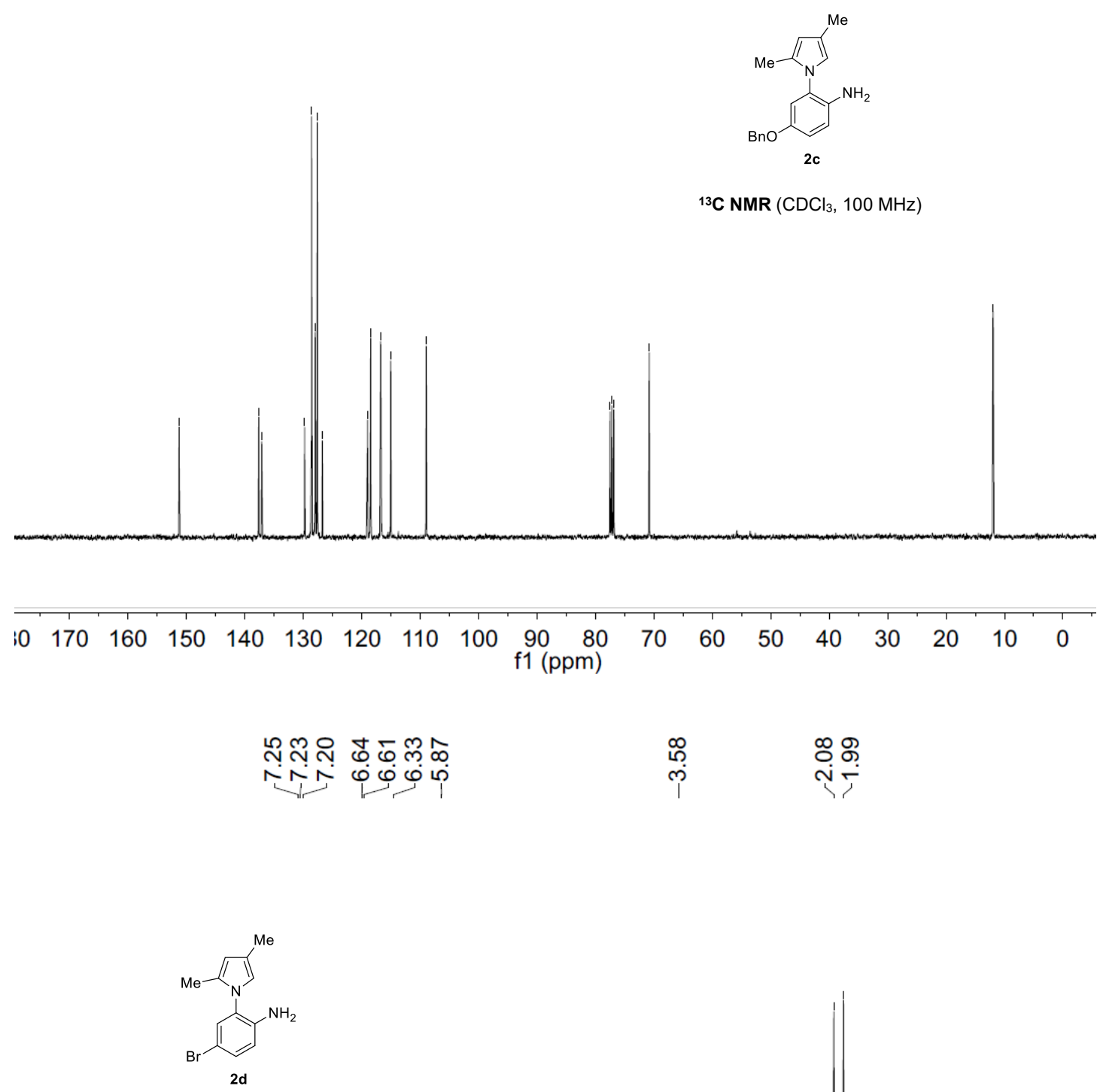

${ }^{13} \mathrm{C} \mathrm{NMR}\left(\mathrm{CDCl}_{3}, 100 \mathrm{MHz}\right)$

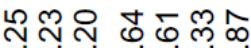

NN

${ }^{1} \mathrm{H} \mathrm{NMR}\left(\mathrm{CDCl}_{3}, 400 \mathrm{MHz}\right)$

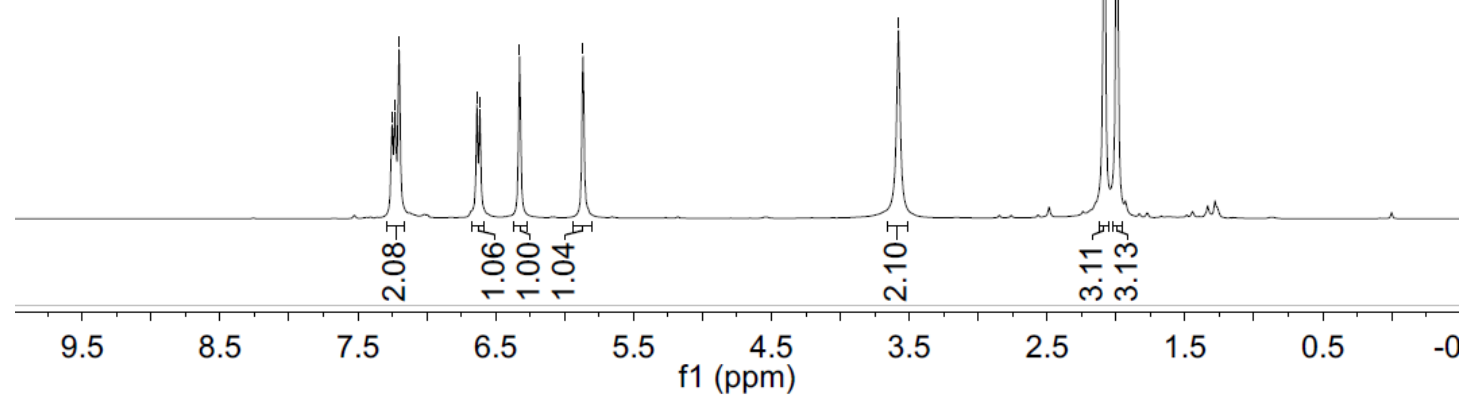


๙ิ ๓

ণ্்

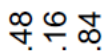

NN心

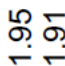

产

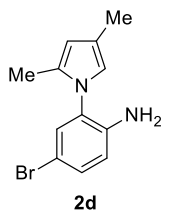

${ }^{13} \mathrm{C}$ NMR $\left(\mathrm{CDCl}_{3}, 100 \mathrm{MHz}\right)$

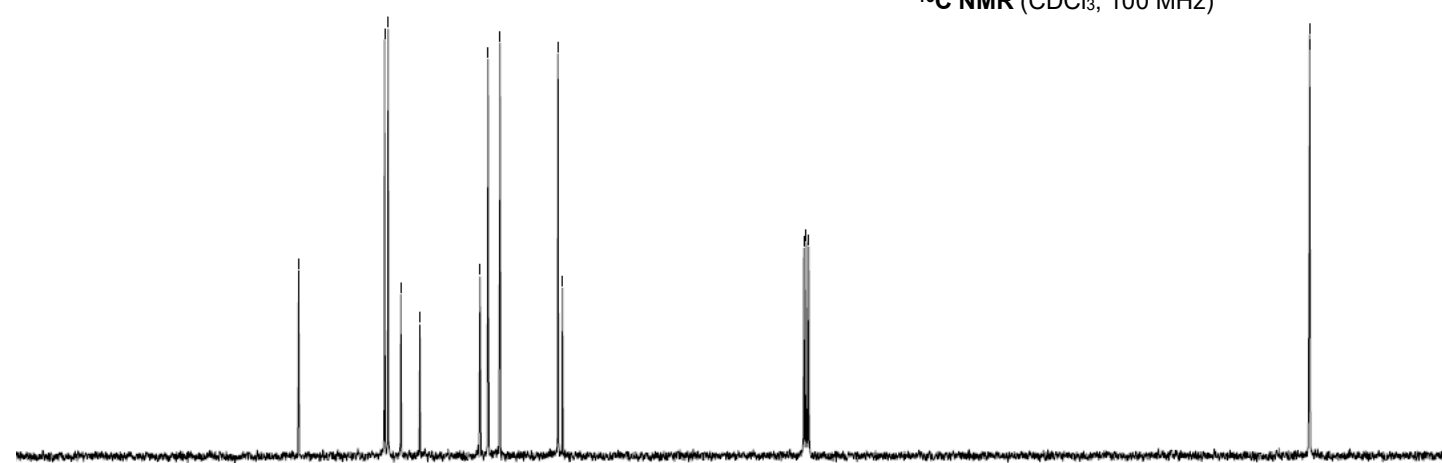

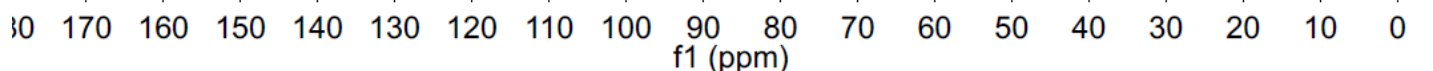

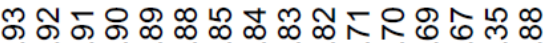

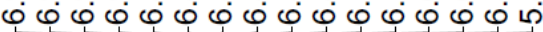

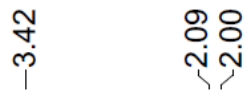

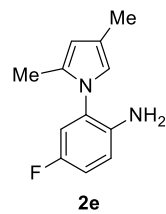

${ }^{1} \mathrm{H}$ NMR $\left(\mathrm{CDCl}_{3}, 400 \mathrm{MHz}\right)$

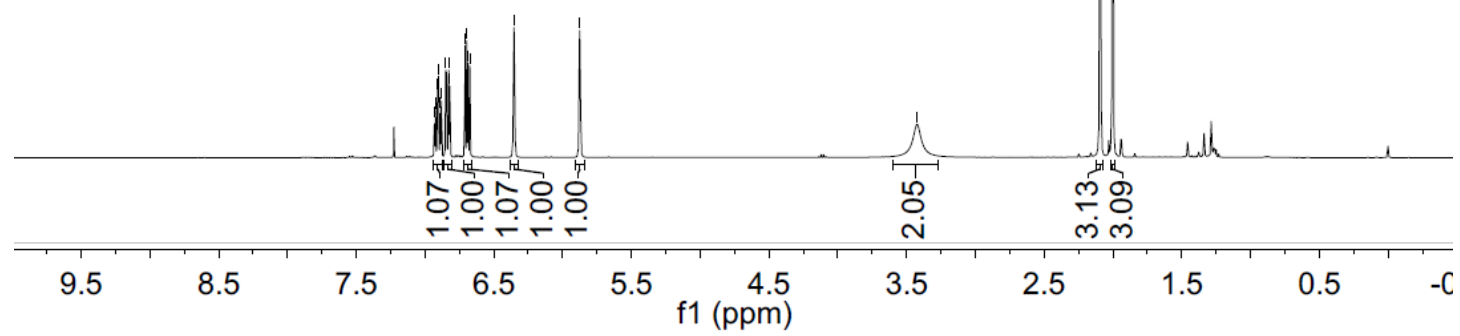




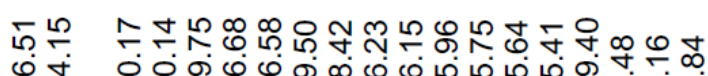

ம்

กิळ

Е

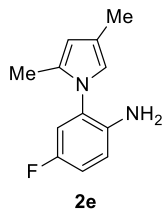

${ }^{13} \mathrm{C} \mathrm{NMR}\left(\mathrm{CDCl}_{3}, 100 \mathrm{MHz}\right)$

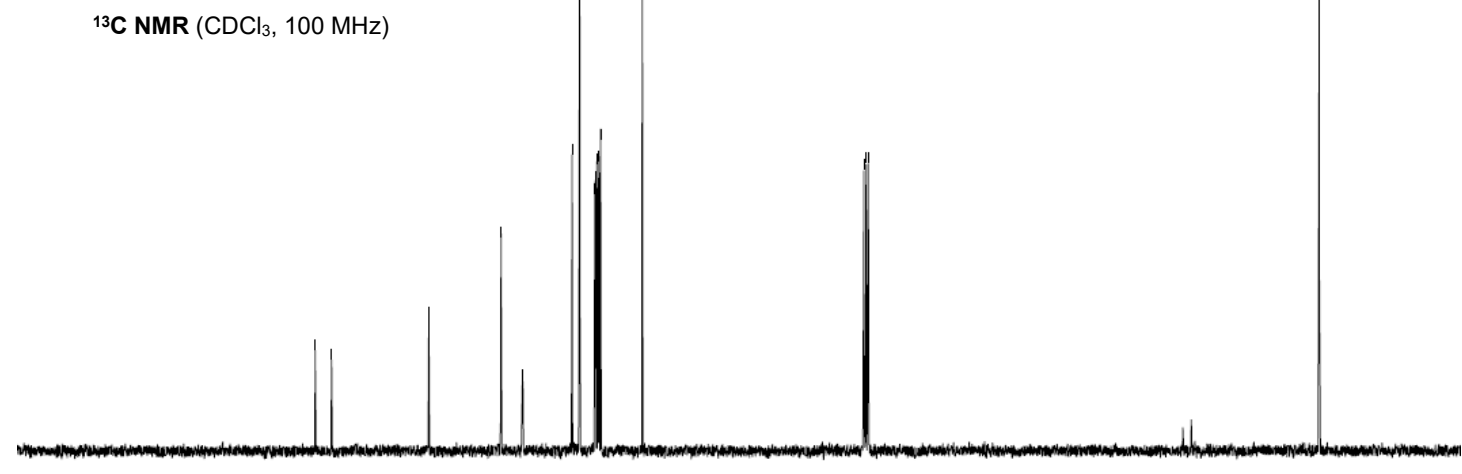

$\begin{array}{lllllllllllllllllllll}190 & 180 & 170 & 160 & 150 & 140 & 130 & 120 & 110 & \begin{array}{c}100 \\ \mathrm{f} 1(\mathrm{ppm})\end{array} & 80 & 70 & 60 & 50 & 40 & 30 & 20 & 10 & 0 & -\end{array}$

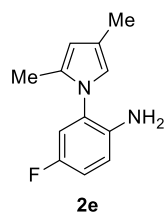

${ }^{19} \mathrm{~F}$ NMR $\left(\mathrm{CDCl}_{3}, 377 \mathrm{MHz}\right)$

$\begin{array}{llllll}0 & -20 & -40 & -60 & -80 & -100\end{array}$
f1 (ppm)

$\begin{array}{llllll}-120 & -140 & -160 & -180 & -200 & -2\end{array}$




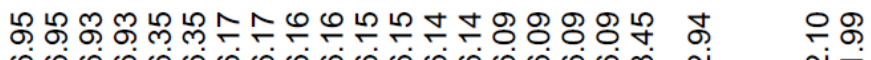

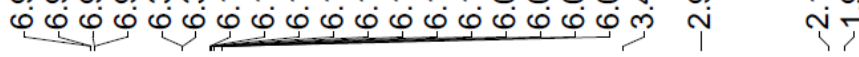

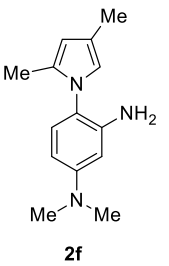

${ }^{1} \mathrm{H}$ NMR $\left(\mathrm{CDCl}_{3}, 400 \mathrm{MHz}\right)$

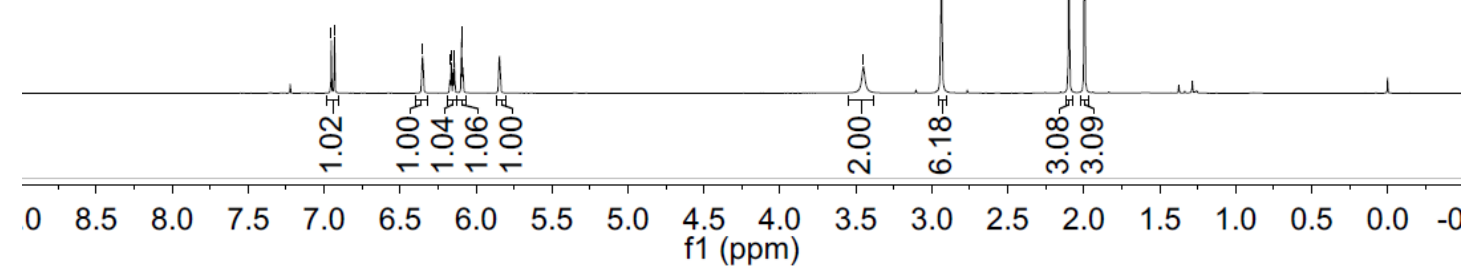

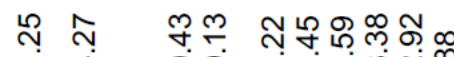

in

广

৫)

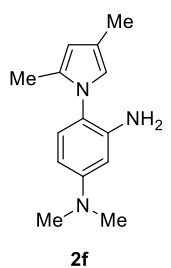

${ }^{13} \mathrm{C} \mathrm{NMR}\left(\mathrm{CDCl}_{3}, 100 \mathrm{MHz}\right)$

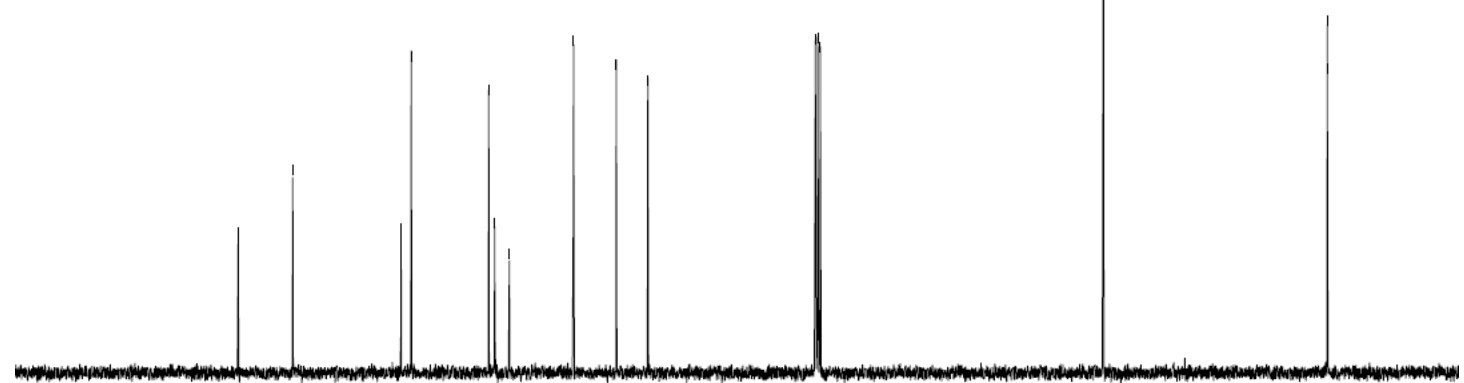

$\begin{array}{lllllllllllllllllll}30 & 170 & 160 & 150 & 140 & 130 & 120 & 110 & 100 & 90 & 80 & 70 & 60 & 50 & 40 & 30 & 20 & 10 & 0\end{array}$ 


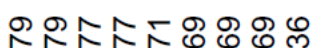

ب

${ }^{1} \mathrm{H}$ NMR $\left(\mathrm{CDCl}_{3}, 400 \mathrm{MHz}\right)$

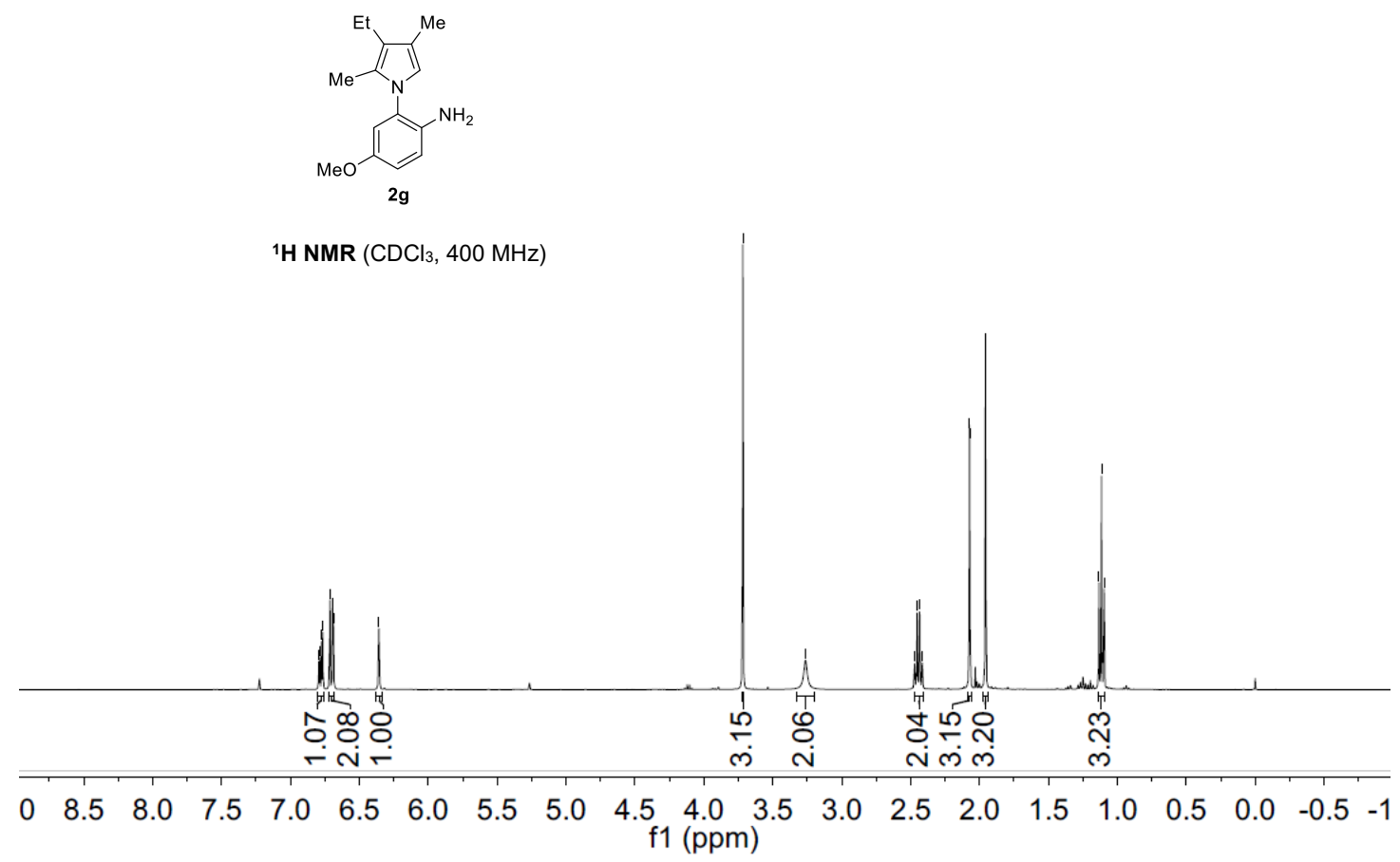

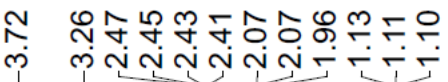

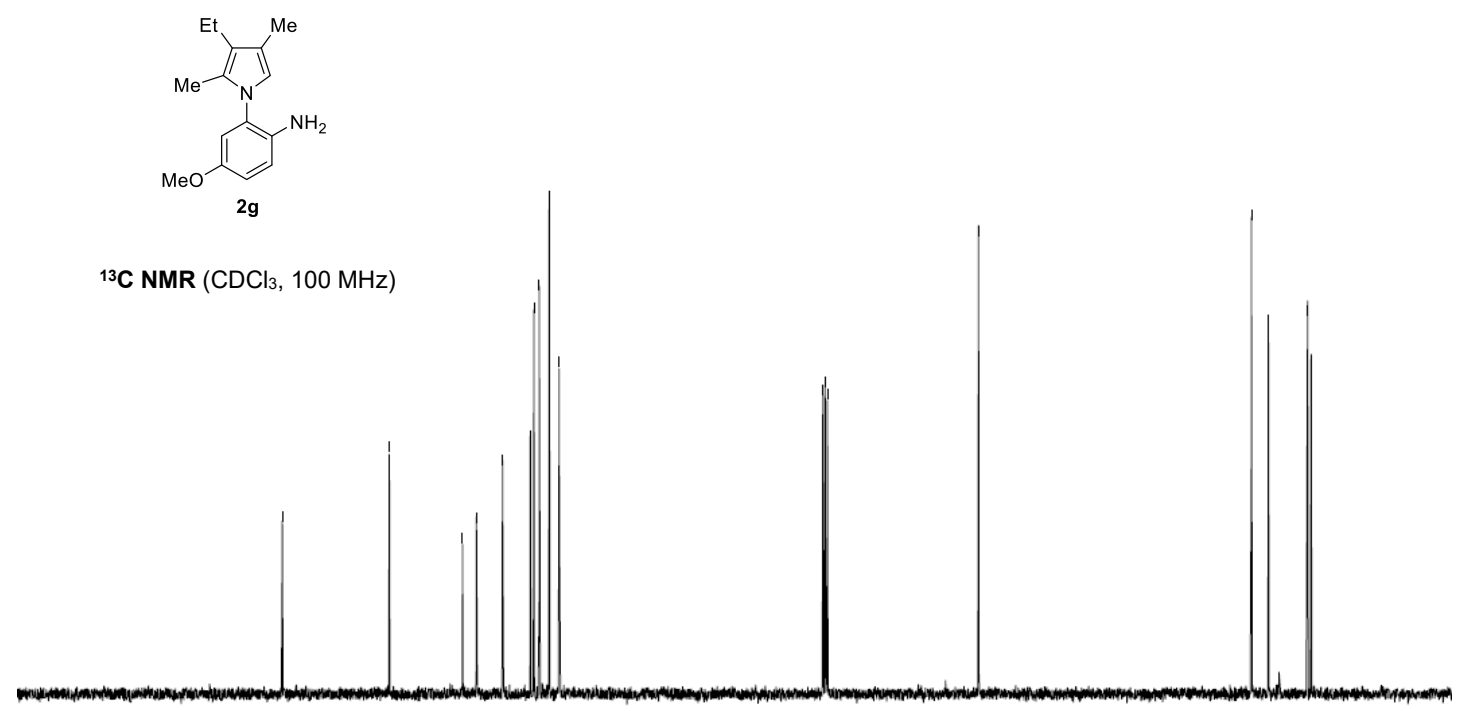

$\begin{array}{llllllllllllllllllll}180 & 170 & 160 & 150 & 140 & 130 & 120 & 110 & 100 & 90 & 80 & 70 & 60 & 50 & 40 & 30 & 20 & 10 & 0 & -1\end{array}$ f1 (ppm) 
ㄲํำ중ํำ

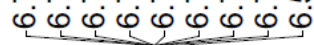

${ }^{1} \mathrm{H}$ NMR $\left(\mathrm{CDCl}_{3}, 400 \mathrm{MHz}\right)$

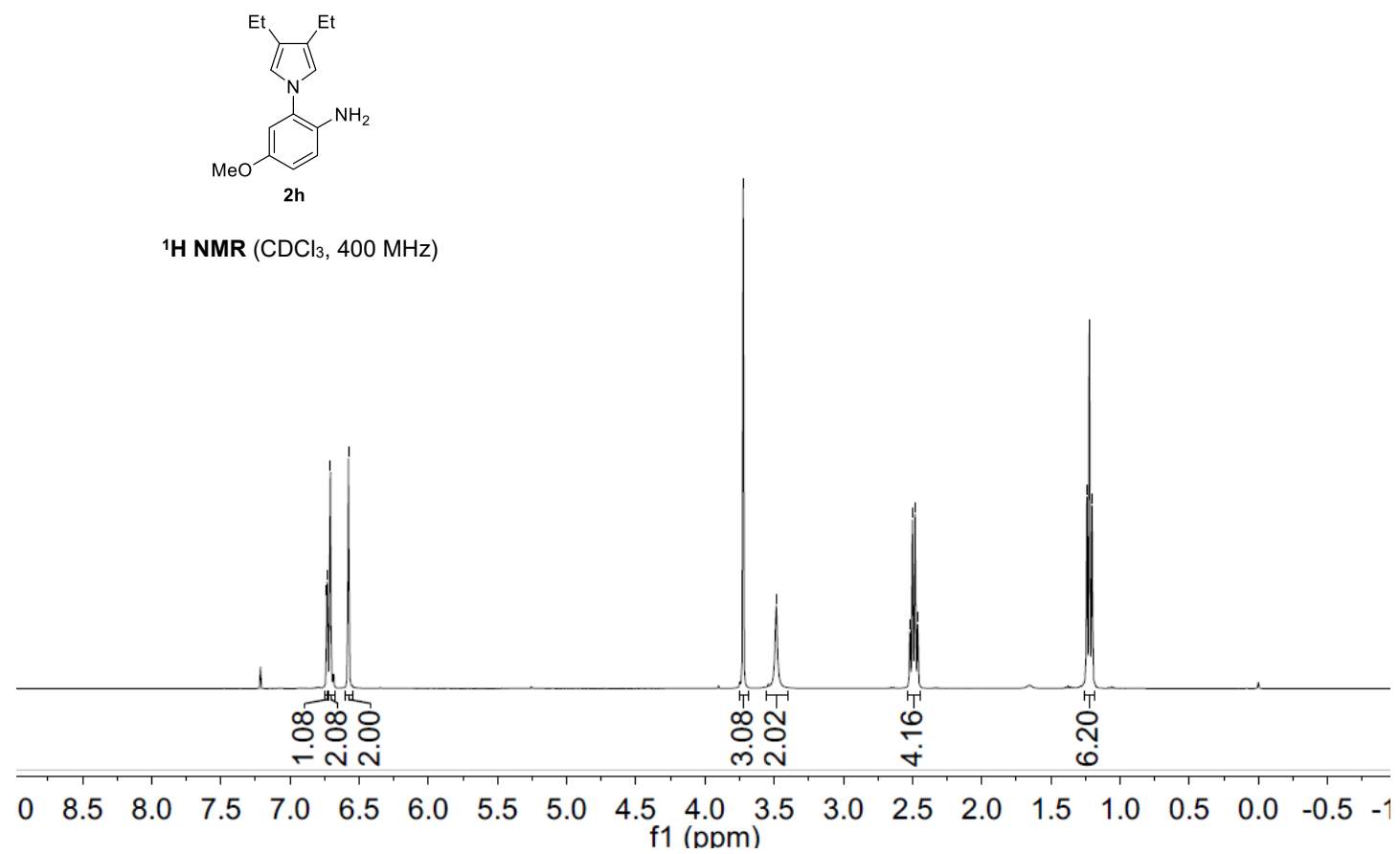

№

m

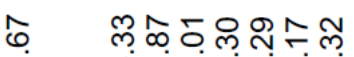

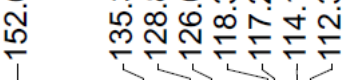

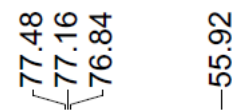

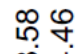

กิ

$\infty$

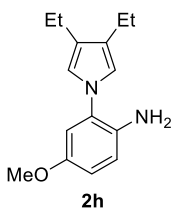

$\left.{ }^{13} \mathrm{C} \mathrm{NMR} \mathrm{(CDCl}, 100 \mathrm{MHz}\right)$

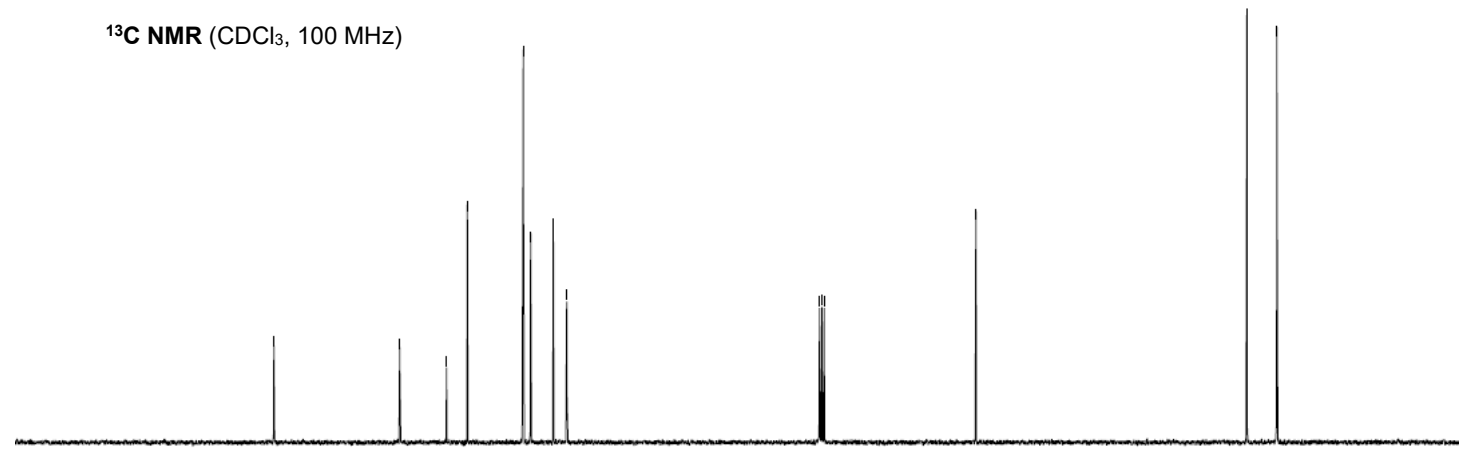

$\begin{array}{llllllllllllllllllll}180 & 170 & 160 & 150 & 140 & 130 & 120 & 110 & 100 & 90 & 80 & 70 & 60 & 50 & 40 & 30 & 20 & 10 & 0 & -1\end{array}$ $\mathrm{f} 1(\mathrm{ppm})$ 


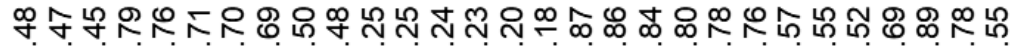

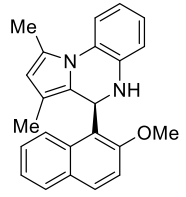

3b

${ }^{1} \mathrm{H}$ NMR $\left(\mathrm{CDCl}_{3}, 400 \mathrm{MHz}\right)$

م 员
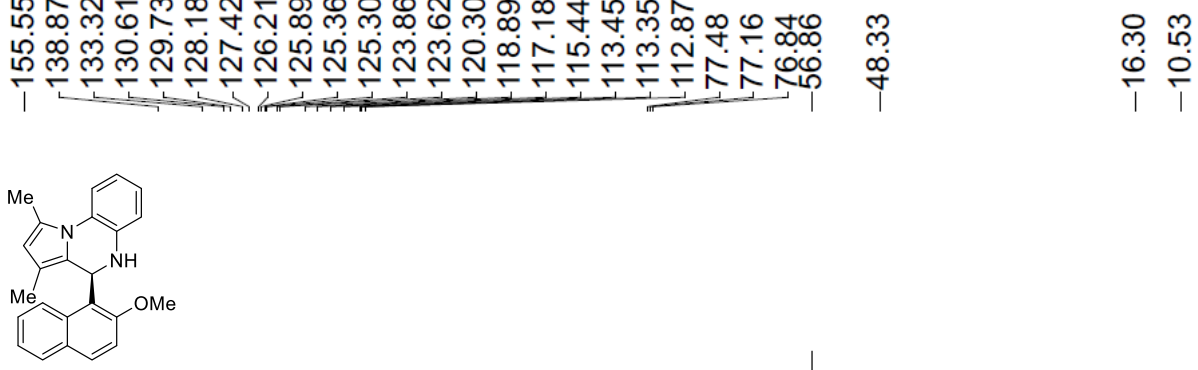

${ }^{13} \mathrm{C}$ NMR $\left(\mathrm{CDCl}_{3}, 100 \mathrm{MHz}\right)$

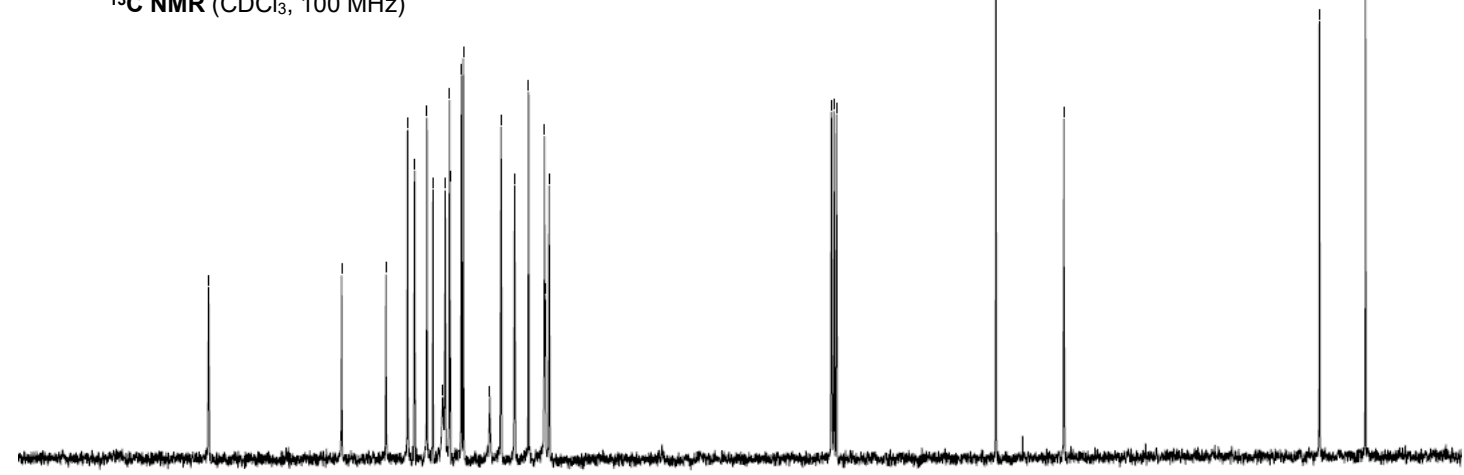

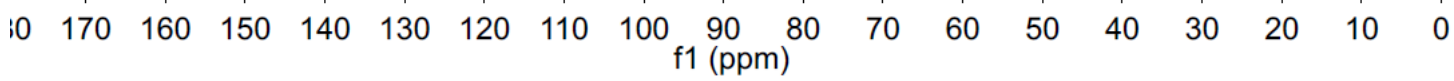




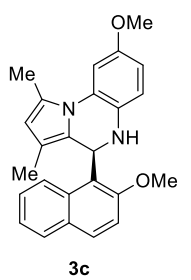

${ }^{1} \mathrm{H} \mathrm{NMR}\left(\mathrm{CDCl}_{3}, 400 \mathrm{MHz}\right)$

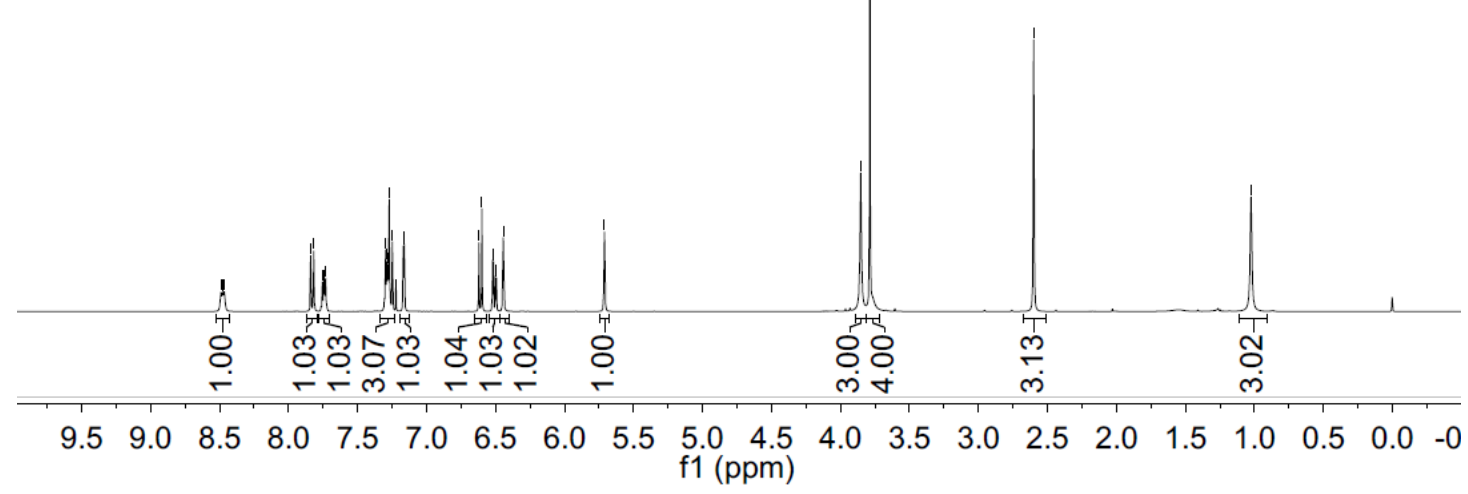

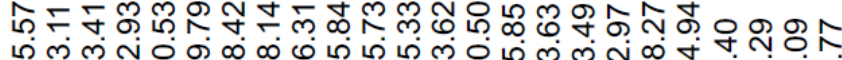
岇

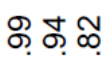

மก

$\stackrel{\infty}{\leftarrow} \stackrel{0}{+}$

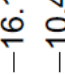

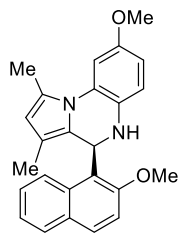

3c

${ }^{13} \mathrm{C} \mathrm{NMR}\left(\mathrm{CDCl}_{3}, 100 \mathrm{MHz}\right)$

$\begin{array}{llllllllllllllllll}30 & 170 & 160 & 150 & 140 & 130 & 120 & 110 & 100 \underset{\mathrm{f} 1}{\stackrel{90}{(\mathrm{ppm})}} \mathbf{8 0} & 70 & 60 & 50 & 40 & 30 & 20 & 10 & 0\end{array}$ 


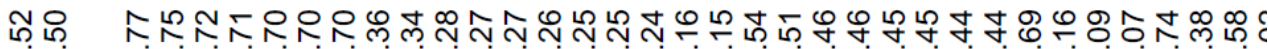

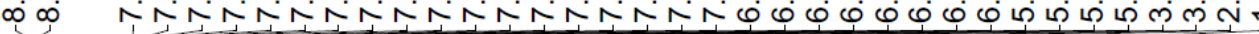

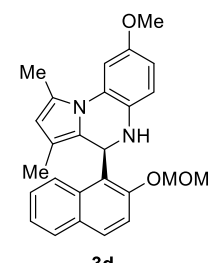

${ }^{1} \mathrm{H}$ NMR $\left(\mathrm{CDCl}_{3}, 400 \mathrm{MHz}\right)$

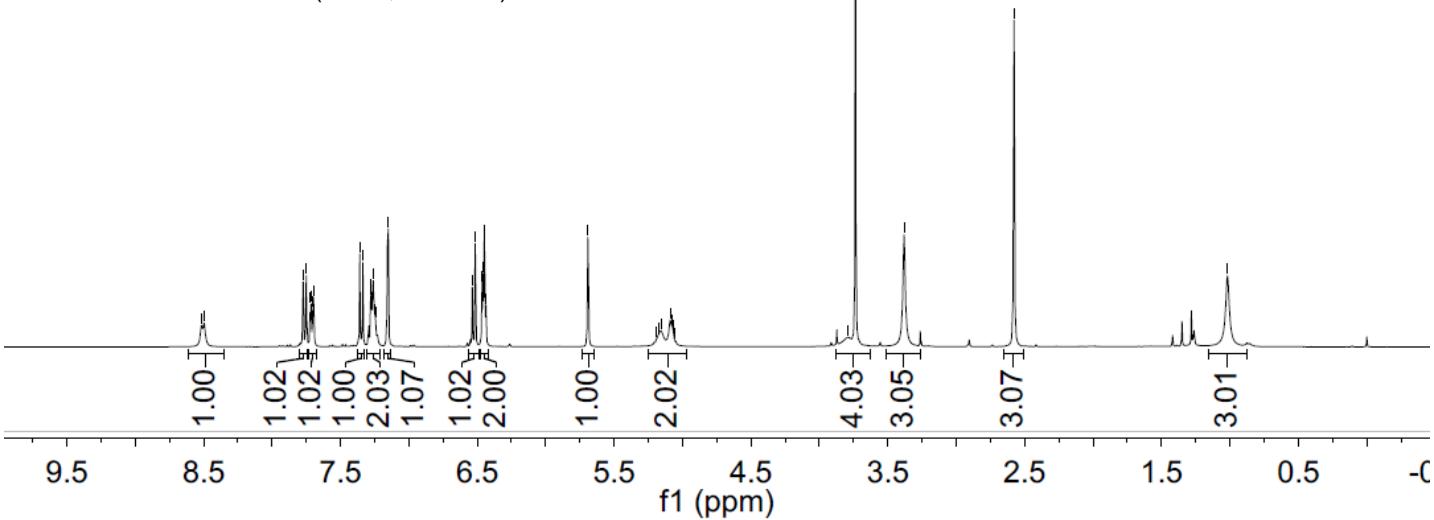

以ூ 负

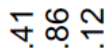

它官守

$\stackrel{m}{\circ}$ 웅

$\stackrel{1}{1}$

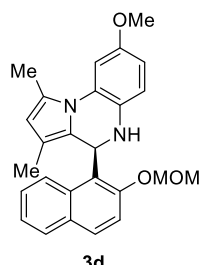

3d

${ }^{13} \mathrm{C} \mathrm{NMR}\left(\mathrm{CDCl}_{3}, 100 \mathrm{MHz}\right)$

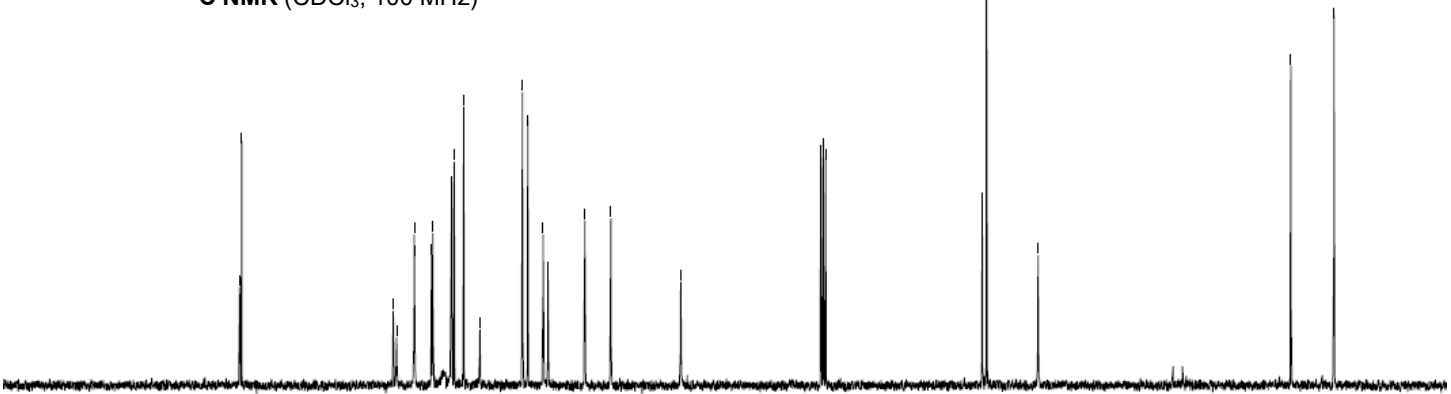

$\begin{array}{lllllllllllllllllll}180 & 170 & 160 & 150 & 140 & 130 & 120 & 110 & 100 \underset{\mathrm{f} 1}{\underset{(\mathrm{ppm})}{90}} \mathbf{8 0} & 70 & 60 & 50 & 40 & 30 & 20 & 10 & 0\end{array}$ 


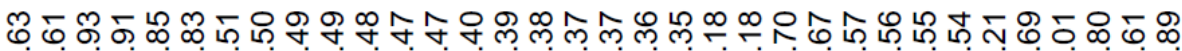

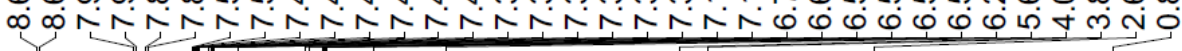

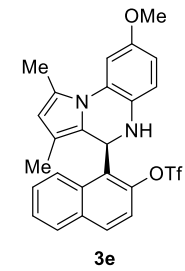

${ }^{1} \mathrm{H}$ NMR $\left(\mathrm{CDCl}_{3}, 400 \mathrm{MHz}\right)$

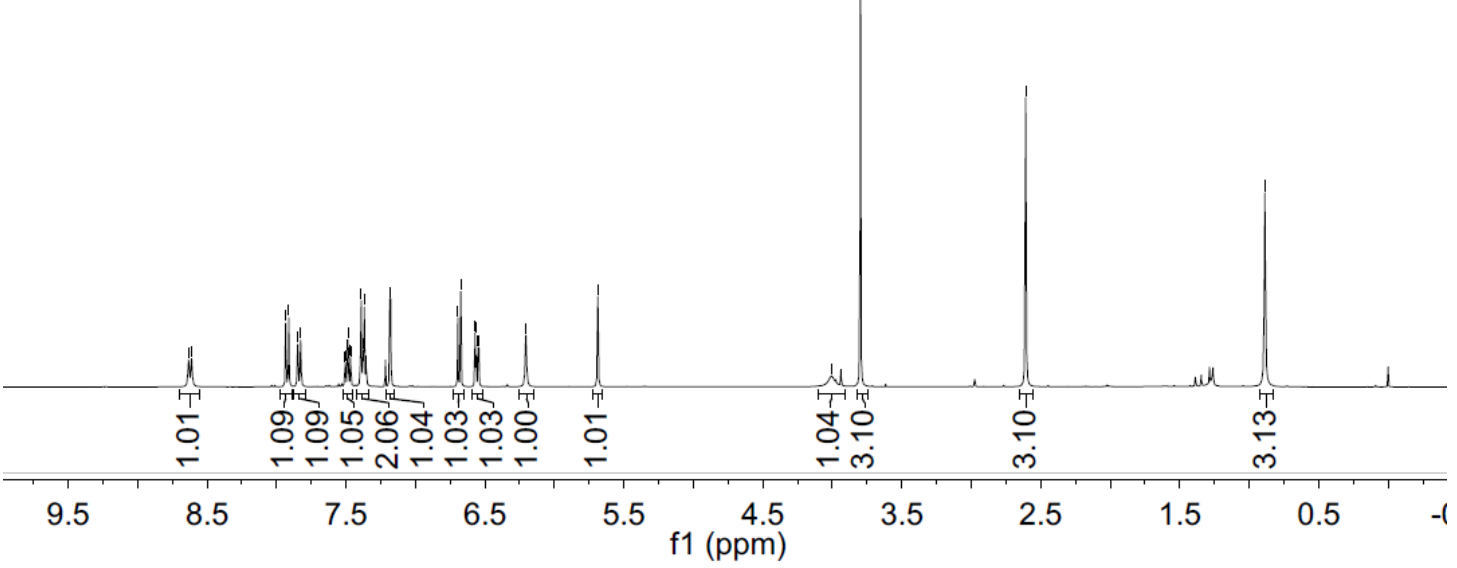

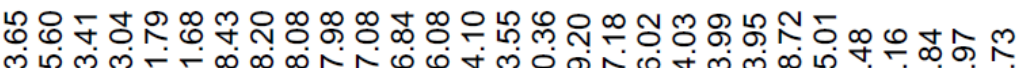

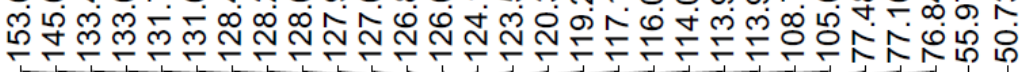

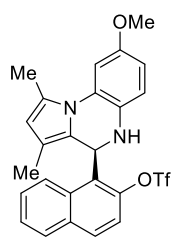

3e

${ }^{13} \mathrm{C} \mathrm{NMR}\left(\mathrm{CDCl}_{3}, 100 \mathrm{MHz}\right)$

$\begin{array}{llllllllllllllllllll}0 & 180 & 170 & 160 & 150 & 140 & 130 & 120 & 110 & 100 \\ \mathrm{f} 1(\mathrm{ppm}) & 80 & 80 & 70 & 60 & 50 & 40 & 30 & 20 & 10 & 0\end{array}$ 


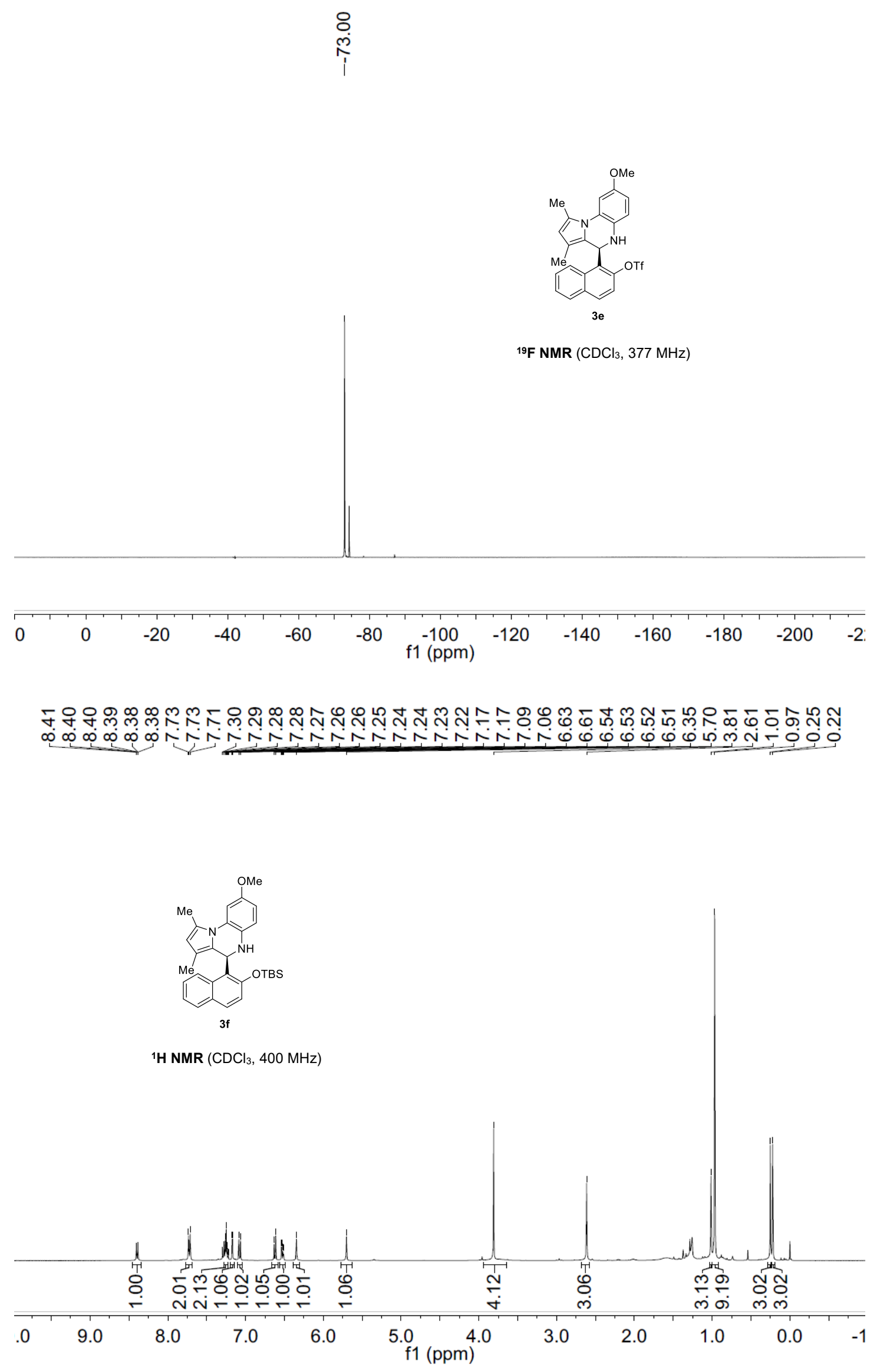




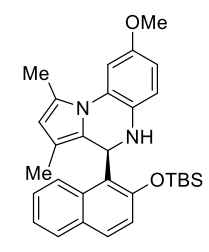

$3 f$

${ }^{13} \mathrm{C} \mathrm{NMR}\left(\mathrm{CDCl}_{3}, 100 \mathrm{MHz}\right)$

$\begin{array}{lllllllllllllllllllll}0 & 170 & 160 & 150 & 140 & 130 & 120 & 110 & 100 & 90 & \underset{\mathrm{f} 1}{(\mathrm{ppm})} \mathbf{8 0} & 70 & 60 & 50 & 40 & 30 & 20 & 10 & 0 & -10\end{array}$

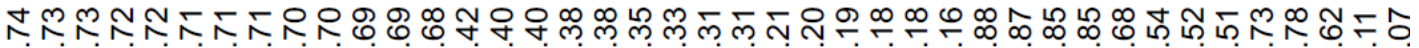

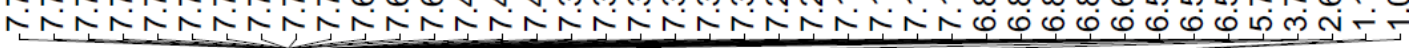

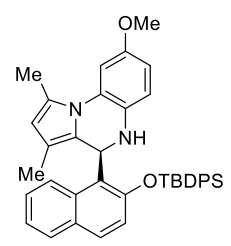

$3 g$

${ }^{1} \mathrm{H}$ NMR $\left(\mathrm{CDCl}_{3}, 400 \mathrm{MHz}\right)$

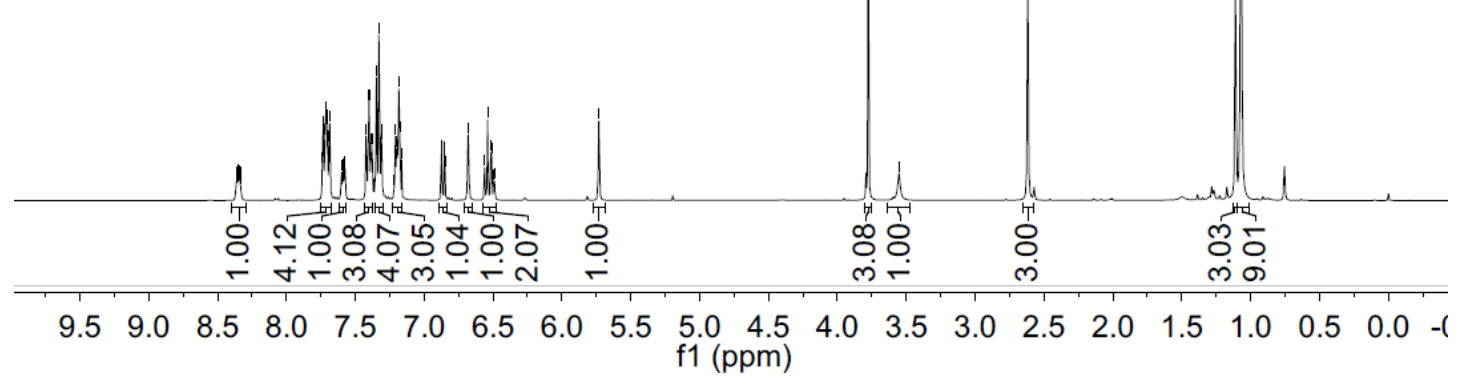


\&

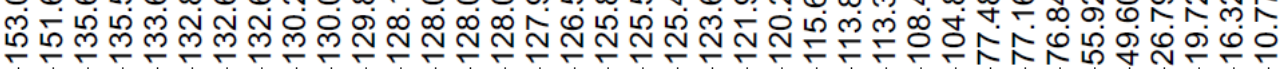
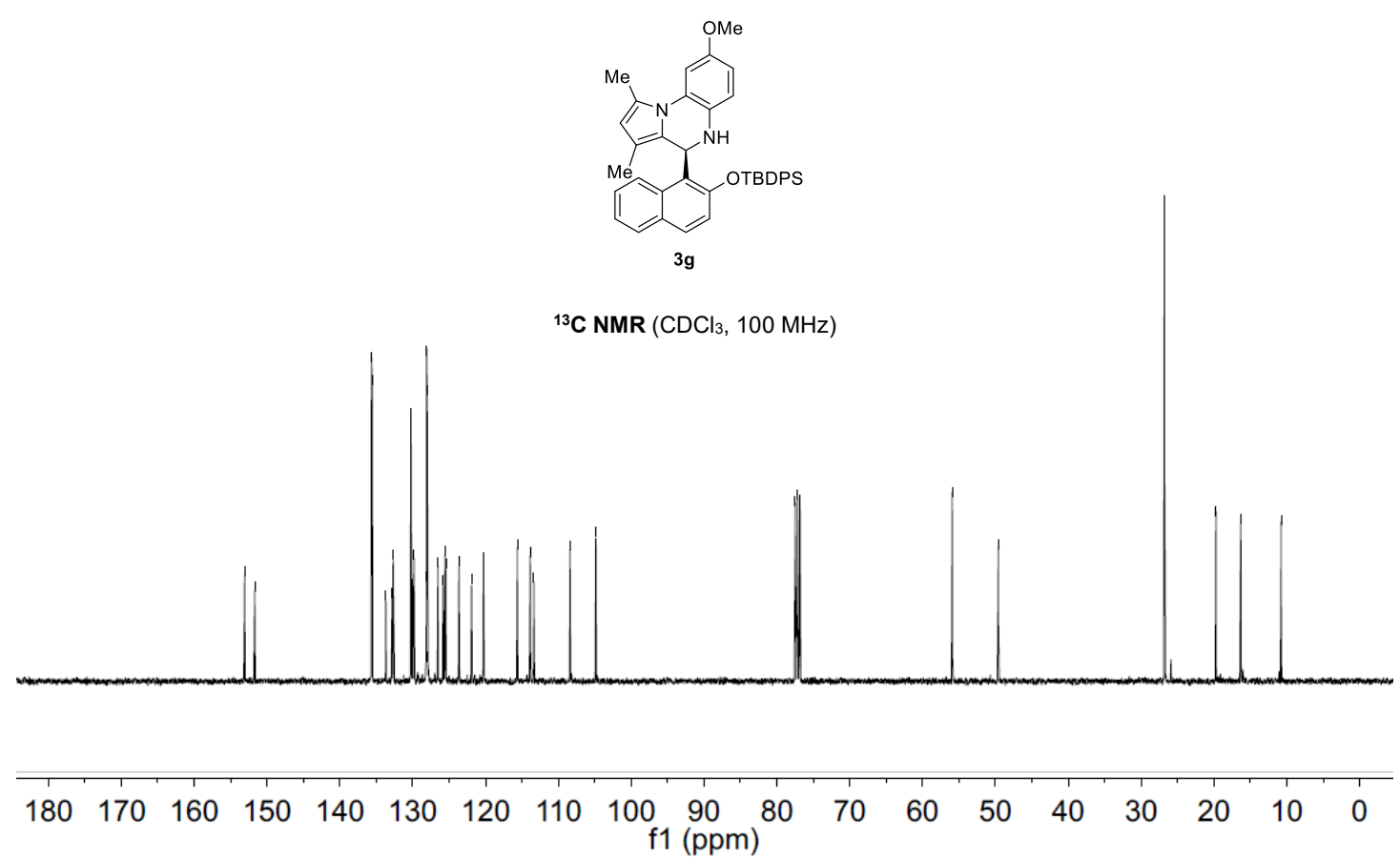

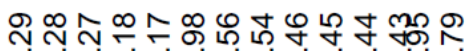

N N N

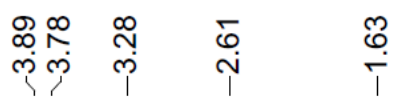

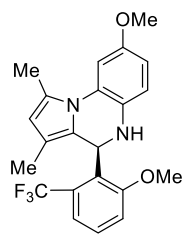

$3 \mathrm{~h}$

${ }^{1} \mathrm{H}$ NMR $\left(\mathrm{CDCl}_{3}, 400 \mathrm{MHz}\right)$

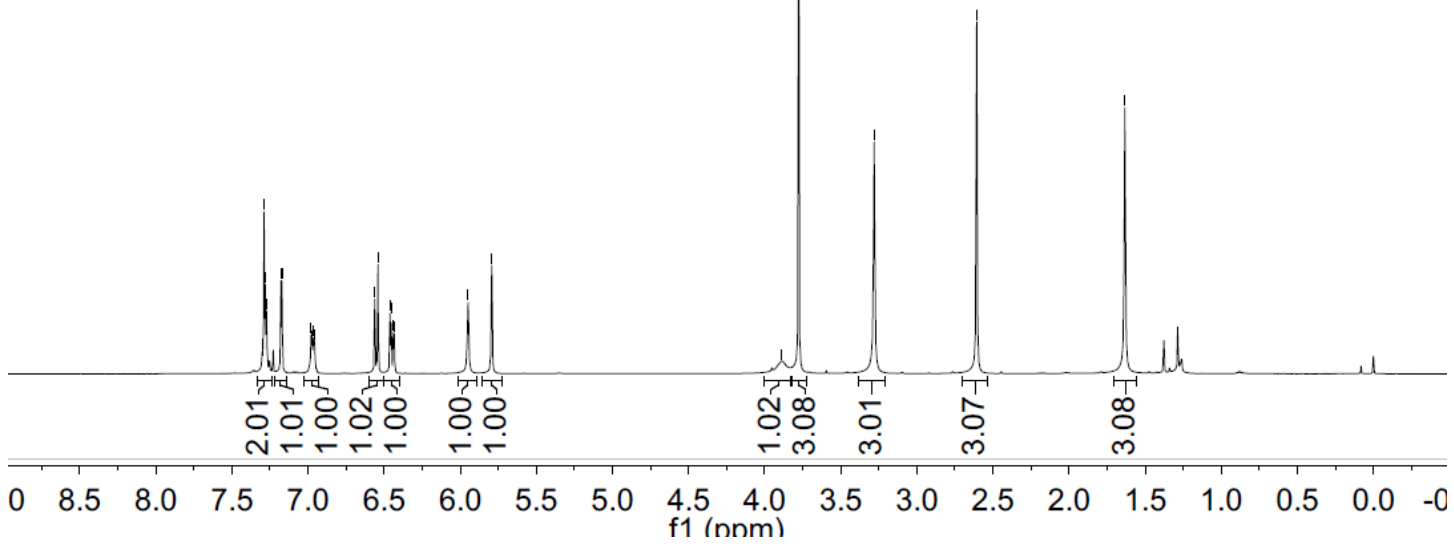




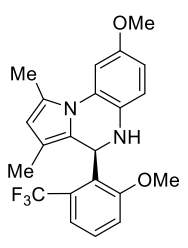

3h

${ }^{13} \mathrm{C} \mathrm{NMR}\left(\mathrm{CDCl}_{3}, 100 \mathrm{MHz}\right)$
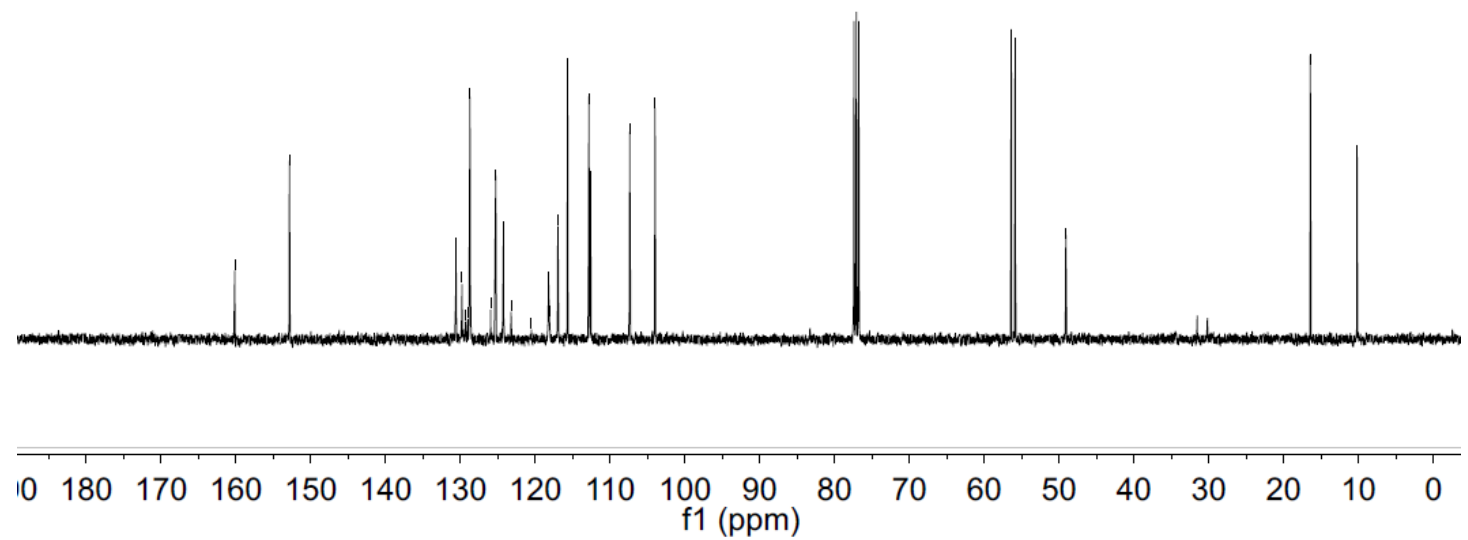

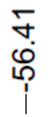

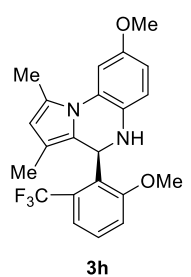

${ }^{19} \mathrm{~F}$ NMR $\left(\mathrm{CDCl}_{3}, 377 \mathrm{MHz}\right)$

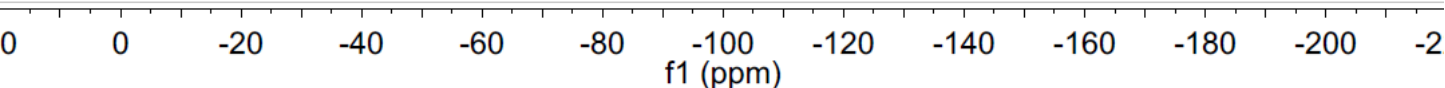




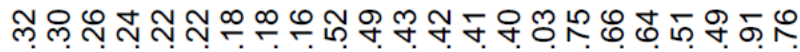

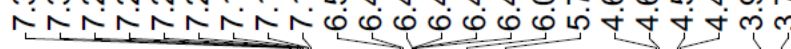

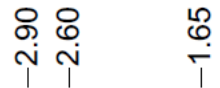

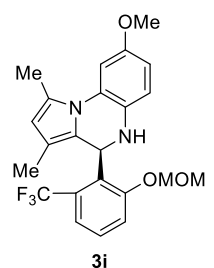

${ }^{1} \mathrm{H}$ NMR $\left(\mathrm{CDCl}_{3}, 400 \mathrm{MHz}\right)$

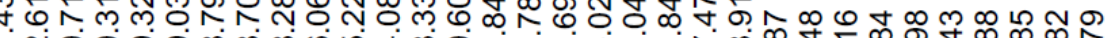

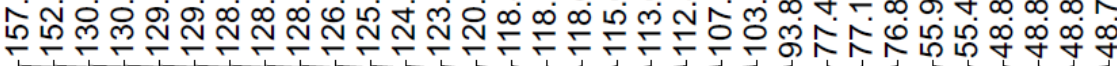

(ृ)

$\stackrel{6}{1}$

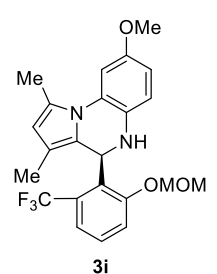

${ }^{13} \mathrm{C} \mathrm{NMR}\left(\mathrm{CDCl}_{3}, 100 \mathrm{MHz}\right)$

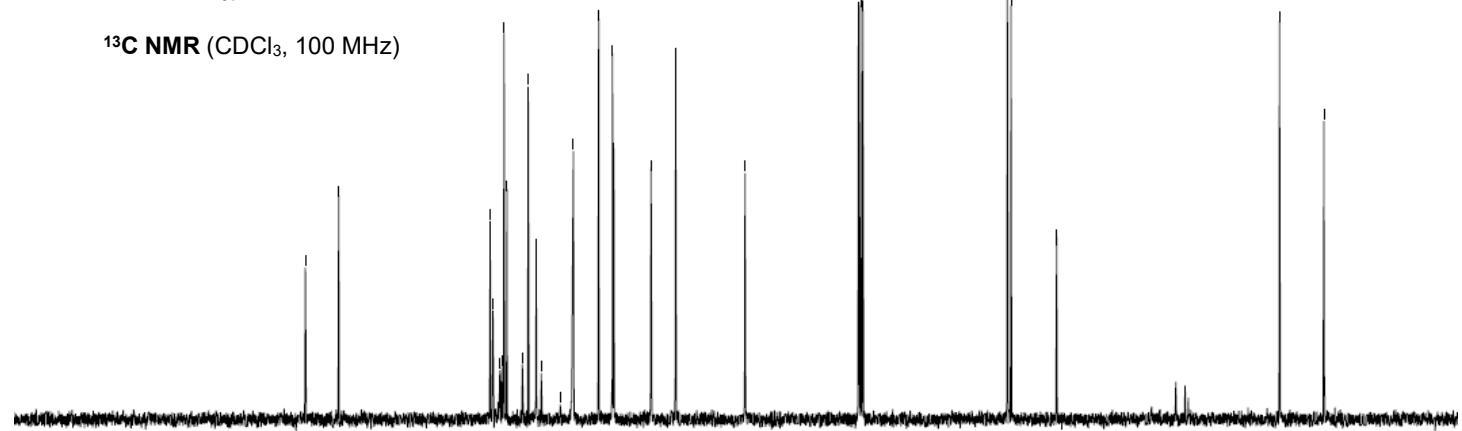

$\begin{array}{llllllllllllllllllllll}10 & 190 & 180 & 170 & 160 & 150 & 140 & 130 & 120 & 110 & \begin{array}{l}100 \\ \mathrm{f} 1(\mathrm{ppm})\end{array} & 80 & 70 & 60 & 50 & 40 & 30 & 20 & 10 & 0 & -\end{array}$ 


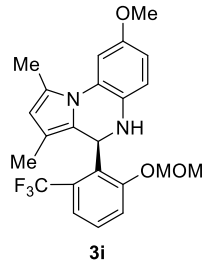

${ }^{19} \mathrm{~F} \mathrm{NMR}\left(\mathrm{CDCl}_{3}, 377 \mathrm{MHz}\right)$

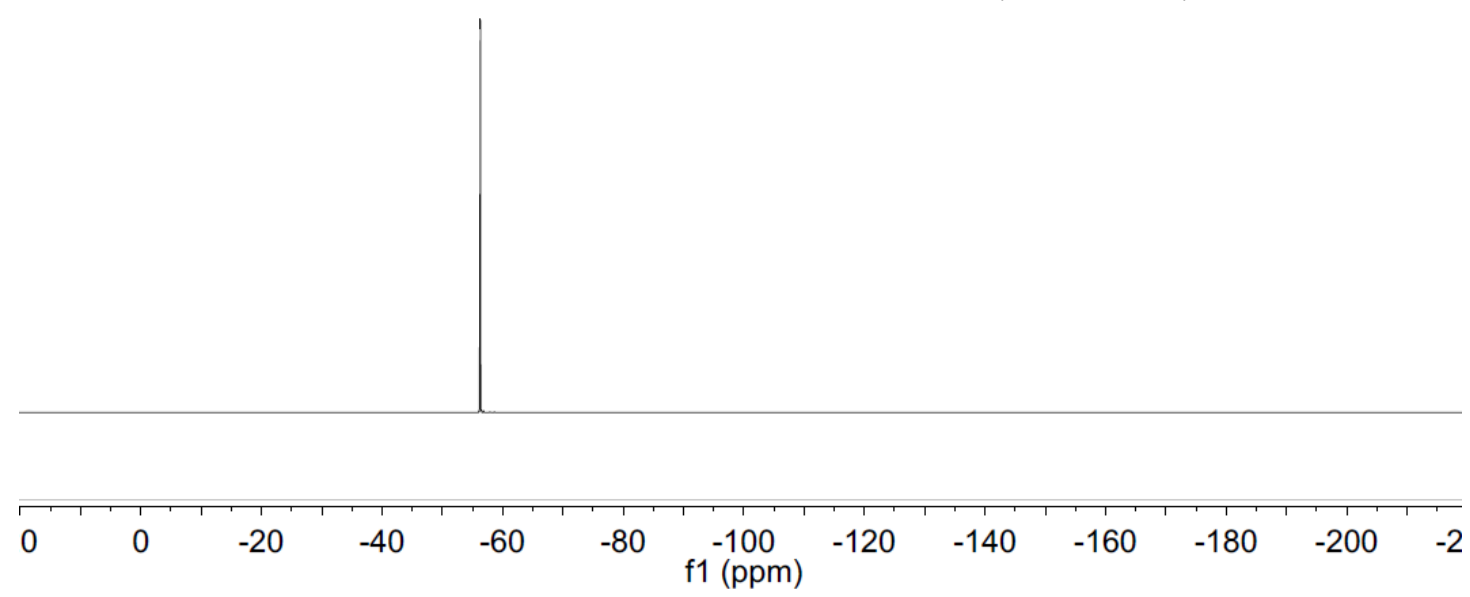

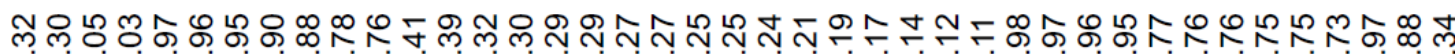

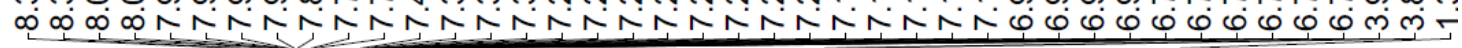

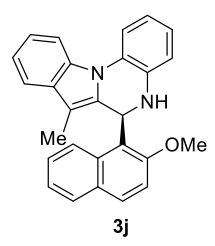

${ }^{1} \mathrm{H}$ NMR $\left(\mathrm{CDCl}_{3}, 400 \mathrm{MHz}\right)$

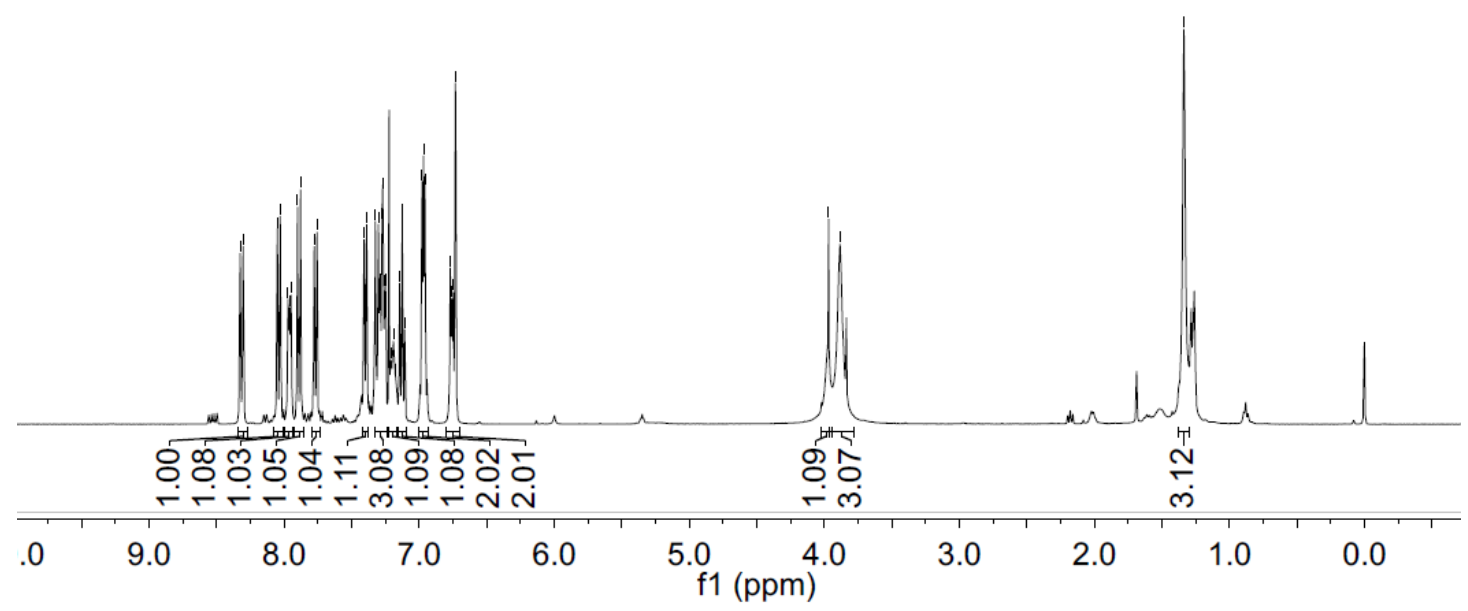


ำกำำ மึ

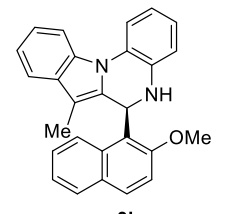

3j

${ }^{13} \mathrm{C} \mathrm{NMR}\left(\mathrm{CDCl}_{3}, 100 \mathrm{MHz}\right)$

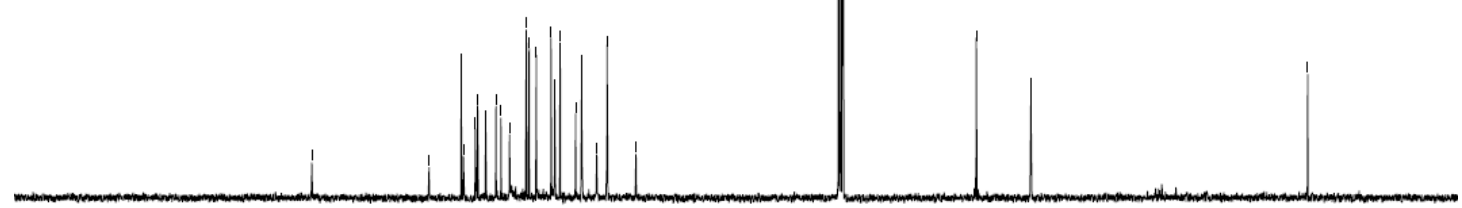

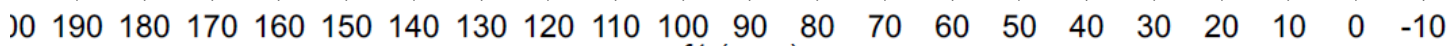
f1 (ppm)

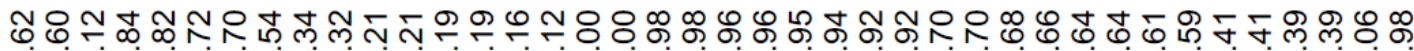

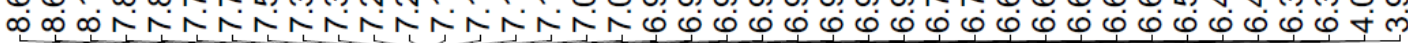

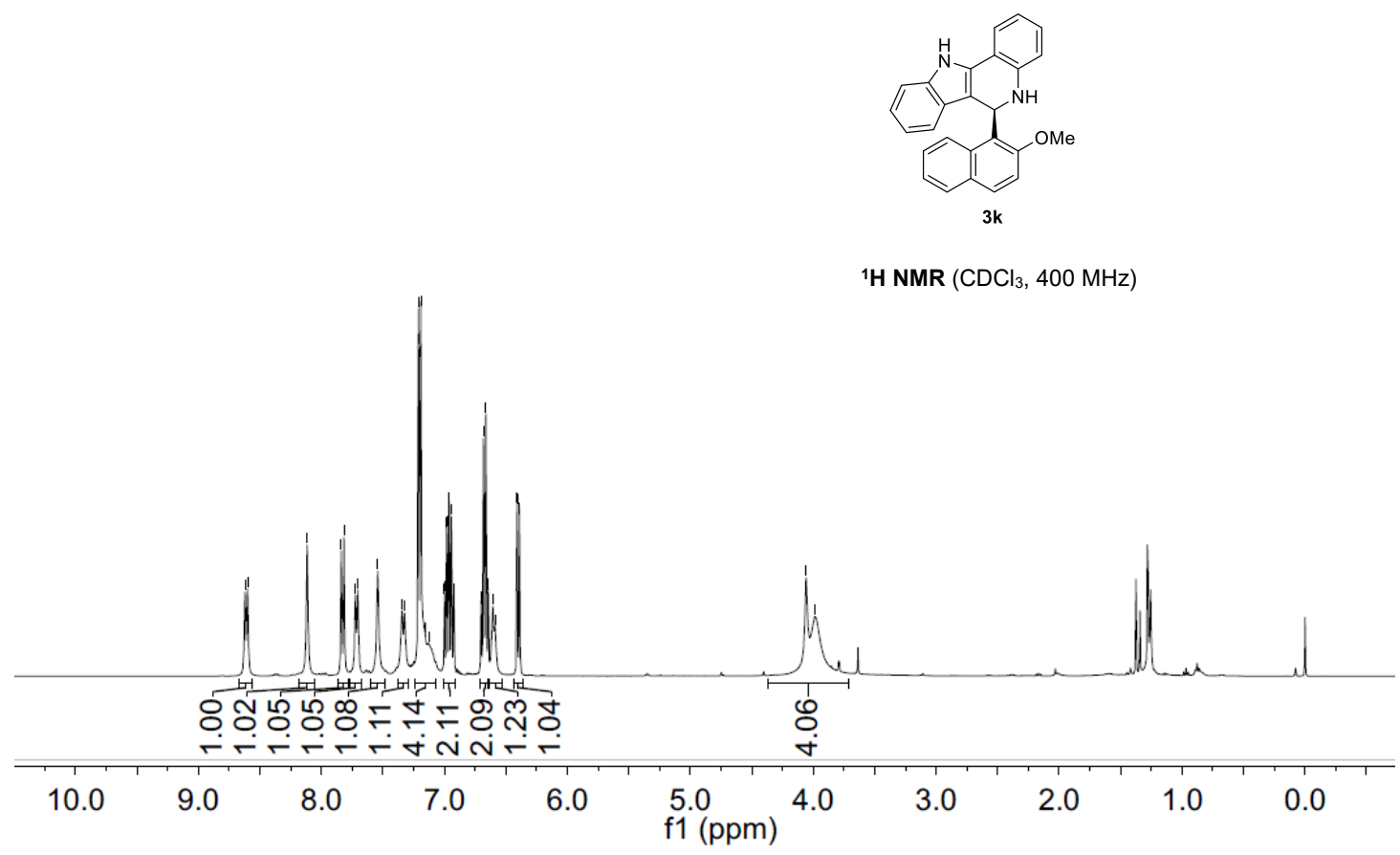


๓

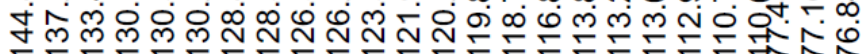

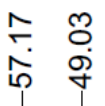

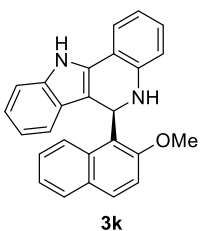

${ }^{13} \mathrm{C} \mathrm{NMR}\left(\mathrm{CDCl}_{3}, 100 \mathrm{MHz}\right)$

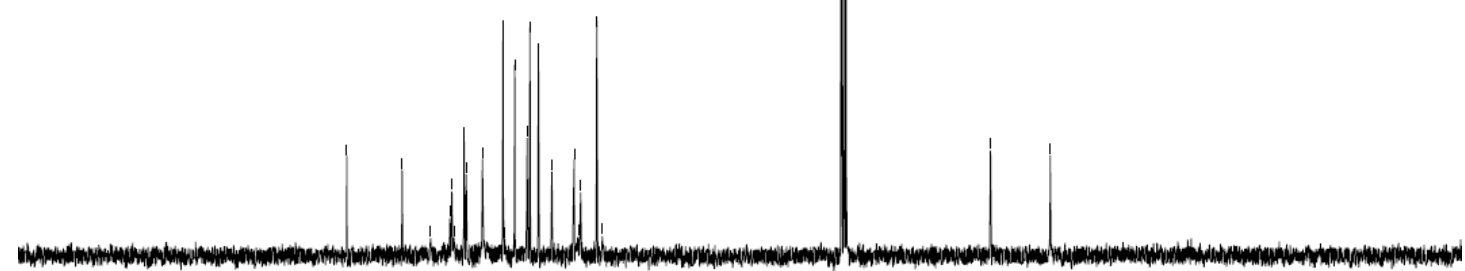

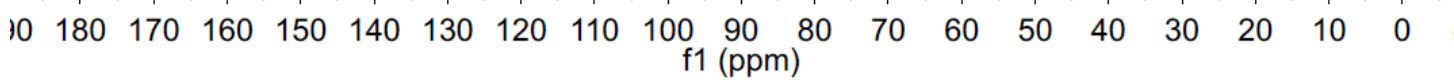

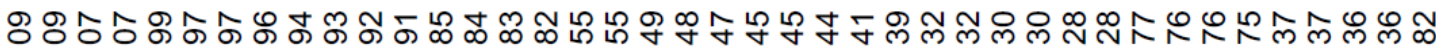
क

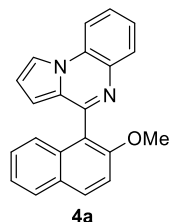

${ }^{1} \mathrm{H}$ NMR $\left(\mathrm{CDCl}_{3}, 400 \mathrm{MHz}\right)$

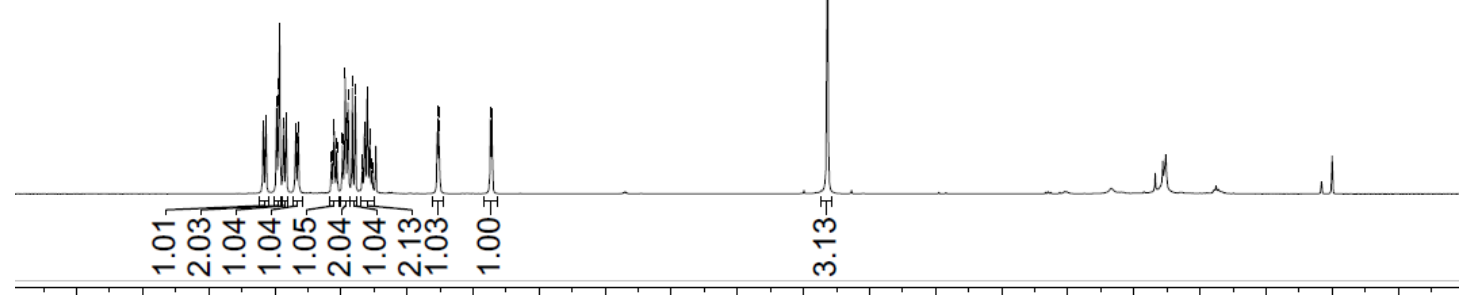

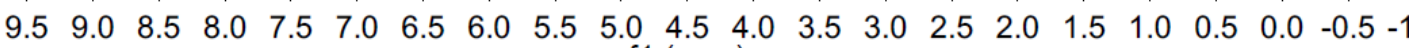
f1 (ppm) 


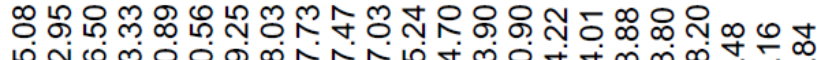

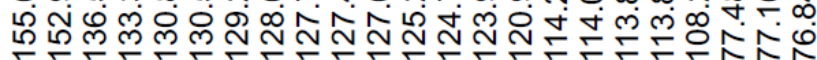

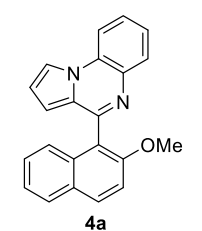

${ }^{13} \mathrm{C} \mathrm{NMR}\left(\mathrm{CDCl}_{3}, 100 \mathrm{MHz}\right)$

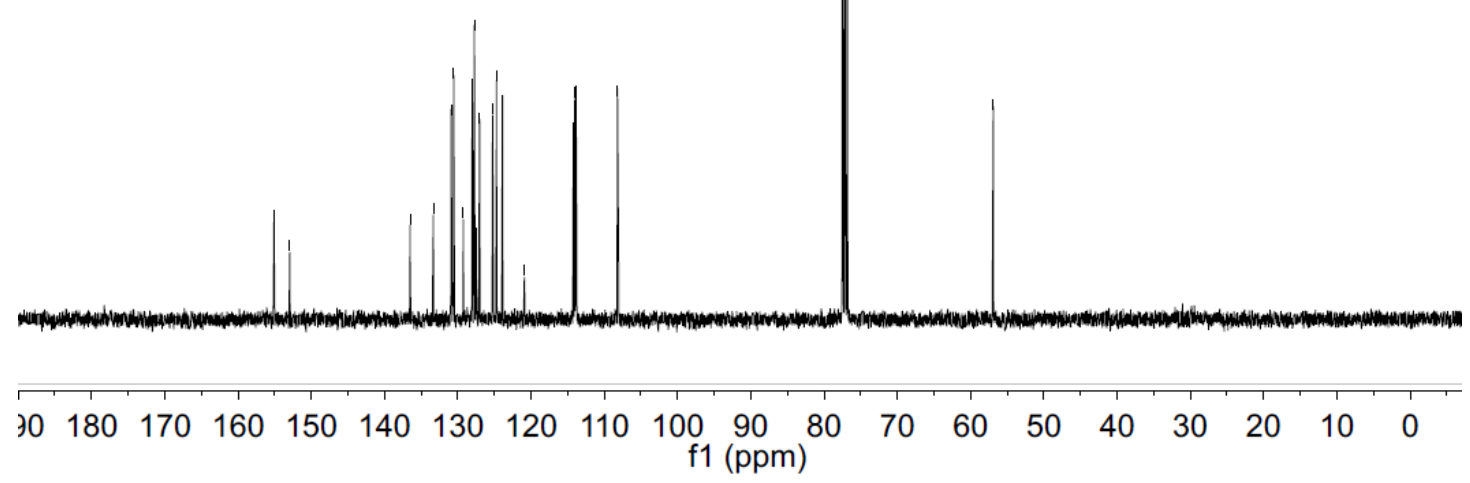

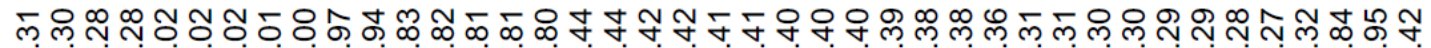

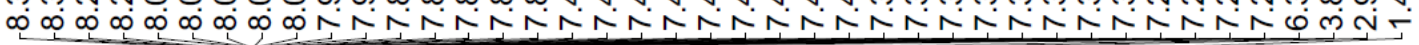

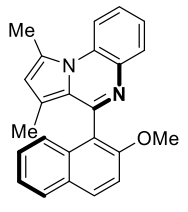

4b

${ }^{1} \mathrm{H}$ NMR $\left(\mathrm{CDCl}_{3}, 400 \mathrm{MHz}\right)$

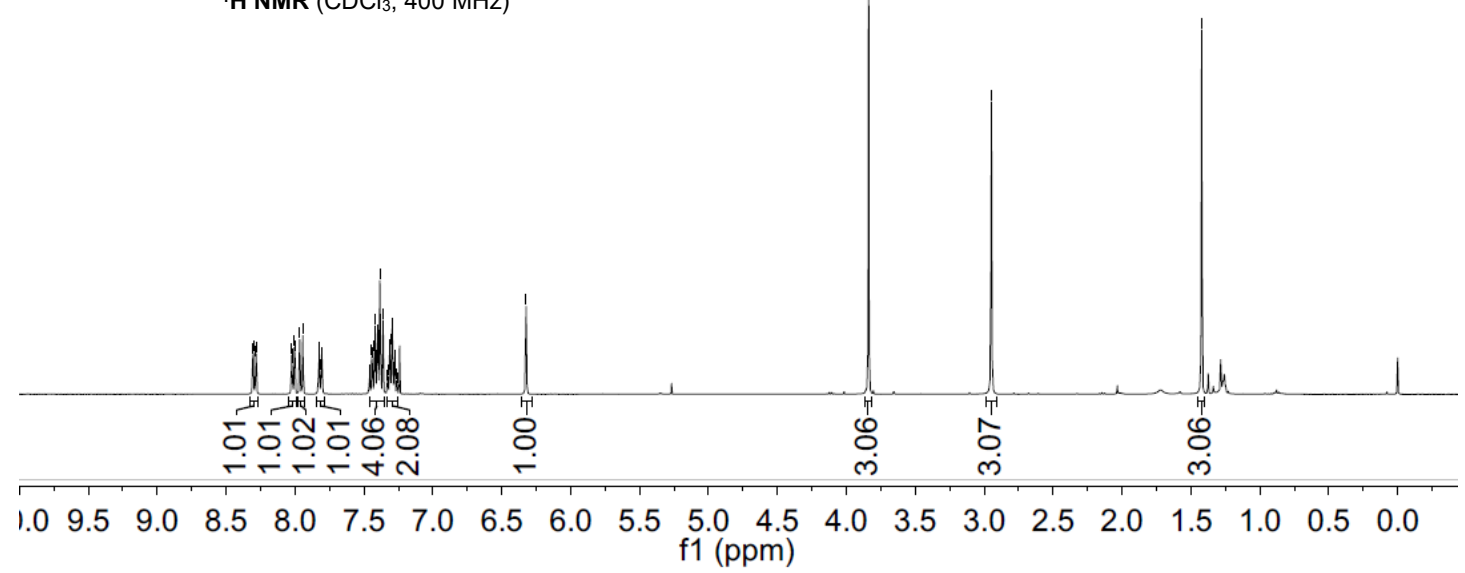




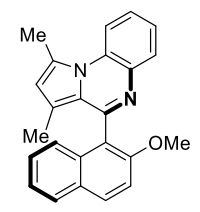

${ }^{13} \mathrm{C}$ NMR $\left(\mathrm{CDCl}_{3}, 100 \mathrm{MHz}\right)$

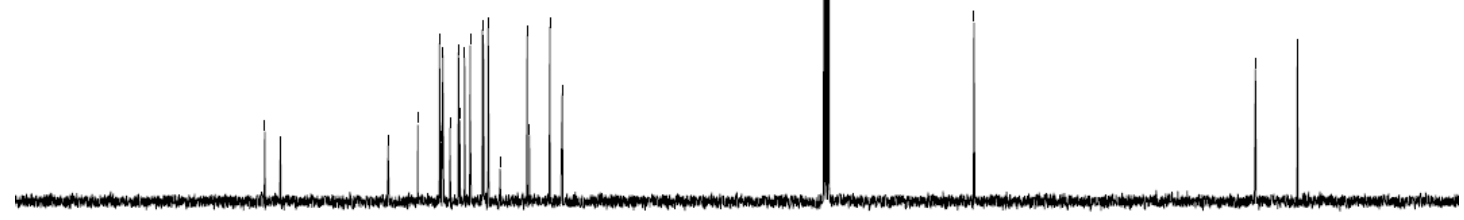

$\begin{array}{llllllllllllllllllll}180 & 170 & 160 & 150 & 140 & 130 & 120 & 110 & 100 & 90 & 80 & 70 & 60 & 50 & 40 & 30 & 20 & 10 & 0 & -1\end{array}$ $\mathrm{f1}(\mathrm{ppm})$

\&

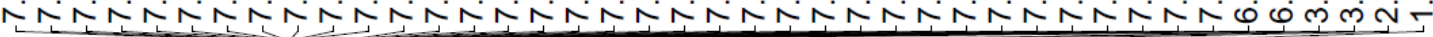

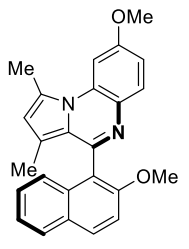

$4 \mathrm{c}$

${ }^{1} \mathrm{H}$ NMR $\left(\mathrm{CDCl}_{3}, 400 \mathrm{MHz}\right)$

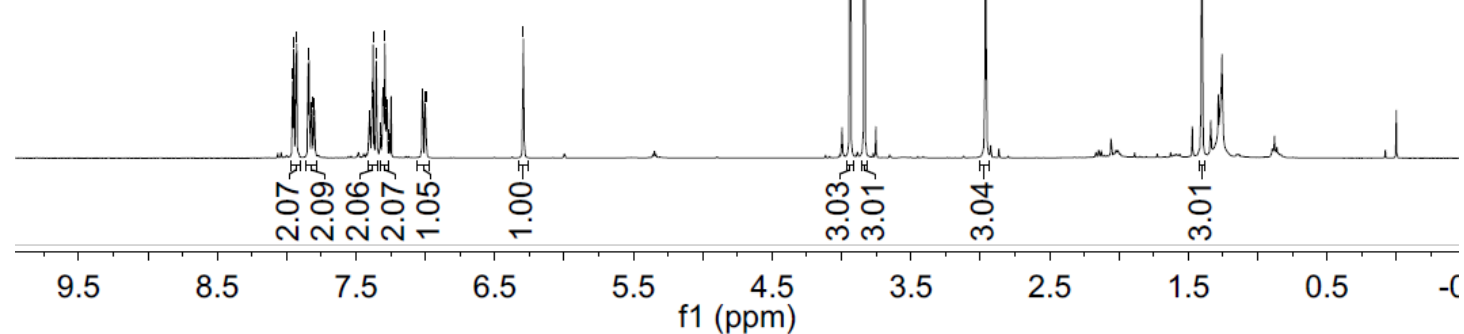


年定

i.

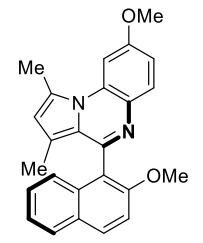

$4 \mathrm{c}$

${ }^{13} \mathrm{C}$ NMR $\left(\mathrm{CDCl}_{3}, 100 \mathrm{MHz}\right)$

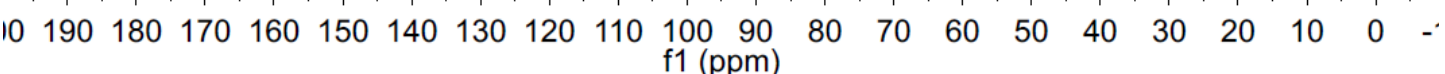

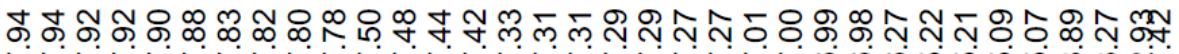

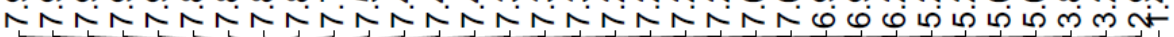

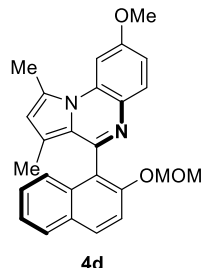

${ }^{1} \mathrm{H} \mathrm{NMR}\left(\mathrm{CDCl}_{3}, 400 \mathrm{MHz}\right)$

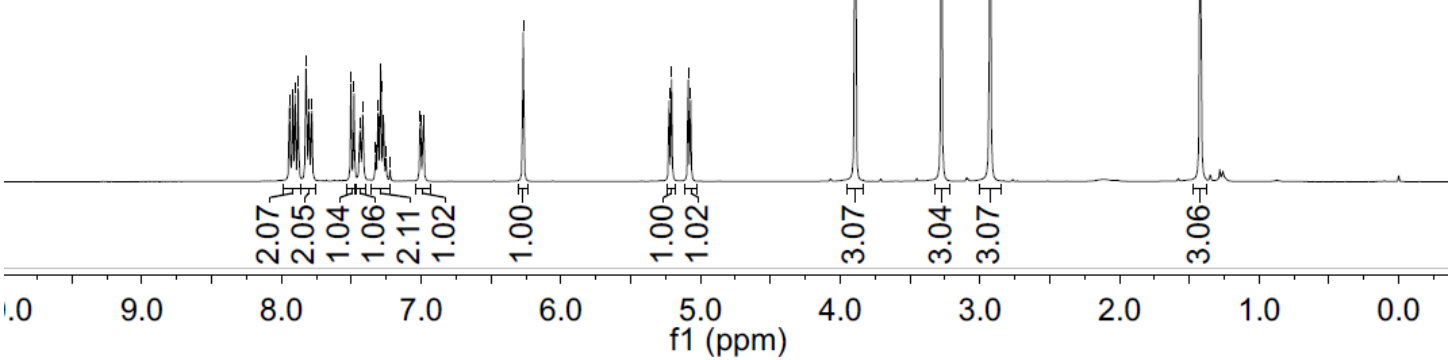




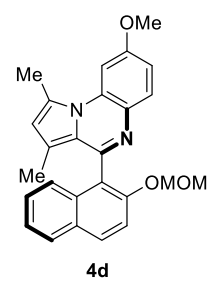

${ }^{13} \mathrm{C}$ NMR $\left(\mathrm{CDCl}_{3}, 100 \mathrm{MHz}\right)$

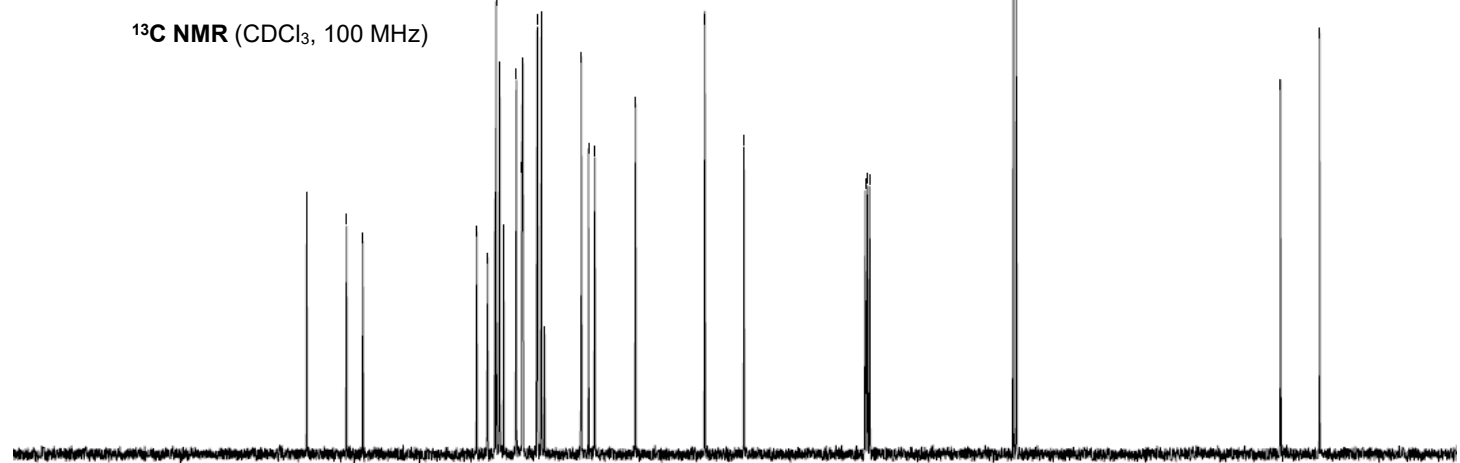

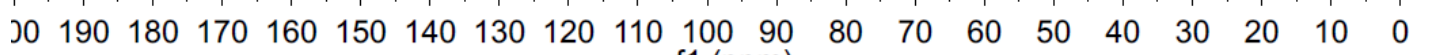

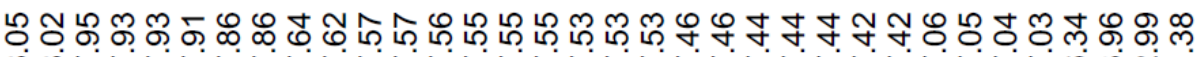

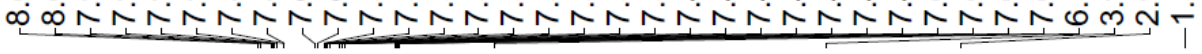

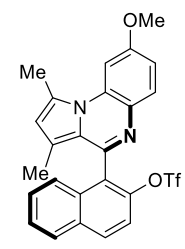

4 e

${ }^{1} \mathrm{H}$ NMR $\left(\mathrm{CDCl}_{3}, 400 \mathrm{MHz}\right)$

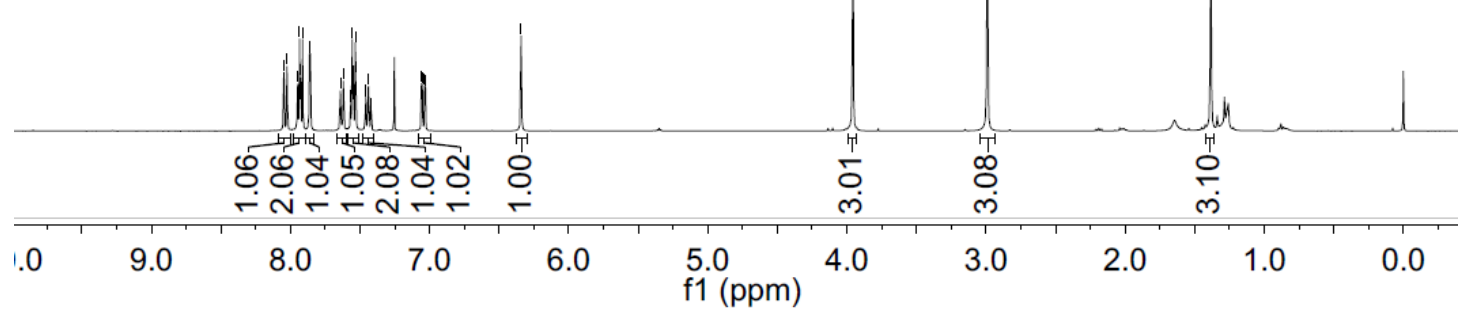




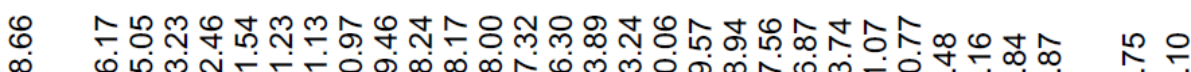

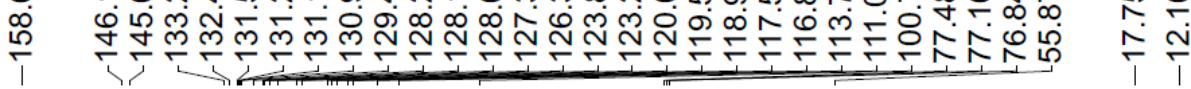

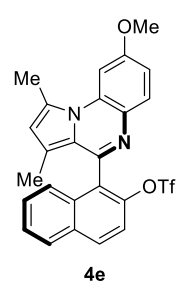

${ }^{13} \mathrm{C}$ NMR $\left(\mathrm{CDCl}_{3}, 100 \mathrm{MHz}\right)$
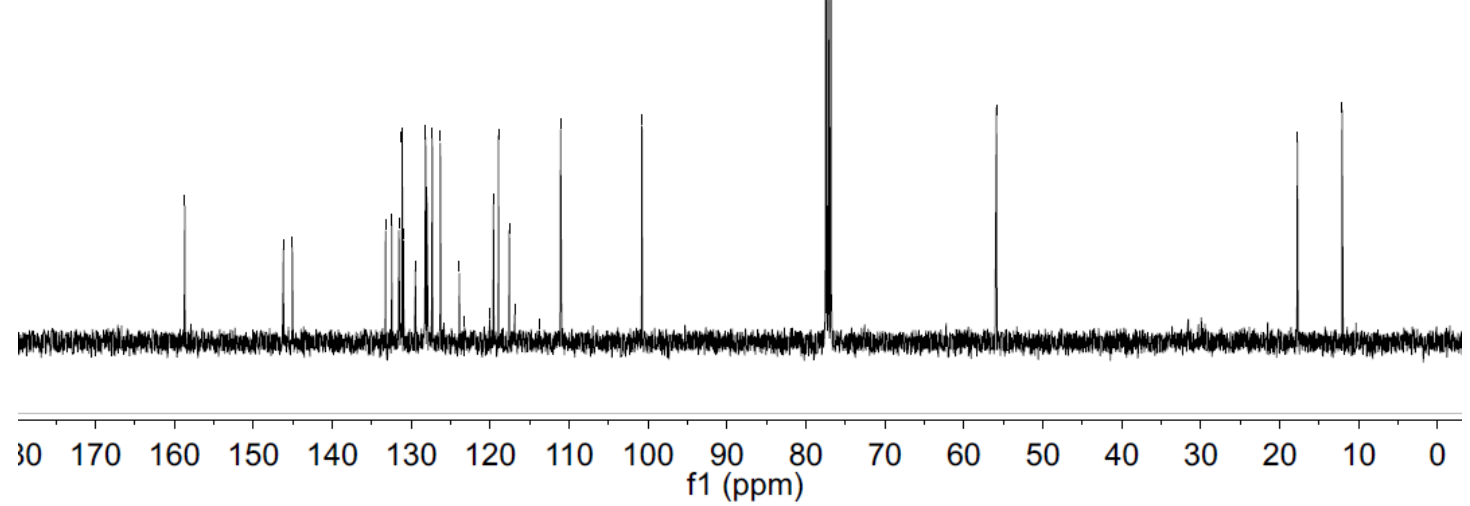

$\frac{5}{i}$

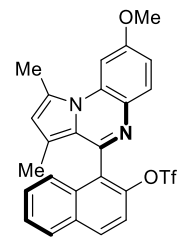

$4 \mathrm{e}$

${ }^{19} \mathrm{~F} \mathrm{NMR}\left(\mathrm{CDCl}_{3}, 377 \mathrm{MHz}\right)$

\begin{tabular}{lllllllllll|l|l|l|l|}
\hline 0 & 0 & -20 & -40 & -60 & -80 & -100 & -120 & -140 & -160 & -180 & -200 & $-2 ;$
\end{tabular}




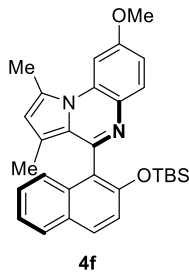

${ }^{1} \mathrm{H}$ NMR $\left(\mathrm{CDCl}_{3}, 400 \mathrm{MHz}\right)$

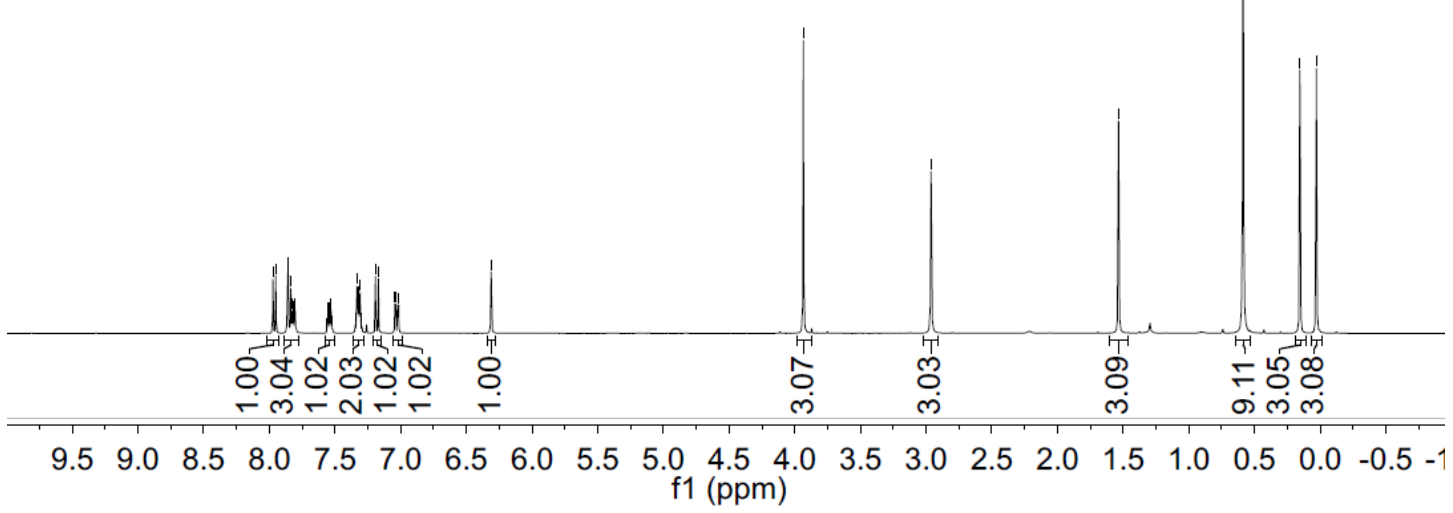

œ

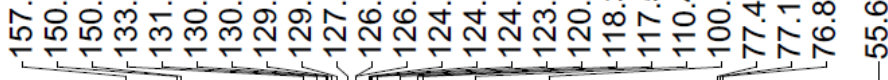

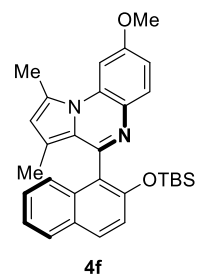

${ }^{13} \mathrm{C} \mathrm{NMR}\left(\mathrm{CDCl}_{3}, 100 \mathrm{MHz}\right)$

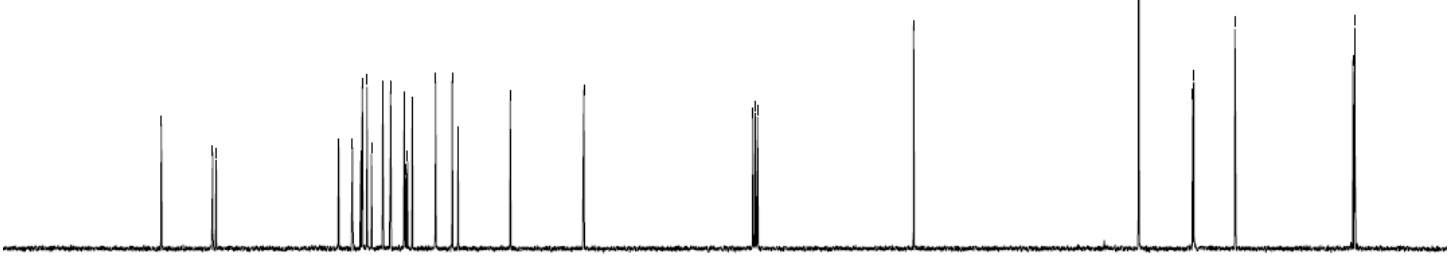

$\begin{array}{llllllllllllllllllll}10 & 170 & 160 & 150 & 140 & 130 & 120 & 110 & 100 & 90 & \underset{\mathrm{f} 1}{(\mathrm{ppm})} \mathrm{80} & 70 & 60 & 50 & 40 & 30 & 20 & 10 & 0 & -10\end{array}$ 
๑ొ

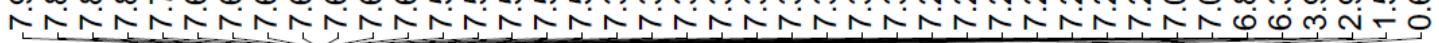

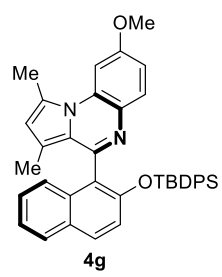

${ }^{1} \mathrm{H} \mathrm{NMR}\left(\mathrm{CDCl}_{3}, 400 \mathrm{MHz}\right)$

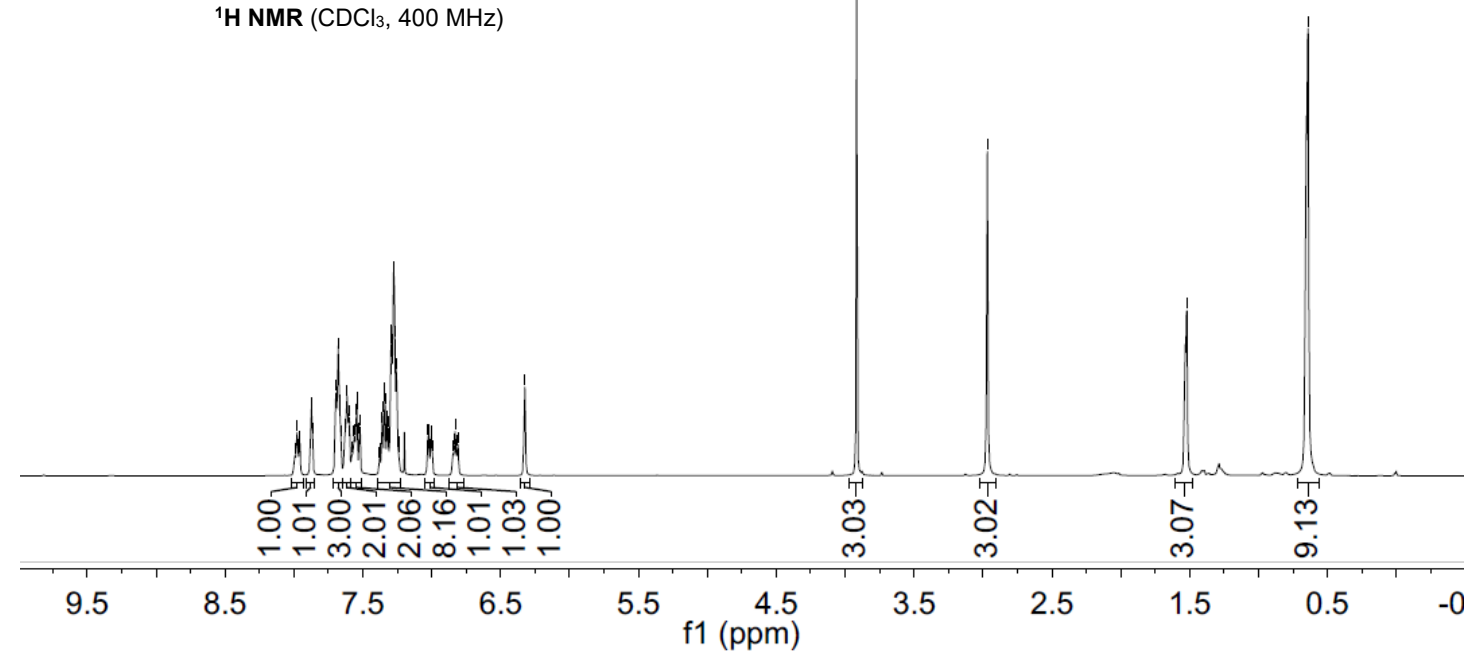

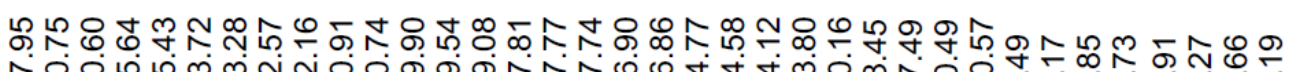
மุ

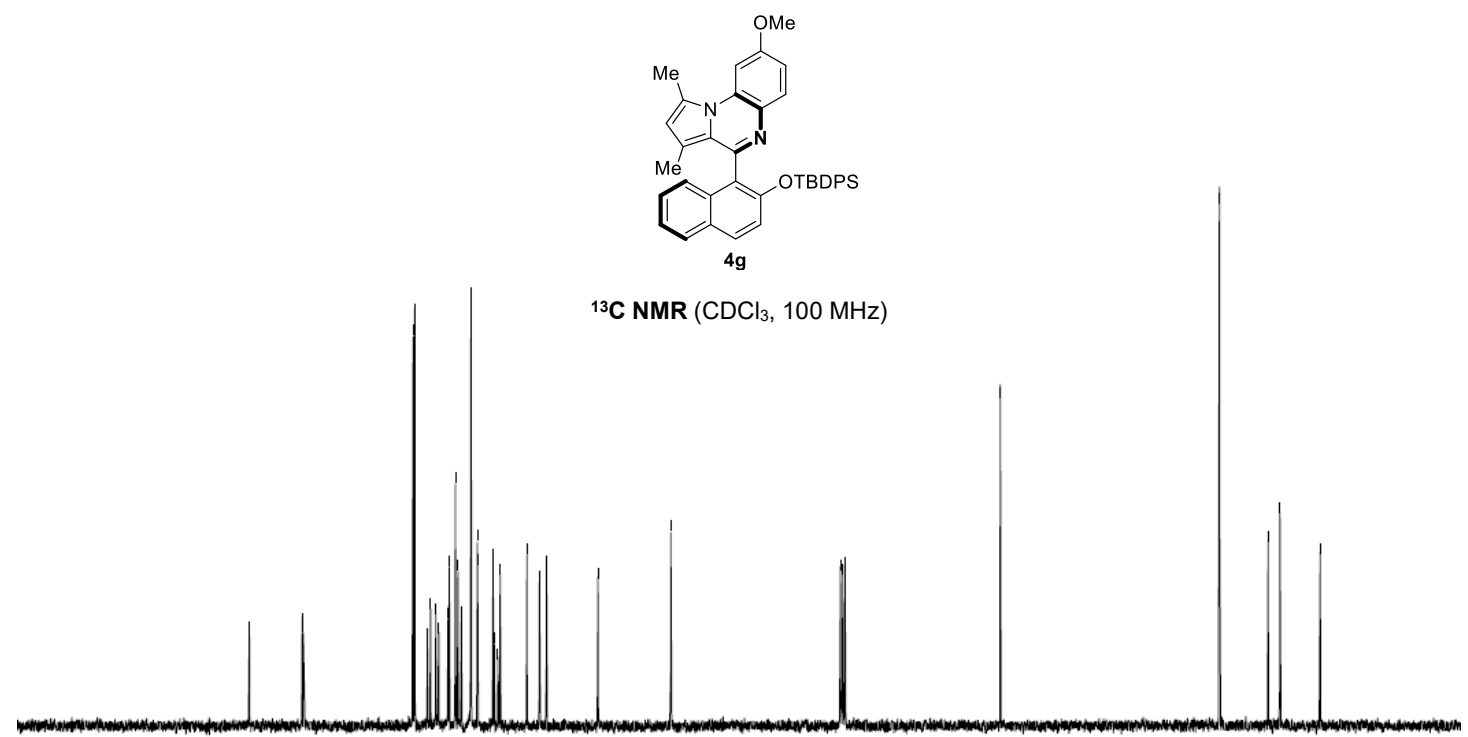

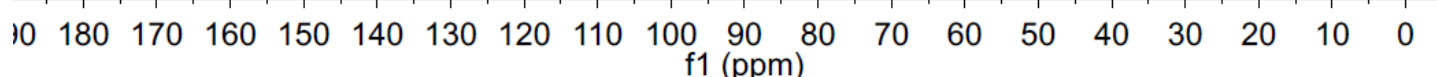




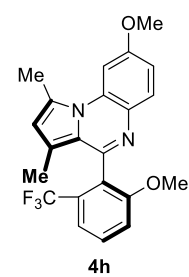

${ }^{1} \mathrm{H}$ NMR $\left(\mathrm{CDCl}_{3}, 400 \mathrm{MHz}\right)$

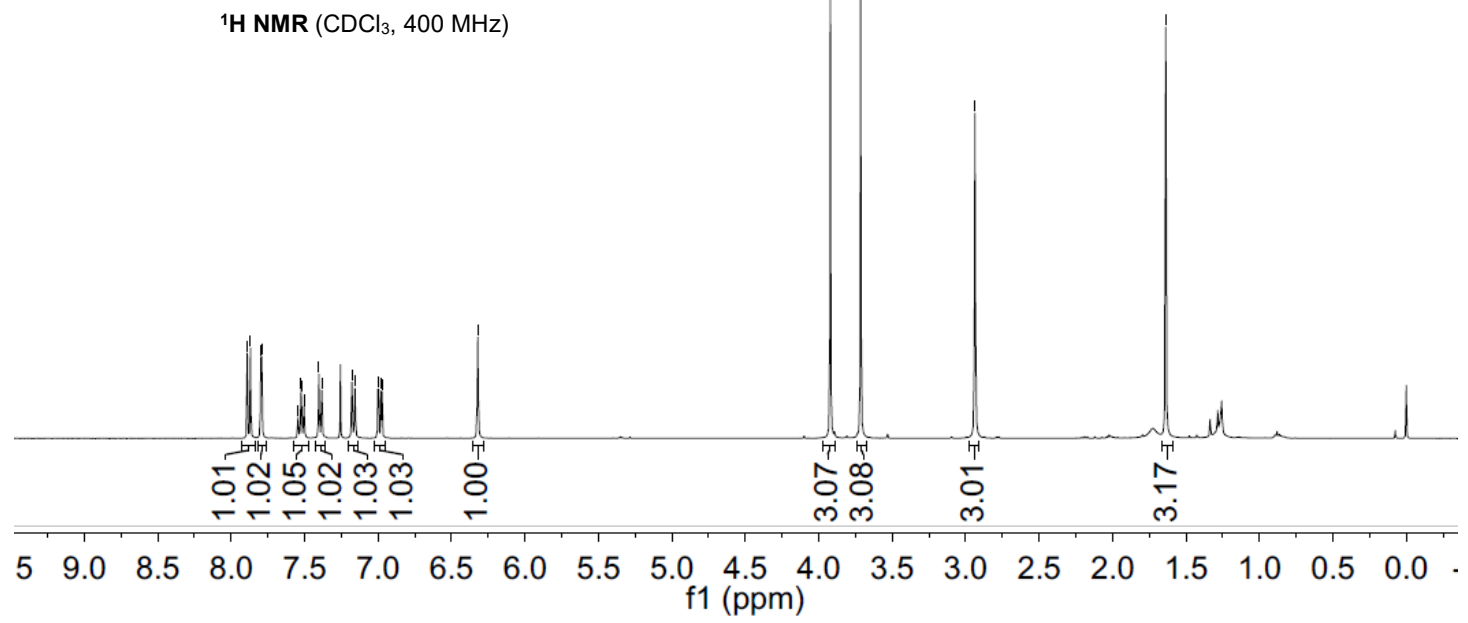

ㄴำ

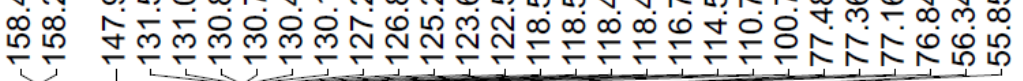

N

ㄷํㄴ

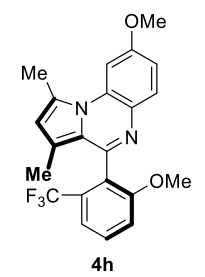

${ }^{13} \mathrm{C} \mathrm{NMR}\left(\mathrm{CDCl}_{3}, 100 \mathrm{MHz}\right)$

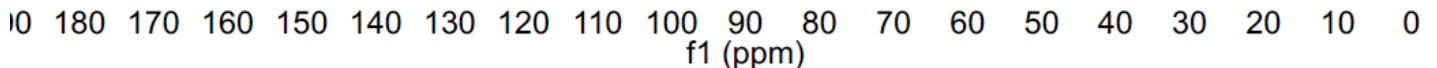




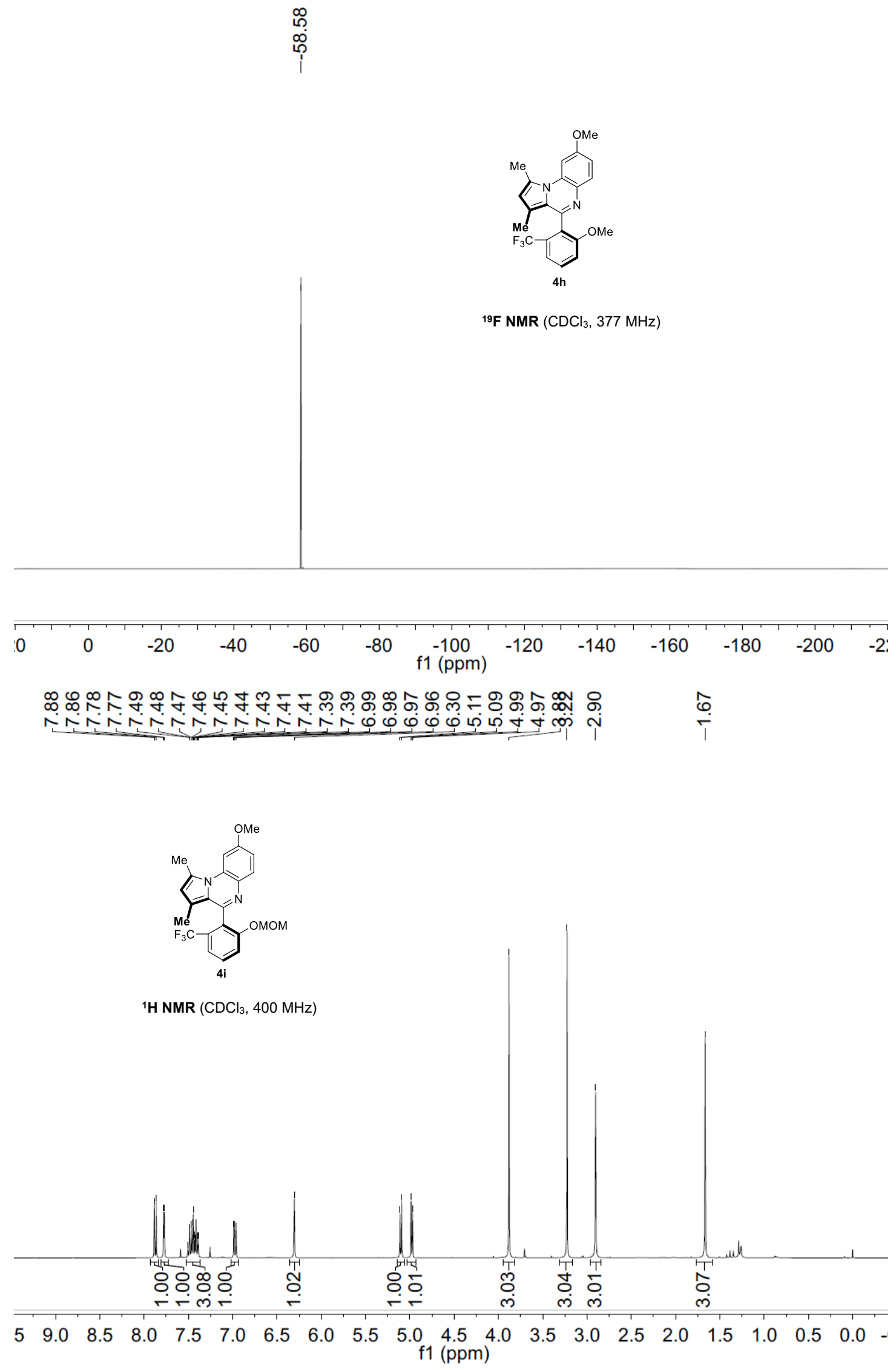




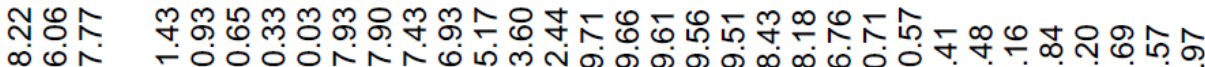

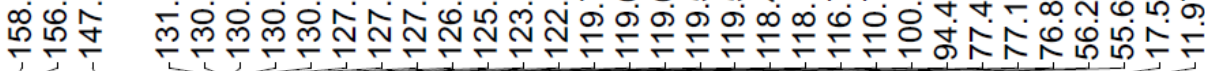

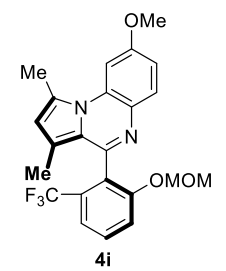

${ }^{13} \mathrm{C}$ NMR $\left(\mathrm{CDCl}_{3}, 100 \mathrm{MHz}\right)$

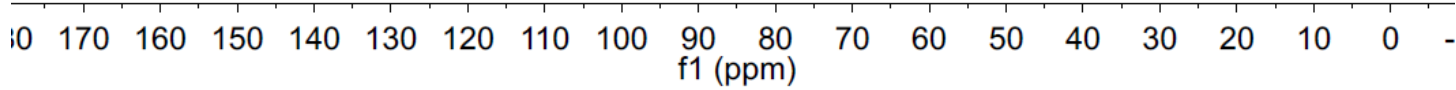

ஜ

i

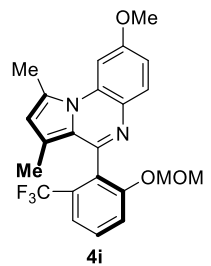

${ }^{19} \mathrm{~F} \mathrm{NMR}\left(\mathrm{CDCl}_{3}, 377 \mathrm{MHz}\right)$

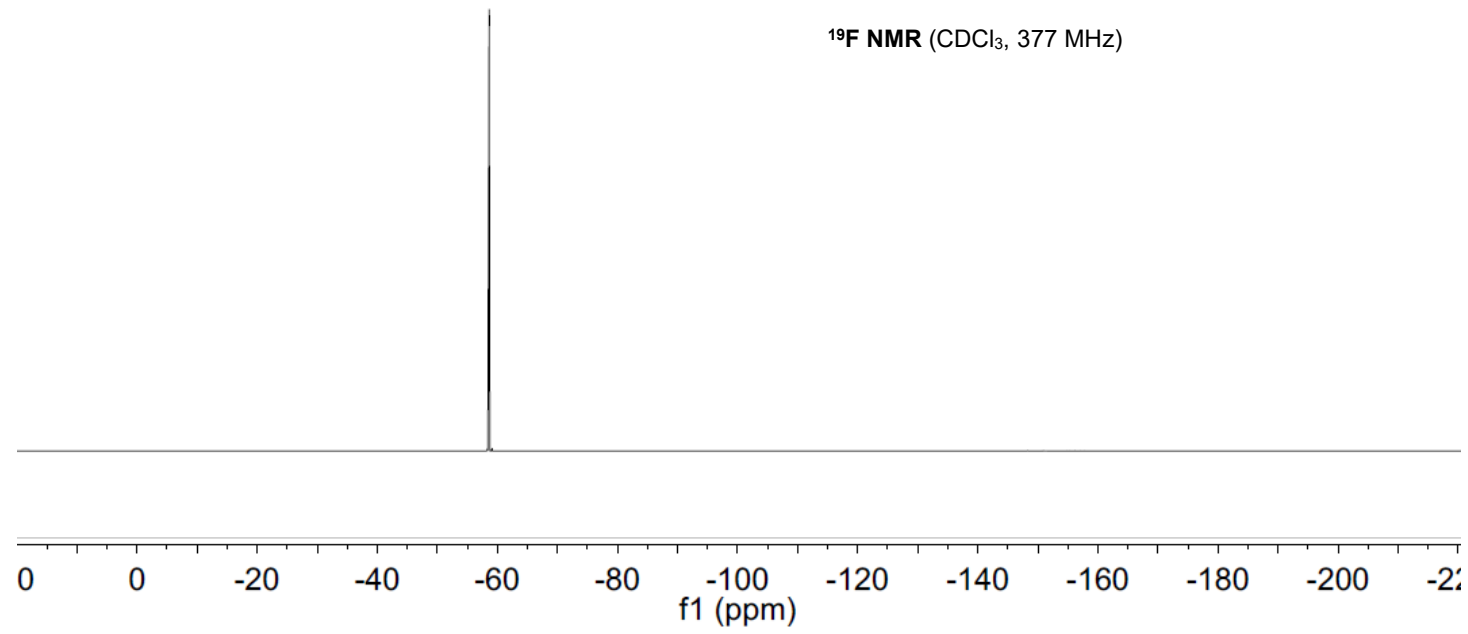


๒ 車

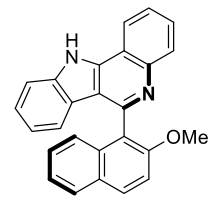

4k

${ }^{1} \mathrm{H}$ NMR ((DMSO- $\left.d_{6}, 400 \mathrm{MHz}\right)$

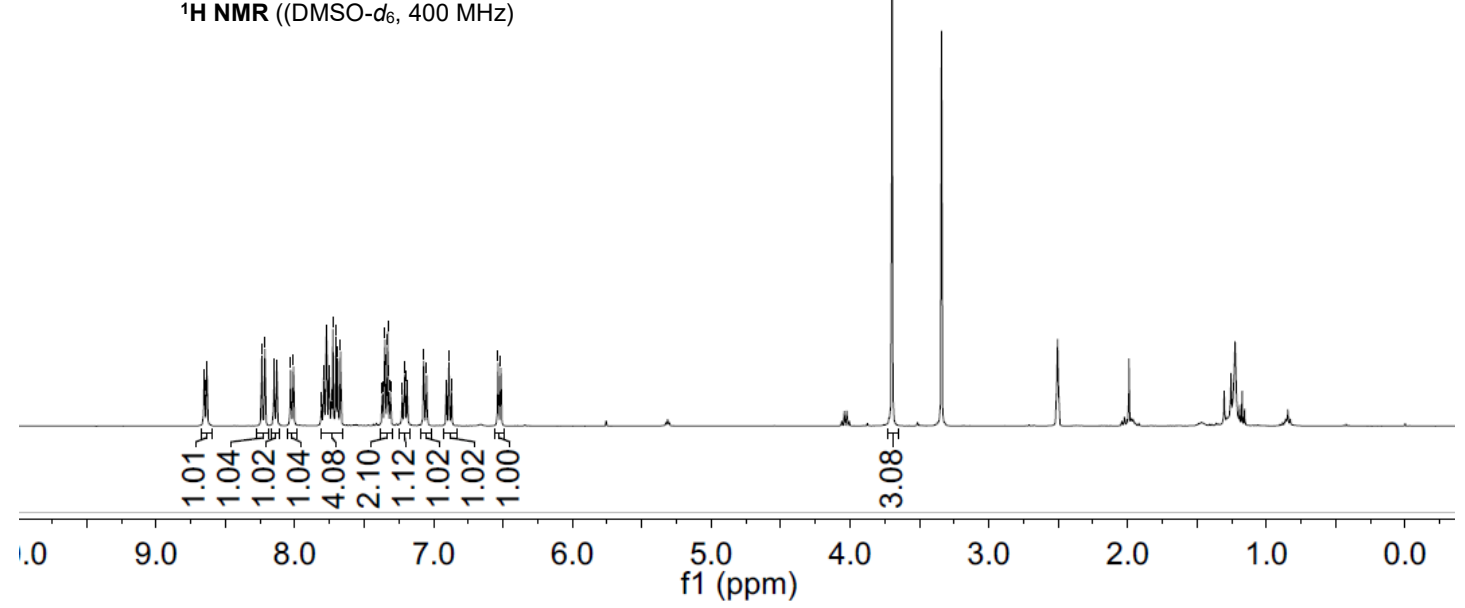

ำ ㅇำ 苔

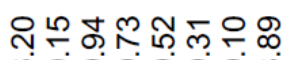

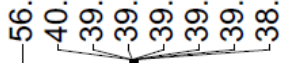

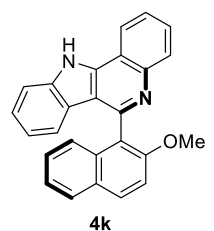

$4 \mathbf{k}$

${ }^{13} \mathrm{C}$ NMR (DMSO- $\left.d_{6}, 100 \mathrm{MHz}\right)$

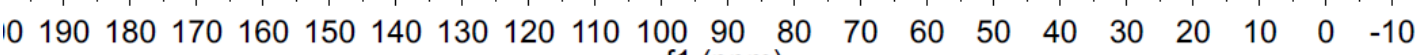
f1 (ppm) 


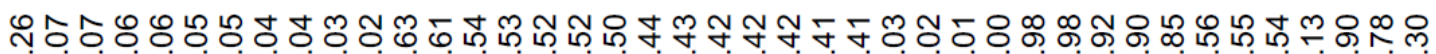

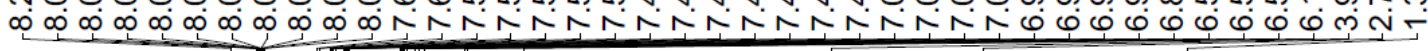

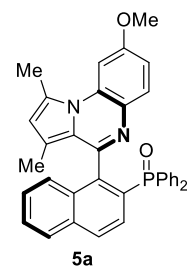

${ }^{1} \mathrm{H} \mathrm{NMR}\left(\mathrm{CDCl}_{3}, 400 \mathrm{MHz}\right)$

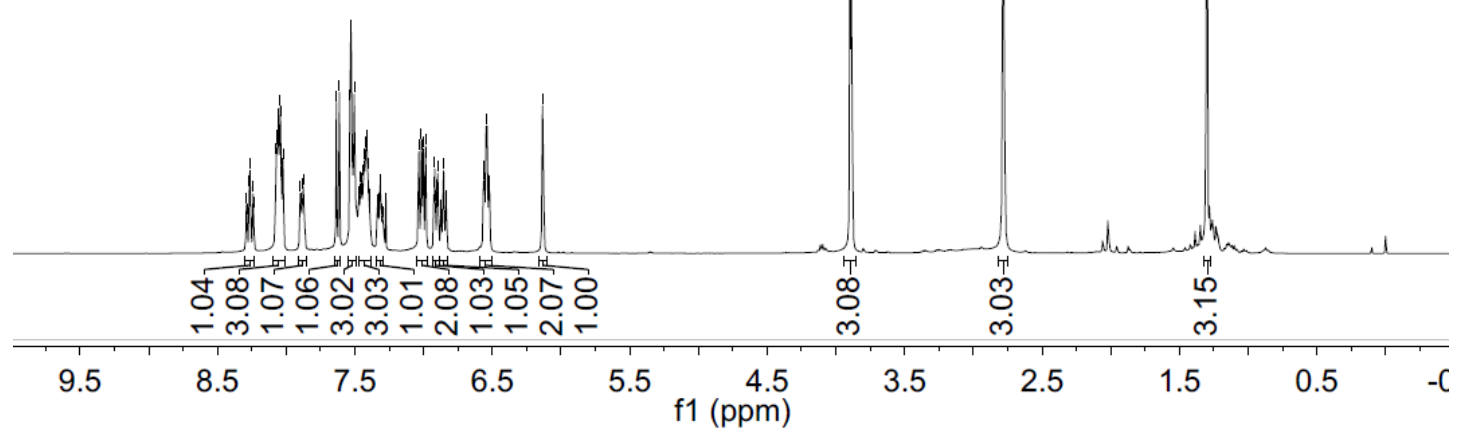

ใิ

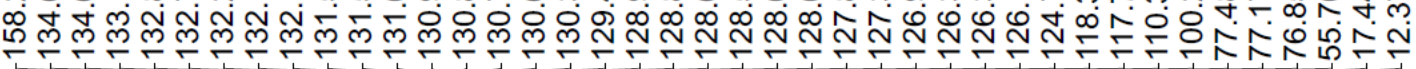

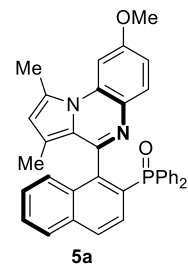

${ }^{13} \mathrm{C} \mathrm{NMR}\left(\mathrm{CDCl}_{3}, 100 \mathrm{MHz}\right)$

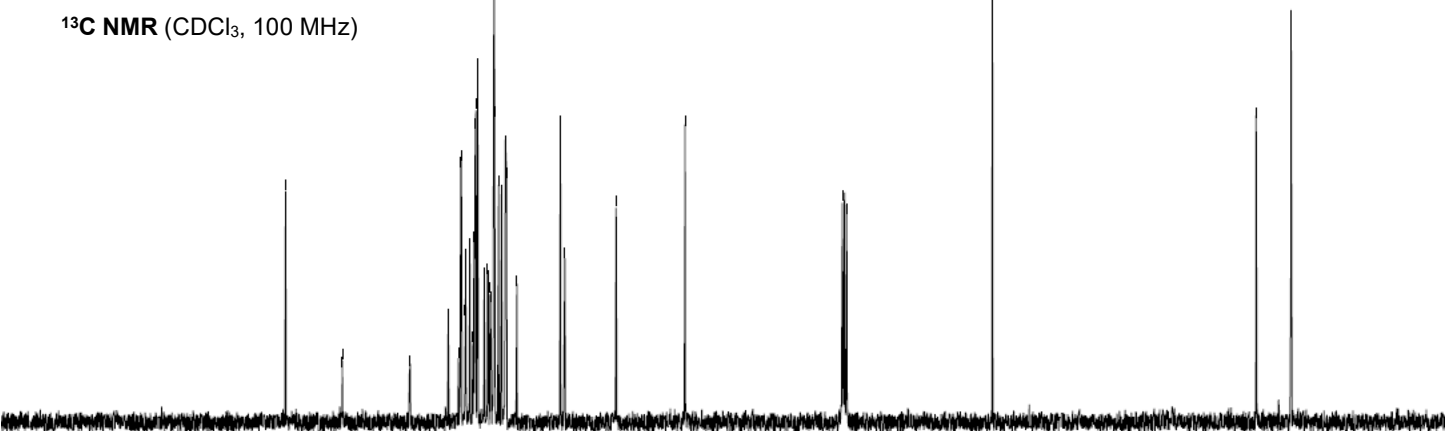

$\begin{array}{llllllllllllllllllllll}10 & 190 & 180 & 170 & 160 & 150 & 140 & 130 & 120 & 110 & \begin{array}{l}100 \\ \mathrm{f} 1(\mathrm{ppm})\end{array} & 80 & 70 & 60 & 50 & 40 & 30 & 20 & 10 & 0 & -1\end{array}$ 


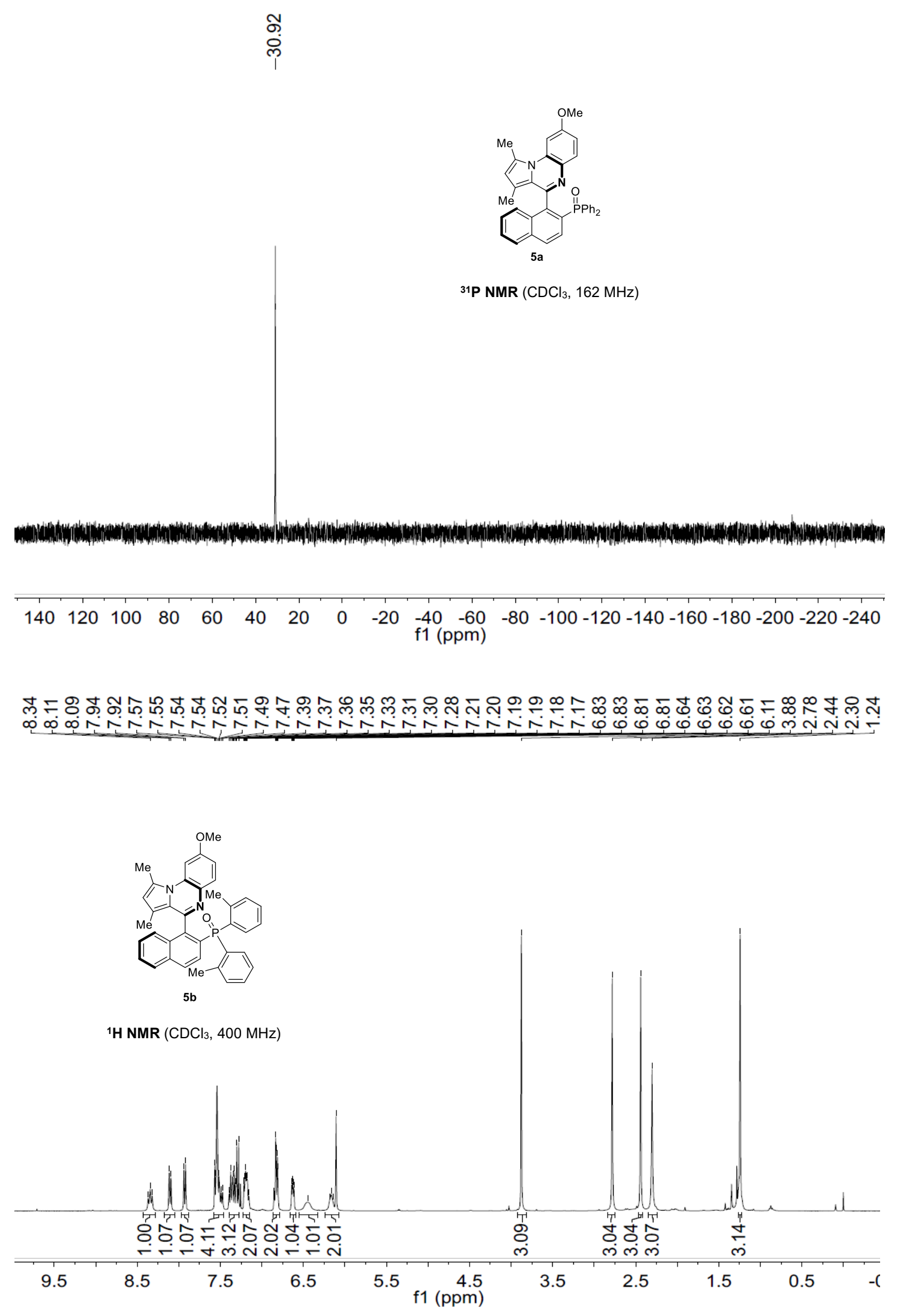




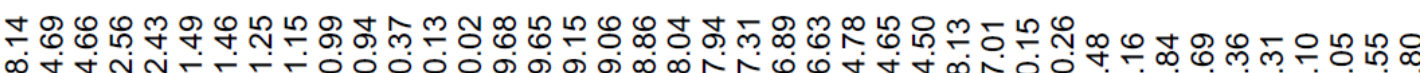

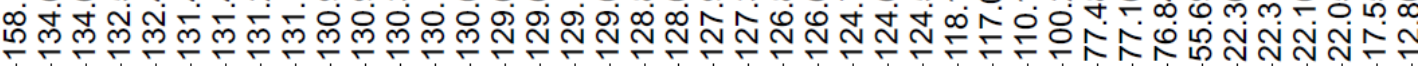

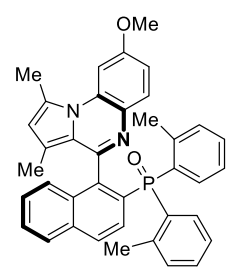

$5 \mathbf{b}$

${ }^{13} \mathrm{C} \mathrm{NMR}\left(\mathrm{CDCl}_{3}, 100 \mathrm{MHz}\right)$

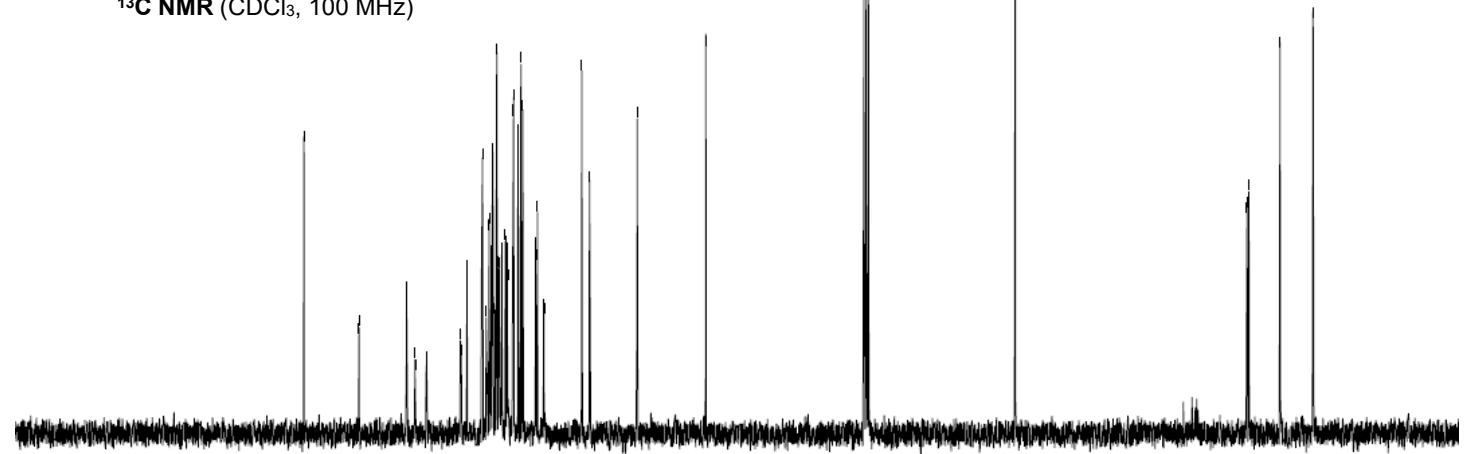

$10190180170160150140130120 \quad 110 \begin{array}{llllllllllll}100 & 90 & 80 & 70 & 60 & 50 & 40 & 30 & 20 & 10 & 0\end{array}$

ơ

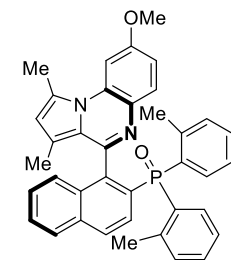

5b

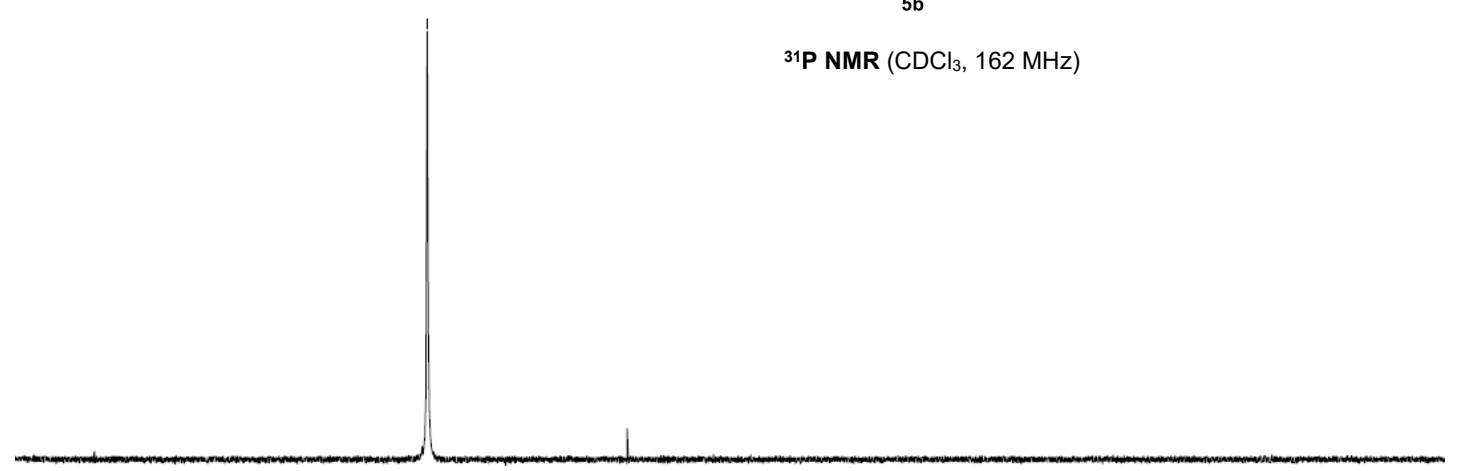

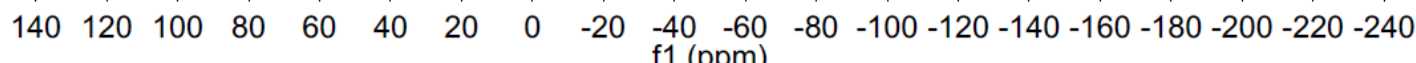


సิ సิ సุด

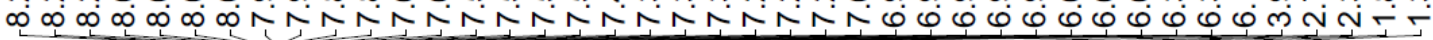

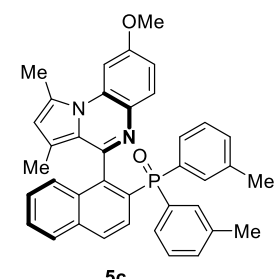

${ }^{1} \mathrm{H}$ NMR $\left(\mathrm{CDCl}_{3}, 400 \mathrm{MHz}\right)$

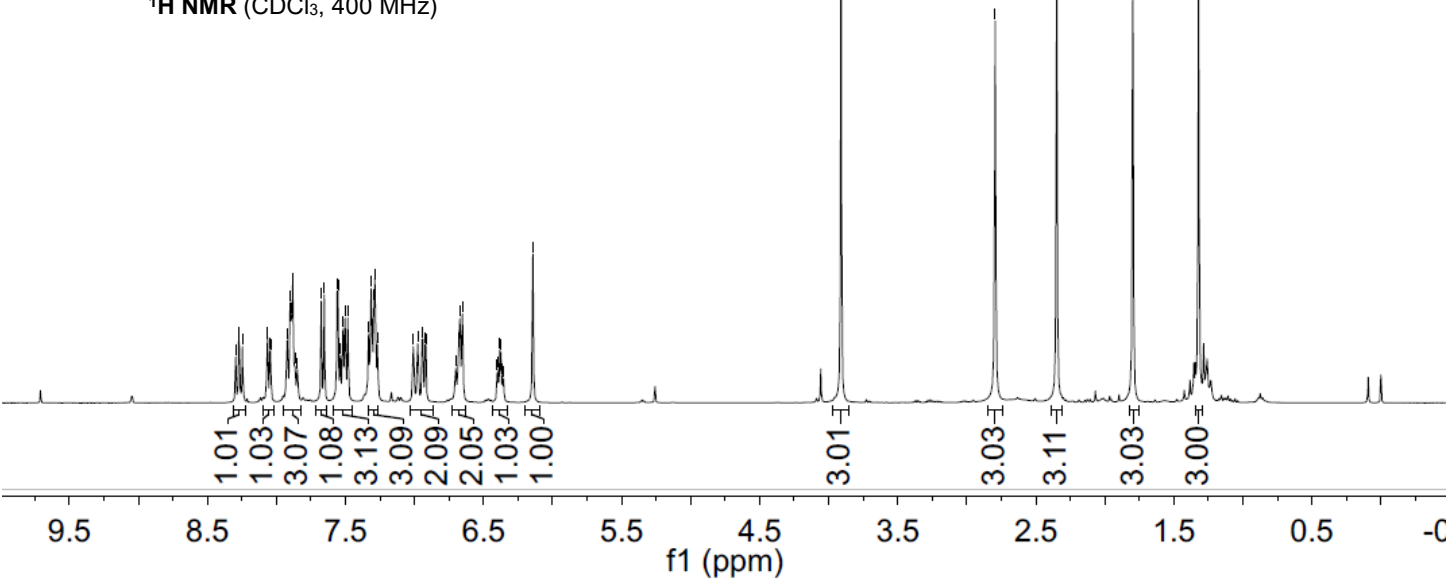

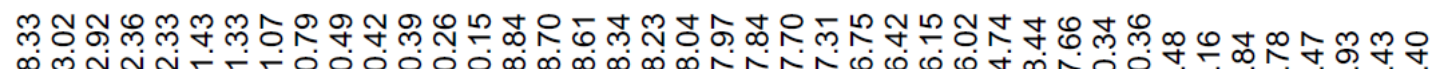
员

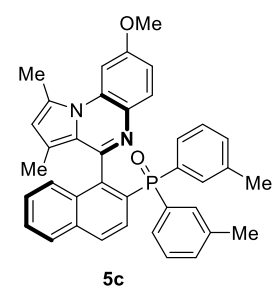

${ }^{13} \mathrm{C} \mathrm{NMR}\left(\mathrm{CDCl}_{3}, 100 \mathrm{MHz}\right)$

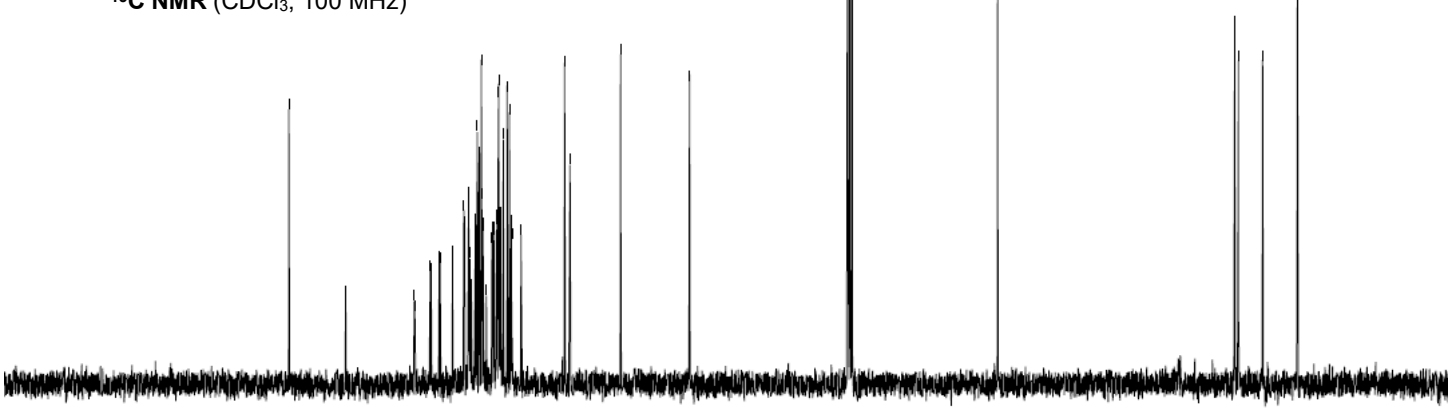

$\begin{array}{lllllllllllllllllllll}10 & 190 & 180 & 170 & 160 & 150 & 140 & 130 & 120 & 110 & \begin{array}{l}100 \\ \mathrm{f} 1(\mathrm{ppm})\end{array} & 80 & 70 & 60 & 50 & 40 & 30 & 20 & 10 & 0 & -\end{array}$ 


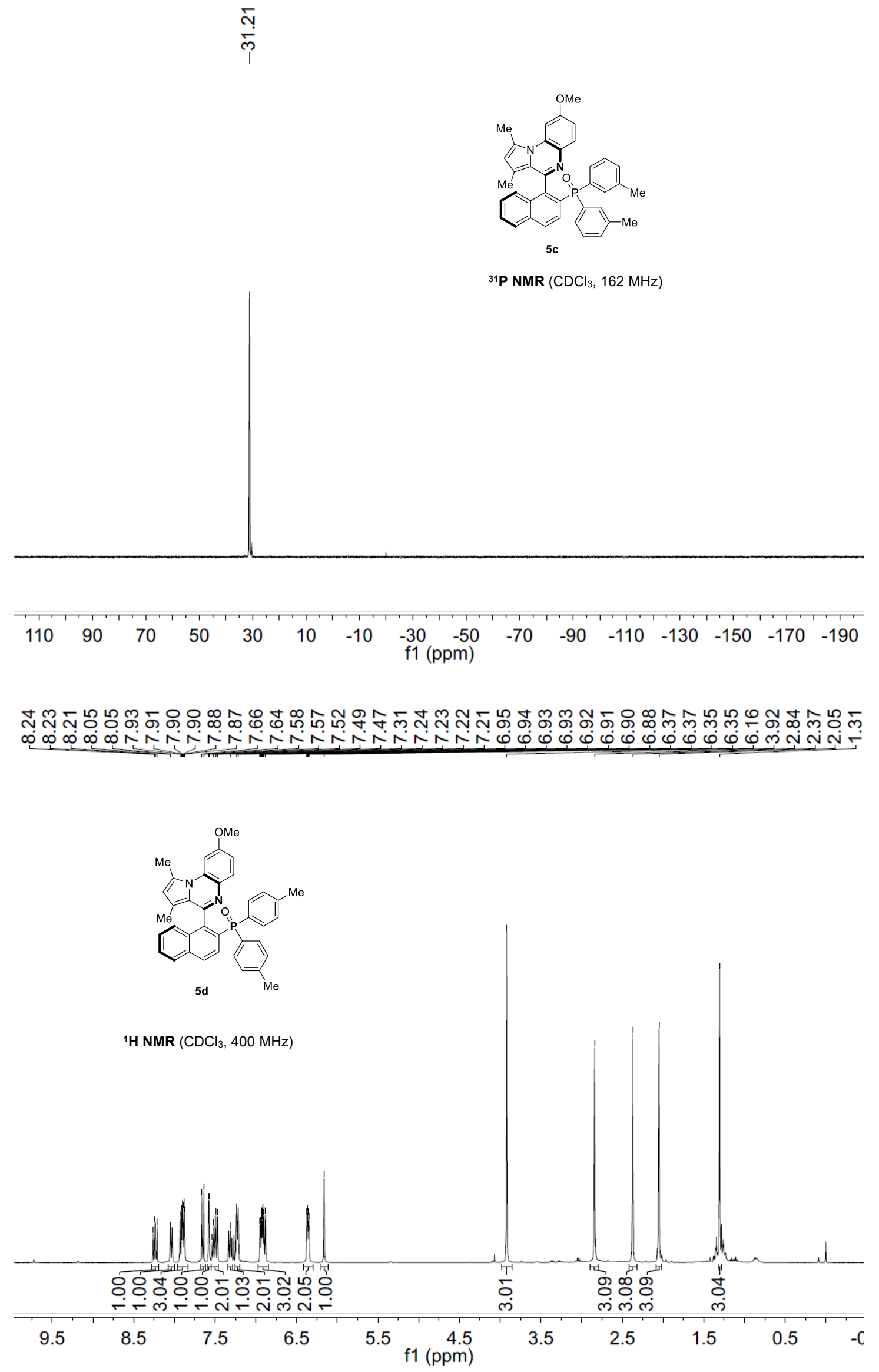


సุ

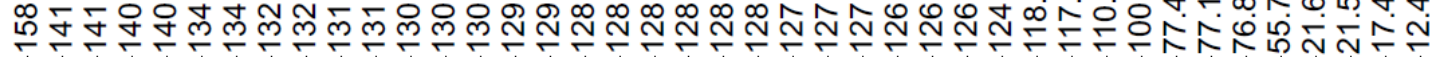

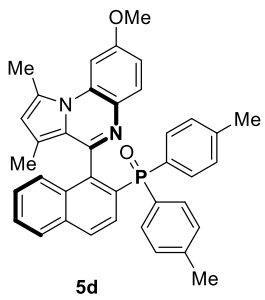

${ }^{13} \mathrm{C}$ NMR $\left(\mathrm{CDCl}_{3}, 100 \mathrm{MHz}\right)$

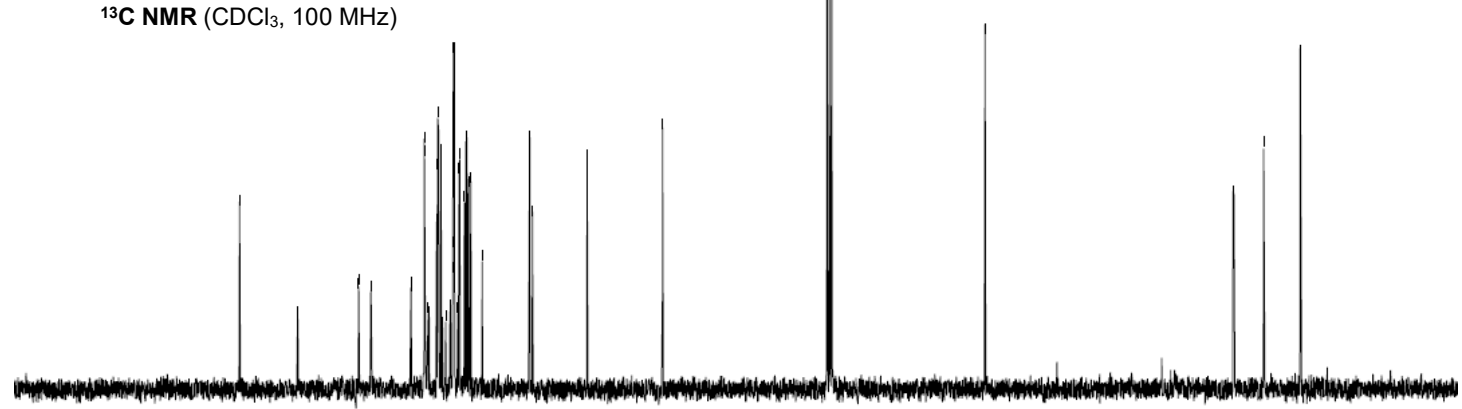

$\begin{array}{lllllllllllllllllllll}10 & 180 & 170 & 160 & 150 & 140 & 130 & 120 & 110 & 100 & \begin{array}{c}90 \\ \mathrm{f} 1\end{array}(\mathrm{ppm}) & 80 & 70 & 60 & 50 & 40 & 30 & 20 & 10 & 0 & -\end{array}$

م.

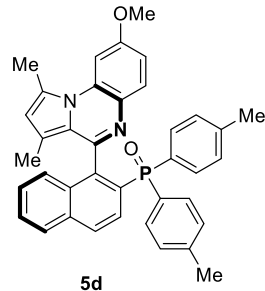

${ }^{31} \mathrm{P}$ NMR $\left(\mathrm{CDCl}_{3}, 162 \mathrm{MHz}\right)$

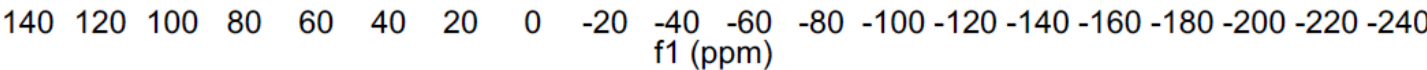


లై 유 \&

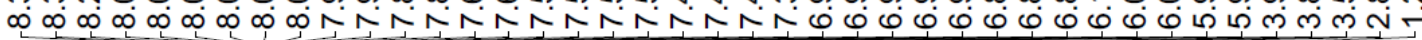

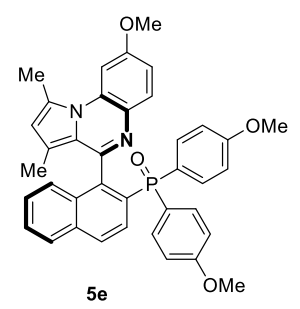

${ }^{1} \mathrm{H} \mathrm{NMR}\left(\mathrm{CDCl}_{3}, 400 \mathrm{MHz}\right)$

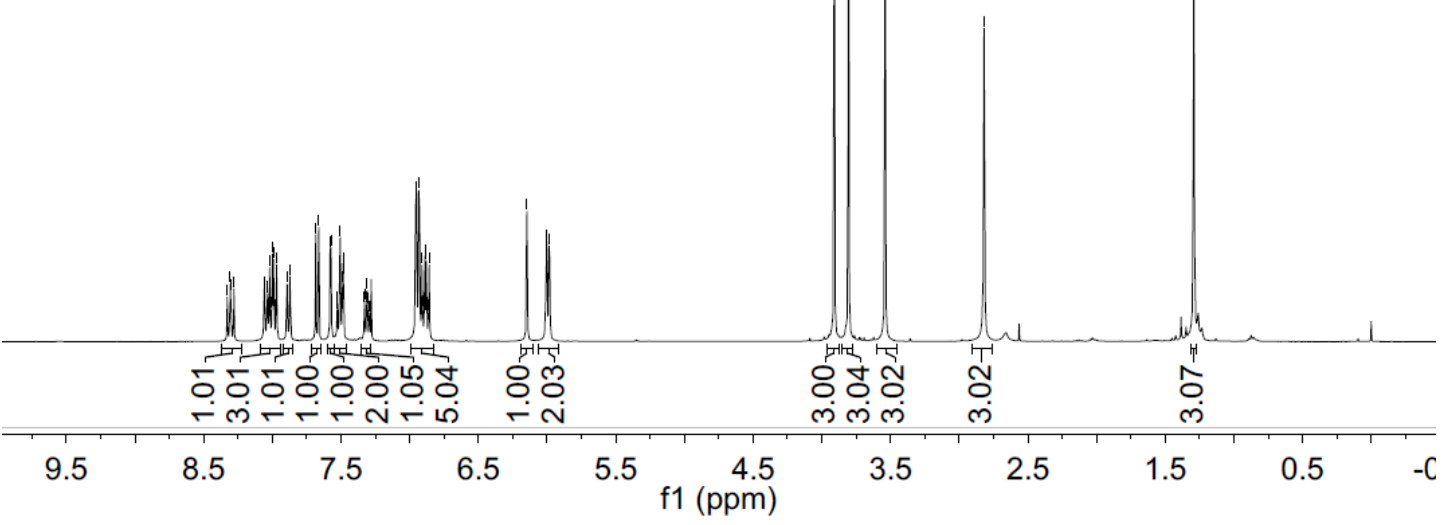

은

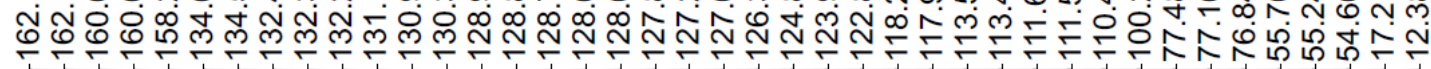

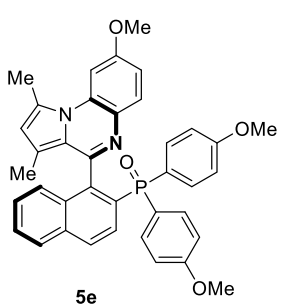

${ }^{13} \mathrm{C} \mathrm{NMR}\left(\mathrm{CDCl}_{3}, 100 \mathrm{MHz}\right)$

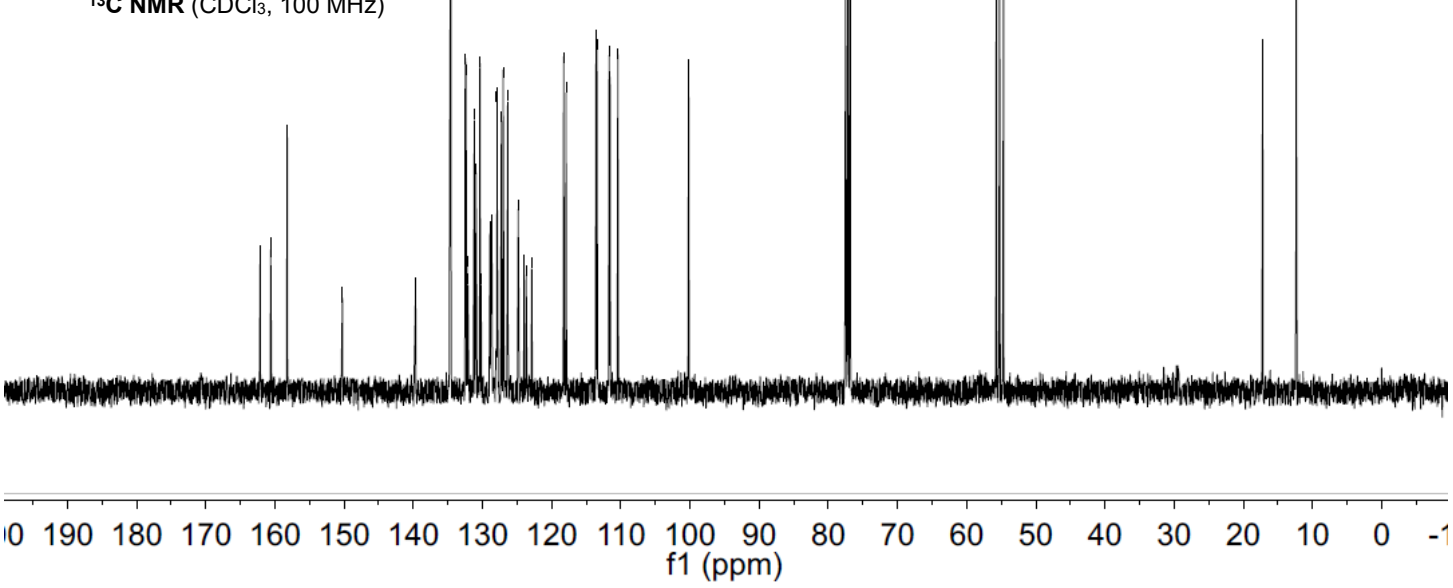


๖̊.

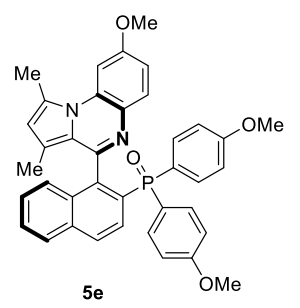

${ }^{31} \mathrm{P}$ NMR $\left(\mathrm{CDCl}_{3}, 162 \mathrm{MHz}\right)$

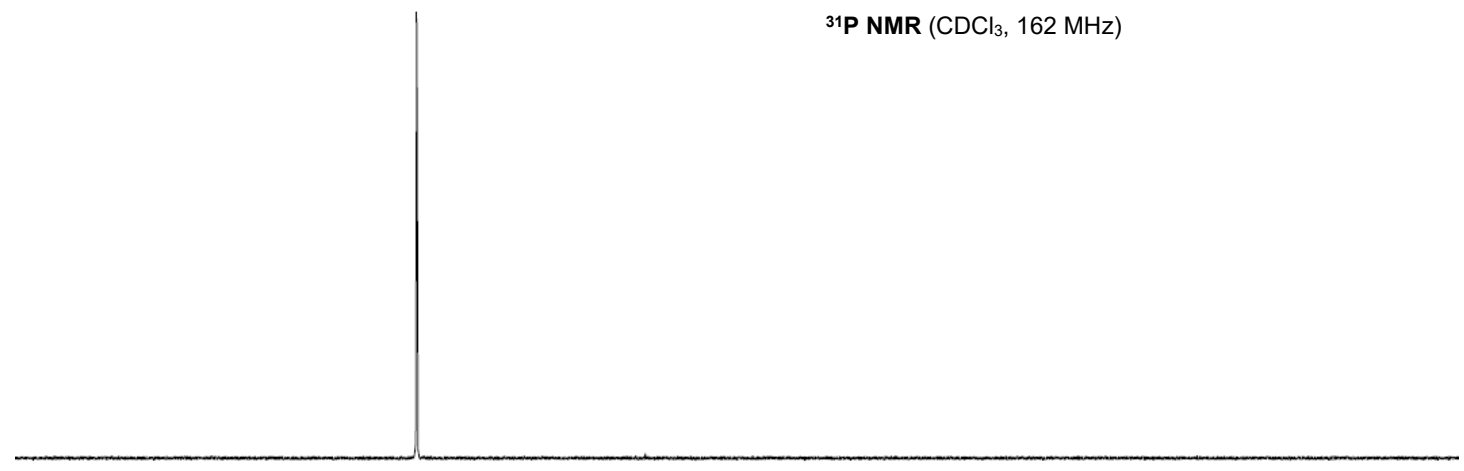

$\begin{array}{lllllllllllllllll}110 & 90 & 70 & 50 & 30 & 10 & -10 & \begin{array}{c}-30 \\ \mathrm{f} 1(\mathrm{ppm})\end{array} & -50 & -70 & -90 & -110 & -130 & -150 & -170 & -190\end{array}$

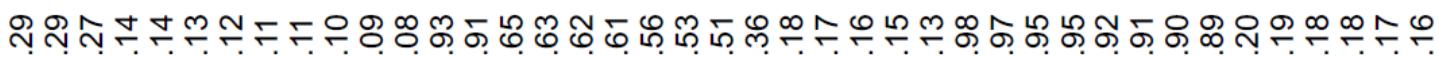

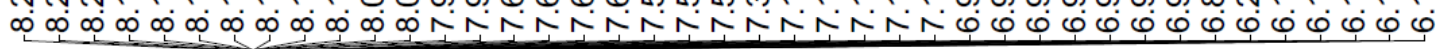

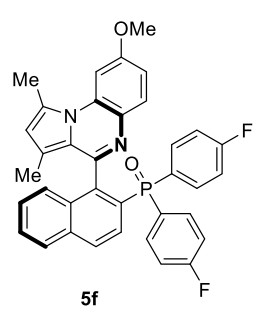

${ }^{1} \mathrm{H}$ NMR $\left(\mathrm{CDCl}_{3}, 400 \mathrm{MHz}\right)$

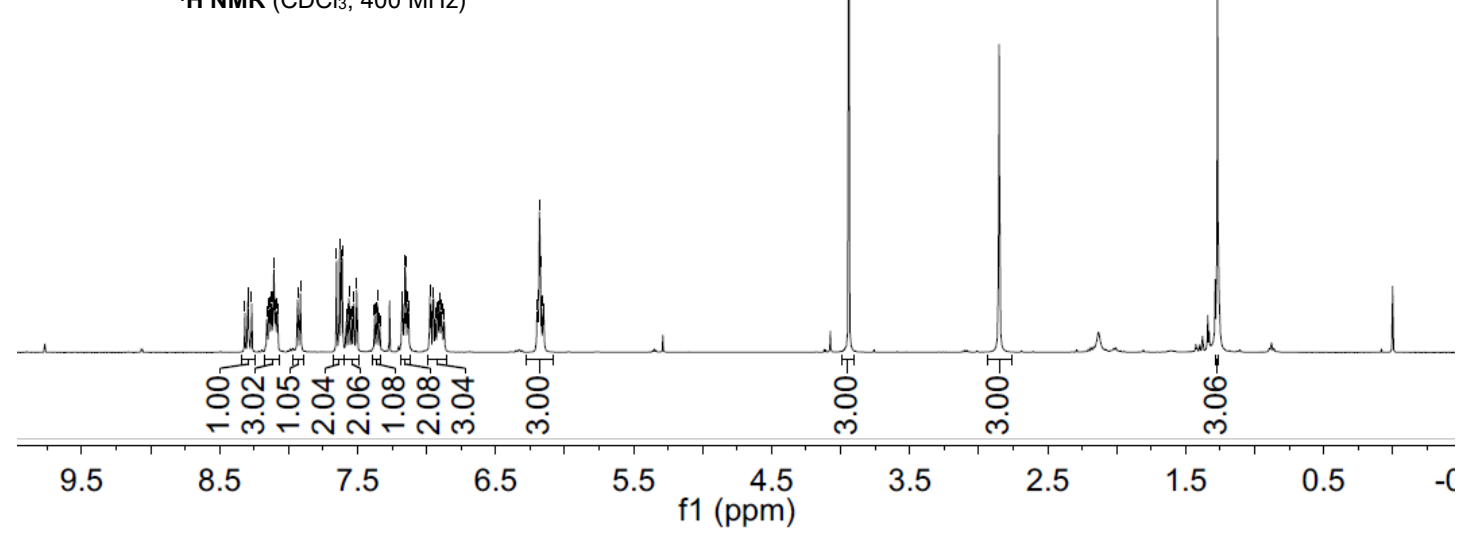




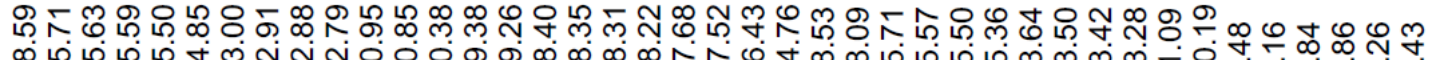
负

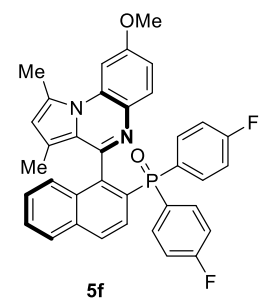

${ }^{13} \mathrm{C} \mathrm{NMR}\left(\mathrm{CDCl}_{3}, 100 \mathrm{MHz}\right)$

$\begin{array}{lllllllllllllllllllllllll}0 & 190 & 180 & 170 & 160 & 150 & 140 & 130 & 120 & 110 & \begin{array}{l}100 \\ \mathrm{f} 1(\mathrm{ppm})\end{array} & 80 & 70 & 60 & 50 & 40 & 30 & 20 & 10 & 0 & -1\end{array}$ ธิ กำ 동요

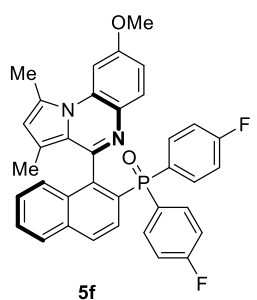

${ }^{19} \mathrm{~F} \mathrm{NMR}\left(\mathrm{CDCl}_{3}, 377 \mathrm{MHz}\right)$

\begin{tabular}{|c|c|c|c|c|c|c|c|c|}
\hline-20 & -40 & -60 & -80 & $\begin{array}{c}-100 \\
\mathrm{f} 1(\mathrm{ppm})\end{array}$ & -120 & -140 & -160 & -180 \\
\hline
\end{tabular}



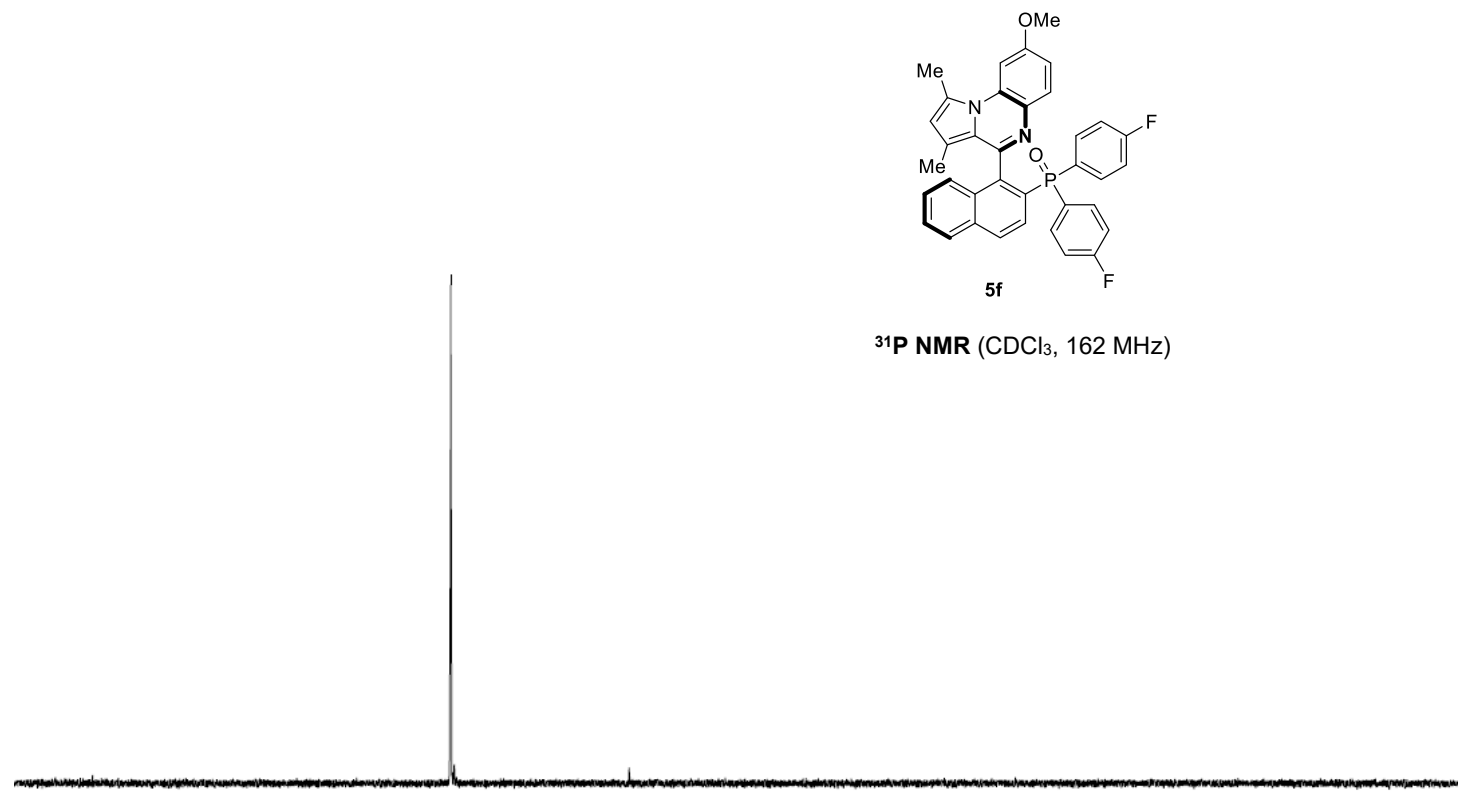

${ }^{31} \mathrm{P}$ NMR $\left(\mathrm{CDCl}_{3}, 162 \mathrm{MHz}\right)$

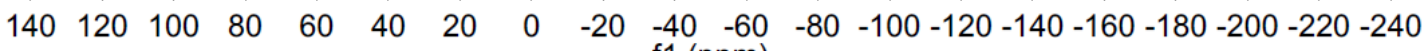
f1 (ppm)

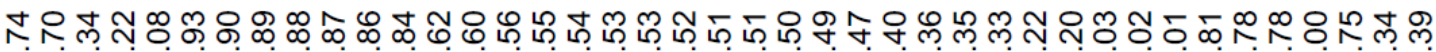
更

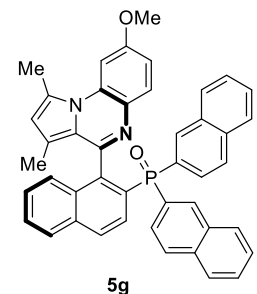

${ }^{1} \mathrm{H}$ NMR (CDCl $\left.3,400 \mathrm{MHz}\right)$

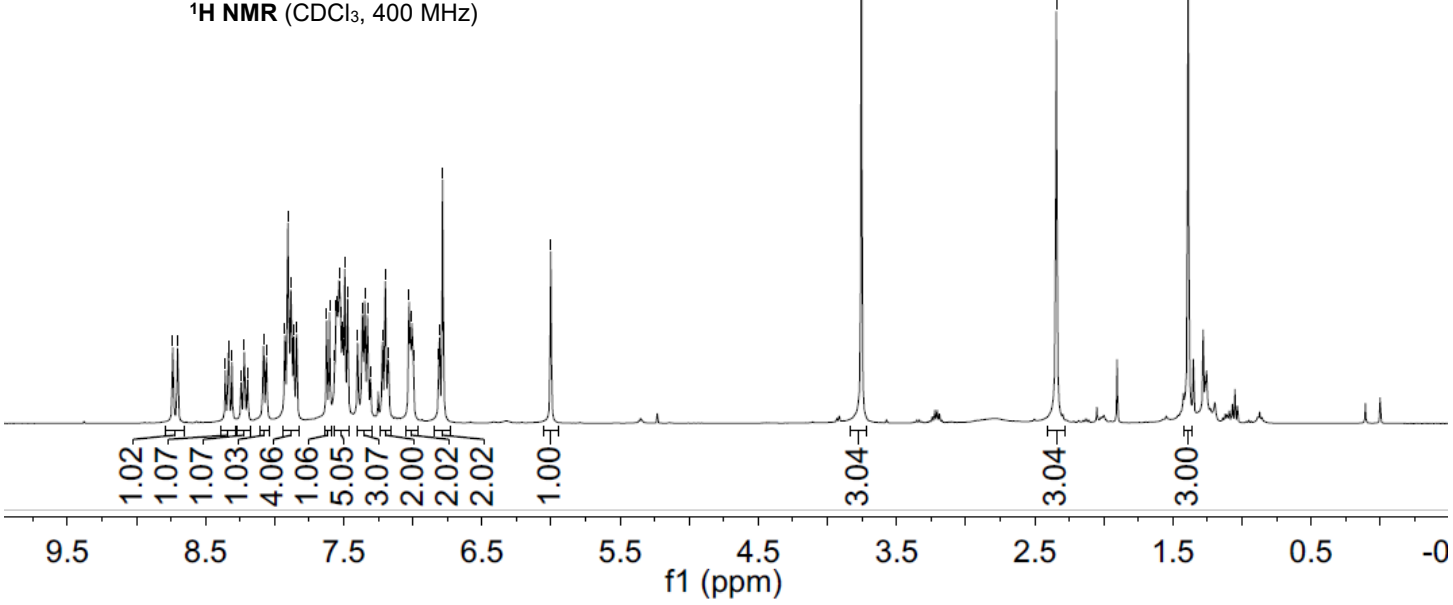




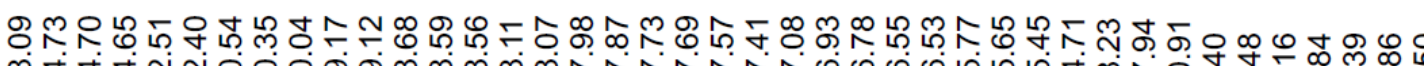

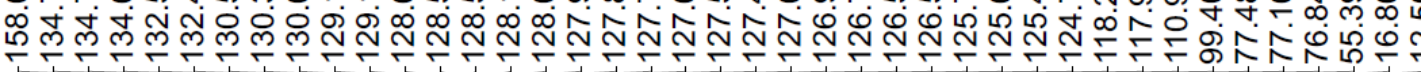

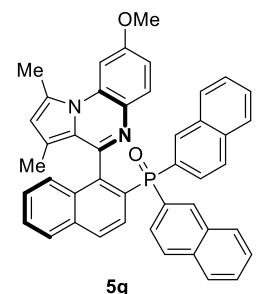

${ }^{13} \mathrm{C} \mathrm{NMR}\left(\mathrm{CDCl}_{3}, 100 \mathrm{MHz}\right)$

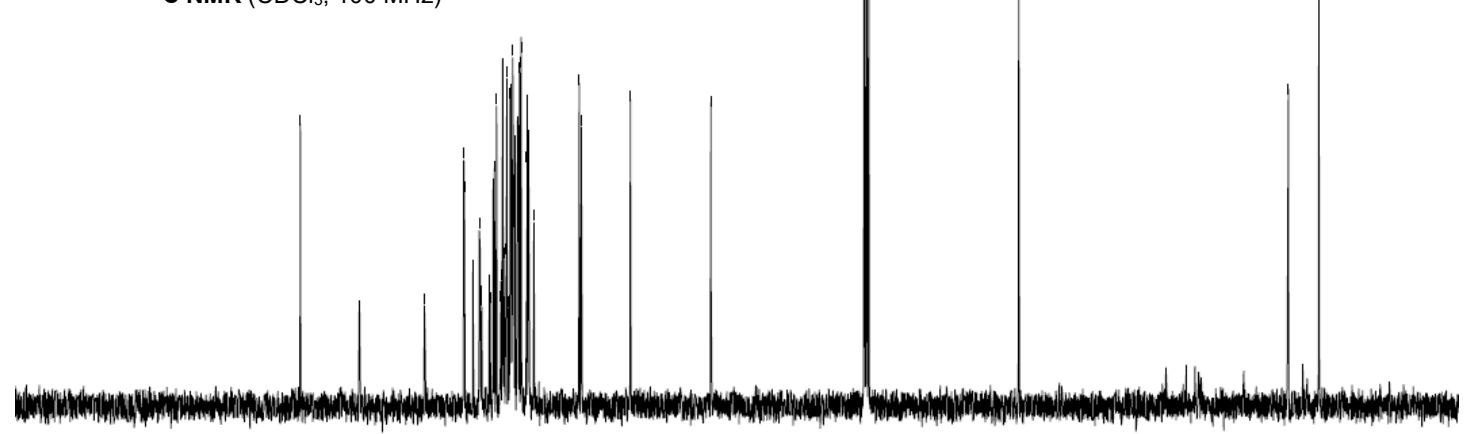

$\begin{array}{llllllllllllllllllll}190 & 180 & 170 & 160 & 150 & 140 & 130 & 120 & 110 & \begin{array}{l}100 \\ \mathrm{f} 1(\mathrm{ppm})\end{array} & 80 & 70 & 60 & 50 & 40 & 30 & 20 & 10 & 0\end{array}$.

$$
\text { 임 }
$$
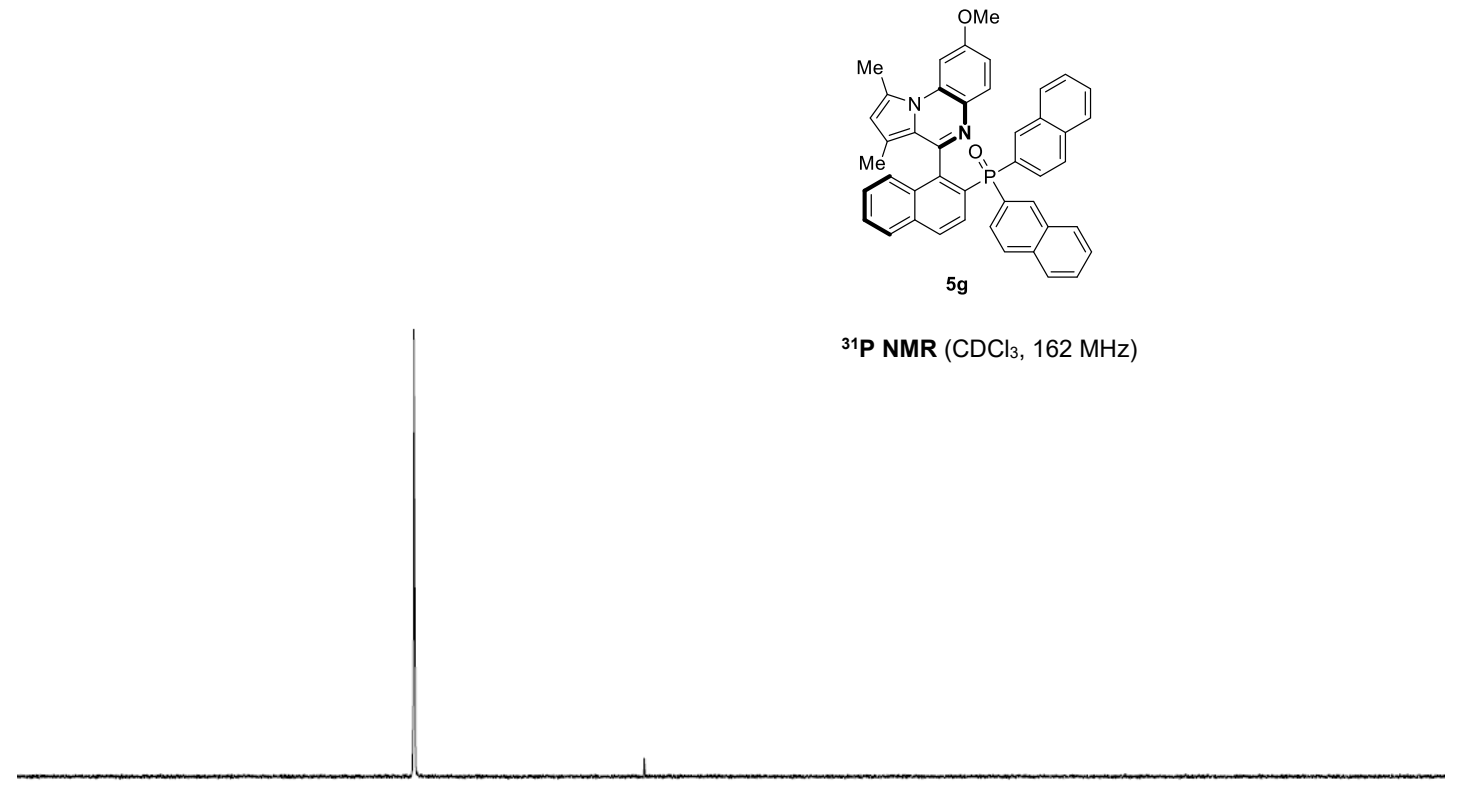

$\begin{array}{llllllllllllllll}110 & 90 & 70 & 50 & 30 & 10 & -10 & \begin{array}{l}-30 \\ \mathrm{f} 1(\mathrm{ppm})\end{array} & -50 & -70 & -90 & -110 & -130 & -150 & -170 & -190\end{array}$




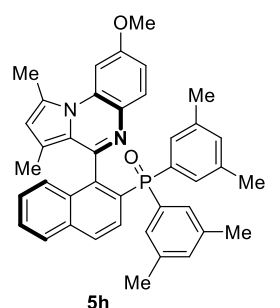

$5 h$

${ }^{1} \mathrm{H}$ NMR $\left(\mathrm{CDCl}_{3}, 400 \mathrm{MHz}\right)$

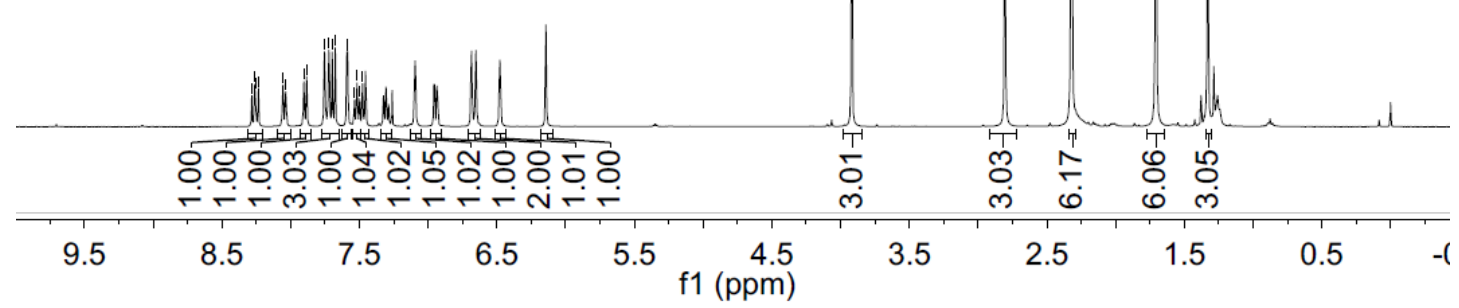

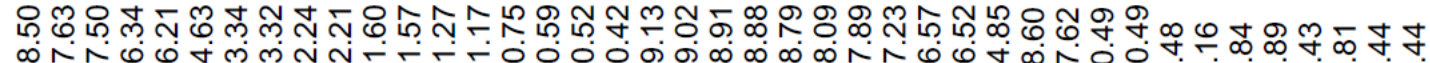
吕

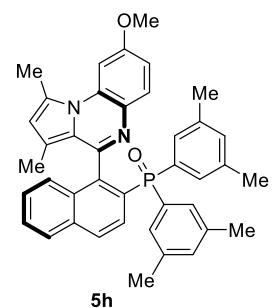

${ }^{13} \mathrm{C} \mathrm{NMR}\left(\mathrm{CDCl}_{3}, 100 \mathrm{MHz}\right)$

$10190180170160150140130120110 \quad 100 \quad 90$ $\begin{array}{lllllllll}80 & 70 & 60 & 50 & 40 & 30 & 20 & 10 & 0\end{array}$ f1 (ppm) 


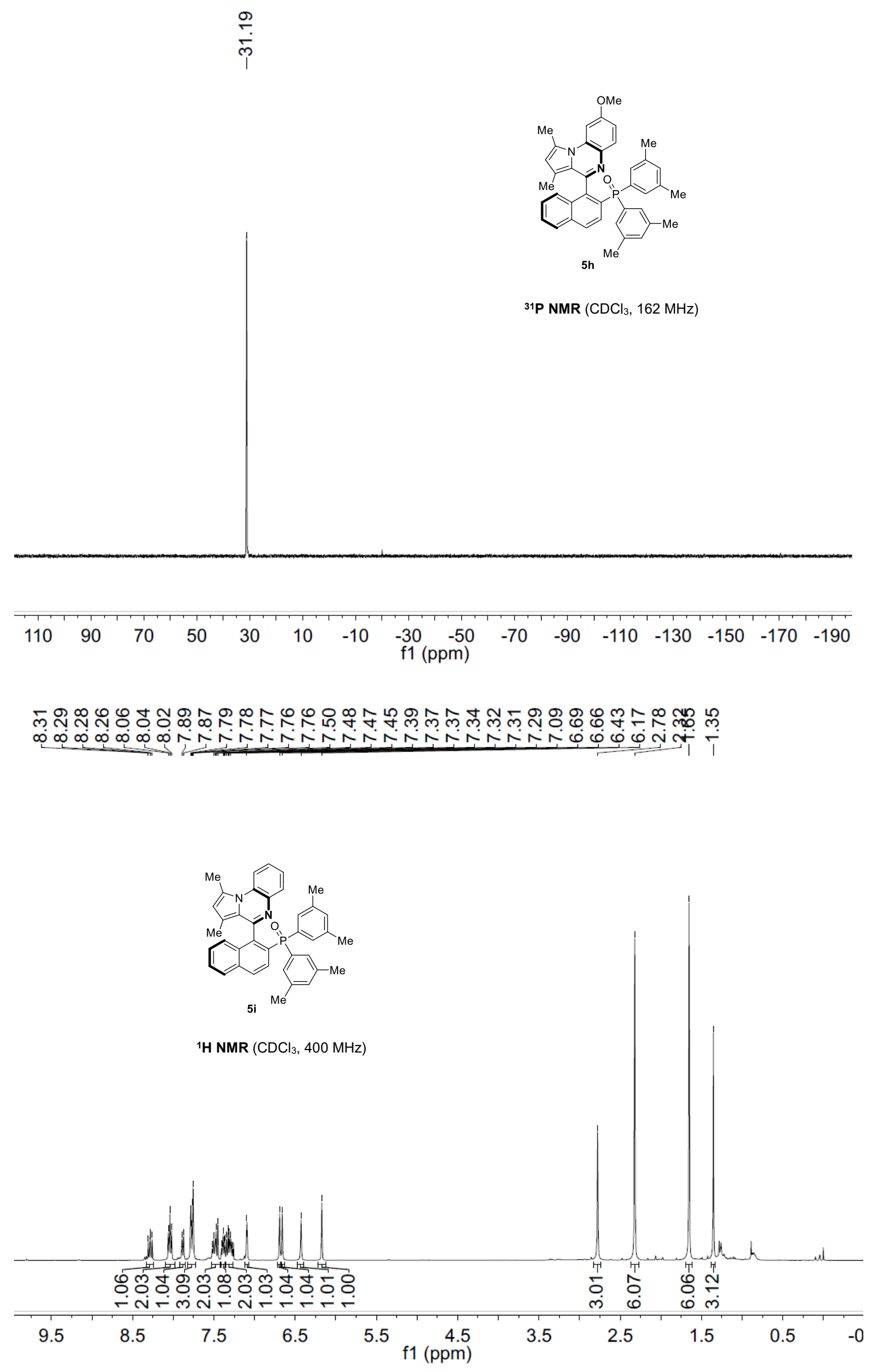




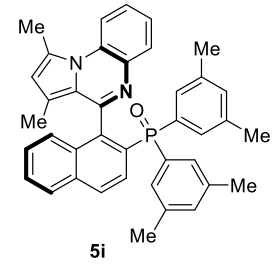

${ }^{13} \mathrm{C} \mathrm{NMR}\left(\mathrm{CDCl}_{3}, 100 \mathrm{MHz}\right)$

$\begin{array}{lllllllllllllllllllll}10 & 180 & 170 & 160 & 150 & 140 & 130 & 120 & 110 & 100 \underset{\mathrm{f} 1}{(\mathrm{ppm})} & 90 & 80 & 70 & 60 & 50 & 40 & 30 & 20 & 10 & 0 & -\end{array}$

ஜ़ं

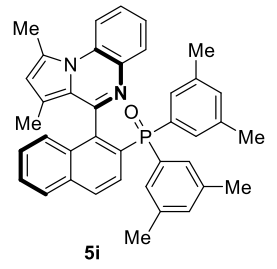

${ }^{31} \mathrm{P}$ NMR $\left(\mathrm{CDCl}_{3}, 162 \mathrm{MHz}\right)$

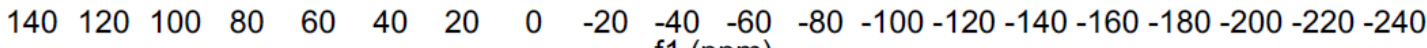
f1 (ppm) 


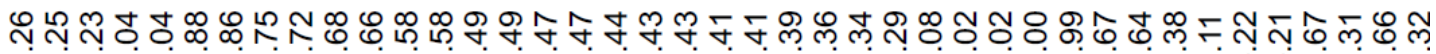

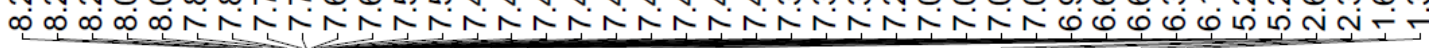

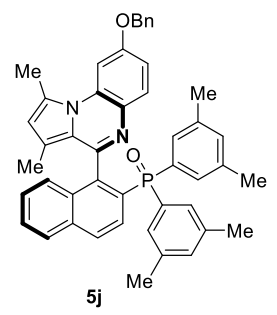

${ }^{1} \mathrm{H}$ NMR $\left(\mathrm{CDCl}_{3}, 400 \mathrm{MHz}\right)$

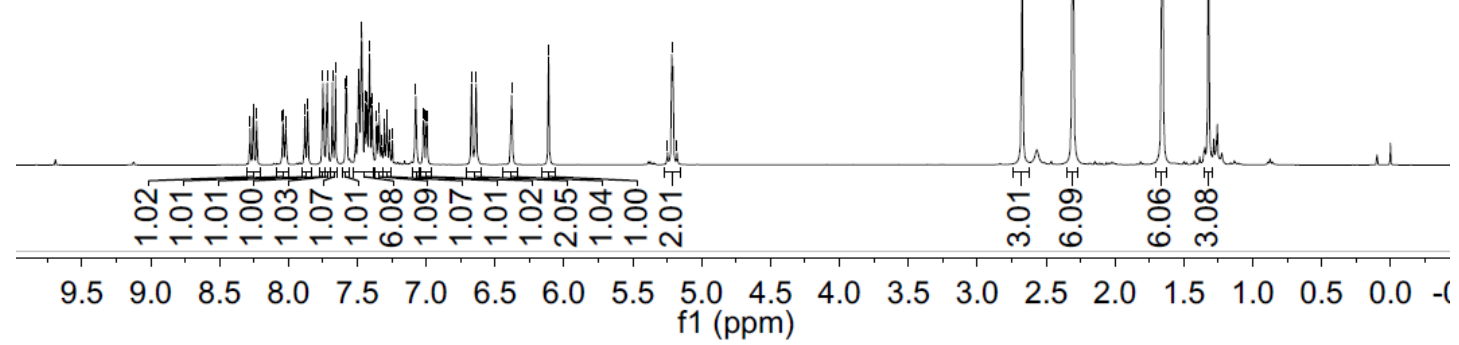

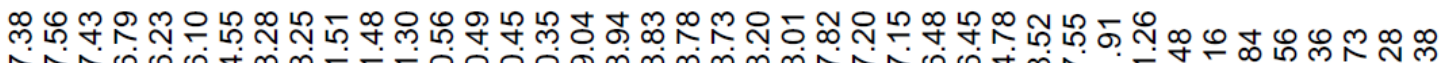
占

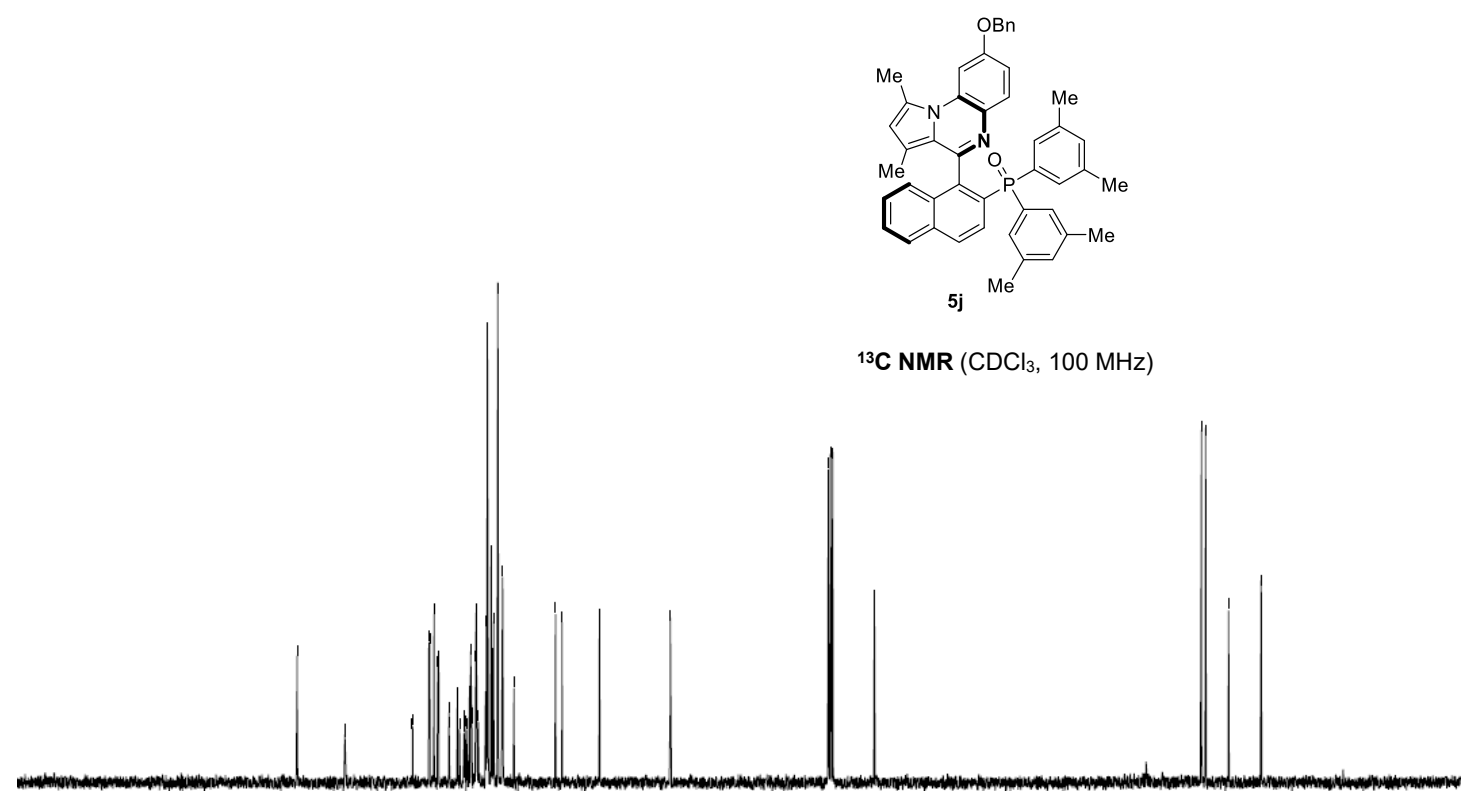

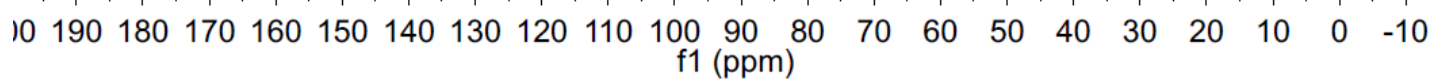




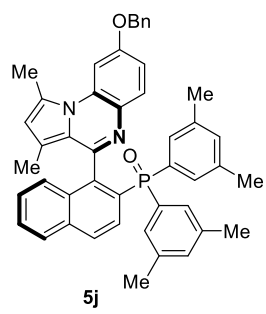

${ }^{31} \mathrm{P}$ NMR $\left(\mathrm{CDCl}_{3}, 162 \mathrm{MHz}\right)$

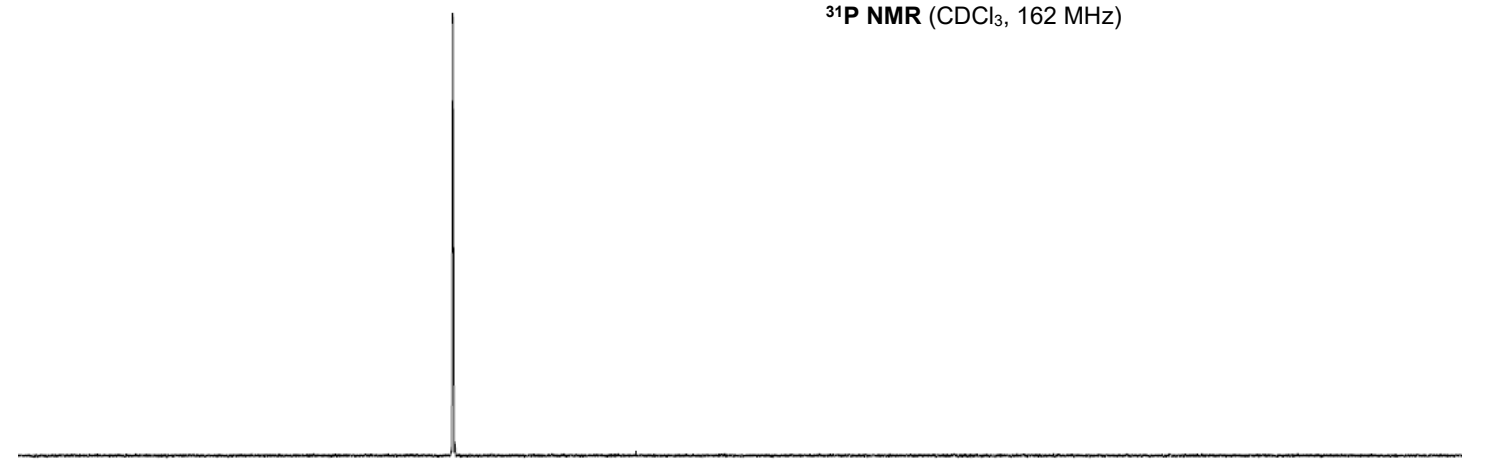

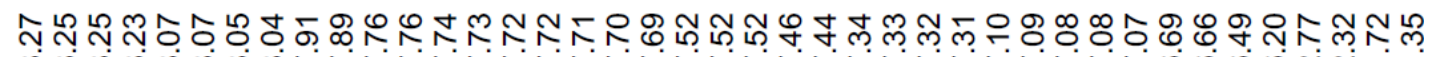

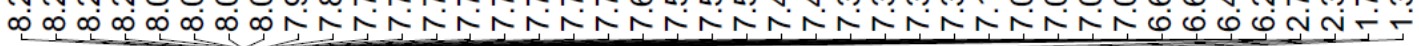

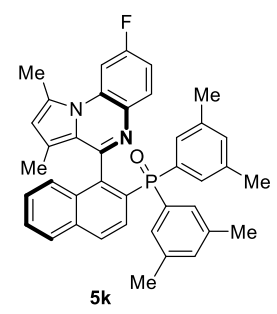

${ }^{1} \mathrm{H}$ NMR $\left(\mathrm{CDCl}_{3}, 400 \mathrm{MHz}\right)$

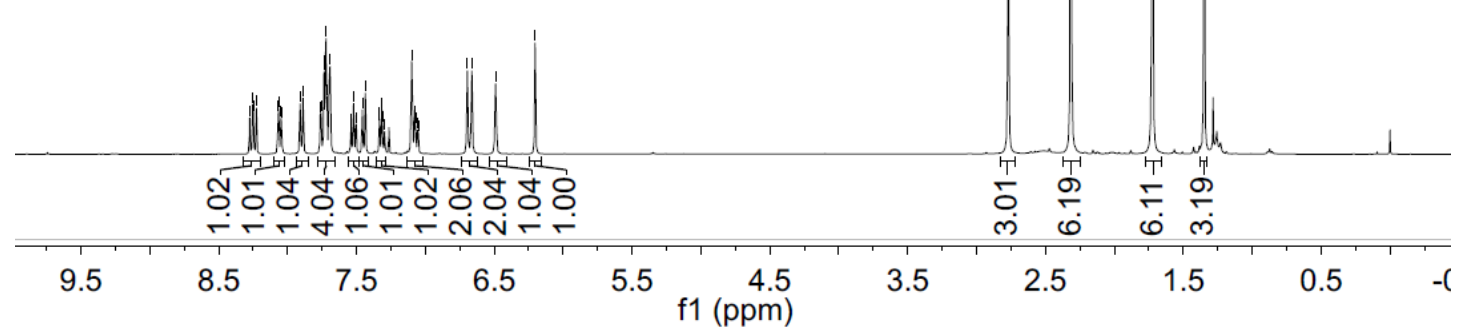




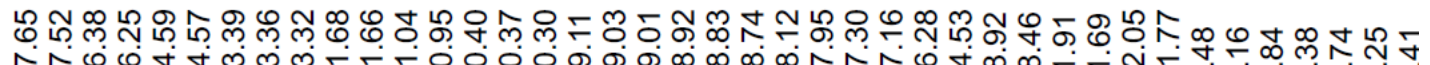

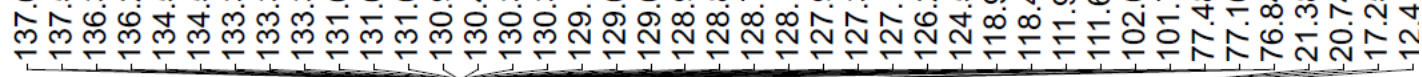

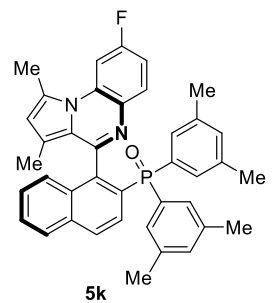

${ }^{13} \mathrm{C}$ NMR $\left(\mathrm{CDCl}_{3}, 100 \mathrm{MHz}\right)$

$\begin{array}{llllllllllllllllllllll}10 & 190 & 180 & 170 & 160 & 150 & 140 & 130 & 120 & 110 & \begin{array}{c}100 \\ \mathrm{f} 1(\mathrm{ppm})\end{array} & 90 & 70 & 60 & 50 & 40 & 30 & 20 & 10 & 0 & -\end{array}$

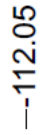

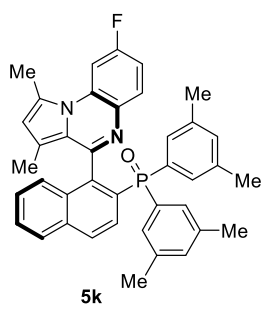

${ }^{19} \mathrm{~F} \mathrm{NMR}\left(\mathrm{CDCl}_{3}, 377 \mathrm{MHz}\right)$

\begin{tabular}{|c|c|c|c|c|c|c|c|c|c|c|c|}
\hline 0 & 0 & -20 & -40 & -60 & -80 & $\begin{array}{c}-100 \\
\mathrm{f} 1(\mathrm{ppm})\end{array}$ & -120 & -140 & -160 & -180 & -200 \\
\hline
\end{tabular}


के
oे
i

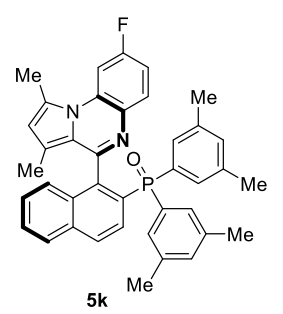

${ }^{31} \mathrm{P}$ NMR $\left(\mathrm{CDCl}_{3}, 162 \mathrm{MHz}\right)$

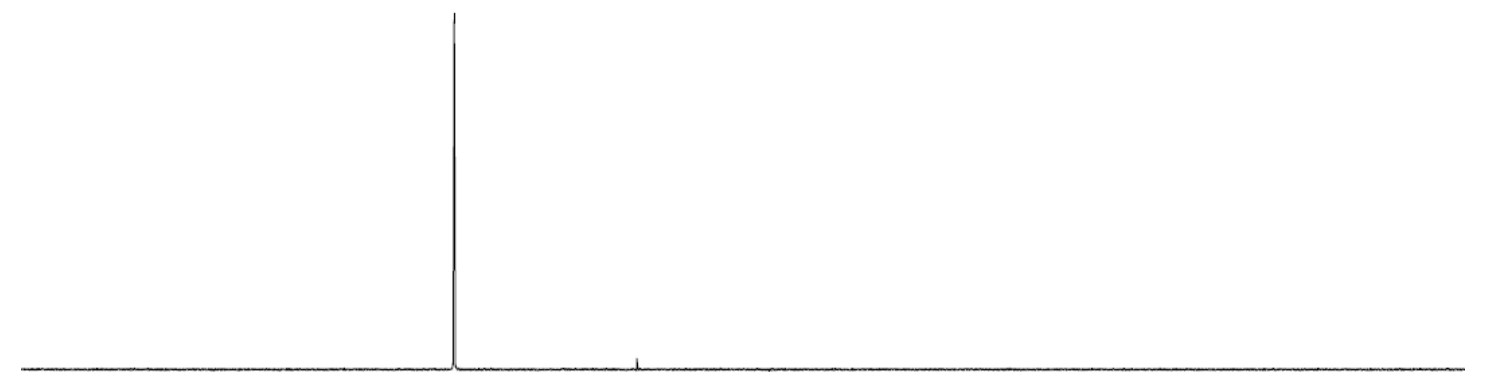

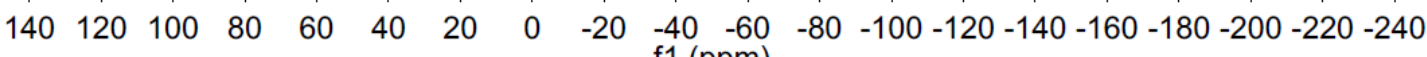
f1 $(\mathrm{ppm})$

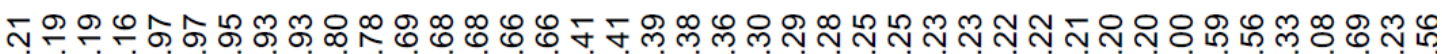

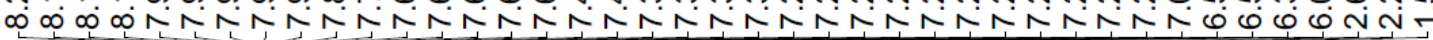

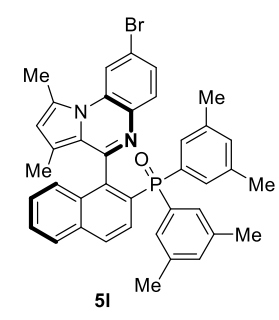

${ }^{1} \mathrm{H}$ NMR $\left(\mathrm{CDCl}_{3}, 400 \mathrm{MHz}\right)$

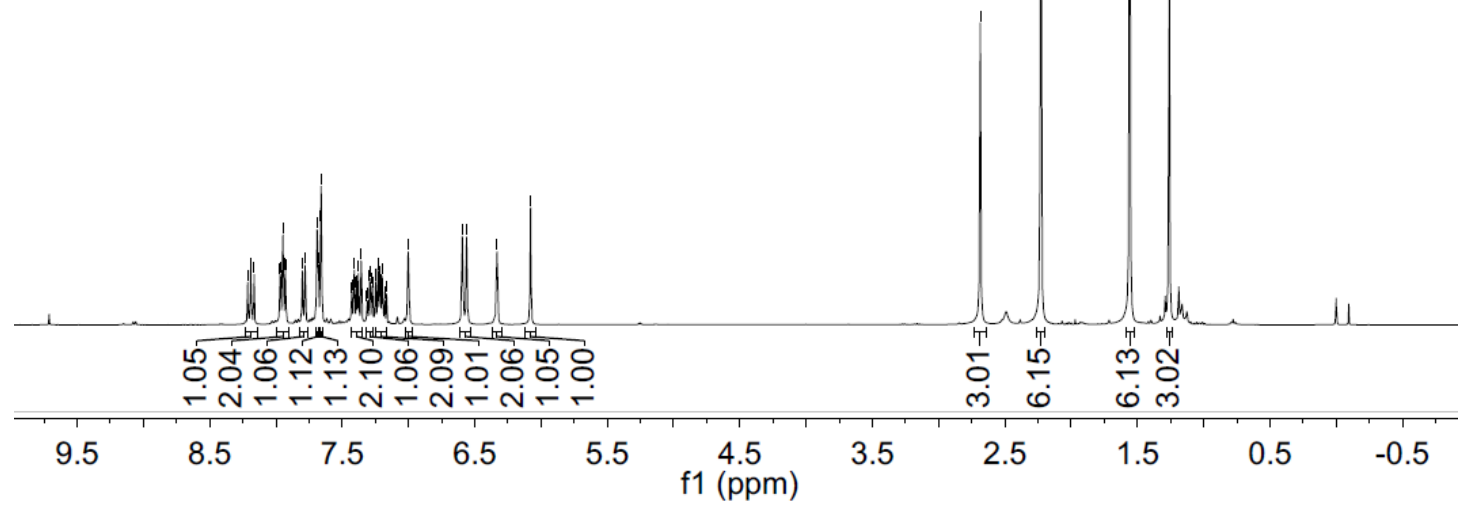




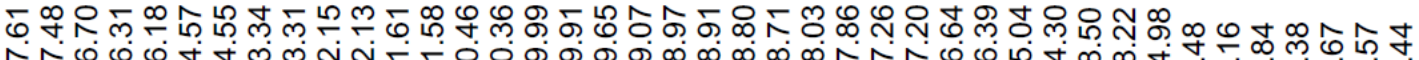

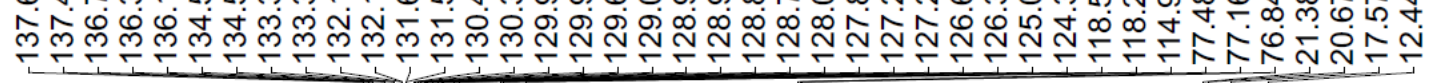

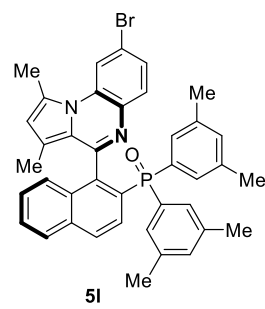

${ }^{13} \mathrm{C}$ NMR $\left(\mathrm{CDCl}_{3}, 100 \mathrm{MHz}\right)$

$\begin{array}{llllllllllllllllllllllll}10 & 180 & 170 & 160 & 150 & 140 & 130 & 120 & 110 & 100 & 90 & 80 & 70 & 60 & 50 & 40 & 30 & 20 & 10 & 0 & -\end{array}$

음

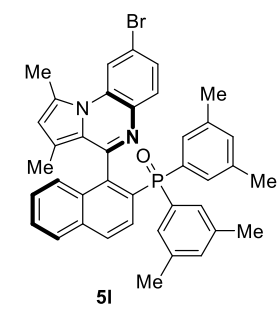

${ }^{31} \mathrm{P}$ NMR $\left(\mathrm{CDCl}_{3}, 162 \mathrm{MHz}\right)$

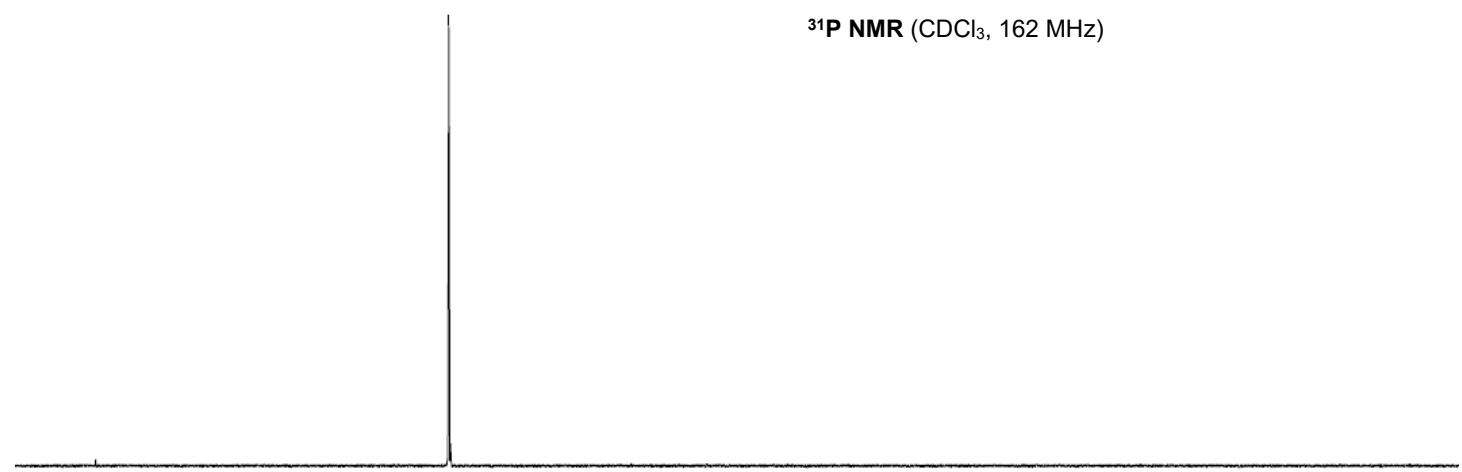

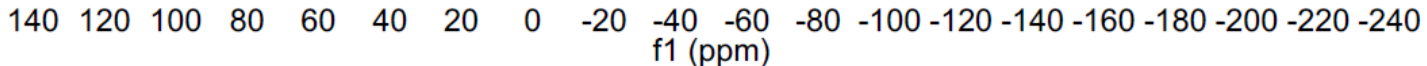




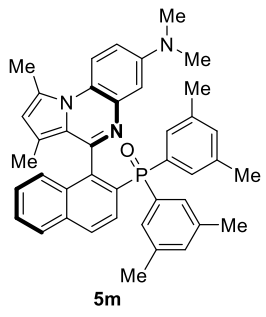

${ }^{1} \mathrm{H}$ NMR $\left(\mathrm{CDCl}_{3}, 400 \mathrm{MHz}\right)$

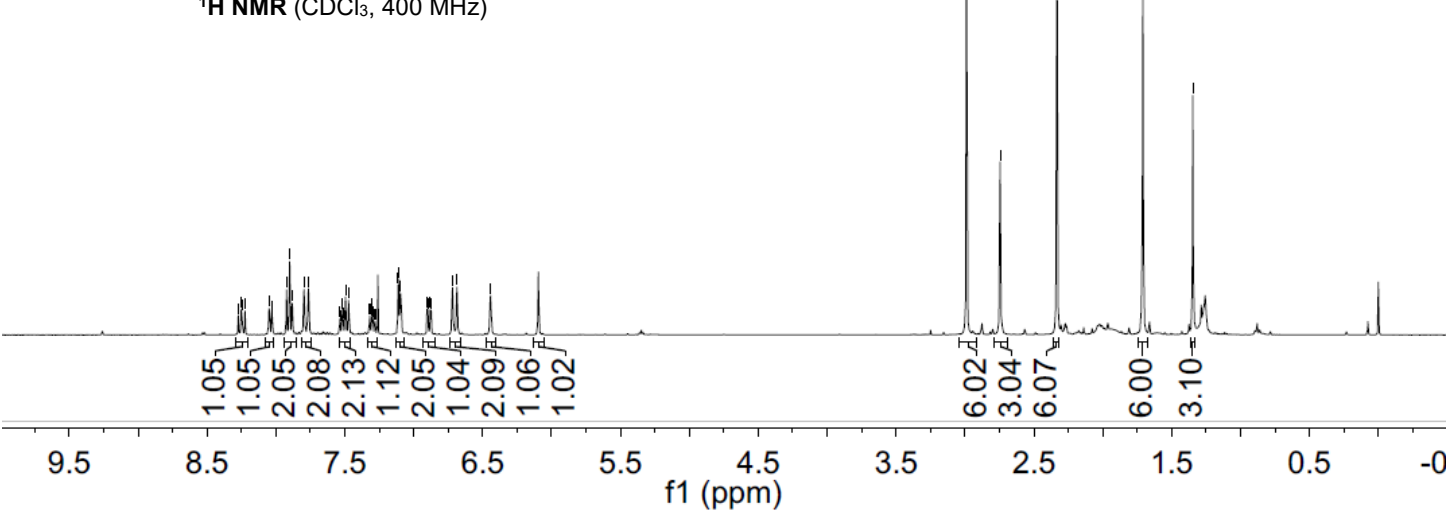

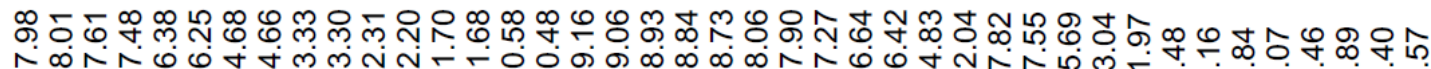

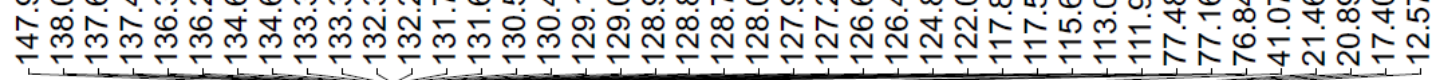

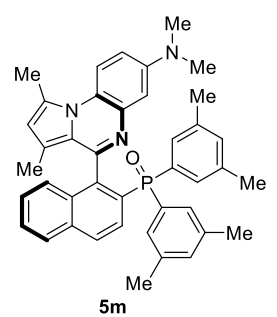

${ }^{13} \mathrm{C} \mathrm{NMR}\left(\mathrm{CDCl}_{3}, 100 \mathrm{MHz}\right)$

$\begin{array}{lllllllllllllllllllll}10 & 180 & 170 & 160 & 150 & 140 & 130 & 120 & 110 & 100 & \begin{array}{c}90 \\ \mathrm{f} 1(\mathrm{ppm})\end{array} & 70 & 60 & 50 & 40 & 30 & 20 & 10 & 0 & -10\end{array}$


৪
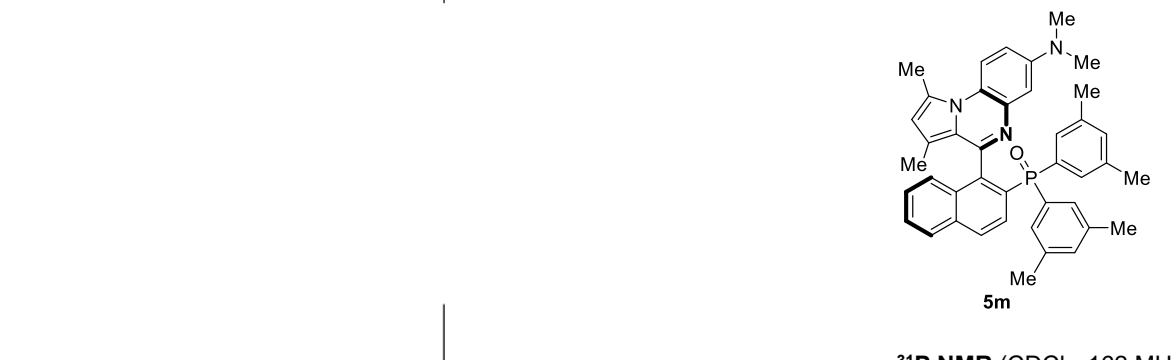

${ }^{31} \mathrm{P}$ NMR $\left(\mathrm{CDCl}_{3}, 162 \mathrm{MHz}\right)$

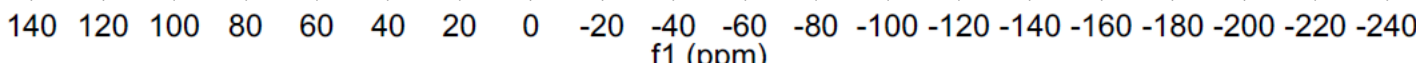

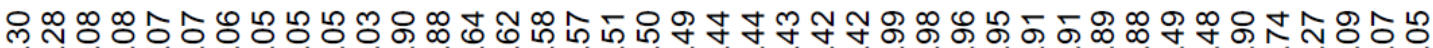

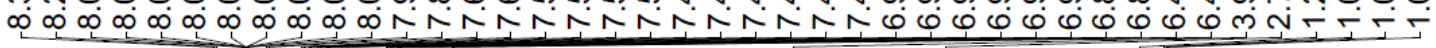

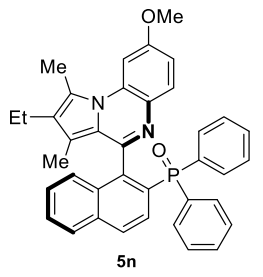

${ }^{1} \mathrm{H} \mathrm{NMR}\left(\mathrm{CDCl}_{3}, 400 \mathrm{MHz}\right)$

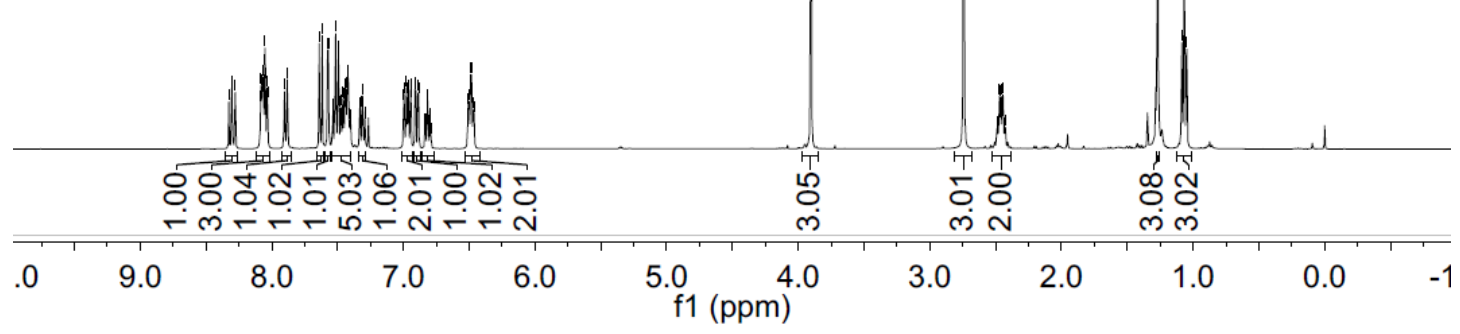




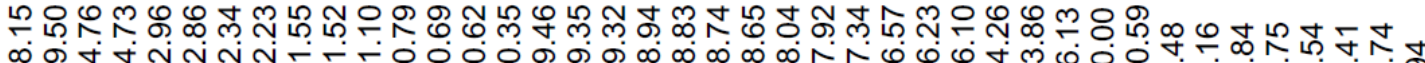

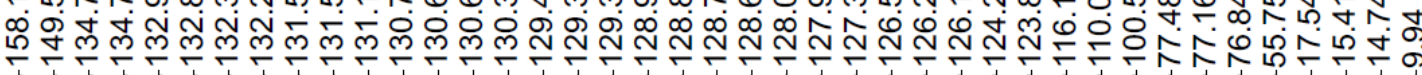

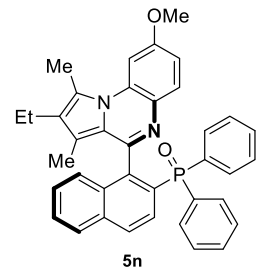

${ }^{13} \mathrm{C}$ NMR $\left(\mathrm{CDCl}_{3}, 100 \mathrm{MHz}\right)$

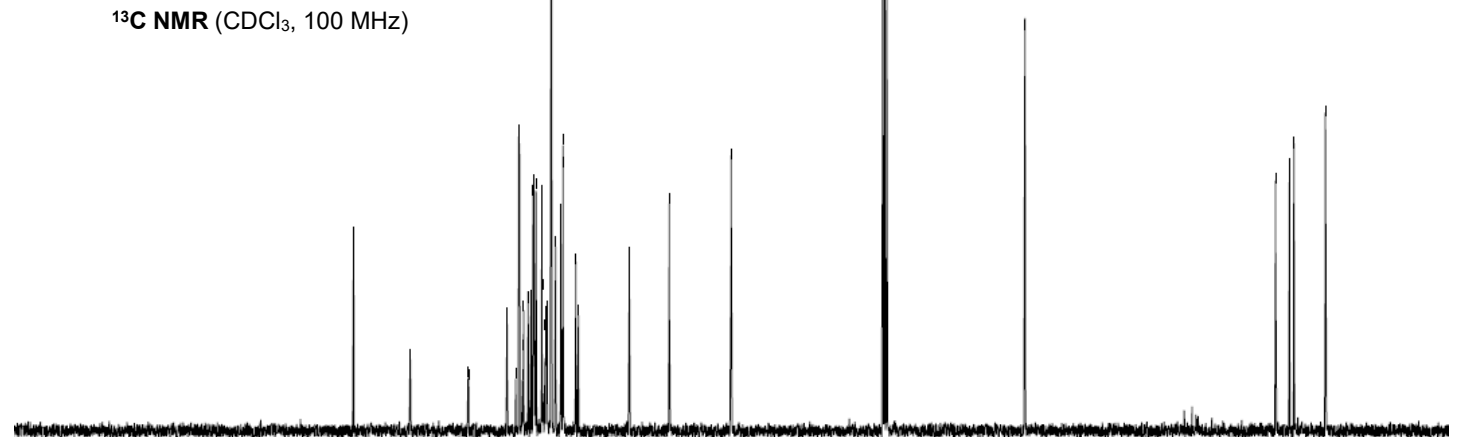

$10200190180170160150140130120 \begin{gathered}110 \quad \begin{array}{l}100 \\ \mathrm{f} 1(\mathrm{ppm})\end{array} \\ 90\end{gathered}$ $\stackrel{+}{\dot{0}}$

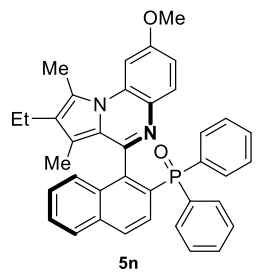

${ }^{31} \mathrm{P}$ NMR $\left(\mathrm{CDCl}_{3}, 162 \mathrm{MHz}\right)$

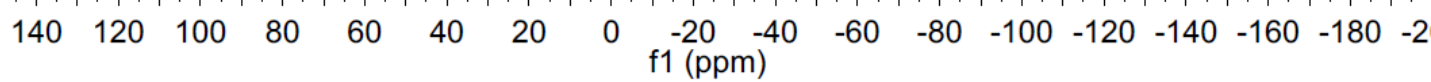


సุำ

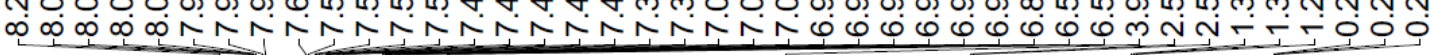

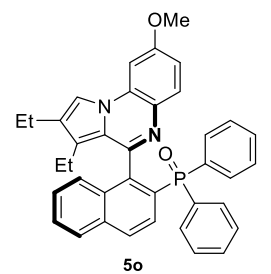

${ }^{1} \mathrm{H}$ NMR $\left(\mathrm{CDCl}_{3}, 400 \mathrm{MHz}\right)$

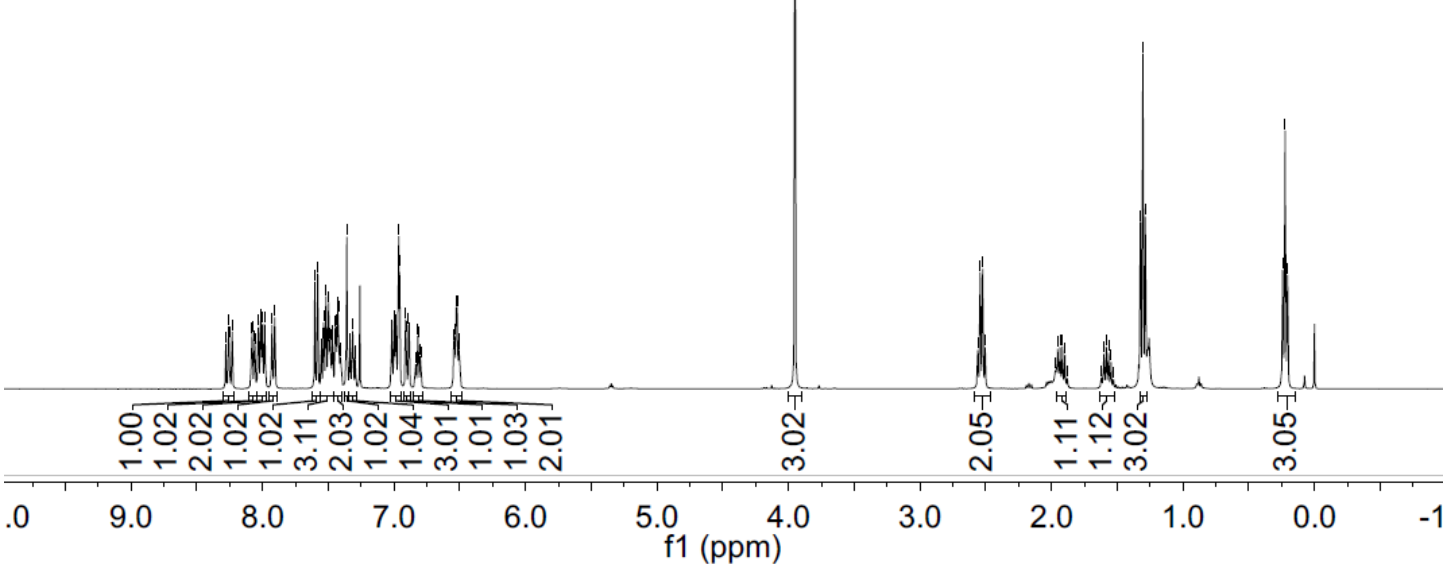

๑ొ ஜ

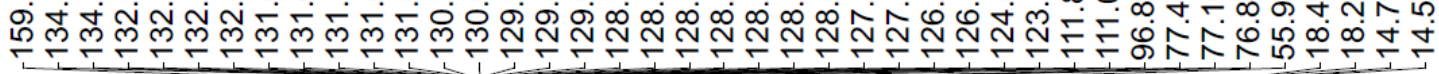

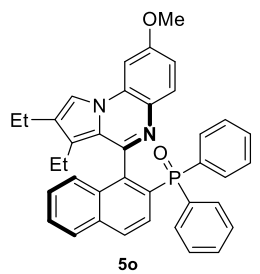

${ }^{13} \mathrm{C} \mathrm{NMR}\left(\mathrm{CDCl}_{3}, 100 \mathrm{MHz}\right)$

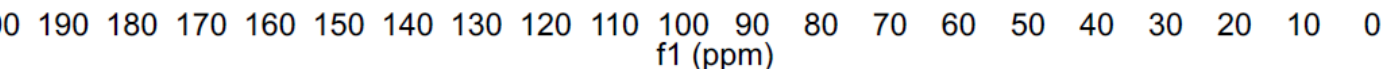




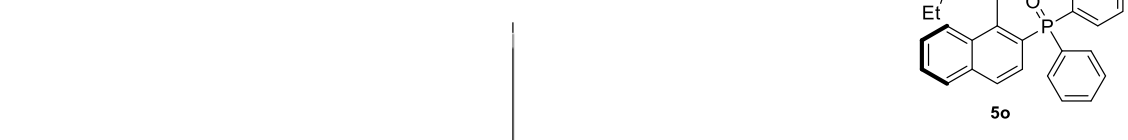

${ }^{31} \mathrm{P}$ NMR $\left(\mathrm{CDCl}_{3}, 162 \mathrm{MHz}\right)$

$\begin{array}{llllllllllllllllll}140 & 120 & 100 & 80 & 60 & 40 & 20 & 0 & \begin{array}{c}-20 \\ \mathrm{f} 1(\mathrm{ppm})\end{array} & -40 & -60 & -80 & -100 & -120 & -140 & -160 & -180 & -\varepsilon\end{array}$

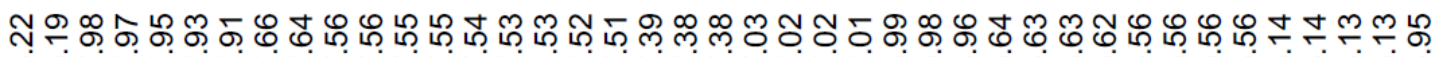

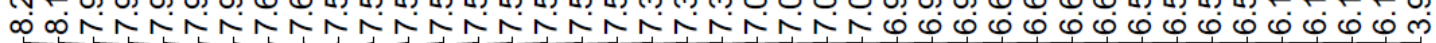

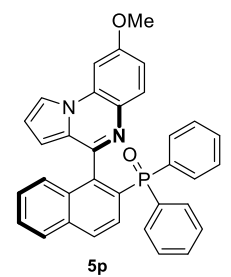

${ }^{1} \mathrm{H}$ NMR $\left(\mathrm{CDCl}_{3}, 400 \mathrm{MHz}\right)$

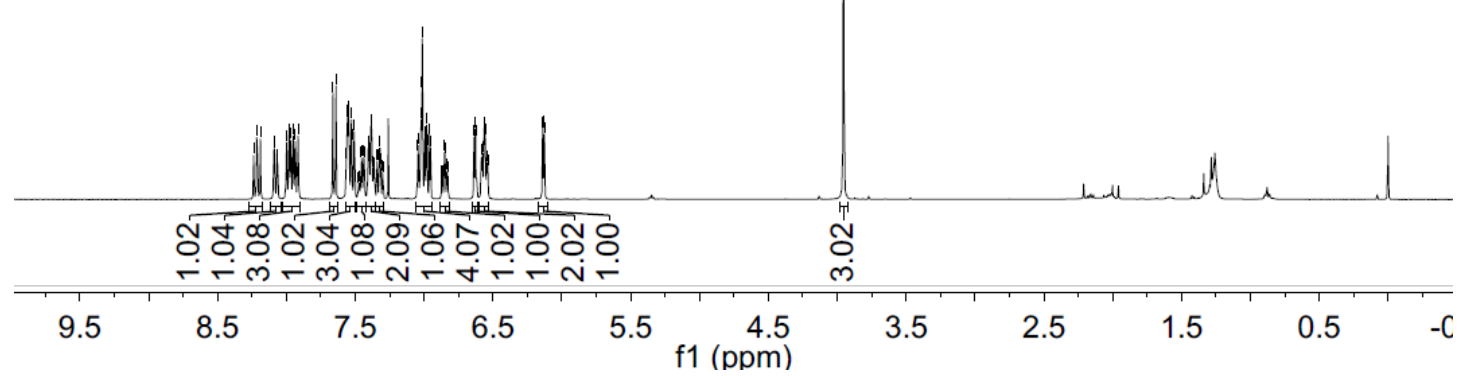


꾼 চூ่

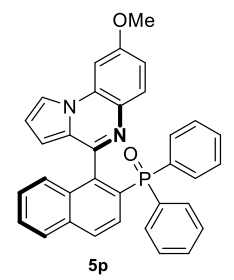

${ }^{13} \mathrm{C} \mathrm{NMR}\left(\mathrm{CDCl}_{3}, 100 \mathrm{MHz}\right)$

$10190180170160150140 \quad 130120110 \begin{array}{lllllllllllll}100 & 90 & 80 & 70 & 60 & 50 & 40 & 30 & 20 & 10 & 0 & -1\end{array}$

厄̊

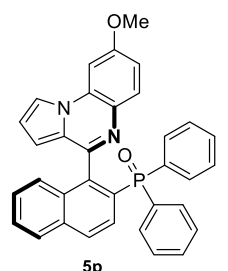

31P NMR $\left(\mathrm{CDCl}_{3}, 162 \mathrm{MHz}\right)$

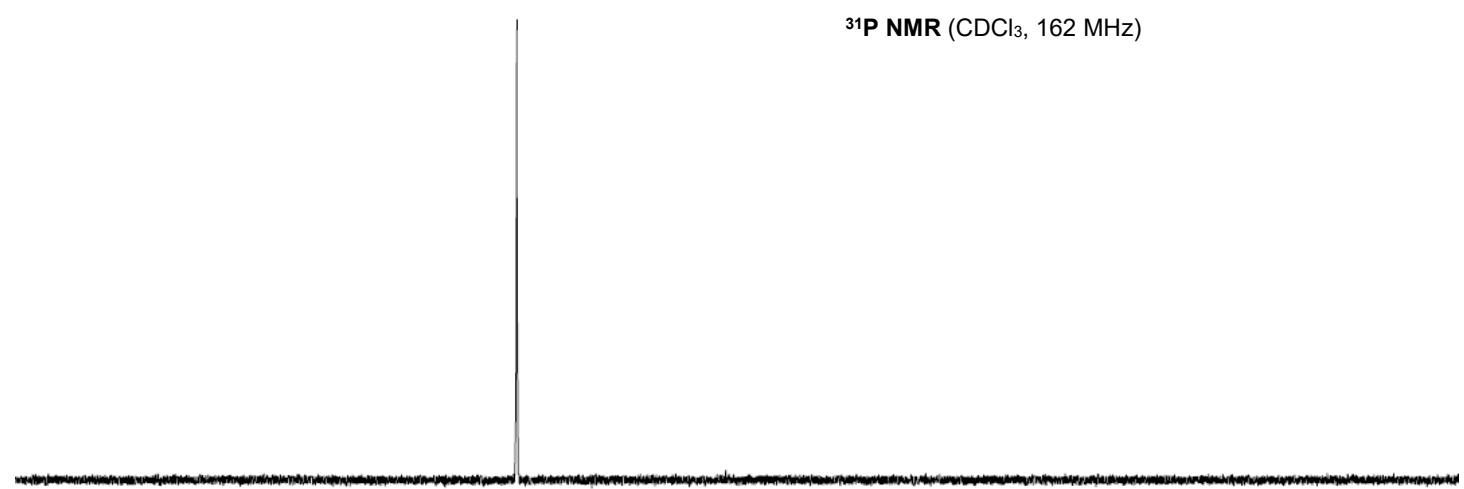

$\begin{array}{lllllllllllllllllll}140 & 120 & 100 & 80 & 60 & 40 & 20 & 0 & -20 & -40 & -60 & -80 & -100 & -120 & -140 & -160 & -180 & -2\end{array}$ f1 (ppm) 


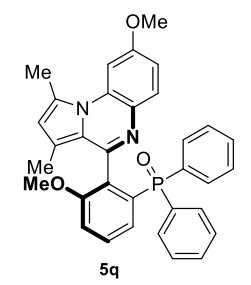

${ }^{1} \mathrm{H}$ NMR $\left(\mathrm{CDCl}_{3}, 400 \mathrm{MHz}\right)$

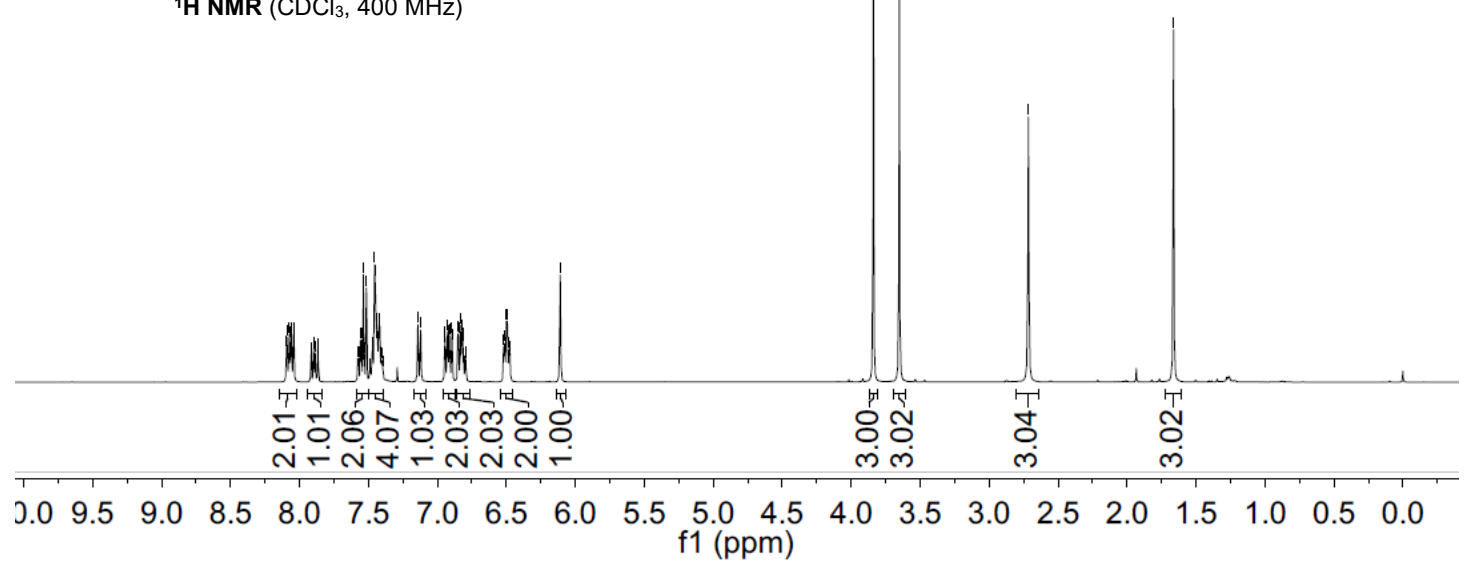

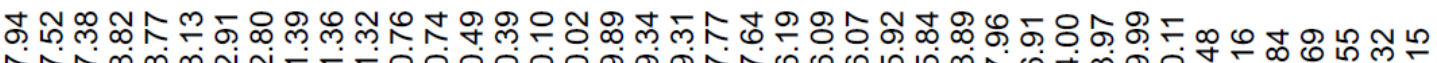

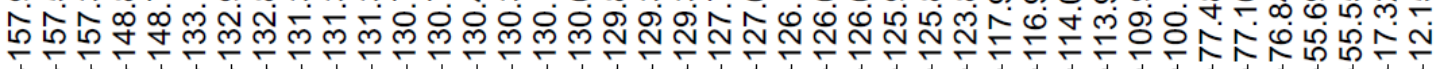

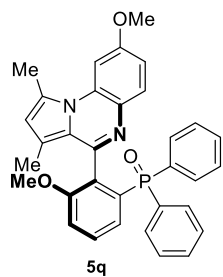

${ }^{13} \mathrm{C}$ NMR $\left(\mathrm{CDCl}_{3}, 100 \mathrm{MHz}\right)$

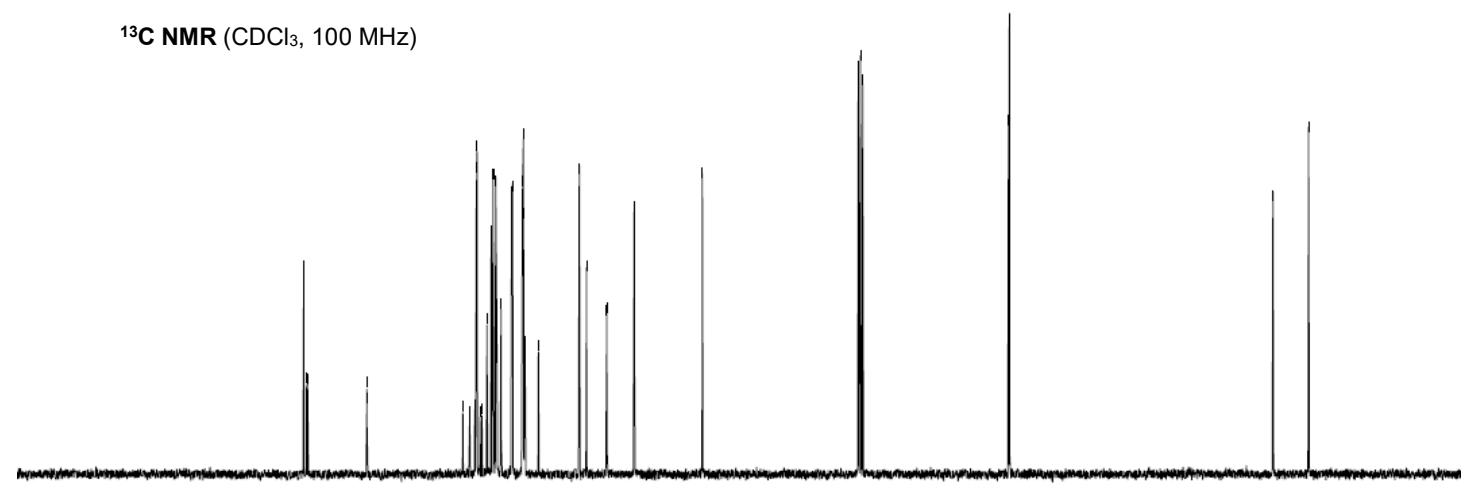

$10190180170160150140130120110 \begin{gathered}100 \\ f 1(\mathrm{ppm})\end{gathered}$ 
$\stackrel{\infty}{\infty}$

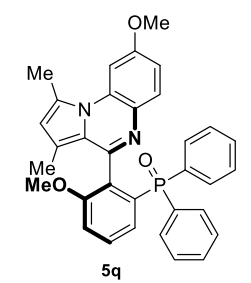

${ }^{31} \mathrm{P}$ NMR $\left(\mathrm{CDCl}_{3}, 162 \mathrm{MHz}\right)$

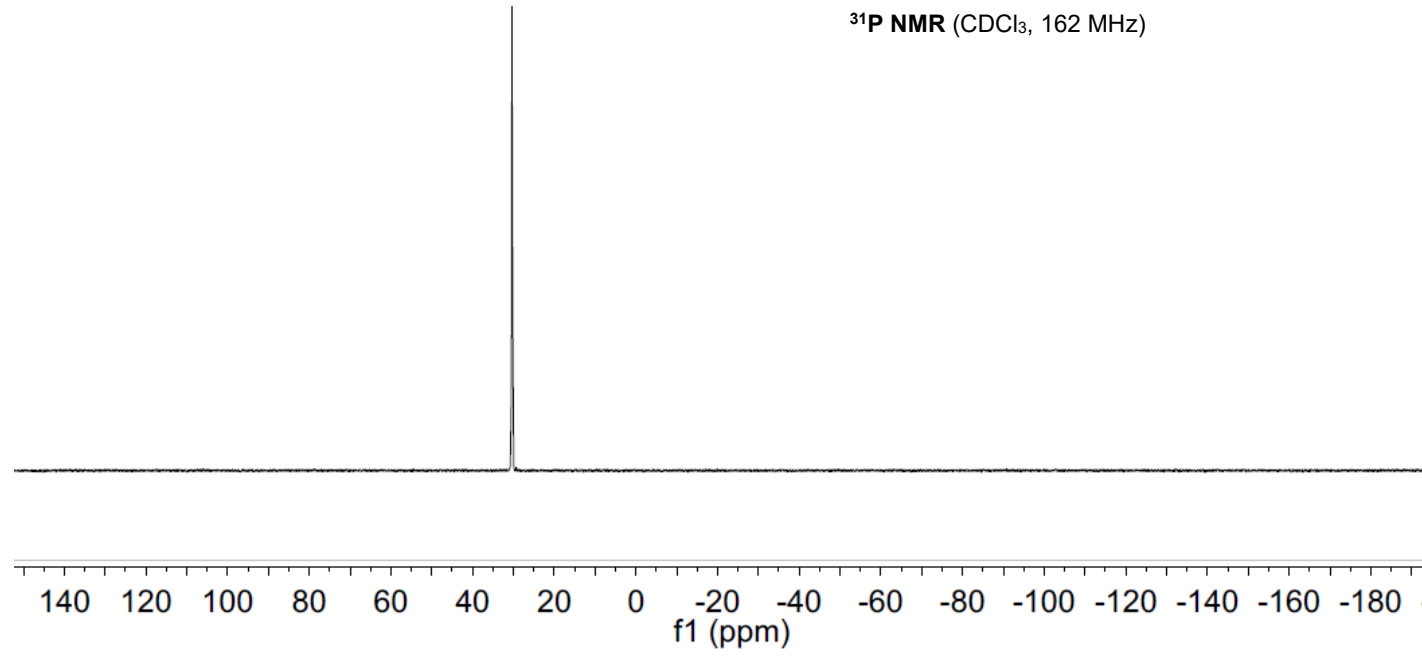

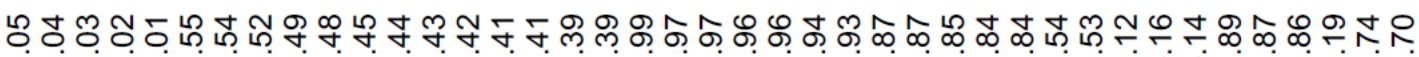

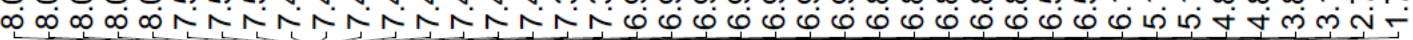

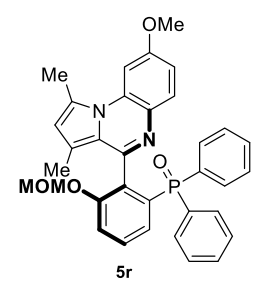

${ }^{1} \mathrm{H}$ NMR $\left(\mathrm{CDCl}_{3}, 400 \mathrm{MHz}\right)$

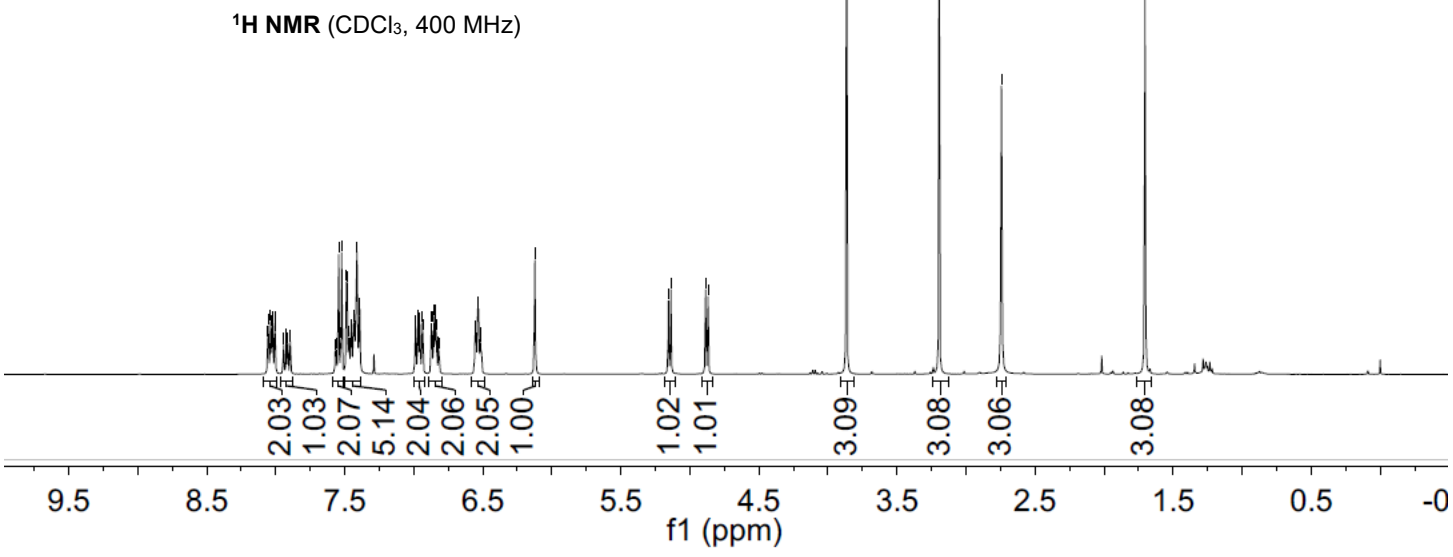




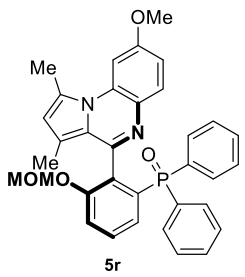

${ }^{13} \mathrm{C} \mathrm{NMR}\left(\mathrm{CDCl}_{3}, 100 \mathrm{MHz}\right)$

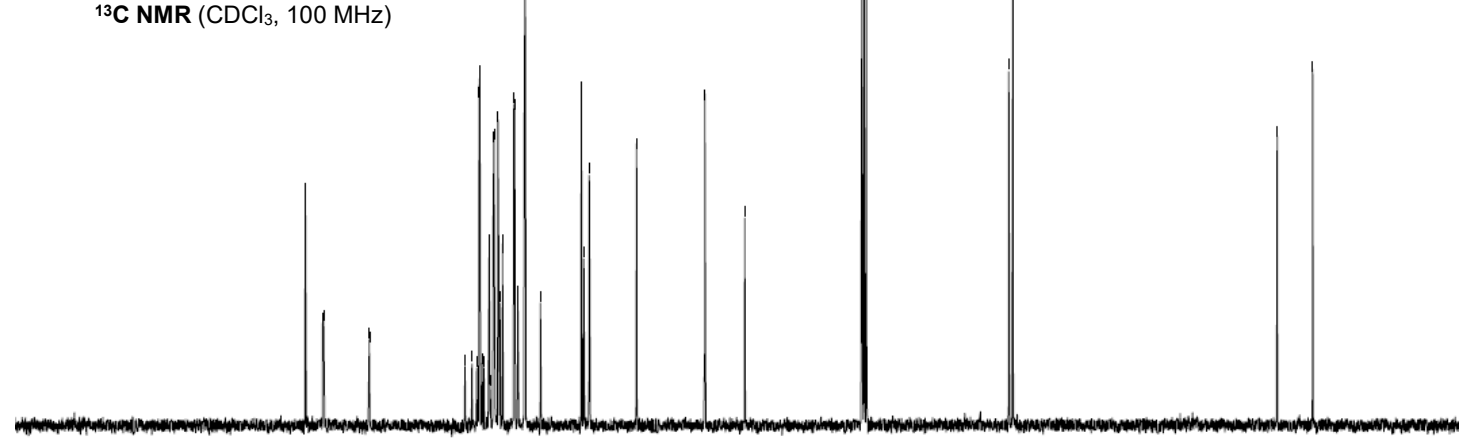

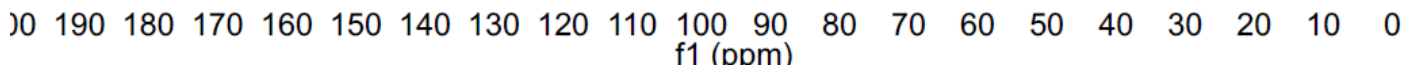

๙্ণ

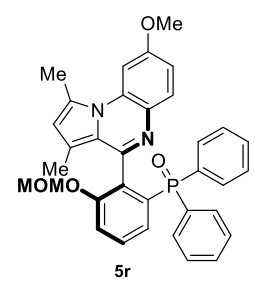

31P NMR ( $\left.\mathrm{CDCl}_{3}, 162 \mathrm{MHz}\right)$

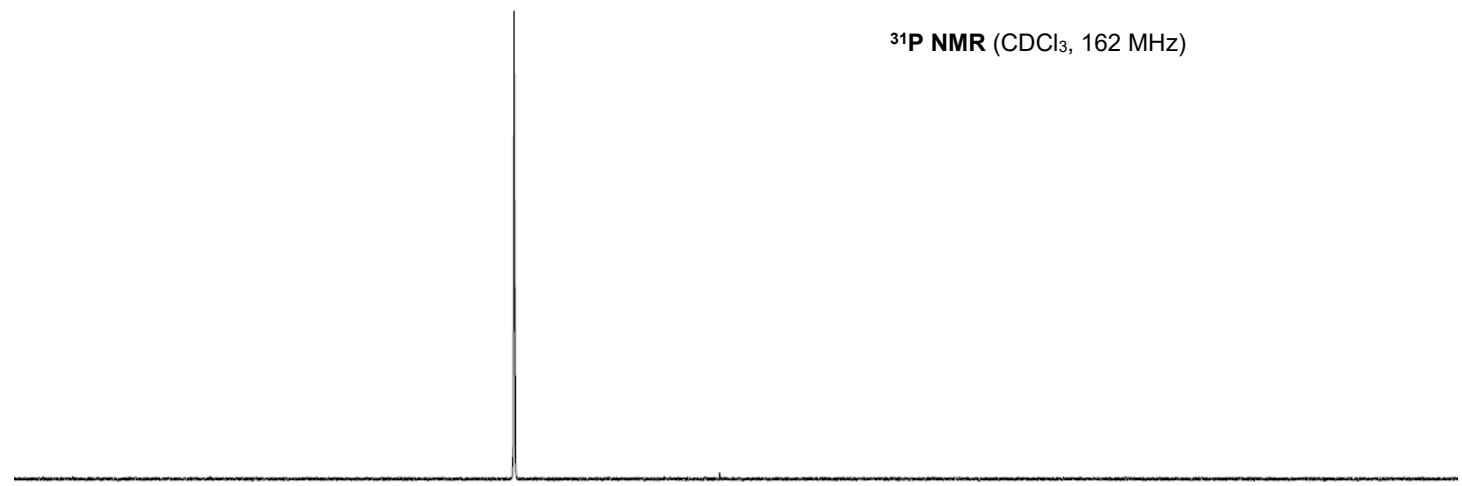

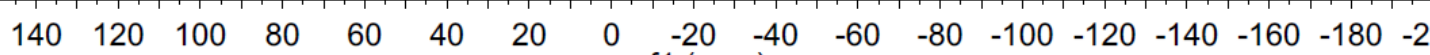
f1 (ppm) 


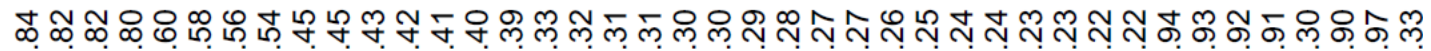

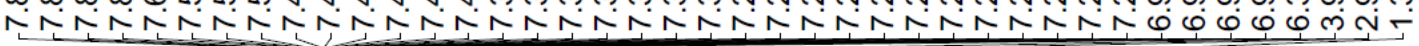

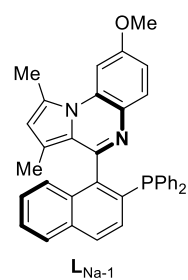

${ }^{1} \mathrm{H}$ NMR $\left(\mathrm{CDCl}_{3}, 400 \mathrm{MHz}\right)$

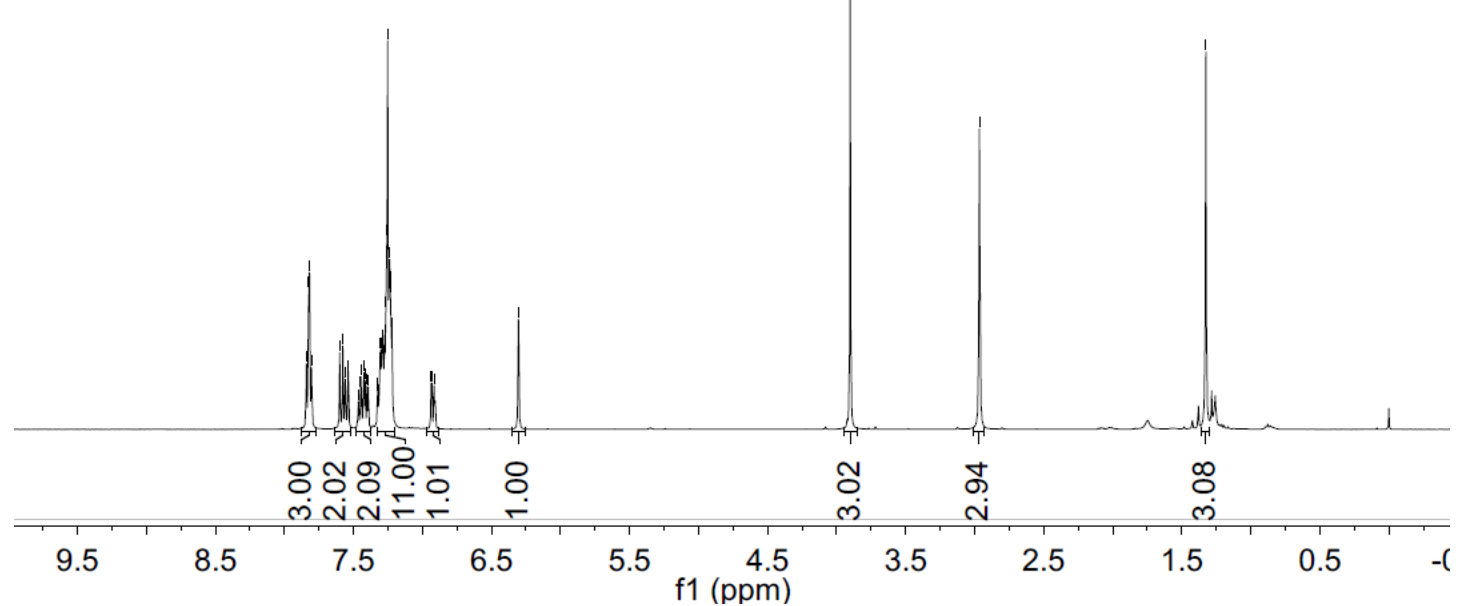

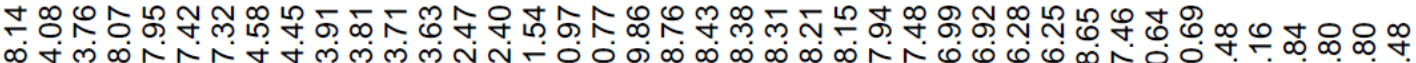

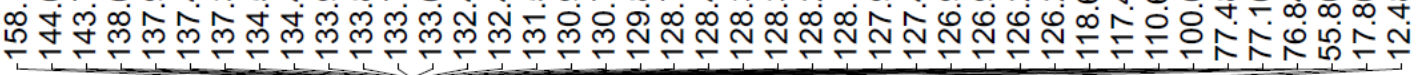

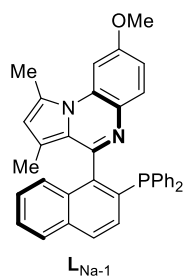

${ }^{13} \mathrm{C} \mathrm{NMR}\left(\mathrm{CDCl}_{3}, 100 \mathrm{MHz}\right)$

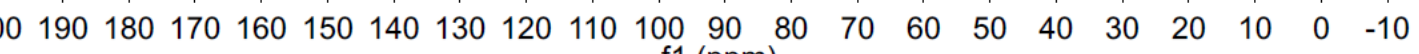
f1 (ppm) 


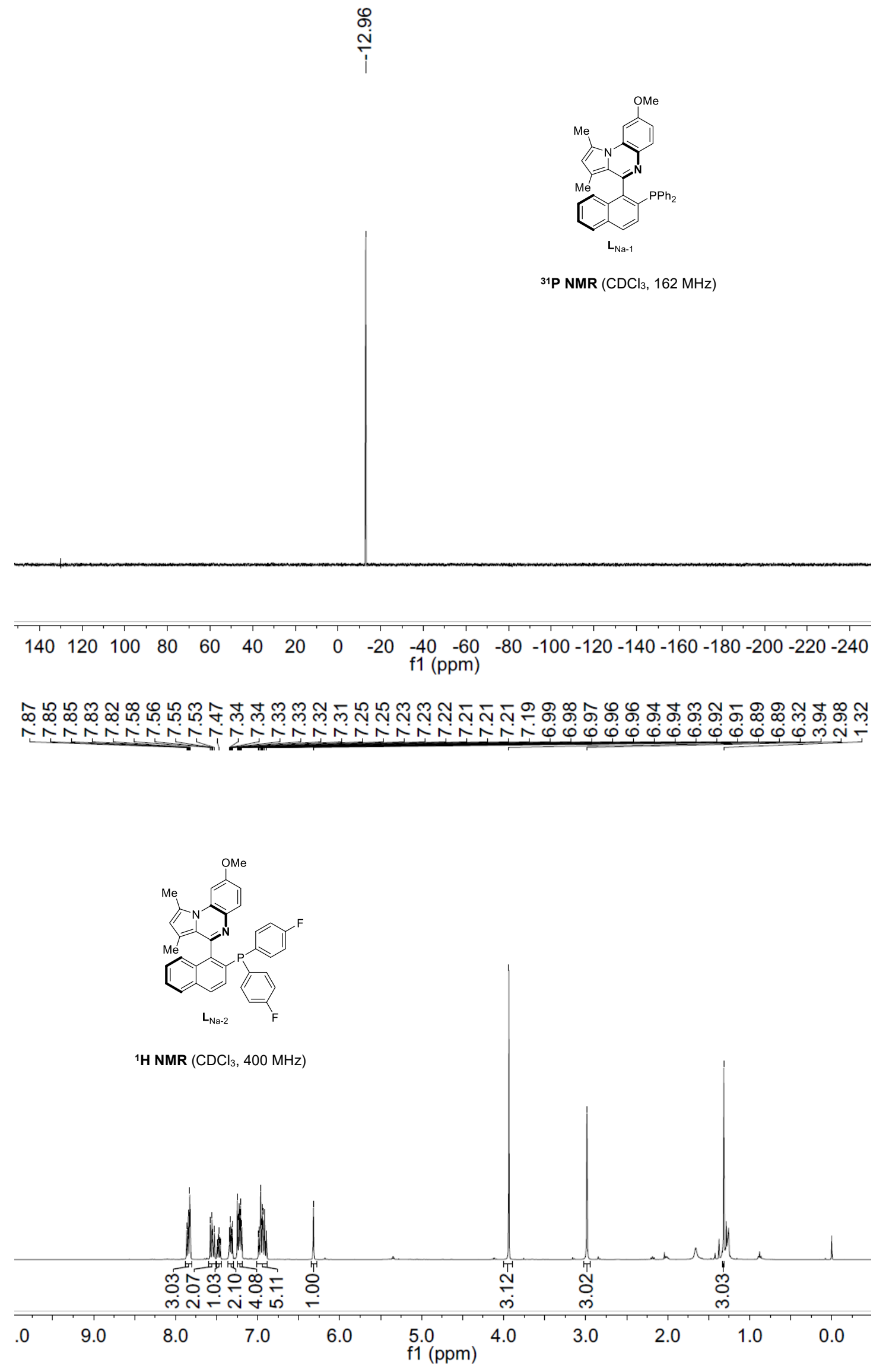




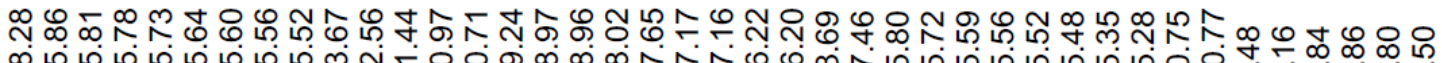

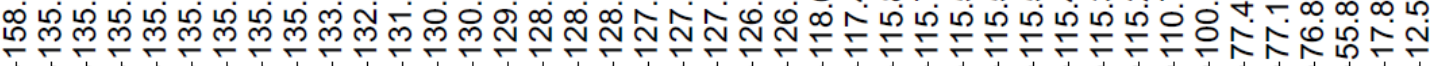

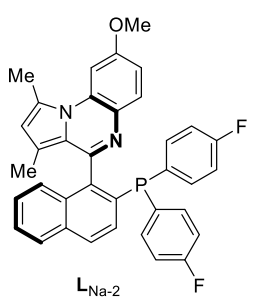

${ }^{13} \mathrm{C} \mathrm{NMR}\left(\mathrm{CDCl}_{3}, 100 \mathrm{MHz}\right)$

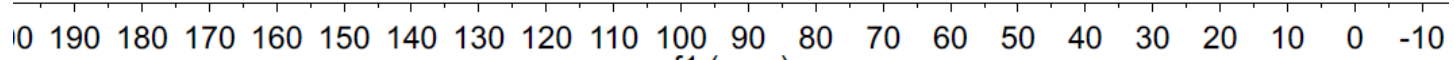
f1 (ppm)

ํㅜㅇ울

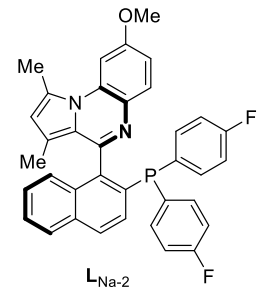

${ }^{19} \mathrm{~F} \mathrm{NMR}\left(\mathrm{CDCl}_{3}, 377 \mathrm{MHz}\right)$

\begin{tabular}{|c|c|c|c|c|c|c|c|c|c|}
\hline & -20 & -40 & -60 & & \multicolumn{2}{|r|}{-120} & -140 & -160 & $-180 \quad-2$ \\
\hline
\end{tabular}




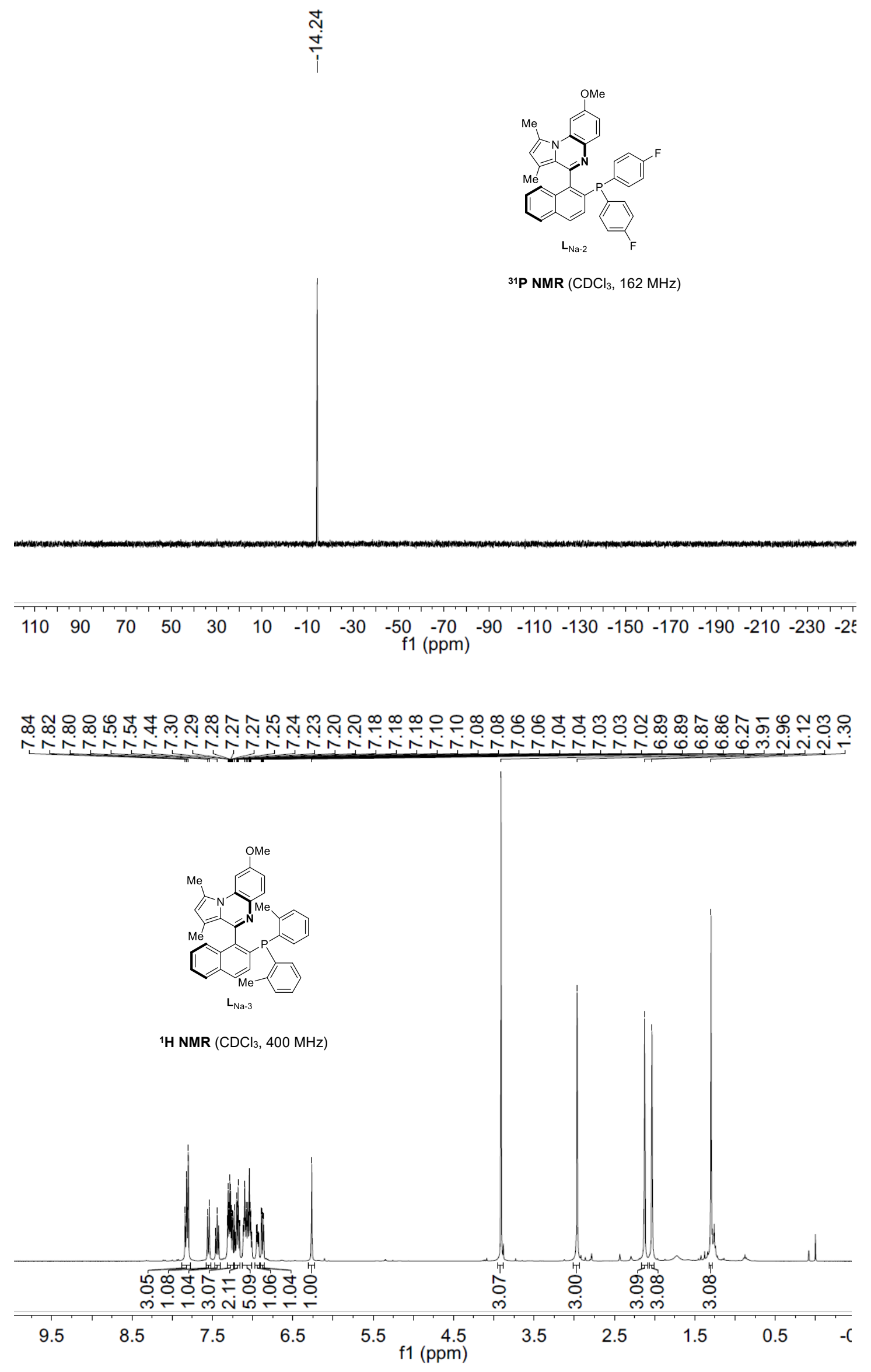




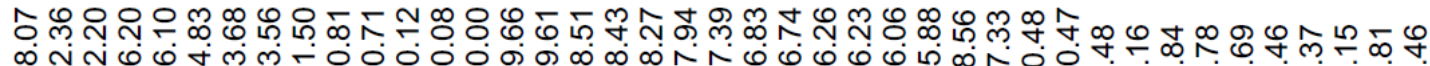

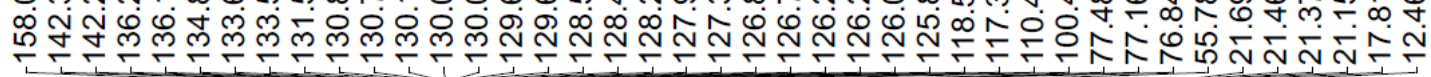

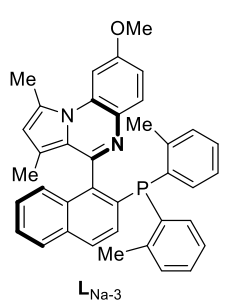

${ }^{13} \mathrm{C}$ NMR $\left(\mathrm{CDCl}_{3}, 100 \mathrm{MHz}\right)$

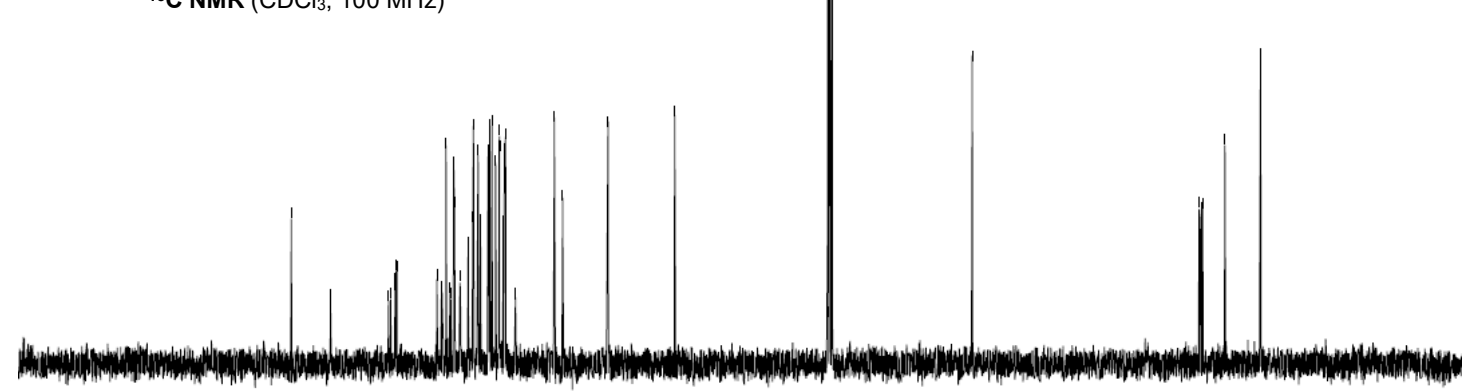

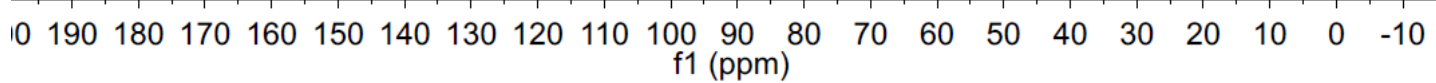

্ֻণ

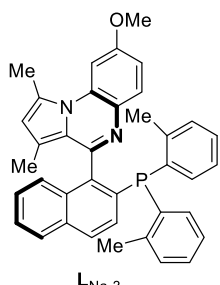

$\mathrm{L}_{\mathrm{Na}-3}$

${ }^{31} \mathrm{P}$ NMR $\left(\mathrm{CDCl}_{3}, 162 \mathrm{MHz}\right)$

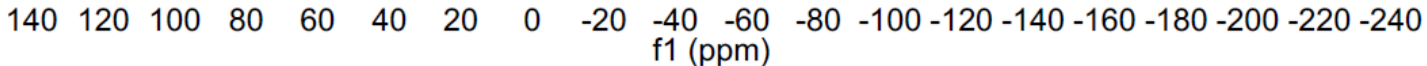




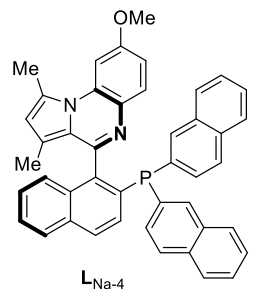

${ }^{1} \mathrm{H}$ NMR $\left(\mathrm{CDCl}_{3}, 400 \mathrm{MHz}\right)$

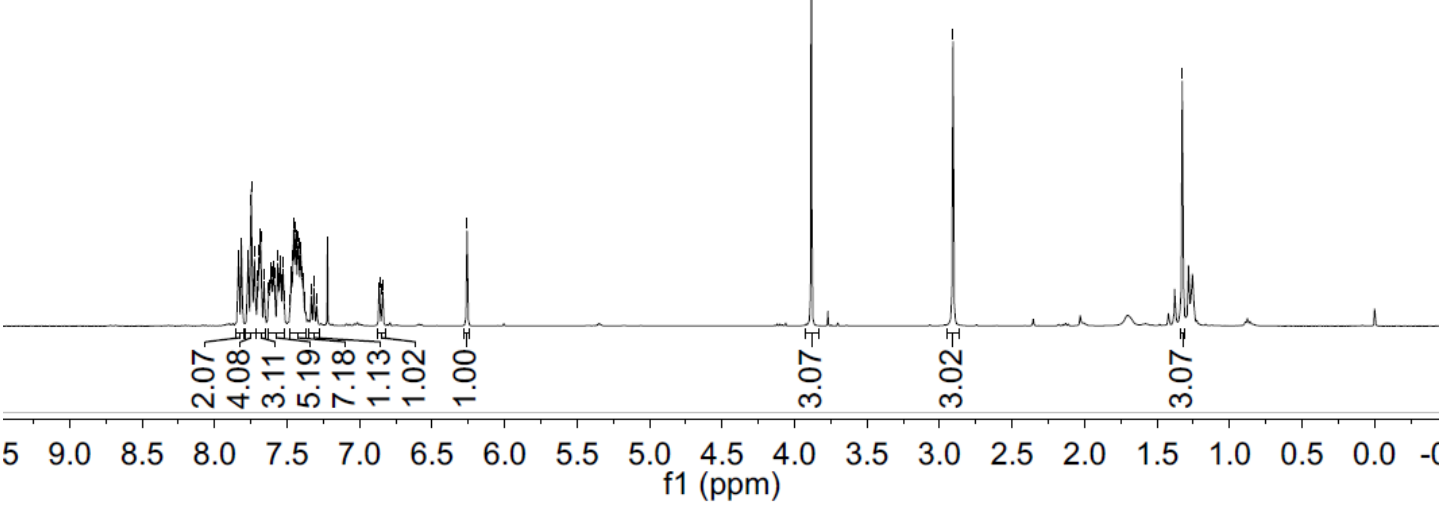

군

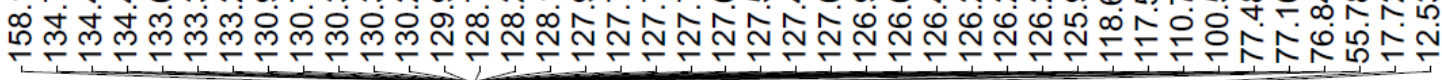

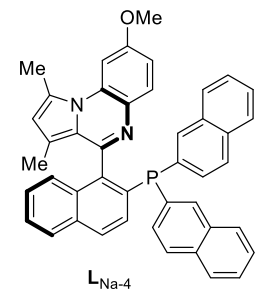

${ }^{13} \mathrm{C} \mathrm{NMR}\left(\mathrm{CDCl}_{3}, 100 \mathrm{MHz}\right)$

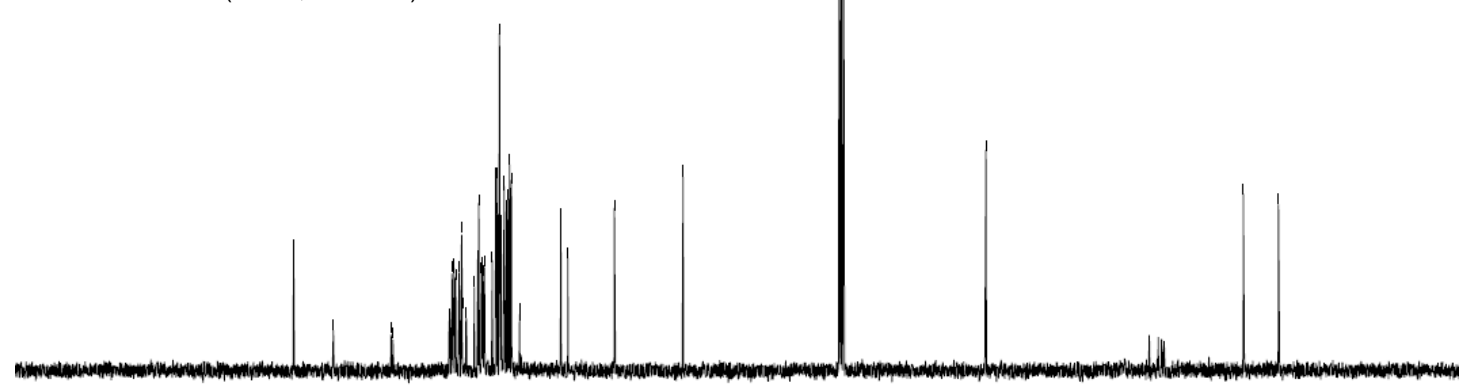

$10190180170160150140130120110 \begin{gathered}100 \underset{f 1(\mathrm{ppm})}{90} \\ 80\end{gathered}$ 


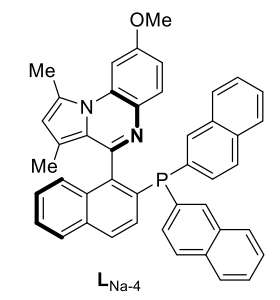

${ }^{31} \mathrm{P}$ NMR $\left(\mathrm{CDCl}_{3}, 162 \mathrm{MHz}\right)$

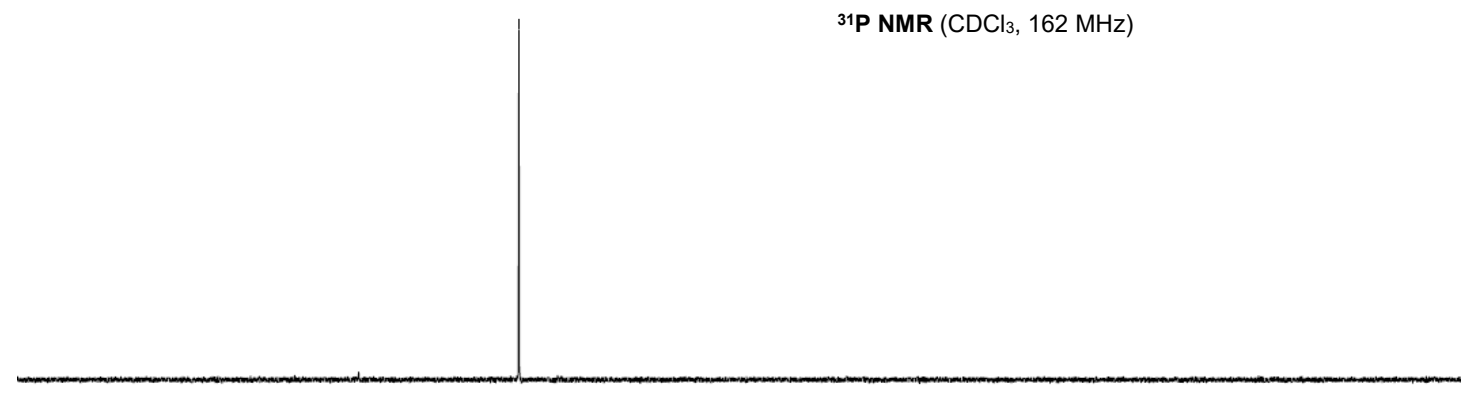

$\begin{array}{llllllllllllllllllllllll}110 & 90 & 70 & 50 & 30 & 10 & -10 & -30 & -50 & -70 & -90 & -110 & -130 & -150 & -170 & -190 & -210 & -230 & -2 \text { s }\end{array}$

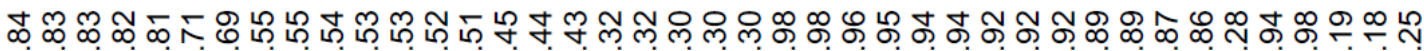

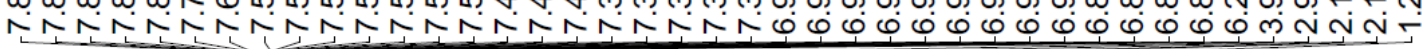

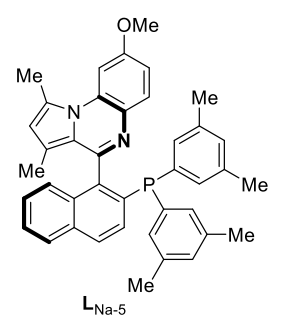

${ }^{1} \mathrm{H}$ NMR $\left(\mathrm{CDCl}_{3}, 400 \mathrm{MHz}\right)$

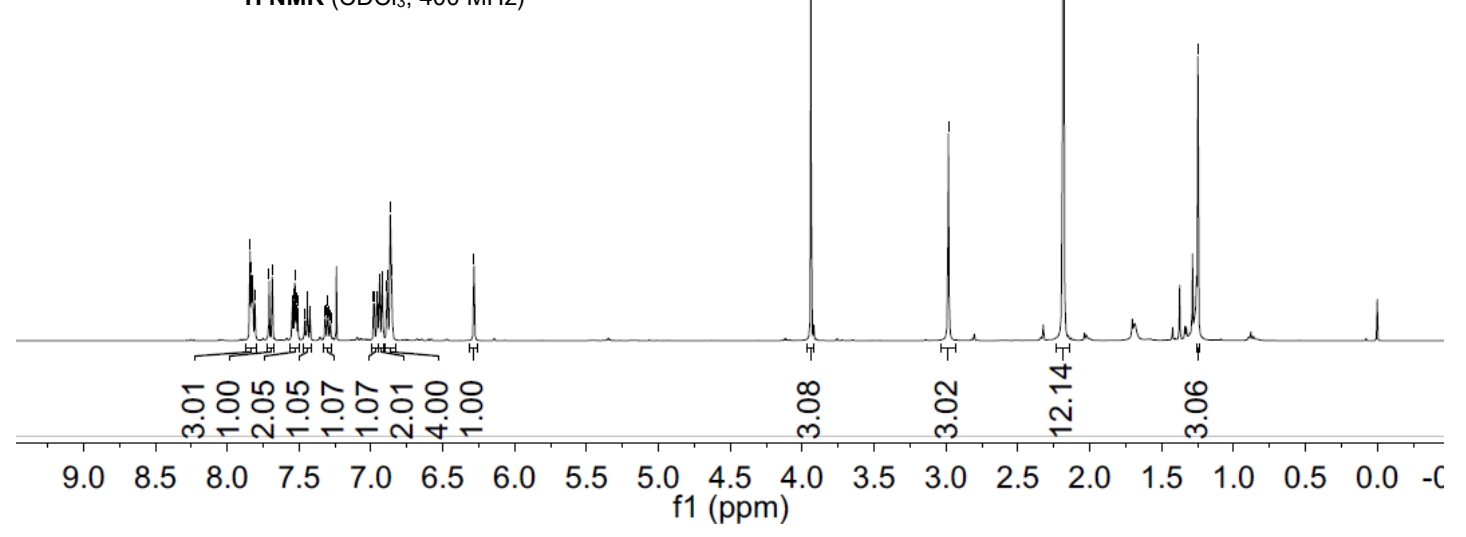




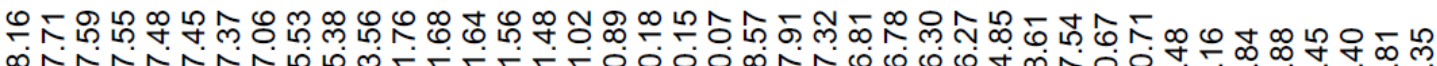
品

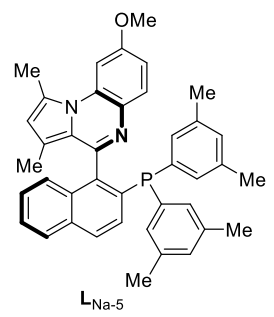

${ }^{13} \mathrm{C}$ NMR $\left(\mathrm{CDCl}_{3}, 100 \mathrm{MHz}\right)$

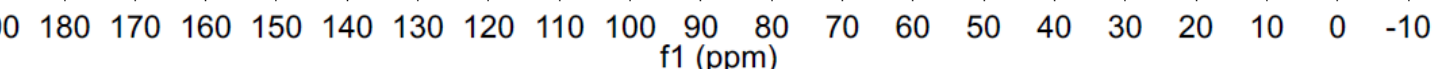

$\stackrel{2}{2}$

$\stackrel{\text { i }}{i}$
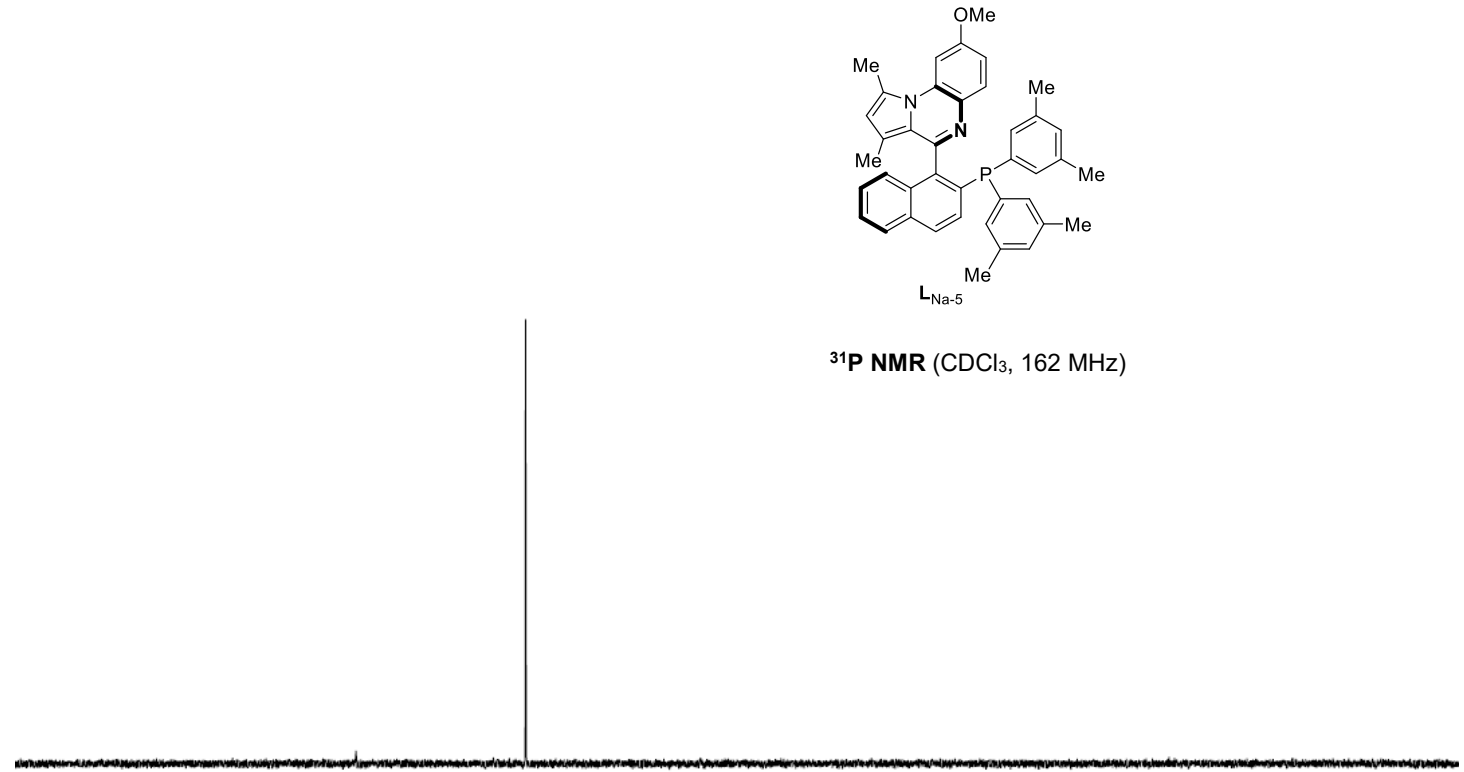

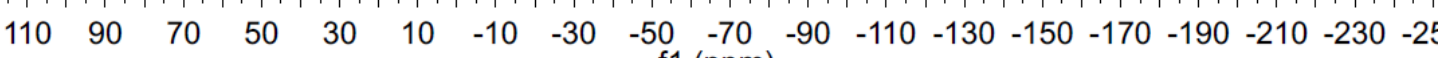
f1 (ppm) 


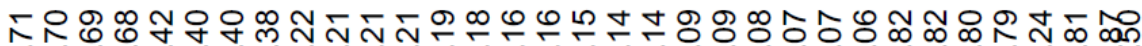

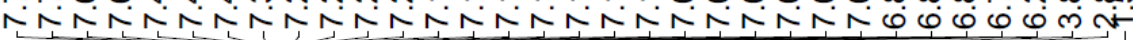

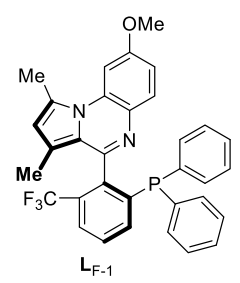

${ }^{1} \mathrm{H}$ NMR $\left(\mathrm{CDCl}_{3}, 400 \mathrm{MHz}\right)$

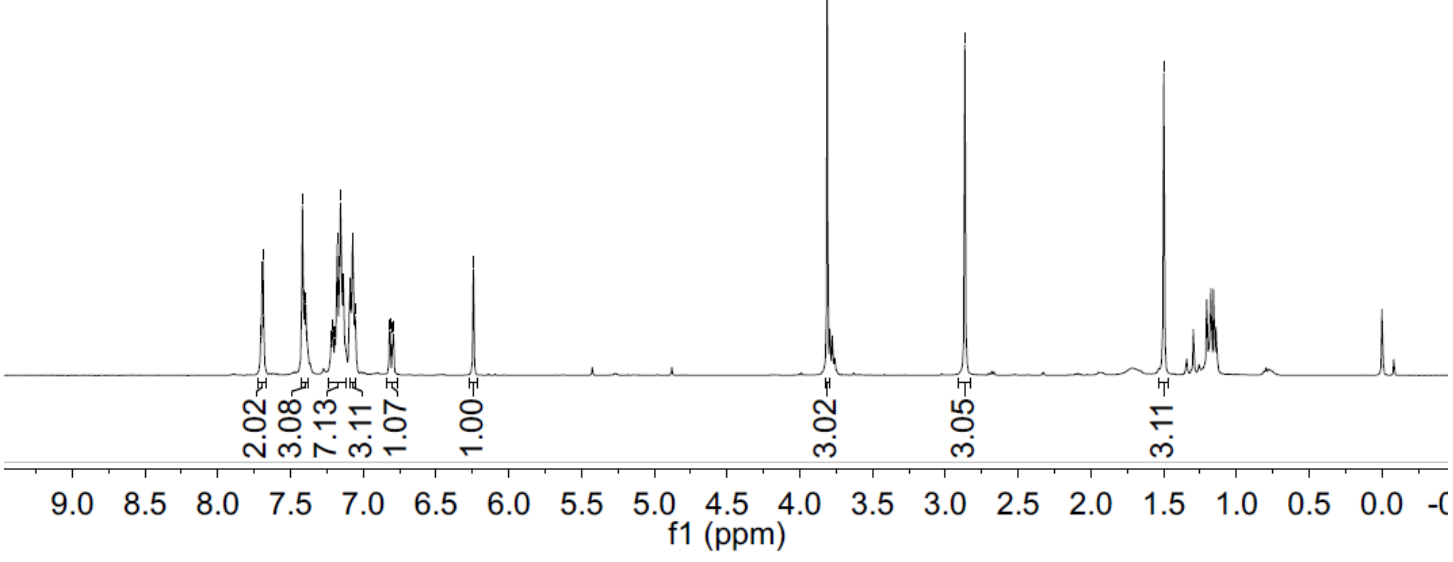

సุำ눈

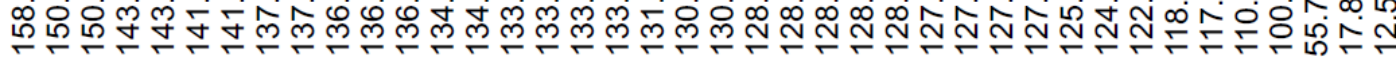

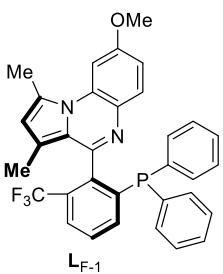

${ }^{13} \mathrm{C} \mathrm{NMR}\left(\mathrm{CDCl}_{3}, 100 \mathrm{MHz}\right)$

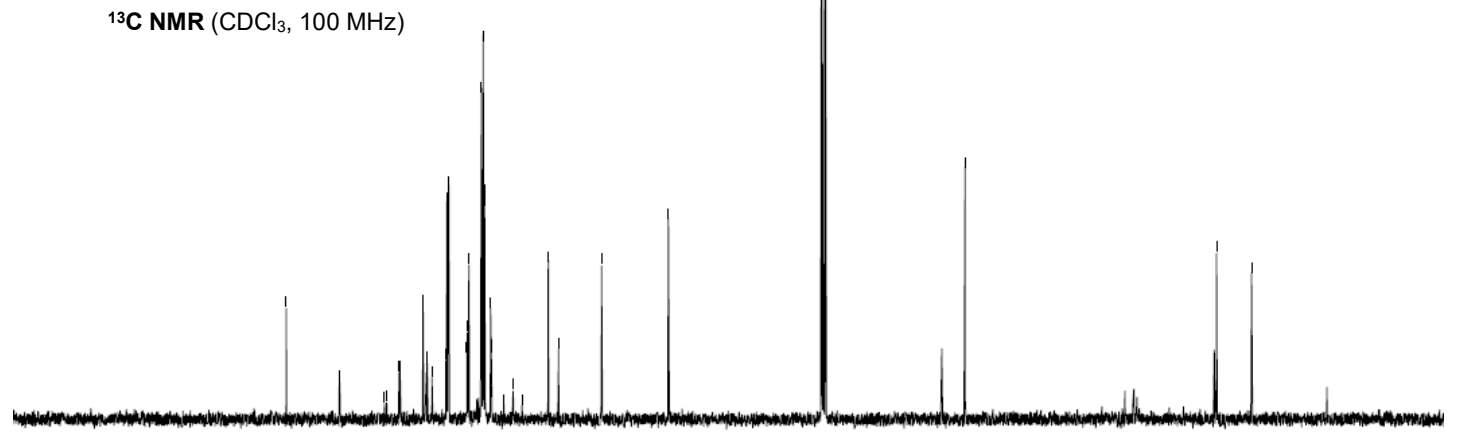

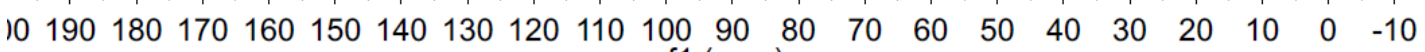
f1 (ppm) 

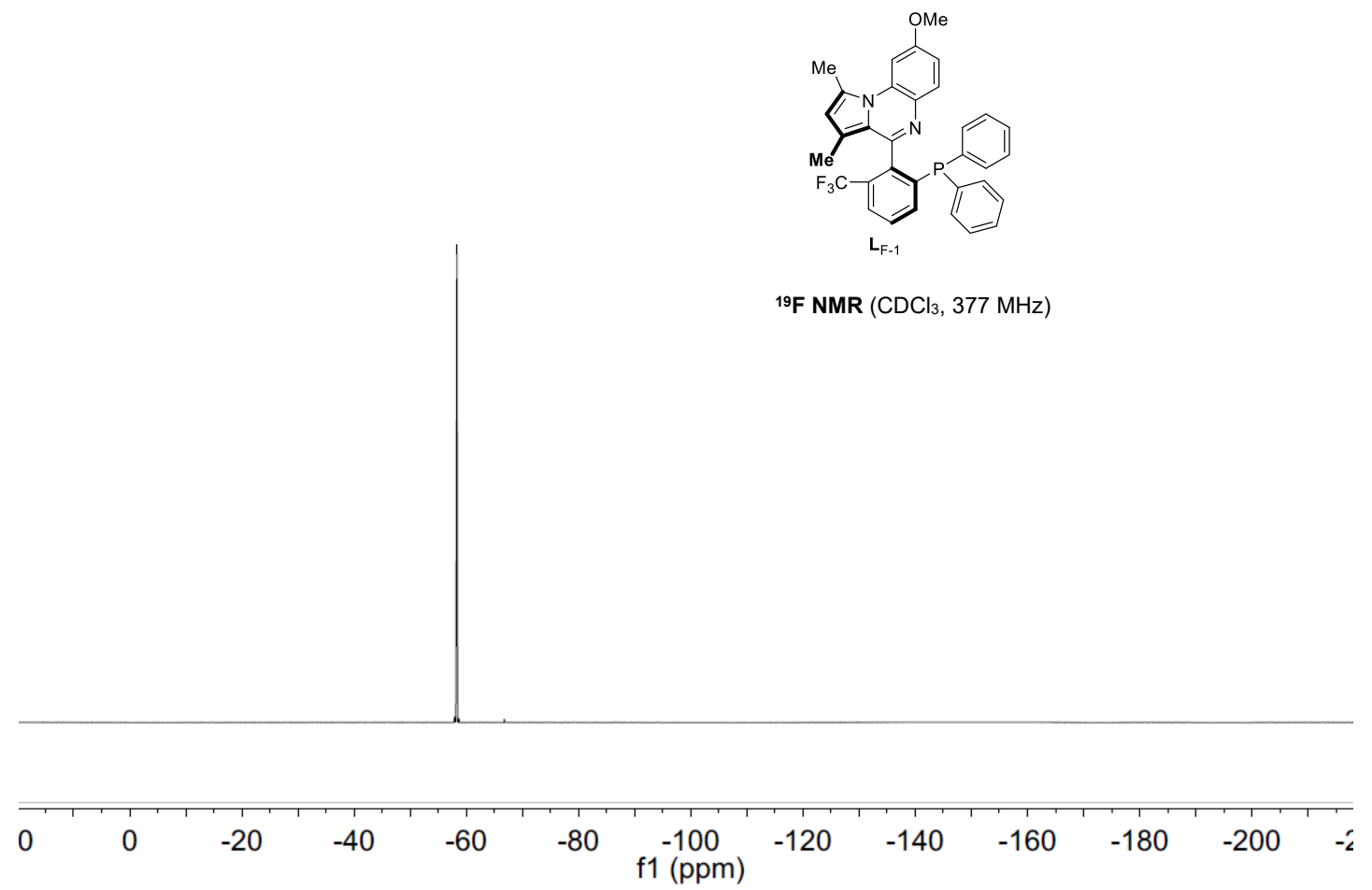

${ }^{9} \mathrm{~F}$ NMR $\left(\mathrm{CDCl}_{3}, 377 \mathrm{MHz}\right)$

$\frac{N}{i}$
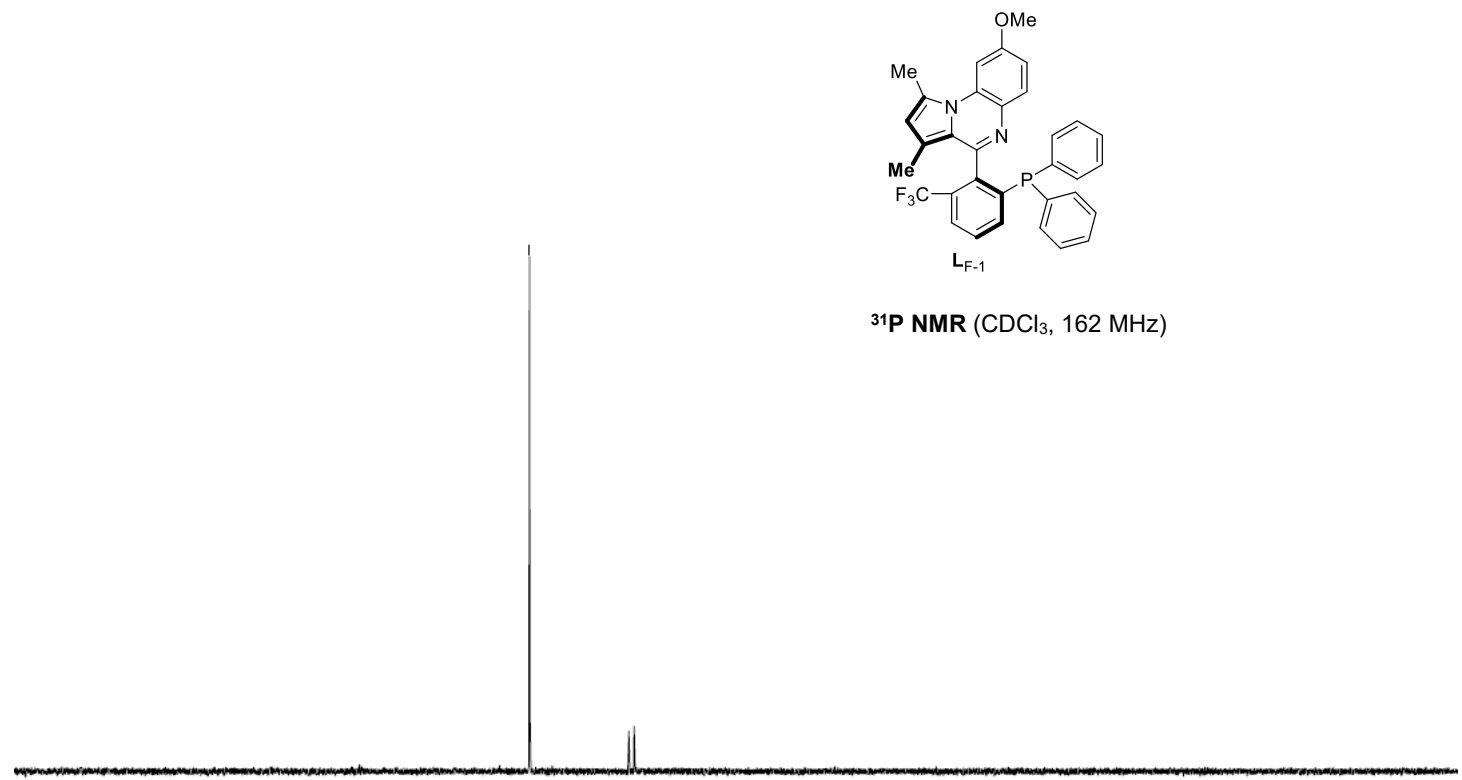

31P NMR $\left(\mathrm{CDCl}_{3}, 162 \mathrm{MHz}\right)$

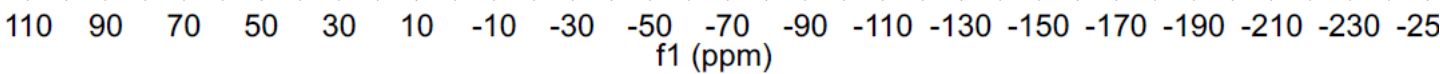




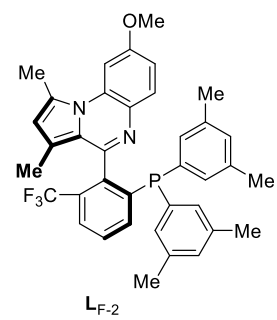

${ }^{1} \mathrm{H}$ NMR $\left(\mathrm{CDCl}_{3}, 400 \mathrm{MHz}\right)$

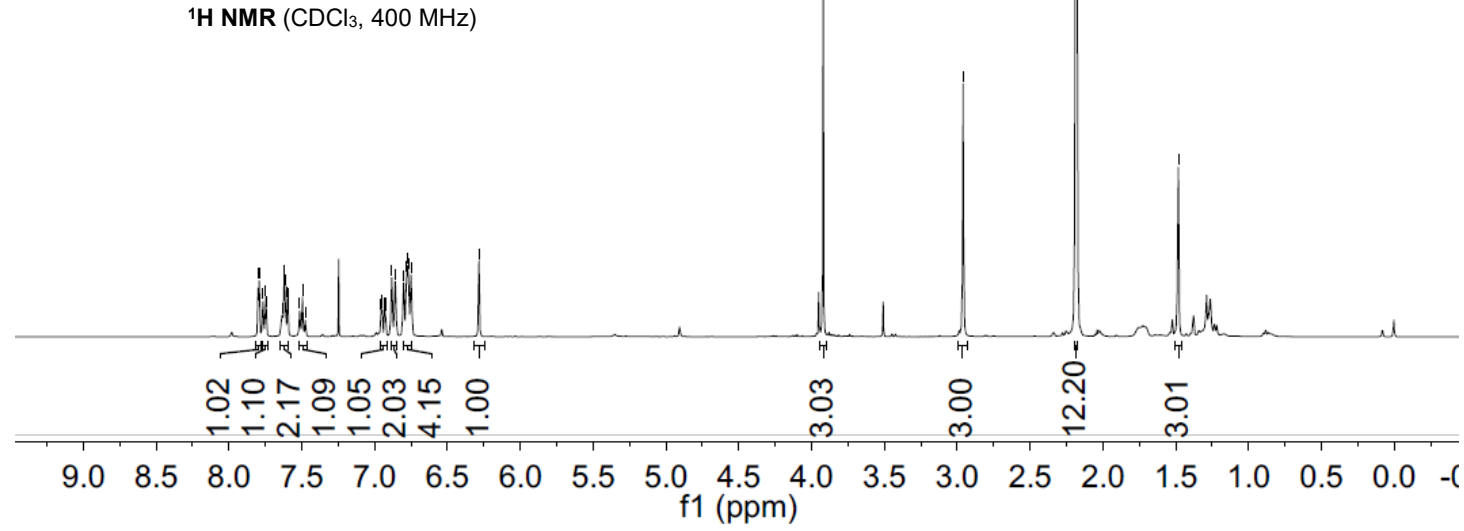

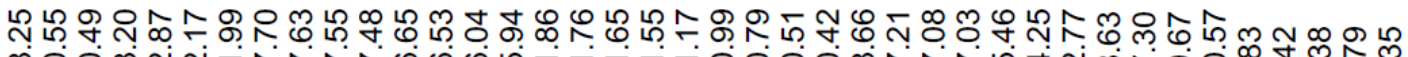
员

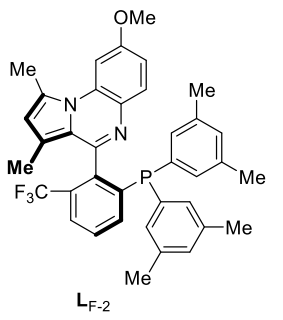

${ }^{13} \mathrm{C}$ NMR $\left(\mathrm{CDCl}_{3}, 100 \mathrm{MHz}\right)$

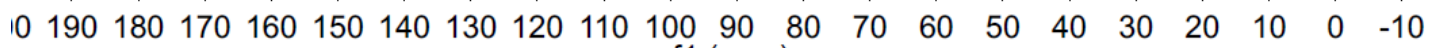

$$
\text { f1 (ppm) }
$$




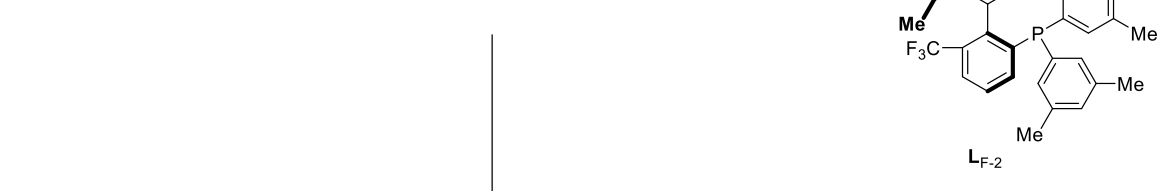

${ }^{19} \mathrm{~F}$ NMR $\left(\mathrm{CDCl}_{3}, 377 \mathrm{MHz}\right)$

\begin{tabular}{|c|c|c|c|c|c|c|c|c|c|c|c|c|}
\hline 0 & 0 & -20 & -40 & -60 & -80 & $\begin{array}{c}-100 \\
\mathrm{f} 1(\mathrm{ppm})\end{array}$ & -120 & -140 & -160 & -180 & -200 & -2 \\
\hline
\end{tabular}

$\stackrel{\substack{\infty\\}}{\stackrel{i}{i}}$

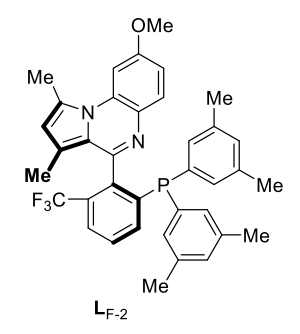

${ }^{31} \mathrm{P}$ NMR $\left(\mathrm{CDCl}_{3}, 162 \mathrm{MHz}\right)$

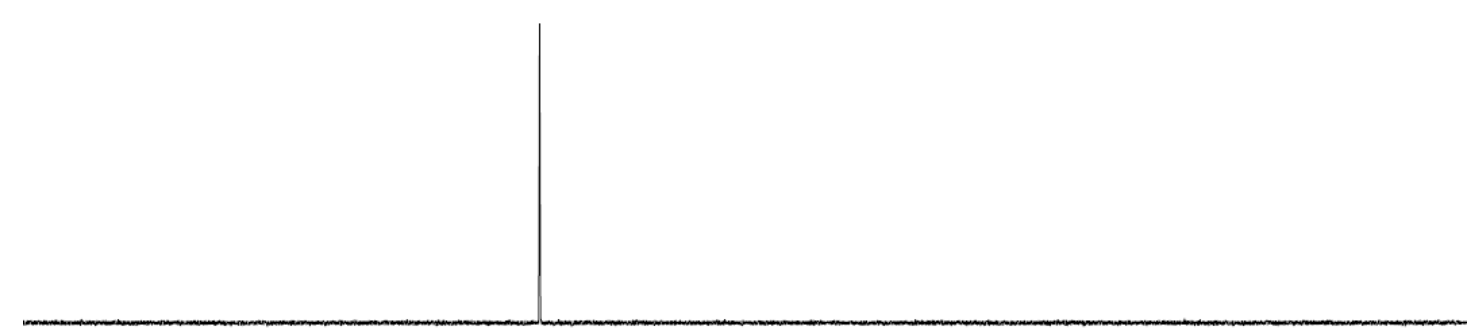

$\begin{array}{llllllllllllllllllllll}110 & 90 & 70 & 50 & 30 & 10 & -10 & -30 & -50 & -70 & -90 & -110 & -130 & -150 & -170 & -190 & -210 & -230 & -25\end{array}$ f1 (ppm) 


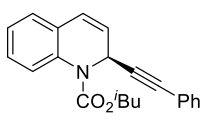

7

${ }^{1} \mathbf{H}$ NMR $\left(\mathrm{CDCl}_{3}, 400 \mathrm{MHz}\right)$

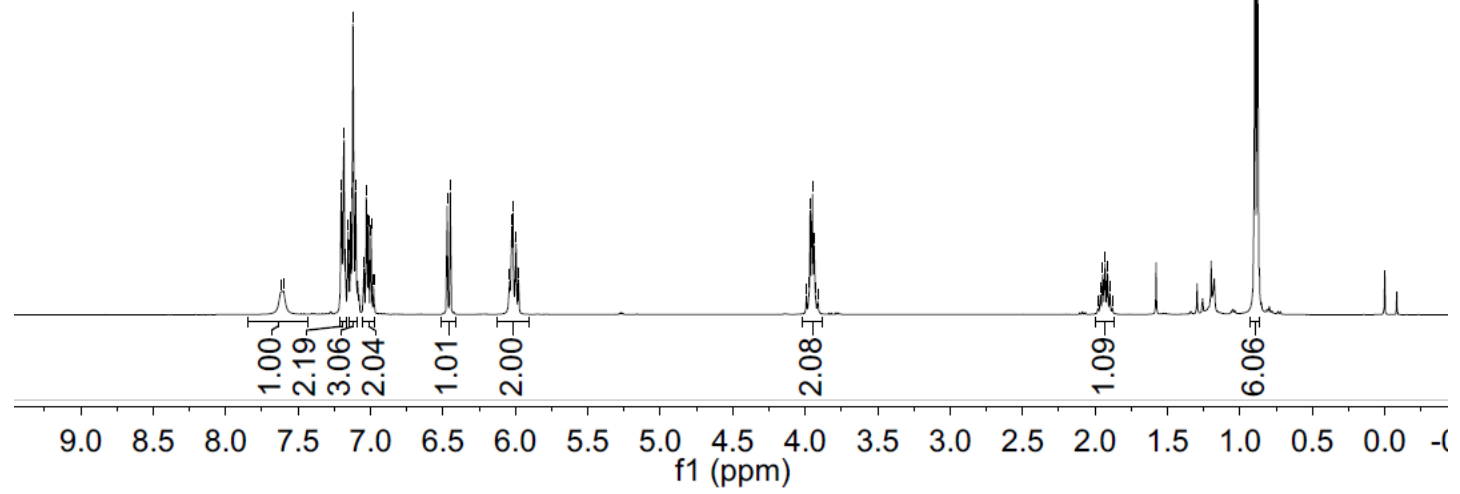

\&

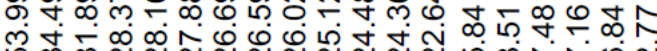

菅

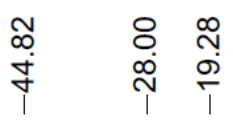

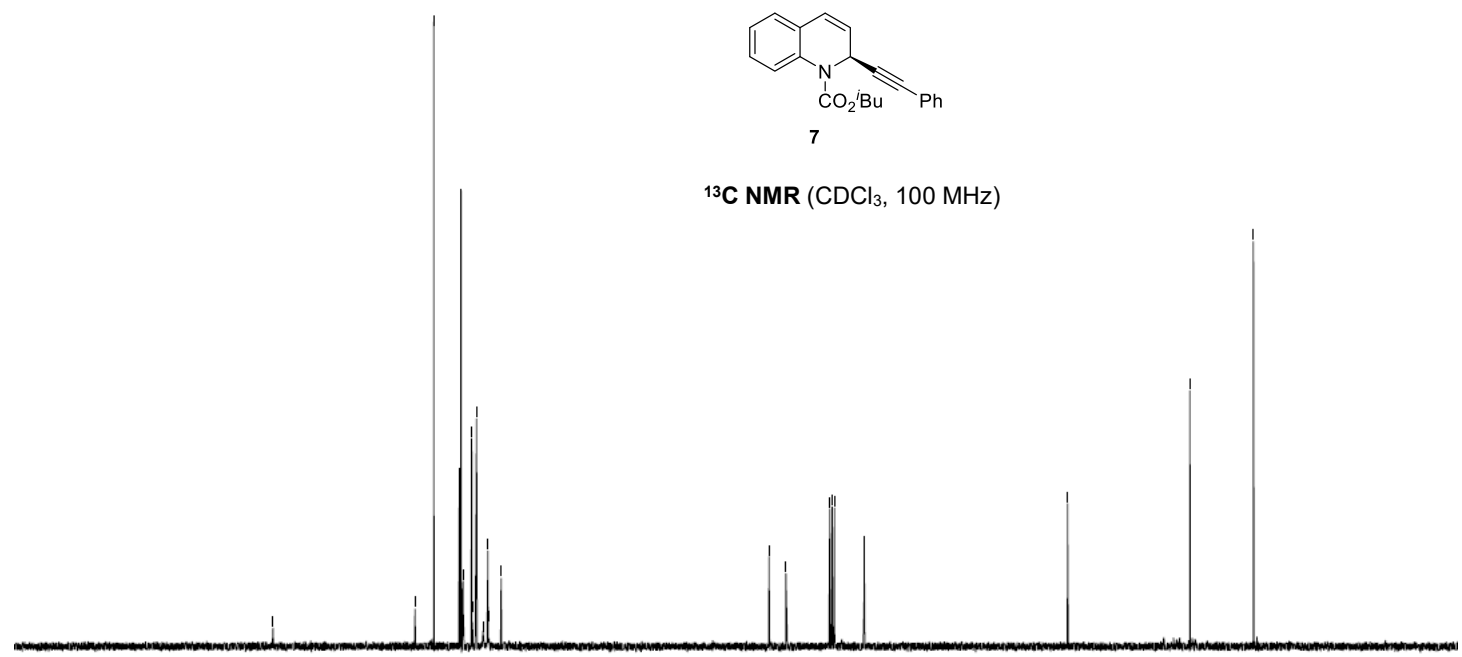

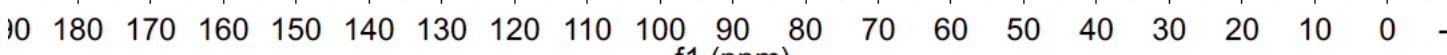
f1 (ppm) 


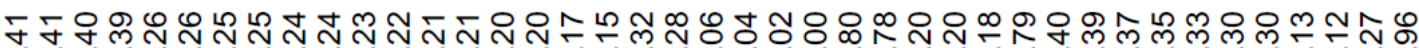

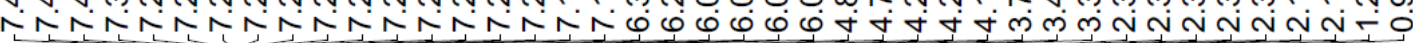

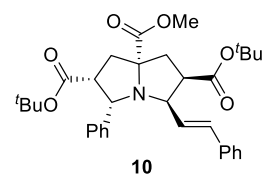

${ }^{1} \mathrm{H}$ NMR $\left(\mathrm{CDCl}_{3}, 400 \mathrm{MHz}\right)$

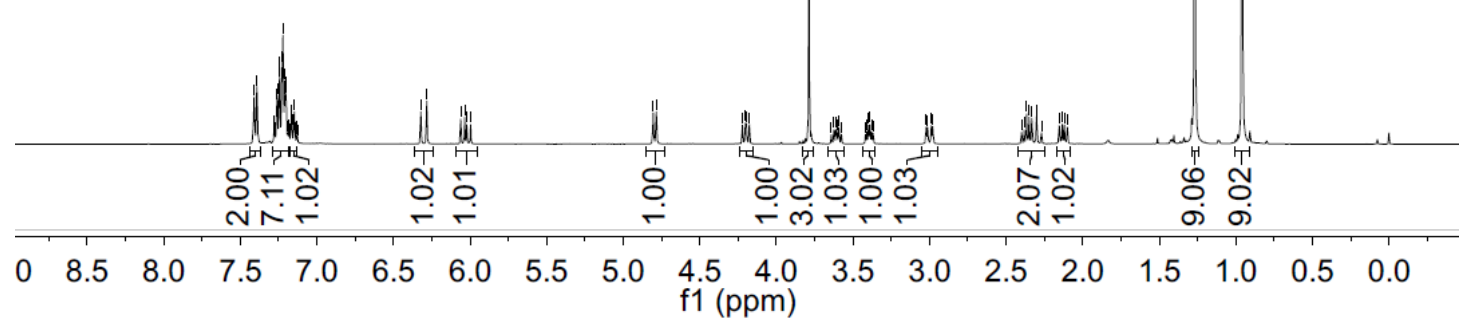

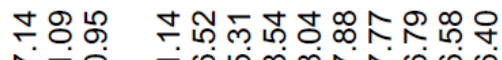

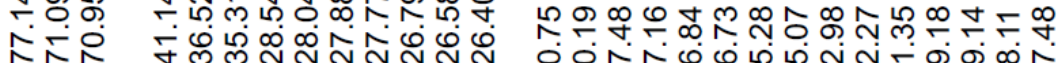

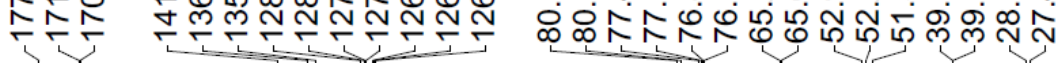

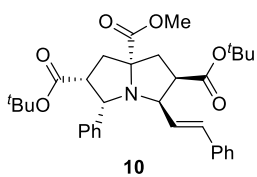

${ }^{13} \mathrm{C} \mathrm{NMR}\left(\mathrm{CDCl}_{3}, 100 \mathrm{MHz}\right)$

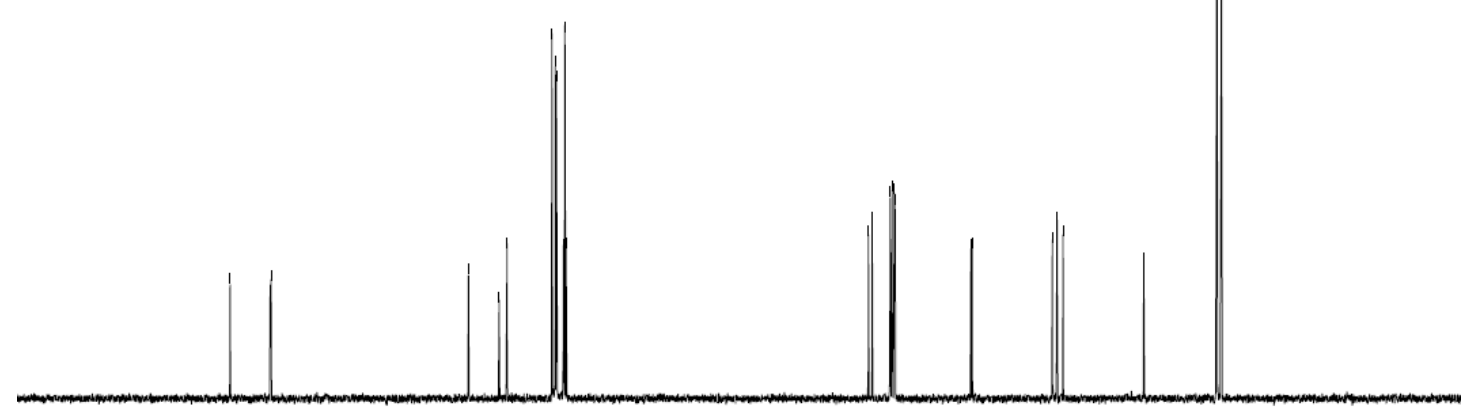

$0200190180170160150140130120 \begin{gathered}110 \begin{array}{c}100 \\ \mathrm{f} 1(\mathrm{ppm})\end{array} \\ 00\end{gathered}$ 